MARTIN PFAFF / DIETMAR WASSENER ASTRID STERZEL / THOMAS NELDNER

\title{
ANALYSE \\ POTENTIELLER \\ AUSWIRKUNGEN EINER \\ AUSWEITUNG DES \\ PHARMAVERSANDES IN \\ DEUTSCHLAND
}




\author{
MARTIN PFAFF / DIETMAR WASSENER \\ ASTRID STERZEL / THOMAS NELDNER
}

\title{
ANALYSE POTENTIELLER AUSWIRKUNGEN EINER AUSWEITUNG DES PHARMAVERSANDES IN DEUTSCHLAND
}

Im Jahre 1999 betrugen die GKV-Ausgaben für apothekenpflichtige Arzneimittel im Rezept DM 36,15 Mrd. Der Vertriebskostenanteil in Form der Apothekenbetriebsspannebetrug 27,3\% des Bruttoumsatzes. DieLeistungsausgaben der gesetzlichen Krankenkassen im Rahmen des Versorgungsauftrages für Arzneimittel aus öffentlichen Apotheken sind in den folgenden Jahren weiter angestiegen. Vor diesem Hintergrund wird argumentiert, die Vertriebswege der Arzneimittel vom Hersteller über den Großhandel bis hin zur Apotheke seien ineffizient. Bei der Diskussion um Einsparpotentiale im Arzneimittelbereich kann eine Ausweitung des Pharmaversandes auf verschreibungspflichtige Medikamente sowohl ein Instrument zur Qualitätsverbesserung für bestimmte Zielgruppen als auch ein Ansatzpunkt zur Erschließung von Wirtschaftlichkeitsreserven im Gesundheitswesen sein.

Martin Pfaff (Jahrgang 1939), Mitglied des Deutschen Bundestages (SPD) seit 1990 stellvertretender Gesundheitspolitischer Sprecher der SPD-Bundestagsfraktion und Bundesvorsitzender der Arbeitsgemeinschaft Sozialdemokratinnen und Sozialdemokraten im Gesundheitswesen (ASG).

Dietmar Wassener (Jahrgang 1963) Gesundheitsökonom und Sozialwissenschaftler an der Universität Augsburg und am Internationalen Institut für Empirische Sozialökonomie (INIFES) bis Herbst 2001. Seit Herbst 2001 tätig im Bereich Marktforschung bei Pharmafakt/Gesellschaft für Datenverarbeitung mbH.

Astrid Sterzel (Jahrgang 1972), Wissenschaftliche Mitarbeiterin am Internationalen Institut für Empirische Sozialökonomie (INIFES) bis 2001.

Thomas Neldner (Jahrgang 1969), Wissenschaftlicher Mitarbeiter am Lehrstuhl für Volkswirtschaftslehre an der Universität Augsburg und Wissenschaftlicher Mitarbeiter am Internationalen Institut für Empirische Sozialökonomie (INIFES). 
Analyse potentieller Auswirkungen einer Ausweitung des Pharmaversandes in Deutschland 


\section{ALLOKATION IM MARKTWIRTSCHAFTLICHEN SYSTEM \\ Herausgegeben von \\ Heinz König, Hans-Heinrich Nachtkamp, Ulrich Schlieper, Eberhard Wille}

\section{Band 45}

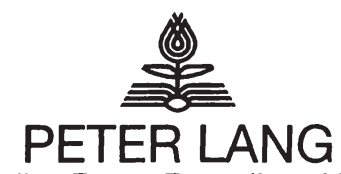

Frankfurt am Main · Berlin · Bern · Bruxelles - New York · Oxford · Wien 


\section{MARTIN PFAFF/DIETMAR WASSENER ASTRID STERZEL/THOMAS NELDNER}

\section{ANALYSE \\ POTENTIELLER \\ AUSWIRKUNGEN \\ EINER AUSWEITUNG DES \\ PHARMAVERSANDES \\ IN DEUTSCHLAND}

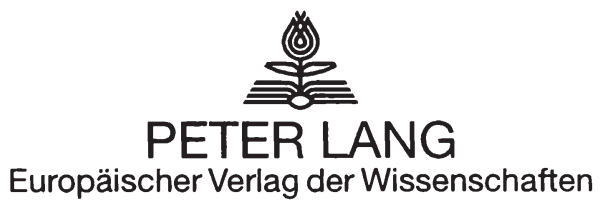


Die Deutsche Bibliothek - CIP-Einheitsaufnahme

Analyse potentieller Auswirkungen einer Ausweitung des Pharmaversandes in Deutschland / Martin Pfaff .... - Frankfurt am Main ; Berlin ; Bern ; Bruxelles ; New York ; Oxford ; Wien : Lang, 2002

Open Access: The online version of this publication is published on www.peterlang.com and www.econstor.eu under the international Creative Commons License CC-BY 4.0. Learn more on how you can use and share this work: http://creativecommons. org/licenses/by/4.0.

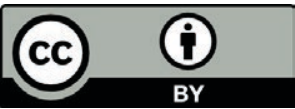

This book is available Open Access thanks to the kind support of ZBW - Leibniz-Informationszentrum Wirtschaft.

(Allokation im marktwirtschaftlichen System ; Bd. 45)

ISBN 3-631-39231-1

Gedruckt auf alterungsbeständigem, säurefreiem Papier.

\author{
ISSN 0939-7728 \\ ISBN3-631-39231-1 \\ ISBN 978-3-631-75554-9 (eBook) \\ (C) Peter Lang GmbH \\ Europäischer Verlag der Wissenschaften \\ Frankfurt am Main 2002 \\ Alle Rechte vorbehalten.
}

Das Werk einschließlich aller seiner Teile ist urheberrechtlich geschützt. Jede Verwertung außerhalb der engen Grenzen des

Urheberrechtsgesetzes ist ohne Zustimmung des Verlages unzulässig und strafbar. Das gilt insbesondere für Vervielfältigungen, Übersetzungen, Mikroverfilmungen und die Einspeicherung und Verarbeitung in elektronischen Systemen.

Printed in Germany 124567

www.peterlang.de 


\section{Vorwort}

Schon im Jahre 1999 betrugen die GKV-Ausgaben für apothekenpflichtige Arzneimittel auf Rezept DM 36,15 Mrd. Der Apothekenumsatz insgesamt für Arzneimittel zu Endverbraucherpreisen (inkl. MwSt.) belief sich auf DM 58 Mrd., der Vertriebskostenanteil in Form der Apothekenbetriebsspanne betrug 27,3\% des Bruttoumsatzes. Die Leistungsausgaben der gesetzlichen Krankenkassen im Rahmen des Versorgungsauftrages für Arzneimittel aus öffentlichen Apotheken sind in den folgenden Jahren weiter angestiegen. Für das Jahr 2001 prognostizierten die Krankenkassen Arzneimittelausgaben bis zu DM 42 Mrd. (21,5 Mrd. Euro).

Vor diesem Hintergrund wird argumentiert, die Vertriebswege der Arzneimittel vom Hersteller über den Großhandel bis hin zur Apotheke seien zum Teil ineffizient. Bei der Diskussion um Einsparpotentiale im Arzneimittelbereich kann eine Ausweitung des Pharmaversandes auf verschreibungspflichtige Medikamente sowohl ein Instrument zur Qualitätsverbesserung für bestimmte Zielgruppen als auch ein Ansatzpunkt zur Erschließung von Wirtschaftlichkeitsreserven im Gesundheitswesen sein.

Für viele spielen dabei nicht nur die genannten Zielsetzungen eine Rolle: Ihnen geht es grundsätzlicher um das gesteuerte Aufbrechen verkrusteter Distributionswege und die intelligente Anwendung neuer Informationstechnologien. Denn die historische Erfahrung zeigt, dass sich solche technologischen Entwicklungen und Möglichkeiten früher oder später selbst - im Zweifelsfalle auch unkontrolliert - den Weg bahnen. Darüber hinaus wird erwartet, dass der Europäische Gerichtshof Einschränkungen des freien Warenverkehrs im EG-Raum auch für den Bereich pharmazeutischer Produkte nicht mehr lange hinnehmen wird.

Eine umfassende, wissenschaftliche Analyse potentieller Auswirkungen einer Ausweitung des Pharmaversandes in Deutschland muss - basierend auf den im Ausland bereits vorliegenden Erfahrungen - einerseits die monetären Einsparpotentiale einer Ausweitung des Pharmaversandes quantifizieren; sie muss andererseits aber auch Aspekte der allgemeinen Akzeptanz, der Patientengerechtigkeit, sowie der Medikamenten- und Versorgungssicherheit angemessen berücksichtigen und dem monetarisierten Einsparpotential gegenrechnen.

Die Autoren haben in der vorliegenden Studie, erstmals für die Bundesrepublik Deutschland, die in der Öffentlichkeit diskutierte Argumente pro und contra Pharmaversand unvoreingenommen hinterfragt und empirisch überprüft. Ziel der am Internationalen Institut für Empirische Sozialökonomie (INIFES) durchgeführten Untersuchung ist, die potentiellen Auswirkungen einer Ausweitung des Pharmaversandes in Deutschland zu untersuchen. Hierbei werden sowohl quantitative, finanzielle als auch qualitative Aspekte berücksichtigt. Im Vordergrund der Studie stehen nationale Modelle des Arzneimittelversandes, die u.a. explizit eine Rezeptübermittlung durch den Arzt beinhalten; die bereits bestehenden Internet-Apotheken sind daher nicht zentraler Gegenstand der Studie.

Die Studie beinhaltet vier zentrale Elemente:

1) Die derzeitigen institutionellen und rechtlichen Rahmenbedingungen des Pharmaversandhandels in Deutschland und Europa werden dargestellt. Dies schließt die Erfassung und Analyse internationaler Erfahrungen mit dieser Art der 
Leistungserbringung ein. Zudem ist das Konzept des hier untersuchten "Pharmaversandes" - und somit der Gegenstand der durchzuführenden Modellrechnungen und Befragungen - definiert.

2) Die monetären Einsparpotentiale, die sich aus einer Ausweitung des Pharmaversandes für Deutschland ergeben können, werden simuliert und verschiedene Szenarien der Ausweitung des Pharmaversandes entwickelt. Dazu erfolgt zum einen eine Segmentierung des Marktes für einen zukünftigen Versand, zum anderen die Herleitung von Betriebs- und Kostenstrukturen für drei verschiedene Umsatzgrößenklassen von Versandapotheken.

3) Die strukturellen und qualitativen Auswirkungen einer Ausweitung des Pharmaversandes auf Anbieter und Nachfrager werden anhand von Primärerhebungen und Expertengesprächen (Focusgruppen) abgeschätzt und dargestellt. Dabei wird z.B. darauf abgezielt, welche Akzeptanz des Versandhandels bei Patienten, Ärzten und Apothekern zu erwarten ist, welche Auswirkungen der Versandhandel auf die Zielgruppen (soziale Kontakte, Beratung, Probleme bei weiteren Verordnungen etc.) hat oder auch, wie die These, durch den Versandhandel könne ggf. der umfassende Versorgungs- und Sicherstellungsauftrag der Apotheken beeinflusst werden, zu bewerten ist.

4) Abschließend werden Szenarien zur Zukunft des Pharmaversandes in Deutschland erarbeitet und vorgestellt, wobei auch die Ergebnisse eines Workshops mit Vertretern der Apotheker- und Ärzteschaft, des Pharmazeutischen Großhandels, der Pharmaindustrie sowie Wissenschaftlern und Politikern am 17. September 2001 in Berlin berücksichtigt werden.

Die Verfasser der Studie kommen zu dem Ergebnis, dass innerhalb des durch Versandapotheken bedienbaren Segments der Dauermedikation dieser komplementäre Vertriebsweg durchaus zu erheblichen Kosteneinsparungen gegenüber der derzeitigen stationären Medikamentendistribution führen kann. In kurz- bis mittelfristiger Sicht erscheinen Einsparpotentiale von DM 400 Mio. bis ca. DM 500 Mio. als realisierbar. In langfristiger Perspektive könnten diese Wirtschaftlichkeitspotentiale annähernd eine Verdopplung erfahren.

Die Implementierung des Versandhandels als zusätzlicher Vertriebsweg ist daher durchaus zu begrüßen, allerdings unter der Voraussetzung bestimmter qualitativer Rahmenbedingungen sowie der Gewährleistung der Patienten- und Arzneimittelsicherheit.

Den Experten, die sich bereit erklärten die Autoren im Rahmen von Gesprächen und Interviews bei der Erstellung der Studie zu unterstützen, danken wir herzlich. Zu besonderem Dank sind wir Frau Tanja Kesselheim, Frau Doris Lerch, Frau Susanne Stiegler und Frau Daniela Schneider verpflichtet, die sich an der Durchsicht des Manuskriptes beteiligt haben und dieses vorbildlich für den Druck vorbereiteten. Schließlich gilt unser Dank dem Marktforschungsinstitut TNS EMNID GmbH \& Co., Bielefeld, für die kompetente Betreuung und Durchführung der repräsentativen Telefoninterviews bei Ärzten, Apothekern und der Bevölkerung innerhalb kürzester Zeit. 


\section{INHALTSVERZEICHNIS}

Tabellenverzeichnis $\quad$ XII

Abbildungsverzeichnis $\quad X V$

Übersichtsverzeichnis $\quad X I X$

Abkürzungsverzeichnis $\quad X X$

0. Einleitung 1

0.1 Problemhintergrund 1

$\begin{array}{lll}0.2 & \text { Zielsetzung } & 8\end{array}$

$\begin{array}{lll}0.3 & \text { Aufbau der Arbeit } & 9\end{array}$

1. Institutionelle Rahmenbedingungen des Arzneimittelvertriebs 10

1.1 Monetäre Entwicklungen und Regulierungen im Arzneimittelmarkt $\begin{array}{ll}\text { in Deutschland } & 10\end{array}$

1.1.1 Monetăre Entwicklung der Arzneimittelausgaben und Einflussfaktoren 11

1.1.2 Maßnahmen zur Ausgabendämpfung im Arzneimittelbereich 13

$\begin{array}{lll}\text { 1.1.3 Zwischenfazit } & 19\end{array}$

$\begin{array}{llr}1.2 & \text { Arzneimittelvertrieb } & 20\end{array}$

1.2.1 Klassifikation von Arzneimitteln - Apothekenpflicht bedingt Vertriebsweg 20

1.2.2 Akteure des Arzneimittelvertriebs 23

1.2.2.1 Pharmazeutische Industrie - Herstellung 23

1.2.2.2 Distributionsstufe pharmazeutischer Großhandel 24

1.2.2.3 Vertriebsinstanz öffentliche Apotheke 28

1.2.2.4 Versandhande/sverbot für apothekenpflichtige Arzneimittel 34

1.2.2.5 Vertriebswege für Arzneimittel im europäischen Vergleich 34

1.2.3 Kosten des Vertriebsweges 36

1.2.3.1 Preisbildung der Pharmazeutischen Industrie 36

1.2.3.2 Festlegung der Preisbildung von Großhandel und Apotheken
durch die Arzneimittelpreisverordnung

1.2.3.3 Übersicht über den Vertriebskostenanteil am Endverkaufspreis $\quad 40$

1.2.3.4. Blick nach Europa: Arzneimittelpreise sowie Vertriebskosten-
Anteile

1.2.4 Feststellung eines Reformbedarfs im Arzneimittelvertriebssystem 44 
$1.3 \quad$ Erfahrungen mit Versandapotheken im Ausland 45

1.3.1 Europa: Beispiel Schweiz 46

$\begin{array}{lll}\text { 1.3.2 Boispiel USA } & 48\end{array}$

1.4 Zwischenfazit und Diskussion 51

2. Quantitative Analyse der Einsparpotentiale 57

2.1 Modell Versandapotheke im Überblick 57

2.1.1 Modellentwicklung Versandapotheke 58

2.1.2 Exkurs: Elektronisches Rezept $\quad 62$

2.2 Annahmen und Methodik 64

2.2.1 Vorgehensweise der Modellrechnungen 64

2.2.2 Datengrundlagen zum Arzneimittelmarkt in Deutschland 68

2.3 Definition des Zielsegments für einen Pharmaversand 69

2.3.1 Vorgehensweise: Basis, Zielgruppenbildung und Operationalisierung 69

$\begin{array}{ll}\text { 2.3.2 Ausprägungen der Zielsegmente } & 77\end{array}$

2.3.2.1 Zielsegment Variante A: Messgröße "Packungsgroßße ab
$50 D D D$

2.3.2.2 Zielsegment Variante B: Messgröße "Packungsgröße N3" 79

2.3.2.3 Zie/segment Variante C: Messgröße "Packungsgroßße ab
35DDD

2.3.3 Zwischenfazit: Überblick Über Zielsegmente 83

2.4 Ökonomische Restriktionen - Entwicklung einer Betriebsund Kostenstruktur für Versandapotheken

2.4.1 Die Kostenstruktur einer Prăsenzapotheke 86

2.4.2 Betriebsstrukturen von Versandapotheken unter Qualităts- und Effizienzmaßstäben

2.4.2.1 Allgemeine Modellannahmen, insbesondere zur Personalund Raumstruktur

2.4.2.2. Patientensicherheit: Fachliche Beratung durch Versandapotheken

2.4.2.3 Bezugs- und Zustellsicherheit: Voraussetzung einer Funktionierende B-2-C Logistik der Versandapotheken

2.4.3 Die Kostenstruktur einer "kleinen" Versandapotheke mit 5 Mio. DM Umsatz (Variante I)

2.4.4 Die Kostenstruktur einer "großen" Versandapotheke mit 20 Mio. DM Umsatz (Variante II)

2.4.5 Die Kostenstruktur einer Versandapotheke mit integrierter Großhandelsfunktion mit 487 Mio. DM (Variante III) 
2.5 Markt- und Einsparpotentiale des Pharmaversandes 138

2.5.1 Vorgehensweise der Berechnungen 139

2.5.2 Ausprägungen der Markt- und Einsparpotentiale 140

2.5.2.1. Markt- u. Einsparpotential für Zielsegmentvariante A von 41\% 140

2.5.2.2. Markt- $u$. Einsparpotential für Zielsegmentvariante B von $47 \% \quad 141$

2.5.2.3. Markt- u. Einsparpotential für Zielsegmentvariante C von 57\% 143

2.5.3. Zwischenfazit zu den Markt- und Einsparpotentialen 144

2.6 Berücksichtigung einer durchschnittlichen Betriebsrendite in den Modellrechnungen

2.6.1 Einflüsse einer durchschnittlichen Betriebsrendite auf das Einsparpotential

2.6.2 Ausprägungen der Einsparpotentiale bei durchschnittlicher Betriebsrendite

2.6.2.1. Einsparpotential für Zielsegmentvariante $A$ von $41 \%$ (bei durchschnittlicher Betriebsrendite)

2.6.2.2. Einsparpotential für Zielsegmentvariante B von $47 \%$ (bei durchschnittlicher Betriebsrendite)

2.6.2.3. Einsparpotential für Zielsegmentvariante $C$ von $57 \%$ (bei durchschnittlicher Betriebsrendite)

2.6.3 Zwischenfazit zur Berücksichtigung einer durchschnittlichen Betriebsrendite in den Modellrechnungen

2.7 Berücksichtigung zusätzlicher einheitlicher Kassenrabatte in den Modelirechnungen

2.7.1 Einflüsse zusätzlicher einheitlicher Kassenrabatte auf das Markt- und Einsparpotential

2.7.2 Ausprägungen der Markt- und Einsparpotentiale bei Berücksichtigung zusätzlicher einheitlicher Kassenrabatte

2.8 Überlegungen zu realisierbaren Marktanteilen

2.9 Zusammenfassung

2.9.1 Zielsegment

2.9.2 Betriebs- und Kostenstruktur

2.9.3 Markt- und Einsparpotentiale

3. Einstellungen zum Pharmaversand

\section{$3.1 \quad$ Einleitung}


3.2 Aufbau der Erhebung 190

$\begin{array}{llr}3.2 .1 & \text { Ziele } & 190\end{array}$

$\begin{array}{ll}\text { 3.2.2 Erhebungstechnik } & 191\end{array}$

$\begin{array}{ll}\text { 3.2.3 Ablauf der Erhebung } & 199\end{array}$

3.3 Inhalte der Befragungen 201

3.3.1 Bevölkerungsbefragung 205

3.3.2 Apothekerbefragung 206

$\begin{array}{ll}\text { 3.3.3 Ärztebefragung } & 207\end{array}$

3.4 Ergebnisse der Befragungen 208

$\begin{array}{ll}3.4 .1 & \text { Vorbemerkungen } \\ & 208\end{array}$

3.4.2 Apothekerbefragung 209

3.4.2.1 Angaben zur Stichprobe 209

3.4.2.2 Vertriebskanal Apotheke 211

$\begin{array}{ll}\text { 3.4.2.3 Alternative Distributionswege } & 217\end{array}$

3.4.2.4 Einstellung der Apotheker zum Modell der Versandapotheke 218

3.4.2.5 Zielgruppe einer Versandapotheke aus Sicht der Apotheker 223

3.4.2.6 Potentielle Auswirkungen eines Versandhandels 225

$\begin{array}{ll}\text { 3.4.2.7 Wirtschaftliche Folgen einer Legalisierung von } & \\ & \text { Versandapotheken }\end{array}$

$\begin{array}{lll}\text { 3.4.2.8 Reformoptionen } & 230\end{array}$

$\begin{array}{lll}3.4 .2 .9 & \text { Fazit } & 232\end{array}$

3.4.3 Ärztebefragung $\quad 232$

3.4.3.1 Angaben zur Stichprobe 232

3.4.3.2 Die Einstellung der Ärzte zum Vertriebskanal Apotheke 234

3.4.3.3 Alternative Distributionswege 236

3.4.3.4 Die Einstellung der Ärzte zum Modell der Versandapotheke 238

3.4.3.5 Zielgruppe einer Versandapotheke 241

3.4.3.6 Erwartete potentielle Auswirkungen eines Versandhande/s 242

3.4.3.7 Die Einstellung der Ärzte zu Reformoptionen 244

$\begin{array}{lll}\text { 3.4.3.8 Fazit } & 246\end{array}$

$\begin{array}{lll}\text { 3.4.4 Bevollkerungsbefragung } & 246\end{array}$

3.4.4.1 Angaben zur Stichprobe 246

3.4.4.2 Gesundheitszustand der Bevölkerung 248

3.4.4.3 Die Einstellung der Bevölkerung zum Vertriebskanal Apotheke 250

3.4.4.4 Einstellung zu alternativen Distributionswegen 255

3.4.4.5 Einstellung zum Modell der Versandapotheke 259

3.4.4.6 Anforderungen an eine Versandapotheke 262

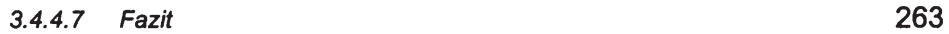

3.5 Zusammenfassung und Schlussfolgerung 264

$x$ 


\section{Zusammenfassung und Bewertung der Projekt- ergebnisse}

4.1 Zusammenfassung der Projektergebnisse 275

4.1.1 Modell einer Versandapotheke, das der Studie zugrunde liegt 278

4.1.2 Zusammenfassung der quantitativen Projektergebnisse 282

4.1.3 Zusammenfassung der qualitativen Projektergebnisse 309

$\begin{array}{lll}\text { 4.2 Bewertung der Projektergebnisse } & 319\end{array}$

4.2.1 Kostenvorteile und Einsparpotentiale 320

4.2.2 Warensortiment, Preisvergleich und Vorwurf des „Rosinenpickens“ 326

4.2.3 Einflüsse auf die Versorgungsdichte und Arbeitsmarkteffekte 330

4.2.4 Nutzerfreundlichkeit, Erreichbarkeit und Zustellsicherheit 332

4.2.5 Sicherstellung von Beratung, Service und Information 337

4.2.6 Bewertung der sozialen Funktion von Arzt und Apotheke 341

4.3 Abschließende Thesen zur Implementierung eines Arzneimittelversandes in Deutschland

$\begin{array}{ll}\text { Anhang } & 346\end{array}$

Anhang I: Nebenrechnungen zu den Marktpotentialen 346

Ohne Berücksichtigung anderer Faktoren $\quad 346$

Bei Berücksichtigung einer durchschnittlichen Betriebsrendite 353

Bei Berücksichtigung zusătzlicher einheitlicher Kassenrabatte 360

Anhang II: Befragungsinstrumente $\quad 370$

Fragebögen der Telefonbefragung: Ärzte, Apotheker, Bevölkerung 370

Leitfaden zu Expertengesprächen $\quad 386$

$\begin{array}{ll}\text { Literaturverzeichnis } & 388\end{array}$ 


\section{TABELLENVERZEICHNIS}

Tabelle I- 1: $\quad$ Amtliche Höchstaufschlagsätze für den Großhandel $\quad 38$

Tabelle I-2: $\quad$ Amtliche Festzuschläge für Apotheken 39

Tabelle I - 3: $\quad$ Prozentuale Anteile der Hersteller, des Großhandels, der Apotheken und der Steuern an den Arzneimittelpreisen 1998

Tabelle I - 4: $\quad$ Mehrwertsteuerzuschläge (Stand 1.1.2000)

Tabelle II - 1: $\quad$ Anteil der akuten und chronischen Verordnungen sowie solcher des Zwischenbereichs (Unterteilung durch pharmazeutische und medizinische Experten)

Tabelle II - 2: $\quad$ Zähleinheiten je Normpackung ausgewählter Indikationen

Tabelle II - 3: $\quad$ Umsatzanteil der Indikationsgruppen mit einer

Packungsgröße ab 50 DDD je Verordnung

Tabelle II - 4: $\quad$ Umsatzanteil der Indikationsgruppen mit einer Packungsgröße N3 (für 1998)

Tabelle II - 5: $\quad$ Umsatzanteil der Indikationsgruppen mit einer Packungsgröße ab 35 DDD je Verordnung

Tabelle II - 6: $\quad$ Personalkosten und Arbeitszeit nach Berufsgruppen in Apotheken (West)

Tabelle II - 7: $\quad$ Umsatz-, Kosten- und Ertragsstruktur einer

Präsenzapotheke

Tabelle II - 8: $\quad$ Kosten für pharmazeutisches Personal nach Tätigkeitsbereichen (West)

Tabelle II - 9: $\quad$ Mietpreise für Gewerbe- und Lagerflächen in Abhängigkeit vom Standort

Tabelle II - 10: $\quad$ Angenommene Verteilung der Öffnungszeiten wie der Telefonanrufe auf Basis eines Jahres

Tabelle II - 11: $\quad$ Überblick über Gesprächsdauer und Gesprächskosten ausgewählter medizinischer Telefon-Hotlines

Tabelle II - 12: $\quad$ Umsatz- und Kostenstruktur einer kleinen Versandapotheke vor Berücksichtigung der Logistikstückkosten von 3,48 DM je Verordnung

Tabelle II - 13: $\quad$ Berechnung der prozentualen Logistikkosten je durchschnittlichen Verordnungswert in Abh. von der Marktpotentialvariante

Tabelle II - 14: Betriebsergebnis kleine Versandapotheke in Abhängigkeit der Marktpotentialvariante 
Tabelle II - 15: $\quad$ Umsatz- und Kostenstruktur einer großen Versandapotheke vor Berücksichtigung der Logistikstückkosten von 4,79 DM je Verordnung

Tabelle II - 16: $\quad$ Berechnung der prozentualen Logistikkosten je durchschnittlichen Verordnungswert in Abh. von der Marktpotentialvariante für die große Versandapotheke

Tabelle II - 17: Betriebsergebnis große Versandapotheke in Abhängigkeit der Marktpotentialvariante

Tabelle II - 18: Umsatz- und Kostenstruktur einer Versandapotheke mit integrierter Großhandelsfunktion vor Berücksichtigung der Logistikstückkosten von 4,79 DM je Verordnung

Tabelle II - 19: $\quad$ Berechnung der prozentualen Logistikkosten je durchschnittlichen Verordnungswert in Abh. von der Marktpotentialvariante für die Versandapotheke mit integrierter Großhandelsfunktion

Tabelle II - 20: $\quad$ Betriebsergebnis Versandapotheke mit integrierter Großhandelsfunktion in Abhängigkeit der Marktpotentialvariante

Tabelle II - 21: $\quad$ Ausprägungen des Marktpotentials in Abhängigkeit der Umsatzgröße des Zielsegments A

Tabelle II - 22: $\quad$ Ausprägungen des Einsparpotentials in Abhängigkeit der Umsatzgröße des Zielsegments A

Tabelle II - 23: $\quad$ Ausprägungen des Marktpotentials in Abhängigkeit der Umsatzgröße des Zielsegments B

Tabelle II - 24: Ausprägungen des Einsparpotentials in Abhängigkeit der Umsatzgröße des Zielsegments B

Tabelle II - 25: $\quad$ Ausprägungen des Einsparpotentials in Abhängigkeit der Umsatzgröße des Zielsegments B Hochrechnung für 1999

Tabelle II - 26: $\quad$ Ausprägungen des Marktpotentials in Abhängigkeit der Umsatzgröße des Zielsegments C

Tabelle II - 27: $\quad$ Ausprägungen des Einsparpotentials in Abhängigkeit der Umsatzgröße des Zielsegments C

Tabelle II - 28: Berechnung einer durchschnittlichen Betriebsrendite für Versandapotheken

Tabelle II - 29: $\quad$ Ausprägungen des Marktpotentials und der Kostenersparnis bei einer durchschnittlichen Betriebsrendite von $3,5 \%$ für Zielsegment $A$ 
Tabelle II - 30: Ausprägungen des Einsparpotentials bei einer durchschnittlichen Betriebsrenditerendite von 3,5\% für Zielsegment A

Tabelle II - 31: $\quad$ Ausprägungen des Marktpotential und der Kostenersparnis bei einer durchschnittlichen Betriebsrendite von $3,5 \%$ für Zielsegment $B$

Tabelle II - 32: Ausprägungen des Einsparpotentials bei einer durchschnittlichen Betriebsrenditerendite von 3,5\% für Zielsegment B

Tabelle II - 33: Ausprägungen des Einsparpotentials bei einer durchschnittlichen Betriebsrendite von 3,5\% für Zielsegment B Hochrechnung für 1999

Tabelle II - 34: $\quad$ Ausprăgungen des Marktpotentials und der Kostenersparnis bei einer durchschnittlichen Betriebsrendite von 3,5\% für Zielsegment C

Tabelle II - 35: $\quad$ Ausprägungen des Einsparpotentials bei einer durchschnittlichen Betriebsrenditerendite von 3,5\% für Zielsegment C

Tabelle III - 1: $\quad$ Ausgewählte Primärerhebungen zum Arzneimittelvertrieb 184

Tabelle III - 2: $\quad$ Studien zu: Arzneimittel im Internet 185

Tabelle III - 3: $\quad$ Sozio-Demographie der Apotheker 210

Tabelle III - 4: $\quad$ Sozio-Demographie der Ärzte 233

Tabelle III - 5: $\quad$ Sozio-Demographie der Bevölkerung 247

Tabelle III - 6: $\quad$ Bewertung verschiedener Aussagen zur Versandapotheke

Tabelle III - 7: $\quad$ Rahmenbedingungen für die Akzeptanz einer Versandapotheke 


\section{ABBILDUNGSVERZEICHNIS}

Abbildung 0 - 1: $\quad$ Ausgabenanstieg der GKV für Arzneimittel 1

Abbildung 0 - 2: $\quad$ Arzneimittelausgaben der GKV je Mitglied und je Rentner, sowie Rentneranteil an der Versichertenstruktur 1994 bis 1998 (jeweils inklusive mitversicherte Familienangehörige)

Abbildung 1 - 1: $\quad$ Gesundheitsausgaben (1998) in \% des BIP 10

Abbildung I - 2: $\quad$ GKV-Ausgaben für Arzneimittel aus Apotheken,1991-1999 11

Abbildung 1 - 3: Komponenten der Ausgabenentwicklung auf dem GKV - Arzneimittelmarkt 13

Abbildung I - 4: $\quad$ Übersicht zu Regulierungen im Arzneimittelmarkt 14

Abbildung I - 5: $\quad$ Regulierungsmechanismen der Arzneimittelpolitik der GKV 15

Abbildung 1-6: Arzneimittelausgaben im europäischen Vergleich (Stand 1997) 19

Abbildung 1 - 7: Zulassungskriterien für neue Arzneimittel 20

Abbildung I- 8 Apothekenpflicht, Verschreibungspflicht und GKV - Erstattungsfähigkeit 21

Abbildung 1-9: Vertriebsweg apothekenpflichtiger Arzneimittel 23

Abbildung I - 10: Pharmagroßhandel in Deutschland (Stand 1999) 26

Abbildung 1-11: Apothekendichte in Deutschland (Stand 1998) 28

Abbildung I - 12: Lage und Größe der Apotheken (Befragung 1995) 29

Abbildung I-13: Anzahl der rezeptpflichtigen und zugelassenen Arzneimittel (Stand1997/98) 31

Abbildung 1-14: Direktlieferungen von Arzneimitteln an den Kunden nach Hause (1995) 33

Abbildung 1-15: Regelungen zum Versandhandelsverbot in Deutschland 34

Abbildung 1-16: Apothekendichte (Stand 1998/1997) 35

Abbildung 1-17: EU-Fremd- und Mehrbesitzverbot 35

Abbildung 1-18: Schweizer Modell einer Versandapotheke, Beispiel MediService $\quad 47$

Abbildung I - 19: Vorteile / Hauptziele der Versandapotheke MediService 48

Abbildung 1-20: PBM und Versandapotheke 50

Abbildung 1-21: Diskussionsansätze pro und contra Arzneimittelversand 51

Abbildung I - 22: Bekanntheit des Arzneimittelbezugs via Internet in Deutschland (Stand 1999) 55

Abbildung II - 1: $\quad$ Einfaches Grundmodell einer Versandapotheke 58

Abbildung II - 2: $\quad$ Gesamtmodell einer unabhängigen Versandapotheke 60

Abbildung II - 3: Begriffsbestimmung und Darstellung der Vorgehensweise bei den Modellrechnungen $\quad 65$ 
Abbildung II - 4: Organigramm für die Berechnung des Zielsegments

Abbildung II - 5: Verteilung der Normpackungsgrößen in den Altersklassen 1991

Abbildung II - 6: Darstellung der Varianten $A$ bis $C$ des Zielsegments

Abbildung II - 7: $\quad$ Darstellung der durchschnittlichen Verordnungswerte in Abhängigkeit zur Größe des potentiellen Zielsegmentes des Pharmaversandes

Abbildung II - 8: Kalkulationsschema Logistikkosten Versandapotheke Variante I

Abbildung II - 9: Umsatzberechnung für Versandapotheke mit integriertem Großhandel

Abbildung II - 10: Wertschöpfungsanteile für Hersteller und Vertrieb

Abbildung II - 11: Verwendung der Apothekenbetriebsspanne - Skaleneffekt der Kosten und des Ergebnisses / der Kostenersparnis in Abhängigkeit der Umsatzgrößenklassen der Apotheke

Abbildung II - 12: Marktpotentialvarianten

Abbildung II - 13: Einsparpotentiale im Überblick

Abbildung II - 14: Veränderung der Kostenersparnis / Rabattgewährung durch Berücksichtigung einer durchschnittlichen Betriebsrendite

Abbildung II - 15: Veränderung der Marktpotentiale durch Berücksichtigung einer durchschnittlichen Betriebsrendite

Abbildung II - 16: Veränderung der Einsparpotentiale des Pharmaversandes durch Berücksichtigung einer durchschnittlichen Betriebsrendite

Abbildung II - 17: Veränderung der Marktpotentiale bei Berücksichtigung eines zusätzlichen einheitlichen Kassenrabatts (in Abhängigkeit der Höhe des zusätzlichen Kassenrabatts)

Abbildung II - 18: Veränderung der Einsparparpotentiale bei Berücksichtigung eines zusätzlichen einheitlichen Kassenrabatts (in Abhängigkeit der Höhe des zusätzlichen Kassenrabatts)

Abbildung II - 19: Einsparvolumina bei einem realisierten Marktanteil von 15\% 167

Abbildung II - 20: Einsparvolumina bei einem realisierten Marktanteil von 15\% (mit Betriebsrendite)

Abbildung II - 21: Einsparvolumina und Kostenersparnisse im Vergleich ohne bzw. mit Berücksichtigung einer durchschnittlichen Betriebsrendite bei $15 \%$ Marktanteil

Abbildung II - 22: Einsparvolumina bei Berücksichtigung zusätzlicher einheitlicher Kassenrabatte und einem Marktanteil von 15\%

Abbildung II - 23: Darstellung der Varianten A bis $\mathrm{C}$ des Zielsegments

Abbildung II - 24: Marktpotentialvarianten 
Abbildung III - 1: Chronische Krankheitsbilder und deren pharmazeutische Therapie

Abbildung III - 2: "Heimlieferungen" pro Woche

Abbildung III - 3: Grad der Wichtigkeit verschiedener Kriterien bei der Auswahl der Apotheke

Abbildung III - 4: Bewertung der Marktchancen verschiedener Distributionswege

Abbildung III - 5: Assoziationen mit dem Begriff "Versandapotheke"

Abbildung III - 6: Bewertung der Vorteilhaftigkeit einer Versandapotheke in bezug auf verschiedene Zielgruppen

Abbildung III - 7: Bewertung verschiedener Aussagen zur Versandapotheke

Abbildung III - 8: Persönliche Umsatzbeeinflussung durch Versandapotheken

Abbildung III - 9: Sonstige vorstellbare Reformoptionen

Abbildung III - 10: Direkte Rezeptweiterleitung an die Apotheke

Abbildung III - 11: Grad der Wichtigkeit verschiedener Kriterien bei der Auswahl der Apotheke

Abbildung III - 12: Bewertung verschiedener Distributionswege von Medikamenten für die Zukunft

Abbildung III - 13: Assoziationen mit dem Begriff "Versandapotheke"

Abbildung III - 14: Bewertung der Vorteilhaftigkeit einer Versandapotheke in bezug auf verschiedene Patientengruppen

Abbildung III - 15: Bewertung verschiedener Aussagen zur Versandapotheke

Abbildung III - 16: Sonstige vorstellbare Reformoptionen (offene Frage)

Abbildung III - 17: Art der dauerhaften, vom Arzt behandelten Gesundheitsstörungen

Abbildung III -18: Häufigkeit der Einlösung von Rezepten in der Apotheke

Abbildung III - 19: Grad der Wichtigkeit verschiedener Kriterien bei der Auswahl der Apotheke

Abbildung III - 20: Beratung bei der Aushändigung von Medikamenten

Abbildung III - 21: Vorstellbarkeit des Bezugs von Medikamenten über eine Versandapotheke

Abbildung III - 22: Vorstellbarkeit des Bezugs von Medikamenten über eine Versandapotheke in Abhängigkeit vom Gesundheitszustand

Abbildung III - 23: Anforderungen an eine Versandapotheke

Abbildung III - 24: Beurteilung der Apothekendichte

Abbildung III - 25: Grad der Wichtigkeit verschiedener Kriterien bei der Auswahl der Apotheke 
Abbildung III - 26: Bewertung der Vorteilhaftigkeit einer Versandapotheke in bezug auf verschiedene Zielgruppen

Abbildung III - 27: Assoziationen mit dem Begriff "Versandapotheke" 270

Abbildung III - 28: Bewertung verschiedener Aussagen zur Versandapotheke 273

Abbildung IV - 1: Gesamtmodell einer unabhängigen Versandapotheke

Abbildung IV - 2: Begriffsbestimmung und Darstellung der Vorgehensweise bei den Modellrechnungen

Abbildung IV - 3: Darstellung der Varianten A bis $C$ des Zielsegments

Abbildung IV - 4: Verwendung der Handelsspanne - Skaleneffekt der Kosten und des Betriebsergebnisses (= Kostenersparnis) in Abhängigkeit der Umsatzgrößenklassen der Versandapotheke

Abbildung IV - 5: Marktpotentialvarianten

Abbildung IV - 6: Einsparpotentiale im Überblick

Abbildung IV - 7: Veränderung der Marktpotentiale durch Berücksichtigung einer Betriebsrendite

Abbildung IV - 8: Veränderung der Einsparpotentiale des Pharmaversandes durch Berücksichtigung einer Betriebsrendite

Abbildung IV - 9: Veränderung der Marktpotentiale bei Berücksichtigung eines zusätzlichen einheitlichen Kassenrabatts (in Abhängigkeit der Höhe des zusätzlichen Kassenrabatts)

Abbildung IV - 10: Veränderung der Einsparparpotentiale bei Berücksichtigung eines zusätzlichen einheitlichen Kassenrabatts (in Abhängigkeit der Höhe des zusätzlichen Kassenrabatts)

Abbildung IV - 11: Einsparvolumina bei einem realisierten Marktanteil von 15\% (ohne Betriebsrendite)

Abbildung IV - 12: Einsparvolumina bei einem realisierten Marktanteil von 15\% (mit Betriebsrendite)

Abbildung IV - 13: Einsparvolumina bei Berücksichtigung zusätzlicher einheitlicher Kassenrabatte und einem Marktanteil von $15 \%$

Abbildung IV - 14: Beurteilung der Apothekendichte

Abbildung IV - 15: Grad der Wichtigkeit verschiedener Kriterien bei der Auswahl der Apotheke

Abbildung IV - 16: Bewertung der Vorteilhaftigkeit einer Versandapotheke in bezug auf verschiedene Zielgruppen

Abbildung IV - 17: Assoziationen mit dem Begriff "Versandapotheke" 


\section{ÜBERSICHTENVERZEICHNIS}

$\begin{array}{lll}\text { Übersicht III - 1: } & \text { Arbeitshypothesen } & 204\end{array}$

Übersicht IV - 1: Kosteneinsparung $\quad 321$

Übersicht IV - 2: Marktpotential und Marktanteil 323

Übersicht IV - 3: $\quad$ Einsparvolumen für die GKV 324

Übersicht IV - 4: Anreizsysteme für Krankenkassen und Versicherte $\quad 325$

Übersicht IV - 5: Arzneimittelsicherheit $\quad 327$

Übersicht IV - 6: $\quad$ Notversorgung 328

Übersicht IV - 7: Warensortiment und Preisvergleich 330

Übersicht IV - 8: Versorgungsdichte $\quad 331$

Übersicht IV - 9: $\quad$ Arbeitsmarkteffekte 332

Übersicht IV - 10: Nutzerfreundlichkeit 333

Übersicht IV - 11: Telefonische Erreichbarkeit 335

Übersicht IV - 12: Zustellsicherheit 336

Übersicht IV - 13: Sicherstellung der Beratung 338

Übersicht IV - 14: Rezepterinnerung $\quad 339$

Übersicht IV - 15: Elektronisches Rezept $\quad 340$

Übersicht IV - 16: Soziale Funktionen $\quad 341$ 


\section{ABKÜRZUNGSVERZEICHNIS}

\begin{tabular}{|l|l|}
\hline Abkürzung & Bedeutung \\
\hline ABDA & Bundesvereinigung Deutscher Apothekerverbände \\
\hline AEK & Apothekeneinkaufspreis \\
\hline AMG & Arzneimittelgesetz \\
\hline AMPreisV & Arzneimittelpreisverordnung \\
\hline ApBetrO & Apothekenbetriebsordnung \\
\hline ApoG & Gesetz über das Apothekenwesen \\
\hline ATC & Anatomisch Therapeutischer Code \\
\hline AVK & Apothekenverkaufspreis \\
\hline B-2-B & Business to Business \\
\hline B-2-C & Business to Consumer \\
\hline BfArM & Bundesinstitut für Arzneimittel und Medizinprodukte \\
\hline BGBI. & Bundesgesetzblatt \\
\hline BIP & Brutto-Inlands-Produkt \\
\hline BKK & Betriebskrankenkasse \\
\hline BMG & Bundesministerium für Gesundheit \\
\hline BPI & Bundesverband der Pharmazeutischen Industrie \\
\hline bzW. & beziehungsweise \\
\hline CATI & Computer Assisted Telephone Interviewing \\
\hline DDD & Defined Daily Dose \\
\hline d.h. & das heißt \\
\hline DM & Deutsche Mark \\
\hline EU & Europäische Union \\
\hline GKV & Gesetzliche Krankenversicherung \\
\hline GSG & Gesundheitsstruktur Gesetz \\
\hline HMO & Health Maintenance Organization \\
\hline HWG & Heilmittelwerbegesetz \\
\hline & \\
\hline & \\
\hline
\end{tabular}




\begin{tabular}{|l|l|}
\hline Abkürzung & Bedeutung \\
\hline MwSt. & Mehrwertsteuer \\
\hline NOG & Neuordnungsgesetz \\
\hline N1- Packungen & $\begin{array}{l}\text { Kleine Packungsgröße nach Zuzahlungsverord- } \\
\text { nung }\end{array}$ \\
\hline N2- Packungen & $\begin{array}{l}\text { Mittlere Packungsgröße nach Zuzahlungsverord- } \\
\text { nung }\end{array}$ \\
\hline N3- Packungen & $\begin{array}{l}\text { Große Packungsgröße nach Zuzahlungsverord- } \\
\text { nung }\end{array}$ \\
\hline OTC & over the counter \\
\hline PBM & Pharmacy-Benefits-Management \\
\hline PEI & Paul-Ehrlich-Institut \\
\hline PKA & Pharmazeutisch-Kaufmännische Angestellte \\
\hline PKV & Private Krankenversicherung \\
\hline PTA & Pharmazeutisch-Technische Assistentin \\
\hline SGB V & Sozialgesetzbuch V \\
\hline VFA & Verband Forschender Arzneimittelhersteller \\
\hline Vgl. & Vergleiche \\
\hline ZzVO & $\begin{array}{l}\text { Verordnung über die Zuzahlung bei der Abgabe von } \\
\text { Arznei- und Verbandmitteln in der vertragsärztli- } \\
\text { chen Versorgung - Zuzahlungsverordnung }\end{array}$ \\
\hline &
\end{tabular}


Martin Pfaff, Dietmar Wassener, Astrid Sterzel and Thomas Neldner - 978-3-631-75554-9 Downloaded from PubFactory at 01/11/2019 03:35:36AM via free access 


\section{Einleitung}

\subsection{Problemhintergrund}

In Deutschland wurden im Jahr 2000 im Rahmen des Versorgungsauftrages der gesetzlichen Krankenkassen ca. 15,3\% der gesamten Leistungsausgaben für Arzneimittel aus öffentlichen Apotheken aufgewendet. Dies entspricht einem Betrag von 37,8 Mrd. DM. 1999 waren insgesamt 36,15 Mrd. DM für die Erstattung von Arzneimitteln aus Apotheken bestimmt, 1998 ca. 33,6 Mrd. DM. Diese Entwicklung bedeutete einen Anstieg von ca. 4,4\% im Jahr 2000 bzw. 7,4\% im Jahr 1999 im Vergleich zum Vorjahr.

Damit verzeichnet der Arzneimittelbereich "die mit Abstand problematischste Entwicklung unter allen Ausgabenbereichen ${ }^{{ }^{2}}$ der GKV und liegt zudem deutlich über den Wachstumsraten des Bruttoinlandsproduktes. ${ }^{3}$ Der aufgezeigte Ausgabenanstieg der letzten drei Jahre kann auch für vorhergehende Zeiträume als kontinuierlich eingestuft werden (nur kurz unterbrochen durch die gesetzlichen Maßnahmen von 1993 bzw. 1997), wie ein Blick auf Abbildung 0 - 1 verdeutlicht:

Abbildung 0 - 1: Ausgabenanstieg der GKV für Arzneimittel

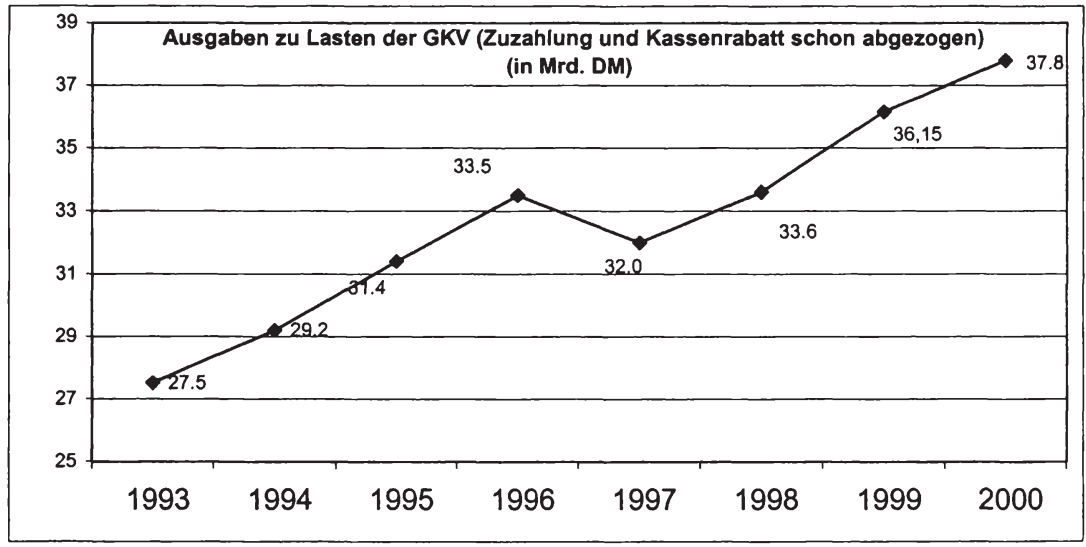

Quelle: INIFES, eigene Darstellung nach BKK (2000), Arzneimittelvertragspolitik, März 2000, S. 2.

Ohne Zuzahlungen der Patienten und Kassenrabatt.

Bundesministerium für Gesundheit (2000), Knapp eine Milliarde Überschuß und stabiles Beitragsniveau: Finanzentwicklung der gesetzlichen Krankenversicherung in 1999 überaus positiv! In: www.bmgesundheit.de, Pressemitteilung Nr. 20, Zugriff am 15.03.2000.

3

Diese lag im Jahre 1999 bei nominal 2,5\% bzw. real 1,5\%, siehe: Institut der deutschen Wirtschaft Köln, (2000), Zahlen zur wirtschaftlichen Entwicklung der Bundesrepublik Deutschland, S. 26. 
Um diesem Ausgabenanstieg entgegenzuwirken, wurden, wie bereits angedeutet, in der Vergangenheit Kostendämpfungsmaßnahmen in Form von Arzneimittelbudgets, Negativlisten oder Zuzahlungsregelungen in das System implementiert. Trotzdem konnte, abgesehen von kurzfristigen Dämpfungseffekten (siehe Abbildung 0 - 1), eine längerfristige Konsolidierung der Arzneimittelausgaben bislang nicht erreicht werden. Zudem sind nach der Ankündigung der Abschaffung der Arzneimittelbudgets durch die Politik vom Februar 2001 die Arzneimittelausgaben der GKV um 9,7\% im ersten Quartal gegenüber dem Vorjahr angewachsen. ${ }^{5}$ Damit ist das Ziel der Beitragssatzstabilität im System der GKV stark gefährdet. Jüngste Ankündigungen von Beitragssatzerhöhungen durch verschiedene Kassen sind Ausdruck der angespannten Situation.

Soll das Ziel, die Beitragssätze für die Versicherten stabil zu halten, nicht aufgegeben werden, müssen zukünftige Reformmaßnahmen auf eine effizientere Nutzung der bereits vorhandenen Ressourcen bei Sicherung der Qualität der medikamentösen Versorgung ausgerichtet werden.

Dabei führt die Diskussion um Reformoptionen im Arzneimittelsektor auch in die Richtung des Distributionskanals Apotheke. Der Vertrieb von Medikamenten ist bisher streng vom Pharmahersteller über den pharmazeutischen Großhandel bis zur Apotheke reguliert: unter die Apothekenpflicht fallende Präparate (in Deutschland ca. 45.000, darunter fungieren automatisch alle rezeptpflichtigen Arzneimittel) dürfen grundsätzlich nur in den Räumen einer öffentlichen Apotheke an den Patienten abgegeben werden. ${ }^{6}$ Andere Formen des Vertriebs, wie sie für freiverkäufliche Arzneimittel, Verband- und Heilmittel oder Medizinprodukte auch in Deutschland durchaus legal und üblich sind, werden für apothekenpflichtige Medikamente nach § 43, I AMG gesetzlich ausgeschlossen.

Aus der reglementierten Distribution über mehrere vertikale Handelsstufen resultieren Vertriebskostenanteile am Medikamentenpreis: zwischen 30\% und 40\% der Medikamentenkosten entfallen in Deutschland auf den Vertrieb über Großhandel und öffentliche Apotheken. Allein für öffentliche Apotheken, die im Jahre 1999 insgesamt 50,3 Mrd. DM (ohne MwSt.) ${ }^{7}$ bzw. ca. 58 Mrd. DM brutto ${ }^{8}$ umsetzten, resultiert bei

Siehe entsprechend in Kapitel 1.

BKK Bundesverband, (2001), Pressemitteilung vom 27.06.2001 GKV: Krankenkassen gegen die Abschaffung von Arznei- und Heilmittelbudgets - Ausgaben explodieren - wachsende Beitragssătze, siehe unter www.bkk.de, Zugriff am 27.06.01.

Siehe § 43 AMG sowie $\S 17$ Apothekenbetriebsordnung (Ausnahme in Einzelfällen durch $\S 17,11)$ ABDA, (1999), Die Apotheke Zahlen, Daten, Fakten, Abbildung 10: davon entfielen auf Arzneimittel 47 Mrd. DM. 
Mischkalkulation ein Vertriebskostenanteil in Form der Apothekenbetriebsspanne von ca. $27,3 \%$ des Bruttoumsatzes.

Vor diesem Hintergrund wird argumentiert, die Vertriebswege der Arzneimittel vom Hersteller über den Großhandel bis hin zur Apotheke seien zum Teil ineffizient. So wird immer lauter hinterfragt, worin der Nutzen für die Beitragszahler besteht, ganze Versorgungsbereiche vom Wettbewerb auszunehmen, wie derzeit die Arzneimittelversorgung. ${ }^{10}$ Reformvorschläge in der Arzneimitteldistribution umfassen unter anderem eine stärkere Öffnung der Krankenhausapotheken für die ambulante Versorgung, eine Verringerung des Mehrwertsteuersatzes auf Arzneimittel, eine Modifizierung der Arzneimittelpreisverordnung wie auch eine (zumindest teilweise) Freigabe des Versandhandels mit Arzneimitteln. Nicht nur die Kostenträger, auch immer mehr politische Verantwortungsträger werben dafür, Alternativen zur öffentlichen Apotheke zumindest rechtlich zuzulassen. In der Praxis würde sich zeigen, ob und in welchem Umfang diese Versorgungsformen in Anspruch genommen werden."

Die Liberalisierung des Vertriebsweges durch potentielle Versandapotheken rückt dabei in den Vordergrund der gesundheitspolitischen Diskussion. Befürworter sehen insbesondere für chronisch Kranke und pflegebedürftige Personen die Möglichkeit der Inanspruchnahme von Versandapotheken als vorteilhaft an. Die Zulassung der Versandapotheken sollte dabei unter Berücksichtigung von Lieferbereitschaft und Service erfolgen. Eine Ausweitung des Pharmaversandes auf verschreibungspflichtige Medikamente könnte demnach die Erschließung von Wirtschaftlichkeitsreserven fördern. Zudem erscheinen durch eine Modifizierung des Apothekenzuschlages sowie die Möglichkeit erheblicher Rabatte beim direkten Bezug vom Pharmahersteller, erhebliche Preisvergünstigung pro Medikament als möglich. Die Kostenträger veröffentlichten bisher Schätzungen zur finanziellen Entlastung des sozialen Krankenversicherungssystems zwischen 800 Mio. $\mathrm{DM}^{12}$ bis $1.700 \mathrm{Mio}^{\mathrm{D}} \mathrm{DM}^{13}$, die durch den Versandhandel möglich wären. Diese Zahlen stützen sich insbesondere auf Erfahrungen aus Ländern, die den Distributionskanal Versand legalisiert haben und ca. $10 \%$ bis $15 \%$ aller Verordnungen auf diese Art und Weise vertreiben, bei Kosteneinsparungen zwischen $10 \%$ bis $30 \%$. ${ }^{14}$ Dazu soll an dieser Stelle ausdrücklich angemerkt werden, dass eine Anlehnung der erzielbaren Kostenvorteile im Ausland aus verschiedenen Gründen nicht 1:1 für Deutschland möglich ist aufgrund von Unterschieden in den

ABDA, (1999), Die Apotheke Zahlen, Daten, Fakten, Abbildung 13, sowie Wirtz, K., (2000), Bericht über die Ergebnisse des Betriebsvergleiches der Apotheken im Jahre 1998, in: Mitteilungen des IFH Köln, Jahrgang 52, S. 25.

10

11

12

13

14

Hovermann, E., zitiert in: Kieselbach, K. (2001), Internet-Apotheken erhalten jetzt Unterstützung, in: DIE WELT vom 12.06.2001, S.16.

Bundesverband der Betriebskrankenkassen, Arzneimittelvertragspolitik März 2000, S. $15 \mathrm{ff}$.

Schleert, D. (2000), zitiert in: Zander, B., Invasion der @-Pillen, in: STERN 24/2000, S 198.

Wissenschaftliches Institut der AOK, (2001), Versandapotheke - eine preiswerte Alternative, Aktuelle Modellrechnung des WidO berechnet Einsparpotential, siehe unter www.wido.de, Zugriff am 25.06.2001.

Berechnungen dieser Art fokussieren insbesondere auf Versender in der Schweiz, den Niederlanden oder den USA. 
Gesundheitssystemen ebenso wie in den nationalen gesundheitspolitischen Rahmenbedingungen. Hier sei z.B. nur die Art und Weise der Preissetzung für Arzneimittel erwähnt. Vergleiche dieser Art unterliegen der Problematik, dass eruierte Wirtschaftlichkeitspotentiale nicht ausschließlich ursächlich auf die Implementierung von Versandapotheken zurückzuführen sind, sondern auch weitere Komponenten, wie z.B. die Ausnutzung geringerer Preisniveaus für Arzneimittel im Ausland, beinhalten können. Sicherlich wird eine Diskussion um eine Liberalisierung der Vertriebswege und damit die Zulassung von mehr Wettbewerb, nicht die Problematik der Preisregulierung ausschließen können, geführt - dies spiegelt auch die angeführte Agenda von Reformoptionen wieder -, die eine Modifizierung der derzeit gültigen Arzneimittelpreisverordnung ${ }^{15}$ beinhaltet. Andererseits ist eine Änderung der Arzneimittelpreisverordnung nicht allein ursächlich mit der Freigabe des Versandhandels verbunden, diese Problematik würde sich allgemein bei der Liberalisierung des Vertriebsweges, wie z.B. auch bei der Aufgabe des Fremd- und Mehrbesitzverbotes, stellen. Zudem werden durch neue Vertriebsformen neue betriebswirtschaftliche Kosten induziert. ${ }^{16}$ Aus volkswirtschaftlicher Sicht sind potentielle Wirtschaftlichkeitsreserven deshalb nicht ohne weiteres auf Basis ausländischer Quellen zu eruieren und in genaue Zahlen zu fassen. Als Orientierungs- und Anhaltspunkte können sie gleichwohl fungieren.

Im Gegensatz zu den genannten Zahlen der Kostenträger befürchten die Akteure des derzeitigen Distributionsweges wie z.B. der Großhandel allerdings Einnahmeverluste, falls teure und häufig verkaufte Arzneimittel per Versandhandel vertrieben würden. Dies gefährde die flächendeckende und schnelle Versorgung mit selten benötigten Arzneimitteln und verteuere sie drastisch. Auch der Apothekerverband bestreitet einen volkswirtschaftlichen Nutzen durch Versandhandel. Kritik an zu hohen Vertriebskosten durch Apotheken sei vollkommen unberechtigt. ${ }^{17}$ Zudem weisen Vertreter der Apothekerverbände ${ }^{18}$ darauf hin, dass eine Legalisierung des Arzneimittelversandes eine Änderung des existierenden Systems bedingt. Sämtliche Eingriffe in bestehende Systeme sind mit Auswirkungen auf das Existente verbunden, eine Änderung des Gesamtsystems sehen die Verfasser der Studie jedoch nicht.

Die Diskussion über neue Vertriebswege, wie bisher erfolgt, nur unter Wirtschaftlichkeits- und Kostenargumenten zu führen, würde den Anspruch der Gewährleistung einer qualitativ hochwertigen Arzneimittelversorgung untergraben. So betont das

Siehe auch Forderung der gesundheitspolitischen Arbeitsgruppe der Bundestagsfraktion der SPD, in: PHAGRO, (2001), Arbeitsgruppe der SPD-Fraktion fordert Änderungen in der Arzneimitteldistribution, www.phagro.de, Zugriff am 02.05.01.

16

17

18

4

Z.B. Kosten für Logistik, Telefonberatung, etc. müssten neu kalkuliert werden. Andererseits können sich Einsparungen bei anderen Kostenpositionen ergeben.

Vgl. zu diesen kritischen Stimmen z.B.: Rücker, D., Politik Versandhandel Einsparungen im Promillebereich, aus: www.pharmazeutische-zeitung.de, Zugriff am 20.09.99.

Ergebnis der von INIFES geführten Expertengespräche und Aussage auf dem Workshop am 17.09.2001 in Berlin. 
BMG, die oberste Priorität der Regierung sei der Schutz des Verbrauchers und die Sicherstellung einer ordnungsgemäßen Arzneimittelversorgung.

Die Apotheker ihrerseits argumentieren, dass bei Medikamentenbezug via Versand keine oder nur kaum Beratung stattfinden könnte und führen in diesem Zusammenhang die Beratung des Kunden in der Apotheke als Wettbewerbsvorteil gegenüber anderen potentiellen Anbietern, so auch Versandapotheken, an. ${ }^{19}$

Eine Gefährdung der Beratung ist allerdings auch heute schon - und zwar in Präsenzapotheken und nicht nur in Einzelfällen - zu vermuten, da aktuelle demografische wie epidemiologische Entwicklungen die Gewährleistung des face-to-face Kontaktes auch im aktuellen Vertriebssystem an Grenzen führen. Wie in den meisten Industrienationen leben in Deutschland viele ältere Menschen, chronisch Kranke oder Multimorbide: im Jahr 2000 ist fast jeder vierte Deutsche über 60 Jahre alt, 2040 wird es mehr als ein Drittel der Deutschen sein. Aufgrund einer altersbedingten höheren Krankheitswahrscheinlichkeit belastet der daraus resultierende hohe Arzneimittelbedarf in Zukunft nicht nur verstärkt die Sozialkassen (s. Abbildung $0-2$ ), sondern bedingt ebenfalls eine hohe Notwendigkeit von Apothekenbesuchen zur Deckung des Arzneimittelbedarfs des Patienten. ${ }^{20}$ Dem steht jedoch aufgrund der alters- wie epidemiologisch bedingten Beeinträchtigungen eine häufig anzutreffende Immobilität entgegen, die den Weg in die Apotheke nur schwerlich oder gar nicht möglich erscheinen lässt. Diese Patienten sind dann auf die Hilfe aus der Familie wie aus anderen externen Quellen angewiesen, um die Arzneimittel aus der Apotheke zu erhalten.

Je nach Befragung ergibt sich konsequenterweise auch, dass $15 \%$ bis $50 \%$ der Apothekenkunden Besorger, d.h. „Rezeptüberbringer" und „Medikamentenbesorger" für andere Menschen mit Medikamentenbedarf sind. Eine direkte Beratung des Patienten durch den Apotheker kann in solchen Fällen nicht erfolgen. Die Begründung der Abgabe in den Apothekenräumen nach $\S 43$, I ApBetrO aufgrund der Erklärungsbedürttigkeit apothekenpflichtiger Medikamente und der daraus abzuleitenden Beratungspflicht des Apothekers verfehlt ihre Intension.

19

Nach $\S 20$ Apothekenbetriebsordnung obliegt dem Apotheker aus Gründen der Arzneimittelsicherheit die Pflicht, dem Apothekenkunden informierend und beratend zur Seite zu stehen. Allerdings darf die Beratung nicht die Therapie des verordnenden Arztes beeinträchtigen.

Drei Viertel der Apothekenkunden sind kranke Menschen, mehr als jeder sechste leidet an einer chronischen Krankheit mit stetigem Arzneimittelbedarf. Zwei Drittel der Apothekenkunden sind Dauerpatienten, die regelmäßig mindestens einmal monatlich Medikamente aus der Apotheke beziehen müssen, davon sind fast alle mehr als 60 Jahre alt. Siehe: Riegl \& Partner $\mathrm{GmbH}$, Institut für Management im Gesundheitsdienst, Das Image der deutschen Apotheke, Augsburg 1995. 
Abbildung 0-2: Arzneimittelausgaben der GKV je Mitglied und je Rentner, sowie Rentneranteil an der Versichertenstruktur 1994 bis 1998 (jeweils inklusive mitversicherte Familienangehörige)

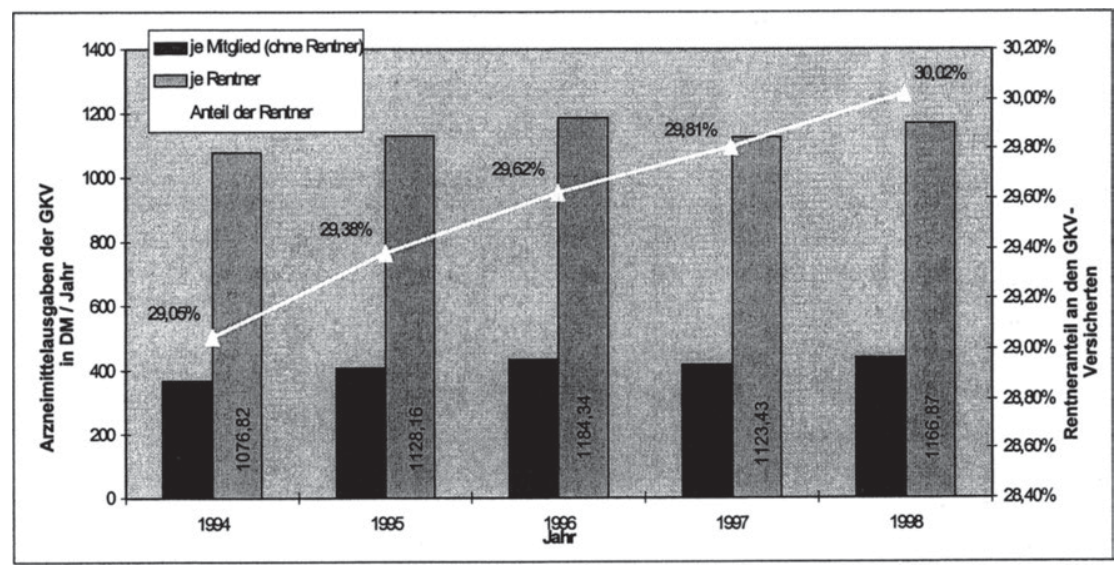

Quelle: ABDA, (1998) Die Apotheke Zahlen, Daten, Fakten

Diese Scherensituation, einerseits eine nichtmobile, kranke und ältere Kundschaft, andererseits die Begrenzung der Arzneimittelabgabe auf die Apothekenräume versuchen viele Apotheker durch die Ausnahmeregelung des §17, II ApBetrO zu lösen. Dieser billigt in begründeten Einzelfällen eine Versendung aus der Apotheke oder die Zustellung durch einen Boten an den Arzneimittelempfänger zu. Hier existiert eine Grauzone. Befragungen ergaben, dass Hausbelieferung durch Apotheken gängige Praxis ist. Im Durchschnitt beliefert jede Apotheke ca. 30 Kunden pro Woche. ${ }^{21}$ Das Agieren der Apotheken ist ein deutliches Zeichen dafür, dass mit der Heimlieferung ein echtes Kundenbedürfnis vorhanden ist, dem - der Wirtschaftstheorie folgend die Anbieter (Apotheken) im Rahmen ihrer (Un)Möglichkeiten versuchen, zu entsprechen.

An diesem Punkt, nämlich der Fokussierung auf die Struktur der Apothekenkunden, kann ein Vergleich mit ausländischen Versandapotheken weit mehr als ein reiner Kostenvergleich von Nutzen sein. Es zeigt sich nämlich, dass das Nachfragerpotential bzw. die Kunden von Versandapotheken in den verschiedenen Ländern ähnliche Merkmale aufweisen. Versandapotheken sind hauptsächlich für Langzeitpatienten ein interessanter Bezugsweg. So ergab eine Befragung in der Schweiz, dass Patienten über 60 Jahre mehr als $65 \%$ der Kundenklientel einer Versandapotheke ausmachen. Zudem benötigen von diesen mehr als $60 \%$ regelmäßig 2-4 Medikamente pro Tag, ca. $15 \%$ sogar täglich mehr als 5 Präparate. Weiterhin profitierten davon Menschen in ländlichen Gebieten. Die Versandapotheke wird zu $59 \%$ von Menschen beansprucht, die in Orten mit weniger als 10.000 Einwohnern

Schöffski, O., Die Regulierung des deutschen Apothekenwesens. Eine ökonomische Analyse. Baden-Baden 1995, S. 238. 
Menschen beansprucht, die in Orten mit weniger als 10.000 Einwohnern leben. ${ }^{22}$ In den USA werden sogenannte Mail-Order-Pharmacies hauptsächlich durch ältere Menschen frequentiert, insbesondere durch Frauen im Alter von über 65 Jahren, die im Gegensatz zu älteren Männern öfter allein im Haushalt leben und damit auf eine Eigenversorgung angewiesen sind. ${ }^{23}$

Erfahrungen mit Versandapotheken im Ausland belegen, dass aufgrund epidemiologischer und demografischer Entwicklungen nachfrageseitig Potentiale in Form neuer Zielgruppen für Versandapotheken zu existieren scheinen. Dies sind insbesondere Menschen mit einer Dauermedikation. Andererseits würden Patienten mit Akutkrankheiten durch eine Versandapotheke nicht adäquat versorgt, bzw. die Kosten einer Expresslieferung stünden in keinem Verhältnis zur Abgabe in der Präsenzapotheke. Eine flächendeckende Versorgung aller Versicherten und Patienten durch Versandapotheken ist damit ausgeschlossen und es zeigt sich, dass Versandapotheken nicht substitutiv, sondern ausschließlich komplementär als ein Glied neuer Vertriebsstrukturen für verordnungspflichtige Arzneimittel wirken können.

Für Deutschland liegen noch keine umfassenden wissenschaftlichen Untersuchungen zu den potentiellen Auswirkungen der Einführung des Arzneimittelversandes vor, die als Grundlage für eine sachliche Diskussion um Pro und Contra dieser Vertriebsform dienen könnten. Fest steht allerdings, dass im Zuge der stetigen Ausgabensteigerungen im Arzneimittelsektor und der knappen Ressourcen der Kostenträger auch im Sinne der angestrebten Beitragssatzstabilität - nach Wegen gesucht werden muss, mögliche Wirtschaftlichkeitspotentiale nutzbar zu machen. Ein Vertriebskostenanteil von durchschnittlich fast einem Drittel der Arzneimittelausgaben der gesetzlichen Krankenversicherung ist dabei zweifellos in Einsparungsüberlegungen einzubeziehen.

Gleichzeitig müssen aber die zentralen Aspekte der Medikamentensicherheit sowie der Akzeptanz des Versandhandels bei Patienten, Ärzten und anderen Betroffenen angemessen berücksichtigt werden. Volkswirtschaftlich und sozialpolitisch akzeptabel können neue Vertriebswege im Arzneimittelsektor nur dann sein, wenn Wirtschaftlichkeitsreserven ausgeschöpft werden können, ohne das bestehende hohe Niveau der Arzneimittelversorgung zu gefährden. Unter diesen Prämissen sind Versandapotheken, wie im Folgenden dargestellt, als Vertriebsformen in Zukunft verstärkt in eine kritische und ergebnisoffene Diskussion um liberalisierte Wege in der Arzneimitteldistribution zu integrieren.

22

Institut für Sozial- und Präventivmedizin der Universität Zürich; Schweizerische PatientenOrganisation (SPO); Schweizerische Patientendienststelle (DVSP), Zufriedenheit der Kunden

23 der MediService Apotheke - Schlussbericht, Zürich 1999.

FIND/SVP, The International Market for Mail Service Pharmaceuticals, New York 1996. 


\subsection{Zielsetzung}

Im Rahmen der INIFES-Studie werden die in der Öffentlichkeit diskutierten Argumente pro und contra Pharmaversand unvoreingenommen hinterfragt und empirisch überprüft. Es ist Ziel der Studie, die potentiellen Auswirkungen einer Ausweitung des Pharmaversandes in Deutschland zu untersuchen. Hierbei sollen sowohl quantitative als auch qualitative Aspekte berücksichtigt werden. Nicht thematisiert werden die mit einer Legalisierung des Versandhandels verbundenen Sekundäreffekte ${ }^{24}$ sowie deren langfristigen Folgewirkungen.

Im Vordergrund der Studie steht explizit ein nationales Modell des Arzneimittelversandes für Deutschland. Dies ermöglicht eine Berücksichtigung bestehender nationaler Regelungen, wie z.B. das Sozialgesetzbuch, die Arzneimittelpreisverordnung und eine Kontrolle durch staatliche Behörden, auch für Versandapotheken. Die sich aufgrund des EG-Vertrages langfristig zu enwartender Folgewirkungen und Umsetzungen bereits bestehender Europäischer Richtlinien werden im Folgenden nur angedeutet.

Die notwendigen Arbeiten beinhalten folgende zentrale Elemente:

- Die institutionellen Rahmenbedingungen des Arzneimittelmarktes wie des Arzneimittelvertriebes werden dargestellt. Dies schließt die Erfassung und Analyse internationaler Erfahrungen (am Beispiel Schweiz, USA, etc.) mit Versandapotheken ein. Zudem ist das Konzept des hier untersuchten "Pharmaversandes" - und somit der Gegenstand der durchzuführenden Modellrechnungen und Befragungen - präzise zu definieren.

- Die monetären Einsparpotentiale, die sich aus einer Ausweitung des Pharmaversandes für die gesetzliche Krankenkasse ergeben können, werden simuliert. Hierbei wird in Abhängigkeit der verfügbaren Daten das Marktpotential und die Kostenersparnis an den Medikamentenkosten durch Einführung eines Versandhandels entwickelt. Aus der Kombination von beiden Faktoren ergibt sich das monetäre Einsparpotential.

- Die strukturellen und qualitativen Auswirkungen einer Ausweitung des Pharmaversandes auf Anbieter und Nachfrager werden anhand von Primärerhebungen und Expertengesprächen abgeschätzt. Dabei wird z.B. darauf abgezielt, welche Akzeptanz des Versandhandels bei Patienten, Ärzten und Apotheken zu erwarten ist, welche Auswirkungen der Versandhandel auf die Zielgruppen (soziale Kontakte, Beratung, Probleme bei weiteren Verordnungen etc.) hat oder auch, wie die These, durch den Versandhandel könne ggf. der umfassende Versorgungs- und Sicherstellungsauftrag der Apotheken beeinflusst werden, zu bewerten ist. ropa-Rechtliche Regelungen. 
- Abschließend werden aus den quantitativen und qualitativen Ergebnissen Szenarien zur Zukunft des Pharmaversandes in Deutschland entwickelt und anhand einer Pro- und Contra-Diskussion dargestellt.

Die Ergebnisse dieser Studie wurden der Öfentlichkeit im Rahmen eines Workshops am 17. September 2001 vorgestellt. Die vorliegende Studie greift Argumente der Podiumsdiskussion auf und berücksichtigt diese, sofern dies die Verfasser für erachtenswert hielten, an den entsprechenden Stellen.

\subsection{Aufbau der Arbeit}

Kapitel 1 befaßt sich mit den institutionellen Rahmenbedingungen des Arzneimittelvertriebs, u.a. mit einer Darstellung des derzeitigen Apothekenkanals in seiner Ausrichtung und den Vertriebskosten, aber auch mit Erfahrungen, die im Ausland bezüglich Versandapotheken zu beobachten sind. Es erfolgt eine Abgrenzung des in der aktuellen Diskussion häufig verwendeten Begriffs der Internetapotheke von den hier ausschließlich betrachteten Versandapotheken.

Kapitel 2 stellt das Modell einer Versandapotheke für verschreibungspflichtige Arzneimittel vor, auf die die weiteren Betrachtungen einer quantitativen wie qualitativen Analyse aufbauen. In diesem Kapitel erfolgt die quantitative Analyse der Einsparpotentiale für die GKV anhand von Zahlen für das Jahr 1999. Dazu werden zum einen Marktpotentiale anhand einer Zielgruppenfokussierung auf chronische und/oder langfristige Medikation aus dem GKV-Fertigarzneimittelmarkt eruiert. Des weiteren wird die Betriebsstruktur einer Versandapotheke entwickelt. Um Skaleneffekte darzustellen, wird von drei Umsatzgrößenklassen ausgegangen. Die resultierenden Kostenvorteile gegenüber Präsenzapotheken ergeben in Bezug auf das Marktpotential das für die GKV zu erwartende Einsparpotential bei einer Einführung des Versandhandels für verschreibungspflichtige Arzneimittel.

Kapitel 3 widmet sich der qualitativen Analyse des Versandhandels. Es werden Ergebnisse einer eigens durchgeführten repräsentativen Umfrage (in Kooperation mit TNS EMNID) vorgestellt: diese umfassen das Meinungsspektrum der Bevölkerung als potentielle Nutzer, aber auch die Einstellung der Ärzte und Apotheker als Betroffene bei einer möglichen Implementierung von Versandapotheken.

Kapitel 4 faßt die quantitativen wie qualitativen Ergebnisse zusammen und liefert eine Bewertung wesentlicher Pro- und Contra-Argumente des Versandhandels, die in der öffentlichen Diskussion häufig zu finden sind. Dazu werden abschließende Thesen formuliert. 


\section{Institutionelle Rahmenbedingungen des Arzneimittel- vertriebs}

\subsection{Monetäre Entwicklungen und Regulierungen im Arzneimittel- markt in Deutschland}

Der Arzneimittelmarkt ist ein Wachstumssektor und dürfte auch zukünftig zu den welt- und europaweit am stärksten expandierenden Märkten zählen: die hauptsächlich in den Industrieländern zunehmende Lebenserwartung, weltweit steigende Bevölkerungszahlen, das Auftreten neuer Krankheiten, der medizinisch-technische Fortschritt u.v.a.m. sind Gründe dafür. Dabei ist das Marktvolumen von wenigen Ländern bestimmt: so entfielen allein auf Deutschland, Frankreich, Italien und Großbritannien mehr als 80 Prozent der Gesamtnachfrage nach Arzneimitteln. ${ }^{1}$ Auch im Vergleich der gesamten Gesundheitsausgaben gibt keine andere Nation in Europa heute, gemessen an der Wirtschaftsleistung, mehr Geld für Gesundheit aus als die Deutschen: rund 10,5\% des Bruttoinlandsproduktes.

Abbildung I - 1: Gesundheitsausgaben 1998 in \% des BIP

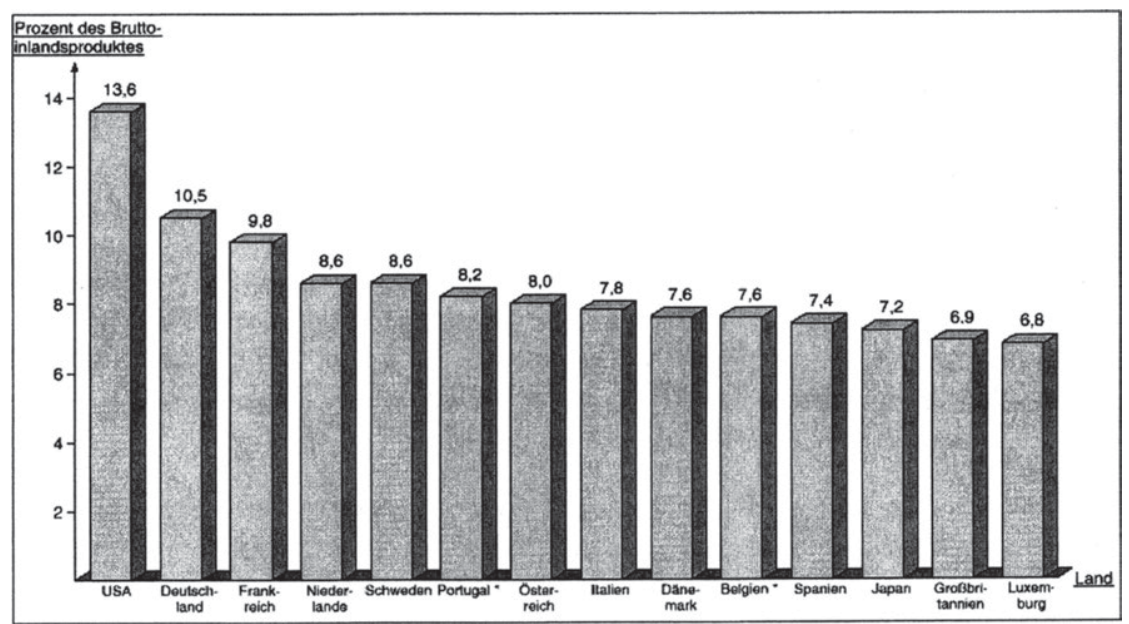

Quelle: OECD Health Data 1998, ") OECD Health Data 1997

Boroch, W. (1994), Internationale Wettbewerbsfähigkeit der EU - Arzneimittelindustrie, Hamburg, S. $10 f$. 


\subsubsection{Monetäre Entwicklung der Arzneimittelausgaben und Einflussfaktoren}

Von den Gesundheitskosten entfielen für den selben Zeitraum ca. 54 Mrd. DM brutto auf Arzneimittel, die in öffentlichen Apotheken abgegeben wurden. Dies betrifft neben Arzneimitteln auf Rezept auch solche ohne ärtliche Verordnung. Ca. 36,15 Mrd. DM dieser Summe, also ca. $66 \%$ des Apothekenumsatzes mit Arzneimitteln, finanzierten die gesetzlichen Krankenkassen. Zudem zahlten die gesetzlich Versicherten ca. 4,2 Mrd. DM in Form von Zuzahlungen, 2,1 Mrd. DM entfielen auf den Apothekenrabatt. ${ }^{2}$ Ca. 4 Mrd. DM betrugen die Ausgaben anderer Kostenträger (v.a. der PKV) für Arzneimittel ihrer Versicherten sowie ca. 7,5 Mrd. DM gaben die Apothekenkunden für nicht verordnete Medikamente aus Eigenmitteln aus (Selbstmedikation $^{3}$ ).

Träger der Gesundheits- und damit auch Arzneimittelkosten ist in erster Linie die GKV mit insgesamt ca. 240 Mrd. DM Leistungsausgaben für $1999^{4}$ bzw. 36,15 Mrd. DM an Arzneimittelausgaben, welche überwiegend durch die Beitragszahlungen der Versicherten bestritten wurden.

Die Arzneimittelausgaben der gesetzlichen Krankenversicherung - als wesentlicher Finanzierungsbaustein des Apothekenumsatzes für Arzneimittel - ergeben für die letzten zehn Jahre unterschiedliche Tendenzen. So ist zum einen ein Rückgang in der Menge der verordneten Verpackungen zu verzeichnen. Andererseits stieg der Arzneimittelverbrauch nach Umsatz kontinuierlich an:

Abbildung I - 2: GKV-Ausgaben für Arzneimittel aus Apotheken, von 1991 bis 1999
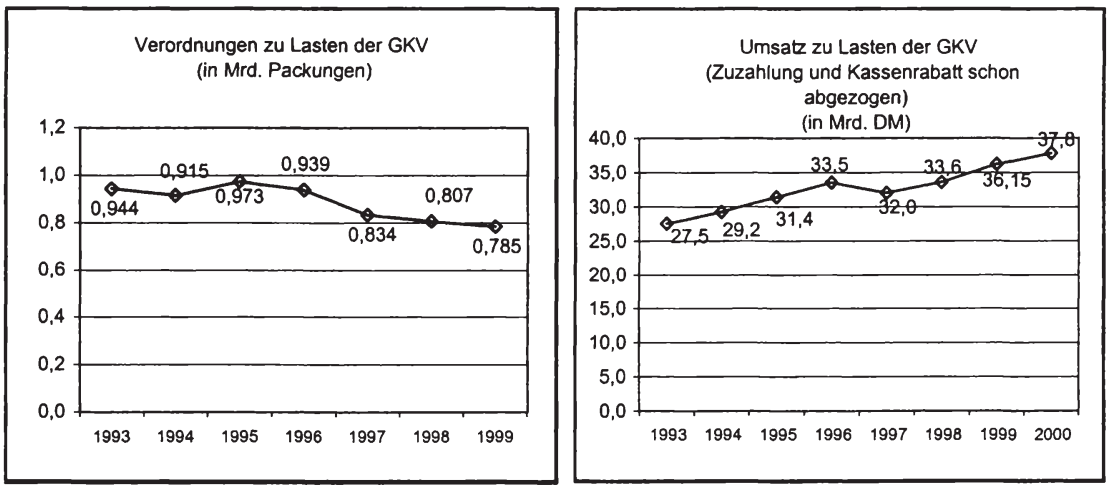

Quelle: INIFES, eigene Darstellung nach:

GKV-Arzneimittelindex;

BMfG, Pressemitteilung Nr. 20 vom 03.03.2000, in: www.bmgesundheit.de;

BKK (2000), Arzneimittelvertragspolitik, März 2000, S. 2.

ABDA, (1999), Die Apotheke Zahlen Daten Fakten, Abbildung 12.

Zur Begriffsbestimmung siehe Kapitel 1.2.1.

Institut der deutschen Wirtschaft Köln, (2000), Zahlen zur wirtschaftlichen Entwicklung der Bundesrepublik Deutschland, Abbildung 95. 
Anhand der beiden gegenläufigen Entwicklungen in Verordnungsmenge und Umsatz ist erkennbar, dass zwar weniger, aber dafür teurer durch die Ärzte zu Lasten der gesetzlichen Krankenversicherung verordnet wird.

Quantitative Einsparungen im Arzneimittelbereich, also ein Rückgang der Verordnungszahlen, zeigen sich durch eine negative Mengenkomponente, die 1998 und 1999 bei ca. minus 3,0\% lag. ${ }^{5}$ Das Preisniveau für Arzneimittel lag 1999 mit einer $0,8 \%$ igen Steigerung moderat über dem Vorjahrespreisanstieg von $0,4 \%$, wobei im festbetragsfreien Markt die Preise überdurchschnittlich um 1,9\% gestiegen sind, während sie im Festbetragsmarkt sogar um 0,3\% rückläufig waren. Der Ausgabenanstieg für Arzneimittel anhand dieser Daten ist also nicht aus den Komponenten Menge und allgemeines Preisniveau zu erklären. Den Ausgabenänderungen liegen vor allem Änderungen in der Konsumstruktur zugrunde: rund ein Drittel der durchschnittlichen Steigerungen der GKV-Arzneimittelausgaben seit 1979 werden dem Struktureffekt zugeschrieben. 1998 war die Steigerung der Arzneiausgaben nahezu vollständig auf den Struktureffekt mit 8,1\% zurückzuführen. ${ }^{6}$ Dies trifft auch für $1999 \mathrm{zu}$, wenn auch abgedämpft mit 5,5\%. ${ }^{7}$ Dabei kann man den Struktureffekt wiederum in Intra- und Intermedikamenteneffekt unterteilen. Beim Intramedikamenteneffekt folgen Ausgabenänderungen bei c.p. gleichen Preisen und Verordnungen aufgrund sich ändernder nachgefragter Packungsgrößen, Darreichungsformen und Dosierungen identischer Arzneimittel desselben Marken- oder Handelsnamen. Der Intramedikamenteneffekt fält dabei weit weniger ins Gewicht als der Intermedikamenteneffekt. Vom Intermedikamenteneffekt spricht man, wenn die Ausgabenänderungen durch einen Wechsel der nachgefragten Medikamente innerhalb oder zwischen Therapiegruppen sowie durch Substitution wirkstoffidentischer Arzneimittel anderer Hersteller, z.B. durch Generika, induziert werden. ${ }^{8}$ Der Intermedikamenteneffekt fällt in den vergangenen Jahren aufgrund der Verordnung teurerer Medikamente sehr hoch aus, obwohl im Markt eine immer stärkere Substitution von Original- zu Generikapräparaten zu verzeichnen ist. In den nächsten zehn Jahren verlieren weltweit Arzneimittel mit einem Umsatz von derzeit ca. 50 Milliarden DM ihren Patentschutz.

BKK (2000), Arzneimittelvertragspolitik März 2000, S. 3.

Bundesverband der Pharmazeutischen Industrie e.V. (1999), Broschüre Pharmadaten 99, Frankfurt, S. 67.

BKK (2000), Arzneimittelvertragspolitik Mărz 2000.

Mehnert, A. (1998), Regulierung auf europäischen Arzneimittelmärkten, Bern, S. 131. www.ksk-pharma.de, Zugriff am 03.02.2000. 
Abbildung I - 3: Komponenten der Ausgabenentwicklung auf dem GKV-Arzneimittelmarkt

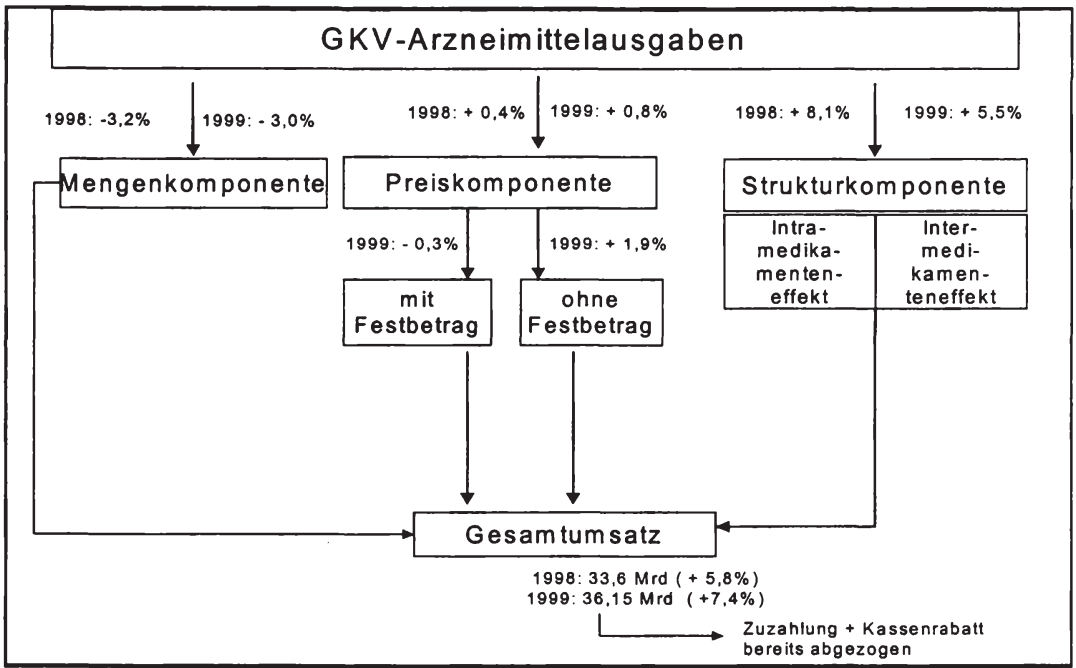

Quelle: INIFES, eigene Darstellung nach BKK (2000): Arzneimittelvertragspolitik, März 2000.

\subsubsection{Maßnahmen zur Ausgabendämpfung im Arzneimittelbereich}

Der Arzneimittelmarkt hat sich in Deutschland zu einem regulierten Markt entwickelt. Seit 1977 gibt es zahlreiche Versuche, die Ausgabensteigerung in der GKV zu bremsen, Beitragssatzstabilităt zu gewährleisten und Anreizmechanismen zum wirtschaftlichen Handeln der Marktteilnehmer zu implementieren. Eine Reihe von Kostendämpfungsmaßnahmen wurde in Form von Gesetzen erlassen. Mit dem Gesundheitsstrukturgesetz (GSG) 1993 und den darauffolgenden Neuordnungsgesetzen (NOG) 1996/97 kam es im Arzneimittelbereich zu Leistungsbegrenzungen. 
Abbildung I - 4: Übersicht zu Regulierungen im Arzneimittelmarkt

\begin{tabular}{|c|c|}
\hline Regelung & Inhalte der Regelung \\
\hline $\begin{array}{c}\text { GRG } \\
1989\end{array}$ & $\begin{array}{l}\text { - } \quad \text { Einführung der Festbetragsregelung } \\
\text { - } \quad \text { Erhöhung der Zuzahlung bei Arzneimitteln } \\
\text { - } \quad \text { Einführung der Negativliste } \\
\text { - Einführung von Richtgrößen für die Verordnung von Arznei- und Hilfsmittein }\end{array}$ \\
\hline $\begin{array}{c}\text { GSG } \\
1993\end{array}$ & $\begin{array}{l}\text { - Einführung eines globalen Arzneimittelbudgets und indikationsbezogener } \\
\text { Richtgrößen } \\
\text { - Senkung der Herstellerpreise für festbetragsfreie verschreibungspflichtige } \\
\text { Arzneimittel um } 5 \% \text {, für OTC-Produkte um } 2 \% \text {, Einfrieren der Preise bis } \\
\text { 1.1.1995 } \\
\text { - Einführung einer preisabhängigen Zuzahlung für Arzneimittel, Umstellung der } \\
\text { Zuzahlung auf packungsgrößenbezogene Zuzahlung } \\
\text { - Einführung einer Positivliste geplant, wird aber wieder verworfen }\end{array}$ \\
\hline $\begin{array}{l}\text { 1.NOG } \\
\text { 2.NOG } \\
1997\end{array}$ & $\begin{array}{l}\text { - } \quad \text { Arztindividuelle Richtgrößen lösen Arzneimittelbudgets ab } \\
\text { - } \quad \text { Erhöhung der Zuzahlungen für Arzneimittel }\end{array}$ \\
\hline $\begin{array}{l}\text { GKV- } \\
\text { Solidaritäts- } \\
\text { gesetz } \\
1999\end{array}$ & $\begin{array}{l}\text { - } \quad \text { Arzneimittelglobalbudget für } 1999 \\
\text { - } \quad \text { Senkung der Zuzahlungen für Arzneimittel (N1: } 8 \text { DM, N2: } 9 \text { DM, N3: } 10 \text { DM) } \\
\text { - } \quad \text { Uöhe der Festbeträge neu festgelegt: } \\
\text { - } \quad \text { Vermstellung der Medikation auf Generikaverordnungen im unteren Preisdrittel } \\
\text { - } \quad \text { Zweitmeinungsverfahren bei umstrittenen oder geringfügig nützlichen Arznei- } \\
\text { mitteln } \\
\text { - } \quad \text { Ausschluss von Bagatellarzneimitteln aus der Erstattung }\end{array}$ \\
\hline $\begin{array}{l}\text { GKV-2000- } \\
\text { Strukturge- } \\
\text { setzentwurf }\end{array}$ & $\begin{array}{l}\text { - Einführung einer Positivliste vorgesehen, dies wurde aber wieder verworfen } \\
\text { - Arzneimittelbudgets (konkretisiert durch GKV-SolG und Aktionsprogramm), im } \\
\text { Februar } 2001 \text { wurde allerdings eine Abschaffung der Arzneimittelbudgets an- } \\
\text { gekündigt }\end{array}$ \\
\hline
\end{tabular}

Quelle: INIFES, eigene Darstellung.

Erkennbar wurden die Leistungsbegrenzungen in Abbildung I - 2 anhand der Kurvenverläufe in den jeweiligen Zeiträumen. So fielen die Verordnungen von über einer Milliarde Packungen im Jahr 1992 auf 944 Millionen im Jahr 1993. Der Umsatz verringerte sich im gleichen Zeitraum um 4 Mrd. DM. Verstärkt wurde der Effekt durch eine Erhöhung der Zuzahlungen für Arzneimittel. Allerdings stiegen die Arzneimittelausgaben der GKV trotz Kostendämpfungsmaßnahmen langfristig wieder an.

Je nach der Ausrichtung der Kostendämpfung können die bisherigen Maßnahmen der Mengen- oder Preissteuerung zugeordnet werden. 
Abbildung I - 5: Regulierungsmechanismen der Arzneimittelpolitik der GKV

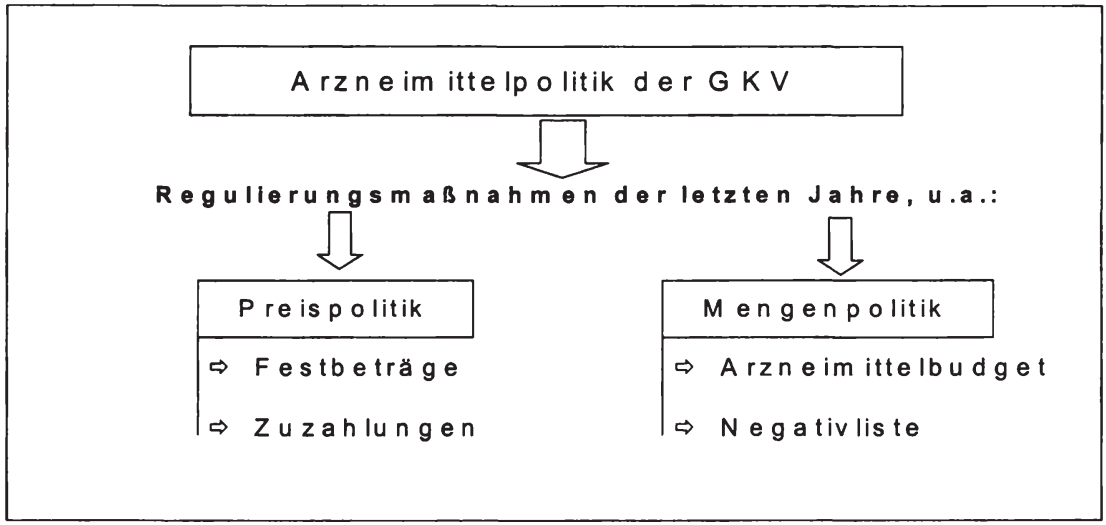

Quelle: INIFES, eigene Darstellung.

\section{$\rightarrow$ Festbeträge}

Ein Grund dafür, dass der Anstieg des Arzneimittelpreisniveaus in den letzten Jahren moderat verlief bzw. stagnierte (siehe Abbildung I - 3), ist in dem Regulierungsinstrument der Festbeträge zu suchen. Festbeträge stellen bei mehreren gleichwertigen zur Auswahl stehenden Arzneimitteln (Festbetragsgruppen) einen bestimmten, durch die GKV zu erstattenden, Höchstwert dar. ${ }^{10}$ Für den Patienten bedeutet dies, dass bei Auftreten einer Unterdeckung zwischen erstattungsfähiger Höchstgrenze und Medikamentenpreis dieser Teil des Medikamentenpreises selbst zu finanzieren ist. Allerdings waren $96,4 \%$ der ca. 24.500 Packungen unter Festbetragsregelung ohne Selbstbeteiligung erhältlich. Der festbetragsbezogene Umsatz entspricht ca. $47 \%$ der GKV-Arzneimittelausgaben bei einem Anteil von ca. $64 \%$ aller Verordnungen (Stand 1999). ${ }^{11}$ Nach Berechnungen des BKK Bundesverbandes können durch Festbeträge jährlich rund drei Milliarden DM eingespart werden. ${ }^{12}$ Allerdings stehen die Festbeträge in ihrer Umsetzung bzw. Anpassung für den Zeitraum bis 2003 noch in der Diskussion zwischen Politik, Krankenkassen, Pharmaindustrie, Großhandel und Apotheken.

Die Einführung dieser Regelung ist erst durch das Auftreten von Generika am Arzneimittelmarkt möglich geworden. Denn Originalpräparate, die noch dem Patentschutz unterliegen, werden nicht in Festbetragsgruppen erfasst. Für neue Arzneimittel unter Patentschutz können die Hersteller ihre Preise frei setzen, dies gilt auch für nicht apothekenpflichtige Arzneimittel. Die Festbetragsregelung greift in diesen Segmenten nicht.

BKK in: www.bkk.de/information, 14.03.00.

www.bkk.de, Zugriff am 13.01.00. 


\section{$\rightarrow$ Zuzahlungen, Selbstbeteiligungen}

Rezeptpflichtige festbetragsfreie Arzneimittel werden von den gesetzlichen Krankenkassen bei Erstattungsfähigkeit im vollen Umfang erstattet. Ansonsten befreit die Erstattungsfähigkeit eines rezeptpflichtigen Medikaments den Patienten häufig nicht vollständig von einer anteiligen finanziellen Selbstbeteiligung. Deren Ziel ist nicht nur die Erreichung der Beitragssatzstabilität, sondern auch die Schaffung einer Anreizwirkung zu einer ökonomischen Verhaltensweise. ${ }^{13}$ So hat der Patient bei verschreibungspflichtigen Arzneimitteln mit Festbeträgen z.T. eine Selbstbeteiligung zu tragen.

Eine von den oben genannten Mechanismen unabhängige Patientenselbstbeteiligung an den Arzneikosten für verordnete Medikamente erfolgt durch eine fixe Zuzahlung pro Medikamentenpackung. Im Zeitraum zwischen 1992 und 1997 wurde dieser Zuzahlungsbetrag viermal erhöht. 1999 erfolgte eine Senkung auf 8 DM, 9 DM oder $10 \mathrm{DM}$, welche eine Verringerung der entsprechenden finanziellen Mittel der GKV durch Zuzahlungen von 1998 mit rd. 5,5 Mrd. DM auf 4,0 Mrd. DM 1999 zur Folge hatte.

Dabei existiert in Deutschland wie auch in allen weiteren Mitgliedstaaten eine Sozialoder Überforderungsklausel für chronisch Kranke, Alte sowie soziale Härtefälle, um eine Überbelastung durch erhöhte Selbstbeteiligungen zu vermeiden. Diese Regelung befreit sowohl von der Packungszuzahlung als auch von der Selbstbeteiligung bei Übersteigen des Festbetrages vollständig oder teilweise. Dies entspricht dem Solidarprinzip der GKV. So waren 1998 35\% der Verordnungen zuzahlungsbefreit, 2000 sei sogar auf jede zweite Verordnung keine Zuzahlung entrichtet worden! $!^{15}$ Eine teilweise Befreiung nach § 62 SGB V erfolgte 1997 für etwa 24 Mio. Kinder, 10 Mio. Zuzahlungsbefreite, 250.000 Härtefälle sowie 65.000 Chroniker. Die Werte für 1998 werden aller Voraussicht nach höher liegen.

\section{$\rightarrow$ Arzneimittelbudgets}

Diese Regulierungsmaßnahme fungiert als Obergrenze der zu Lasten der GKV verschreibbaren Arzneimittel, und setzt damit an der Handlungsweise des Arztes an. Arzneimittelbudgets wurden erstmals mit dem GSG 1993 eingeführt und auf nationaler Ebene gedeckelt, bevor sie 1994 auf die einzelnen Regionen aufgeteilt wurden. Bei Überschreitung sollten die Ärzte in der Region haften. 1997 wurden die Budgets durch arztindividuelle Richtgrößen ersetzt, welche eine Individualhaftung der Ärzte vorsahen. Dabei blieb es jedoch nicht. Seit 1999 wurde durch das Aktionsprogramm zur Sicherung der Arznei- und Heilmittelbudgets erneut ein globales Arzneimittelbudget eingeführt, welches sich am Budgetvolumen von 1996 plus einer Zuwachsrate orientierte. Bei Überschreitung des Budgets müssten die Ärzte dieser Kassenärztli-

13

14

15

Wăhling, S., Schulenburg, J.-M. (1996), Regulierung und Eingriffshäufigkeit im Arzneimittelmarkt. Analyse anhand eines Simultatiosmodells, in: ifo Studien, Jg. 42, S. 469.

BKK (2000), Arzneimittelvertragspolitik März 2000, in: www.bkk.de /information/presse.

o. V. (2001), 55 Prozent der Rezepte ohne Zuzahlung, in: ApothekerZeitung, Jg. 17, Nr. 16/17 vom 23.04.01. 
chen Vereinigung bis maximal $5 \%$ des Budgets haften, weitergehende Überschreitungen gehen voll zu Lasten der Krankenkassen.

Allerdings sind die Arzneimittelbudgets seit Februar 2001 durch die Ankündigung einer Budgetabschaffung von Seiten des Bundesgesundheitsministeriums faktisch außer Kraft gesetzt und damit auch ihre kostendämpfende Wirkung. Entsprechend erhöhten sich die Arzneimittelausgaben der GKV gegenüber dem Vorjahr um 9,7\%.

\section{$\rightarrow$ Positiv-, Negativliste}

Die Erstattungsfähigkeit apothekenpflichtiger Arzneimittel durch die GKV wird anhand von Listen niedergelegt, die neben Deutschland in jedem EU-Staat existieren. Für die Erstattungsfähigkeit können grundsätzlich zwei Verfahren der Auflistung zur Anwendung gelangen: Negativlisten, welche die gelisteten Arzneimittel von der Erstattung ausschließen, und Positivlisten, die dagegen alle erstattungsfähigen Medikamente enthalten. In Deutschland existiert seit Jahren eine Negativliste. Kriterien für die Aufnahme eines Medikamentes in die Erstattungsfähigkeit sind im allgemeinen vom sozialen, wirtschaftlichen und therapeutischen Nutzen abhängig. Dabei bestehen in der Interpretation des wirtschaftlichen und therapeutischen Nutzens eines Präparates erhebliche Spielräume. In Deutschland gelten Arzneimittel als unwirtschaftlich und damit nicht erstattungsfähig, wenn ihr therapeutischer Nutzen nicht nachgewiesen ist und sie Stoffe beinhalten, die nicht mit ausreichender Sicherheit beurteilt werden können (§ 34, III SGB V). Viele Versuche einer neuen Gesundheitsgesetzgebung hin zu einer Positivliste scheiterten bisher an konträren Interessen der Beteiligten.

\section{$\rightarrow$ Exkurs: Regulierungsmaßnahmen in Europa}

Alle europäischen Länder haben im Laufe der 90er Jahre regulierend in den Arzneimittelmarkt eingegriffen. Unterschiede bestehen dabei hauptsächlich in der Intensität: In erster Linie konzentrierten sich die kostensenkenden Maßnahmen auf erstattungsfähige Arzneimittel:

- Die überwiegende Mehrzahl der Länder implementierte Positivlisten zur Regulierung der Erstattungsfähigkeit; Negativlisten existieren neben Deutschland nur noch in Großbritannien.

- Festbeträge existieren neben Deutschland derzeit noch in Dänemark, den Niederlanden und Schweden. Alle anderen Länder bedienen sich nicht dieses preispolitischen Instrumentes.

- Selbstbeteiligungen werden überall, allerdings in unterschiedlicher Form (z.B. als Fixbetrag, gestaffelt oder in Form prozentualer Beteiligungen) erhoben und orientieren sich zumeist an der Indikation, aber auch an den Arzneimittelpreisen.

- Einige Länder arbeiten zudem wie Deutschland mit Arzneimittelbudgets. Allerdings sehen die Sanktionsadressaten und -mechanismen jeweils recht unterschiedlich aus. 
Es zeigten sich folgende Resultate ${ }^{16}$ (Stand 1997/98):

- Die Kosten für Arzneimittel wurden vermehrt auf die Patienten in Form von Selbstbeteiligungen abgewälzt.

- Durchschnittlich wurden weniger Arzneimittel verordnet. In Italien und Deutschland zeigte sich eine Reduktion, in den übrigen Ländern nahm die Anzahl der verordneten Arzneimittel leicht zu, jedoch war der Zuwachs deutlich geringer.

- Die durchschnittlichen Ausgaben pro Verordnung nahmen aufgrund einer steigenden Anzahl mehr neuer und hochpreisiger Arzneimittel zu.

- Länder mit hohen Zuzahlungsregelungen wiesen ein niedriges Verordnungsniveau auf.

Die Regulierungsstrategien zeigten dabei typische „Pendelbewegungen": mit der Ausgangslage unzufrieden wurden grundlegende Veränderungen eingeleitet, das Pendel schlug in die entgegengesetzte Richtung aus. Zeigten sich dann Mängel und folgten daraufhin Maßnahmen, bewegte sich das Pendel wieder in Richtung Ausgangsposition. Zur Zeit befinden sich die Mitgliedsstaaten in unterschiedlichen Phasen dieses Zyklus. Einige kehren zu staatlichen Interventionen zurück, andere versuchen es mit marktwirtschaftlichen Instrumenten. Zudem bestätigt sich die eher kurzfristige Wirksamkeit von Maßnahmen: Jeder regulierende Ansatz weist Schlupflöcher auf und wirkt deshalb immer nur für begrenzte Zeit, eben so lange, bis die Marktbeteiligten sich mit den neuen Bedingungen arrangiert haben. Daraus leitet sich die $\mathrm{Er}$ kenntnis $a b$, dass keines der bisherigen Systeme zur Kostendämpfung dauerhaft wirkt. ${ }^{17}$

Osterreichisches Bundesinstitut für Gesundheitswesen (1998), Arzneimittel, Steuerung der Mărkte in neun europăischen Ländern, Wien, S. III f.

Osterreichisches Bundesinstitut für Gesundheitswesen (1998), Arzneimittel Steuerung der Märkte in neun europăischen Ländern, Wien, S IIIf. 
Abbildung I - 6: Arzneimittelausgaben im europäischen Vergleich (Stand 1997)

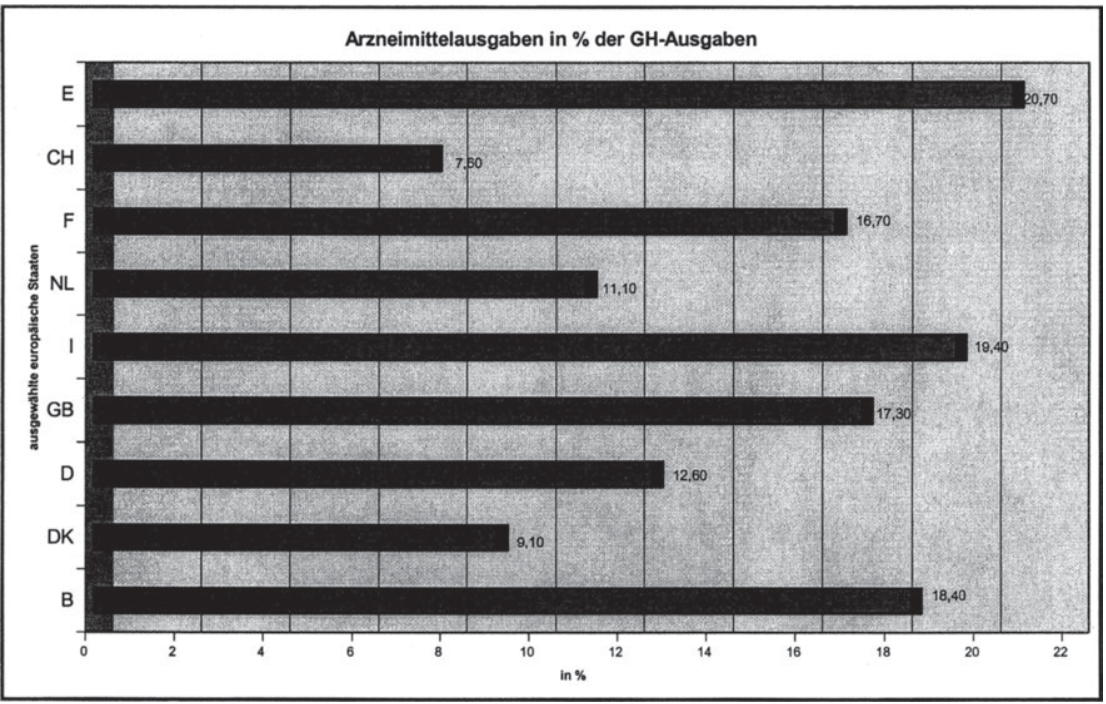

Quelle: INIFES, eigene Darstellung nach: VFA, (2000), Statistics 2000, S. $47 f$.

\subsubsection{Zwischenfazit}

Die bisherigen Maßnahmen in Form von Festbetragsregelungen, Zuzahlungen, Arzneimittelbudgets und Negativlisten konnten in Deutschland die Preise für Arzneimittel stabilisieren sowie die Anzahl der Verordnungen senken.

Eine langfristige Konsolidierung des Ausgabenniveaus der GKV für Arzneimittel - und damit die Gewährleistung einer wesentlichen Voraussetzung zur Beitragssatzstabilität - wurde jedoch nicht erreicht. Es gilt also, weitere Effizienzreserven im Arzneimittelmarkt aufzudecken, um dem kontinuierlichen Ausgabenanstieg entgegen zuwirken. Die bisherigen Regulierungsmaßnahmen fokussierten dabei im wesentlichen auf das Nachfrageverhalten der Akteure Arzt und Patient sowie auf die Preissetzung der Pharmahersteller.

In den Mittelpunkt der aktuellen Diskussion rücken nun verstärkt die im Vertriebsweg für Arzneimittel geltenden Regulierungen. Diese werden Gegenstand der nächsten Kapitel sein. Es gilt, deren möglichen Einfluss auf Ineffizienzen im System und damit auf hohe Arzneimittelausgaben zu untersuchen.

Denn „die Versicherten (haben) einen berechtigten Anspruch auf eine qualitativ hochwertige und kosteneffiziente Arzneimittelversorgung“. ${ }^{18}$

18

BKK (2000): Arzneimittelvertragspolitik, März 2000, S. 17. 


\subsection{Arzneimittelvertrieb}

Die wesentlichen Regelungen für den Vertrieb von Arzneimitteln finden sich in der Großhandelsbetriebs- und der Apothekenbetriebsordnung, im Apotheken und Arzneimittelgesetz, im Sozialgesetzbuch sowie in der Arzneimittelpreisverordnung. Daraus resultieren zum einen die streng regulierte Richtung bzw. Reihenfolge der Instanzen des Vertriebsweges vom Hersteller über den Großhandel in die Apotheke, zum anderen aber auch die Höhe der derzeitigen Vertriebskosten. Letztere werden in Abschnitt 1.2.3 behandelt. Im Folgenden geht es grundsätzlich um die einzelnen Vertriebsinstanzen und entsprechende nicht preisbezogene Regulierungen (Abschnitt 1.2.2).

Als erstes ist dabei zu klären, welche Arzneimittel über diesen Vertriebsweg distribuiert werden.

\subsubsection{Klassifikation von Arzneimitteln - Apothekenpflicht bedingt Vertriebsweg}

Was ist eigentlich ein Arzneimittel? § 2 AMG dazu: Arzneimittel sind Stoffe und Zubereitungen aus Stoffen zur Verhütung, Erkennung, Linderung, Beseitigung von Krankheiten, Beschwerden und Körperschäden von Mensch und Tier, wozu u.a. auch ärztliche, zahnärztliche oder tierärztliche Instrumente zur einmaligen Anwendung, Verbandstoffe, Pflaster, Watte, Diagnostika, Schwangerschaftsverhütungs- und Desinfektionsmittel zählen. Keine Arzneimittel sind: Lebensmittel, Tabakerzeugnisse, Kosmetika, Futtermittel, Medizinprodukte oder Organe.

In Deutschland sind danach ca. 52.000 Arzneimittel verkehrsfähig. ${ }^{19}$ In der EU beruhen mittlerweile alle nationalen Zulassungsregelungen für neue Arzneimittel, so auch das deutsche, auf einheitlichen Richtlinien. Mit dem Antrag auf Zulassung eines Medikaments für den Arzneimittelmarkt muss der Hersteller die erforderliche Qualität, Wirksamkeit und Unbedenklichkeit des Präparates nachweisen, die folgendes zum Inhalt haben:

Abbildung I - 7: Zulassungskriterien für neue Arzneimittel

\begin{tabular}{|l|l|l|l|}
\hline Kriterium & \multicolumn{1}{|c|}{ Wirksamkeit } & \multicolumn{1}{c|}{ Unbedenklichkeit } & \multicolumn{1}{c|}{ Qualität } \\
\hline $\begin{array}{l}\text { Begriffsbe- } \\
\text { deutung }\end{array}$ & $\begin{array}{l}\text { Bei bestimmungsmäßigem } \\
\text { Gebrauch Stiftung eines für } \\
\text { den Patienten therapeuti- } \\
\text { schen Nutzens }\end{array}$ & $\begin{array}{l}\text { Bei bestimmungsmäßigem } \\
\text { Gebrauch wird die Sicher- } \\
\text { heit des Patienten nicht ge- } \\
\text { făhrdet }\end{array}$ & $\begin{array}{l}\text { Keine Abweichung } \\
\text { von der Zusammen- } \\
\text { setzung nach Art und } \\
\text { Menge }\end{array}$ \\
\hline
\end{tabular}

Quelle: INIFES, eigene Darstellung.

18

ÖBIG (1998), Arzneimittelvertrieb in Europa, Wien, S. 63. 
Seit Inkrafttreten des AMG 1978 müssen alle neuen Arzneimittel ein Zulassungsverfahren beim Bundesinstitut für Arzneimittel und Medizinprodukte (BfArM) bzw. beim Paul-Ehrlich-Institut (PEI) durchlaufen, ehe sie in den Handel gebracht werden. Arzneimittel, die bereits vor 1978 auf dem Markt waren, müssen das erforderliche Zulassungsverfahren bis zum Jahr 2004 nachbringen. In Deutschland waren demnach 1998 nur 19.000 Arzneimittel nach AMG zugelassen, dazu kamen rund 30.000 sogenannte „Alt-Arzneimittel“, die unter die beschriebene Übergangsfrist fallen. Damit waren zum damaligen Zeitpunkt mehr als die Hälfte aller auf dem deutschen Markt befindlichen Arzneimittel noch nicht auf Wirksamkeit, Unbedenklichkeit, Qualität und Wirtschaftlichkeit untersucht. ${ }^{20}$

Die oben genannte Allgemeindefinition allein ist für die weiteren Betrachtungen nicht ausreichend. Eine Segmentierung des Arzneimittelbegriffes tut Not, insbesondere im Hinblick auf weiterführende Darstellung bezüglich der Arzneimitteldistribution über den Vertriebskanal Apotheke sowie der Finanzierung durch die gesetzliche Krankenversicherung. Damit rücken die Begriffe der Apothekenpflicht, der Verschreibungspflicht und der Erstattungsfähigkeit in den Vordergrund (siehe Abbildung I-8).

Abbildung I - 8: Apothekenpflicht, Verschreibungspflicht und GKV-Erstattungsfähigkeit

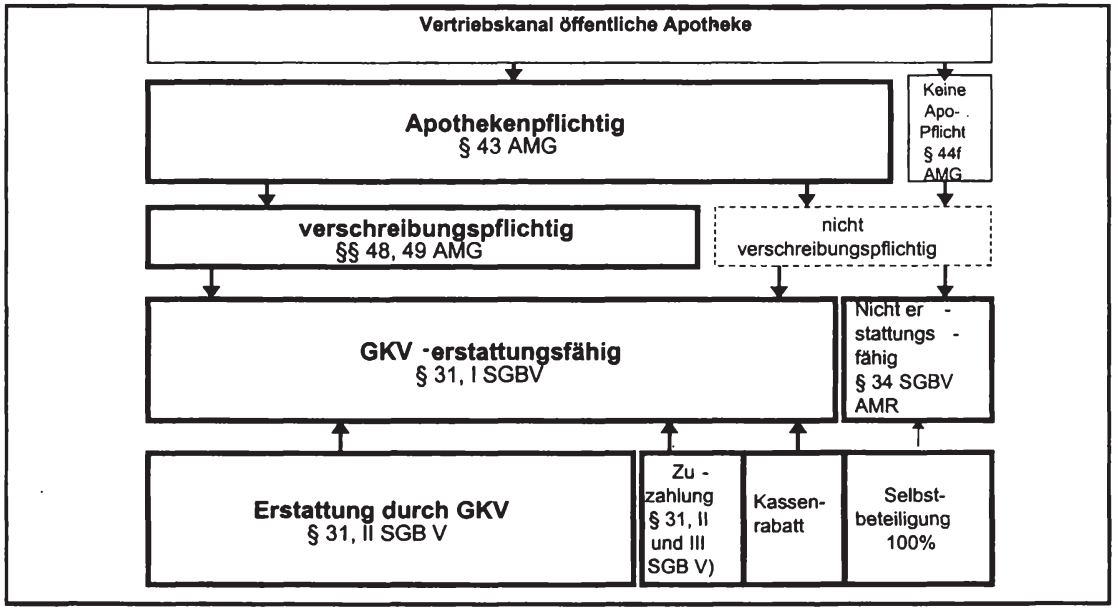

Quelle: INIFES, eigene Darstellung.

Für den Vertriebsweg der Arzneimittel ist entscheidend, ob die Arzneimittel der Apothekenpflicht nach § 43 AMG unterliegen. Fast alle apothekenpflichtigen Arzneimittel werden zu 99\% durch pharmazeutische Hersteller industriell gefertigt und als Fertigarzneimittel bezeichnet. Daneben stellen die Apotheken, wenn auch nur äußerst sel-

20

OBIG (1998), Arzneimittelvertrieb in Europa, Wien, S. 63. 
ten, anhand von Verordnungen der Ärzte sogenannte Magistralzubereitungen in eigenen Laboratorien her. Unter die Apothekenpflicht fallen alle Medikamente, die erklärungsbedürftig sind und Risiken beinhalten können: so alle verschreibungspflichtigen Medikamente nach §§ 48, 49 AMG, aber auch einige nicht verschreibungspflichtige Arzneimittel. Als einziger Vertriebsweg fungieren hier die Apotheken. Arzneimittel, für die keine Notwendigkeit der Beratung bzw. Risiken existieren, gelten als freiverkäuflich und können neben Apotheken z.B. auch in Drogerien vertrieben werden.

Mit der Verschreibungs- und Apothekenpflichtigkeit eines Arzneimittels eng verbunden ist die Erstattungsfähigkeit durch die GKV. Nach § 31, I SGB V haben gesetzlich Versicherte Anspruch auf die Versorgung mit apothekenpflichtigen Arzneimitteln, sofern diese nach § 34 SGBV nicht von der Erstattung ausgeschlossen sind. ${ }^{21} 1999$ gaben die gesetzlichen Krankenkassen dafür 36,15 Mrd. DM aus, im Jahr 2000 waren es 37,8 Mrd. DM (siehe Abbildung I-9). Unter die Erstattungsfähigkeit fielen ca. 40.000 bis 45.000 der über 52.000 Arzneimittel am Markt. ${ }^{22}$

Von den apothekenpflichtigen Arzneimitteln sind de facto nur die rezeptfreien Selbstmedikationen von der Erstattung nach SGB V ausgeschlossen. Aus Gründen der Kostendämpfung resultierten jedoch Ausschlüsse aus der Erstattungs- und damit auch Verschreibungsfähigkeit, basierend auf dem Wirtschaftlichkeitsgebot des $\S 12$ SGB V.

Mit der Klassifikation der Arzneimittel in solche mit und andere ohne Apothekenpflicht nach § 43, I AMG ist der Vertriebsweg vom Hersteller bis zum Nachfrager vorgegeben. Daraus resultieren eine klare Struktur und Richtung der Distribution vom Anbieter zum Nachfrager.

$\S 34$, (1) SGB V: ausgeschlossen sind u.a. Arzneimittel für Erkältungskrankheiten und grippale Infekte, Schnupfenmittel, Schmerzmittel, hustendämfende und -lösende Mittel, Mund- und Ra-

OBIG (1998), Arzneimittelvertrieb in Europa, Wien, S. 63. 
Abbildung I - 9: Vertriebsweg apothekenpflichtiger Arzneimittel

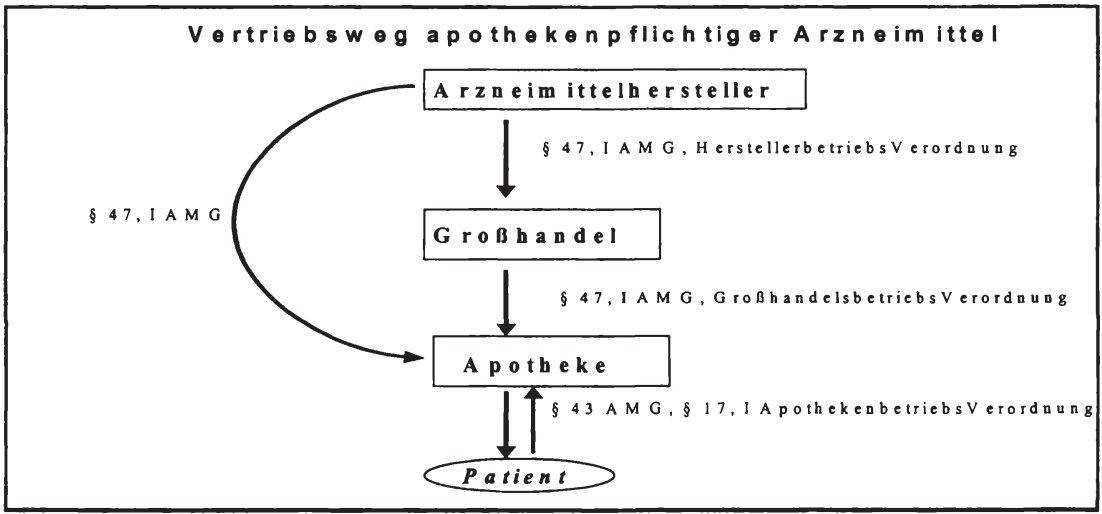

Quelle: INIFES, eigene Darstellung.

Die Herstellung und der Vertrieb beruhen dabei auf einer komplexen Anbieterkette. Deren vertikale Ausrichtung auf drei Ebenen wird durch das AMG und die sich darauf stützenden Pharma-, Großhandels- und Apothekenbetriebsordnung zementiert. Danach dürfen die Arzneimittelhersteller apothekenpflichtige Medikamente außer an Apotheken nur an andere pharmazeutische Unternehmen und Großhändler abgeben. $^{23}$ Ein Direktvertrieb der Präparate an den Patienten ist somit weder dem Arzneimittelhersteller noch dem Großhändler gestattet, sondern ausschließlich der Letztinstanz Apotheke. Diese Beschränkung des Vertriebswegs ist eine logische Konsequenz der Apothekenpflicht nach § 43,I AMG.

\subsubsection{Akteure des Arzneimittelvertriebs}

\subsubsection{Pharmazeutische Industrie - Herstellung}

Derzeit gibt es in Deutschland ca. 1100 Arzneimittelhersteller. ${ }^{24}$ Dabei variiert die Unternehmensgröße zwischen kleinen Herstellern bis zu multinationalen Konzernen, wobei die Mehrzahl kleine und mittelständige Firmen sind. Anders als kleine und Generika herstellende Unternehmen, die häufig nur einen Standort besitzen und nur über ein begrenztes Absatzgebiet verfügen, unterhalten große, vor allem forschende Unternehmen Standorte in verschiedenen Ländern und bieten ihre Produkte global an. $^{25}$ Die pharmazeutische Industrie zählte $1999 \mathrm{ca} .113 .000$ Mitarbeiter, die Arzneimittelproduktion lag zu Herstellerpreisen bei $36 \mathrm{Mrd}$. DM. Auf Arzneimittel, die über

23

Bestimmte Produkte wie Infusionslösungen, Impfstoffe oder Blutprodukte dürfen auch an andere, z.B. Ärzte, Krankenhäuser, Impfzentren usw. abgegeben werden.

24 Schleert, D. (1998), Strukturwandel der phramazeutischen Industrie - Auswirkungen auf den GKV - Arzneimittelmarkt, in: Die BKK, Jg. 86, Heft 11, S. 567.

Mehnert, A. (1998), Regulierung auf ... a.a.O., S. 81. 
öffentliche Apotheken vertrieben wurden, entfielen ca. 29 Mrd. DM, davon ca. 22 Mrd. DM (zu Herstellerpreisen) für den GKV-Arzneimittelmarkt. ${ }^{26}$

Deutschland ist ein Pharmaexportland, der Exportüberschuss lag 1999 bei 7,2 Mrd. DM. Die wichtigsten Abnehmerländer sind neben den USA die Schweiz und Frankreich. ${ }^{27}$ Im Arzneimittelmarkt als europäischen wie globalen Wachstumssektor - so sind z.B. mit Hilfe der Gen- und Biotechnologie für die Zukunft weitere Fortschritte in der Bekämpfung von Aids, Rheuma, Krebs und anderen chronischen Krankheiten zu erkennen - sehen sich Pharmahersteller einem zunehmenden Wettbewerb und Kostendruck gegenüber. Hauptsächlich die Größe eines Unternehmens wird in Zukunft über Erfolg und Misserfolg im Pharmageschäft entscheiden, denn die Kosten für die Forschung und neue Technologien steigen stetig. Führende Unternehmen bringen jedes Jahr bis zu drei neue Medikamente auf den Markt. Allein die Entwicklung eines einzigen erfolgreichen Arzneimittel kostet ca. 500 Millionen Dollar. ${ }^{28}$ Folge sind nationale wie internationale Konzentrationswellen. Vor allem in den USA, dem wichtigsten Medikamentenmarkt, laufen große Fusionswellen.

Die pharmazeutische Industrie kann nach den gesetzlichen Regelungen in Deutschland den pharmazeutischen Großhandel, die öffentlichen Apotheken, wie auch Drogerien oder Reformhäuser beliefern, letztere jedoch nur mit freiverkäuflichen Arzneimitteln. In der Praxis werden also direkt durch den Hersteller im wesentlichen die Instanzen außerhalb der öffentlichen Apotheken beliefert, der Apothekenmarkt selbst wird über die Zwischenhandelsstufe des pharmazeutischen Großhandels versorgt.

Die Preisbildung erfolgt gesondert in Kapitel 1.2.3.1.

\subsubsection{Distributionsstufe pharmazeutischer Großhandel}

Der pharmazeutische Großhandel nimmt bei apothekenpflichtigen Arzneimitteln die Distributionsstufe zwischen den Pharmaherstellern und den Apotheken ein. In der Regel ist der Großhandel der Abnehmer der Pharmazeutischen Industrie, sofern die Apotheken bei einigen Produkten - z.B. bei sogenannten rezeptfreien Schnelldrehern - nicht den Direktbezug vom Hersteller wählen. ${ }^{29}$ Die meisten deutschen Großhändler sind „full-line-wholesaler“, d.h. der Hersteller vertreibt seine Produkte über mehrere Großhändler. ${ }^{30}$

Ein Fremd- und Mehrbesitz im pharmazeutischen Großhandel ist zulässig, d.h. ein Pharmagroßhändler kann mehrere Großhandelsunternehmen besitzen. Zudem kön-

BPI, (2000), PharmaDaten 2000, S. 9.

BPI, (2000), PharmaDaten 2000, S. 5 ff.

Salz, J. (2000), Bedeutung verloren, in: Wirtschaftswoche Nr.4 / 20.01.2000, S. 60.

1996/97 betrug der Direktbezug vom Hersteller ca. 7\% des Arzneimittelumsatzes der Apotheken, in: Boström, H.C., Eichholz-Klein, S. (1998), Apotheke 2010, in: BBE Handelsbericht, S. 99.

Meyer, H.J. (1995), Pharmazeutischer Großhandel in Europa im Wandel, in: Pharmazeutische Industrie, Jg. 57, Heft 6, S. 432. 
nen auch Branchenfremde, also z.B. Pharmahersteller, im Besitz von Großhandelsunternehmen sein.

Auf Basis der deutschen Betriebsverordnung für Arzneimittelgroßhandelsbetriebe ist der Großhandelsvertrieb von Arzneimitteln jede Tätigkeit, die in der :

- Beschaffung,

- Lagerung,

- Lieferung oder

- Ausfuhr

von Arzneimitteln besteht.

Nach § 47 AMG darf keine Abgabe apothekenpflichtiger Medikamente durch den Großhandel an die Öffentlichkeit erfolgen. Diese ist den Apotheken vorbehalten. Kunden des Großhandels sind demnach nur andere Großhändler und Apotheken.

Die Breite des Sortiments an Arzneispezialitäten, mit denen in Apotheken gearbeitet wird (es war von ca. 45.000 Präparaten die Rede, siehe 0.1), hat zu einer weitgehenden Ausgliederung der Lagerhaltungsfunktion aus den Apotheken und zur Übernahme dieser Funktion durch den Großhandel geführt. Aufgaben wie die Sortimentierung, Lagerhaltung und Gewährleistung einer schnellen und unverzüglichen Belieferung der Apotheken gehören neben der Beschaffung zum Kerngeschäft des Großhandels. Bezüglich Dienstleistungsfunktionen für die vorgelagerte pharmazeutische Industrie werden die Lieferung von Marktdaten, die Unterstützung bei Marketingaktivitäten bis hin zur Übernahme der Zentrallagerfunktion für den Hersteller immer bedeutender. $^{32}$

Beide Gesetze, Großhandelsbetriebsverordnung wie AMG, verfügen darüber hinaus über weitere wesentliche Inhalte zum Handel mit Medikamenten, so z.B. über:

- die Qualifikation des Personals,

- die Sicherstellung der zuverlässigen Durchführung von Arzneimittelrückrufen (Notstandsplan),

- die Gewährleistung der Arzneimittelqualität während der Distribution,

- die Vorhaltung geeigneter und ausreichender Räumlichkeiten, Anlagen und Einrichtungen für die ordnungsgemäße Lagerung und den Vertrieb der Arzneimittel oder

- die Dokumentation aller Vorgänge.

Die meisten pharmazeutischen Großhändler in Deutschland sind regional begrenzt tätig, nur vier Unternehmen agieren bundesweit. ${ }^{33}$ Dabei bilden den Kern des Groß-

Weitere Ausnahmen siehe $\S 47, \mathrm{I}, 2 \mathrm{ff}$.

Meyer, H.J. (1995), Pharmazeutischer Großhandel ... a.a.O., S. 433.

OBIG (1998), Arzneimittelvertrieb in Europa, Wien, S. 67. 
handels in Deutschland 20 vollsortierte Unternehmen mit 106 Betriebstätten. Diese weisen im Schnitt etwa 100.000 Lagerpositionen auf, wovon rund 70.000 Positionen auf Arzneimittel und 30.000 Positionen auf das Randsortiment entfallen. ${ }^{34}$ Ist eine Apotheke bei einem Vollsortierer Kunde, wird sie in der Regel mehrmals täglich binnen weniger Stunden beliefert. Dies bedeutet für den Großhandel aufgrund der unverzüglichen Auftragserledigung und der relativ geringen Stückmengen einen hohen logistischen Aufwand. Vollsortierer zeichnen sich durch ein regionales, oft aber auch überregionales Vertriebsnetz aus.

Die vier Pharmagroßhändler Gehe, Sanacorp, Anzag und Phoenix teilen den deutschen Zuliefermarkt fast vollständig untereinander auf, insgesamt entfällt auf diese mehr als drei Viertel des Arzneimittelmarktes.

Abbildung I - 10: Pharmagroßhandel in Deutschland (Stand 1999)

\begin{tabular}{|l|c|}
\hline \multicolumn{1}{|c|}{ Pharma-Großhändler } & Marktanteile \\
\hline Gehe AG & $20 \%$ \\
\hline Sanacorp & $13 \%$ \\
\hline Noweda & $8 \%$ \\
\hline Anzag & $16 \%$ \\
\hline Phönix & $28 \%$ \\
\hline andere & $15 \%$ \\
\hline
\end{tabular}

Quelle: INIFES, eigene Darstellung nach: GEHE AG (2000), Geschäftsbericht 1999, S. 37.

Der vollsortierte Großhandel in Deutschland ist demzufolge hochkonzentriert. Der Marktführer Phönix AG ist international tätig, auch unterhält er einige Produktionsstätten für OTC-Präparate. An zweiter Stelle liegt die GEHE AG, welche nicht nur wie in Deutschland aufgrund der rechtlichen Situation ein pharmazeutischer Großhändler ist, sondern international auch im Apothekeneinzelhandel (in Großbritannien, Italien und Tschechien) ihre Geschäftsfelder besetzt. Bis 1999 war GEHE zudem im Versandhandel tätig. ${ }^{35}$ Den dritten und vierten Platz nehmen ANZAG bzw. SANACORP ein, hier halten jeweils Apotheker Beteiligungen am Unternehmen. ${ }^{36}$

Im teilsortierten Großhandel, der durch ein beschränktes Sortiment, eine höhere durchschnittliche Absatzhäufigkeit und des Wegfalls von Serviceleistungen wie Nacht- oder Sonntagbereitschaft gekennzeichnet ist, können aufgrund geringerer Stückkosten günstigere Preise an Apotheken offeriert werden. In der Regel führen diese keine seltenen Medikamente, Produkte mit geringer Haltbarkeit oder eilige Bestellungen. Die Teilsortimentierung kann entweder auf bewussten absatzpolitischen

\footnotetext{
34

Schöffski, O. (1995), Die Regulierung des deutschen Apothekenwesens, Baden-Baden, S. $165 f$.

35

GEHE AG (2000), Geschäftsbericht GEHE AG 1999, S. 3 u. S. 61.

ÖBIG (1998), Arzneimittelvertrieb in Europa, Wien, S. 69.
} 
Entscheidungen beruhen oder die Folge einer unzureichenden Kapitalausstattung sein; sie kann aber auch dadurch bedingt sein, dass zahlreiche Hersteller, vor allem der Groß- und Mittelindustrie, den Kreis der Großhandelskunden nicht ausweiten. ${ }^{37}$

Einige Großhändler betreiben Pharmahandel mit Arzneimitteln auch über die Ländergrenzen hinaus in Form von Reimporten und Parallelimporten. Deren Bedeutung am gesamten deutschen Arzneimittelmarkt beläuft sich auf ca. $1 \%{ }^{38}$, wobei auch Zahlen von bis zu $5 \%{ }^{39}$ kursieren. Unter "Reimport" versteht man dabei Arzneimittel, die ursprünglich im Hochpreisland selbst hergestellt und für das Zielland entsprechend verpackt wurden, durch den Hersteller und seine Vertriebskanäle in Niedrigpreisländer verbracht und dort auf dem Arzneimittelmarkt verkauft werden. Der ReImporteur kauft diese Medikamente unter Ausnutzung der Arbitragespanne vom Großhändler im Niedrigpreis- und importiert sie ins Einkaufsland. Beim „Parallelimport" läuft dieses Schema identisch ab, nur handelt es sich hier um Arzneimittel, die durch multinationale Pharmahersteller in einem EU-Mitgliedsstaat hergestellt wurden und in ausländischer Verpackung für den dortigen Markt bestimmt sind. Dabei ist Voraussetzung für einen Parallel- oder Reimport nach Deutschland, dass die betreffenden Arzneimittel, die aus dem EU-Mitgliedsstaat importiert werden, mit denen in der Bundesrepublik auch nach Warenkennzeichen identisch sind. ${ }^{40}$ Die europaweiten Regelungen für Parallel-/Re-Importeure werden als ausreichend für die Arzneimittelsicherheit angesehen und bilden damit die Grundlage für die gleichberechtigte Abgabe von Importprodukten aus EU-Mitgliedsstaaten. ${ }^{41}$ Der in Deutschland nach $\S \S 43$, 47 AMG vorgeschriebene Vertriebsweg ist dabei unbedingt einzuhalten, d.h. nach wie vor ist die Apotheke Abnehmer und Adressat des Parallel-/Re-Importeurs. Beide Fakten finden ihren Niederschlag in $\S 129$, I SGBV, der die Apotheker zur Abgabe günstiger Arzneimittel verpflichtet. $\mathrm{Da}$ die Re-/Parallelimportprodukte einen günstigeren Preis - im Durchschnitt sind sie zwischen $10 \%$ bis $15 \%$ billiger als inländische Originalprodukte ${ }^{42}$ - aufweisen, werden sie durch diese Klausel gefördert.

Die Preisbildung des pharmazeutischen Großhandels wird gesondert in Kapitel 1.2.3.2 dargestellt.

37

Ziegler, B. (1980), Arzneimittelversorgung und Wettbewerb: eine Analyse von Marktstruktur, Marktverhalten und Marktergebnis, Vandenhoeck und Ruprecht, Gottingen, S. 92.

Nach Angaben der Parallelimporteure und des Bundesverband der Arzneimittelimporteure.

Laut pharmazeutischer Industrie.

May, O. (1994), Parallelimporte - Eine Möglichkeit zur Sicherstellung der Arzneimittelversorgung? - Eine Möglichkeit zur Wettbewerbsbelebung auf dem Arzneimittelmarkt? in: Medizin Mensch Gesellschaft, Jg. 9, S. 85.

Schefold, C.P. (1997), Der "graue" Gemeinsame Markt pharmazeutischer Markenartikel, Aachen, S. $74 f$.

42

Rahner, E. (1989), Qualităt und Sicherheit von Arzneimittelimporten. Beanstandungen der Apotheker, in: Pharmazeutische Industrie, Jg. 51, Heft 12, S. 1332. 


\subsubsection{Vertriebsinstanz öffentliche Apotheke}

\section{$\rightarrow$ Strukturdaten:}

In Deutschland liegt mit einer allgemein hohen Apothekendichte eine polypolistische Marktstruktur mit vielen Verbrauchern und vielen Anbietern ('99: 21.590 Apotheken) vor. Eine Niederlassungsbeschränkung für Apotheker gibt es seit 1960 nicht mehr. Zwischen den einzelnen Bundesländern variiert die Apothekendichte relativ stark. Während 1999 im Saarland eine Apotheke durchschnittlich 2980 Personen versorgte und damit bundesweit die höchste Apothekendichte aufwies, waren dies in Brandenburg mit der geringsten Apothekendichte 4970 Einwohner, also vergleichsweise ca. zwei Drittel mehr Einwohner je Apotheke. Damit hat sich zwar in den letzten Jahren die Apothekendichte der neuen Bundesländern erhöht, allerdings noch nicht auf das Niveau der alten Bundesländer.

Abbildung I - 11: Apothekendichte in Deutschland, Stand 1998

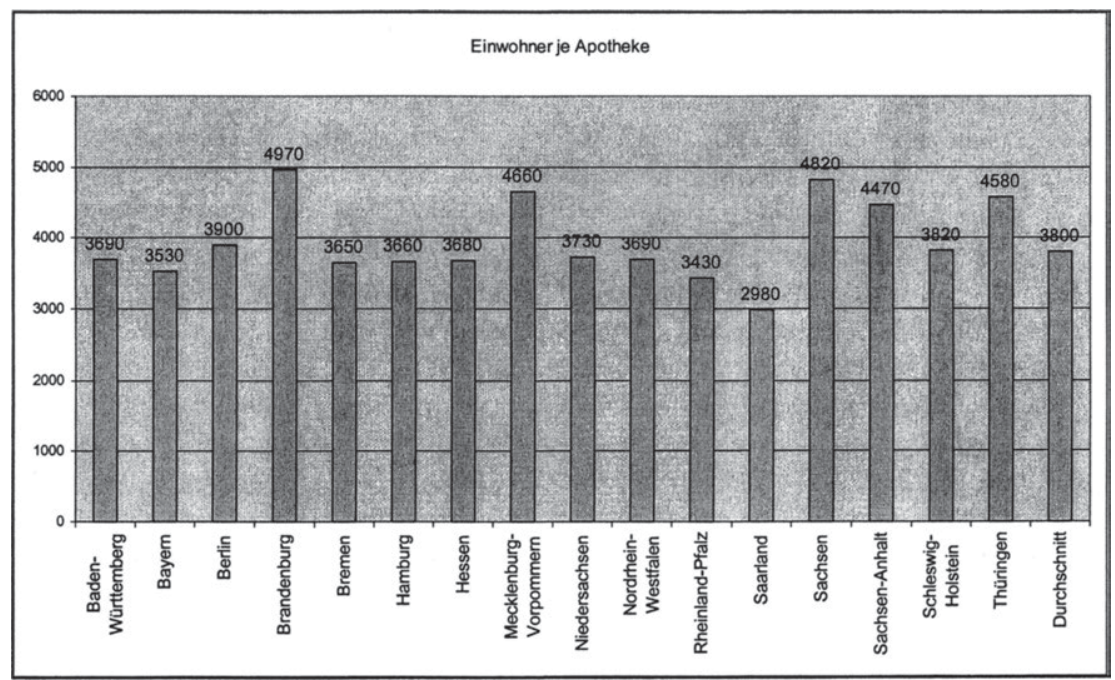

Quelle: ABDA (1999), Apotheke 1999: Zahlen, Daten, Fakten.

Weiterhin gibt es unter regionalen Gesichtspunkten in den Innenstadtbereichen viele Apotheken, in ländlichen Gebieten dagegen sind die Verbraucher oft an eine Apotheke gebunden. So ergaben Befragungen, dass $40,5 \%$ aller Apotheken in den Innenstadtlagen, aber nur $26,6 \%$ in ländlichen Gebieten operieren. Damit tendiert auf dem Land die polypolistische Struktur eher zu monopolistischen Verhaltensweisen. Weiterhin liegen nach dieser Befragung mehr als die Hälfte aller Apotheken in mittelgroßen Städten mit 5.000 bis 100.000 Einwohnern, nur jede zehnte Apotheke hatte 
sich in großen Städten mit über 100.000 Einwohnern niedergelassen. ${ }^{44}$ Damit ist der Wettbewerb in mittelgroßen Städten am größten, nicht wie meist angenommen in den Großstädten. Der Umsatz und damit auch die Größe der jeweiligen Apotheken stützen diese Beobachtung.

Abbildung I - 12: Lage und Größe (in Umsatzzahlen) der Apotheken (Apothekenbefragung 1995)

\begin{tabular}{|l|c|c|}
\hline \multirow{2}{*}{ Lage der Apotheke } & \multicolumn{2}{|c|}{ Größe der Apotheke } \\
& $<1,5$ Mio. Umsatz & $>1,5$ Mio. Umsatz \\
\hline Gemeinden mit weniger als 5.000 Einw. & $65,4 \%$ & $34,6 \%$ \\
\hline Gemeinden zwischen 5.000 und 20.000 Einw. & $45,0 \%$ & $55,0 \%$ \\
\hline Gemeinden über 20.000 bis 100.000 Einw. & $35,8 \%$ & $64,2 \%$ \\
\hline Gemeinden über 100.000 bis 500.000Einw. & $45,7 \%$ & $54,3 \%$ \\
\hline Gemeinden über 500.000 Einw. & $59,1 \%$ & $40,9 \%$ \\
\hline
\end{tabular}

Quelle: Schöffski, O (1995), Die Regulierung des deutschen Apothekenwesens, Baden-Baden, S. 222.

Umsatzstärkere Apotheken sind also nicht in den Großstädten überrepräsentiert, sondern vor allem in mittelgroßen Städten mit 20.000 bis 100.000 Einwohnern. In kleinen Gemeinden befinden sich tendenziell überwiegend die Apotheken mit geringem Umsatz, aber auch in den Großstädten mit über 500.000 Einwohnern. Hier scheint demzufolge die Polarisierung der Apotheken am stärksten zu Tage zu treten: den Großapotheken in den Innenstadtbereichen stehen viele kleine in den Außenbezirken gegenüber.

1999 lag der Umsatz laut ABDA im Durchschnitt bei ca. 2,2 Mio. DM netto je Apotheke (alte Bundesländer) ${ }^{45}$ bzw. bei ca. 2,5 Mio. DM Bruttoumsatz. Die Gewinne der Apotheken sind in etwa proportional zu deren Umsatz. So erzielen insbesondere die Apotheken hohe Gewinne, die in günstigen Lagen große Umsätze erzielen können, also vor allem in mittelgroßen Gemeinden und Innenstadtlagen. ${ }^{46}$ Die Umsatzrendite (das betriebswirtschaftliche Ergebnis), also das Ergebnis nach Abzug aller Kosten von der Apothekenbetriebsspanne, lag 1999 bei ca. $0,3 \%$ bis $0,4 \%$ vom Umsatz.

Fusionen mehrerer Apotheken, z.B. um Synergieeffekte bei den Kosten zu erzielen, sind in Deutschland im Gegensatz zu den Pharmaherstellern und den pharmazeutischen Großhandel, die einem wachsenden Wettbewerb sowie Kostendruck ausgesetzt sind, nicht erlaubt. Das sogenannte Fremd- und Mehrbesitzverbot des $\S 2$ Apothekenbetriebsordnung besagt, dass eine Apotheke durch den Apothekeneigentü-

\footnotetext{
44 Schöffski, O. (1995), Die Regulierung des deutschen Apothekenwesens, Baden - Baden, S. 221.

45 ABDA (1999), Die Apotheke Zahlen Daten Fakten, Abbildung 7.

Schöffski, O. (1996), Gedanken zur Deregulierung des deutschen Apothekenwesens, in: Zeitschrift für Wirtschaftspolitik (Lucius \& Lucius, Stuttgart), Jg. 45, Heft 2, S. 233.

47 ABDA (1999), Die Apotheke Zahlen Daten Fakten, Abbildung 13.
} 
mer, der nach $\S 2$ Gesetz über das Apothekenwesen die Betriebserlaubnis zum Führen einer Apotheke nur als approbierter Apotheker erhält, persönlich und eigenverantwortlich zu führen ist. D.h. eine Apotheke darf nur mit der pharmazeutischen Kompetenz eines Apothekers geführt werden (nicht jedoch z.B. durch ein Großhandelsunternehmen), zudem darf ein Apotheker nicht mehrere Apotheken führen. Beide Tatbestände werden zudem im $\S 7$ und $\S 2$ des Gesetzes über das Apothekenwesen detailliert geregelt. Diese restriktiven Regelungen gelten, wie schon angeklungen, nicht für den gesamten Vertriebsweg. Der Großhandel kennt weder Fremd- noch Mehrbesitzverbot. Jedoch ergab eine Apothekerbefragung von $1995^{48}$ eine große Mehrheit der Apotheker für das Fremd- wie Mehrbesitzverbot. Auch geht man heute von der Vermutung aus, dass bereits eine Reihe von Apothekern beherrschenden Einfluss auf mehr als eine Apotheke ausüben, z.B. durch sehr kurzfristige Mietverträge für Apothekenräumlichkeiten, deren Konditionen den Betriebsergebnissen der Apotheke angepasst werden. ${ }^{48}$ Der Hauptgrund für den mehrheitlichen Wunsch der Apotheker nach Beibehaltung der Verbote wird in der Besitzstandswahrung vermutet. Es zeigt sich eine „Herr-im-Haus"-Mentalität, eine Trennung zwischen Management und Besitz scheint für Apotheken kein Thema zu sein.

\section{$\rightarrow$ Warensortiment:}

Die Apotheke kann nicht als normales Einzelhandelsgeschäft bezeichnet werden. Sie ist weder in ihrer Sortiments- ${ }^{51}$, noch in ihrer Preisgestaltung frei. Zusätzlich werden an das Personal, die Lagerhaltung u.v.a.m. gesetzliche Bedingungen geknüpft. Auf der anderen Seite sichert die Apothekenpflicht ein Warenmonopol für die entsprechenden Präparate. Apotheken vertreiben in diesem Segment exklusiv, ohne den Wettbewerb weiterer Einzelhandelsunternehmen fürchten zu müssen.

Das Warenmonopol für Apotheken im Bereich der apothekenpflichtigen Arzneimittel ist dabei nicht spezifisch für Deutschland, sondern europaweit anzutreffen. In allen EU-Staaten existiert derzeit ein Warenmonopol aufgrund der Apothekenpflicht. Je nach Umfang der Apothekenpflicht fällt dabei der Umfang des Warenmonopols aus. Rezeptpflichtige Arzneimittel unterliegen überall der Apothekenexklusivität im Vertrieb. Viele Länder haben die Apothekenpflicht auf die gesamten Arzneimittel ausge-

\footnotetext{
48 Schöffski, O. (1995), Die Regulierung des deutschen Apothekenwesens, Baden - Baden, S. $201 \mathrm{ff}$

Schöffski, O. (1995), Die Regulierung des deutschen Apothekenwesens, Baden - Baden, S. 110.

Schöffski, O. (1995), Die Regulierung des deutschen Apothekenwesens, Baden - Baden, S. $231 \mathrm{ff}$.

Durch die umfassende Apothekenpflicht wird den Apotheken zwar ein Warenmonopol zugesichert, andererseits sind sie damit in ihrer zusätzlichen Sortimentsgestaltung stark eingeschränkt. Dinge, die Apotheken über die apothekenpflichtigen Arzneimittel hinaus anbieten möchten, werden durch das Randsortiment nach §25 ApoBO gesetzlich begrenzt.

So sehen $50 \%$ der Apotheker die Apothekenpflicht als Wettbewerbsvorteil, während dies nur 45\% der Kunden tun. Das Hauptklientel der Apotheken, Männer und Frauen über 60 Jahre, bejahen dies sogar nur zu 38\%. Aus: Riegl \& Partner GmbH, Institut für Management im Gesundheitsdienst (1995), Das Image der deutschen Apotheke, Augsburg, S. 135.
} 
dehnt. Das bedeutet, dass grundsätzlich jedes Medikament nur in Apotheken, nicht jedoch im übrigen Einzelhandel bezogen werden kann. Allerdings ist in den meisten Ländern die Definition für Arzneimittel und solche mit Rezeptpflichtigkeit bei weitem nicht so umfassend wie in Deutschland. So liegt die Anzahl der zugelassenen und erstattungsfähigen Arzneimittel in den anderen europäischen Ländern in der Regel bei maximal einem Fünftel der in Deutschland verkehrsfähigen 52.000 Präparate.

Abbildung I - 13: Anzahl der rezeptpflichtigen und zugelassenen Arzneimittel (Stand 1997/98)

\begin{tabular}{|l|c|c|}
\hline Land & $\begin{array}{c}\text { Rezeptpflichtige } \text { /*) erstattungsfähige }^{\text {Arzneimittel }} \\
\text { Niederlande }\end{array}$ & $\begin{array}{c}\text { Verkehrsfähige / zugelassene } \\
\text { Arzneimittel }\end{array}$ \\
\hline Schweden & 4.800 & 5.500 \\
\hline Dänemark & 2.346 & 3.502 \\
\hline Schweiz & 3.534 & 4.030 \\
\hline Frankreich & 4.816 & 7.764 \\
\hline Italien & 2.900 & 7.700 \\
\hline Osterreich & 6.643 & 9.064 \\
\hline GB & 6.285 & 10.236 \\
\hline Deutschland & 9.604 & 14.021 \\
\hline
\end{tabular}

Quelle: Osterreichisches Bundesinstitut für Gesundheitswesen (1998), Arzneimittel - Steuerung der Märkte in neun Europäischen Ländern, Wien.

Die öffentlichen Apotheken müssen jederzeit die Arzneimittelversorgung der Bevölkerung nach $\S 1$ Gesetz über das Apothekenwesen sicherstellen und die Verschreibungen des Arztes sofort ausführen. Dazu müssen die Arzneimittel nach $\S 15$ ApBetrO in einer dem Durchschnittsbedarf einer Woche vorgeschriebenen Menge vorrätig sein. Das bedeutet für die Apotheken eine äußerst komplexe Lagerhaltung. Diese wird allerdings immer häufiger, wie bereits erwähnt, durch den Großhandel übernommen. Um die Qualität der Arzneimittel zu garantieren, werden an die Betriebs- und Lagerräume der Apotheken nach § 4 ApBetrO hohe Anforderungen gestellt. Auch die Ausstattung mit Geräten ist vorgeschrieben, selbst wenn deren Bedeutung aufgrund des nur marginalen Anteils der Magistralzubereitungen irrelevant und damit eher kostentreibend ist.

\section{$\rightarrow$ Medikamentenabgabe und Beratung:}

Neben Vorschriften für den Apothekeneigentümer existieren weitere für das Apothekenpersonal ( $\$ 3 \mathrm{ApBetrO}$ ), welches aus pharmazeutischem Personal [approbierte Apotheker, pharmazeutisch-technischen Assistenten (PTA), Apothekerassistenten] und nichtpharmazeutischem Personal [Apothekenhelfer, Apothekenfacharbeiter, pharmazeutisch-kaufmännischen Angestellten (PKA)] besteht. In einer Apotheke arbeiten im Durchschnitt ca. 6,7 Beschäftigte (inklusive leitender Apotheker), keine Vollzeitangaben, davon sind ca. 2,5 approbierte Apotheker (inklusive leitender Apo- 
theker); 1,6 Beschäftigte arbeiten als PTA; 1,5 als PKA; die anderen verteilen sich auf andere Aufgabenbereiche.

Die Abgabe von apothekenpflichtigen Arzneimitteln darf nach $\S 17$ ApBetrO zum einen nur durch pharmazeutisches Personal wie Apotheker und PTA (diese eingeschränkt unter Anwesenheit eines Apothekers), und zum anderen nur in den Apothekenbetriebsräumen erfolgen. Wie bereits unter 1.1.1. Klassifikation von Arzneimitteln aufgeführt, basiert die Begründung einer Apothekenpflicht auf den Risiken eines Medikamentes wie dem Erklärungsbedarf. Mit dieser Regulierung des Vertriebswegs soll vor der Einnahme durch den Patienten eine pharmazeutische Beratung ermöglicht werden.

Gesetzlich geregelt ist diese Beratungspflicht in $\S 20$ ApBetrO: aus Gründen der Arzneimittelsicherheit muss der Apothekenkunde bei der Abgabe des Arzneimittels beraten werden. Durch die weitere Restriktion der Abgabe des Arzneimittels in den Räumen einer Apotheke wird auf eine persönliche Kommunikation face-to-face zwischen pharmazeutischem Personal und Patienten abgestellt. Mit der Beratung darf jedoch nicht bei Verordnung auf Rezept die Therapie des verordnenden Arztes beeinträchtigt werden, d.h. eine Kritik der ärztlichen Diagnose oder Verschreibung ist den Apotheken nicht gestattet. Die selbständige Beratung des Apothekers erstreckt sich im wesentlichen auf die Selbstmedikation.

Allerdings zeigen Patientenbefragungen im Bereich der Selbstmedikation, dass es an Beratung in den Apotheken mangelt. Eine Umfrage im Jahre 1995 ergab, dass nur $30 \%$ der Apotheken ihre Beratung verbessern und intensivieren möchten, um drohenden Umsatzeinbußen, damals aufgrund des GSG, begegnen zu können. Fast ein Viertel der Apotheker hatte diesbezüglich keinerlei Ideen. ${ }^{54}$ Auch die Stiftung Warentest stieß in ihrem Apothekentest 1999 auf lustlose Berater. So verkaufte jede dritte Apotheke ohne Beratung, manchmal fühlten sich die Kunden sogar zum Kauf gedrängt. Auf Preisunterschiede wurde nie hingewiesen. Die Beratung, falls erfolgt, wurde in weniger als der Hälfte der Fälle als gut eingeschätzt. Die Frage nach Symptomen, Krankheiten und Lebensumständen wurde, wenn überhaupt, nur oberflächlich behandelt. Zudem bestand bei den Apothekern eine sehr hohe Schwelle zum Anraten eines Arztbesuches.

Mit der Gewährleistung der Beratung einher geht neben der pharmazeutischen Kompetenz des Apothekenpersonals der zweite Aspekt des $\S 17 \mathrm{ApBetrO}$, nämlich dass die Abgabe des Medikamentes und damit zeitlich zusammenfallend auch die Beratung in den Räumen der Apotheke zu erfolgen hat. Das heißt, der Patient hat einer

53

Hasan-Boehme, U., u.a., Tätigkeits- und Kostenprofile der Apothekenmitarbeiter, in: www.pharmazeutische-zeitung.de/pza/1999-39, Tabelle 1 sowie

54 www.pharmazeutische-zeitung.de/pza/1999-35, Tabelle 1, Zugriff am 21.11.00.

Schöffski, O. (1995), Die Regulierung des deutschen Apothekenwesens, Baden - Baden, S. $240 f$.

O.V. (1999), Beratung in Apotheken - Risiken und Nebenwirkungen, Apothekentest bei Präparaten der Selbstmedikation, in: Stiftung Warentest 7/99, S. 94-99. 
Anwesenheitspflicht in der Apotheke Folge zu leisten, um die verordneten Arzneimittel zu empfangen und eine Beratung zu erhalten.

Aus Befragungsergebnissen ist jedoch der Umstand abzuleiten, dass Kunden der Apotheke zu 60\% kranke Menschen sind. Mehr als jeder sechste Apothekenkunde hat dabei eine chronische Krankheit mit stetigem Arzneimittelbedarf. ${ }^{56}$ Aufgrund der auf die Apothekenräume begrenzten Abgabe muss er trotz gesundheitlicher Beeinträchtigungen diesen Weg absolvieren, sofern er keinen „Besorger" für die Medikamente zur Hand hat. $77 \%$ der Kunden müssen regelmäßig monatlich mindestens einmal zur Apotheke. Davon sind $87 \%$ Dauerpatienten, ca. $80 \%$ sind Menschen über 60 Jahre.

Viele Apotheker greifen deshalb auf die Ausnahmeregelungen des $\S 17$, II ApBetrO, welcher in begründeten Einzelfällen eine Versendung aus der Apotheke oder die Zustellung durch einen Boten ${ }^{58}$ zubilligt, zurück. Hier existiert eine Grauzone. Befragungen haben ergeben, dass Hausbelieferungen durch Apotheken gängige Praxis, und nicht, wie gesetzlich intendiert, eine Einzelfallösung ist. Im Durchschnitt beliefern Apotheken ca. 30 Kunden pro Woche. Hier kann nicht mehr von begründeten Einzelfällen die Rede sein. Der Spitzenwert lag bei 1.200 Kundenbelieferungen pro Woche, hier könnte man schon fast von einem Versandhandel sprechen. ${ }^{59}$ Wie bereits unter 0.1 thematisiert, stellt sich hier allerdings die Frage der Gewährleistung einer Beratung. Der Bote, der die Arzneimittel bringt, ist häufig Student oder Rentner, also nicht zur Beratung befugt. ${ }^{60}$ War aber der Patient also zur Einlösung des Rezeptes nicht selbst in der Apotheke anwesend ${ }^{61}$, erfolgt in solchen Fällen keine direkte Beratung des Patienten durch die Apotheke.

Abbildung I - 14: Direktlieferungen von Arzneimitteln an den Kunden nach Hause (1995)

\begin{tabular}{|l|c|c|c|c|c|}
\hline & $\begin{array}{c}\text { Keine Hausbe- } \\
\text { lieferung }\end{array}$ & $\begin{array}{c}\text { Bis zu } 5 \mathrm{mal} \\
\text { pro Woche }\end{array}$ & $\begin{array}{c}\text { Bis zu } 10 \mathrm{mal} \\
\text { pro Woche }\end{array}$ & $\begin{array}{c}11 \text { und } 50 \mathrm{mal} \\
\text { pro Woche }\end{array}$ & $\begin{array}{c}>50 \text { mal pro } \\
\text { Woche }\end{array}$ \\
\hline $\begin{array}{l}\text { In \% der Apo- } \\
\text { theken }\end{array}$ & 5,4 & 28,1 & 26,7 & 32,3 & 7,7 \\
\hline
\end{tabular}

Quelle: Schöffski, O. (1995), Die Regulierung des deutschen Apothekenwesens. Eine ökonomische Analyse, Nomos Verlagsgesellschaft, Baden-Baden, S. 238.

Die Preisbildung der Apotheke wird unter 1.2.3.2 dargestellt.

\footnotetext{
56

Riegl \& Partner GmbH, Institut für Management im Gesundheitsdienst (1995), Das Image der deutschen Apotheke, Augsburg, S. 35.

Riegl \& Partner GmbH, Institut für Management im Gesundheitsdienst (1995), Das Image der deutschen Apotheke, Augsburg, S. 55.

Dieser muss nicht zum Apothekenpersonal gehören. Schöffski, O., Die Regulierung des deutschen Apothekenwesens, Baden - Baden S. 124. werden die Auslieferungen vom Apothekenpersonal übernommen.

61 Besorgerproblematik, siehe 0.1 .
} 


\subsubsection{Versandhandelsverbot für apothekenpflichtige Arzneimittel}

Auch wenn auf die Einzelfallösung des $\S 17$ ApBetrO durch die öffentlichen Apotheken in mehr als nur den „begründeten Einzelfällen“ zurückgegriffen wird, und dies sicherlich aus gutem Grund, wie ein Blick auf die Struktur der Apothekenkunden bestätigte, darf dieser Umstand nicht darüber hinwegtäuschen, dass durch den § 43,I AMG wie den $\S 17$, I ApBetrO die Abgabe von apothekenpflichtigen Arzneimitteln außerhalb der Betriebsräume einer Apotheke nicht gestattet ist.

In Deutschland dürfen damit auch alle verordneten Arzneimittel nur in Apotheken und nicht im Wege des Versandes an den Patienten gebracht werden. Konsequenterweise ist auch eine Werbung, die darauf abzielt, apothekenpflichtige Arzneimittel im Wege des Versandes in Deutschland zu beziehen, nach $\S 8$ Heilmittelwerbegesetz (HWG) unzulässig. Beide Regelungen verankern das Versandhandelsverbot für apothekenpflichtige Arzneimittel in Deutschland.

Abbildung I - 15: Regelungen zum Versandhandelsverbot in Deutschland

Fundament des Versandhandelsverbotes für apothekenpflichtige Arzneimittel

§ 43, I AMG, § 17, I ApBetrO:

Apothekenpflichtige Arzneimittel dürfen an den Endverbraucher nur in Apotheken und nicht im Wege des Versandes abgegeben werden.
§ 8 HWG:

Unzulässig ist eine Werbung, die darauf hinwirkt, apothekenpflichtige Arzneimittel im Wege des Versandes zu beziehen.

Quelle: INIFES, eigene Darstellung.

Erlaubt und üblich ist der Versandhandel in Deutschland derzeit nur für Verband- und Heilmittel, Medizinprodukte sowie freiverkäufliche Arzneimittel.

\subsubsection{Vertriebswege für Arzneimittel im europäischen Vergleich}

Schon die Darstellung der Anzahl der verkehrsfähigen Präparate brachte extreme Differenzen im Sortimentumfang zwischen den einzelnen europäischen Staaten zutage. Deutschland liegt mit $\mathbf{5 2 . 0 0 0 ~ v e r k e h r s f a ̈ h i g e n ~ A r z n e i m i t t e l n ~ a n ~ d e r ~ S p i t z e , ~ a n - ~}$ dere Länder weisen nur ein Fünftel bis ein Zehntel des deutschen Arzneimittelspektrums auf. Zudem differiert der Umfang der rezeptpflichtigen und erstattungsfähigen Präparate. Gegen die immense Anzahl verkehrsfähiger und apothekenpflichtiger Arzneimittel, wie sie in Deutschland anzutreffen ist, sprechen im wesentlichen Produkttransparenzkriterien. So erscheint es nicht verwunderlich, dass die wenigsten Medikamente - nämlich insgesamt nur ca. 2000 Präparate - eine entscheidende Rolle bei der Umsatzbildung am Arzneimittelmarkt spielen: auf sie entfallen ca. $90 \%$ der Arzneimittelausgaben. ${ }^{62}$ Trotz der Harmonisierung von Zulassungsverfahren in Euro- 
pa sind also noch erhebliche Unterschiede auszumachen, die einem einheitlichen Binnenmarkt für Arzneimittel hinderlich sind.

Zudem unterscheiden sich die europäischen Staaten hinsichtlich ihrer Apothekendichte. Deutschland weist allgemein ein dichtes Netz an Apotheken auf und liegt im europäischen Vergleich im vorderen Feld. Im Vergleich dazu versorgen Apotheken in Dänemark, den Niederlanden oder Schweden fast das Drei- bis Fünffache an Einwohnern. Die Schweiz oder Belgien haben die höchste Apothekendichte.

Abbildung I - 16: Apothekendichte Stand 1998 / 1997*

\begin{tabular}{|l|c|c|}
\hline \multicolumn{1}{|c|}{ Land } & Einwohner je Apotheke & Niederlassungsbeschränkungen \\
\hline Belgien & $1.934^{\star}$ & JA \\
\hline Dänemark & $17.869^{*}$ & JA \\
\hline Deutschland & 3.800 & NEIN \\
\hline Frankreich & 2.589 & JA \\
\hline GB & 4.769 & NEIN \\
\hline Italien & 3.579 & JA \\
\hline Niederlande & 10.146 & NEIN \\
\hline Schweden & 9.860 & JA \\
\hline Spanien & $2.115^{\star}$ & JA \\
\hline Schweiz & 1.400 & NEIN \\
\hline
\end{tabular}

Quelle: INIFES, eigene Darstellung nach:

Osterreichisches Bundesinstitut für Gesundheitswesen (1998), Arzneimittel. Steuerung der Märkte in neun europäischen Ländern, Wien.

Die Ausprägung der Apothekendichte ist auf nationale Regelungen zur Niederlassungsfreiheit zurückzuführen, wie auf die Handhabung des Fremd- und Mehrbesitzverbotes in den einzelnen Ländern. Einige Länder beanspruchen für sich eine sehr strikte Regelung durch das gleichzeitige Bestehen eines Fremd- und Mehrbesitzverbotes. Andere wieder verbieten den Fremdbesitz, lassen aber einen Mehrbesitz zu. In einigen wenigen Staaten ist Fremd- wie Mehrbesitz an Apotheken gesetzlich erlaubt.

Abbildung I - 17: EU - Fremd- und Mehrbesitzverbot

\begin{tabular}{|l|c|c|}
\hline Land & Fremdbesitzverbot & Mehrbesitzverbot \\
\hline Belgien & $\mathrm{X}$ & $\mathrm{X}$ \\
\hline Dänemark & $\mathrm{X}$ & $\mathrm{X}$ \\
\hline Deutschland & $\mathrm{X}$ & \\
\hline Frankreich & & \\
\hline GB & $\mathrm{X}$ & \\
\hline Italien & $\mathrm{X}$ & $\mathrm{X}$ \\
\hline Niederlande & $\mathrm{X}$ & \\
\hline Spanien & & \\
\hline Schweiz & & \\
\hline
\end{tabular}

Quelle: INIFES, eigene Darstellung nach: Osterreichisches Bundesinstitut für Gesundheitswesen (1998), Arzneimittel Vertrieb in Europa, Wien. 
Ein Fremd- wie Mehrbesitzverbot besteht neben Deutschland auch in Dänemark, Frankreich oder Spanien. Eine Kettenbildung oder z.B. ein Fremdbesitz von Apotheken durch den Pharmagroßhandel oder -hersteller ist hier nicht möglich. Liberaler gestaltet sich der Besitz in den Niederlanden und Italien. Hier ist entweder ein Fremdbesitzverbot oder ein Mehrbesitzverbot geregelt. Weder ein Fremd- noch ein Mehrbesitzverbot herrschen in Belgien oder Großbritannien vor. Damit ist eine Apothekenkettenbildung, aber auch der Fremdbesitz durch andere Akteure des Pharmasektors wie durch Krankenkassen legal. So wird in Großbritannien der Apothekenmarkt durch die Kette Boots stark dominiert. Aber z.B. auch deutsche Großhändler sind in Großbritannien am Apothekenmarkt aktiv. So unterhält die GEHE AG dort eine eigene Apothekenkette.

Ein Versandhandelsverbot für rezeptpflichtige Arzneimittel existiert wie in Deutschland in den meisten Staaten der EU. Zwar ist von Seiten der europäischen Rechtssprechung in Form des Fernabsatzgesetzes der Versandhandel von Arzneimitteln grundsätzlich legalisiert, allerdings steht es den einzelnen Ländern zu, im Rahmen der Sicherung der Arzneimittelversorgung der Bevölkerung eigene Regelungen zu erlassen. Nur die Niederlande, Großbritannien und die Schweiz haben Versandapotheken unter der Bedingung der Einhaltung wesentlicher Standards gesetzlich legitimiert. Schweden wird ab 2002 den Versandhandel im Segment der rezeptpflichtigen Arzneimittel zulassen. Alle anderen Länder erlauben keine Versandapotheken.

Allerdings ist im Zuge des Internets und des länderübergreifenden elektronischen Handels insbesondere im Bereich der Arzneimitteldistribution erkennbar, dass nationale Regelungen zur Regulierung des Vertriebsweges ihre Wirkung einbüßen. Grenzüberschreitender Versand von Arzneimittein aus europäischen Ländern, in denen ein Versandhandel mit rezeptpflichtigen Arzneimitteln erlaubt ist, nach Deutschland finden mehr und mehr über dieses Medium statt. Auf diese Art des Versandhandels, der im wesentlichen auf dem Medium Internet basiert, wird im Abschnitt 1.4 eingegangen.

\subsubsection{Kosten des Vertriebsweges}

\subsubsection{Preisbildung der pharmazeutischen Industrie}

Die Herstellerpreise unterliegen in Deutschland keiner direkten gesetzlichen Preisfestsetzung durch den Staat. Bei der Preisfindung der Arzneimittelhersteller finden in der Regel nicht nur die Preise der jeweiligen Konkurrenzunternehmen Berücksichtigung, sondern auch die Qualität der Arzneimittel, unterschiedliche Behandlungsmethoden und Krankheitsstrukturen mit Auswirkungen auf den Verbrauch und damit den Umsatz der Arzneimittel.

Die Preissetzung ist jedoch indirekt beeinflusst durch Regelungen der GKVErstattungsfähigkeit, wie z.B. der unter 1.1.2. dargestellten Maßnahme der Festbeträge. Durch das Festlegen von Erstattungshöchstgrenzen werden die Herstellerprei- 
se indirekt beeinflusst und das Preisniveau für Arzneimittel relativ konstant gehalten (siehe dazu Punkt 1.1.1.). Versuche der Hersteller, ihre Produkte preislich über den Festbeträgen zu positionieren, führten aufgrund der erhöhten Zuzahlung der Patienten zu deutlichen Umsatzrückgängen. ${ }^{63}$ Des weiteren fanden im Zuge der Kostendämpfung generelle Preisstopps und Preiszu- bzw. abschläge ihre Anwendung. So schrieb das GSG einen auf zwei Jahre befristeten Preisstopp sowie eine Preissenkung von 5\% für verschreibungspflichtige Arzneimittel für den Zeitraum 1993 bis 1995 vor.

\subsubsection{Festlegung der Preisbildung von Großhandel und Apotheken durch die Arzneimittelpreisverordnung}

Auch wenn die Hersteller in ihrer Preissetzung relativ unabhängig sind, so bedeutet dies nicht, dass auch innerhalb der Arzneimitteldistribution die Preise durch die Großhändler wie die Apotheken frei gesetzt werden können. Die Arzneimittelpreisverordnung, gültig für alle apothekenpflichtigen Arzneimittel, setzt hier einen restriktiven Rahmen. Freiverkäufliche Arzneimittel unterliegen dieser Preissetzung nicht, hier können die Preise durch den Großhandel wie die Apotheken frei gesetzt werden.

\section{Preisbildung Großhandel:}

In Deutschland wird der Großhandelspreis für apothekenpflichtige Arzneimittel gestaffelt und durch die Arzneimittelpreisverordnung (AMPreisV) festgelegt. Danach errechnet sich für alle apothekenpflichtigen Arzneimittel der Großhandelspreis (= Apothekeneinkaufspreis) aus dem Herstellerabgabepreis plus einem Höchstzuschlag. Die AMPreisV besitzt auf der Großhandelsstufe den Charakter einer Höchstpreisverordnung, d.h. es werden maximale Sätze bzw. DM-Beträge genannt, die auf den Herstellerabgabepreis aufgeschlagen werden dürfen. Geringere Zuschläge sind jederzeit möglich. Die maximal zulässigen Zuschlagssätze verlaufen degressiv, d.h. mit steigender Basis wird der Zuschlagssatz geringer, trotzdem steigt der absolute Zuschlag. ${ }^{64}$ Die festgelegten Aufschlagsätze für den Großhandel wurden mit der Änderung der AMPreisV im Juli 1998 im höherpreisigen Arzneimittelsegment ab 1339 DM abgesenkt. 
Tabelle I - 1: Amtliche Höchstaufschlagsätze für den Großhandel ab Juli 1998

\begin{tabular}{|c|c|c|c|c|c|}
\hline \multicolumn{2}{|c|}{ Herstellerpreis } & \multirow{2}{*}{$\begin{array}{l}\text { Großhandelsauf- } \\
\text { schlag in \% d. Her- } \\
\text { stellerpreises. }\end{array}$} & \multirow{2}{*}{$\begin{array}{c}\text { Großhandels- } \\
\text { spanne in \% d. } \\
\text { AEK } \\
\end{array}$} & \multirow{2}{*}{ Zuschläge in DM } & \multirow{2}{*}{$\begin{array}{c}\text { Apothekeneinkaufs- } \\
\text { preis (AEK) }\end{array}$} \\
\hline Von (in DM) & bis (DM) & & & & \\
\hline 0,00 & 1,65 & $21,0 \%$ & $17,4 \%$ & $0,00 \mathrm{DM}$ bis $0,35 \mathrm{DM}$ & 0,00 bis 2,00 \\
\hline 1,66 & 1,73 & & & $0,35 D M$ & 2,01 bis 2,08 \\
\hline 1,74 & 3,33 & $20,0 \%$ & $16,7 \%$ & $0,35 \mathrm{DM}$ bis $0,67 \mathrm{DM}$ & 2,09 bis 4,00 \\
\hline 3,34 & 3,42 & & & $0,67 D M$ & 4,01 bis 4,09 \\
\hline 3,43 & 5,02 & $19,5 \%$ & $16,3 \%$ & $0,67 \mathrm{DM}$ bis $0,98 \mathrm{DM}$ & 4,10 bis 6,00 \\
\hline 5,03 & 5,15 & & & $0,98 D M$ & 6,01 bis 6,13 \\
\hline 5,16 & 7,14 & $19,0 \%$ & $16,0 \%$ & $0,98 \mathrm{DM}$ bis $1,36 \mathrm{DM}$ & 6,14 bis 8,50 \\
\hline 7,15 & 7,34 & & & $1,36 \mathrm{DM}$ & 8,51 bis 8,70 \\
\hline 7,35 & 11,81 & $18,5 \%$ & $15,6 \%$ & $1,36 \mathrm{DM}$ bis $2,19 \mathrm{DM}$ & 8,71 bis 14,00 \\
\hline 11,82 & 12,14 & & & $2,19 D M$ & 14,01 bis 14,33 \\
\hline 12,15 & 17,80 & $18,0 \%$ & $15,3 \%$ & $2,19 \mathrm{DM}$ bis $3,20 \mathrm{DM}$ & 14,34 bis 21,00 \\
\hline 17,81 & 21,36 & & & $3,20 \mathrm{DM}$ & 21,01 bis 24,56 \\
\hline 21,37 & 86,96 & $15,0 \%$ & $13,0 \%$ & $3,20 \mathrm{DM}$ bis $13,04 \mathrm{DM}$ & 24,57 bis 100,00 \\
\hline 86,97 & 108,71 & & & $13,04 D M$ & 100,01 bis 121,75 \\
\hline 108,72 & 1339,28 & $12,0 \%$ & $10,7 \%$ & $\begin{array}{c}13,04 \mathrm{DM} \text { bis } 160,71 \\
\mathrm{DM}\end{array}$ & 121,76 bis 1499,99 \\
\hline 1339,29 & & $3,0 \%+120,53 D M$ & & $A b 160,71 D M$ & Ab 1500 \\
\hline
\end{tabular}

Quelle: INIFES, eigene Darstellung nach Arzneimittelpreisverordnung vom 1.7.1998.

\section{Preisbildung Apotheke:}

Die Apothekenaufschlagsätze auf den Apothekeneinkaufspreis unterliegen ebenfalls einer amtlichen Regelung. Sie sind auch in der AMPreisV vom 1.Juli 1998 in Form von Festzuschlägen geregelt und unterliegen einem degressiven Verlauf. Die Festzuschläge wurden mit der neuen Verordnung für höherpreisige Arzneimittel abgesenkt.

Die Festzuschläge bei vom pharmazeutischen Großhandel beziehbaren Arzneimitteln werden immer auf den maximal möglichen Apothekeneinkaufspreis bezogen. Nur bei Arzneimitteln, die ausschließlich vom Hersteller bezogen werden können, gilt der Herstellerabgabepreis als Basis für die Festzuschläge. Durch den Bezug der Festzuschläge auf die maximalen Apothekeneinkaufspreise wird das im Arzneimittelgesetz formulierte Ziel der bundesweit einheitlichen Apothekenverkaufspreise für apothekenpflichtige Arzneimittel (§ $78 \mathrm{AMG}$ ) erreicht. Egal, zu welchem tatsächlichen Preis Arzneimittel auf den unterschiedlichen Handelsstufen gehandelt werden, es ergeben sich für identische Arzneimittel immer identische Letztverbraucherpreise ${ }^{65}$, unabhängig davon, wo innerhalb Deutschlands der Patient die Arzneimittel bezieht. 
Tabelle I - 2: Amtliche Festzuschläge für Fertigarzneimittel durch Apotheken ab Juli 1998

\begin{tabular}{|c|c|c|c|c|c|}
\hline \multicolumn{2}{|c|}{ AEK } & $\begin{array}{c}\text { Apothekenauf- } \\
\text { schlag in } \% \\
\text { des AEK }\end{array}$ & $\begin{array}{c}\text { Apotheken- } \\
\text { spanne in } \% \\
\text { des AVK }\end{array}$ & Zuschlag in DM & Apothekenpreis (AVK) \\
\hline 0,00 & 2,40 & $68,0 \%$ & $40,5 \%$ & 0,00 bis $1,63 \mathrm{DM}$ & 0,00 bis $4,03 \mathrm{DM}$ \\
\hline 2,41 & 2,63 & & & $1,63 \mathrm{DM}$ & $4,04 \mathrm{DM}$ bis $4,26 \mathrm{DM}$ \\
\hline 2,64 & 7,60 & $62,0 \%$ & $38,3 \%$ & $1,63 \mathrm{DM}$ bis $4,71 \mathrm{DM}$ & $4,27 \mathrm{DM}$ bis $12,31 \mathrm{DM}$ \\
\hline 7,61 & 8,26 & & & $4,71 \mathrm{DM}$ & $12,32 \mathrm{DM}$ bis $12,97 \mathrm{DM}$ \\
\hline 8,27 & 14,28 & $57,0 \%$ & $36,3 \%$ & $4,71 \mathrm{DM}$ bis $8,14 \mathrm{DM}$ & $12,98 \mathrm{DM}$ bis $22,42 \mathrm{DM}$ \\
\hline 14,29 & 16,96 & & & $8,14 \mathrm{DM}$ & $22,43 \mathrm{DM}$ bis $25,10 \mathrm{DM}$ \\
\hline 16,97 & 23,75 & $48 \%$ & $32,4 \%$ & $8,14 \mathrm{DM}$ bis $11,40 \mathrm{DM}$ & $25,11 \mathrm{DM}$ bis $35,15 \mathrm{DM}$ \\
\hline 23,76 & 26,51 & & & $11,40 \mathrm{DM}$ & $35,16 \mathrm{DM}$ bis $37,91 \mathrm{DM}$ \\
\hline 26,52 & 38,00 & $43,0 \%$ & $30,1 \%$ & $11,40 \mathrm{DM}$ bis $16,34 \mathrm{DM}$ & $37,92 \mathrm{DM}$ bis $54,34 \mathrm{DM}$ \\
\hline 38,01 & 44,16 & & & $16,34 \mathrm{DM}$ & $54,35 \mathrm{DM}$ bis $60,50 \mathrm{DM}$ \\
\hline 44,17 & 57,00 & $37,0 \%$ & $27,0 \%$ & $16,34 \mathrm{DM}$ bis $21,09 \mathrm{DM}$ & $60,51 \mathrm{DM}$ bis $78,09 \mathrm{DM}$ \\
\hline 57,01 & 70,30 & & & $21,09 \mathrm{DM}$ & $78,09 \mathrm{DM}$ bis $91,39 \mathrm{DM}$ \\
\hline 70,31 & 1063,81 & $30,0 \%$ & $23,1 \%$ & $21,09 \mathrm{DM}$ bis $319,14 \mathrm{DM}$ & $91,40 \mathrm{DM}$ bis $1382,96 \mathrm{DM}$ \\
\hline 1063,82 & & $8,263 \%$ & & $319,15 \mathrm{DM}$ & Ab 1382,97 DM \\
\hline
\end{tabular}

Quelle: INIFES, eigene Darstellung nach Arzneimittelpreisverordnung vom 1.7.1998.

\section{Mehrwertsteuer:}

Die Apothekenverkaufspreise sind um die Mehrwertsteuer von $16 \%$ zu erhöhen (= Endverkaufspreis). Deutschland belegt alle Arzneimittel, auch die über die gesetzliche Krankenversicherung erstattungsfähigen Präparate, mit dem Normalsteuersatz. Damit ist Deutschland neben Dänemark europaweit das einzige Land, welches Arzneimittel nicht bzw. nicht einmal teilweise von der Mehrwertsteuerbelastung ausnimmt.

\section{Apothekenrabatt für GKV-erstattungsfähige Arzneimittel:}

Des weiteren sind die Apotheken gesetzlich verpflichtet, auf durch die GKV erstattungsfähige Arzneimittel einen Kassenrabatt von 5\% des Endverkaufspreises (inklusive Mehrwertsteuer) zu gewähren. Im Handverkauf und bei privatversicherten Patienten sind die Apotheker dagegen verpflichtet, den vollen Endverkaufspreis zu verlangen, ein Rabatt ist hier nicht nur nicht vorgeschrieben, er ist sogar verboten.

\section{Großhandelsrabatte für Apotheken:}

Im Preiswettbewerb um die Apotheken haben die Großhändler Rabattsysteme wie Skonti, Mengenrabatte, Sondernachlässe, Warenrückvergütungen, etc. entwickelt, die ihnen die Regelung der Höchstzuschlagssätze ermöglicht. Schätzungen zufolge werden bis zu $15 \%$ des Apothekeneinkaufspreises vom Großhandel an die Apothe- 
ken gewährt. $^{68}$ So ist auch aufgrund der verschärften Wettbewerbssituation das Rabattniveau auch 1999 deutlich gestiegen. ${ }^{69}$ Vermutlich werden jährlich zwischen 1,5 und 2 Mrd. DM Rabatte von den Arzneimittelherstellern und -großhändlern an Apotheken gewährt. ${ }^{70}$ Aufgrund des Gebotes der bundeseinheitlichen Endverbraucherpreise werden die an die Apotheken gewährten Rabatte nicht an den Endverbraucher Patient weitergegeben, sondern verbleiben bei den Apotheken und erhöhen die in der AMPreisV ausgewiesenen Apothekenspannen.

\subsubsection{3. Übersicht über den Vertriebskostenanteil am Endverkaufspreis}

Der Vertrieb von Arzneimitteln ist, wie gezeigt, strikt in vertikaler Ausrichtung wie auch in der Setzung der Preise reglementiert. Aus der Struktur der Arzneimittelpreisverordnung ergeben sich folgende Spannen an Zuschlagsätzen für Großhandel und Apotheken:

- $12 \%$ bis $21 \%$ Großhandelsaufschlag auf den Herstellerpreis

- $30 \%$ bis $68 \%$ Apothekenaufschlag auf den Apothekeneinkaufspreis

Der Herstellerabgabepreis plus die aufgeführten Zuschläge und der Mehrwertsteuer von $16 \%$ ergeben den Endverkaufspreis eines Medikamentes. Werden die in der AMPreisV genannten Zuschläge prozentual auf den Endverkaufspreis, der gleichzusetzen ist mit den Medikamentenkosten des entsprechenden Präparates, bezogen, berechnen sich die anteiligen Herstell-, Vertriebs- und Mehrwertsteuerkosten:

Die Zahlen der VFA Statistics 2000 weisen folgende durchschnittliche Anteile aus ${ }^{71}$ :

- $\varnothing 54,8 \%$ Herstellkosten (pharmazeutische Industrie)

- $~ Ø 31,4 \%$ Vertriebskosten (Großhandels- und Apothekenbetriebsspanne)

- $\varnothing 13,8 \%$ Mehrwertsteuer (Staat)

Andere Quellen nennen eine ähnliche Verteilung der durchschnittlichen Anteile ${ }^{72}$ :

- $\varnothing 53,7 \%$ Herstellkosten (pharmazeutische Industrie)

- $\varnothing 32,5 \%$ Vertriebskosten (Großhandels- und Apothekenbetriebsspanne)

- $\varnothing 13,8 \%$ Mehrwertsteuer (Staat)

68

Schöffski, O. (1995), Die Regulierung des deutschen Apothekenwesens, Baden - Baden, S. $167 f$.

GEHE AG, (2000), Geschäftsbericht 1999, S. 37.

Cassel, D. (2000), Effiziente Distributionswege, S. 308, in: Wille, E., Albring, M. (Hrsg.), (2000), Rationalisierungsreserven im Gesundheitswesen, Peter Lang Verlag, Frankfurt Main, S. 307 318.

VFA (2000), Statistics 2000 - Die Arzneimittelindustrie in Deutschland, S. 22.

Z. B. die Arbeitsgemeinschaft der Verbraucherverbände e.V. (2000), Internethandel mit Medikamenten: In Europa Qualităt sichern \& Chancen für Verbraucher nutzen, siehe unter www.agv.de, Zugriff am 24.01.2001. 
Auf die Apothekenbetriebsspanne entfallen danach durchschnittlich $27,3 \%$ bis $27,5 \%$, auf die Großhandelsspanne zwischen $4 \%$ und $5 \%$ des Endverkaufspreises.

Über die Erstattungsfähigkeit der Arzneimittel schlagen sich Vertriebskosten auf die GKV-Arzneimittelausgaben nieder. Die GKV-Arzneimittelausgaben für 1999 lagen bei 36,15 Mrd. DM. Diese Summe kann in der Diskussion um eine effiziente Ausgabensteuerung im Arzneimittelsektor nicht ausgespart werden. Angesichts eines Vertriebskostenanteils von fast einem Drittel an den GKV-Arzneimittelausgaben stellt sich die Frage, ob der Arzneimittelvertrieb unter Beibehaltung der Arzneimittelsicherheit wirtschaftlicher organisiert werden könnte. ${ }^{73}$

\subsubsection{Blick nach Europa: Arzneimittelpreise sowie Vertriebskostenanteile}

Derzeit unterscheidet sich das Arzneimittelpreisniveau innerhalb der EU trotz harmonisierter Zulassungsverfahren, auf die hier nicht näher eingegangen werden soll, erheblich. Dieses resultiert u.a. aus unterschiedlichen Preisbildungsmechanismen auf der Ebene der Hersteller, der Organisation des Vertriebsweges wie auch der steuerlichen Belastung auf Arzneimittel.

Ausdruck dieses Preisgefälles in Europa sind nicht zuletzt die unter 1.2.2.2. genannten Parallel- und Reimporte, die einen Preisvorteil von bis zu 15\% aufweisen können.

Unter den Bedingungen einer gemeinsamen europäischen Währung könnte die Preistransparenz jedoch erleichtert und damit eine bessere Vergleichbarkeit der Arzneimittelpreise europaweit ermöglicht werden.

Derzeit wird in den meisten europäischen Staaten bereits auf der Herstellerebene in die Preisbildung regulierend eingegriffen, z.T. nur bei erstattungsfähigen Präparaten, z.T. aber auch für alle apothekenpflichtigen Arzneimittel. Die staatliche Preisregulierung auf dieser Stufe kann anhand unterschiedlicher Vorgehensweisen erfolgen, die hier nicht Gegenstand sein sollen.

Es zeigt sich konsequenterweise bereits bei der anteiligen Herstellerwertschöpfung am Arzneimittelpreis eine erhebliche Schwankungsbreite zwischen den aufgeführten Staaten. Deutschland weist den geringsten Herstelleranteil auf, was indirekt auf den höchsten Anteil an nicht herstellungsbedingten Kosten - also Vertrieb und Steuern schließen lässt.

73 FIND/SVP (1996), The International Market for Mail Service Pharmaceuticals. A Market Intelligence Report, S. $166 \mathrm{ff}$. 
Tabelle 1 - 3: Prozentuale Anteile der Hersteller, des Großhandels, der Apotheken und der Steuern an den Arzneimittelpreisen - Stand 1998

\begin{tabular}{|l|c|c|c|c|c|}
\hline & Hersteller & Großhandel & Apotheke & Vertrieb gesamt & Steuer \\
\hline $\mathrm{D}^{1)}$ & $54,8 \%$ & $4,1 \%$ & $27,3 \%$ & $31,4 \%$ & $13,8 \%$ \\
$\mathrm{DK}$ & $56,5 \%$ & $4,3 \%$ & $19,2 \%$ & $23,5 \%$ & $20,0 \%$ \\
$\mathrm{~B}$ & $56,6 \%$ & $8,5 \%$ & $29,2 \%$ & $37,7 \%$ & $5,7 \%$ \\
$\mathrm{CH}$ & $57,2 \%$ & $8,2 \%$ & $32,4 \%$ & $40,6 \%$ & $2,0 \%$ \\
$2^{2)}$ & $60,6 \%$ & $6,1 \%$ & $24,3 \%$ & $30,4 \%$ & $9,1 \%$ \\
$\mathrm{E}$ & $61,7 \%$ & $7,6 \%$ & $26,8 \%$ & $34,4 \%$ & $3,9 \%$ \\
$\mathrm{NL}^{2)}$ & $63,5 \%$ & $11,6 \%$ & $19,2 \%$ & $30,8 \%$ & $5,7 \%$ \\
$\mathrm{~GB}^{2)}$ & $65,8 \%$ & $9,4 \%$ & $24,8 \%$ & $34,2 \%$ & $0,0 \%$ \\
$\mathrm{~F}^{3)}$ & $66,5 \%$ & $7,1 \%$ & $24,3 \%$ & $31,4 \%$ & $2,1 \%$ \\
\hline
\end{tabular}

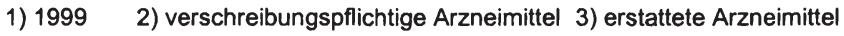

Quelle: EFPIA, zitiert aus VFA (2000), Statistics 2000 - Die Arzneimittelindustrie in Deutschland, S. 23.

Analog zu Deutschland wird auf die Herstellerebene aufbauend der Anteil der Vertriebskosten ermittelt. Der Vertrieb rezeptpflichtiger Arzneimittel durchläuft in allen europäischen Mitgliedsstaaten die für Deutschland aufgezeigten Handelsstufen des Großhandels und der Apotheke mit der Folge, dass in keinem Land die Preisbildung auf den Handelsstufen freigestellt ist. Dabei schwanken die Handelsspannen für den Vertrieb erheblich, zwischen 23\% (Dänemark) und 40\% (Schweiz). Deutschland liegt mit $31,4 \%$ bei den hier verglichenen Ländern im Mittelfeld, Länder wie die Schweiz, Belgien, Spanien und Großbritannien weisen höhere anteilsmäßige Vertriebskosten auf. Allgemein kann festgehalten werden, dass die Distributionskosten in den aufgeführten Staaten mit durchschnittlich ca. einem Drittel der Medikamentenkosten zu Buche schlagen (siehe Tabelle I-3).

Schließlich existieren stark voneinander abweichende einzelstaatliche Mehrwertsteuersätze für Arzneimittel, die somit die Arzneimittelpreisniveaus in Europa weiter auseinander driften lassen. Einige Staaten erheben demnach keine Mehrwertsteuer auf Arzneimittel, einige andere wie Deutschland den vollen Mehrwertsteuersatz. 
Tabelle I - 4: Mehrwertsteuerzuschläge (Stand 1.1.2000)

\begin{tabular}{|l|c|c|}
\hline Land & Mehrwertsteuersatz für Arzneimittel & $\begin{array}{c}\text { Allgemeines } \\
\text { Mehrwertsteuerniveau }\end{array}$ \\
\hline D & $16 \%$ & $16 \%(7 \%)$ \\
\hline DK & $25 \%$ & $25 \%$ \\
\hline B & $6 \%$ & $21 \%$ \\
\hline CH & $2 \%$ & $7,6 \%$ \\
\hline I & $10 \%$ & $20 \%$ \\
\hline E & $4 \%$ & $16 \%$ \\
\hline NL & $6 \%$ & $17,5 \%$ \\
\hline GB & $0 \%$ für erstattungsfähige Arzneimittel, \\
sonst 17,5\% & $17,5 \%$ \\
\hline F & $2,1 \%$ für erstattungsfähige Arzneimittel, \\
sonst 5,5\%
\end{tabular}

Quelle: BPI (2000), PharmaDaten 2000, S. 27.

Diese Darstellungen sagen nichts über die absolute Höhe der Arzneimittelpreise aus. Ein multilateraler Vergleich der Arzneimittelpreise ist allerdings höchst umstritten, da z.B. aufgrund unterschiedlicher Herstellerpreisbildungen, differierender wirtschaftlicher Komponenten (Kaufkraft, Einkommen, Wechselkurs), heterogener Vertriebsstrukturen oder Reglementierungen der Handelsspannen seine Aussagewirkung stark begrenzt sein würde. Allerdings kann beim Vergleich der jeweiligen Wertschöpfungsanteile für jedes einzelne europäische Land festgestellt werden, dass der Anteil der nicht herstellungsbedingten Kosten am Endpreis sehr hoch ist.

Aufgrund dessen hat die Organisation Association Internationale de la Mutualute (AIM) bereits 1996 eine Analyse der in einigen europäischen Ländern (Belgien, Deutschland, Frankreich, Niederlande, Schweiz) praktizierten Reglementierungen im Arzneimittelmarkt veröffentlicht. Dabei traf sie auf Basis ihres Benchmarking die Empfehlungen, Distributionskosten für Arzneimittel zu senken und im Bereich der Apotheken versuchsweise neue Vertriebsformen zu testen. ${ }^{74}$

So hat z.B. die Schweiz, die mit ihrem Vertriebskostenanteil von $40 \%$ an den Arzneimittelkosten an der Spitze der aufgeführten Länder liegt, seit Ende der neunziger Jahre mit der Liberalisierung des Arzneimittelvertriebs begonnen und den Versandhandel für Medikamente unter bestimmten Zertifizierungsvoraussetzungen zugelassen. Arzneimittel, die über den Versandweg an den Patienten abgegeben werden, weisen einen Kostenvorteil von bis zu 15\% der Medikamentenkosten auf (siehe dazu Kapitel 1.3).

74

Fink-Anthe, C. (1997), Die Kirschen aus Nachbars Garten, in: Pharmazeutische Industrie, Jg. 59, Nr. 3, S. III/69. 


\subsubsection{Feststellung eines Reformbedarfs im Arzneimittelvertriebssystem}

In Deutschland führt der Status quo der Arzneimitteldistribution mit den Komponenten

- Regulierung des Vertriebsweges aufgrund der Apothekenpflicht (Kapital 1.2.1. u. 1.2.2.)

- Gesetzliche Preisbildungsmechanismen nach AMPreisV (Kapitel 1.2.3.)

zu beträchtlichen Vertriebskosten von fast einem Drittel am Arzneimittelpreis. Zudem wirkt die Belastung des Apothekenverkaufspreises mit dem vollen MwSt-Satz kostentreibend.

Folgerichtig bietet die Arzneimitteldistribution von apothekenpflichtigen Medikamenten ein großes wirtschafts- und gesundheitspolitisches Diskussionspotential. Reformvorschläge umfassen unter anderem eine Verringerung des Mehrwertsteuersatzes auf Arzneimittel, eine Modifizierung der Arneimittelpreisverordnung wie auch eine (zumindest teilweise) Freigabe des Versandhandels mit Arzneimitteln. Die ersten zwei Punkte seien hier im Sinne einer Vollständigkeit zwar erwähnt, jedoch im Rahmen dieser Studie nicht umfassend dargestellt. Das Hauptaugenmerk liegt im folgenden auf dem dritten Punkt, der Freigabe eines Versandhandels für apothekenpflichtige Arzneimittel.

- Mehrwertsteuer:

Die Mehrwertsteuererhebung auf Arzneimittel von 16\% belastet die gesetzlichen Krankenkassen mit ca. 5 Mrd. DM jährlich. Ob es sinnvoll ist, den Beitragszahlern der parastaatlichen gesetzlichen Krankenversicherung auch die Kosten der auf Arzneimittel erhobenen Mehrwertsteuer und damit eine Form der Doppelbesteuerung aufzubürden, wird kritisch hinterfragt. Angestrebt werden sollte hier eine einheitliche europäische Lösung.

- Arzneimittelpreisverordnung:

In Deutschland erhöht sich der Preis eines Arzneimittels vom Hersteller bis in die Offizin des Apothekers enorm. Inzwischen wird manche ärztliche Behandlung des Patienten einschließlich der Verordnung einer Arznei geringer vergütet als die Abgabe des Präparates in der Apotheke. ${ }^{76}$ Der Sachverständigenrat für die Konzertierte Aktion im Gesundheitswesen hält in seinem Sondergutachten 1995 „Gesundheitsversorgung und Krankenversicherung 2000" für erstattungsfähige Arzneimittel eine Umstellung von den derzeitigen degressiven Festspannen auf Fix-

75

Schöffski, O. (1996), Gedanken zur Deregulierung des deutschen Apothekenwesens, Baden Baden, S. 239f.

Schleert, D., Kaesbach, W. (1997), Arzneimitteldistribution - value for money? Konsequenz aus der Reformdiskussion um die Arzneimittelpreisverordnung, in: Forum für Gesellschaftspolitik, Februar 1997, S. 53. 
zuschläge je Packung, gegebenenfalls gestaffelt, für möglich. Dies unterstützen auch die Krankenkassen und die EU-Kommission."

- Arzneimittelversand:

Ein weiterer Reformvorschlag, dem im weiteren Verlauf der Studie das Hauptaugenmerk folgt, ist die Lieferung von rezeptpflichtigen Arzneimitteln durch Versandapotheken. Ein Indikator für Fehlsteuerungen des derzeitigen Systems liegt in dem bereits derzeit sehr hohen Maße von Unterlaufungen. Die umfassende Nutzung des $\S 17$, II ApBetrO durch die Apotheken bezüglich des Heimlieferservice ist bereits unter Abschnitt 1.2.2.3 erwähnt worden.

Die gesetzlichen Krankenkassen als wesentlicher Kostenträger im Arzneimittelmarkt halten aufgrund der heutigen Kosten der Arzneimitteldistribution eine Liberalisierung der Vertriebswege für Arzneimittel für erforderlich und erreichbar. Weder werde eine flächendeckende Versorgung über das gesamte Arzneimittelangebot durch Versandapotheke beabsichtigt noch erwartet, allerdings würde sich diese Vertriebsform insbesondere für chronisch Kranke oder immobile Patienten anbieten. Da in Deutschland derzeit nicht erlaubt, soll ein Blick über die Landesgrenzen den Begriff der Versandapotheke näher bringen.

\subsection{Erfahrungen mit Versandapotheken im Ausland}

Die EU-Kommission, die von der Legalität der Versandapotheken ausgeht, hat die Verantwortung für die Entscheidung über die Erlaubnis von Versandapotheken in die Kompetenz der einzelnen Mitgliedsstaaten gelegt. So haben die Niederlande, Großbritannien und die Schweiz Versandapotheken gesetzlich legitimiert, Schweden folgt Anfang 2002. Alle anderen europäischen Länder erlauben keine Versandapotheken. In den USA ist der Versandhandel ebenfalls, allerdings schon wesentlich länger als in Europa, legalisiert.

Die im Folgenden dargestellten Modelle der Schweiz sowie der USA werden im Rahmen der INIFES-Studie bezüglich einer technischen Umsetzbarkeit und einer Zielgruppendefinition für einen Versandhandel untersucht, um somit einen Ansatzpunkt zu den eigenen Überlegungen zu liefern. Eine Übertragung beider Modelle ist für Deutschland aufgrund von Unterschieden in den Gesundheitssystemen ebenso wie in den nationalen gesundheitspolitischen Rahmenbedingungen nicht möglich und für die INIFES-Modellrechnungen auch nicht notwendig.

Schleert, D., Kaesbach, W. (1997), Arzneimitteldistribution - value for money? Konsequenz aus der Reformdiskussion um die Arzneimittlelpreisverordnung, in: Forum für Gesellschaftspolitik, Februar 1997, S. 53. 


\subsubsection{Europa: Beispiel Schweiz}

In der Schweiz fand in der Vergangenheit eine anhaltende kontroverse Diskussion über die Zulässigkeit von Versandapotheken statt. Die kantonalen Zuständigkeiten für den Gesundheits- und Arzneimittelsektor verhinderten lange eine einheitliche Regelung, bis die erste Versandapotheke 1997 ihren Betrieb in einigen Kantonen aufnahm. Seit Herbst 1999 ist die Versandapotheke, die in Kooperation mit einer Reihe von Krankenkassen arbeitet, in allen Teilen der Schweiz zugelassen und bewährt sich in einem Markt, der in seiner Apothekendichte und dem Niveau der Vertriebskosten für Medikamente über dem des deutschen liegt. Per Gesetz kann in der Schweiz seitdem eine Versandapotheke dann zugelassen werden, wenn diese alle vorgeschriebenen Kriterien bezüglich Arzneimittelsicherheit, usw. nachweislich erfüllt.

Dabei sieht sich die Versandapotheke als Ergänzung zu den traditionellen Apotheken und als neuer Dienstleister. Zum Service zählen u.a. eine kostenlose 24-StundenTelefonhotline mit Kundendienst und Beratung durch geschultes Personal, ein persönlicher Medikamentenpass, der zusammen mit den Medikamenten zugestellt wird und wichtige Informationen zu den Arzneimitteln enthält. Zudem werden die Patienten auf Basis von Patientendossiers daran erinnert, wann ein Rezept erneuert werden muss.

Dies entspricht vor allem den Bedürfnissen der Langzeitpatienten. Eine Befragung ergab, dass Patienten über 60 Jahre mehr als 65\% der Kundenklientel ausmachen. Zudem benötigen von diesen mehr als $60 \%$ regelmäßig 2-4 Medikamente pro Tag, ca. $15 \%$ sogar täglich mehr als 5 Präparate. Damit sind die Hauptnutzer der Versandapotheke ältere Patienten mit Dauermedikation. Weiterhin profitierten davon Menschen in ländlichen Gebieten. Die Versandapotheke wird zu 59\% von Menschen beansprucht, die in Orten mit weniger als 10.000 Einwohnern leben. $^{78}$

Der Ablauf des Arzneimittelbezuges per Versand gestaltet sich folgendermaßen: Nach dem Arztbesuch schickt der Arzt das Rezept entweder direkt an die Versandapotheke oder gibt es dem Patienten selbst mit. Damit obliegt es der Entscheidung des Patienten, ob er sich an eine traditionelle Präsenzapotheke wendet oder das $\mathrm{Re}-$ zept bei der Versandapotheke einreicht. Nach Eingang des Rezeptes bei der Versandapotheke sowie nach computergestützter Interaktionsprüfung auf Wechsel- und Nebenwirkungen, Wirkstoffallergien oder Dosierungsanweisungen stellt die Versandapotheke die Arzneimittel verordnungsgetreu zusammen. Bei Unklarheiten wird der verordnende Arzt kontaktiert. Die Abrechnung der Arzneikosten erfolgt direkt zwischen Versandapotheke und kooperierender Krankenkasse.

Der Versand erfolgt innerhalb der Schweiz für gewöhnlich an die Privatadresse, es kann aber auch die Arbeitsstelle oder der Ferienort sein. Die Medikamente werden

78

Institut für Sozial- und Präventivmedizin der Universität Zürich; Schweizerische PatientenOrganisation (SPO); Schweizerische Patientendienststelle (DVSP), Zufriedenheit der Kunden der MediService Apotheke - Schlussbericht, Zürich 1999. 
neutral verpackt und i.d.R. innerhalb eines Arbeitstages portofrei zugestellt. Dabei bedient man sich eines Zustellservices, der gemeinsam mit der Schweizer Post entwickelt wurde. Die Abgabe an der Haustür erfolgt nur gegen eigenhändige Unterschrift des Patienten, eine Deponierung im Briefkasten oder bei anderen Hausbewohnern wird nicht vorgenommen. Bei kurzer Abwesenheit erfolgt eine erneute kostenlose Zweitzustellung. Ist die Sendung länger nicht zustellbar, wird sie von der Versandapotheke zurückgenommen.

Abbildung I - 18: Schweizer Modell einer Versandapotheke am Beispiel Mediservice

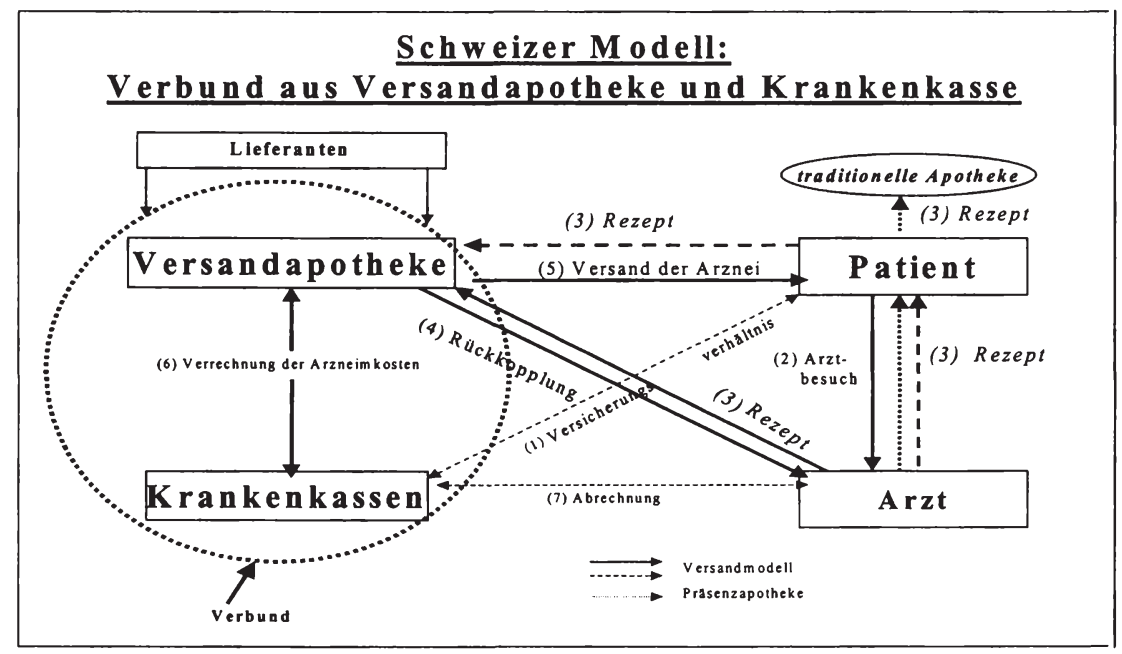

Quelle: INIFES, eigene Darstellung.

Neben dem Versorgungsaspekt konnten Erfolge bei der Kostenminimierung erzielt werden: bisherige Einsparungen belaufen sich - gemäss eigener Angaben der Versandapotheke - auf $10 \%$ bis $15 \%$ der Medikamentenkosten. Gezielt wird demnach an den Kosten angesetzt. Die Medikamentenpreise für über die Versandapotheke vertriebene Präparate richten sich nach der Schweizer Preisverordnung und sind identisch denen der Präsenzapotheken. Als Anreizsystem zu mehr Kosteneinsparung wurde ein Rückvergütungssystem entwickelt, welches einen Teil der eingesparten Kosten direkt an die Apothekenkunden und die Kostenträger erstattet. Über eine Verringerung der Beiträge kommt dieses wiederum allen Versicherten zu Gute.

Abschließend sollen die aus Sicht der Versandapotheke argumentierten Vorteile bzw. Hauptziele dieses Vertriebsweges aufgeführt werden. 
Abbildung 1-19: Vorteile / Hauptziele der Versandapotheke MediService in der Schweiz

\begin{tabular}{|c|c|c|c|}
\hline Kosteneinsparung & Qualität / Sicherheit & Dienstleistungen & Freiwilligkeit \\
\hline $\begin{array}{l}\text { - optimaler Einkauf } \\
\text { - Effizienzsteigerung } \\
\text { - verbesserte Logistik } \\
\text { - erhöhte Compliance } \\
\text { - ständige Informatio- } \\
\text { nen an den Patienten }\end{array}$ & $\begin{array}{l}\text { - mehrfache Rezeptprü- } \\
\text { fung } \\
\text { - standardisierte compu- } \\
\text { tergestützte Abläufe } \\
\text { - systematische Kontrolle } \\
\text { des Patientendossiers } \\
\text { auf Interaktionen } \\
\text { - Medikamentenpass } \\
\text { - sichere Lieferung } \\
\text { - sofortige Rücksprache } \\
\text { von Arzt und Patient bei } \\
\text { Unregelmäßigkeiten }\end{array}$ & $\begin{array}{l}\text { - verbesserte und } \\
\text { maßgeschneiderte } \\
\text { Dienstleistung } \\
\text { - sichere Heimlieferung } \\
\text { als Erleichterung für } \\
\text { Langzeitpatienten } \\
\text { - kostenlose 24- } \\
\text { Stunden-Telefon- } \\
\text { Hotline } \\
\text { - Rezepterinnerung } \\
\text { - kostenlose Zustellung }\end{array}$ & $\begin{array}{l}\text { eigene Entschei- } \\
\text { dung des Patien- } \\
\text { ten für die Ver- } \\
\text { sandapotheke } \\
\text { - jederzeitige Opti- } \\
\text { on für Patienten, } \\
\text { andere Apothe- } \\
\text { ken zu nutzen }\end{array}$ \\
\hline
\end{tabular}

Quelle: INIFES, eigene Darstellung nach:

Krähenbühl, J.-P., (1997) Mediservice - Eine Dienstleistung mit Kosteneinsparungspotential, in: EUROFORUM Pharma-Fachtagung. Versandhandel von Arzneimitteln in der europäischen Union.

\subsubsection{Beispiel USA}

An dieser Stelle soll ein Blick in die USA folgen. Dies weniger unter dem Gesichtspunkt der Vergleichbarkeit mit europäischen Versandmodellen - das ist aufgrund der Verschiedenartigkeit der Gesundheitssysteme nicht sinnvoll - als vielmehr unter dem Aspekt, dass hier die Wiege des Versandhandels für Arzneimittelstand stand. In den USA existieren verschiedene Vertriebskanäle für verschreibungspflichtige Medikamente: Einzelapotheken, Versandapotheken (Mail-Order-Pharmacies), Drogerie- und Apothekenketten, der Großhandel und herkömmliche Supermärkte.

Die Arzneimittelnachfrage in Versandapotheken läuft über Medien wie Telefon, Fax oder E-Mail. So bieten fast alle eine Rund-um-die-Uhr-Telefonberatung durch eigenes Apothekerpersonal an. "Mail-Order-Pharmacies" haben sich zu "Mail-ServicePharmacies" gewandelt. Die Rolle des Apothekers, der Wert seiner Dienstleistung und deren Vergütung ist dabei neu zu definieren. Befürworter von Mail-ServicePharmacies argumentieren für eine Telefonberatung aufgrund einer höheren Spezialisierung, besserer Patientendossiers und einer diskreteren Beratung durch einen professionell geschulten Telefonberatungsservice. Verfechter herkömmlicher Apotheken möchten auf die langjährige face-to-face-Interaktion zwischen Patient und 
Apotheker sowie dessen persönliches Wissen über den Patienten und dessen Leiden nicht verzichten.

Mail-Order-Pharmacies haben ihre Wurzeln im Jahre 1946. Die erste kommerzielle Versandapotheke wurde 1963 gegründet, der Durchbruch kam allerdings erst in den 80er Jahren. Der Umsatzanteil des Arzneimittelversandes an den Arzneimittelumsätzen für verschreibungspflichtige Präparate lag 1995 bei $9,2 \%$. Für das Jahr 2000 spricht man bereits von Umsatzanteilen von $15 \%{ }^{80}$, Tendenz stark steigend.

Mail-Service-Pharmacies selbst differieren stark in Größe, Eigentümerverhältnissen, Strategien und Kundenpotential: allein das Unternehmen Medco hält die Hälfte am Gesamtumsatz des Mail-Service-Marktes, die top ten insgesamt $90 \%$. Die verbleibenden $10 \%$ werden von über 150 kleinen Versandapotheken bedient. ${ }^{81}$ Die Bereitschaft der Inanspruchnahme wird durch Anreizmechanismen bezüglich der Erstattungsfähigkeit unterstützt. So erstatten einige Versicherungsunternehmen nur die vollen Medikamentenkosten, wenn der Bezug über Mail-Order-Apotheken erfolgte.

Der immer stärker werdende Zwang zu Kostendämpfungsmaßnahmen, eine zu beobachtende höhere Konkurrenz auf Seiten der Hersteller und Vertreiber von Arzneimitteln sowie der Einsatz günstiger Ressourcen wie Generika wirken fördernd auf die Implementierung von Mail-Order-Pharmacies. US-Studien sprechen von ca. $25 \%{ }^{83}$ Einsparung bei den Arzneimittelausgaben durch Versandapotheken. Diese können den Bedarf ihrer Kunden durch billige Großeinkäufe und die konsequente Abgabe von Generika anstelle von Originalpräparaten günstiger decken als Einzelapotheken. So liegt der Anteil der Generika am Umsatz von verordnungspflichtigen Arzneimitteln in Versandapotheken bei ca. $72 \%$, in Präsenzapotheken nur bei $56 \%{ }^{84}$ Dabei sind Versandapotheken gar nicht mehr nur durch geringere Einkaufskosten profitabel, sondern auch durch Wettbewerbsvorteile bei Personal- und Betriebskosten. Versandapotheken unterhalten oft große Verteilerzentren mit immensen Absatzvolumina in relativ niedrigpreisigen Gebieten. Ein hoher Automatisierungsgrad führt zusätzlich zu höherer Produktivität.

Der Begriff Versandapotheke wird in den USA häufig zusammen mit PatienceBenefit-Management (PBM) Unternehmen gebraucht: Mail-Order-Pharmacies bilden dabei häufig einen Vertriebsarm für PBM-Unternehmen.

79

FIND/SVP (1996), The International Market for Mail Service Pharmaceuticals., New York, S. 48 .

80

Ahrens, H.J., (2001), Interview des Vorstandsvorsitzenden der AOK mit der Zeitschrift impulse, Rubrik Drei Fragen an ..., siehe: impulse / märz 2001, S. 168.

FIND/SVP (1996). The International Market for Mail Service Pharmaceuticals, New York, S. 87.

O. V. (1993), Mail-Order Pharmacy in Europe, in: Marketletter, July 26, 1993, S. 13.

Schöffski, O. (1995), Die Regulierung des deutschen Apothekenwesens, Baden - Baden, S. 133.

Schöffski, O. (1995), Die Regulierung des deutschen Apothekenwesens, Baden - Baden, S. 133. 


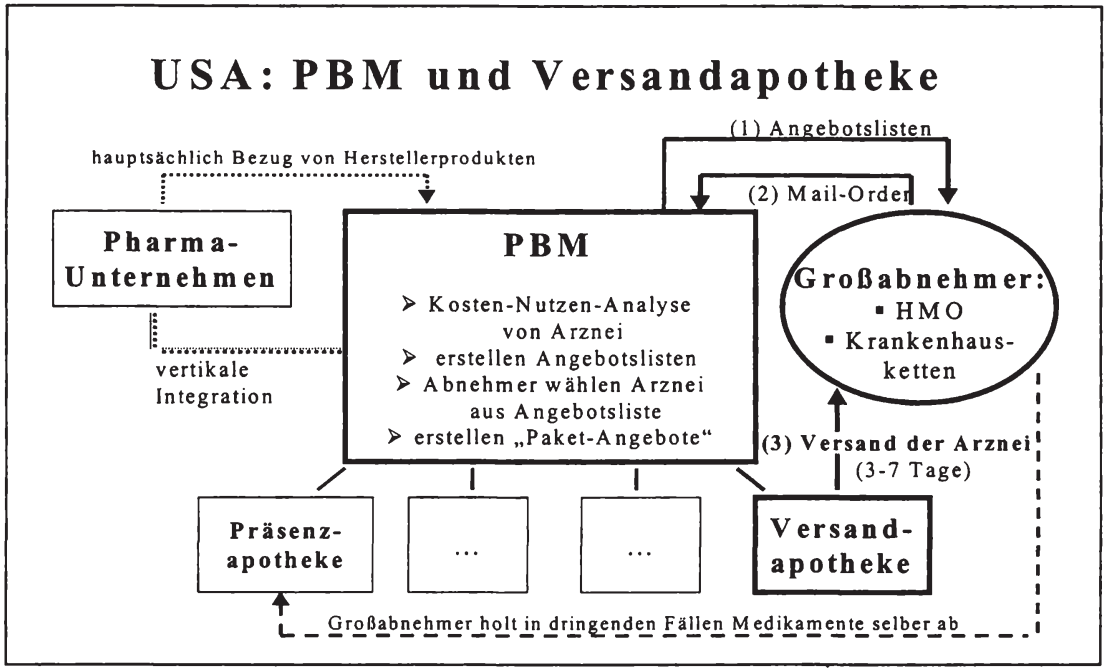

Quelle: INIFES, eigene Darstellung.

PBMs organisieren für Großabnehmer wie $\mathrm{HMOs}^{86}$ oder Krankenhausketten eine rationale Arzneimittelversorgung durch die systematische Auswertung von KostenNutzen-Analysen für Arzneimittel. Darauf basierend erstellen sie Kauflisten für ihre Großabnehmer. In einigen Fällen können sie sogar „Capitation-Programme" anbieten. Damit verpflichten sich die PBMs, für einen fest vereinbarten Preis alle nötigen Medikamente zur Behandlung einer bestimmten Krankheit den Krankenhäusern oder Versicherten einer HMO zur Verfügung zu stellen. Dies ist innen durch exakt kalkulierte Kosten, die auf großen Datensammlungen basieren, möglich. ${ }^{87}$ Problematisch ist, dass durch die PBM-Unternehmen nicht nur Rezeptdaten erfasst, sondern mit den innen vorliegenden Datensammlungen das Geschehen am Pharmamarkt bedeutend beeinflusst wird.

Die Struktur, die Arbeitsweise wie die daraus abzuleitenden Kosteneinsparungen und Marktanteile amerikanischer Versandapotheken zeigen, dass ein Vergleich zu europäischen Versandmodellen - wie dem der Schweiz - wesentlichen Einschränkungen unterliegt. Übereinstimmungen ergeben sich allerdings beim Versorgungspotential. So belegen Studien, dass Mail-Order-Pharmacies hauptsächlich durch ältere Menschen frequentiert werden, insbesondere durch Frauen über 65 Jahren, die im $\mathrm{Ge}$ gensatz zu älteren Männern öfter allein im Haushalt leben und damit auf eine Eigen-

86

Health Maintenance Organization - börsennotierte Krankenversicherungsunternehmen.

Schöffski, O. (1995), Die Regulierung des deutschen Apothekenwesens, Baden - Baden, S. 132 . 
versorgung angewiesen sind. 1995 waren zwei Drittel des Umsatzes am Mail-OrderMarkt Produkte der Dauermedikation.

Das Angebot in den Versandapotheken ist daher hauptsächlich auf chronische Leiden ausgerichtet und verzeichnet nach wie vor in diesen Therapiegruppen hohe $\mathrm{Zu}$ wächse. Neben älteren Menschen nutzen auch Berufstätige den Service von Versandapotheken.

Für das Sortiment eher ungeeignet erscheinen auch in den USA Arzneimittel zur Behandlung akuter Krankheiten, die unmittelbar nach der Verordnung durch den Arzt verfügbar sein müssen: „... This overcomes an important problem facing online and mail order pharmacies: timely delivery of acute drugs - medications to be given within hours of the physician visit. About half of all prescriptions are for acute drugs."

\subsection{Zwischenfazit und Diskussion}

Die Diskussion um eine Liberalisierung des Arzneimittelvertriebs in Deutschland wird derzeit sehr kontrovers geführt. Dies zeigt einerseits die hohe ökonomische und politische Brisanz dieses Themas, andererseits ist es ein Hinweis auf die Tragweite, die eine Veränderung der Strukturen für alle Beteiligten - positiv wie negativ - mit sich bringen würde. Die im folgenden dargestellten Standpunkte geben nur einen kleinen Einblick in die Pro- und Contra-Debatte wieder:

Abbildung I - 21: Diskussionsansätze pro und contra Arzneimittelversand

\begin{tabular}{|l|l|}
\hline Pro Arzneimittelversand & Contra Arzneimittelversand \\
\hline $\begin{array}{l}\text { Kostenvorteil: } \\
\begin{array}{l}\text { Finanzielles Einsparpotential } \\
\text { Economies of scale, }\end{array}\end{array}$ & $\begin{array}{l}\text { "Rosinenpicken“, Warensortiment: } \\
\text { Mischfinanzierung der Apotheken wird einge- } \\
\text { schränkt, Teil- versus Vollsortiment }\end{array}$ \\
\hline $\begin{array}{l}\text { Nutzerfreundlichkeit / Erreichbarkeit / Zustellsi- } \\
\text { cherheit: }\end{array}$ & Beratung, Service und Information: \\
$\begin{array}{l}\text { Lieferung frei Haus - kein Apothekengang not- } \\
\text { wendig (Ältere; chronisch Kranke, Immobile) } \\
\text { telefonische Erreichbarkeit rund um die Uhr, } \\
\text { bzw. direkt über den verordnenden Arzt } \\
\begin{array}{l}\text { Sicherstellung der rechtzeitigen Lieferung, Ge- } \\
\text { fahr des Verlusts von Sendungen }\end{array}\end{array}$ & $\begin{array}{l}\text { Gefahren für Beratung, da kein persönliches } \\
\text { Notfallversorgung gefährdet }\end{array}$ \\
\hline $\begin{array}{l}\text { Apotheken-Nersorgungsdichte: } \\
\text { Zu hoher Apothekendichte wird kostentreiben- } \\
\text { de Funktion unterstellt }\end{array}$ & $\begin{array}{l}\text { Integration, Berücksichtigung der Gesamtsitu- } \\
\text { ation, flexible Reaktion auf akute Erkrankun- } \\
\text { gen }\end{array}$ \\
\hline
\end{tabular}

Quelle: INIFES, eigene Darstellung.

88

Buhle, L. (1999), Potential for Profit, Potential for Real Change, in: www.managedcaremag.com, Zugriff in 07/1999. 
Die Pharmahersteller unterstützen ausdrücklich ein Versandhandelsverbot für Arzneimittel und eine Festschreibung im Arzneimittelgesetz. Dies soll den unkontrollierten Arzneimittelversand aus dem Ausland verhindern und das Risiko minimieren, dass gefälschte Arzneimittel eingeschleust werden. ${ }^{89}$

Die Großhändler befürchten Einnahmeverluste, wenn teure und häufig verkaufte Arzneimittel über einen Versandhandel vertrieben würden. Diese gefährden nach ihrer Ansicht die flächendeckende und schnelle Versorgung mit selten benötigten Arzneimitteln und verteuere sie drastisch. „Wenn man die Rosinen herauspickt, kann das übrige System nicht mehr funktionieren. ${ }^{100}$

Der Apothekerverband ABDA warnt vor Versandhandel und Apothekenketten. Sicherheit und Qualität der Arzneimittelversorgung seien die wichtigsten Aufgaben gegenüber Patienten und Kunden. ${ }^{91}$ Ohne den Dolmetscher Apotheker bleibe der Beipackzettel für viele Patienten unverständlich. Die Kritik an zu hohen Vertriebskosten durch die Apotheken sei vollkommen unberechtigt. ${ }^{92}$

Die Betriebskrankenkassen fordern, statt den Versandhandel von Arzneimitteln zu verbieten, sollten Regelungen gefunden werden, die geeignet sind, den Versand von Medikamenten in geordnete Bahnen zu lenken. Vor allem bei teuren Medikamenten sei die Abgabe aufgrund der Aufschläge über den Versand weitaus günstiger als in einer Präsenzapotheke. ${ }^{93}$ Gerade hier liegen im Versandhandel preiswertere und qualitativ gleichwertige Angebote. ${ }^{94}$ Versandapotheken, die vertraglich an die Krankenkassen gebunden sind, könnten für die Versorgung bettlägeriger und pflegebedürftiger Patienten sowie chronisch Kranker zugelassen werden. Denn diese Patienten erhalten regelmäßig dasselbe Medikament und benötigen deshalb keine Einzelberatung durch den Apotheker. Mit den Ersparnissen aus dem Versandhandel will man die Ausgaben für Arzneimittel senken.

Das Bundesministerium für Gesundheit erwähnt, dass es billiger wäre, umsatzstarke Arzneimittel per Versandhandel zu vertreiben. Allerdings kann auf die sofortige Versorgung durch Apotheken mit allen akut benötigten Arzneimittel rund um die Uhr nicht verzichtet werden. Die Regelungen des AMG schützen die Patienten vor Risi-

89

90

92

93

94

95

VFA (1997), Einblicke 1997, Jahresbericht, S. 16.

Rücker, D. (1999), Politik Versandhandel: Einsparungen im Promillebereich, www.pharmazeutische-zeitung.de, Zugriff am 20.09.99.

O.V. Apotheker warnen neue Regierung: kein Versandhandel und Ketten, in: dpa vom 30.09.98, S.3.

Rücker, D. (1999), Politik Versandhandel: Einsparungen im Promillebereich, in: www.pharmazeutische-zeitung.de, Zugriff am 20.09.99.

Rücker, D. (1999), Politik Versandhandel Einsparungen im Promillebereich, in: www.pharmazeutische-zeitung.de, Zugriff am 20.09.99.

Voss, V. (1998), Arzneimittel / Der Versandhandel ist ein Geschäft voller Risiken und Nebenwirkungen. Mit der Gesundheit zahlen, in: Rheinischer Merkur, S. 13.

Voss, V. (1998), Arzneimittel / Der Versandhandel ist ein Geschäft voller Risiken und Nebenwirkungen. Mit der Gesundheit zahlen, in: Rheinischer Merkur, S. 13. 
ken, die bei der Einnahme von Arzneimitteln unvermeidlich bestehen und die eine fachliche Beratung in der Apotheke notwendig machen, besonders auch wegen der Nebenwirkungen und Wechselwirkungen. Vor allem bei älteren Menschen, die mehrere Arzneimittel zugleich einnehmen, die sie z.T. von verschiedenen Ärzten verschrieben bekommen, ist die fachliche Beratung in der Apotheke wichtig. Allerdings sind die Mehrkosten durch die Apothekenpflichtigkeit nur in dem Maße gerechtfertigt, in dem die Apotheker ihrer Verantwortung gerecht werden und die Beratung durchführen.

Erfahrungen mit Versandapotheken im Ausland belegen, dass aufgrund epidemiologischer und demografischer Entwicklungen nachfrageseitig Potentiale in Form neuer Zielgruppen für Versandapotheken zu existieren scheinen. Auch Deutschland unterliegt diesen Bevölkerungs- und epidemiologischen Entwicklungen hin zu chronischen Erkrankung oder Multimorbidität. Zudem ist im Jahr 2000 ist fast jeder vierte Deutsche über 60 Jahre alt, 2040 wird es mehr als ein Drittel der Deutschen sein. Aufgrund einer altersbedingten höheren Krankheitswahrscheinlichkeit belastet der daraus resultierende hohe Arzneimittelbedarf in Zukunft nicht nur verstärkt die Sozialkassen, sondern bedingt ebenfalls eine hohe Notwendigkeit von Apothekenbesuchen zur Deckung des Arzneimittelbedarfs des Patienten ${ }^{97}$, denen jedoch aufgrund der alters- wie epidemiologisch bedingten Beeinträchtigungen eine häufig anzutreffende Immobilităt entgegensteht, die den Weg in die Apotheke nur schwerlich oder gar nicht möglich erscheinen läßt. Diese Patienten sind auf die Hilfe aus der Familie wie aus anderen externen Quellen angewiesen. Je nach Befragung ergibt sich, dass $15 \%$ bis $50 \%$ der Apothekenkunden Besorger, d.h. „Rezeptüberbringer" und „Medikamentenbesorger" für andere Menschen mit Medikamentenbedarf sind. Es scheint also auch hier ein Nachfragepotential entsprechend der Schweiz oder den USA für Versandapotheken zu existieren.

Versandapotheken werden dabei nicht substitutiv, sondern ausschließlich komplementär als ein Glied neuer Vertriebsstrukturen für verordnungspflichtige Arzneimittel wirken können.

Problematischer gestaltet sich eine Ableitung von Einsparpotentialen für Deutschland anhand der ausländischen Erfahrungen. Bisher veröffentlichte Schätzungen zur finanziellen Entlastung des sozialen Krankenversicherungssystems zwischen 800 Mio. $\mathrm{DM}^{98}$ bis 1.700 Mio. DM ${ }^{99}$ stützen sich insbesondere auf ca. $10 \%$ bis $15 \%$ Marktanteil

BMfG (1998), Warum ist in Deutschland der Versandhandel mit Arzneimitteln verboten - könnte man dadurch nicht viel Geld sparen?, in: www.bmgesundheit.de/aktuell/frage von 31/98.

97

Drei Viertel der Apothekenkunden sind kranke Menschen, mehr als jeder sechste leidet an einer chronischen Krankheit mit stetigem Arzneimittelbedarf. Zwei Drittel der Apothekenkunden sind Dauerpatienten, die regelmäßig mindestens einmal monatlich Medikamente aus der Apotheke beziehen müssen, davon sind fast alle mehr als 60 Jahre alt. Siehe: Riegl \& Partner $\mathrm{GmbH}, \mathrm{In}-$ stitut für Management im Gesundheitsdienst, Das Image der deutschen Apotheke, Augsburg. 1995.

Schleert, D.(2000), zitiert in: Zander, B., Invasion der @-Pillen, in: STERN 24/2000, S. 198. 
und Kosteneinsparungen zwischen $10 \%$ bis $30 \% .{ }^{100}$ Dazu soll an dieser Stelle noch einmal ausdrücklich angemerkt werden, dass eine Anlehnung der erzielbaren Kostenvorteile im Ausland aus verschiedenen Gründen nicht 1:1 auf Deutschland übertragbar ist. Dagegen sprechen Unterschiede in den Gesundheitssystemen ebenso wie nationale gesundheitspolitische Rahmenbedingungen.

Fest steht allerdings, dass im Zuge der stetigen Ausgabensteigerungen im Arzneimittelsektor und der knappen Ressourcen der Kostenträger - auch im Sinne der angestrebten Beitragssatzstabilität - nach Wegen gesucht werden muss, mögliche Wirtschaftlichkeitspotentiale nutzbar machen. Ein Vertriebskostenanteil von fast einem Drittel der Arzneimittelausgaben der gesetzlichen Krankenversicherung ist dabei zweifellos in Einsparungsüberlegungen einzubeziehen. Gleichzeitig müssen aber die zentralen Aspekte der Patientengerechtigkeit, der Medikamentensicherheit, der flächendeckenden Versorgungssicherheit sowie der Akzeptanz des Versandhandels bei Patienten Ärzten und anderen Betroffenen angemessen berücksichtigt, und mit dem potentiellen monetarisierten Einsparpotential gegengerechnet werden.

Volkswirtschaftlich und sozialpolitisch akzeptabel können neue Vertriebswege im Arzneimittelsektor nur dann sein, wenn Wirtschaftlichkeitsreserven ausgeschöpft werden können, ohne das bestehende hohe Niveau der Arzneimittelversorgung zu gefährden. Unter diesen Prämissen sind Versandapotheken, wie hier dargestellt, als Vertriebsformen in Zukunft verstärkt in eine kritische und ergebnisoffene Diskussion um liberalisierte Wege in der Arzneimitteldistribution einzubeziehen.

\section{SExkurs: Internetapotheken}

In der aktuellen Diskussion über die Implementierung eines Arzneimittelversandes mit verschreibungspflichtigen Arzneimitteln findet sich hauptsächlich der Begriff „Internetapotheke“, die mit dem Begriff der Versandapotheke häufig synonym gebraucht wird, sich jedoch von dieser, insbesondere in der Art der Kommunikation zwischen Apotheke und Patienten, unterscheidet. Inhalt dieser Studie sind explizit Versandapotheken, keine Internetapotheken.

Immer häufiger wird in Deutschland das Internet zur Bestellung von Arzneimitteln auch verschreibungspflichtigen - aus dem Ausland benutzt. Das besondere Merkmal der Internetapotheken ist, dass neben der hauptsächlichen Nutzung des Mediums Internet als Kommunikationsbasis Anbieter (Apotheken) und Nachfrager (Patient) nicht beide in Deutschland ansässig sind. Dieser Umstand ist daraus geboren, dass das deutsche Versandhandelsverbot innerhalb Deutschlands einen Versandhandel für apothekenpflichtige Arzneimittel verbietet. Jenseits dieser Formalia ist jedoch Fakt, dass Internetapotheken durch Patienten in Deutschland nachgefragt werden. Einer

99

Wissenschaftliches Institut der AOK, (2001), Versandapotheke - eine preiswerte Alternative, Aktuelle Modellrechnungen des WidO berechnet Einsparpotential, siehe unter www.wido.de, Zugriff am 25.06.2001. 
Studie des BPI zum Bekanntheitsgrad und zur Nutzung der Internetbestellung von Medikamenten ergab folgende Ergebnisse: Mehr als die Hälfte aller Deutschen kennt diese Möglichkeit des Arzneimittelbezuges noch nicht. Immerhin 20\% der Befragten haben diese Form bereits genutzt bzw. halten sich für die Zukunft eine Nutzung offen.

Abbildung I-22: Bekanntheit des Arzneimittelbezugs via Internet in Deutschland (Stand 1999)

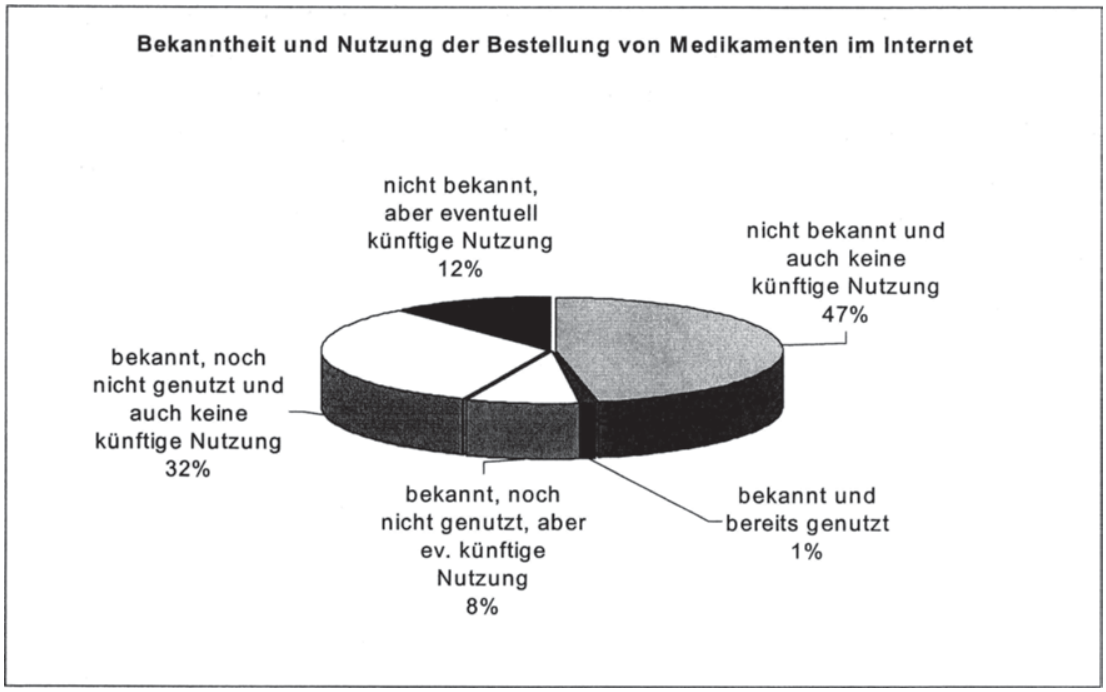

Quelle: BPI, Surfen in gefährlichen Gewässern, in: www.bpi.de.

Es stellt sich in diesem Zusammenhang die Problematik der Kontroll- und Sanktionsmöglichkeiten im world-wide-web. Testbestellungen verschiedener Organisationen in der Vergangenheit zeigten, dass häufig die Beipackzettel fehlen, bestellte Arzneimittel nicht beim Patienten ankommen, z.T. aber auch gravierende Qualitätsmängel und Arzneimittelfälschungen vorliegen. ${ }^{101}$ Dies trifft insbesondere für den Versand aus Staaten außerhalb der EU zu. Innerhalb der EU sind die Zulassungsvorschriften weitgehend harmonisiert und die Arzneimittelsicherheit der im Internet innerhalb der EU-Staaten vertriebenen Medikamente nicht grundsätzlich anzuzweifeln, sonst müsste dies wohl auch für Parallel- und Reimporte gelten. Allerdings ist aufgrund der weltweiten Ausbreitung des Internets und der angesprochenen fehlenden Kontrollmöglichkeiten die Differenzierung zwischen seriösen und nicht seriösen Anbietern relativ schwierig. Dieser Umstand sowie eine Umgehung nationaler Gege-

101 Bundesverband der Pharmazeutischen Industrie, Arzneimittel im Internet, www.bpi.de, Zugriff: Dezember 1999. 
benheiten macht deshalb den Arzneimittelvertrieb via Internet allgemein (noch) angreifbar.

Ob die Versendung von Arzneimitteln aus anderen EU-Staaten nach Deutschland aufgrund der genannten Probleme legal ist oder nicht, soll hier nicht Gegenstand sein. Darum streiten derzeit die verschiedenen Interessensvertreter im Arzneimittelmarkt.

In Anbetracht des rasanten technischen Fortschritts sind allerdings alle Maßnahmen auf europäischer und nationaler Ebene zu unterstützen, die einerseits die Arzneimittelsicherheit gewährleisten, andererseits mögliche Effizienzvorteile eines für den Verbraucher sicheren Versandhandels nutzen. So wäre denkbar, dass technisch versierte Nachfrager den "Umweg" des Arzneimittelversands via Internet aus dem Ausland nur solange als Alternative nutzen, wie Versandapotheken in Deutschland nicht zugelassen sind. 


\section{Quantitative Analyse der Einsparpotentiale}

\subsection{Modell Versandapotheke im Überblick}

Die Diskussion um eine Liberalisierung des Arzneimittelvertriebs in Deutschland wird derzeit sehr kontrovers und oftmals interessengeleitet geführt. Dies zeigt einerseits die hohe ökonomische und politische Brisanz dieses Themas, andererseits ist es ein Hinweis auf die Tragweite, die eine Veränderung der Strukturen für alle Beteiligten positiv wie negativ - mit sich bringen würde. Unumstritten ist, dass im Zuge der stetigen Ausgabensteigerungen im Arzneimittelsektor und der knappen Ressourcen der Kostenträger - auch im Sinne der angestrebten Beitragssatzstabilität - nach Wegen gesucht werden muss, mögliche Einsparpotentiale nutzbar zu machen. Ein Vertriebskostenanteil von über einem Drittel der Arzneimittelausgaben der gesetzlichen Krankenversicherung ist dabei zweifellos in die Überlegungen einzubeziehen.

Im Kapitel 1 wurden die wesentlichen Rahmenbedingungen im derzeitigen Arzneimittelvertrieb dargestellt. Das Versandhandelsverbot des § 43, I AMG untersagt derzeit den Versandhandel für apothekenpflichtige Arzneimittel in Deutschland. Ein Blick in die Schweiz und die USA, in denen die Vertriebsform der Versandapotheken neben Ländern wie Großbritannien, den Niederlanden oder demnächst Schweden bereits komplementär zu den Präsenzapotheken existiert, informierte über die Funktionsweise, wesentliche Strukturkomponenten, Service- und Dienstleistungsangebote, aber auch über realisierte Kosteneinsparungen, Marktanteile und Hauptnutzergruppen. Zudem wurde der wesentliche Unterschied zum Internetversand von Arzneimitteln hervorgehoben, der derzeit aufgrund der Rechtslage (noch) gleichzusetzen ist mit einem grenzüberschreitenden Versand.

An dieser Stelle sei betont, dass sich die Ausführungen der folgenden Kapitel zwei, drei und vier auf Versandapotheken im nationalen Kontext, nicht auf Internetapotheken mit grenzüberschreitendem Pharmaversand beziehen. Die Aufhebung des nationalen Versandhandelsverbotes nach $\S 43$, I AMG wird entsprechend unterstellt. Ansonsten sind Versandapotheken im Sinne dieser Studie zugelassene Apotheken mit allen Anforderungen an die Arzneimittelsicherheit und Arzneimittelqualität. Im Gegensatz zu den Präsenzapotheken wird hier der neue Vertriebsweg des Versandes eingeführt. Ein weiterer wesentlicher Unterschied besteht in der Kommunikationsform zwischen Arzt, Apotheke und Patient (Beratung und Rezeptübermittlung).

Das im Folgenden zu entwickelnde Modell einer Versandapotheke ist die Basis für alle weiteren Kapitel. Es liegt der quantitativen Analyse der Einsparpotentiale des Kapitels zwei ebenso zugrunde wie der qualitativen Analyse der Einstellungen zum Pharmaversand des Kapitels drei. 


\subsubsection{Modellentwicklung Versandapotheke}

Es ist wenig hilfreich, den Begriff der "Internetapotheken", also des Internetversandes von Arzneimitteln aus dem Ausland mit dem Begriff der "Versandapotheken" gleichzusetzen bzw. synonym zu gebrauchen, wie es derzeit oft getan wird. Der reine Internetversand ist oft mit erheblichen Unabwägbarkeiten für den Verbraucher belastet und entzieht sich derzeit staatlicher Kontrolle.

Im Gegensatz hierzu handelt es sich bei dem Modell der Versandapotheke um eine Vertriebsform, an die dieselben gesetzlichen Anforderungen bzgl. der Qualität gestellt werden, wie an die in Deutschland existierenden Präsenzapotheken. Der wesentliche Unterschied besteht in der Kommunikationsform zwischen Arzt, Apotheke und Patient sowie in der Art der Medikamentenabgabe an den Patienten.

Geht man vom Szenario "Versandapotheken für verordnete Medikamente" aus, bietet sich die folgende Modellentwicklung für Deutschland an. Dabei soll die elektronische Rezeptübermittlung vom Arzt an die Apotheke wesentlicher Bestandteil des Modells sein. Als Modellbasis wird im Folgenden grundrissartig die Dreiecksbeziehung zwischen Arzt, Patient und Versandapotheke dargestellt, die auch in den weiterführenden Modellstufen einen tragenden Pfeiler bildet (vgl. Abbildung $\|-1^{2}$ ).

Abbildung II - 1: Einfaches Grundmodell einer Versandapotheke

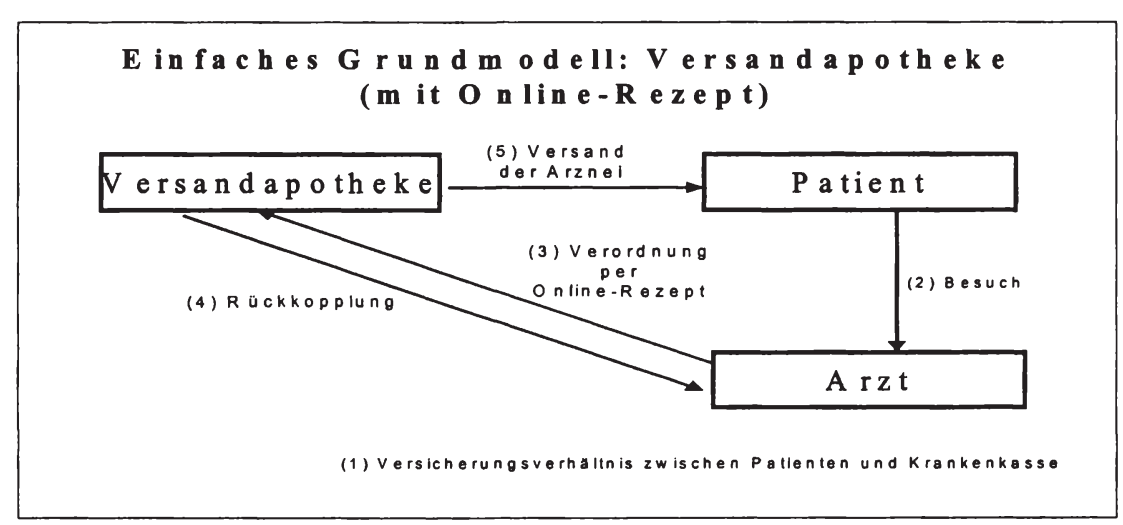

Quelle: INIFES, eigene Darstellung.

Vgl. hierzu: Sterzel, A., Wassener, D. (2001) Arzneimitteldistribution in Deutschland Versandapotheken als Reformoption?, in: Arbeit und Sozialpolitik, Jg. 55, S. 14ff. 
Während eines Arztbesuches (2) werden dem Patienten Arzneimittel verordnet. Das Rezept erstellt der Arzt bei Einverständnis des Patienten elektronisch und schickt es per Datenfernübertragung an eine Versandapotheke (3). Diese wiederum prüft die Rezeptangaben und wendet sich bei Rückfragen an den verordnenden Arzt (4). Sind alle Unklarheiten beseitigt, stellt die Versandapotheke die Medikamente dem Rezept entsprechend zusammen und versendet diese umgehend an den Patienten (5).

Nachdem der Versand der Arznei an den Patienten erfolgt ist (5), verrechnet die Versandapotheke die Arzneimittelkosten mit den Spitzenverbänden der Krankenkassen, bei welchen der Patient versichert ist (6) (vgl. Abbildung II - 2). Die Krankenkassen rechnen die ärztlichen Leistungen nach wie vor mit den Kassenärztlichen Vereinigungen ab (7a); hierbei gibt es also keinerlei Veränderungen, die Zuzahlungen werden ähnlich der Krankenhauszuzahlung an Patienten von der Kasse per Rechnung belastet. Die Versandapotheke bezieht - genau wie die Präsenzapotheke - ihr Arzneimittelsortiment an Original- und Generikaprodukten über Lieferanten aus dem Inland und der EU (8). Allerdings erhofft man sich durch die Versandapotheken einen stärkeren Einsatz von Generika. ${ }^{3}$ Dabei bleibt die Therapiefreiheit des Arztes unangetastet.

Aufbauend auf den vorangegangenen Strukturen entsteht für Deutschland das in Abbildung II - 2 dargestellte Gesamtmodell für einen Arzneimittelversand verordneter Medikamente. Dabei ist hervorzuheben, dass die Versandapotheke selbständig, d.h. nicht im Verbund mit den Krankenkassen agiert. Die Unabhängigkeit der Versandapotheke ermöglicht den Zugang aller Patienten, egal bei welcher Krankenkasse das Versicherungsverhältnis besteht. Welchen Weg das Rezept einschlägt, ob in die Offizin einer Präsenzapotheke oder in eine Versandapotheke, hängt (weiter) von den einzelnen Krankheitsbildern, der Dringlichkeit des Medikamentenbedarfes sowie den Kosten des jeweiligen Vertriebsweges ab. Wie schon erwähnt, eignen sich nicht alle Arzneimittel zum Vertrieb über Versandapotheken. Nur wenn die Medikamente versandfähig und billiger sind, sowie keine akute Dringlichkeit vorliegt, wird der Patient die Versandapotheke als Alternative zur Präsenzapotheke nutzen.

3

Dies ist in den USA zu beobachten. Hier hatten Präsenzapotheken einen Anteil von $56 \%$, Versandapotheken einen Anteil von $72 \%$ der Generika am Umsatz mit verschreibungspflichtigen Arzneimitteln aufzuweisen. Siehe FIND/SVP (1996), The International Market for Mail Service Pharmaceuticals, S. 133. 


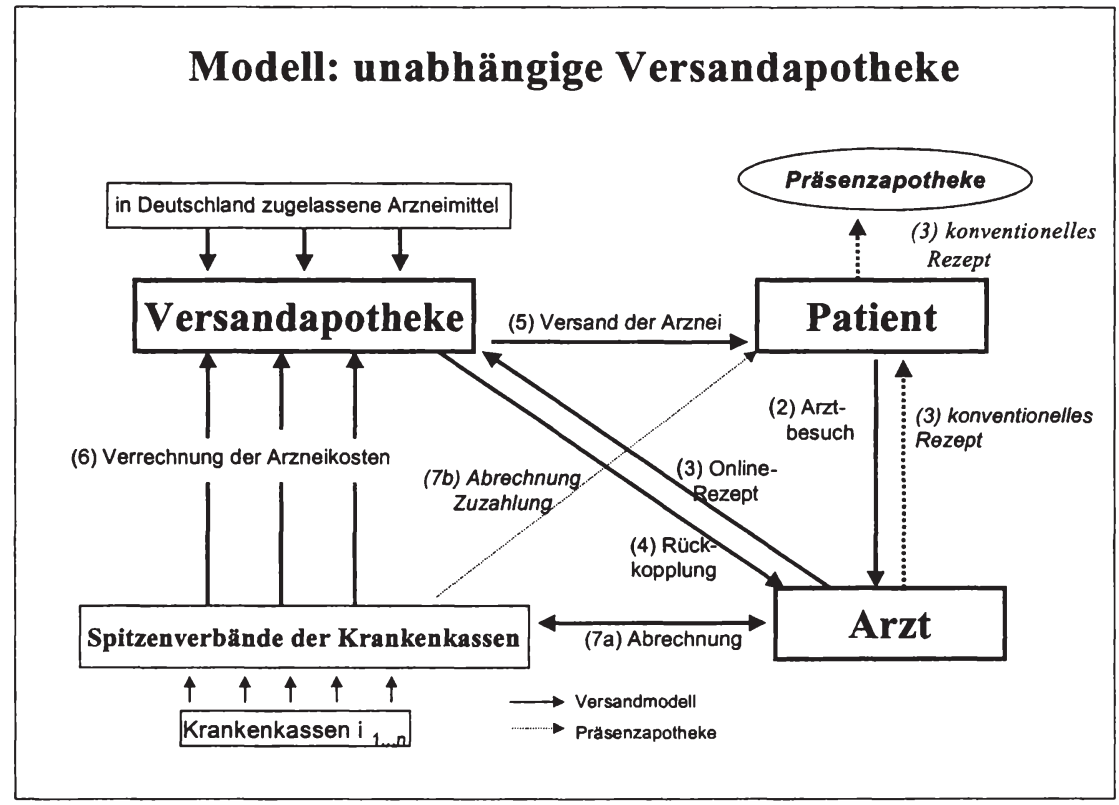

Quelle: INIFES, eigene Darstellung.

In den dargestellten Strukturmodellen bleibt bisher offen, wie die Verrechnung der Arzneimittelzuzahlungen der Versicherten im Rahmen organisiert werden soll. Während der Großteil der Arzneimittelausgaben bei „traditionellen“ wie bei Versandapotheken direkt zwischen Apotheke und Kasse (ggf. unter Einschaltung von Rezeptsammel- und -verrechnungsstellen) abgerechnet wird, werden die Arzneimittelzuzahlungen zwischen Patienten und Apotheken verrechnet. Obwohl davon auszugehen ist, dass die Mehrzahl der potentiellen Nachfrager der Dienstleistungen der Versandapotheken aufgrund chronischer Erkrankungen von Zuzahlungen befreit sein wird ( $\left(61\right.$ ff SGB V) - im Durchschnitt der Bevölkerung sind es derzeit bereits $50 \%{ }^{4}$ muss eine Lösung für die Abrechnung der Zuzahlungen erarbeitet werden. Grundsätzlich bieten sich für Deutschland z.B. folgende Modelle an:

1) Die Zuzahlungen werden den Patienten von den Versandapotheken in Rechnung gestellt und entweder einzeln für jede Lieferung oder Z.B. quartalsweise überwiesen (ähnlich der Zuzahlungsmodalitäten für einen stationären Aufenthalt).

2) Die Zuzahlungen werden von den Versandapotheken übernommen, schmälern somit deren Gewinn; alle Nutzer von Versandapotheken sind zuzahlungsbefreit.

3) Die Arzneimittelzuzahlungen entfallen generell bei der Nutzung von Versandapotheken.

O. V. (2001), Treiben Kassen Missbrauch mit der Rezeptgebühr?, in: uww.medical-tribune, de, Zugriff am 09.07.01.

In Expertengesprächen wurde von Seiten der Kostenträger diese Lösungsmöglichkeit vorgeschlagen. 
Angesichts der derzeitigen Rechtslage, nach der Zuzahlungen explizit nicht nur als Mittel zu Kosteneinsparung für die Krankenkassen, sondern auch als Instrument zur Nachfragesteuerung vorgesehen sind, beinhalten Alternativen 2) und 3) sicherlich einen schwerwiegenderen Systembruch. Vorhaben, wie die von Internet-Versendern geplante Übernahme der Zuzahlungen ${ }^{6}$, sind derzeit (rechtlich) gemäss $\S 31$, III SGB V nicht zulässig.

Im Folgenden wird bei den vorgenommenen Modellrechnungen daher davon ausgegangen, dass die Zuzahlungen auch weiterhin von den Patienten übernommen werden müssen (Alternative 1).

Abschließend ein Hinweis zur Kommunikation zwischen Ärzten und Apothekern: In Deutschland erfolgen derzeit alle Verordnungen auf einem konventionellen Rezept, welches der Arzt ausstellt und dem Patienten überreicht. Allerdings werden mit der Entwicklung neuer Kommunikationswege, wie über die Medien, Überlegungen zum sog. „Elektronischen Rezept" lauter. In anderen Ländern hat diese Diskussion bereits begonnen. In Deutschland wird in Projekten der Einsatz von Kartensystemen zur Erstellung elektronischer Rezepte geprüft, nicht jedoch die hier skizzierte Rezeptübermittlung vom Arzt an die Apotheker via Datenübertragung. Der Arzt bedient sich in unserem Modell - ähnlich den englischen NHS-Pilotprojekten - der Online-Datenübertragung des Rezeptes direkt an die Apotheke.

Im Zusammenhang mit Online-Rezepten werden, wie im gesamten Bereich der elektronischen Übermittlung insbesondere Datensicherheits- und Legitimationsaspekte diskutiert. Nach dem deutschen Signaturgesetz sind digitale Signaturen den handschriftlichen Unterschriften rechtlich noch nicht gleichgestellt. Allerdings hat die EU im Dezember 1999 aufgrund der wachsenden Bedeutung der elektronischen Kommunikation eine Richtlinie über gemeinschaftliche Rahmenbedingungen für elektronische Signaturen verabschiedet. Zudem haben sich auf internationaler Ebene weltweit tätige Unternehmen zusammengeschlossen, um die Implementierung gemeinsam erarbeiteter, technologieneutraler Rechts- und Verfahrensgrundlagen zu fördern. Sie sollen als Grundlage für einheitliche Rahmenbedingungen bzw. einheitliche Regelungen u.a. für die Bereiche Authentisierung und Sicherheit dienen ${ }^{7}$. Denn diese neue Art der Rezeptweitergabe könnte neben Effizienzvorteilen, die sich aus veränderten Distributionswegen ergeben, dazu beitragen, dass sich die bisher streng reglementierten Vertriebswege zu einem verbraucherfreundlichen Nebeneinander von Präsenz- und Versandapotheke entwickeln.

Vgl. Meyer, C (2000), Viagra aus Kerkrade, in: Der Spiegel, Heft 39, S. 88.

O.V. (2000), Online Gesetze, Richtlinien und Rechtsgrundlagen der Internetnutzung, in: www.deutsche-bank.de, Rubrik eCommerce, Zugriff am 17.03.00.

Schäfers, C., Kaesbach, W. (2000), Internethandel mit Arzneimitteln, in: Die BKK. Zeitschrift der Betrieblichen Krankenversicherung, Jg.88, Heft 5, S. $205 f$. 


\subsubsection{Exkurs: Elektronisches Rezept}

Für das dargestellte Modell der Versandapotheke bedarf es grundsätzlich keiner gesonderten technischen Ausstattung zur Übertragung der Rezeptdaten an die Apotheke. So beruht das Konzept der Schweizer Versandapotheke MediService allein auf einer postalischen Rezeptübermittlung durch die Versicherten an die Versandapotheke; eine elektronische Übermittlung der Rezeptdaten zwischen verschreibendem Arzt und Versandapotheke erfolgt nicht ${ }^{9}$.

Allerdings stellt die elektronische Übertragung der Rezeptdaten direkt vom verschreibenden Arzt an die Versandapotheke - insbesondere aus Sicht der Verbraucher - einen besonderen Vorteil dar, da sich so die Transaktionskosten (postalische Versendung der Rezepte durch die Patienten oder den Arzt; persönliche Weitergabe der Rezepte an die Apotheke) erheblich reduzieren. Folgerichtig wird im Zusammenhang mit Versandapotheken nahezu immer von einer elektronischen Rezeptdatenübertragung ausgegangen.

In den folgenden Modellrechnungen kann die derzeit von verschiedenen Seiten ${ }^{10}$ noch nicht abschließend diskutierte technische Umsetzung des elektronischen Rezepts nur kursorisch thematisiert werden. Festzuhalten bleibt allerdings, dass das elektronische Rezept nicht nur im Rahmen der Überlegungen zur Ausweitung des Pharmaversandes eine zunehmend bedeutende Rolle spielt.

Die heutige Form der Datenübermittlung auf Datenträger in Verbindung mit den gesetzlich zugewiesenen Zuständigkeiten bewirkt große Datenvolumina, Belastungsspitzen und Verzögerungen für die weitere Datenverarbeitung. An dem heutigen Rezeptverfahren sind rund 70 Millionen Versicherte, 360 Krankenkassen, 110.000 Ärzte, 53.000 Zahnärzte, 22.000 Apotheken, 2.200 Krankenhäuser und 120.000 weitere Leistungserbringer beteiligt. Es werden jährlich 600 Millionen Rezepte mit 900 Verordnungen und einem Bruttoapothekenumsatz von 40 Milliarden DM ausgestellt ${ }^{11}$.

Allein die Betriebskosten der Apothekerseite belaufen sich auf 160 Millionen DM jährlich für die Annahme, Prüfung und Weiterbearbeitung der Daten in den Apothekenrechenzentren. Nach Expertenschätzungen belaufen sich die bei allen Beteiligten anfallenden Verfahrenskosten auf mindestens 2 Milliarden DM, ohne jedoch eine zufriedenstellende Aktualität und Qualität sicherstellen zu können ${ }^{12}$.

9

Vgl. Krähenbühl, J.-P., (2000), Medikamente direkt nach Hause liefern - eine Innovation im Gesundheitswesen, in: Die BKK. Zeitschrift der Betrieblichen Krankenversicherung, Jg. 88, Heft 5, S. 200.

Einen Überblick über den Diskussionsstand geben die Ergebnisse des Aktionsforums "Telematik im Gesundheitswesen" (ATG), die auch unter www.gvg-koeln.de einzusehen sind. Sendatzki, V. (2001), Elektronisches Rezept gewinnt an Kontur, S. 21.

Sendatzki, V. (2001), Elektronisches Rezept. Startrampe für die Telematikplattform in der GKV, in: Die BKK. Zeitschrift der Betrieblichen Krankenversicherung, Jg. 89, Heft 3, S. 133. 
Auch der Nacherfassungsaufwand für unkorrekt bedruckte Rezeptformulare verursacht Kosten in Höhe von ca. 18 Mio. DM pro Jahr. Allein die GKV-Aufwendungen für die manuelle Nachbereitung und das Einlesen der Belege betragen 60 Mio. DM pro Jahr. Trotz dieses Aufwands wird für die GKV von einem Fehlbetrag von über 500 Mio. DM durch ungerechtfertigte Zuzahlungsbefreiungen ausgegangen ${ }^{13}$.

Der bislang primär abrechnungsorientierte Datenfluss erschwert insgesamt Rückkopplungsinformation und es ergeben sich lange Laufzeiten und Störquellen aufgrund der schrittweisen Aufbereitung der Daten. Schließlich treten häufig Medienbrüche im Datenfluss auf, die zu weiteren Beeinträchtigungen führen. Die Ordnungsmäßigkeit der Daten wird durch zusätzliche Maßnahmen der manuellen Kontrolle und Nachbearbeitung teuer erkauft.

Eine effizientere Netztechnik in Form des elektronischen Rezeptes als übergreifendes Übermittlungsinstrument zwischen Arzt, Patient, Apotheker und Krankenkasse erfüllt die Schaffung effizienter Versorgungsstrukturen, ermöglicht Kosteneinsparungen und erhöht Qualität und Aktualität des gegenwärtigen Verordnungsablaufes.

Die Umstellung auf durchgängig elektronische Kommunikation ermöglichen mittelfristig eine Reduktion der Kosten und eine lückenlose und übergangslose Abbildung des Datenflusses im Gesundheitswesen. Nach Einschätzung von Experten können durch den Einsatz von IT-Standards die Datenströme im Gesundheitswesen wesentlich effizienter gestaltet werden. Die Informationsströme lassen sich über eine Vernetzung zeitnah, valide und bedarfsgerechter darstellen. Das Gesundheitswesen kann somit durch eine effizientere Leistungs- und Kostenstruktur optimiert werden.

Vor diesem Hintergrund relativieren sich auch die zu erwartenden zusätzlichen Investitionskosten die z.B. in Arztpraxen und (Versand-)Apotheken durch die Einführung umfassender elektronischer Kommunikationstechniken entstehen. Diese werden in ersten groben Analysen auf maximal 3.000 DM für die derzeit existierenden Präsenzapotheken und auf "... bis zu 4.000 DM für eine Arztpraxis mit und auf 10.000 DM für eine Arztpraxis ohne EDV-Ausstattung ..." taxiert ${ }^{14}$.

Das Konzept des "elektronischen Rezepts" muss allerdings - und dies gilt auch für die Integration der elektronischen Rezeptdatenübertragung im Rahmen eines Modells der Versandapotheke - strengen Sicherheitsansprüchen genügen ${ }^{15}$ :

- Vertraulichkeit, d.h. nur die definierten Kommunikationspartner (z.B. verschreibender Arzt und im Vorfeld definierte Versandapotheke) können die für sie bestimmten Informationen lesen und verwenden;

13

Aktionsforum Telematik im Gesundheitswesen (2000), Managementpapier "Elektronisches Rezept", Köln 2000, S. 11.

14

Schubert, F., Das Elektronische Rezept: Chancen, Risiken und Gestaltungsmöglichkeiten. Diplomarbeit, Heidelberg 1999, S. 20.

15

Vgl. hierzu auch: Aktionsforum "Telematik im Gesundheitswesen" (2001), Management-Papier zur Sicherheitsinfrastruktur, Köln 2001. 
- Integrität, d.h. es kann nachgewiesen und überprüft werden, dass Nachrichten (Rezeptdaten) richtig, vollständig und unverändert ausgetauscht werden;

- Authentizität, d.h. Absender und Empfänger können eindeutig identifiziert werden, um sicherzustellen, wer wann welche Daten übermittelt hat (bedeutsam z.B. auch zur Garante der Rückkoppelung zwischen Apotheke und Arzt);

- Unabstreitbarkeit, d.h. die Teilnahme definierter Absender und Empfänger an der Kommunikation ist (z.B. im Rahmen der Abrechnung mit den Krankenkassen) belegbar.

Weiterhin muss frühzeitig Öffentlichkeitsarbeit geleistet werden, um die notwendige Zusammenarbeit aller Beteiligten zu gewährleisten. Auch die Definition des rechtlichen ist zwingend, sind doch gegenwärtig gesetzlich Formulare und eine eigenhändige Unterschrift des Arztes bei der Medikamentenverordnung zwingend vorgeschrieben. Es besteht derzeit noch keine Rechtsgrundlage für eine papierlose, ITgestützte Medikamentenverordnung.

\subsection{Annahmen und Methodik}

\subsubsection{Vorgehensweise der Modellrechnungen}

Im Folgenden werden die differenzierten Ergebnisse der Berechnungen eines potentiellen monetären Einsparvolumens einer Ausweitung des Pharmaversandes dargestellt. Grundsätzlich gilt es dabei, folgende Fragen zu beantworten:

(1) In welchem Medikationssegment positionieren sich potentielle Versandapotheken (sogenanntes Zielsegment von Versandapotheken)? Unter epidemiologischen Gesichtspunkten könnte sich der Arzneimittelversand insbesondere für Patienten mit chronischen Erkrankungen bzw. Dauermedikation als sinnvoll erweisen.

(2) Wie stellen sich die Kosten des neuen Vertriebsweges von Arzneimitteln an den Endverbraucher im Vergleich zur derzeitigen Kostenstruktur der Präsenzapotheke dar? Welche betriebsinternen Umsatz-, Kosten- und Ertragsstrukturen potentieller Anbieter bilden sich heraus?

(3) Welches Marktpotential ergibt sich aus der Kombination von Medikationssegment und den jeweiligen betriebsinternen Umsatz-, Kosten- und Ertragsstrukturen?

(4) Welches Einsparpotential resultiert aus der Zulassung eines Pharmaversandes für die gesetzliche Krankenversicherung?

In der Reihenfolge dieser Fragen wird auch bei den Modellrechnungen vorgegangen (siehe dazu Abbildung II - 3): 
Abbildung II - 3: Begriffsbestimmung und Darstellung der Vorgehensweise bei den Modellrechnungen

\section{GKV-Fertigarzneimittelmarkt}

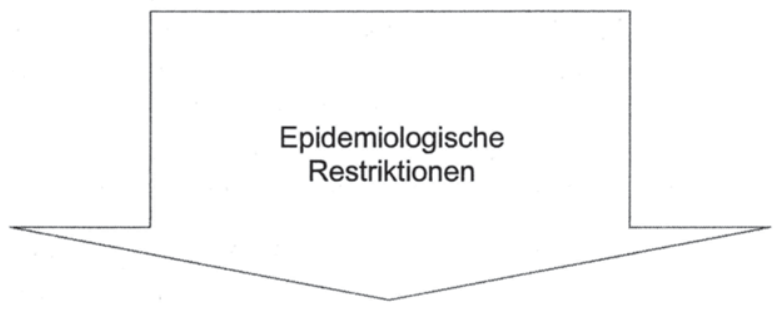

\section{Zielsegment von Versandapotheken (auch Versandpotential genannt)}

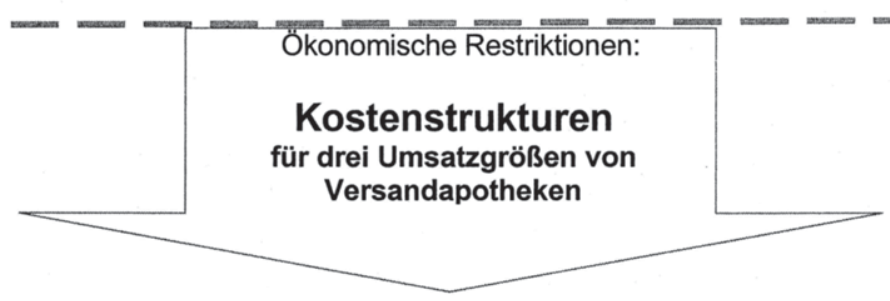

\section{Marktpotentiale $\Rightarrow$ Einsparpotentiale}

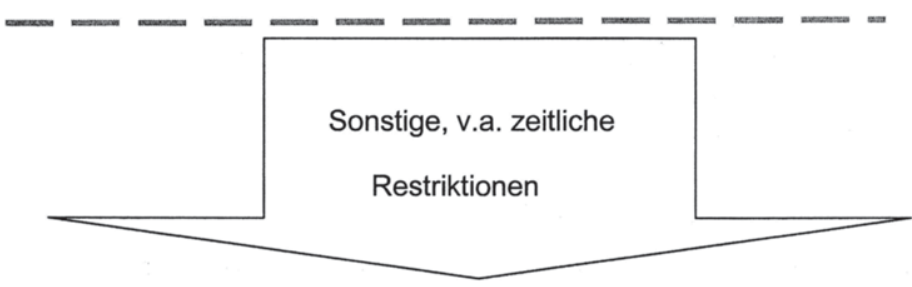

Realisierung von Marktanteilen
Realisierung von

Einsparvolumina 
(1) Entwicklung des Zielsegments anhand epidemiologischer Kriterien aus dem GKV-Fertigarzneimittelumsatz (1999: 36,7 Mrd. DM):

Basierend auf ausländischen Erfahrungen und Expertenmeinungen ist davon auszugehen, dass der Arzneimittelversand insbesondere für Patienten mit chronischen Erkrankungen und Dauermedikation vorteilhaft erscheint, während er auch bei optimaler Logistik im Bereich der Akutmedizin eher eine untergeordnete Rolle spielen wird. Anhand dieser Zielgruppenfokussierung gilt es, die Verordnungen herauszufiltern, die bei Annahme eines planbaren mittel- bis langfristigen Medikamentenbedarfes des Patienten für den Versandweg und der damit verbundenen 24- bis 48-stündigen Lieferzeit als „versendungsfähig“ gelten können. Nicht versendungsfähig sind im Gegensatz dazu Verordnungen, die ohne zeitliche Verzögerung aus der Apotheke zu beziehen sind (Akutmedikation).

Die Analysen ergeben aus einem breiten Spektrum möglicher Varianten drei plausible Zielsegmente (in \% vom GKV-Fertigarzneimittelumsatz): $41 \%$ (Variante A), $47 \%$ (Variante B), $57 \%$ (Variante C).

(2) Ermittlung der betrieblichen Umsatz-, Kosten- und Ertragsstruktur:

Hierzu wird auf Basis zugänglicher Apothekenbetriebsvergleichs-Daten die Kostenstruktur einer durchschnittlichen Präsenzapotheke dargestellt. Unter Zuhilfenahme bestimmter Szenarien zur Betriebsstruktur und weiterer Sekundärdaten (Kapitel 2.4.) erfolgt in einem nächsten Schritt die Entwicklung der Kostenstruktur einer zukünftigen Versandapotheke, allerdings z.T. basierend auf theoriegeleiteten Annahmen. Dies zum einen deshalb, da neue Vertriebsformen neue betriebswirtschaftliche Kosten (für Logistik, Beratungsangebote, usw.) induzieren werden. Andererseits können sich Einsparungen durch günstigere Einkaufskonditionen, Mietkosten, usw. ergeben. Dabei wird die Kosten- und Ertragsstruktur an unterschiedliche Umsatzgrößenklassen von Versandapotheken gekoppelt, um Skaleneffekte bei den Kosten und deren Auswirkung auf das Betriebsergebnis zu analysieren.

Es ergeben sich drei Varianten von Arzneimittelversendern: die "kleine" (Variante VA I), die "große" (Variante VA II) und die "Versandapotheke im Verbund mit einem Großhändler" (Variante VA III).

(3) Berechnung des Marktpotentials:

Das unter (1) definierte Zielsegment wird gemäß der ökonomischen Restriktionen im Punkt (2) zum sogenannten Marktpotential begrenzt. Es ergeben sich aufgrund der Kombinationen aus drei Apothekengrößenklassen und drei Zielsegmentvarianten neun unterschiedliche Marktpotentialvarianten. 
Ob und in welchem Maß dieses Marktpotential in Form von Marktanteilen durch die jeweiligen potentiellen Anbieter realisiert wird, hängt von derem wirtschaftlichen Agieren sowie weiteren Restriktionen ab. Denkbar wären hier insbesondere zeitbezogene Kriterien (Markteintrittsschranken, Überwindung von Akzeptanzhürden bei Patienten und Ärzten).

(4) Darstellung des Einsparpotentials:

Dazu werden die neun Kombinationen des Marktpotentials auf ihre Kosteneinsparungen im Vergleich zur derzeitigen Apothekenbetriebsspanne untersucht. Die neun Varianten möglicher Einsparpotentiale können als Grenzwert i.d.S. betrachtet werden, dass unter Effizienzkriterien keine maximalere monetäre Ausschöpfung von Wirtschaftlichkeitsreserven bei der jeweiligen Kombination möglich ist.

In welchem Maß das Einsparpotential in Form von Einsparvolumina realisiert wird, hängt von der Entwicklung der tatsächlichen Marktanteile ab.

Zusammenfassung der gebrauchten Begriffsdefinitionen:

\section{$\rightarrow$ Zielsegment ( = Versandpotential):}

Umsatzanteil der Medikation der Zielgruppe Chronisch Kranker / Patienten mit Dauermedikation am GKV-Fertigarzneimittelmarkt. Diese bilden die epidemiologischen Restriktionen.

\section{-Umsatz - Kosten - und Ertragsstruktur:}

Interne Betriebsstruktur und deren Auswirkungen auf die internen Kosten und das Betriebsergebnis $^{16}$. Diese bilden die ökonomischen Restriktionen.

\section{-Marktpotential:}

Maximal erreichbarer Umsatzanteil am GKV- Fertigarzneimittelmarkt, der über Versandapotheken unter Berücksichtigung epidemiologischer sowie ökonomischer Restriktionen vertrieben werden könnte.

\section{-Einsparpotential:}

Aus der Kombination aus Marktpotential und Kostenstruktur (bzw. erzielbare Kosteneinsparungen auf den Medikamentenpreis) ergibt sich das Einsparpotential der GKV bei vollständiger Abschöpfung des Marktpotentials.

Y(tatsächlicher / realisierter) Marktanteil: Realisierungsgrad des Marktpotentials.

\section{-Ytatsächliches / realisiertes) Einsparvolumen:}

In Abhängigkeit vom Realisierungsgrad des Marktpotentials erzielbare Einsparungen für die GKV. 


\subsubsection{Datengrundlagen zum Arzneimittelmarkt in Deutschland}

Aufgrund des umfangreichen Bedarfs an Informationen muss auf eine Vielzahl verschiedener Quellen zugrückgegriffen werden. In diesem Zusammenhang sollte erwähnt werden, dass Zahlenangaben über die Arzneimittelversorgung bekanntlich je nach Quelle erheblich differieren. Ferner kann eine Beeinflussung des Zahlenmaterials durch partikuläre Interessen der jeweiligen Akteure am Arzneimittelmarkt nicht grundsätzlich ausgeschlossen werden. Entsprechend schwierig ist es deshalb, vergleichbare Daten zu erhalten und daraus Rückschlüsse zu ziehen. ${ }^{17}$

Ferner wirkt erschwerend, dass zum einen eine Differenzierung der Berechnungen aus volkswirtschaftlicher Perspektive (Zielsegmente, Kapitel 2.3.) und betriebswirtschaftlicher Perspektive (interne Kostenstrukturen von Apotheken, Kapitel 2.4.) nötig ist, und diese wiederum zusammengeführt werden müssen (Markt- und daraus abzuleitende Einsparpotentiale, Kapitel 2.5.).

Die volkswirtschaftliche Berechnung des Zielsegments in Kapitel 2.3 basiert auf der Datenbasis des GKV-Fertigarzneimittelumsatzes. Der Fokus liegt also auf dem GKVfinanzierten Arzneimittelumsatz, der Umsatzanteil anderer Kostenträger wird nicht berücksichtigt. Der GKV-Fertigarzneimittelumsatz wiederum basiert auf Zahlenmaterial des Wissenschaftlichen Instituts der AOK (WIdO), das eine Vier-Promille-Stichprobe aus allen über Apothekenrechenzentren abgerechneten Rezeptblättern zieht, und daraus eine Hochrechnung für die gesamte Bundesrepublik erstellt ${ }^{18}$. Ausgehend von den amtlichen Statistiken des BMG (KV 45), welche Arzneimittelausgaben der GKV für Arzneimittel aus Apotheken für 1999 von 36,15 Mrd. DM ausweisen, errechnet das WIdO einen GKV-Fertigarzneimittelumsatz von 36,7 Mrd. DM.

Der GKV-Fertigarzneimittelumsatz basiert auf 782,6 Mio. Stück Verordnungen bzw. 27.751,4 Mio. Stück definierten Tagesdosen (DDD). ${ }^{19}$ Daraus ergibt sich ein durchschnittlicher Verordnungswert von 47 DM (und 35 DDD je Verordnung) inklusive Mehrwertsteuer und Kassenrabatt.

In Kapitel 2.4. wird für die betriebswirtschaftliche Kalkulation der internen Kostenstruktur von Präsenzapotheken der Apothekenbetriebsvergleich des Instituts für Handelsforschung an der Universität zu Köln (Basisjahr 1998) ${ }^{20}$ zugrundegelegt. Der Betriebsvergleich basiert auf einer Finanzierung des Apothekenumsatzes durch die

17

18

19

20

Reiblich, M. (2000), Die Schlacht der Statistiker im Gesundheitswesen, in: Medikament \& Meinung, Jg. 24, Nr. 8, S. 3.

Schröder, H., Selke, G.W. (2000), Ergänzende statistische Übersicht, in: Schwabe, Paffrath (Hrsg.), Arzneimittelverordnungs-Report, S. 796-881.

Schröder, H., Selke, G.W. (2000), Ergănzende statistische Übersicht, in: Schwabe, Paffrath (Hrsg.), Arzneimittelverordnungs-Report, S. 813.

Büchel, D., (1998), Der neue IfH-Monatsbetriebsvergleich für Apotheken, S. 102, Abb. 10, in: Management in Apotheken, Sonderheft 43 des IFH, S. 87-105, sowie: Kaapke, A. (1998), Controlling in Apotheken mit Hilfe des Jahresbetriebsvergleiches des IFH, S. 75, in: Management in Apotheken, Sonderheft 43 des IFH, S. 57-85. 
GKV sowie anderer Kostenträger (PKV, Selbstzahler). Folgende Wertschöpfungsanteile am Apothekenumsatz werden ausgewiesen ${ }^{21}: 58,7 \%$ Wareneinsatz (Hersteller, Großhandel), 27,5\% Betriebshandelsspanne, 13,8\% Mehrwertsteuer ${ }^{22}$. Im Sinne einer allgemeinen Vergleichbarkeit werden auch für Versandapotheken betriebliche Umsatz- wie Kostenstrukturen entwickelt (Kapitel 2.4.3. bis 2.4.5.), welche auf einer Finanzierung durch unterschiedliche Kostenträger beruhen. Die Herangehensweise an die interne Kostenkalkulation für Versandapotheken ist damit methodisch identisch mit der für Präsenzapotheken.

Die Berechnung der Marktpotentiale aus der Betrachtung des Zielsegments unter Berücksichtigung kostenökonomischer Restriktionen folgt einer differenzierten Betrachtung des durchschnittlichen Verordnungswertes von ca. 47 DM auf der Ebene der einzelnen ATC-Indikationsgruppen (international akzeptierte anatomischtherapeutisch-chemische Kategorien). Bei der Ableitung der Einsparpotentiale aus den Marktpotentialen in Kapitel 2.5. werden die betrachteten Verordnungswerte um den Kassenrabatt von 5\% gekürzt, da dieser Betrag aufgrund seiner Natur als bereits von den Apotheken an die GKV abzuführender Rabatt nicht zukünftigen Einsparpotentialen zugerechnet werden kann.

\subsection{Definition des Zielsegments für einen Pharmaversand}

\subsubsection{Vorgehensweise: Basis, Zielgruppenbildung und Operationalisierung}

Basis der folgenden Ermittlung des Zielsegments, per Definition dem Bereich der Medikation, der unter epidemiologischen Gesichtspunkten als „versandfähig“ definiert werden kann, ist der GKV-Fertigarzneimittelumsatz. Das Merkmal „versendungsfähig" bezieht sich auf die Dringlichkeit der Arzneimittelnachfrage. Geht man davon aus, dass eine sehr dringliche Medikation, also insbesondere Akutmedikation, für den Patienten ohne zeitliche Verzögerung aus der Apotheke zur Verfügung stehen muss, ist diese unter der Restriktion einer 24 bis 48 -Stunden-Zustellfrist ${ }^{23}$ für einen Bezug via Versand (noch) nicht geeignet ${ }^{24}$. Für Medikationen, die keiner akuten Dringlichkeit unterliegen und damit in ihrer Nachfrage planbar sind, ist die Restriktion der Lieferfrist überbrückbar und folglich kein Ausschlusskriterium. Eine repräsentative INIFES - Bevölkerungsbefragung ergab, dass die Befragten, die sich grundsätzlich einen Medikamentenbezug per Versand für ihre eigene Situation vorstellen könnten,

21

Vgl. Angaben ABDA (1998), Zahlen, Daten, Fakten. Sowie: Büchel, D. (1998), Der neue IfHMonatsbetriebsvergleich für Apotheken, S. 102, Abb. 10, in: Management in Apotheken, Sonderheft 43 des IFH, S. 87-105,

22

Zum 1.4. 1998 wurde die Mehrwertsteuer von $15 \%$ auf $16 \%$ angehoben, was im Durchschnitt für 1998 exakt 13,6\% ergeben würde. Da für die folgenden Jahre jedoch $16 \%$ MwSt durchschnittlich 13,8\% ergeben, wird hier bereits für 1998 dieser Wert angeführt.

23

Die üblichen Zustellfristen ausländischer seriöser Versandapotheken liegen laut Expertengesprăch in dieser Frist.

24

Siehe auch Hovermann, E., (2001), Versandhandel versus Einzelapotheke, in: Die BKK. Zeitschrift der betrieblichen Krankenversicherung, Heft 4, S. 177. 
an diesen als wichtige Mindestanforderung eine zügige Medikamentenlieferung binnen 48 Stunden knüpfen.

Die folgenden Berechnungen basieren also auf der restriktiven Annahme, dass Medikation für akute Erkrankungen zunächst kein Potential für den Versand von Arzneimitteln darstellt. Das Zielsegment ist demnach der Teil des GKV-Fertigarzneimittelumsatzes, der durch die Zielfokussierung auf chronische bzw. dauerhafte Medikation abgegrenzt wird, und damit die maximale Obergrenze für eine Option "Versandhandel mit Arzneimitteln" bildet. Alle weiteren Modellschritte, wie die Berechnung des Markt- bzw. des Einsparpotentials (siehe Kapitel 2.5.), werden sich auf das Zielsegment beziehen. In diesem Punkt, d.h. in einer zielgruppenorientierten Betrachtungsweise des Arzneimittelumsatzes, unterscheidet sich die gewählte Vorgehensweise von anderen, zumeist verkürzten Darstellungen in der Fachliteratur, wo zumeist der gesamte Apothekenumsatz bzw. die GKV-Ausgaben für Arzneimittel als Bezugsgröße für Spekulationen über das Volumen des Versandhandels gewählt werden. ${ }^{25}$

Das Zielsegment des Versandhandels wird demnach im weiteren durch die Medikation für chronische Erkrankungen bzw. der Dauermedikation gebildet. Eine präzise, allgemeingültige Abgrenzung dieser Kategorien existiert in der Gesundheitsökonomie nicht. Eine im Krankenhausreport '99 - Schwerpunkt Versorgung chronisch Kranker enthaltene Untersuchung grenzt Patienten aufgrund ihrer Medikation als "chronisch krank" ein, wenn sie eine kontinuierliche Arzneiversorgung aus ein - und derselben Arzneimittelgruppe über mindestens drei Quartale aufweisen. ${ }^{26}$ Das SGB V (§ 62) spricht von chronischer Erkrankung bei Versicherten, die "... wegen derselben Erkrankung in Dauerbehandlung sind...".

Die folgenden Überlegungen nähern sich verschiedenen Abgrenzungsvarianten über die Auswertung von diesbezüglich geführten Expertengesprächen und Sekundärdatenanalysen. Abbildung II - 4 fasst die Überlegungen zusammen.

Siehe auch Hovermann, E., (2001), Versandhandel versus Einzelapotheke, in: Die BKK. Zeitschrift der betrieblichen Krankenversicherung, Heft 4, S. 178. 
Abbildung II - 4: Organigramm für die Berechnung des Zielsegments

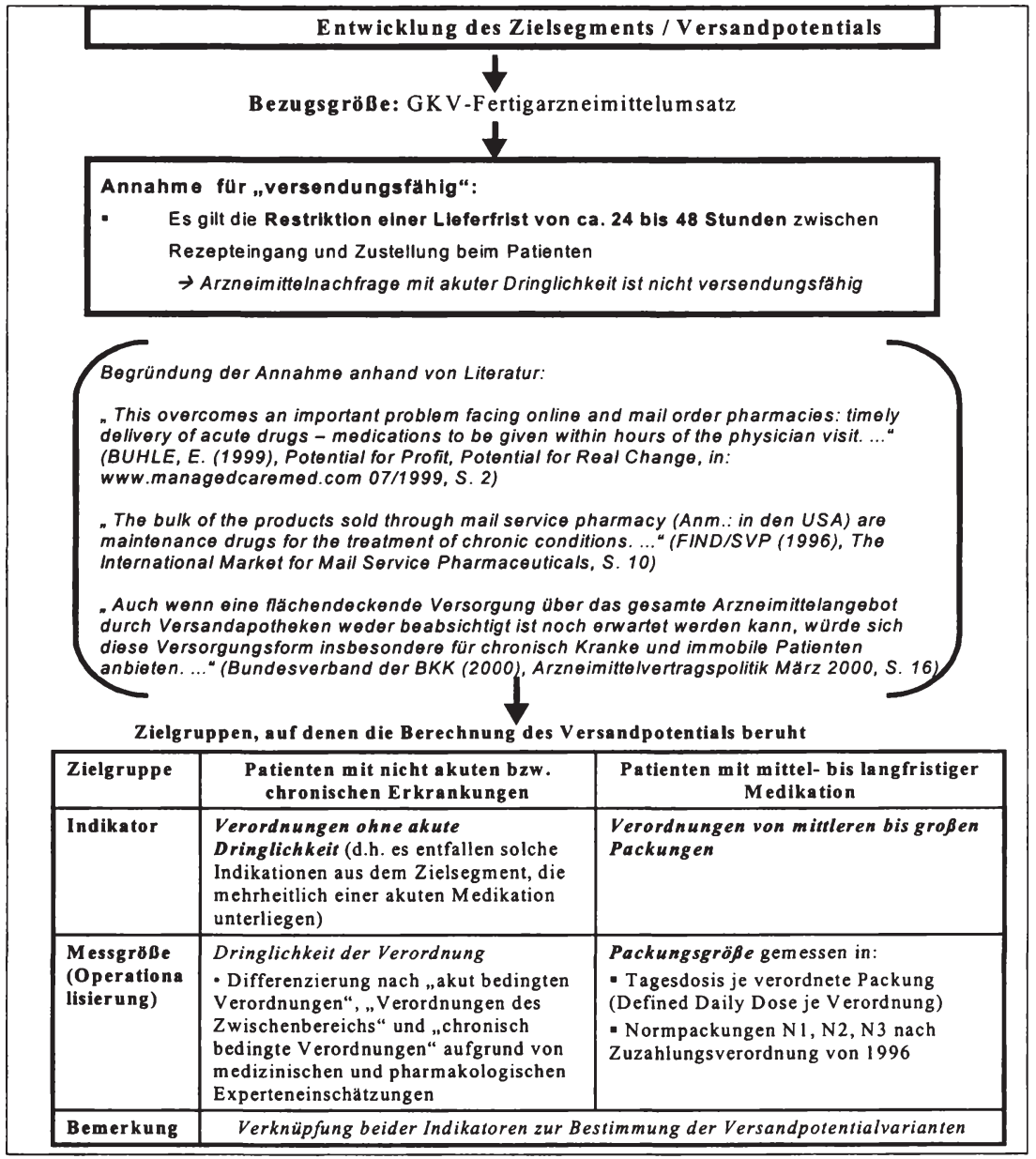

Quelle: INIFES, eigene Darstellung. 
Die Berechnungen des Zielsegments basieren auf dem GKV - Fertigarzneimittelumsatz, gegliedert in einzelne Indikationsgruppen. ${ }^{27}$ Dabei wird von folgenden Sachverhalten ausgegangen:

- In Fällen, in denen eine Akutversorgung mit Medikamenten im Anschluss an den Arztbesuch erfolgen muss, ist ein Bezug aus der Versandapotheke aufgrund der längeren Distributionsdauer (derzeit wird anhand von Erfahrungen im Ausland von ca. 24 bis 48 Stunden ausgegangen) nicht möglich bzw. mit hohen Versandkosen behaftet (Expressversand), die einen Direktversand kleinerer Arzneimittelmengen an den Endverbraucher ökonomisch nicht sinnvoll erscheinen lassen. Die Arzneimittelabgabe durch die Präsenzapotheke bleibt Priorität.

- Daraus kann allerdings auch abgeleitet werden, dass bei allen nicht akuten Diagnosen grundsätzlich die Alternative Arzneimittelvertrieb ins Auge gefasst werden kann, da der Arzneimittelbedarf im Gegensatz zu Akutkrankheiten nicht plötzlich eintritt, sondern aufgrund bestimmter Indikationen für den Arzt und Patienten für einen gewissen mittel- bis längerfristigen Zeitraum planbar wird.

Mit diesen Annahmen kristallisieren sich die Zielgruppen heraus:

- Bezieher von mittel- und langfristiger Medikation bzw.

- Patienten, die unter langwierigen chronischen Krankheiten leiden.

Es gilt, die Zielgruppen anhand von Indikatoren quantitativ zu operationalisieren und als Zielsegment vom GKV-Arzneimittelumsatz abzubilden. Dies geschieht mit den Indiatoren „Verordnungen / Medikation ohne akute Dringlichkeit" und „Verordnungen / Medikation von mittleren bis großen Packungen".

\section{Zielgruppe Patienten mit chronischen Erkrankungen}

Es wurde der Indikator „Verordnungen ohne akute Dringlichkeit" gewählt. Da eine klare Definition der Begriffe akut bzw. chronisch nicht existiert und nur von einem "common sense" unter den medizinischen wie pharmazeutischen Experten ausgegangen werden kann, wurden Expertenmeinungen von Ärzten und Apothekern eingeholt. Dabei sollte je Indikationsgruppe eruiert werden, wie hoch der Anteil der Verordnungen ist, die auf akut bedingte Medikation und auf chronisch bedingte Medikation entfallen. Die Dringlichkeit der Nachfrage wurde in diesen beiden Kategorien, um eine einheitliche Basis des Begriffsverständnisses zu ermöglichen, anhand der zeitlichen Dauer der Medikation und daran, ob es sich um eine voraussichtlich permanente Arzneimittelnachfrage handelt, operationalisiert. ${ }^{28}$

Diese werden dem Arzneiverordnungsreport entnommen. Vgl. Schröder, H., Selke, G.W. (2000), Ergănzende statistische Übersicht, in: Schwabe, U., Paffrath, D. (Hrsg) (2000), Arzneiverordnungsreport 1999, S. 722ff (Tabelle 53.4.).

Ein möglicher Missbrauch von Arzneimitteln durch Suchtverhalten wird hier vernachlässigt, maßgeblich ist ausschließlich die medizinische Notwendigkeit. 
- Unter „akut" wird eine Medikation von nur wenigen Tagen bzw. Wochen verstanden, die in diesem Zeitraum zu einer Heilung beiträgt, und deshalb danach als nicht mehr medizinisch nötig bezeichnet werden kann.

- "Chronische“ Medikation dagegen wird mindestens über viele Monate bzw. Jahre verordnet und führt in den seltensten Fällen zu einer endgültigen Heilung des Leidens, sondern vielmehr „nur" zu einer Zustands- oder Befindlichkeitsverbesserung. Die Nachfrage nach chronischer Medikation ist überwiegend lebenslänglich determiniert.

Da eine exakte Abgrenzung zwischen „akut“ und „chronisch“ nicht definiert werden kann, offensichtlich aber durch die jeweiligen Zeitangaben ein Übergangsbereich existiert, wurde die Kategorie „Zwischenbereich“ eingeführt. Hier befinden sich zum einen

- Verordnungen, die häufig mit einer akuten Medikation beginnen und danach in eine mittel- bis längerfristige Arzneitherapie münden,

- zum anderen aber auch Verordnungen, deren Medikationsdauer nicht von vornherein klar definiert ist.

- Zudem werden darunter Verordnungen verstanden, die in ihrer Medikation mittelfristig angelegt sind, also länger als einige Tage verabreicht werden, jedoch nach einer gewissen Anwendungszeit wieder abgesetzt werden können.

Es ergaben sich aufgrund des Expertenrates folgende Verordnungsanteile für akute und chronische Medikation ${ }^{29}$, differenziert je Indikationsgruppe nach AnatomischTherapeutischer-Klassifizierung (ATC-Code):

28

Die ATC - Klassifizierung H01 (Hypophysen- und hypothalamische Hormone) wurde nach den Expertenmeinungen zwar einstimmig als chronische Medikation definiert, jedoch aufgrund ihrer besonderen Eigenschaften wird nicht unter das Zielsegment eines Versandes an den Endverbraucher Patient subsumiert. 
Tabelle II - 1: Anteil der akuten und chronischen Verordnungen sowie solcher des Zwischenbereichs (Unterteilung durch pharmazeutische und medizinische Experten)

\begin{tabular}{|c|c|c|c|}
\hline ATC-Klassifikation & Akut & $\begin{array}{l}\text { Zwischen- } \\
\text { bereich }\end{array}$ & $\begin{array}{l}\text { Chro- } \\
\text { nisch }\end{array}$ \\
\hline A01 (Stomat Präparate) & $40 \%$ & $10 \%$ & $50 \%$ \\
\hline $\begin{array}{l}\text { A02 (Antacida / Ulkustherapeutika, } \\
\text { Karminativa) }\end{array}$ & $30 \%$ & $0 \%$ & $70 \%$ \\
\hline $\begin{array}{l}\text { A03 (Spasmolytika, } \\
\text { Anticholinergika) }\end{array}$ & $80 \%$ & $20 \%$ & $0 \%$ \\
\hline $\begin{array}{l}\text { A04 (Antiemetika/Mittel gegen } \\
\text { Übelkeit) }\end{array}$ & $85 \%$ & $0 \%$ & $15 \%$ \\
\hline $\begin{array}{l}\text { A05 (Gallen- und } \\
\text { Lebertherapeutika) }\end{array}$ & $15 \%$ & $0 \%$ & $85 \%$ \\
\hline A06 (Laxantia) & $30 \%$ & $0 \%$ & $70 \%$ \\
\hline $\begin{array}{l}\text { A07 (Antidiarrhoika/intestale } \\
\text { Antiphlogistika) }\end{array}$ & $90 \%$ & $10 \%$ & $0 \%$ \\
\hline A09 (Digestiva, inklusive Enzyme) & $0 \%$ & $30 \%$ & $70 \%$ \\
\hline A10 (Antidiabetika) & $5 \%$ & $0 \%$ & $95 \%$ \\
\hline A11 (Vitamine) & $30 \%$ & $20 \%$ & $50 \%$ \\
\hline A12 (Mineralstoffe) & $60 \%$ & $40 \%$ & $0 \%$ \\
\hline B01 (Antikoagulantia) & $30 \%$ & $0 \%$ & $70 \%$ \\
\hline B02 (Antihämorrhagika) & $20 \%$ & $20 \%$ & $60 \%$ \\
\hline B03 (Antianămika) & $20 \%$ & $30 \%$ & $50 \%$ \\
\hline $\begin{array}{l}\text { B05 (Plasmaersatzmittel, } \\
\text { Infusionslösungen) }\end{array}$ & $100 \%$ & $0 \%$ & $0 \%$ \\
\hline C01 (Herztherapie) & $15 \%$ & $0 \%$ & $85 \%$ \\
\hline C02 (Antihypertonika) & $15 \%$ & $0 \%$ & $85 \%$ \\
\hline C03 (Diuretika) & $15 \%$ & $0 \%$ & $85 \%$ \\
\hline C04 (periphere Vasodilitatoren) & $20 \%$ & $0 \%$ & $80 \%$ \\
\hline C05 (Vasoprotektoren) & $15 \%$ & $0 \%$ & $85 \%$ \\
\hline $\begin{array}{l}\text { C06 (andere Herz- und } \\
\text { Kreislaufpräparate) }\end{array}$ & $15 \%$ & $0 \%$ & $85 \%$ \\
\hline C07 (Betarezeptorenblocker) & $15 \%$ & $0 \%$ & $85 \%$ \\
\hline C08 (Kalziumkanalblocker) & $15 \%$ & $0 \%$ & $85 \%$ \\
\hline $\begin{array}{l}\text { C09 (Hemmstoffe des Renin- } \\
\text { Angiotensin-Systems) }\end{array}$ & $10 \%$ & $0 \%$ & $90 \%$ \\
\hline C10 (Lipidsenkende Mittel) & $0 \%$ & $15 \%$ & $85 \%$ \\
\hline D01 (Dermat. Antimykotika) & $55 \%$ & $10 \%$ & $35 \%$ \\
\hline D02 (Emollentia, Hautschutzmittel) & $0 \%$ & $0 \%$ & $100 \%$ \\
\hline $\begin{array}{l}\text { D03 (Therapeutika b. } \\
\text { Wunden/Geschwüren }\end{array}$ & $75 \%$ & $10 \%$ & $15 \%$ \\
\hline $\begin{array}{l}\text { D04 (Antipruriginosa,topische } \\
\text { Antihistaminika, Anästhetika) }\end{array}$ & $80 \%$ & $10 \%$ & $10 \%$ \\
\hline D05 (Antipsoriatika) & $5 \%$ & $0 \%$ & $95 \%$ \\
\hline $\begin{array}{l}\text { D06 (Topische } \\
\text { Antibiotika/Chemotherapeutika) }\end{array}$ & $90 \%$ & $10 \%$ & $0 \%$ \\
\hline D07 (Topische Corticosteriode) & $50 \%$ & $40 \%$ & $10 \%$ \\
\hline D08 (Dermat. Antiseptiva) & $70 \%$ & $20 \%$ & $10 \%$ \\
\hline $\begin{array}{l}\text { D09 (Arzneistoffhaltige } \\
\text { Verbandmittel) }\end{array}$ & $70 \%$ & $20 \%$ & $10 \%$ \\
\hline D10 (Aknemittel) & $35 \%$ & $40 \%$ & $25 \%$ \\
\hline D11 (andere Dermatika) & $20 \%$ & $30 \%$ & $50 \%$ \\
\hline $\begin{array}{l}\text { G01 (Gyn. } \\
\text { Antiinfektiva/Antiseptika) }\end{array}$ & $100 \%$ & $0 \%$ & $0 \%$ \\
\hline G02 (andere Gynăkologika) & $80 \%$ & $10 \%$ & $10 \%$ \\
\hline $\begin{array}{l}\text { G03 (Sexualhormone, Modulatoren } \\
\text { des Genitalsystems) }\end{array}$ & $0 \%$ & $15 \%$ & $85 \%$ \\
\hline G04 (Urologika) & $55 \%$ & $0 \%$ & $45 \%$ \\
\hline
\end{tabular}

\begin{tabular}{|c|c|c|c|}
\hline ATC-Klassifikation & Akut & $\begin{array}{l}\text { Zwischen- } \\
\text { bereich }\end{array}$ & $\begin{array}{l}\text { Chro- } \\
\text { nisch }\end{array}$ \\
\hline $\begin{array}{l}\text { H01 (Hypophysen- } \mathrm{u} . \\
\text { hypothalamische Hormone, } \\
\text { Analoga) } \\
\end{array}$ & $0 \%$ & $0 \%$ & $100 \%$ \\
\hline $\begin{array}{l}\mathrm{H} 02 \\
\text { (Corticosteroide, systemisch) }\end{array}$ & $10 \%$ & $70 \%$ & $20 \%$ \\
\hline $\begin{array}{l}\text { HO3 } \\
\text { (Schilddrüsentherapeutika) }\end{array}$ & $10 \%$ & $0 \%$ & $90 \%$ \\
\hline H05 (Calciumstoffwechsel) & $5 \%$ & $25 \%$ & $70 \%$ \\
\hline J01 (Syst. Antibiotika) & $90 \%$ & $10 \%$ & $0 \%$ \\
\hline J02 (Syst. Antimykotika) & $90 \%$ & $10 \%$ & $0 \%$ \\
\hline $\begin{array}{l}\text { J04 (Antimykobakterielle } \\
\text { Pharmaka) }\end{array}$ & $80 \%$ & $20 \%$ & $0 \%$ \\
\hline J05 (syst. Antivirale Mittel & $100 \%$ & $0 \%$ & $0 \%$ \\
\hline J06 (Immunseren/Immunglobuline) & $90 \%$ & $10 \%$ & $0 \%$ \\
\hline J07 (Impfstoffe) & $100 \%$ & $0 \%$ & $0 \%$ \\
\hline L01 (Antineoplastische Mittel) & $70 \%$ & $0 \%$ & $30 \%$ \\
\hline L02 (Endokrine Therapie) & $10 \%$ & $10 \%$ & $80 \%$ \\
\hline L03 (Immunstimulantien) & $35 \%$ & $0 \%$ & $65 \%$ \\
\hline L04 (Immunsuppressiva) & $10 \%$ & $0 \%$ & $90 \%$ \\
\hline $\begin{array}{l}\text { M01 (Antiphlogistika, } \\
\text { Antirheumatika) }\end{array}$ & $15 \%$ & $15 \%$ & $70 \%$ \\
\hline $\begin{array}{l}\text { M02 (Substanzen bei Gelenk- und } \\
\text { Muskelschmerzen, topisch) }\end{array}$ & $60 \%$ & $20 \%$ & $20 \%$ \\
\hline M03 (Muskelrelaxantien) & $70 \%$ & $0 \%$ & $30 \%$ \\
\hline M04 (Gichtmittel) & $15 \%$ & $0 \%$ & $85 \%$ \\
\hline $\begin{array}{l}\text { M05 (Mittel zur Behandl v } \\
\text { Knochenkrankheiten) }\end{array}$ & $0 \%$ & $15 \%$ & $85 \%$ \\
\hline $\begin{array}{l}\text { M09 (and. Mittel gg. Störungen d. } \\
\text { Bewegungsapparates) }\end{array}$ & $0 \%$ & $0 \%$ & $100 \%$ \\
\hline N01 (Anästhetika) & $100 \%$ & $0 \%$ & $0 \%$ \\
\hline N02 (Analgetika) & $25 \%$ & $35 \%$ & $40 \%$ \\
\hline N03 (Antiepileptika) & $10 \%$ & $0 \%$ & $90 \%$ \\
\hline N04 (Antiparkinsonmittel) & $10 \%$ & $0 \%$ & $90 \%$ \\
\hline N05 (Psychopharmaka) & $10 \%$ & $20 \%$ & $70 \%$ \\
\hline N06 (Psychoanaleptika) & $40 \%$ & $60 \%$ & $0 \%$ \\
\hline $\begin{array}{l}\text { N07 (andere Mittel für das } \\
\text { Nervensystem) }\end{array}$ & $20 \%$ & $0 \%$ & $80 \%$ \\
\hline P02 (Anthelmintika) & $90 \%$ & $10 \%$ & $0 \%$ \\
\hline P03 (Ektoparasitizide) & $90 \%$ & $10 \%$ & $0 \%$ \\
\hline R01 (Rhinoligika) & $80 \%$ & $20 \%$ & $0 \%$ \\
\hline R02 (Halsschmerzmittel) & $100 \%$ & $0 \%$ & $0 \%$ \\
\hline R03 (Antiasthmatika) & $15 \%$ & $20 \%$ & $65 \%$ \\
\hline $\begin{array}{l}\text { R04 } \\
\text { (Brusteinreibungen/Inhalate) }\end{array}$ & $100 \%$ & $0 \%$ & $0 \%$ \\
\hline $\begin{array}{l}\text { R05 } \\
\text { (Husten/Erkăltungspräparate) }\end{array}$ & $100 \%$ & $0 \%$ & $0 \%$ \\
\hline $\begin{array}{l}\text { R06 (syst. Antihistaminika d. } \\
\text { Respirationssyst.) }\end{array}$ & $55 \%$ & $0 \%$ & $45 \%$ \\
\hline S01 (Ophtalmika) & $50 \%$ & $35 \%$ & $15 \%$ \\
\hline S02 (Otologika) & $70 \%$ & $15 \%$ & $15 \%$ \\
\hline $\begin{array}{l}\text { S03 } \\
\text { (komb.Ophtamologika/Otologika }\end{array}$ & $80 \%$ & $20 \%$ & $0 \%$ \\
\hline V01 (Allergene) & $60 \%$ & $10 \%$ & $30 \%$ \\
\hline Umsatz am GKV-Markt 1999 & $30 \%$ & $11 \%$ & $59 \%$ \\
\hline
\end{tabular}

Quelle: INIFES, eigene Darstellung. 


\section{Zielgruppe „Patienten mit mittel- und langfristiger Medikation“}

Als Indikator eignen sich Verordnungen mittlerer und großer Packungen. Die Packungsgröße fließt in die Berechnungen nach zwei möglichen Methoden ein:

- Zum einen kann die Frage gestellt werden, wie viele Tage eine verordnete Packung durchschnittlich vorliegt. Dafür bedarf es Angaben zur sogenannten „defined daily dose“ (DDD), welche aussagt, wie viele Tagesdosen die Verordnung umfasst. Eine durchschnittliche Packung beinhaltete 1999 ca. 35 Tagesdosen. Je mehr Tagesdosen also eine Verordnung umfasst, umso größer ist die Packung. In einem solchen Fall kann davon ausgegangen werden, dass es sich tendenziell eher um Dauermedikation als um eine akute Medikation handelt.

- Des weiteren kann die Packungsgröße nach Normpackungen, basierend auf der im Zuge des Gesundheitsstrukturgesetzes (GSG) 1993 und den damit veränderten Zuzahlungsregelungen erlassenen Zuzahlungsverordnung (ZzVO) Änderung am 12.9.1997 - , gemessen werden. Die Packungsgrößen N1 (klein), N2 (mittel), N3 (groß) sind in sechs Anlagen zur ZzVO aufgeführt, differenziert nach Darreichungsformen, Indikationsgebieten und Therapierichtungen. Sie geben die jeweils maximale Packungsgröße einer Zuzahlungsstufe an.

Indikationsgruppen mit einem hohen Anteil an N3-Packungen lassen grundsätzlich auf eine medikamentöse Langzeittherapie schließen. Die Eindeutigkeit der Aussage, je mehr N3-Packungen verschrieben wurden, desto eher ist diese Indikationsgruppe eine der Dauermedikation, unterliegt einigen Begrenzungen. So hat bereits die Art der Indikation Einfluss auf die Normierung der Packungsgröße: für typisch chronische Krankheiten (z.B. Hypertonie, Diabetes) enthalten die einzelnen Packungen mehr Tabletten als für typisch akute Krankheiten (z.B. Schmerzmittel). Damit stellt sich das Problem, dass bei einigen Indikationsgruppen die N2-Packungen mehr Zähleinheiten (z.B. Tabletten, Dragees, Suppositorien, etc.) enthalten als die N3-Packungen anderer Indikationsgruppen (vgl. Tabelle II - 2).

Tabelle II - 2: Zähleinheiten (nicht zu verwechseln mit DDD) je Normpackung ausgewählter Indikationen

\begin{tabular}{|l|c|c|c|}
\hline $\begin{array}{l}\text { Normpackung nach ZzVO } \\
\text { (Zähleinheiten nach Anlage 1) }\end{array}$ & N1 & N2 & N3 \\
\hline Antihypertonika & 30 & 60 & 100 \\
\hline Antidiabetika & 30 & 120 & - \\
\hline Analgetika & 10 & 30 & 50 \\
\hline Hypophysen-, Hypothalamische Hormone & 5 & 10 & 30 \\
\hline Analgetika & 10 & 30 & 50 \\
\hline
\end{tabular}

Quelle: Nach Zuzahlungsverordnung von 1993, Anlage 1. 
Zudem existiert bei der Verordnung durch den Arzt häufig das Verhaltensmuster, größere Packungen aus dem Grund zu verschreiben, dass der Patient bei N3Packungen an der Zuzahlung spart, weil diese als fester Betrag pro Packungsgröße definiert ist, und nicht vom Packungspreis abgeleitet wird. In diesen Fällen ist die Verordnung großer Packungen kein Zeichen für das Auftreten von Dauermedikation, sondern eher die Ausnutzung des "Mengenrabatts" großer Packungen. Die geschilderten Probleme sollten nicht unerwähnt bleiben, beschränken den Indikator Packungsgröße nach Normpackungen jedoch nicht in seiner grundsätzlichen Aussagekraft.

Ein großer Anteil an N3-Packungen als Indikator für Dauermedikation korreliert mit zunehmendem Alter und trat vor allem in den Altersklassen ab 60 Jahren auf. Die Verteilung der Packungsgröße auf die einzelnen Altersstufen ist in Abbildung II - 5 für das Jahr 1991 wiedergegeben.

Abbildung II - 5: Verteilung der Normpackungsgrößen in den Altersklassen 1991

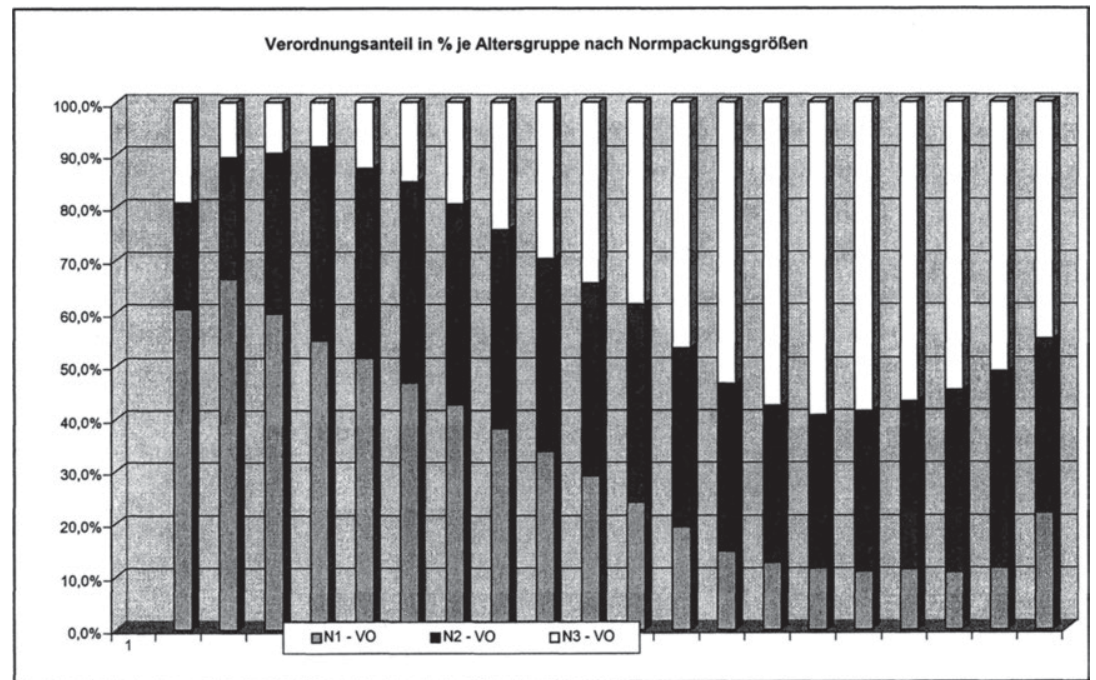

Quelle: INIFES, eigene Darstellung nach: Klauber, J., Selke, G.W., (1993), Auswirkungen des Gesundheitsstrukturgesetzes, in: Schwabe, U., Paffrath, D. (Hrsg,), (1993), Arzneiverordnungs-Report 1993, S. 486.

Die Indikatoren „Packungsinhalt nach DDD“ bzw. „Packungsinhalt nach Normpackungsgröße" müssen in einem weiteren Schritt operationalisiert und entsprechend messbar gemacht werden. Dem widmet sich der nächste Punkt.

30

Umgehen lassen sich diese praktischen Probleme eher durch die Messung der Packungsgröße nach mittleren Tagesdosen je Packung, wie bereits dargestellt. Diese rechnerische Große wird seit langem als Basis für Preisvergleiche, auch im Rahmen des GKV-Arzneimittelindex oder international von der WHO, genutzt. Nach: Schwabe, U., Paffrath, D. (1993), Arzneiverordnungsreport 1993, S. 484. 


\subsubsection{Ausprägungen der Zielsegmente}

Wie bereits enwähnt, stellt das Zielsegment grundsätzlich den Anteil aller als "versendungsfähig" definierten Verordnungen am gesamten GKV-Markt dar, differenziert nach Indikationsgruppen. Die Betonung liegt hier auf der Ebene der Indikationsgruppen. Diese bietet den Vorteil einer Operationalisierung / Quantifizierung von messbaren Zielsegmenten für den Versand.

Entsprechend wird auf den durchschnittlichen Verordnungswert je Indikationsgruppe fokussiert, nicht auf das einzelne Präparat. Dabei umfasst eine Indikationsgruppe eine Vielzahl von Präparaten, in einigen Fällen einige hundert und mehr. Der Verordnungswert je Indikationsgruppe stellt entsprechend den Durchschnittswert über alle verordneten Präparate dieser Gruppe dar.

In diesem Zusammenhang ist zu betonen, das eine Operationalisierung der Zielsegmente auf der Ebene der einzelnen Präparate unbestritten wünschenswert gewesen wäre, jedoch aufgrund der unüberschaubaren Präparateanzahl von über 45.000 bis 50.000 Stück bei einem zudem kontinuierlichen pharmazeutischen Fortschritt sich als nicht umsetzbar gestaltet. Zudem gründet diese hohe Anzahl an Präparaten z.T. auf unterschiedlichen Darreichungsformen (z.B. Tabletten, Suppositorien, Ampullen, etc.) für ein und dasselbe Arzneimittel.

Die Abschätzung eines zukünftigen Zielsegments für den Versandhandel kann unter Berücksichtigung der Komplexität des Arzneimittelmarktes nur erste Eckpunkte liefern, d.h. die Ausprägungen der Zielsegmente unterliegen gewissen Schwankungsbreiten. Dies ist u.a. auch damit zu begründen, dass die gewählten Indikatoren in ihrer Validität gewissen Einschränkungen unterliegen (siehe dazu die Ausführungen zu den Indikatoren in diesem Kapitel). Diese Einschränkungen werden allerdings durch den Vorteil aufgewogen, dass sie eine quantitative Messbarkeit von Zielsegmentvarianten ermöglichen. Eine quantitative Messbarkeit ist wiederum die Voraussetzung für alle weiteren Modellrechnungen.

Der höchste durchschnittliche Verordnungswert je Indikationsgruppe im GKV-Segment liegt im übrigen zählt dieser nicht zum erhobenen Zielsegment eines Versandes - im Jahre 1999 bei ca. 1005 DM (H01) AVK brutto. Entsprechend der Staffelung der Arzneimittelpreisverordnung liegt dieser zudem nicht in der höchsten Preisstufe (die Kappung der Arzneimittelpreisverordnung 1998 ist entsprechend ohne Einfluss). Der höchste durchschnittliche Verordnungswert einer als versendungsfähig eingestuften Indikation liegt bei ca. 550 DM (LO2) AVK brutto. 


\subsubsection{Zielsegment Variante A: Messgröße „Packungsgröße ab 50 DDD“}

Ausgehend von dem Indikator Packungsgröße wird der Umsatzanteil der Indikationsgruppen mit einer Anzahl von mindestens 50 Tagesdosen (DDD) je Verordnung (verordneter Packung) am GKV-Markt herangezogen. Begründet ist dieses Vorgehen in dem Definitionsversuch von Wolters, der eine Medikamentenpackung ab ca. 50 DDD als groß bezeichnet. ${ }^{32}$

Der genannte Indikator wird zudem den Einschätzungen der Experten (siehe Tabelle II - 1) gegenübergestellt. Das heißt, über diesen Indikator erhobene Indikationsgruppen werden zudem daraufhin untersucht, ob möglichenweise eine aufgrund der Packungsgröße von mindestens 50 DDD und mehr als versendungsfähig eingestufte Indikationsgruppe von Seiten der Experten eher dem akuten bzw. dem Zwischenbereich zugeordnet wird. Entsprechend entfallen die Indikationen J06 (Immunseren I Immunglobuline) mit einem Anteil von 90\% akuten Verordnungen, V01 (Allergene) mit 60\% akuten Verordnungen und J04 (Antimykobakterielle Pharmaka) mit 80\% akuten Verordnungen aus dem Zielsegment, obwohl sie mindestens 50 DDD je Packung aufweisen. ${ }^{33}$.

INIFES - Berechnungen ergeben nach der Modifizierung ein Zielsegment von $41 \%$ am GKV - Fertigarzneimittelumsatz auf Basis der Messgröße „Packungsgröße ab 50 DDD“ (vgl. Tabelle II - 3).

Wolters, J., Simon, S.T., Sochert, R. (1998), Arzneimitteldaten - Vorteile ihrer Einbeziehung in die betriebliche Gesundheitsberichterstattung, in: Die Betriebskrankenkasse 86(1998)11, S. 558-565.

Deshalb erscheinen diese auch nicht in der Tabelle $\|-3$. 
Tabelle II - 3: Umsatzanteil der Indikationsgruppen mit einer Packungsgröße ab 50 DDD je Verordnung

\begin{tabular}{|c|c|r|r|r|r|}
\hline ATC & Umsatz Mio DM & DDD Mio & VO in Mio & DDD / VO & DM / VO \\
\hline L02 & 604,50 & 84,80 & 1,10 & 77 & 549,55 \\
\hline M05 & 285,70 & 55,20 & 0,80 & 69 & 357,13 \\
\hline C10 & $1.699,50$ & 717,80 & 9,40 & 76 & 180,80 \\
\hline D05 & 86,40 & 63,90 & 0,80 & 80 & 108,00 \\
\hline C09 & $2.490,80$ & $1.908,60$ & 25,80 & 74 & 96,54 \\
\hline B03 & 477,70 & 283,10 & 5,20 & 54 & 91,87 \\
\hline C02 & 453,10 & 356,90 & 5,00 & 71 & 90,62 \\
\hline A10 & $1.772,80$ & $1.124,60$ & 20,60 & 55 & 86,06 \\
\hline B01 & 671,30 & 705,00 & 9,60 & 73 & 69,93 \\
\hline C08 & $1.282,00$ & $1.193,90$ & 19,70 & 61 & 65,08 \\
\hline C07 & $1.399,50$ & $1.161,30$ & 21,70 & 54 & 64,49 \\
\hline G03 & $1.253,40$ & $1.825,20$ & 24,70 & 74 & 50,74 \\
\hline C01 & $1.247,50$ & $1.739,40$ & 30,40 & 57 & 41,04 \\
\hline C03 & 657,30 & $1.419,10$ & 16,70 & 85 & 39,36 \\
\hline A11 & 165,60 & 511,00 & 5,80 & 88 & 28,55 \\
\hline M04 & 112,20 & 307,70 & 5,70 & 54 & 19,68 \\
\hline H03 & 311,00 & $1.309,00$ & 16,80 & 78 & 18,51 \\
\hline A01 & 81,70 & 578,20 & 5,60 & 103 & 14,59 \\
\hline Versandapotential & $15.052,00$ & $15.344,70$ & 225,40 & 68 & 66,78 \\
\hline GKV-Markt & $36.773,80$ & $27.751,40$ & 782,60 & 35 & 46,99 \\
\hline in \% des GKV-Markt & $41 \%$ & & & $192 \%$ & $142 \%$ \\
\hline
\end{tabular}

Quelle: INIFES, eigene Berechnungen nach Arzneiverordnungsreport 2000.

Die nach ATC klassifizierten Indikationsgruppen ${ }^{34}$ wurden in absteigender Reihenfolge des Verordnungswertes in DM geordnet.

Der Wert einer durchschnittlichen Verordnung liegt bei durchschnittlich 66,78 DM und einem Packungsinhalt von 68 DDD. Damit sind die Packungen nach dieser Operationalisierung des Zielsegments zwar um 42\% teurer, jedoch um 92\% größer als die durchschnittliche GKV-Verordnung. Bezogen auf die Kosten einer Tagesdosis sind die Verordnungen des Zielsegments (0,98 DM je DDD) relativ billiger als der GKVDurchschnitt (1,33 DM je DDD).

\subsubsection{Zielsegment Variante B: Messgröße "Packungsgröße N3"}

Die folgende Variante zur Abgrenzung des Zielsegments setzt ebenfalls an dem Indikator Packungsgröße an, allerdings nun operationalisiert nach Normpackungsgröße laut Zuzahlungsverordnung. Die Berechnung folgt Datenquellen des WIdO ${ }^{35}$. Diese weisen die verschiedenen Normpackungsgrößen N1, N2 und N3 in der Datenstruktur auf, klassifizieren die Daten jedoch anstelle der ATC-Codes nach Roter Liste. Für die

Siehe Tabelle 5.5.4 in: Schröder, H., Ergänzende statistische Übersicht, S. 796-881, in: Schwabe, U., Paffrath, D. (2000), Arzneiverordnungs-Report 1999. Schwerpunkt Naturheilmittel, Berlin, Heidelberg, New York.

Diese Daten liegen bei INIFES vor. 
Vergleichbarkeit der Varianten bedeutet dies allerdings keine wesentliche Einschränkung. Wie bereits dargestellt, liegen Daten zur Verteilung der Normpackungen am GKV-Umsatz aktuell für das Jahr 1998, nicht für 1999, vor. Allerdings kann von einer relativen Konstanz der Anteilswerte ausgegangen werden.

N3-Packungen gelten per Zuzahlungsverordnung als Packungsgröße für langfristige Medikation. Indikationsgruppen mit einem hohen Anteil an N3-Packungen ${ }^{36}$ lassen also grundsätzlich auf eine mittel- bis langfristige Medikation schließen. Entsprechend wird der Umsatzanteil der N3-Packungen am GKV-Markt herangezogen.

Allerdings erfolgt auch hier eine Gegenüberstellung des Indikators Packungsgröße N3 mit den Expertenmeinungen (siehe Tabelle II - 1). Entsprechend wird eine Modifizierung bei solchen Indikationen ${ }^{37}$ vorgenommen, welche durch diese als überwiegend akut bedingt bzw. als sich im Zwischenbereich zwischen akut und chronisch befindend, eingestuft werden. Folglich werden diese Indikationsgruppen nicht unter diese Zielsegmentvariante subsumiert.

INIFES - Berechnungen ergeben nach der Modifizierung ein Zielsegment von $47 \%$ am GKV - Fertigarzneimittelumsatz auf Basis der Messgröße „Packungsgröße N3 ${ }^{{ }^{38}}$ (vgl. Tabelle II - 4).

Der Wert einer durchschnittlichen N3-Verordnung liegt bei 64,85 DM mit ca. 80 bis 100 Zähleinheiten Packungsinhalt. Damit ist eine Verordnung nach dieser Operationalisierung zwar um $46 \%$ teurer im Vergleich zum durchschnittlichen Verordnungswert nach dieser Klassifikation von 44,45 DM (1998), jedoch annähernd doppelt so groß wie eine durchschnittliche GKV-Verordnung mit 50 bis 60 Zähleinheiten.

Die nach Roter Liste klassifizierten Indikationsgruppen wurden in absteigender Reihenfolge des Verordnungswertes in DM geordnet. (Eine umfassende Aufschlüsselung ist im Anhang I - Tabelle 4 enthalten).

Bzw. bei A10 Antidiabetika der Anteil der N2-Packungen, da hier keine N3-Normpackungen existieren (siehe auch Tabelle II - 2).

Hypophysen-, hypothalamische Hormone, Anthelminthika, Immunmodulatoren, Zytostatika, Antibiotika / Antiinfektiva, Entwöhnungsmittel, Tuberkulosemittel, Antiallergika, Muskelrelaxantia, Cholinergika, Antimykotika, Wundbehandlungsmittel, Spasmolytika, Corticoide (Interna), Antiemetika / Antivertiginosa, Infusionslösungen, Diagnostika, Antitussiva / Expektorantia, Desinfizentia / Antiseptika, Rhinologika / Sinusitismittel, Lokalanästhetika / Neuraltherapeutika, Mund- und Rachentherapeutika, Umstimmungsmittel (siehe Tabelle II -4, vollständige Ausführung im Anhang I).

Bei den Antidiabetika existieren keine N3-Packungen, Begründung siehe unter 2.2. Deshalb wurden hier innerhalb dieser Indikation die N2-Packungen für die Berechnung herangezogen. 
Tabelle II - 4: Umsatzanteil der Indikationsgruppen mit einer Packungsgröße N3 (für 1998), Auszug:

\begin{tabular}{|c|c|c|c|c|c|}
\hline $\begin{array}{c}\text { Indikationsgruppe } \\
\text { Nach Roter Liste bzw. } \\
\text { Zuzahlungsverordnung }\end{array}$ & $\begin{array}{c}\text { Umsatz } \\
\text { gesamt } \\
\text { in } \\
\text { Mio. DM }\end{array}$ & $\begin{array}{l}\text { Umsatz N3 } \\
\text { in } \\
\text { Mio. DM }\end{array}$ & \begin{tabular}{|c|} 
Anteil N3- \\
Umsatz am \\
Gesamtumsat \\
$z$
\end{tabular} & $\begin{array}{l}\text { N3-Vo } \\
\text { in } \\
\text { Mio. Stück }\end{array}$ & $\begin{array}{c}\text { DM/VO } \\
\text { je N3 -Packung }\end{array}$ \\
\hline Diätetika & 5,56 & 1,13 & $20,28 \%$ & 0,004 & 308,61 \\
\hline Lipidsenker & $1.495,58$ & 1085,14 & $72,56 \%$ & 5,43 & 199,92 \\
\hline .... & \multicolumn{5}{|c|}{ Siehe im Anhang I Tabelle 2 und Tabelle 4.} \\
\hline $\begin{array}{l}\text { Zielsegment } \\
\text { Indikation "A10": } \\
\text { N2 als N3 }\end{array}$ & & $16.698,5$ & & 257,49 & 64,85 \\
\hline $\begin{array}{l}\text { Gesamt GKV-Umsatz } \\
1998\end{array}$ & $35.611,3$ & & & & 44,45 \\
\hline In \% des GKV-Marktes & & $47 \%$ & & $32 \%$ & $146 \%$ \\
\hline
\end{tabular}

Quelle: INIFES, eigene Berechnungen nach WIdO - Daten mit Normpackungsstruktur.

\subsubsection{Zielsegment Variante C: Messgröße „Packungsgröße ab 35 DDD”}

1999 lag der durchschnittliche Wert einer GKV-Verordnung bei ca. 35 Tagesdosen. Ausgehend von dem Indikator Packungsgröße wird für eine weitere Operationalisierung des Zielsegments der Umsatzanteil der Indikationsgruppen mit einer überdurchschnittlichen Anzahl an Tagesdosen (DDD) je Verordnung herangezogen. Dieser Ansatz basiert auf der Annahme, dass überdurchschnittlich große Packungen definitionsgemäß eine zeitlich gesehen überdurchschnittlich lange Medikation garantieren. Deshalb können diese Indikationsgruppen als versendungsfähig gelten. Entsprechend werden alle Indikationsgruppen mit 35 und mehr Tagesdosen je Verordnung als versendungsfähig eingestuft. Variante $C$ umfasst entsprechend Variante $A$ als Schnittmenge.

Der Indikator „Packungsgröße, operationalisiert mit einem Wert mindestens 35 DDD je Verordnung" zur Abschätzung einer Versendungsfähigkeit der einzelnen Verordnungen wird ebenfalls anhand der Expertenmeinungen zur Dringlichkeit der Medikation modifiziert. Es entfallen wie bei Zielsegmentvariante A die Indikationen J06 (Immunseren / Immunglobuline), V01 (Allergene), L01 (Antineoplastische Mittel), J04 (Antimykobakterielle Pharmaka), sowie hier zusätzlich noch die Indikationsgruppe G02 (andere Gynäkologika). (siehe Tabelle II - 1). Deshalb werden diese Indikationsgruppen nicht unter diese Zielsegmentvariante subsumiert.

INIFES - Berechnungen ergeben nach der Modifizierung ein Zielsegment von circa $57 \%$ am GKV - Fertigarzneimittelumsatz auf Basis der Messgröße „Packungsgröße ab 35 DDD" (vgl. Tabelle II - 5). 
Die nach ATC klassifizierten Indikationsgruppen ${ }^{39}$ wurden in absteigender Reihenfolge des Verordnungswertes in DM geordnet.

Der Wert einer durchschnittlichen Verordnung liegt bei 61,36 DM und umfasst ca. 59 DDD Packungsinhalt. Damit sind die Packungen nach dieser Operationalisierung des Zielsegments zwar um 31\% teurer, jedoch um 69\% größer als eine durchschnittliche GKV-Verordnung. Bezogen auf die Kosten einer Tagesdosis sind die Verordnungen des Zielsegments mit 1,07 DM je DDD relativ billiger als der GKV-Durchschnitt mit 1,33 DM je DDD.

Tabelle II - 5: Umsatzanteil der Indikationsgruppen mit einer Packungsgröße ab 35 DDD je Verordnung

\begin{tabular}{|c|c|c|c|c|c|}
\hline ATC & Umsatz Mio. DM & DDD Mio. & VO in Mio & DDD / VO & DM / VO \\
\hline L02 & 604,50 & 84,80 & 1,10 & 77 & 549,55 \\
\hline M05 & 285,70 & 55,20 & 0,80 & 69 & 357,13 \\
\hline C10 & $1.699,50$ & 717,80 & 9,40 & 76 & 180,80 \\
\hline D05 & 86,40 & 63,90 & 0,80 & 80 & 108,00 \\
\hline $\mathrm{CO9}$ & $2.490,80$ & $1.908,60$ & 25,80 & 74 & 96,54 \\
\hline B03 & 477,70 & 283,10 & 5,20 & 54 & 91,87 \\
\hline $\mathrm{CO} 2$ & 453,10 & 356,90 & 5,00 & 71 & 90,62 \\
\hline N03 & 434,50 & 173,80 & 4,90 & 35 & 88,67 \\
\hline No7 & 325,90 & 163,40 & 3,70 & 44 & 88,08 \\
\hline A10 & $1.772,80$ & $1.124,60$ & 20,60 & 55 & 86,06 \\
\hline G04 & 712,40 & 402,80 & 9,60 & 42 & 74,21 \\
\hline R03 & $1.935,50$ & $1.302,30$ & 27,60 & 47 & 70,13 \\
\hline $\mathrm{B} 01$ & 671,30 & 705,00 & 9,60 & 73 & 69,93 \\
\hline $\mathrm{CO8}$ & $1.282,00$ & $1.193,90$ & 19,70 & 61 & 65,08 \\
\hline $\mathrm{Co7}$ & $1.399,50$ & $1.161,30$ & 21,70 & 54 & 64,49 \\
\hline N06 & $1.421,00$ & 815,20 & 23,20 & 35 & 61,25 \\
\hline $\mathrm{G03}$ & $1.253,40$ & $1.825,20$ & 24,70 & 74 & 50,74 \\
\hline $\mathrm{C} 01$ & $1.247,50$ & $1.739,40$ & 30,40 & 57 & 41,04 \\
\hline $\mathrm{CO3}$ & 657,30 & $1.419,10$ & 16,70 & 85 & 39,36 \\
\hline $\mathrm{CO6}$ & 37,50 & 41,20 & 1,00 & 41 & 37,50 \\
\hline $\mathrm{HO2}$ & 271,30 & 299,80 & 7,50 & 40 & 36,17 \\
\hline A11 & 165,60 & 511,00 & 5,80 & 88 & 28,55 \\
\hline $\mathrm{A} 06$ & 108,70 & 194,90 & 4,30 & 45 & 25,28 \\
\hline D11 & 74,00 & 119,50 & 3,30 & 36 & 22,42 \\
\hline S01 & 624,50 & $1.312,60$ & 28,70 & 46 & 21,76 \\
\hline $\mathrm{D} 02$ & 87,30 & 171,30 & 4,40 & 39 & 19,84 \\
\hline $\mathrm{MO4}$ & 112,20 & 307,70 & 5,70 & 54 & 19,68 \\
\hline $\mathrm{HO3}$ & 311,00 & $1.309,00$ & 16,80 & 78 & 18,51 \\
\hline $\mathrm{A} 01$ & 81,70 & 578,20 & 5,60 & 103 & 14,59 \\
\hline Zielsegment & $21.084,60$ & $20.341,50$ & 343,60 & 59 & 61,36 \\
\hline GKV-Markt & $36.773,80$ & $27.751,40$ & 782,60 & 35 & 46,99 \\
\hline $\begin{array}{l}\text { In } \% \text { des } \\
\text { GKV Marktes }\end{array}$ & $57 \%$ & & & $169 \%$ & $131 \%$ \\
\hline
\end{tabular}

Quelle: INIFES, eigene Berechnungen nach Arzneiverordnungsreport 2000. Schwabe, U., Paffrath, D. (2000), Arzneiverordnungs-Report 1999. Schwerpunkt Naturheilmittel, Berlin, Heidelberg, New York. 


\subsubsection{Zwischenfazit: Überblick über Zielsegmente}

Im Folgenden werden die bisher gewonnenen Erkenntnisse zum maximal möglichen Potential eines Arzneimittelversandes zusammengefasst. Die Herleitung möglicher Zielsegmente basierte dabei auf der Begriffsbestimmung "versandfähig", die sich auf die Ebene der Indikationsgruppe bezieht.

Die Ebene der Präparate, die für eine Differenzierung nach Länge der Dauermedikation bzw. nicht akuter Medikation sicherlich die optimale Analyseebene wäre, kann aufgrund der immensen Anzahl der Präparate auf dem deutschen Apothekenmarkt die Zahl wird auf 50.000 und mehr geschätzt - aus praktischen Gründen nicht erfolgen. Zudem differieren Präparate oft in ihrer Darreichungsform und Dosierung. All diese Komponenten zu berücksichtigen, ist nicht durchführbar.

Die Berechnungen basieren deshalb auf der (nächsthöheren) Stufe der Indikationsgruppen. Sekundärdaten zum Arzneimittelmarkt liegen häufig in der Klassifikation der Roten Liste vor. Diesen Daten fehlen allerdings ein für die INIFES - Berechnungen wichtiges Detail: die Angabe der DDDs (definierten Tagesdosen). Deshalb wird auf die - für "Praktiker" etwas ungewöhnliche - ATC-Klassifizierung in den Varianten $A$ und $C$ zurückgegriffen, die auch das WIdO zur Aufarbeitung seiner Arzneiverordnungsreports nutzt.

In der gemeinsamen Betrachtung aller drei Ausprägungen des Zielsegments zeigt sich, dass ca. die Hälfte des GKV-Umsatzes auf die Verordnung langfristiger Medikation entfält und damit als generell versendungsfähig eingestuft werden kann. Andererseits bedeutet dies auch, das ca. die Hälfte der gesamten GKV-Ausgaben für Arzneimittel nicht für einen Versandweg geeignet ist. Alle weiteren Betrachtungen beziehen sich nur auf das versandfähige Segment.

Abbildung II - 6: Darstellung der Varianten $A$ bis $C$ des Zielsegments

\begin{tabular}{|c|l|c|c|}
\hline $\begin{array}{c}\text { Versand } \\
\text { potential } \\
\text { Variante }\end{array}$ & \multicolumn{1}{|c|}{$\begin{array}{c}\text { Messgröße / } \\
\text { Operationalisierung }\end{array}$} & $\begin{array}{c}\text { Basisparameter: } \\
\text { GKV-Fertigarznei- } \\
\text { mittelumsatz }\end{array}$ & $\begin{array}{c}\text { Zielsegment } \\
\text { in \% }\end{array}$ \\
\hline A & Packungsgröße ab 50 DDD je Verordnung (modifiziert) & 1999 & $41,0 \%$ \\
\hline B & Packungsgröße N3-Packungen (modifiziert) & 1998 & $47,0 \%$ \\
\hline C & Packungsgröße ab 35 DDD je Verordnung (modifiziert) & 1999 & $57,0 \%$ \\
\hline
\end{tabular}

Quelle: INIFES, eigene Darstellung.

An dieser Stelle erscheint es noch einmal notwendig, darauf hinzuweisen, dass diese Indikatoren natürlich gewissen Einschränkungen unterliegen, aber für eine Operationalisierung unumgänglich erscheinen. Insbesondere deshalb, da für den Begriff "chronisch" keine allgemeingültige Definition in der Wissenschaft existiert, sondern allgemein eher von einem "common sense" auszugehen ist. Zudem ist die Begriffs-

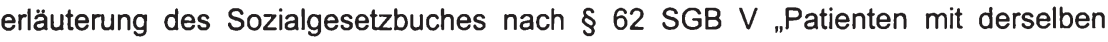


Erkrankung in Dauerbehandlung" für eine Quantifizierung eines entsprechenden Arzneimittelverbrauchs nicht aussagekräftig genug.

Auch die Eruierung der Expertenmeinungen zur Dringlichkeit der Medikation mit Fokussierung auf die Definition der Versendungsfähigkeit ergab, dass diese individuellen Einschätzungen bezüglich einer akuten oder chronischen Dringlichkeit nicht unwesentlichen Schwankungen in Abhängigkeit des medizinischen wie pharmakologischen Fachgebietes des Experten unterliegen.

Zum anderen wird auch die Einschätzung der Versandfähigkeit von der Entwicklung des Qualitätsniveaus der pharmazeutischen Logistik, d.h. wie sicher und wie schnell eine Zusendung in Zukunft erfolgen kann, abhängig sein.

Es ist an dieser Stelle jedoch wiederholt darauf hinzuweisen, dass die ermittelten Ausprägungen von Zielsegmenten für einen Pharmaversand nicht mit Marktanteilen zu verwechseln sind. Sie ermöglichen in einem ersten Modellschritt lediglich eine Aussage darüber, welcher Anteil des GKV- Fertigarzneimittelumsatzes anhand der gewählten epidemiologischen Indikatoren überhaupt als „versendungsfähiges" Segment betrachtet und damit als Grundlage weiterer Modellentwicklungen gelten kann.

Damit unterscheidet sich diese Herangehensweise gegenüber den oftmals in Diskussionen geäußerten verkürzten Berechnungen zum möglichen Umfang eines Arzneimittelversandes, welche die gesamten GKV-Ausgaben als Grundlage für Marktberechnungen heranziehen (z.B. 15\% erwarteter Marktanteil des Versandes an den GKV-Ausgaben aufgrund der Erfahrungen in den USA), ohne dabei eine Zielgruppenbildung anhand epidemiologischer Kriterien vorzunehmen. Das würde bedeuten, dass alle Verordnungen unabhängig von ihrer jeweiligen Dringlichkeit und pharmakologischen Zusammensetzung als versendungsfähig angesehen werden. Dem widersprechen die angeführten Literaturquellen sowie Erfahrungen im Ausland.

Die hier gewählte zielgruppenorientierte Differenzierung des GKV-Marktes nach epidemiologischen Kriterien zeigt für das Zielsegment eines Versandhandels, dass die jeweiligen Umsätze je Verordnung zwar über dem GKV-Durchschnitt von 46,99 DM liegen, dem jedoch überproportional größere Packungsinhalte gegenüberstehen (resultierend aus der Annahme langfristiger Medikation). Das heißt, in Relation zum Umsatz einer Tagesdosis liegen die versendungsfähigen Verordnungen unter dem Durchschnitt aller GKV-Verordnungen. Zudem fallen unter das Zielsegment des Pharmaversandes aufgrund der gewählten Indikatoren auch solche Indikationsgruppen, die mit ihrem Verordnungswert unter dem GKV-Durchschnitt liegen.

Das Zielsegment wird, wie Abbildung II - 7 zu entnehmen ist, aufgrund der individuellen Umsatz- und Kostenstrukturen der Versandapotheken determiniert. Diesem weiteren Modellschritt widmet sich das folgende Kapitel 2.4. 
Abbildung II - 7: Darstellung der durchschnittlichen Verordnungswerte in Abhängigkeit zur Größe des potentiellen Zielsegmentes des Pharmaversandes

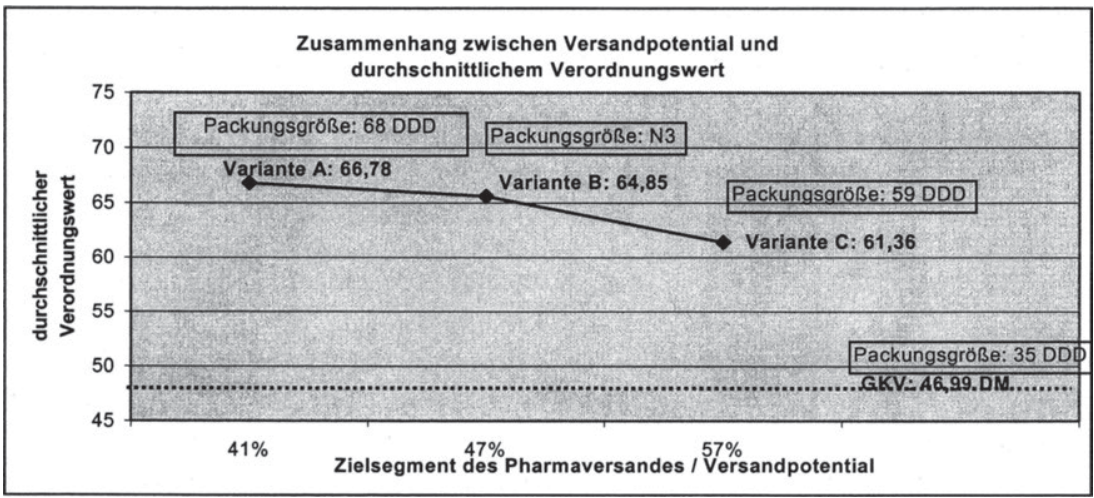

Quelle: INIFES, eigene Darstellung.

\section{4. Ökonomische Restriktionen - Entwicklung einer Betriebs- und Kostenstruktur für Versandapotheken}

Das Vorgehen in Kapitel 2.3. segmentierte die Verordnungen bezüglich ihrer Versandfähigkeit aufgrund epidemiologischer Kriterien.

In diesem Kapitel gilt es nun zu untersuchen, welche ökonomischen Restriktionen bei einem Versand zu beachten sind. Das heißt, unter der Annahme der Erstattungsfähigkeit der im GKV-Fertigarzneimittelindex erfassten Verordnungen muss eine Deckung der entstehenden Kosten für den Vertriebsweg Versand aus der Betriebshandelsspanne der Apotheken erfolgen bzw. darf nicht zu finanziellen Mehrbelastungen der GKV führen. Damit ergibt sich die Konsequenz, dass einige, aus epidemiologischen Gesichtspunkten dem Zielsegment zuzuweisende Verordnungen, unter Geltung ökonomischer Restriktion, für den Vertriebsweg Versand nicht geeignet sind.

Im Folgenden wird deshalb der Versuch unternommen, die interne Kostenstruktur einer Versandapotheke zu entwickeln. An dieser Stelle erfolgt also eine betriebswirtschaftliche Analyse auf der Ebene der einzelnen Anbieter (d.h. der potentiellen Versandapotheken), die in den weiteren Kapiteln zur Darstellung von Markt- wie Einsparpotentialen für einen Versandhandel mit Arzneimitteln Einfluss finden wird. Zudem wird die Darstellung der Kostenstruktur an unterschiedliche Umsatzgrößenklassen von Versandapotheken gekoppelt, um Skaleneffekte bei den Kosten und deren Auswirkung auf das betriebswirtschaftliche Ergebnis zu analysieren. Es handelt sich um folgende drei Varianten der Umsatzgröße von Versandapotheken: 
1. Variante VA I: Versandapotheke mit ca. 5 Mio DM Umsatz. Diese Modellvariante beschreibt eine Versandapotheke, die aus den Strukturen der Präsenzapotheke aus Punkt 2.4.1 entwickelt wird (Punkt 2.4.3.).

2. Variante VA II: Versandapotheke mit ca. 20 Mio. DM Umsatz (Punkt 2.4.4).

3. Variante VA III: Versandapotheke mit ca. $\mathbf{4 8 7}$ Mio. DM Umsatz: Diese Modellvariante beschreibt eine Versandapotheke, die neben der pharmazeutischen Kompetenz die Großhandelsfunktion vereint. Aus einem Großhandelsumsatz von 300 Mio. DM wird nach der gültigen Preisspannenverordnung ein Apothekenumsatz brutto von 487 Mio. DM der Kalkulation zugrunde gelegt (Punkt 2.4.4).

Die Entwicklung der internen Kostenstruktur erfolgt zu großen Teilen aus den veröffentlichten Ergebnissen des alljährlichen Betriebsvergleichs für Präsenzapotheken des Instituts für Handelsforschung an der Universität zu Köln (IFH Köln). Die Darstellung der Kostenstruktur einer Präsenzapotheke soll deshalb als Einleitung und Fundament für weitere Entwicklungsschritte am Anfang der Ausführungen stehen. Die IFH - Zahlen werden, bis auf einige Ausnahmen, in ihrer Höhe (in Relation zum Umsatz gemessen) übernommen und beziehen sich auf das Jahr $1998 .^{40}$

\subsubsection{Die Kostenstruktur einer Präsenzapotheke}

Die interne Struktur einer durchschnittlichen Präsenzapotheke wird als Referenzmaß benötigt. Einerseits werden Strukturmerkmale der Präsenzapotheke insbesondere im Modell der Variante VA I Versandapotheke mit 5 Mio. DM Umsatz herangezogen, andererseits dienen die Angaben zur Struktur einer durchschnittlichen Präsenzapotheke der später durchzuführenden Ermittlung möglicher Einsparpotentiale.

\section{UMSATZ}

- Nach IFH wie ABDA ${ }^{41}$ beträgt der Bruttoumsatz einer Apotheke ca. 2,56 Mio. DM.

\section{WARENEINSATZ UND BETRIEBSHANDELSSPANNE}

- Der Wareneinsatz einer Apotheke bestimmt sich aus den der Apothekenstufe vorgelagerten Umsatzanteilen des Großhandels und der Hersteller vom Bruttoumsatz, und wird damit determiniert durch die Preisspannenverordnung für apothekenpflichtige Präparate.

Wirtz, K., (2000), Bericht über die Ergebnisse des Betriebsvergleiches der Apotheken im Jahre 1998, in: Mitteilungen des IFH Köln, Jahrgang 52, S. 25.

ABDA (1999), Die Apotheke Zahlen, Daten, Fakten. 
- Der Wareneinsatz betrug 1998 ca. $58,6 \%{ }^{42}$ bzw. $58,7 \%{ }^{43}$. Nach Abzug der Mehrwertsteuer von $13,8 \%{ }^{44}$ ergibt sich die durchschnittliche Betriebshandelsspanne von $27,6 \%$ bzw. 27,5\% (bereits nach Abzug des gesetzlichen GKV-Rabattes ${ }^{45}$ ). Für die Berechnungen werden die IFH Zahlen $58,7 \%$ und $27,5 \%$ verwendet.

\section{TATSÄCHLICHE KOSTEN}

\section{A) Personalkosten}

- Die Personalkosten ergeben sich aus der Personalstruktur der Apotheke nach $\S 3$ ApBetrO, welche aus pharmazeutischem (approbierte Apotheker, Apothekerassistenten, PTA) und nichtpharmazeutischem Personal (PKA, Helfer) besteht. Pharmazeutische Aufgaben (also z.B. die Arzneimittelabgabe oder Beratung) dürfen nur vom pharmazeutischen Personal ausgeführt werden.

- Die Apotheke ist ein mittelständiger Betrieb, der Apothekenleiter arbeitet auf Selbständigenbasis. Der Unternehmerlohn wird deshalb nicht unter die Personalkosten gefasst, sondern als kalkulatorische Kosten abgerechnet.

- Nach einer Erhebung der Treuhand Hannover zu Tätigkeits- und Kostenprofilen der Apothekenmitarbeiter ${ }^{47}$ arbeiten ca. 5,7 Mitarbeiter (ohne Leiter) in einer Apotheke. Nach Vollzeitstellen (ohne Leiter) wären dies 4,1 Vollbeschäftigte. Tabelle II - 6 gibt den Ergebnissen der Erhebung folgend einen Überblick über das Personalkostenprofil einer durchschnittlichen Apotheke für 1998 / 1999 in Westdeutschland.

- Bezogen auf den durchschnittlichen Bruttoumsatz belaufen sich die Personalkosten auf ca. $11 \%$. Diese Prozentangabe ist identisch mit der Angabe des Apothekenbetriebsvergleiches des IFH Köln.

ABDA (1999), Die Apotheke Zahlen, Daten, Fakten.

Wirtz, K. (2000), Bericht über die Ergebnisse des Betriebsvergleiches der Apotheken im Jahre 1998, in: Mitteilungen des IFH Köln, Jg. 52, S. 25.

Zum 1.4.98 erfolgte eine Erhöhung der Mehrwertsteuer von 15\% auf $16 \%$. Damit errechnet sich speziell für 1998 ein Durchschnittswert von 13,6\%; für alle folgenden Jahre jedoch von 13,8\%. Aus diesem Grund wird hier bereits mit 13,8\% kalkuliert.

Dies wurde nach Nachfrage beim IFH Köln durch dieses ausdrücklich bestätigt.

$\S 3$ ApBetro fordert bei der Beratung der Patienten durch eine(n) PTA lediglich die „Beaufsichtigung durch einen Apotheker".

Hasan-Boehme, U., u.a., Tătigkeits- und Kostenprofile der Apothekenmitarbeiter, in: uww.pharmazeutische-zeitung.de/pza/1999-39, Tabelle 1, und www.pharmazeutische-zeitung.de/pza/1999-35, Tabelle 1, Zugriff am 21.11.00.

Müller-Hagedorn, L., (1998), Strategische Optionen für die Apotheke, S. 47, Aufsatz S. 33 bis 55, in: Management in Apotheken, Sonderheft 43 des IFH Köln. 
Tabelle II - 6: Personalkosten und Arbeitszeit nach Berufsgruppen in Apotheken (West)

\begin{tabular}{|c|c|c|c|c|c|}
\hline $\begin{array}{l}\text { Präsenzapotheke } \\
\text { (Basis 1998/1999) }\end{array}$ & $\begin{array}{l}\text { Arbeitszeit je } \\
\text { Woche }\end{array}$ & $\begin{array}{l}\text { Köpfe je } \\
\text { Apotheke }\end{array}$ & $\begin{array}{l}\text { Personalkosten/ } \\
\text { Vollzeitstelle p.a. in DM }\end{array}$ & Arbeitszeit*) & $\begin{array}{c}\text { Verrechnete } \\
\text { Personalkosten } \\
\text { p.a. je } \\
\text { Apotheke }\end{array}$ \\
\hline \multicolumn{6}{|l|}{ Apotheker } \\
\hline Angestellte & 27,5 & 1,52 & 96.500 & $72 \%$ & 106.486 \\
\hline Leiter & & 1,00 & kalk. UN-Lohn & & kalk. UN-Lohn \\
\hline Apo-Assistenten & 19,1 & 1,06 & 74.500 & $50 \%$ & 39.657 \\
\hline PTA & 28,4 & 1,57 & 58.000 & $75 \%$ & 68.259 \\
\hline PKA / Helfer & 27,8 & 1,54 & 48.300 & $73 \%$ & 63.979 \\
\hline $\begin{array}{l}\text { Beschäftigte } \\
\text { insgesamt (Teilzeit) }\end{array}$ & & 6,7 & & & \\
\hline $\begin{array}{l}\text { Mitarbeiter (ohne } \\
\text { Leiter) }\end{array}$ & & 5,7 & 57.900 & $72 \%$ & 278.381 \\
\hline
\end{tabular}

*) Bezogen auf eine Vollzeitstelle (38 Wochenstunden und 45 Wochen: 1.710 Arbeitsstunden p.a.)

Quelle: INIFES, eigene Darstellung, nach: Hasan-Boehme, U., u.a., Tätigkeits- und Kostenprofile der Apothekenmitarbeiter, in:

www.pharmazeutische-zeitung.de/pza/1999-39, Tabelle 1,

www.pharmazeutische-zeitung.de/pza/1999-35, Tabelle 1, Zugriff am 21.11.00.

\section{B) Mietkosten}

- Laut IFH Köln haben Präsenzapotheken durchschnittlich ca. 165 qm Geschäftsraum.

- Im Durchschnitt liegt der Mietpreis der Apothekenräume bei ca. $25 \mathrm{DM}$ je qm. ${ }^{49}$

- Auf die Miete werden $28 \%{ }^{50}$ für Sach- bzw. Mietnebenkosten aufgeschlagen.

- Die gesamten Mietkosten pro Jahr ergeben so ca. 2,5\%.

- Diese Zahl korrespondiert mit den Prozentangaben des IFH Köln.

\section{C) Abschreibungen und Zinsen}

- Laut IFH Köln betragen die jährlichen Abschreibungen ca. 1,2\% vom Umsatz.

- Die Fremdkapitalzinsen betragen nach IFH Köln ca. 0,8\% vom Umsatz. ${ }^{51}$ Dabei hat die Höhe des Lagerbestandes einen Einfluss auf die Höhe der Fremdkapital-

Büchel, D., (1998), Der neue IfH-Monatsbetriebsvergleich für Apotheken, S. 102, Abb. 10, in: Management in Apotheken, Sonderheft 43 des IFH, S. 87 - 105, sowie: Kaapke, A., (1998), Controlling in Apotheken mit Hilfe des Jahresbetriebsvergleiches des IFH, S. 75, in: Management in Apotheken, Sonderheft 43 des IFH Köln, S. 57 - 85.

U.a. in Anlehnung an Kaapke, A., (1998), Controlling in Apotheken mit Hilfe des Jahresbetriebsvergleiches des IFH Köln, S. 75, in: Management in Apotheken, Sonderheft 43 des IFH Köln, S. $57-85$. 
zinsen, sofern dieser zumindest teilweise fremdfinanziert wird. Ein optimaler Lagerbestand trägt zu einer Senkung der Fremdkapitalzinsen aufgrund eines geringeren gebundenen Kapitalstocks in Lagerwerten bei.

\section{D) Laufender Geschäftsbetrieb: Werbung, KFZ, übrige Kosten ${ }^{52}$}

- Werbung darf von den Apotheken nach dem Heilmittelwerbegesetz nur für nicht apothekenpflichtige Arzneimittel der Selbstmedikation vorgenommen werden. Für verordnungspflichtige Arzneimittel ist keine Öffentlichkeitswerbung erlaubt, nur Fachkreise wie z.B. Ärzte dürfen beworben werden. Die Werbungskosten betragen nach IFH Köln $0,7 \%$ vom Umsatz.

- Die KFZ-Kosten für den Fuhrpark betragen nach IFH Köln ca. 0,4\% vom Umsatz.

- Die übrigen Kosten betragen nach IFH Köln 2,9\% vom Umsatz. Eine Spezifizierung, was darunter subsumiert wird, kann nach der Literatur nicht eruiert werden.

\section{GEWERBESTEUER}

- Die Gewerbesteuer ist abhängig von dem jeweiligen Hebesatz der Gemeinde. Allgemein wird sie ermittelt, indem die Differenz aus Betriebshandelsspanne und tatsächlichen Kosten mit ca. $16 \%$ verrechnet wird.

\section{KALKULATORISCHE KOSTEN}

- Kalkulatorische Kosten stellen keine tatsächlichen Kosten dar. Darunter werden der Unternehmerlohn des Apothekenleiters und die Eigenkapitalzinsen subsumiert. Absolute Angaben über die Höhe des kalkulatorischen Unternehmerlohns des Apothekenleiters sind kaum transparent. In einer Literaturquelle werden diese mit durchschnittlich 111 TDM bis 147 TDM angegeben. ${ }^{53}$

- Das IFH Köln weist den Unternehmerlohn mit den Personalkosten gemeinsam aus (17,5\% vom Umsatz), so dass eine eindeutige Angabe hier nicht zu ermitteln ist, jedoch im Zusammenhang mit anderen Zahlen ${ }^{54}$ auf ca. $6 \%$ schließen lässt.

- Die Eigenkapitalzinsen betragen nach IFH Köln 0,4\% vom Umsatz. ${ }^{55}$

Wirtz, K., (2000), Bericht über die Ergebnisse des Betriebsvergleiches der Apotheken im Jahre 1998, S. 25, in: Beiträge zur betriebswirtschaftlichen Planung und Kontrolle in Apotheken, Jahrgang 52, Januar 2000, S. 23 - 25.

Wirtz, K., (2000), Bericht über die Ergebnisse des Betriebsvergleiches der Apotheken im Jahre 1998, S. 25, in: Beiträge zur betriebswirtschaftlichen Planung und Kontrolle in Apotheken, Jahrgang 52, Januar 2000, S. 23 - 25.

Schöffski, O. (1995), Die Regulierung des deutschen Apothekenwesens. Eine ökonomische Analyse, Baden-Baden, S.175.

54

So wird an anderer Stelle des IFH - Sonderheftes von ca. $11 \%$ Personalkosten gesprochen. Siehe Müller-Hagedorn, L., (1998), Strategische Optionen für die Apotheke, S. 47, Aufsatz S. 33 - 55, in: Management in Apotheken, Sonderheft 43 des IFH Köln. 
- Zu berücksichtigen ist, dass die kalkulatorischen Kosten für kleine und große Apotheken ähnlich hoch sind, und damit die betriebswirtschaftlichen Ergebnisse bei umsatzstarken Apotheken geringer belasten.

\section{BETRIEBSWIRTSCHAFTLICHES ERGEBNIS}

- Das Ergebnis nach Gewerbeertrag um die kalkulatorischen Kosten gekürzt, ergibt die Rendite. In der hier untersuchten Größenumsatzklasse der Apotheken liegt die Rendite bei ca. $0,4 \%$ bis $1 \%$ vom Umsatz.

In der folgenden Tabelle II - 7 sind die zentralen Angaben zur Struktur einer durchschnittlichen Präsenzapotheke bei Mischkalkulation nochmals zusammengefasst.

Tabelle II - 7: Umsatz-, Kosten- und Ertragsstruktur einer Präsenzapotheke

\begin{tabular}{|l|c|c|}
\hline & $\begin{array}{c}\text { in } \% \text { des } \\
\text { Apothekenumsatzes }\end{array}$ & $\begin{array}{c}\text { in } \% \text { der } \\
\text { Handelsspanne }\end{array}$ \\
\hline Umsatz brutto in TDM & $100,0 \%$ & \\
Mehrwertsteuer 16\% & $13,8 \%$ & \\
Wareneinsatz & $58,7 \%$ & $100,0 \%$ \\
\hline BETRIEBSHANDELSSPANNE & $\left.27,5 \%^{*}\right)$ & $70,9 \%$ \\
\hline TATSÄCHLICHE KOSTEN & $19,5 \%$ & $40,0 \%$ \\
Personalaufwand & $\left.11,0 \%{ }^{*}\right)$ & $9,1 \%$ \\
Mietaufwand & $2,5 \%$ & $7,3 \%$ \\
Abschreibungen, Zinsen & $\left.2,0 \%^{*}\right)$ & $14,5 \%$ \\
Werbung, KfZ, übrige Kosten & $\left.4,0 \%{ }^{*}\right)$ & $29,1 \%$ \\
\hline Ergebnis vor Steuern & $8,0 \%$ & $4,7 \%$ \\
\hline GEWERBESTEUER & $1,3 \%$ & $22,9 \%$ \\
\hline KALKULATORISCHE KOSTEN & $6,3 \%$ & $21,5 \%$ \\
Kalkulatorischer Unternehmerlohn & $5,9 \%$ & $1,4 \%$ \\
\hline Eigenkapitalzinsen & $\left.0,4 \%^{*}\right)$ & $1,5 \%$ \\
\hline BETRIEBSWIRTSCHAFTLICHES ERGEBNIS & $0,4 \%^{58}$ & \\
\hline
\end{tabular}

*) In Anlehnung an die Betriebsvergleichsergebnisse der Apotheken des IFH Köln für 1998.

Quelle: INIFES, eigene Berechnungen.

Wirtz, K., (2000), Bericht über die Ergebnisse des Betriebsvergleiches der Apotheken im Jahre 1998, S. 25. in: Beiträge zur betriebswirtschaftlichen Planung und Kontrolle in Apotheken, Jahrgang 52, Januar 2000, S. 23 - 25.

56

Schöffski, O. (1995), Die Regulierung des deutschen Apothekenwesens. Eine ökonomische Analyse, Baden-Baden, S.190.

Müller-Hagedorn, L. (1998), Strategische Optionen für die Apotheke, S. 38, Aufsatz S. 33 bis 55, in: Management in Apotheken, Sonderheft 43 des IFH Köln.

Das betriebswirtschaftliche Ergebnis laut IFH beträgt $0 \%$. Diese Differenz zwischen der INIFES-Berechnung und der des IFH entsteht aufgrund marginal abweichender Zahlen bezüglich der Mietkosten, der kalkulatorischen Kosten (diese werden beim IFH nicht explizit aufgeführt) und der Gewerbesteuer. Ein Hinweis darauf findet sich in den jeweiligen Positionen. 
Nachdem die Kostenstrukturen einer durchschnittlichen Präsenzapotheke analysiert worden sind, werden diesen im Folgenden die Strukturen (2.4.2.) und die daraus abzuleitenden Betriebskosten von Versandapotheken (2.4.3. bis 2.4.5.) gegenüber gestellt.

Für Positionen, in denen sich Präsenz- und Versandapotheken strukturell unterscheiden (z.B. in der Art der Distribution oder der Sicherstellung der Beratung) muss an einigen Stellen mangels öffentlich verfügbarer Daten auf Annahmen oder vertrauliche Informationen zurückgegriffen werden.

\subsubsection{Betriebsstrukturen von Versandapotheken unter Qualitäts- und Effizienz- maßstäben}

Für die Akzeptanz von Versandapotheken seitens der Patienten ist das Kriterium der Versorgungsqualität einschließlich höchstmöglicher Sicherheitsstandards von wesentlicher Bedeutung. Neben der Arzneimittelsicherheit muss zusätzlich garantiert sein, dass

- die Patienten sachgemäß beraten werden (Patientensicherheit) sowie

- die Zustellung der Medikamente zuverlässig erfolgt (Versand-/ Zustellsicherheit).

Die Erfahrungen mit seriösen Versandapotheken im Ausland zeigen, dass dies möglich ist: in der Schweiz bescheinigen Verbraucher- und Patientenorganisationen neben staatlichen Kontrollstellen, Wettbewerbskommissionen und dem obersten Bundesgericht, dass der Versandhandel den Präsenzapotheken in Qualität und Sicherheit in nichts nachsteht. Dies gilt nicht nur für die Qualität, sondern auch für die Effizienz: Einsparungen bis zu 15\% der Arzneikosten konnten erzielt werden. ${ }^{59}$

Dies setzt entsprechende betriebliche Strukturen und Prozessabläufe in den Versandapotheken voraus, z.B. dokumentiert durch eine Zertifizierung nach den Qualitätsstandards der ISO 9002. In der Praxis bedeutet dies eine hochwertige EDV, Endkontrollen der Versendungen durch examinierte Pharmazeuten, adäquate Informations- und Beratungsangebote für Patienten via Telefon, E-Mail / Internet durch qualifizierte Fachleute sowie funktionierende Logistikstrukturen.

Es erscheint deshalb sinnvoll, vor der Darstellung der Kostenentwicklung einige wesentliche Modellannahmen, die betriebliche Struktur von Versandapotheken betreffend, voranzustellen. Zum einen, da sich diese hier in ihrem Leistungsangebot gegenüber Präsenzapotheken abgrenzen, zum anderen, da davon ein Einfluss auf die Kostenstruktur zu erwarten ist:

- Annahmen zur Personal- und Raumstruktur (siehe 2.4.2.1.)

- Annahmen zur fachlichen Beratung der Patienten (siehe 2.4.2.2.)

- Annahmen zur Gewährleistung einer funktionierenden B-2-C-Logistik (siehe 2.4.2.3.)

59

Hovermann, E., (2001), Versandhandel versus Einzelapotheke, S. 177f, in: Die BKK 4/2001, S. 174-181. 


\subsubsection{Allgemeine Modellannahmen, insbesondere zur Personal- und Raum- struktur}

Der Verkauf bzw. die Medikamentenabgabe in Präsenzapotheken beansprucht viel Zeit und verursacht entsprechend hohe Kosten, insbesondere dann, wenn die Beschäftigten, die in dem Bereich tätig sind, überdurchschnittliche Gehälter bekommen (Durchschnitt s. Tabelle II - 7), beispielsweise approbierte Apotheker. Der Kostenanteil des Verkaufsbereiches an den Personalkosten ist demzufolge am höchsten. (vgl. Tabelle II-8).

Tabelle II - 8:Kosten für pharmazeutisches Personal nach Tätigkeitsbereichen (West)

\begin{tabular}{|l|c|c|}
\hline $\begin{array}{l}\text { Personalkosten } \\
\text { Pharmazeutisches Personal }\end{array}$ & $\begin{array}{c}\text { Personalkosten in \% nach } \\
\text { Tätigkeitsbereichen }\end{array}$ & $\begin{array}{c}\text { Durchschnittskosten je } \\
\text { Arbeitsstunde }\end{array}$ \\
\hline Verkauf & 62,9 & 42 \\
\hline Rezeptur & 15,1 & 40 \\
\hline Einkauf / Warenwirtschaft & 12,9 & 38 \\
\hline Sonstiges & 9,1 & 44 \\
\hline
\end{tabular}

Quelle: Hasan-Boehme, U., u.a., Tätigkeits- und Kostenprofile der Apothekenmitarbeiter, in: www.pharmazeutische-zeitung.de/pza/1999-39, Tabelle 2, Zugriff am 21.11.00.

Im Vergleich zu den Präsenz - entstehen in Versandapotheken mit dem Wegfall der face-to-face-Interaktion zwischen Apotheke und Patient veränderte Ablaufstrukturen, die eine relative zeitliche Verschiebung der Gewichtigkeit von Tätigkeiten zur Folge haben. Niederschlag finden diese in der Personalstruktur bzw. den Personalkosten:

- Die Abgabe der Arzneimittel an den Patienten erfolgt per Versand. Die Zusammenstellung der Sendungen kann demnach nach dem Vorbild seriöser ausländischer Versender anhand des Rezeptes durch eine PTA vorgenommen werden. Dem Apotheker obliegt dabei nach wie vor die Endkontrollfunktion. Eine qualitative Minderung ist nicht zu erwarten.

Jedoch ist daraus abzuleiten, dass neben einem niedrigeren Zeitanteil für die Medikamentenzusammenstellung durch standardisierte Abläufe der (im Verhältnis zum gesamten pharmazeutischen Personal) verstärkte Einsatz von mittlerem pharmazeutischen Personal (beispielsweise PTA) zu niedrigeren Personalkosten in diesen Bereichen führt: So weist die Mitarbeiterstruktur in deutschen Präsenzapotheken ein Verhältnis angestellte approbierte Apotheker (ohne Leiter 1,52 Beschäftigte) zu Apothekenassistenten (1,06 Beschäftigte) und PTA (1,57

60

Hasan-Boehme, U., u.a., Tätigkeits- und Kostenprofile der Apothekenmitarbeiter, in: www.pharmazeutische-zeitung.de/pza/1999-39 und www.pharmazeutische-zeitung.de/pza/1999-39, Zugriff am 21.11.00. 
Beschäftigte) ein Verhältnis von $1: 1,7$ auf (entnommen Tabelle II - 6), während es in einer (Versand)Apotheke ohne Offizin bei $1: 2,6^{61}$ und mehr liegen kann.

- Entsprechend der Verschiebung wie Standardisierung der Tätigkeitsbereiche und -abläufe ist für das pharmazeutische Personal der Versandapotheken, insbesondere für die approbierten Apotheker, eine höhere Produktivität, d.h. ein höherer pro - Kopf - Umsatz zu erwarten. Der Umsatz pro angestellten approbierten Apotheker liegt in den Versandapotheken per Annahme 1,7 bis 2 mal höher als in Präsenzapotheken.

- Dem Patienten wird nach wie vor eine pharmazeutische Beratung zuteil. Hier entstehen neue Dienstleistungsangebote wie zeitlich unbefristet verfügbare Telefonberatungen (siehe Punkt 2.4.2.2.). Eine entsprechende Nachfrage nach zusätzlichem qualifiziertem Personal für diesen Tätigkeitsbereich ist anzunehmen.

Der Wegfall der face-to-face-Interaktion hat neben der Personalstruktur auch Auswirkungen auf die räumliche Struktur in Vergleich zwischen Präsenz- und Versandapotheken: Die Modellrechnungen für Versandapotheken gehen davon aus, dass in den Räumen der Versandapotheke kein Kundenverkehr stattfindet, bzw. dass der Kundenverkehr auf ein Mindestmass beschränkt bleibt. Ein Niederschlag auf die Raumkosten ist zu erwarten:

- Versandapotheken sind im Gegensatz zu Präsenzapotheken nicht an eine gute Geschäftslage, z.B. in teuren Innenstadtlagen, gebunden. Ihr Standort kann ohne Umsatzeinbußen auch eine Randlage (Gewerbegebiet) oder der ländliche Bereich sein.

- Zudem ist die Vorhaltung einer Offizin nicht notwendig. (Von den ca. $165 \mathrm{qm}^{63}$ Gesamtfläche einer Präsenzapotheke entfallen ca. $40 \%$ auf die Offizin ${ }^{64}$ ).

- Jedoch werden für die Telefonberatung entsprechende räumliche Kapazitäten benötigt (siehe dazu ausführlich Punkt 2.4.2.2.).

61

Siehe MediService: 8 Apotheker, 21 Pharmazeutische Assistenten, unter www.mediservice.ch, Zugriff am 08.08.2001.

Bei einem Umsatz der Präsenzapotheke von ca. 2,56 Mio. DM und einer Anzahl angestellte Apotheker von 1,52 (siehe Tabelle II - 2: Wert in Teilzeit) ergibt dies eine Produktivität von ca. 1,65 Mio. DM je Apotheker. Der Beitrag des leitenden Apothekers wird bewusst nicht einkalkuliert, da dies mit wachsendem Umsatz zu Verzerrungen führt. Dessen Tätigkeitsbereich wird mit steigender Umsatzgröße eher weg von der pharmazeutischen Versorgung hin zu Managementaufgaben verlagert. Unter der Annahme der Produktivitätsverdopplung werden demzufolge 3,3 Mio. DM Umsatz je angestellter Apotheker p.a. in derselben Arbeitszeit (c.p.) erwirtschaftet. Ähnliches trifft ebenso für die PTA zu.

Nach § 4 Apothekenbetriebsordnung (ApBetrO) muss eine Präsenzapotheke mindestens eine Fläche von $110 \mathrm{qm}^{63}$ Geschäftsraum aufweisen.

Berechnet nach den Angaben des IFH Köln (2000), Hüsgen, Die öffentliche Apotheke im Gesundheitsmarkt. Darstellung der volkswirtschaftlichen Situation und der gesundheitsökonomischen Bedeutung, S. 9-31, S. 29, in: Management, in Apotheken, Sonderheft 43 des IFH. 
- Um die Abgabe der Arzneimittel via Versand organisieren zu können, müssen in den Versandapotheken Verpackungsräume vorgehalten werden. Eine räumliche Integration in die Lagerkapazitäten ist denkbar.

- Versandapotheken steht durch den Wegfall von Offizinräumlichkeiten eine relativ größere Lagerfläche zur Verfügung.

- Durch die geänderte Raumstruktur von Versandapotheken sowie die Möglichkeit einer Ansiedlung in Randlagen ist mit geringeren Mietkosten zu rechnen: für 1998 werden in den Berechnungen ca. 12 DM je qm an Mietkosten bzw. Mietwert angesetzt. $^{65}$ (siehe auch die in Tabelle II - 9 dargestellten Mietpreise):

Tabelle II - 9: Mietpreise für Gewerbe- und Lagerflächen in Abhängigkeit vom Standort

\begin{tabular}{|l|c|c|c|c|}
\hline Standort & \multicolumn{2}{|c|}{$\begin{array}{c}\text { Mietpreis Gewerbeflächen in } \\
\text { DM/qm }\end{array}$} & $\begin{array}{c}\text { Mietpreis Lager- und Logistikflä- } \\
\text { chen in DM/qm }\end{array}$ \\
\hline & Stadt & Umland & Stadt & Umland \\
\hline Hamburg & $9-15$ & $9-15$ & $7-11$ & $6-9$ \\
\hline Berlin & $12-18$ & $12-15$ & $8-13$ & $7-12$ \\
\hline Leipzig & $6-12$ & $6-10$ & $6-10$ & $5-8$ \\
\hline Ruhrgebiet & $7-12$ & $5-10$ & $6-8$ & $4-6$ \\
\hline Düsseldorf & $10-15$ & $7-12$ & $9-12$ & $5-8$ \\
\hline Frankfurt/Main & $10-22$ & $10-16$ & $12-16$ & $10-15$ \\
\hline Stuttgart & $10-14$ & $10-12$ & $8-12$ & $6-10$ \\
\hline München & $12-18$ & $12-16$ & $10-12$ & $9-12$ \\
\hline
\end{tabular}

Quelle: Unterreiner, F. (2001), Die günstigsten Standorte für Mieter und Bauherren, in: Zeitschrift Impulse 2/2001, S. 53.

Den Mietkosten werden (wie für Präsenzapotheken) 28\% für Sach- bzw. Mietnebenkosten zugeschlagen.

Neben einer veränderten räumlichen Struktur (Fokussierung auf Gewerbe- und Lagerflächen) sowie geringerer Mietkosten ist die Optimierung des Lagerbestandes anhand der Kennzahlen Lagerumschlag (definiert als Maß, wie oft der Lagerbestand verkauft wird) und Lagerwert (in DM) bedeutsam. ${ }^{66}$ Ziel ist ein höherer Umsatz je qm Lager sowie der Effekt, Fremdkapitalzinsen auf den Lagerbestand zu sparen:

Z.B. siehe Mietspiegel für ländliche Kommunen der IHK Darmstadt, Gewerbliche Mietpreise 2000 im Bezirk der IHK Darmstadt - DM/qm, www.ink.darmstadt.gmd.de, Zugriff 30.11.00: ländlicher Bereich -Büros 8-12 DM/qm, Hallenflächen (beheizt) 4-8 DM/qm, Gernsheim: Büros 10-15 DM/qm, Hallenflächen (beheizt) 5-12 DM/qm. 
- Für Versandapotheken wird laut Annahme von einem Lagerwert pro Jahr von $8 \%$ des Nettoumsatzes ausgegangen, der 8,5 mal pro Jahr umgeschlagen wird (d.h. ein Umschlag der Waren auf Lager aller 6 Wochen) Diese Zahlen basieren auf Angaben zur Optimierung der Lagerplanung in Präsenzapotheken ${ }^{67}$.

- Im Gegensatz zu den optimierten Werten weisen Präsenzapotheken allerdings durchschnittlich einen Lagerwert von $10 \%$ des Nettoumsatzes auf, woraus sich ein Lagerwert von ca. 220 TDM berechnet ${ }^{68}$ (in der Literatur finden sich Durchschnittsangaben von ca. 200 TDM gebundenes Kapital als Lagerbestand ${ }^{69}$ ), der ca. 6,8 mal pro Jahr umgeschlagen wird (d.h. ein Umschlag der Waren auf Lager aller 8 Wochen). Bezogen auf die Lagerfläche $^{70}$ resultieren ca. 2,93 TDM Lagerbestand je qm Lager.

- Der Wert Lagerbestand je qm Lager wird für Versandapotheken übernommen, um den anhand der genannten Parameter optimierten gesamten Lagerwert $(8 \%$ Lagerwert, 8,5 mal Lagerumschlag p.a.) im selben Maße je qm Lagerfläche zu konzentrieren wie in Präsenzapotheken auch.

- Aus der Übernahme des Lagerbestandes je qm Lager für Versandapotheken in Kombination mit einer Optimierung der Umschlaghäufigkeit des gesamten Lagerbestandes resultiert, dass in Versandapotheken je qm Lagerfläche ein höherer Umsatz erreicht werden kann als in den Präsenzapotheken.

- Eine zusätzliche Umsatzsteigerung durch Lagerbestandsoptimierung könnte aus der Raumstruktur der Versandapotheken (Gewerbe- und Lagerräume, s.o.) resultieren: so wäre es möglich, dass das Lager aufgrund der Gegebenheiten eher in die Höhe als in die Breite expandiert. Entsprechend würde sich der Lagerwert je Quadratmeter Lager erhöhen, eine zusätzliche Umsatzsteigerung wäre realisierbar. Damit könnten weitere Mietkosten gespart werden.

O.V. (1999), Kapitel Lagerorientierte Kennziffern, in: Wirtschaftshandbuch für die Apotheke, in: Leseprobe www.govi.de/rubriken/buchart7buecher/covers/0003.htm, Zugriff am 05.12.00.

Bei 2,5 Mio. DM ergibt sich ein Nettoumsatz von ca. 2,2 Mio. DM, darauf werden $10 \%$ berechnet.

So z.B. in: Hüsgen, Die öffentliche Apotheke im Gesundheitsmarkt. Darstellung der volkswirtschaftlichen Situation und der gesundheitsökonomischen Bedeutung, S. 9-31, S. 27. in: Management in Apotheken, Sonderheft 43 des IFH Köln.

Ca. $45 \%$ von 165 qm Geschäftsfläche ergeben 75 qm Lagerfläche, resultiert aus $40 \%$ Offizin, ca. $9 \%$ Laboratorium (15 qm von 165 qm), ca. 6\% Nachtdienstzimmer / Büro (Annahme: $10 \mathrm{qm}$ von $165 \mathrm{qm})$. 


\subsubsection{Patientensicherheit: fachliche Beratung durch Versandapotheken}

Nach § 20 ApBetrO muss aus Gründen der Arzneimittelsicherheit der Apothekenkunde bei der Abgabe des Arzneimittels pharmazeutisch beraten werden. Die Pflicht zur Sicherstellung einer qualifizierten Beratung als Bestandteil der pharmazeutischen Tätigkeit gilt demzufolge für alle Apotheken, unabhängig von der Vertriebsart. Dabei soll nicht zur Diskussion stehen, ob die pharmazeutische Beratung in Präsenzapotheken ausreichend erbracht wird: „Der Aufbau eines seriösen Versandhandels eröffnet somit Chancen der nachhaltigen Debatte um Beratungsstandards sowohl im Versandhandel als auch im niedergelassenen Apothekensegment sowie um eine dementsprechende Aktivierung der Verbrauchernachfrage in der Angebotssteuerung."

Da die Medikamentenabgabe, und damit die Kommunikation, zwischen der Versandapotheke und dem Kunden nicht wie in einer Präsenzapotheke face-to-face bei persönlicher Anwesenheit erfolgt, muss die Beratung durch eine andere Art der Kommunikation garantiert werden. Wesentliches Kommunikationsmedium von Versandapotheken, so zeigen Erfahrungen im Ausland, ist das Telefon. Vorteilhaft ist, dass annähernd jeder Haushalt über ein Telefon verfügt und die Handhabung allgemein vertraut ist. Häufig kann der Beratungsbedarf zusätzlich auch via Internet in Form von "Gesundheitsportalen" über die Homepage der Versandapotheke geäußert werden. Diese Art der Kommunikation ist allerdings an die Verfügbarkeit eines Internetzugangs gekoppelt und damit im Vergleich zum Telefon auch auf längere Sicht nicht jedem Patienten zugänglich bzw. vertraut. In der Funktion eines Dienstleisters für den oftmals gesundheitlich - wie mobilitätsbedingt - beeinträchtigten Kunden bieten die im Ausland zugelassenen seriösen Versandapotheken Telefon-Hotlines mit Kundendienst und pharmazeutischer Beratung an, die in den meisten Fällen rund um die Uhr an 365 Tagen im Jahr erreichbar sind. Dieser Service ist i.d.R. für den Anrufer kostenlos. Dagegen bieten unseriöse Versandhändler häufig keine oder extrem schlechte Beratungsmöglichkeiten bzw. Telefon-Hotlines. ${ }^{72}$

Deshalb sollte die telefonische Verfügbarkeit sowie deren Umfang nicht in die Entscheidungsgewalt der einzelnen Versandapotheke gestellt werden, sondern im rechtlichen Rahmen vorgegeben sein. So verlangen z.B. die meisten US-Bundesstaaten von Versandapotheken eine kostenfreie Telefonnummer, unter der jederzeit oder in einem bestimmten Stundenumfang eine telefonische Beratung zur Verfügung steht. Dies stößt auf große Nachfrage seitens der Konsumenten, so die Zahlen des Versandhandelsmarktführers Merck-Medco von 1999 in den USA: bei rund 60 Millionen versandten Arzneimitteln wurden insgesamt 48 Millionen Telefongespräche

71

Arbeitsgemeinschaft der Verbraucherverbände e.V. (AgV), (2000), Internethandel mit Medikamenten: In Europa Qualität sichern \& Chancen für Verbraucher nutzen, S. 5, siehe unter unw.agv.de, Zugriff am 24.01.2001.

Arbeitsgemeinschaft der Verbraucherverbände e.V. (AgV), (2000), Internethandel mit Medikamenten: In Europa Qualität sichern \& Chancen für Verbraucher nutzen, S. 6, siehe unter www.agv.de, Zugriff am 24.01.2001. 
gezählt. Allerdings fallen hierunter auch sämtliche Aktivitäten des Benefit Managements, so dass sich diese Zahl nicht nur auf Patientengespräche mit pharmazeutischer Beratung bezieht. ${ }^{73}$

Die Informationsmöglichkeiten für die Bevölkerung über Leistungsangebote im Gesundheitsbereich sowie allgemeine gesundheitliche Fragen sind in der Bundesrepublik im Vergleich zu anderen Ländern deutlich beschränkt. Zudem stehen diesen Informationsangeboten rechtliche Restriktionen bezüglich weitergehender Beratungsleistungen zu medizinischen Themen entgegen. Erst in jüngerer Zeit bieten deshalb telefonische Dienste allgemeine Auskünfte zur gesundheitlichen Versorgung an. Meistens handelt es sich um Adressauskünfte von Ärzten, Apothekern oder anderen Akteuren sowie um Vermittlungen medizinischer Beratungs- und Kontaktmöglichkeiten (z.B. Selbsthilfegruppen) ${ }^{74}$, nicht jedoch um eine therapeutische Beratung.

Die genannten Aspekte erschweren eine Einschätzung der zu erwartenden Kosten für eine fachlich hochwertige pharmazeutische Beratung in den potentiellen Versandapotheken. Im Konsens darüber, dass Versandapotheken für die Zulassung dieselben Qualitätsstandards zu erfüllen haben wie Präsenzapotheken auch ${ }^{75}$, wird es zudem im Sinne des Verbraucherschutzes und der Akzeptanz durch den Patien$\operatorname{ten}^{76}$ unabdingbar sein, dass eine in Deutschland zugelassene Versandapotheke eine Telefon - Hotline mit pharmazeutischem Personal zeitlich unbegrenzt, d.h. täglich rund um die Uhr, und für den Anrufer kostenlos anbietet. Dies muss nicht durch jede Apotheke eigenständig erbracht, sondern kann auch in Kooperationsverbünden sichergestellt werden.

Erfahrungen im Ausland zeigen außerdem, dass die Mehrheit der Anrufe die Versandapotheke während der üblichen Dienstzeit erreicht: innerhalb der Öffnungszeit, die ca. ein Drittel der Jahresstunden beträgt, finden $95 \%$ bis $97 \%$ aller Anrufe statt. Nur $3 \%$ bis $5 \%$ aller Anrufe verteilen sich dagegen auf die Zeit außerhalb der Öffnungszeiten, die den überwiegenden Teil der Jahresstunden (inklusive der Nachstunden) ausmacht.

Blankenberg, K., (2000), www.zukunft.de? - Einblicke in Amerikas legale Online-Apotheken, S. 26, in: Pharmazeutische Zeitung Nr. 49, 145. Jahrgang vom 07.12.2000, S. 25-26.

74

IGES Institut für Gesundheits- und Sozialforschung, (2000), Qualitätstest medizinischer Informationsdienste - Abschlußbericht, S. 7, siehe unter www.almeda.de, Zugriff am 20.03.2001.

Arbeitsgemeinschaft der Verbraucherverbände e.V. (AgV), (2000), Internethandel mit Medikamenten: In Europa Qualität sichern \& Chancen für Verbraucher nutzen, S. 9, siehe unter www.agv.de, Zugriff am 24.01.2001.

Projektbegleitende Umfragen haben ergeben, dass ca. $71 \%$ der Befragten die 24-StundenVerfügbarkeit als Qualitätskriterium fordern.

Arbeitsgemeinschaft der Verbraucherverbände e.V. (AgV), (2000), Internethandel mit Medikamenten: In Europa Qualität sichern \& Chancen für Verbraucher nutzen, S. 11, siehe unter www.agv.de, Zugriff am 24.01.2001. 
Tabelle II - 10: Angenommene Verteilung der Öffnungszeiten wie der Telefonanrufe auf Basis von Jahresstunden

\begin{tabular}{|l|c|c|}
\hline \multicolumn{1}{|c|}{ Versandapotheke } & $\begin{array}{l}\text { Verteilung der Öffnungszeiten p.a. } \\
\text { (in Relation zu den gesamten Jah- } \\
\text { resstunden) }\end{array}$ & Verteilung der Anrufe $^{78}$ \\
\hline $\begin{array}{l}\text { Offnungszeiten / } \\
\text { eingehende Anrufe pro Jahr }\end{array}$ & $28 \%$ & $95 \%-97 \%$ \\
\hline $\begin{array}{l}\text { Keine Öffnungszeiten (inklusive } \\
\text { Nachtstunden)/ } \\
\text { eingehende Anrufe pro Jahr }\end{array}$ & $72 \%$ & $5 \%-3 \%$ \\
\hline $\begin{array}{l}\text { Jahresstunden insgesamt / } \\
\text { Anrufe insgesamt }\end{array}$ & $100 \%$ & $100 \%$ \\
\hline
\end{tabular}

Quelle: INIFES, eigene Darstellung.

Es stellt sich also die Frage, wie Versandapotheken die für den Kunden kostenlose rund-um-die-Uhr Telefonberatung gewährleisten können, durch interne oder externe Call - Center. Dazu bedarf es nicht nur der Kenntnis einer relativen Verteilung der Anrufe auf die Jahresstunden (wie in obiger Tabelle dargestellt), sondern auch der Höhe der zu enwartenden Anrufe pro Stunde (während wie außerhalb der Öffnungszeiten).

Dass die Angabe absoluter Anruferzahlen vage und zudem von vielen Faktoren wie u.a. der Akzeptanz der Kunden (auch bei kostenloser Nutzung) oder den qualitativen Standards des Beratungsangebots abhängig ist, läßt sich erahnen. Allgemein kann jedoch festgestellt werden, dass:

- die Anzahl der zu erwartenden telefonischen Kundenkontakte mit der Gesamtanzahl der Kunden einer Versandapotheke und damit auch mit der Umsatzgröße stark korreliert,

- dass zudem mit wachsender Akzeptanz durch die Nachfrager eine seh rege statt verzagte Nutzung dieser Beratungsmöglichkeit anzunehmen ist ${ }^{79}$.

Eine Eigenerstellung der telefonischen Beratung setzt voraus, dass diese - neben der Gewährleistung der technischen Standards - ausreichend qualifiziertes pharmazeutisches Personal zusätzlich zum üblichen Apothekenbetrieb vorhält. ${ }^{80}$

Informationen liegen INIFES aufgrund von Expertengesprächen vertraulich vor.

Dies belegen verschiedene Informationen und Literaturquellen, die bezüglich der zu erwartenden Anruferzahlen in Abhängigkeit vom Umsatz durch INIFES ausgewertet wurden.

In anderen Branchen (z.B. Reise / Verkehr, Finanzdienstleister, Telefon- oder IT-Gesellschaften) bilden zwei Drittel der Call - Center eigene Abteilungen des jeweiligen Unternehmens, die übrigen Call - Center sind externe Dienstleister. Der Trend zum Outsourcing ist jedoch ungebrochen. Siehe dazu: Hennersdorf, A., Kiani-Kress, R. (2001), Frust am Draht, S. 107, in: Wirtschaftswoche Nr. 21, vom 17.5.2001, S. 105-115. 
Die Gegenüberstellung der Kosten für den Fall der Inanspruchnahme eines externen pharmazeutischen Call-Centers durch die Versandapotheke ist allerdings noch erschwert, da derzeit keine pharmazeutischen Telefonberatungsdienste existieren sowie die am Markt agierenden medizinischen Telefoninformationsdienste ${ }^{81}$ unterschiedlichste Modelle telefonischer Erreichbarkeit wie Abrechnungsmodalitäten praktizieren. Dies ist darin begründet, dass nur wenige Anbieter der Allgemeinheit zugänglich sind und damit einen Preis in Höhe des Marktpreises berechnen, die meisten Anbieter aber im Gegensatz dazu mit Krankenversicherungen zusammenarbeiten (oder diesen gar angeschlossen sind) und damit nur einen auf die Versicherungsmitglieder beschränkten Zugang ermöglichen. Entsprechend werden die Auskunftskosten häufig in den von den Mitgliedern der entsprechenden Kassen zu zahlenden Versicherungsbeiträgen „versteckt“, so dass durch den Anrufer nur die eigene Telefongebühr zu tragen ist bzw. auch diese z.T. entfällt.

INIFES analysierte drei medizinische Telefondienste, die neben einer zeitlich unbegrenzten Verfügbarkeit zudem Auskünfte ${ }^{83}$ erteilten, die über die Mitteilung einer Arzt- oder Apothekenadresse hinaus gingen. Siehe dazu Tabelle II - 11.

Tabelle II - 11: Überblick über Gesprächsdauer und Gesprächskosten ausgewählter medizinischer Telefon-Hotlines

\begin{tabular}{|c|c|c|c|}
\hline medizinis che Telefonhotline & Arzt-Partner & MD Medicus & Mercure Assistance \\
\hline \multicolumn{4}{|l|}{ Zugang für Versicherte: } \\
\hline & unbeschränkt & Continentale & TKK \\
\hline$\therefore$ & & Mannheimer & Iduna-Nova \\
\hline & & BKK Siemens & \\
\hline \multicolumn{4}{|l|}{ Gesprächs dauer( in m in.): } \\
\hline ca. & 5,25 & 7,25 & 4,25 \\
\hline gew ichteter Durchschnitt & & & 5,00 \\
\hline \multicolumn{4}{|l|}{ Gesprächskosten ( in DM): } \\
\hline ca & 19,06 & 1,74 & 1,02 \\
\hline \multicolumn{4}{|c|}{ Kosten je Gesprächsm inute (DM pro Minute): } \\
\hline ca. & 3,63 & 0,24 & 0,24 \\
\hline gew ichteter Durchschnitt & & & 1,30 \\
\hline
\end{tabular}

Quelle: INIFES, eigene Darstellung nach IGES (2000), Qualitätstest medizinischer Informationsdienste - Abschlußbericht, S. 79ff, siehe unter www.almeda.de, Zugriff am 20.03.2001.

Auf dieser Basis können die Preise für ein Outsourcing der Telefonberatung an externe Call - Center eruiert werden: eine Gesprächsminute kostet ca.1,30 DM. Bei

81

Betrachtet man diese mangels anderer Anbieter als Grundlage für mögliche monetäre Analysen.

82

IGES Institut für Gesundheits- und Sozialforschung (2000), Qualitätstest medizinischer Informationsdienste - Abschlußbericht, S. 7, siehe unter www.almeda.de, Zugriff am 20.03.2001.

83

So z.B. bei Diabetes: Nennung von Informations- und Kontaktmöglichkeiten, Informationen in Anlehnung an einschlägige Leitlinien wie denen der Deutschen Diabetes-Gesellschaft. Siehe dazu unter IGES (2000), Qualitätstest medizinischer Informationsdienste - Abschlußbericht, S. 27ff, www.almeda.de, Zugriff am 20.03.2001. 
einer durchschnittlichen Dauer eines Telefonkontaktes von fünf Minuten ${ }^{84}$ kostet die Fremdvergabe an externe Call - Center der Versandapotheke ca. 6,50 DM pro Anruf.

Exkurs: Im Vergleich zu anderen Branchen, bei denen der Preiskampf aufgrund der wachsenden Anzahl externer Call - Center zu einem deutlichen Preisverfall in den letzten zwei Jahren geführt hat, sind für einen Anruf von ebenfalls fünf Minuten statt fünf bis acht Mark heute nur noch zwei bis drei Mark üblich. Die Kehrseite ist häufig ein Defizit an Mitarbeiterkompetenz und technischer Ausstattung der Call - Center ${ }^{85}$.

Den Ausführungen folgend soll für die jeweiligen Modelle einer Versandapotheke VA I, VA II und VA III eine Abwägung bezüglich interner und externer Telefonberatung unter Kostenaspekten, differenziert nach Öffnungs- wie Nicht-Öffnungszeiten, getroffen werden.

\section{Innerhalb der Öffnungszeiten einer Versandapotheke:}

Im Durchschnitt sowie bei Zugrundelegung eines linearen Zusammenhangs von Umsatz und Anruferzahl ${ }^{86}$, werden mit Hilfe verschiedener Quellen ${ }^{87}$ folgende Anruferzahlen je Stunde angenommen:

- kleine Versandapotheke (5 Mio. DM Umsatz): ca. 15 Anrufe

- große Versandapotheke (20 Mio DM. Umsatz): ca. 60 Anrufe

- Versandapotheke mit Großhandel (487 Mio. DM Umsatz): ca. 1.300 Anrufe

Multipliziert mit je fünf Minuten, berechnen sich die Gesprächsminuten je Stunde:

- kleine Versandapotheke (5 Mio. DM Umsatz): 75 Gesprächsminuten

- große Versandapotheke (20 Mio DM. Umsatz): 300 Gesprächsminuten

- Versandapotheke mit Großhandel (487 Mio. Umsatz): 6.500 Gesprächsminuten

Bei einem Stundenumfang von sechzig Minuten ergibt sich durch Verrechnung der Gesprächsminuten in Gesprächsstunden (z.B. 75 Gesprächsminuten bei VA I sind ca. 1,3 Gesprächsstunden) die Anzahl des benötigten pharmazeutischen Beratungspersonals je Arbeitsstunde (entsprechend sind 1,3 Telefonberater bei VA I je Arbeitsstunde gleichzeitig notwendig). Durch Hochrechnung der Gesprächsstunden auf ein Jahr wird die Anzahl des nötigen Beratungspersonals pro Jahr unter der Restriktion von 1.710 Arbeitsstunden pro Jahr und Vollzeitstelle ermittelt. Multipliziert

Dies ergaben andere Quellen sowie Informationen aus Expertengesprächen, die INIFES vertraulich vorliegen.

Hennersdorf, A., Kiani-Kress, R. (2001), Frust am Draht, S. 115, in: Wirtschaftswoche Nr. 21, vom 17.5.2001, S. $105-115$. Aufgrund dessen erscheint es im Sinne der Sicherung einer hochwertigen Beratungsqualität geboten, die letztgenannten Zahlen nicht als Grundlage monetärer Analysen für die Bereitstellung pharmazeutischer Telefonberatung in adäquaten externen Call - Centern zu verwenden.

Dieser Zusammenhang wird mit wachsender Umsatzgröße wahrscheinlich nicht linear, sondern eher degressiv wachsend ausgeprägt sein. Dazu gibt es jedoch keine genauen Informationen.

Informationen liegen INIFES auf Basis von Expertengesprăchen vertraulich vor. 
mit den Personalkosten ${ }^{88}$ p.a. ergibt sich der gesamte Personalaufwand eines internen Call - Centers pro Jahr.

Für die technischen Voraussetzungen einer internen Telefonberatung ergeht die Annahmen, dass pro zusätzlich nötigem Arbeitsplatz (Hard- und Software, Telefonausstattung) zudem ein Aufwand von ca. 5.000 DM in Form einer linearen Abschreibungsrate über 4 Jahre zu kalkulieren ist. Zusätzlich wird die ebenfalls über einen Zeitraum von vier Jahren kalkulierte Abschreibungsrate einer sogenannten Call-Distributions-Anlage ${ }^{89}$ in die Aufwendungen für die technische Ausstattung einbezogen.

Ein Vergleich der gesamten internen Telefonberatungskosten mit den Preisen externer Call - Center ${ }^{90}$ ergibt für alle drei Versandapothekenmodelle während der Öffnungszeiten als Optimum den Aufbau einer interne Telefonberatung mit den entsprechende Kapazitäten .

\section{Außerhalb der Dienstzeiten einer Versandapotheke:}

Aufgrund der Verteilung der Anrufe - ca. 3\% bis 5\% entfallen auf ca. $72 \%$ der Jahresstunden $^{91}$ - ist mit nur sehr geringen Anruferzahlen bzw. Gesprächsminuten je Stunde außerhalb der Öffnungszeiten zu rechnen. In der Konsequenz muß in den beiden Versandapothekenmodellen VA I und VA II mehr pharmazeutisches Personal wegen der Länge des zu überbrückenden Zeitraumes vorrätig sein, als durch die Anruferanzahl begründet wäre (Fixkosten). Der Personalaufwand stiege überproportional.

Eine Abwägung aller internen Telefonberatungskosten mit den Preisen externer Call - Center führt dazu, dass nur die Versandapotheke mit Großhandelsfunktion (Umsatz 487 Mio. DM) außerhalb der Öfnungszeiten eine eigene Telefonberatung anbieten wird. Die beiden Versandapotheken VA I und VA II mit geringeren Umsätzen werden die Telefonberatung außerhalb der Öffnungszeiten an externe Call

- Center vergeben oder durch Kooperationsverbünde von Apotheken sicherstellen. ${ }^{92}$

Dies bestätigen internationale Erfahrungen sowie nationale Erfahrungen mit Telefonberatungsservice in anderen Branchen.

Siehe ebenfalls Tabelle II - 6: Den Personalkosten wird entsprechend der geforderten pharmazeutischen Qualifikation des telefonischen Beratungspersonals ein Wert von 58.000 DM (korrespondierend der Qualifikation einer PTA) je Vollzeitstelle zugrunde gelegt. $\rightarrow$ Begründung, warum keine Apothekerqualifikation der Kostenberechnung zugrunde liegt: Rückfrage bei den für den „üblichen" Geschäftsbetrieb zuständigen Apothekern durch den Telefonkundendienst während der üblichen Dienstzeiten einer Versandapotheke jederzeit möglich.

Eisinger, H. (1998), So funktioniert der Versandhandel, Wien, S. 163.

Preis für externes Call - Center p.a. = 6,50 DM Kosten je Anruf * Anrufe p.a.

Siehe Tabelle II - 10.

Siehe entsprechend Kapitel 2.4.2.2. 


\subsubsection{Bezugs- und Zustellsicherheit: Voraussetzung einer funktionierenden B-2-C Logistik der Versandapotheken}

Versandapotheken können im Vergleich zu Präsenzapotheken keine umgehende Lieferung des Medikaments aufgrund der zwischen Abgabe und Empfang befindlichen Versandzeit gewährleisten: eine Lieferfrist von ca. 48 Stunden zwischen Rezepteingang in der Apotheke und Zustellung beim Patienten (s. Abbildung II - 4 unter Kapitel 2.3.) wurde angeführt. Diese Zahl ist den Erfahrungen in der Schweiz entnommen. Es sollte allerdings auch erwähnt werden, dass einige Versender mit einer längeren Lieferfrist von ca. 4 bis 5 Tagen arbeiten.

Grundsätzlich ist eine längere Lieferfrist bezüglich der Definition versendungsfähiger Verordnungen, wie in Kapitel 2.3. vorgenommen, aufgrund der dort gewählten Indikatoren unproblematisch. Allerdings bestätigt sich, dass längere Lieferzeiten kontraproduktiv auf die Inanspruchnahme von Versandapotheken wirken. So ergab die INIFES - Befragung, dass eine zügige Medikamentenlieferung binnen 48 Stunden für die Nutzung von Versandapotheken seitens der Bevölkerung ein wesentliches Akzeptanzkriterium darstellt.

Die Logistik ist also neben der pharmazeutischen Kernkompetenz eine Leistung, die für Versandapotheken einen strategischen Parameter darstellt. Darunter ist die Sicherstellung der Aufgaben zu verstehen, die garantieren, dass das richtige Arzneimittel für den richtigen Patienten rechtzeitig am richtigen Ort und in einwandfreier Qualität unter Einhaltung aller fachlichen und gesetzlichen Anforderungen kostenoptimal zur Verfügung steht. ${ }^{93}$ Im Falle von Versandapotheken handelt es sich um eine Business - to - Customer - Logistik (B-2-C): Adressat der Lieferung ist der Patient, der das Arzneimittel an einem bestimmten Aufenthaltsort (zu Hause, am Arbeitsplatz, am Urlaubsort) erhalten möchte.

Im Unterschied dazu beruht die Logistik des pharmazeutischen Großhandels auf Strukturen der Business - to - Business - Distribution (B-2-B): Adressat der Lieferung ist die öffentliche Apotheke. Das heißt, die Logistik potentieller Versandapotheken wird aufgrund der konsumentenorientierten Fokussierung auf den Apothekenkunden im Vergleich zum pharmazeutischen Großhandel mit Business-Orientierung auf die Einzelapotheke komplexere Liefer- und Zustellmodalitäten sowie vergleichsweise kleinere Auftrags- bzw. Versandwerte aufweisen. Ohne Zweifel stellt dieser Umstand eine Herausforderung für potentielle Versandapotheken dar. Eine Make or - Buy - Entscheidung, d.h. eine Analyse darüber, ob die Logistikkompetenz vom Markt durch externe Logistikunternehmen bezogen werden sollte oder durch die Versandapotheke zu erstellen wäre, ist ein wichtiger Punkt in der strategischen Positionierung.

93

Fenske, D., Krämer, I. (2001), Optimierung der pharmazeutischen Logistik, S. 62, in: Krankenhauspharmazie, Jg. 22, Nr. 2, S. 62- 67. 
Eine Entscheidung darüber hängt u.a. von der angestrebten räumlichen Reichweite des Versandes ab. Unternehmen des pharmazeutischen Großhandels, die neben einigen regional begrenzten Teilsortimentern überwiegend bundesweit versenden, kaufen i.d.R. die Logistikleistung von externen Dienstleistern ein. ${ }^{94}$

Auch ausländische Versandapotheken mit überregionalem bzw. landesweitem Versand verfahren ähnlich: so bedient sich die Versandapotheke in der Schweiz dem Kuriersystem der Schweizer Post für die Versendung und Zustellung der Arzneimittel. Dieser Trend ist in Deutschland in dem bereits für den Versandhandel zugelassenen OTC-Arzneimittelsegment zu beobachten.

Daraus ist zu schließen, dass mit dem Anspruch der Erschließung eines bundesweiten Kundenkreises (was wiederum eine entsprechende Umsatzgrößeklasse induziert) die Logistik einer potentiellen Versandapotheke tendenziell an externe Anbieter outgesourct wird. Zudem wird die Entwicklung des Logistik-Marktes durch E-Commerce oder E-Logistics (um nur einige zu nennen) gefördert, weil die stark gestiegene Nachfrage nach Logistikleistungen insbesondere aus dem Konsumentenbereich umfassende Dienstleistungsangebote erfordert. Zudem führen neue Anbieter zu einem verstärkten Preiswettbewerb.

Mit Blick auf die Entwicklung des europäischen Binnenmarktes, der einschlägigen europäischen Richtlinien oder des Internets ist von einer Reichweite des Versandes über die Landesgrenzen hinaus auszugehen bzw. ist dieser Trend schon offensichtlich, selbst wenn derzeit im Segment der verordnungsfähigen Arzneimittel noch nationale Sozialversicherungs- und Erstattungssysteme eine EU-weite Versendung beeinträchtigen.

Der Aufbau eigener Logistikstrukturen für einen bundesweit ausgerichteten Arneimittelversender erscheint unter diesen Umständen eher unrealistisch als eine Fremdvergabe an externe Anbieter. Deshalb wird für die Modellrechnungen der großen Versandapotheken mit 20 Mio. DM (Variante II) bzw. der Versandapotheke mit Großhandelsfunktion und 487 Mio. DM Umsatz (Variante III) von externen Logistikanbietern ausgegangen. Begründung:

- Erfahrungen in Ländern mit Versandhandel belegen, dass die überwiegende Mehrzahl der Sendungen Normalsendungen sind, d.h. die Arzneimittel erreichen den Patienten in der Regel am nächsten bzw. übernächsten Werktag nach Rezepteingang. Express-Sendungen, die den Patienten noch am selben Tag erreichen müssen, stellen einen marginalen Anteil von unter $5 \%{ }^{95}$ der Sendungen dar.

Auch an dieser Relation wird die Zielgruppe des Versandes bzw. deren Bedürfnisse offensichtlich: der planbare Medikationsbedarf steht im Vordergrund, nicht

Dies bestätigen verschiedene Expertengespräche.

Angaben liegen INIFES vertraulich vor. 
die umgehend benötigte Akutmedizin. Unter Kostenaspekten sind Express-Sendungen nicht nur teuer, auch die qualitativen Vorteile eines bequemen Heimlieferservice werden durch die hohen Logistikkosten negiert. Die weiteren Betrachtungen bauen auf Normalsendung auf.

- Die Medikamente werden neutral verpackt, eine Zustellung in Mehrwegboxen, die von dem Logistikunternehmen nach Auslieferung bei Endverbraucher wieder mitgenommen werden können, ist auch unter Umweltschutzgründen anzustreben.

- Die Versand- und Verpackungskosten für Normalsendungen (inklusive anteiliger Retourenkosten sowie Samstagzuschlag) können anhand fundierter Quellen ${ }^{96}$ mit 7,66 DM je Paket unter 3 kg kalkuliert werden. Der Kostenträger "Sendung" lässt dabei noch offen, wie viele Verordnungen bzw. Packungen eine Sendung beinhaltet.

- Wird je Sendung ein Rezept zugrunde gelegt, dann entspricht die Konstellation Rezept zu Verordnungen für die letzten Jahre einem konstanten Wert von 1,6 verordneten Packungen je GKV-Rezept ${ }^{97}$ : die Versand- und Verpackungskosten je Verordnung ergeben einen Wert von 4,79 DM.

Eine als wahrscheinlich anzunehmende Fokussierung der meisten potentiellen Anbieter auf einen überregionalen bzw. landesweiten Versand schließt jedoch nicht aus, dass es ebenso Versandapotheken geben könnte, deren Einzugsgebiet regional begrenzt ist: geringere räumliche Distanzen könnten die Realisierung möglichst kurzer Lieferzeiten im Gegenteil sogar positiv beeinflussen. Mit der Begrenzung der Zustellung auf Kunden innerhalb eines gewissen geografischen Gebietes kann eine Eigenerbringung der Logistikleistung durch die Versandapotheke durchaus eine Alternative zum Fremdbezug sein. Trotz Versandhandelsverbot zeigen sich entsprechende Praktiken bereits bei den heutigen Präsenzapotheken in Form der Heimlieferungen nach $\$ 17 \mathrm{ApBetrO}$, die - wie schon unter Punkt 0.1 aufgezeigt - nicht nur im Einzelfall durch eigenes Personal sowie Fahrzeuge der Apotheke erbracht werden. Daraus wäre zu schließen, dass der Aufbau einer eigenen Logistikkompetenz bei regionaler Begrenzung des Versandes durchaus eine kostengünstigere Alternative (z.B. fast kostenneutrale Retourenabwicklung) für umsatzschwächere Marktanbieter sein könnte. Zudem, da umsatzschwächere Versender in Preisverhandlungen mit externen Anbietern nur ein vergleichsweise geringeres Versandvolumen "in die Waagschale werfen“ könnten und demzufolge mit höheren Logistikpreisen je Sendung als umsatzstärkere Versandapotheken rechnen müssten.

Im Sinne einer strategischen Positionierung unter Qualitäts- wie auch Kundenbindungskriterien eröffnet ein eigenes Logistiksystem möglicherweise die Besetzung einer Nischenposition, weshalb die Modellrechnung der kleinen Versandapotheke mit 5 Mio. DM Umsatz (Variante I) von der Alternative eigene Auslieferung ausgeht: 
- Die Arzneimittel erreichen den Patienten eventuell sogar am selben, in aller Regel jedoch am nächsten bzw. übernächsten Werktag nach Rezepteingang. D.h. der Zulieferdienst umfasst sechs Wochentage, um Rezepte, die am Freitag in der Versandapotheke eingehen, am Samstag noch zustellen zu können.

- Die Medikamente werden neutral verpackt in Mehrwegboxen zugestellt, die von dem Logistikunternehmen nach Auslieferung wieder mitgenommen werden können.

- Im Falle der Nichtabnahme von Lieferungen durch den Adressaten können die Retouren durch den Apothekenzustelldienst wieder mitgenommen werden.

- Die Auslieferungskosten ergeben bei Kalkulation mit den Angaben in Abbildung II - 8 je Sendung 5,57 DM bzw. 3,48 DM je Verordnung. Damit ist diese Alternative im Vergleich zum Preis externer Anbieter von 4,79 DM kostengünstiger.

Abbildung II - 8: Kalkulationsschema Logistikkosten Versandapotheke Variante I

\begin{tabular}{|l|r|}
\hline \multicolumn{1}{|c|}{$\quad$ Logistikkosten Versandapothe ke Variante I } \\
\hline Umsatz in Mio. DM & 5.000 .000 \\
\hline durchschnittlicher Sendungswert (aus Versandpotential abgeleitet) in DM & 100 \\
\hline Anzahl der auszuliefernden Sendungen p.a. & 50.000 \\
\hline Auszuliefernde Sendungen pro Tag (ohne Sonn- und Feiertage) & 167 \\
\hline Anzahl Ausliefererfahrzeuge*) & 4 \\
\hline Anzahl Auslieferkräfte Vollzeit mit 1710 Std. p.a. & 6 \\
\hline Stundenlohn mit Gemeinkosten in DM**) & 22 \\
\hline monatliche Leasingkosten je Fahrzeug (bei ca. 2,5\% und 36 Monatsraten) & 875 \\
\hline Auslieferungsstrecke je Stunde in km (im Radius) & 20 \\
\hline Dieselkosten je Liter & 1,65 \\
\hline Logistikkosten je Sendung in DM & 5,57 \\
\hline Logistikkosten je Verordnung in DM & 3,48 \\
\hline
\end{tabular}

*) Bei angenommenen 9 Stunden Auslieferung pro Tag und durchschnittlich 4 bis 5 Auslieferpunkten je Stunde (Annahmen basieren auf Rechengrößen anderer Branchen)

**) Annahme basiert auf Rechengrößen anderer Branchen

Quelle: INIFES, eigene Darstellung. 


\subsubsection{Die Kostenstruktur einer "kleinen" Versandapotheke mit 5 Mio. DM Umsatz (Variante I)}

Wie bereits erwähnt, basiert die Umsatz- und Kostenstruktur einer sogenannten "kleinen" Versandapotheke auf den Strukturen einer Präsenzapotheke. Aufgrund der Annahmen einer veränderten und standardisierten Tätigkeitsstruktur in der Versandapotheke, einer gestiegenen Produktivität des pharmazeutischen Personals, einer veränderten Nutzung der Räumlichkeiten (2.4.2.1.), der Art der Bereitstellung einer zeitlich unbefristeten Telefonberatung (2.4.2.2.) sowie der Bereitstellung der Logistikleistung (2.4.2.3.) können folgende „Fundamente" gelegt werden:

- Die kleine Versandapotheke beschäftigt die identische Anzahl an pharmazeutischem Personal (ohne Leiter) wie die Präsenzapotheke auch (s. 2.4.1.). Hierunter zählt nicht das zusäzzlich einzustellende pharmazeutische Personal für die Telefonhotline. Dieses wird extra erwähnt.

- Es wird aufgrund der erhöhten Produktivität des pharmazeutischen Personals sowie eines höheren durchschnittlichen Verordnungswertes ein Umsatz von 5 Mio. DM brutto p.a. erzielt.

- Mit der Zunahme der Umsatzstärke ist allgemein, d.h. auch analog zu den Einkaufsmodalitäten der Präsenzapotheken, eine Rabattgewährung des pharmazeutischen Großhandels an die Versandapotheken anzunehmen. Dies gilt für alle drei folgenden Versandapothekenmodelle. Beispielhaft soll hier anhand von VA I auch für die Modelle VA II und VA III argumentiert werden.

Von Seiten des Großhandels wie der Hersteller sind bezüglich der Rabattgewährung keine öffentlich zugänglichen Daten verfügbar. ${ }^{98}$ Deshalb fokussieren sich die Annahmen auf vertrauliche Expertengespräche wie auf die wenigen zu dieser Thematik verfügbaren Literaturquellen:

- Allgemein ist von einer regionalen Abhängigkeit der Höhe der Großhandelsrabatte auszugehen. In Regionen mit einer stärkeren Wettbewerbsstruktur des Großhandels werden höhere Rabatte an Apotheken gewährt als Regionen mit einigen wenigen marktdominierenden Großhändlern.

- Laut Expertenmeinung erhalten Präsenzapotheken mit einem unterdurchschnittlichen jährlichen Umsatz von ca. 1,5 Mio. DM einen Großhandelsrabatt von $11 \%$ auf den Apothekeneinkaufspreis plus Skonto. ${ }^{99}$

- Diese Rabattgewährung wird zudem von der Literatur gestützt, wonach durchschnittlich ca. $15 \%$ des Apothekeneinkaufspreises als Rabattgewährung des Großhandels an die Apotheken fließen.

98 
- Eine andere Literaturquelle geht von bis zu zwei Mrd. DM aus, die vom Großhandel wie den Herstellern jährlich den Apotheken gewährt werden: „Da nach der Arzneimittelpreisverordnung apothekenpflichtige Arzneimittel Medikamente überall den gleichen Endverkaufspreis haben, können die Rabatte nicht im Preiswettbewerb an Kassen und Versicherten weitergegeben werden. Im Reingewinn, der 1998 nach ABDA - Angaben für alle öffentlichen Apotheken zusammen nur 0,4 Mrd. DM betrug, können sich die Rabatte aber ebenso wenig niederschlagen. Bleibt somit nur noch die Vermutung, dass damit der "Schlupf" auf der Apothekenstufe finanziert wird ....."

- Zudem erhalten Apotheken mehr und mehr sogenannte Naturalrabatte, insbesondere bei Generika, von den Herstellern. Insider rechnen mit ca. 750 Mio. DM jährlich: „Der Hersteller [...] stellt dem Apotheker eine bestimmte Menge von Packungen in Rechnung, liefert dann aber schließlich mehr. Dieses Mehr ist der Naturalrabatt. [...] Dass der Apotheker die geschenkte Packung zu Lasten der Krankenkassen verkauft, versteht sich von selbst. ${ }^{102}$

- Durch den Wegfall einer Offizin steht deren Anteil an der Geschäftsfläche anderen Nutzungsmöglichkeiten zur Verfügung. In den Modellrechnungen werden deshalb $2 / 3$ der ursprünglichen Offizin (die 40\% der Gesamtfläche einer Präsenzapotheke umfasst) als zusätzliche Lagerfäche angenommen.

- Die Telefonberatung wird während der Öffnungszeiten durch zusätzliches pharmazeutisches Personal selbst erstellt, sonst an externe Call - Center vergeben bzw. im Kooperationsverbund mit anderen Apotheken garantiert.

- Der Vertrieb / Versand der Arzneimittel wird aufgrund der regionalen Begrenzung des Wirkungskreises der Versandapotheke durch eigene Auslieferungsfahrzeuge und -personal sichergestellt.

Ob in kleinen Versandapotheken zusätzlich der Vertriebskanal einer Präsenzapotheke parallel angeboten wird, ist eine individuelle Unternehmensentscheidung. Entsprechend müsste allerdings die Kostenstruktur kalkuliert werden. Im Folgenden wird nur der Vertriebsweg Versand dargestellt. Es ergeben sich dafür folgende Umsatz und Kostenstrukturen:

Cassel, D. (2000), Effiziente Distributionswege, in: Wille, E., Albring, M. (Hrsg.), Rationalisierungsreserven im Gesundheitswesen, Peter Lang Verlag, Frankfurt / Main, S. 308. 


\section{UMSATZ}

- Gemäß der unter 2.4.2.1. begründeten Annahme der doppelten Umsatzproduktivität je approbierter Apotheker (gegenüber Präsenzapotheken) wird bei gleicher Apothekerbelegung ${ }^{103}$ ein Brutto-Umsatz von ca. 5 Mio. DM p.a. erzielt.

\section{WARENEINSATZ UND BETRIEBSHANDELSSPANNE}

- Der Wareneinsatz wie die Betriebshandelsspanne wird analog zur Präsenzapotheke entwickelt: nach IFH - Angaben $58,7 \%$ bzw. $27,5 \%$.

- Aufgrund des gestiegenen Umsatzvolumen ist eine Rabattgewährung durch die vorgelagerten Hersteller- bzw. Handelsstufen wahrscheinlich. Damit würde sich der Wareneinsatz verringern und die Handelsspanne der Apotheken erhöhen. Anhand der oben angeführten Argumente wird in der Modellrechnungen von einer Rabattgewährung des Großhandels an die Versandapotheken ausgegangen, allerdings sehr moderat von $5 \%$ auf den Apothekeneinkaufspreis. ${ }^{104}$ Aufgrund des Grundsatzes bundesweiter einheitlicher Apothekenverkaufspreise können Rabatte nicht an den Kunden weitergegeben werden und erhöhen so ausschließlich die Handelsspanne der Apotheken.

\section{TATSÄCHLICHE KOSTEN}

\section{A) Personalkosten für den laufenden Apothekenbetrieb}

- Das Personal für die Betreuung der Telefonhotline sowie zur Auslieferung an den Kunden ist in dieser Position nicht eingeschlossen. Es wird in den entsprechenden Positionen "Telefonberatungskosten" sowie "Logistikkosten" extra berücksichtigt.

- Die Personalentwicklung der kleinen Versandapotheke folgt aus der Präsenzapotheke heraus, deshalb beträgt auch hier das Verhältnis zwischen approbierten Apothekern und PTA bzw. Apothekerassistenten 1 : 1,7 (bei Teilzeit).

- Aufgrund der Annahme gestiegener Produktivität des pharmazeutischen Personals (entsprechen 2.4.2.1. bis maximal um das Doppelte) bleibt die Anzahl der angestellten Apotheker, der Apothekerassistenten sowie der PTA gegenüber der Versandapotheke konstant. In der Summe ergibt dies analog zur Präsenzapotheke 4,15 pharmazeutische Mitarbeiter ohne Leiter.

103

104

105

Siehe Tabelle II -6 Personalkosten und Arbeitszeit nach Berufsgruppen in der Apotheke.

Diese Prozentzahlen bestätigen Expertengespräche wie Literaturangaben, u.a. Schoffski, $O$. (1995), Die Regulierung des deutschen Apothekenwesens. Eine ökonomische Analyse, BadenBaden, S. 168.

Siehe insbesondere Cassel, D. (2000), Effiziente Distributionswege, in: Wille, E., Albring, M. (Hrsg.), Rationalisierungsreserven im Gesundheitswesen, Peter Lang Verlag, Frankfurt / Main, S. 308. 
- Das nichtpharmazeutische Personal wird insbesondere für Warenwirtschafts-, Lager- und Versandvorbereitungstätigkeiten benötigt. Die Entwicklung der Mitarbeiteranzahl wächst zwar in Abhängigkeit zur Umsatzsteigerung, allerdings wird aufgrund der Verschiedenartigkeit der Tätigkeiten nicht von einer Verdopplung der Produktivität ausgegangen. Es ergeht die Annahme von 2,6 PKA (bei Verdopplung wäre von 3 PKA auszugehen).

- Die Arbeitszeit sowie die Höhe des Gehalts je angestellte Person wurden ohne Änderungen von der Präsenzapotheke übernommen (siehe Punkt 2.4.1., Tabelle II -6).

- Die kleine Versandapotheke beschäftigt für den laufenden Apothekenbetrieb 6,75 Mitarbeiter (ohne Apothekenleiter).

\section{B) Mietkosten}

- Versandapotheken benötigen durch den Wegfall des Kundenverkehrs keine teuren Innenstadträumlichkeiten als Standort. Anstelle dessen kommen adäquate Gewerbe- und Lagerflächen in Randgebieten mit geringeren Mietpreisen in Betracht. Diese können mit ca. 12 DM je qm kalkuliert werden (siehe Punkt 2.4.2.1., Tabelle II - 9).

- Auf die Mietkosten werden analog den Präsenzapotheken $28 \%$ für Sach- bzw. Mietnebenkosten aufgeschlagen.

- Die benötigte gesamte Geschäftsfläche berechnet sich aus dem Lagerwert in Kombination mit der räumlichen Aufteilung:

Bei Optimierung des Lagerwertes einer Versandapotheke mit $8 \%$ vom Nettoumsatz (siehe 2.4.1.) und einem Umschlag von 8,5 mal p.a. ergibt sich unter Geltung der Annahme eines für Präsenz- wie Versandapotheken einheitlichen Lagerwertes von 2,93 TDM ${ }^{108}$ je qm Lager eine notwendige Lagerfläche von $118 \mathrm{qm}$.

Durch die zusätzliche Lagerfläche aufgrund der geänderten räumlichen Nutzungsmöglichkeiten i.V. zur Präsenzapotheke beträgt deren Anteil an der Gesamtfläche ca. $72 \%$.

Aus der absoluten Angabe 118 qm Lagerfläche, kombiniert mit der Prozentangabe $72 \%$ anteilige Lagerfläche ergibt sich eine Geschäftsfläche von 164 qm für kleine Versandapotheken. Durch die veränderten räumlichen Nutzungsmöglichkeiten und die Optimierung des Lagerbestandes ist es der kleinen Versandapotheke also möglich, den doppelten Umsatz mit derselben Geschäftsfläche wie Präsenzapotheken zu erzielen. 


\section{C) Abschreibungen und Zinsen}

- In der Versandapotheke ist keine Offizinausstattung notwendig. Diese Abschreibungen (detaillierte Angaben liegen nicht vor) würden i.V. zur Präsenzapotheke entfallen. Andererseits muss in eine erhöhte Lagerausstattung sowie die Ausstattung von Telefonberatungsplätzen (siehe unter Telefonberatungskosten) investiert werden, die sich in jährlichen Abschreibungsbeträgen niederschlägt. $\mathrm{Ob}$ diese erstere kompensieren, ist „generell“ nicht zu beantworten.

- Es wird deshalb eine Abschreibungsrate in \% vom Umsatz in derselben Höhe wie in Präsenzapotheken angenommen.

- Fremdkapitalzinsen auf den Lagerbestand können aufgrund der Lageroptimierung in der Versandapotheke bzgl. der Präsenzapotheke verringert werden, allerdings erfolgt hier keine Quantifizierung. Es wird deshalb eine Fremdkapitalzinsbelastung in \% vom Umsatz in derselben Höhe wie in Präsenzapotheken angenommen.

\section{D) Laufender Geschäftsbetrieb: Werbung, KFZ, übrige Kosten}

- Werbung darf von den (Versand)Apotheken nur für nicht apothekenpflichtige Arzneimittel der Selbstmedikation vorgenommen werden. Die Versandapotheke agiert annahmegemäß hauptsächlich im Segment der verordneten Arzneimittel (Zielgruppe: Chroniker, Dauermedikation). Öffentlichkeitswerbung ist in diesem Segment nicht gestattet (identisch mit Präsenzapotheke). Es werden deshalb relative Werbungskosten in \% vom Umsatz in derselben Höhe wie in Präsenzapotheken angenommen.

- Die KFZ-Kosten (hierunter fallen nicht die Kosten der Auslieferfahrzeuge, sondern z.B. das KFZ des Apothekenleiters) entwickeln sich durch die Umsatzverdopplung stark degressiv.

- Für die "übrigen Kosten" wird angenommen, dass sich diese im Verhältnis zum Umsatz unterproportional entwickeln.

\section{E) Neu: Telefonberatungskosten}

- Die Telefonberatung wird während der üblichen Geschäftszeiten der Apotheke (siehe Tabelle II - 10) durch eigenes - ausschließlich pharmazeutisches - Personal sichergestellt. Es kann mit ca. 15 Anrufen à 5 Minuten je Stunde gerechnet werden (dies entspricht 1,25 Gesprächsstunden je Stunde bzw. daraus resultiert die Notwendigkeit von 1,3 gleichzeitig arbeitenden Telefonkräften je Arbeitsstunde).

Wie unter 2.4.1. für Präsenzapotheken ausgewiesen, kann auch hier von 1.710 Arbeitsstunden p.a. je Vollzeitstelle ausgegangen werden: entsprechend sind 1,8 Personen pharmazeutisches Personal bei Vollzeit bzw. 2,5 bei Teilzeit auf die Sicht eines ganzen Jahres zu kalkulieren. 
- Die Arbeitszeit sowie die Höhe der Gehälter für das Telefonberatungspersonal wird ohne Änderungen von der Präsenzapotheke übernommen (siehe Tabelle II - 6). Damit werden hier bewusst höhere Personalkosten kalkuliert als für Call-Center-Personal allgemein üblich ${ }^{107}$, um den qualitativen Standard zu garantieren.

- Wie unter 2.4.2.2. bereits angeführt, wird die technische Ausstattung mit 5.000 DM pro Telefonarbeitsplatz ${ }^{108}$ durch eine lineare Abschreibungsrate über 4 Jahre berücksichtigt. Hinzu kommt die ebenfalls über 4 Jahre linear kalkulierte Abschreibungsrate für eine sogenannten Call-Distributionsanlage von 30.000 DM. $^{109}$

- Außerhalb der üblichen Geschäftszeiten ist kaum mit Anrufen zu rechnen (siehe Tabelle II - 10). Aufgrund der hohen Fixkosten bei Eigenerstellung wird die Telefonbereitschaft während dieser Zeit an ein qualifiziertes externes Call - Center ausgelagert, welches pro Anruf 6,50 DM der Versandapotheke berechnet. Dieses Call - Center könnte bzw. es wäre unter Qualitätsaspekten sogar sinnvoll, wie unter 2.4.2.2. erwähnt, auch als Kooperationsverbund mehrerer (Versand) Apotheken betrieben werden.

\section{F) Neu: Logistikstückkosten}

- Die Logistik der kleinen Versandapotheke kann aufgrund der Annahme einer regional begrenzten Reichweite des Versandes durch diese selbst erstellt werden.

- In dieser Position werden die für eine eigene Auslieferung benötigten Personalwie KFZ - Kapazitäten entsprechend Abbildung II - 8 berücksichtigt: 6 Mitarbeiter in Vollzeit (pharmazeutisches Personal ist dafür nicht erforderlich, die derzeitige Heimliefer- und Botenlieferung der Präsenzapotheken bestätigt dies) bzw. 8,2 in Teilzeit (entsprechend Tabelle II - 6: 72\%) für nicht pharmazeutisches Personal sind dafür zusätzlich nötig.

- Die Logistikstückkosten betragen ca. 3,48 DM je Verordnung bzw. 5,57 DM je Sendung $^{110}$ (eine Sendung entspricht einem Rezept mit 1,6 Verordnungen).

107

Bruttomonatsgehălter liegen je nach Qualifikation bei 2.000 DM bis 4.650 DM für Vollzeitkräfte bzw. zwischen 14 und 22,50 DM für Stundenkräfte. Siehe Eisinger, H. (1998), So funktioniert der Versandhandel, Wien, S. 163.

Orientiert sich an der Anzahl des parallel arbeitenden Telefonpersonals, also zwei.

Eisinger, H. (1998), So funktioniert der Versandhandel, Wien, S. 163.

110

Hier ist ein durchschnittlicher Wert von $100 \mathrm{DM}$ je Sendung (abgeleitet aus den verschiedenen Varianten des Zielsegments) zugrunde gelegt. Die gesamten Logistikkosten in \% vom Umsatz würden entsprechend ca. $5,6 \%$ betragen. Würde der durchschnittliche Sendungswert auf 120 DM geschätzt, entsprächen die Kosten je Sendung ca. 5,75 DM und damit die gesamten Logistikkosten bei 41.667 Sendungen ca. $4,8 \%$ vom Umsatz. Dies zeigt die relativ große Schwankungsbreite der Logistikkosten, je nachdem, welcher durchschnittliche Versendungswert zugrunde gelegt wird. 


\section{GEWERBESTEUER}

- Die Gewerbesteuer ist vom Hebesatz der Gemeinden abhängig. Je nach Wahl des Standortes in Randlagen oder im ländlichen Raum fällt der Hebesatz geringer aus und wirkt damit wieder gewerbesteuersenkend.

- Das Berechnungsschema folgt analog dem der Präsenzapotheke: Betriebshandelsspanne verringert um die tatsächlichen Kosten. Hier sind bei der Berechnung also zusätzlich die Telefonberatungs- und Logistikkosten zu berücksichtigen.

\section{KALKULATORISCHE KOSTEN}

- Der kalkulatorische Unternehmerlohn des selbständigen Apothekenleiters wird in absoluter Höhe übernommen.

- Ebenso wird die prozentuale Rate der Eigenkapitalzinsen von der Präsenzapotheke übernommen.

- Das betriebswirtschaftliche Ergebnis der umsatzstärkeren (Versand)Apotheke wird durch die kalkulatorischen Kosten geringer belastet als das der Präsenzapotheke.

\section{BETRIEBSWIRTSCHAFTLICHES ERGEBNIS}

- Das betriebswirtschaftliche Ergebnis fällt trotz neu entstandener Kosten für Telefonberatung und Logistik bei der kleinen Versandapotheke gegenüber der Präsenzapotheke höher aus. Dies resultiert insbesondere aus den relativ geringeren Kosten für Personal, Miete und kalkulatorische Aufwendungen sowie besseren Einkaufskonditionen (Rabattgewährung).

Es erfolgt in einem Zwischenschritt die Darstellung der Kostenstruktur ohne Logistikkosten, in einem weiteren Schritt werden diese mit einbezogen. Ursächlich ist dafür deren Kostenstruktur als Stückkosten je Verordnung.

Schöffski, O. (1995), Die Regulierung des deutschen Apothekenwesens. Eine ökonomische Analyse, Baden-Baden, S.190. 
Tabelle II - 12: Umsatz- und Kostenstruktur einer kleinen Versandapotheke vor Berücksichtigung der Logistikstückkosten von 3,48 DM je Verordnung

\begin{tabular}{|c|c|c|}
\hline VORLÄUFIGE UMSATZ- UND KOSTENSTRUKTUR & $\begin{array}{l}\text { in \% des } \\
\text { Umsatz }\end{array}$ & $\begin{array}{c}\text { in \% der } \\
\text { Handelsspanne }\end{array}$ \\
\hline Umsatz brutto in TDM & $100,0 \%$ & \\
\hline Mehrwertsteuer $16 \%$ & $13,8 \%$ & \\
\hline Wareneinsatz & $55,7 \%$ & \\
\hline BETRIEBSHANDELSSPANNE ${ }^{112}$ & $30,5 \%$ & $100,0 \%$ \\
\hline TATSÄCHLICHE KOSTEN OHNE LOGISTIKKOSTEN*) & $14,1 \%$ & $46,3 \%$ \\
\hline Personalaufwand & $6,1 \%$ & $20,0 \%$ \\
\hline Mietaufwand & $0,6 \%$ & $2,0 \%$ \\
\hline Abschreibungen, Zinsen & $2,0 \%$ & $6,6 \%$ \\
\hline Werbung, KfZ, übrige Kosten & $3,0 \%$ & $9,8 \%$ \\
\hline Telefonberatungskosten & $2,4 \%$ & $7,9 \%$ \\
\hline Logistikstückkosten 3,48 DM je Verordnung & s. Tabelle II - 13 & \\
\hline GEWERBESTEUER OHNE LOGISTIKKOSTEN*) & $2,6 \%$ & $8,5 \%$ \\
\hline KALKULATORISCHE KOSTEN & $3,4 \%$ & $11,1 \%$ \\
\hline Kalkulatorischer Unternehmerlohn & $3,0 \%$ & $9,8 \%$ \\
\hline Eigenkapitalzinsen & $0,4 \%$ & $1,3 \%$ \\
\hline $\begin{array}{l}\text { BETRIEBSWIRTSCHAFTLICHES ERGEBNIS OHNE } \\
\text { LOGISTIKKOSTEN*) }\end{array}$ & $10,4 \%$ & $34,1 \%$ \\
\hline
\end{tabular}

*) Vorläufige Ergebnisse, mit der folgenden Berechnung der Logistikkosten in Tabelle II - 13 ändern sich diese entsprechend, siehe auch Tabelle II - 14.

Quelle: INIFES, eigene Berechnungen.

Aufgrund der Natur der Logistikkosten als Stückkosten je Verordnung (3,48 DM je Verordnung, unabhängig von der Höhe des Verordnungswertes) können die Logistikkosten der Versandapotheke als prozentuale Ausprägung im Verhältnis zum Umsatz nur auf der Ebene des durchschnittlichen Verordnungswertes bestimmt werden. (siehe Tabelle II - 13), nicht jedoch als Gesamtgröße bezüglich des Umsatzes.

Durch die Festlegung eines Zielsegments (siehe 2.3.2, Variante A bis C) sind entsprechende durchschnittliche Verordnungswerte je Variante für einen Pharmaversand bekannt.

Diese durchschnittlichen Verordnungswerte müssen jedoch in einem weiteren Schritt aufgrund der Berücksichtigung von Logistikstückkosten differenziert werden. Das Inklusive der angenommenen Rabattgewährung des Großhandels an die (Versand)Apotheke. 
heißt, einige der sich im Zielsegment unter epidemiologischen Gesichtspunkten befindlichen Indikationen können aufgrund eines zu niedrigen Verordnungswertes die zusätzlich anfallenden Logistikkosten von 3,48 DM je Verordnung nicht decken. Dies hat zur Konsequenz, dass sie der Umfang des Zielsegments - gemessen in Umsatzzahlen wie in Verordnungszahlen - für jede Zielvariante um diese Indikationsgruppen entsprechend verringert (daraus resultiert entsprechend der Definition in 2.2.1. das Marktpotential). Aus dem Zielsegment können die Indikationsgruppen die Logistikkosten aus ihren durchschnittlichen Verordnungswerten decken, die über einem Minimalverordnungswert von ca. 33 DM liegen. Dieser ergibt sich, indem die Logistikstückkosten von 3,48 ins Verhältnis gesetzt werden zum betriebswirtschaftlichen Ergebnis vor Logistikkosten von 10,4\% (siehe Tabelle II - 12).

Die dieser Restriktion Minimalverordnungswert entsprechenden Indikationen bilden im Vergleich zum ursprünglichen Zielsegment jeweils leicht erhöhte durchschnittliche Verordnungswerte: 75,10 DM für Marktpotentialvariante $A^{114}, 72,57$ DM für Marktpotentialvariante $B^{115}$ und 72,56 DM für Marktpotentialvariante $C^{116}$.

Verringert man diese drei durchschnittlichen Verordnungswerte in einem weiteren Schritt jeweils um den 5\%igen Kassenrabatt (entsprechend A: 71,35 DM, B: 68,94 DM, C: 68,94 DM) und setzt die Logistikstückkosten von 3,48 DM ins Verhältnis zu diesen Werten, ergibt sich der prozentuale Anteil der Logistikkosten am Verordnungswert.

Tabelle II - 13: Berechnung der prozentualen Logistikkosten je durchschnittlichen Verordnungswert in Abh. von der Marktpotentialvariante

\begin{tabular}{|l|c|c|c|}
\hline & $\begin{array}{c}\text { Marktpotential- } \\
\text { variante A }\end{array}$ & $\begin{array}{c}\text { Marktpotential- } \\
\text { variante B }\end{array}$ & $\begin{array}{c}\text { Marktpotential- } \\
\text { variante C }\end{array}$ \\
\hline Durchschnittlicher Verordnungswert & $75,10 \mathrm{DM}$ & $72,57 \mathrm{DM}$ & $72,56 \mathrm{DM}$ \\
\hline Minus 5\% GKV-Rabatt ergibt: & $71,35 \mathrm{DM}$ & $68,94 \mathrm{DM}$ & $68,94 \mathrm{DM}$ \\
\hline Logistikkosten & $3,48 \mathrm{DM}$ & $3,48 \mathrm{DM}$ & $3,48 \mathrm{DM}$ \\
\hline $\begin{array}{l}\text { Logistikkosten in \% vom Verordnungswert } \\
\text { (abzgl. 5\% GKV-Rabatt) }\end{array}$ & $\mathbf{4 , 9 \%}$ & $\mathbf{5 , 0} \%$ & $\mathbf{5 , 0} \%$ \\
\hline Korrektur Gewerbesteuer $^{117}$ auf Logistikkosten & $0,8 \%$ & $0,8 \%$ & $0,8 \%$ \\
\hline
\end{tabular}

Quelle: INIFES, eigene Berechnungen.

\footnotetext{
114 Siehe Nebenrechnung im Anhang I, Tabelle I - 1.

115 Siehe Nebenrechnung im Anhang I, Tabelle I - 2.

116 Siehe Nebenrechnung im Anhang I, Tabelle I - 3.

117 Die Logistikkosten sind tatsächliche Kosten, welche die Gewerbesteuerzahlungen vermindern.

Deshalb muss eine Korrektur der Gewerbesteuer erfolgen.
} 
Die prozentualen Logistikkosten wie die Korrektur der Gewerbsteuer können ohne Einschränkungen auf die Ebene des Apothekenumsatzes übertragen werden. Es ergibt sich nun entgültig das betriebswirtschaftliche Ergebnis der kleiner Versandapotheke VA I nach Berücksichtigung der Logistikkosten in Abhängigkeit von der Marktpotentialvariante (Tabelle II - 14)

Tabelle II - 14: Betriebsergebnis kleine Versandapotheke in Abhängigkeit der Marktpotentialvariante

\begin{tabular}{|c|c|c|c|}
\hline (FORTFÜHRUNG Tabelle II - 12) & $\begin{array}{c}\text { Marktpotential- } \\
\text { variante A }\end{array}$ & $\begin{array}{c}\text { Marktpotential- } \\
\text { variante B }\end{array}$ & $\begin{array}{c}\text { Marktpotential- } \\
\text { variante C }\end{array}$ \\
\hline $\begin{array}{l}\text { Betriebshandelsspanne } \\
\text { (siehe Tabelle II - 12) }\end{array}$ & \multicolumn{3}{|c|}{$30,5 \%$} \\
\hline $\begin{array}{l}\text { Tatsächliche Kosten ohne Logistikkosten } \\
\text { (siehe Tabelle II - 12) }\end{array}$ & \multicolumn{3}{|c|}{$14,1 \%$} \\
\hline $\begin{array}{l}\text { Logistikkosten } \\
\text { (siehe Tabelle II - 13) }\end{array}$ & $4,9 \%$ & $5,0 \%$ & $5,0 \%$ \\
\hline Gewerbesteuer $^{118}$ & $1,8 \%$ & $1,8 \%$ & $1,8 \%$ \\
\hline $\begin{array}{l}\text { Kalkulatorische Kosten } \\
\text { (siehe Tabelle II - 12) }\end{array}$ & \multicolumn{3}{|c|}{$3,4 \%$} \\
\hline Betriebswirtschaftliches Ergebnis & $6,3 \%$ & $6,2 \%$ & $6,2 \%$ \\
\hline
\end{tabular}

Quelle: INIFES, eigene Darstellung.

Nach Berücksichtung der Logistikkosten und der korrigierten Gewerbesteuer ergibt sich ein Betriebsergebnis von 6,2\% bzw. 6,3\%. Dieses stellt den Betrag dar, der von der Apothekenbetriebsspanne nach Abzug der gesamten Kosten der Versandapotheke VA I als positives Ergebnis verbleibt.

\subsubsection{Die Kostenstruktur einer "großen" Versandapotheke mit 20 Mio. DM Umsatz (Variante II)}

Basierend auf den Darstellungen zur Betriebsstruktur von Versandapotheken (Punkte 2.4.2.1. bis 2.4.2.3.) sowie auf den Entwicklungsschritten einer kleinen Versandapotheke kann die Kostenstruktur einer im Vergleich dazu umsatzstärkeren Versandapotheke simuliert werden:

- Der Brutto-Umsatz der "großen“ Versandapotheke beträgt ca. 20 Mio. DM im Jahr. ${ }^{119}$ Dazu sei angemerkt, dass schon heute einige wenige Präsenzapotheken mit Umsatzwerten um ca. 20 Mio. DM existieren. ${ }^{120}$

\footnotetext{
118 Korrigierter Wert: Wert ( siehe Tabelle II - 12) minus Wert (siehe Tabelle II - 13).

119 Dieser Wert orientiert sich an Expertengesprächen mit europäischen Versandapothekenbetreibern, die Umsätze im zweistelligen Millionenbereich (in DM gemessen) erzielen.

120

Statistisches Bundesamt, zitiert in: Hackenbroch, V. (2000), Schwindel auf Rezept, S. 164, in: DER SPIEGEL 33/2000, S. 164-168.
} 
- Die Annahme einer erhöhten Pro-Kopf-Produktivität des pharmazeutischen Personals (2.4.2.1.) gilt in 2.4.4. mindestens im selben Maße wie unter 2.4.3.

- Für die Rabattgewährung des pharmazeutischen Großhandels an die Versandapotheke VA II gelten die selben Argumente wie für VA I unter 2.4.3.

- Die Verschiebung der räumlichen Nutzungsmöglichkeiten zu mehr Lagerkapazitäten durch den Wegfall der Offizin (2.4.2.1.) gilt in 2.4.4. mindestens im selben Maße wie unter 2.4.3.

- Die Telefonberatung wird während der Öffnungszeiten durch zusätzliches pharmazeutisches Personal selbst erstellt, sonst an externe Call-Center vergeben bzw. im Kooperationsverbund mit anderen Apotheken garantiert.

- Der Vertrieb / Versand der Arzneimittel wird aufgrund des überregionalen bzw. bundesweiten Wirkungskreises der Versandapotheke (siehe Argumente unter 2.4.2.3.) durch externe seriöse Logistikunternehmen sichergestellt.

Es ergeben sich folgende Umsatz - und Kostenstrukturen:

\section{UMSATZ}

- Die Berechnungen gehen von einem Umsatz von 20 Mio. DM brutto aus.

\section{WARENEINSATZ UND BETRIEBSHANDELSSPANNE}

- Analog 2.4.3. Versandapotheke Variante VAI.

\section{TATSÄCHLICHE KOSTEN}

\section{A) Personalkosten für den laufenden Apothekenbetrieb}

- Das Personal für die Betreuung der Telefonhotline ist in dieser Position nicht eingeschlossen. Es wird in der entsprechenden Position „Telefonberatungskosten" extra berücksichtigt.

- Die Anzahl des mittleren pharmazeutischen Personals (PTA und Apothekenassistenten) entwickelt sich entsprechend der Umsatzvervierfachung gegenüber Versandapotheke VA I: dort waren analog zur Präsenzapotheke $2,63^{121}$ entsprechende Personen beschäftigt, für die "große“ Versandapotheke ergibt dies analog 10,6 PTA.

- Das Verhältnis zwischen approbierten Apothekern und PTA bzw. Apothekerassistenten liegt bei VA I bei $1: 1,7$ (bei Teilzeit). Entsprechend errechnet sich für die Anzahl der angestellten approbierten Apotheker eine Beschäftigtenzahl von ca. 6,1 Personen.

121

In VA I entspricht die Personalstruktur und -anzahl bei pharmazeutischem Personal den Strukturen der Präsenzapotheke (siehe Tabelle II -6). 
- Die Entwicklung der Anzahl des nicht-pharmazeutischen Personals (PKA, Helferinnen) erfolgt wie in der kleinen Versandapotheke unterproportional zur Umsatzentwicklung: dort erfolgte bei einer Umsatzverdopplung von 2,5 auf 5 Mio. DM Umsatz gegenüber den Präsenzapotheken eine Erhöhung der PKA / Helfer um das 1,7fache von 1,54 auf 2,6. Bei entsprechender Kontinuität der Annahme (d.h. dreimaliger Verdopplung des Umsatzes von VA II gegenüber der Präsenzapotheke) ergibt sich bei 20 Mio. DM Umsatz eine Anzahl von 7,6 PKA / Helferinnen. ${ }^{122}$

- Die Arbeitszeit sowie die Gehälter je Beschäftigten werden ohne Änderungen von der Präsenzapotheke übernommen. (siehe Punkt 2.4.1., Tabelle II - 6).

- Die große Versandapotheke beschäftigt für den laufenden Apothekenbetrieb 24,2 Mitarbeiter (ohne Apothekenleiter). Das zusätzlich notwendige Telefonberatungspersonal ist hier noch nicht berücksichtigt, (s. Punkt "Telefonberatungskosten").

\section{B) Mietkosten}

- Im Durchschnitt werden für 1998 in den Berechnungen - wie bereits bei den Modellrechnungen zur "kleinen" Versandapotheke - ca. 12 DM je qm an Mietkosten bzw. Mietwert angesetzt.

- Den Mietkosten werden analog zu 2.4.1. und 2.4.3. 28\% für Sach- bzw. Mietnebenkosten zugeschlagen.

- Die benötigte gesamte Geschäftsfläche berechnet sich analog zur "kleinen“ Versandapotheke aus dem Lagerwert in Kombination mit der räumlichen Aufteilung:

Bei Optimierung des Lagerwertes einer Versandapotheke mit $8 \%$ vom Nettoumsatz (siehe 2.4.2.1.) und einem Umschlag von 8,5 mal p.a. ergibt sich unter Geltung der Annahme eines für Präsenz- wie Versandapotheken einheitlichen Lagerwertes von 2,93 $\operatorname{TDM}^{123}$ je qm Lager eine notwendige Lagerfläche von $470 \mathrm{qm}$.

Die Lagerfläche wird analog zu kleinen Versandapotheken mit $72 \%$ der Gesamtfläche kalkuliert, wobei hier sicherlich auch ein höherer Anteil denkbar wäre.

Aus der absoluten Angabe $470 \mathrm{qm}$ Lagerfläche, kombiniert mit der Prozentangabe $72 \%$ anteilige Lagerfläche ergibt sich eine Geschäftsfläche von 652 qm für große Versandapotheken.

\footnotetext{
122 Entspricht der Berechnung 1,54 multipliziert mit 1,7

123 Siehe unter 2.4.2.1.
} 


\section{C) Abschreibungen und Zinsen}

- Die Entwicklung erfolgt analog der Argumentation der kleinen Versandapotheke und beläuft sich für beide Positionen auf die Höhe der entsprechenden Umsatzanteile der Präsenzapotheke.

\section{D) Laufender Geschäftsbetrieb: Werbung, KFZ, übrige Kosten}

- Analog zur Argumentation der kleinen Versandapotheke muss auch hier von relativen Werbungskosten in \% vom Umsatz in derselben Höhe wie in Präsenzapotheken ausgegangen werden.

- Die KFZ-Kosten entwickeln sich durch die Umsatzvervierfachung gegen null.

- Die "übrigen Kosten" werden in ihrem prozentualen Wert analog zur Versandapotheke VA I angenommen.

\section{E) Neu: Telefonberatungskosten}

- Die Telefonberatung wird während der üblichen Geschäftszeiten der Apotheke (siehe Tabelle II - 10) durch eigenes pharmazeutisches Personal sichergestellt. Es kann mit ca. 60 Anrufen à 5 Minuten je Stunde gerechnet werden (dies entspricht fünf Gesprächsstunden je Stunde bzw. daraus resultiert die Notwendigkeit von fünf gleichzeitig arbeitenden Telefonkräften je Arbeitsstunde innerhalb der Öffnungszeiten).

Wie unter 2.4.1. für Präsenzapotheken ausgewiesen, kann auch hier von 1.710 Arbeitsstunden p.a. je Vollzeitstelle ausgegangen werden: entsprechend sind 7,2 Personen pharmazeutisches Personal bei Vollzeit bzw. 9,6 bei Teilzeit auf die Sicht eines Jahres zu kalkulieren.

- Die Arbeitszeit sowie die Gehälter für das Telefonberatungspersonal wird ohne Änderungen von der Präsenzapotheke übernommen (siehe Tabelle II - 6).

- Die Kosten für die technische Ausstattung werden analog zur kleinen Versandapotheke entwickelt.

- Außerhalb der üblichen Geschäftszeiten ist auch bei Modell VA II kaum mit Anrufen zu rechnen (siehe Tabelle II - 10). Aufgrund der hohen Fixkosten bei Eigenerstellung wird die Telefonbereitschaft während dieser Zeit an ein qualifiziertes externes Call - Center ausgelagert, welches pro Anruf 6,50 DM der Versandapotheke berechnet. Dieses Call - Center könnte bzw. es wäre unter Qualitätsaspekten sogar sinnvoll, wie unter 2.4.2.2. erwähnt, auch als Kooperationsverbund mehrerer (Versand)Apotheken betrieben werden. 


\section{F) Neu: Logistikstückkosten}

- Die Reichweite des Versandes umsatzstärkerer Versandapotheken wie VA II ist als überregional, mindestens bundesweit einzuschätzen (siehe dazu die Annahmen unter 2.4.2.3.)

- Die Logistik wird deshalb an externe Logistikdienstleister vergeben. Die Logistikkosten je Verordnung werden mit 4,79 DM analog der Herleitung unter 2.4.2.3. kalkuliert.

\section{GEWERBESTEUER}

- Die Gewerbesteuerberechnung erfolgt analog der kleinen Versandapotheke.

\section{KALKULATORISCHE KOSTEN}

- Der kalkulatorische Unternehmerlohn des selbständigen Apothekenleiters wird in absoluter Höhe von der Präsenzapotheke übernommen ${ }^{124}$.

- Ebenso wird die prozentuale Rate der Eigenkapitalzinsen von der Präsenzapotheke übernommen.

- In hohen Umsatzklassen entwickeln sich die kalkulatorischen Kosten gegen den Grenzwert Null.

\section{BETRIEBSWIRTSCHAFTLICHES ERGEBNIS}

- Das betriebswirtschaftliche Ergebnis fält bei großen Versandapotheken trotz höherer Logistikkosten (aufgrund bundesweiten Versands und Outsourcing an externe Logistiker) aufgrund der Kostendegression insbesondere bei den kalkulatorischen und den Personalkosten höher aus als bei kleinen Versandapotheken.

Es erfolgt in einem Zwischenschritt die Darstellung der Kostenstruktur ohne Logistikkosten, in einem weiteren Schritt werden diese mit einbezogen. Ursächlich ist dafür deren Kostenstruktur als Stückkosten je Verordnung.

124 Entsprechend der Umsatzstärke könnten hier auch Kapitalgesellschaften für das Modell VA II denkbar sein. Der kalkulatorische Unternehmerlohn würde dann entsprechend unter die Personalkosten fallen. Allerdings sind über die mögliche Gesellschaftsform solcher Apotheken nur Spekulationen möglich. Deshalb wird das bisher angewendete Schema fortgeführt (Anmerkung: Es wurde bereits angesprochen, dass bereits heute einige wenige Präsenzapotheken solcher Umsatzklassen existieren. Aufgrund rechtlicher Vorschriften dürfen diese nicht als Kapitalgesellschaften geführt werden). 
Tabelle II - 15: Umsatz- und Kostenstruktur einer großen Versandapotheke vor Berücksichtigung der Logistikstückkosten von 4,79 DM je Verordnung

\begin{tabular}{|c|c|c|}
\hline VORLÄUFIGE UMSATZ- UND KOSTENSTRUKTUR & $\begin{array}{l}\text { in \% des } \\
\text { Umsatz }\end{array}$ & $\begin{array}{c}\text { in } \% \text { der } \\
\text { Handelsspanne }\end{array}$ \\
\hline Umsatz brutto in TDM & $100,0 \%$ & \\
\hline Mehrwertsteuer $16 \%$ & $13,8 \%$ & \\
\hline Wareneinsatz & $55,7 \%$ & \\
\hline BETRIEBSHANDELSSPANNE ${ }^{125}$ & $30,5 \%$ & $100,0 \%$ \\
\hline TATSÄCHLICHE KOSTEN OHNE LOGISTIKKOSTEN*) & $13,2 \%$ & $43,3 \%$ \\
\hline Personalaufwand & $5,7 \%$ & $18,7 \%$ \\
\hline Mietaufwand & $0,6 \%$ & $2,0 \%$ \\
\hline Abschreibungen, Zinsen & $2,0 \%$ & $6,6 \%$ \\
\hline Werbung, KfZ, übrige Kosten & $2,6 \%$ & $8,5 \%$ \\
\hline Telefonberatungskosten & $2,3 \%$ & $7,5 \%$ \\
\hline Logistikstückkosten 4,79 DM je Verordnung & s.Tabelle II - 16 & \\
\hline GEWERBESTEUER OHNE LOGISTIKKOSTEN*) & $2,7 \%$ & $8,9 \%$ \\
\hline KALKULATORISCHE KOSTEN & $1,2 \%$ & $3,9 \%$ \\
\hline Kalkulatorischer Unternehmerlohn & $0,8 \%$ & $2,6 \%$ \\
\hline Eigenkapitalzinsen & $0,4 \%$ & $1,3 \%$ \\
\hline $\begin{array}{l}\text { BETRIEBSWIRTSCHAFTLICHES ERGEBNIS OHNE } \\
\text { LOGISTIKKOSTEN*) }\end{array}$ & $13,4 \%$ & $43,9 \%$ \\
\hline
\end{tabular}

*) Vorläufige Ergebnisse, mit der folgenden Berechnung der Logistikkosten in Tabelle II - 16 ändern sich diese entsprechend, siehe auch Tabelle II - 17.

Quelle: INIFES, eigene Berechnungen.

Aufgrund der Natur der Logistikkosten als Stückkosten je Verordnung (4,79 DM je Verordnung, unabhängig von der Höhe des Verordnungswertes) können die Logistikkosten der Versandapotheke als prozentuale Ausprägung im Verhältnis zum Umsatz nur auf der Ebene des durchschnittlichen Verordnungswertes bestimmt werden. (siehe Tabelle II - 16).

Aufgrund der Festlegung eines Zielsegments (siehe 2.3.2, Variante $A$ bis $C$ ) sind entsprechende durchschnittliche Verordnungswerte je Variante für einen Pharmaversand bekannt. $^{126}$ 
Diese durchschnittlichen Verordnungswerte müssen jedoch in einem weiteren Schritt aufgrund der Berücksichtigung von Logistikstückkosten differenziert werden. Das heißt, einige der sich im Zielsegment unter epidemiologischen Gesichtspunkten befindlichen Indikationen können aufgrund eines zu niedrigen Verordnungswertes die zusätzlich anfallenden Logistikkosten von 4,79 DM je Verordnung nicht decken. Dies hat zur Konsequenz, dass sie der Umfang des Zielsegments - gemessen in Umsatzzahlen wie in Verordnungszahlen - für jede Zielvariante um diese Indikationsgruppen entsprechend verringert (daraus resultiert entsprechend der Definition in 2.2.1. das Marktpotential). Aus dem Zielsegment können die Indikationsgruppen die Logistikkosten aus ihren durchschnittlichen Verordnungswerten decken, die über einem Minimalverordnungswert von ca. 35 DM liegen. Dieser ergibt sich, indem die Logistikstückkosten von 4,79 ins Verhältnis gesetzt werden zum betriebswirtschaftlichen Ergebnis vor Logistikkosten von ca. 13,4\% (siehe Tabelle II - 15).

Die dieser Restriktion Minimalwert entsprechenden Indikationen bilden im Vergleich zum ursprünglichen Zielsegment jeweils leicht erhöhte durchschnittliche Verordnungswerte: 75,10 DM für Marktpotentialvariante $A^{127}, 73,87$ DM für Marktpotentialvariante $B^{128}$ und 73,61 DM für Marktpotentialvariante $C^{129}$.

Verringert man diese drei durchschnittlichen Verordnungswerte in einem weiteren Schritt jeweils um den 5\%igen Kassenrabatt (entsprechend für A: 71,35 DM, B: 70,18 DM, C: 69,93 DM) und setzt die Logistikstückkosten von 4,79 DM jeweils ins Verhältnis zu diesen Werten, ergibt sich der prozentuale Anteil der Logistikkosten am Verordnungswert.

Tabelle II - 16: Berechnung der prozentualen Logistikkosten je durchschnittlichen Verordnungswert in Abh. von der Marktpotentialvariante für die große Versandapotheke

\begin{tabular}{|l|c|c|c|}
\hline & $\begin{array}{c}\text { Marktpotential- } \\
\text { variante A }\end{array}$ & $\begin{array}{c}\text { Marktpotential- } \\
\text { variante B }\end{array}$ & $\begin{array}{c}\text { Marktpotential- } \\
\text { variante C }\end{array}$ \\
\hline Durchschnittlicher Verordnungswert & $75,10 \mathrm{DM}$ & $73,87 \mathrm{DM}$ & $73,61 \mathrm{DM}$ \\
\hline Minus 5\% GKV-Rabatt & $71,35 \mathrm{DM}$ & $70,18 \mathrm{DM}$ & $69,93 \mathrm{DM}$ \\
\hline Logistikkosten & $4,79 \mathrm{DM}$ & $4,79 \mathrm{DM}$ & $4,79 \mathrm{DM}$ \\
\hline $\begin{array}{l}\text { Logistikkosten in \% vom Verordnungswert } \\
\text { (abzgl. 5\% GKV-Rabatt) }\end{array}$ & $\mathbf{6 , 7} \%$ & $\mathbf{6 , 8} \%$ & $\mathbf{6 , 9 \%}$ \\
\hline $\begin{array}{l}\text { Korrektur Gewerbesteuer } \\
\text { Logistikkosten }\end{array}$ & $1,1 \%$ & $1,1 \%$ & $1,1 \%$ \\
\hline
\end{tabular}

Quelle: INIFES, eigene Berechnungen.

127

Siehe Nebenrechnung im Anhang I, Tabelle I - 1.

Siehe Nebenrechnung im Anhang I, Tabelle I- 2.

Siehe Nebenrechnung im Anhang I, Tabelle I-3.

Die Logistikkosten sind tatsächliche Kosten, welche die Gewerbesteuerzahlungen vermindern. Deshalb muss eine Korrektur der ausgewiesenen Gewerbesteuer erfolgen. 
Die prozentualen Logistikkosten sowie die Korrektur der Gewerbsteuer können ohne Einschränkungen auf die Ebene des Apothekenumsatzes übertragen werden. Es ergibt sich nun entgültig das betriebswirtschaftliche Ergebnis der großen Versandapotheke VA II nach Berücksichtigung der Logistikkosten in Abhängigkeit von der Marktpotentialvariante (Tabelle II - 17).

Tabelle II-17: Betriebsergebnis große Versandapotheke in Abhängigkeit der Marktpotentialvariante

\begin{tabular}{|l|c|c|c|}
\hline (FORTFÜHRUNG Tabelle II - 15) & $\begin{array}{c}\text { Marktpotential- } \\
\text { variante A }\end{array}$ & $\begin{array}{c}\text { Marktpotential- } \\
\text { variante B }\end{array}$ & $\begin{array}{c}\text { Marktpotential- } \\
\text { variante C }\end{array}$ \\
\hline $\begin{array}{l}\text { Betriebshandelsspanne } \\
\text { (siehe Tabelle II - 15) }\end{array}$ & \multicolumn{3}{|c|}{$30,5 \%$} \\
\hline $\begin{array}{l}\text { Tatsächliche Kosten ohne Logistikkosten } \\
\text { (siehe Tabelle II - 15) }\end{array}$ & \multicolumn{3}{|c|}{$13,2 \%$} \\
\hline $\begin{array}{l}\text { Logistikkosten } \\
\text { (siehe Tabelle II - 16) }\end{array}$ & $6,7 \%$ & $6,8 \%$ & $6,9 \%$ \\
\hline Gewerbesteuer $^{131}$ & $1,6 \%$ & $1,6 \%$ & $1,6 \%$ \\
\hline $\begin{array}{l}\text { Kalkulatorische Kosten } \\
\text { (siehe Tabelle II - 15) }\end{array}$ & & $1,2 \%$ & \\
\hline Betriebswirtschaftliches Ergebnis & $7,8 \%$ & $7,7 \%$ & $7,6 \%$ \\
\hline
\end{tabular}

Quelle: INIFES, eigene Darstellung.

Nach Berücksichtung der Logistikkosten und der korrigierten Gewerbesteuer ergibt sich ein Betriebsergebnis von ca. 7,7\% (Mittelwert aus den drei Varianten). Dieses stellt den Betrag dar, der von der Apothekenbetriebsspanne nach Abzug der gesamten Kosten der Versandapotheke VA II als positives Ergebnis verbleibt.

\subsubsection{Die Kostenstruktur einer Versandapotheke mit integrierter Großhandels- funktion mit 487 Mio. DM (Variante III)}

In verschiedensten Branchen ist erkennbar, dass die Zusammenführung unterschiedlicher Geschäftsfelder neben der Konzentration von qualitativen Kernkompetenzen auch zu Synergie- und Größeneffekten bei den Kosten führt.

So ist auch in der Pharmabranche international wie in Europa, z.B. in Großbritannien oder Italien, mehr und mehr zu beobachten, dass pharmazeutische Großhandelsunternehmen und Einzelapotheken in einer Handelsstufe, soweit gesetzlich möglich, integriert werden. Auch deutsche Pharmagroßhandelsunternehmen, wie z.B. Gehe, betreiben in den genannten Ländern Apothekenunternehmen.

Im Gegensatz dazu ist das Betreiben einer Apotheke in Deutschland an die pharmazeutische Kompetenz des Eigentümers gebunden (§ 2 ApBetrO, $\S \S 2$ und 7 ApoG); nach dem Fremdbesitzverbot darf nur ein approbierter Apotheker eine Apotheke

131

Korrigierter Wert: (Tabelle II - 15) minus (Tabelle II - 16). 
besitzen. Das Führen eines Pharmagroßhandels ist in Deutschland wiederum auch ohne pharmazeutische Kompetenz möglich. Damit ist derzeit rechtlich ausgeschlossen, dass ein Großhandelsunternehmen die Apothekenfunktion übernimmt.

Die Integration beider Handelsfunktionen aufgrund möglicher Kosten- und Synergieeffekte - neben den traditionellen Aufgaben Beschaffung, Lagerung und Distribution baut der Großhandel weitere Funktionen durch eine gezielte Sortimentspolitik aus; neue Technologien zur Optimierung von Distribution und Logistik (z.B. intelligente Lagersysteme, Self-Scanning, Managementinformations- und Warenwirtschaftssysteme) stellen einen wichtigen Bestandteil zur Sicherung der Wettbewerbsfähigkeit $\operatorname{dar}^{132}$ - zu einer Handelsstufe wäre nach derzeitiger Rechtslage nur durch eine vertikale Rückwärtsintegration denkbar.

In Anbetracht der Umsatzstärke des Unternehmens ist es als wahrscheinlich zu betrachten, dass sich in diesem Modell VA III im Gegensatz zu Präsenzapotheken oder auch umsatzschwächeren Versandapotheken wie VA I und VA II die Gesellschaftsform der Versandapotheke in Richtung einer Kapitalgesellschaft entwickelt. In diesem Zusammenhang ist nicht auszuschließen, dass das Fremdbesitzverbot zur Disposition stehen wird, d.h. ein Großhandelsunternehmen eine Versandapotheke betreibt. Jedoch ist es nicht Auftrag der Studie, diese - derzeit noch als spekulativ zu bezeichnenden - rechtlichen Entwicklungen weiter zu verfolgen. Die Benennung möglicher Veränderungen geschieht hier ausschließlich im Sinne einer allgemeinen Strukturbetrachtung.

Den oben genannten Argumenten folgend (die rechtliche Thematik des Zusammenschlusses steht wie erwähnt nicht im Vordergrund), übernimmt die Versandapotheke VA III zusätzlich zur Apotheken- auch die Großhandelsfunktion. Damit wird nicht nur der Distributionskanal vom Hersteller zum Patienten verkürzt, sondern es kommt ebenso zu einer Konzentration der Einkaufsmacht gegenüber den Herstellern. Die in Variante VA I und VA II bereits eingeführte moderate Rabattgewährung von ca. $5 \%$ auf den Apothekeneinkaufspreis könnte hier wesentlich höher ausfallen.

Für die weiteren Ausführungen gilt uneingeschränkt, dass Daten über den pharmazeutischen Großhandel bezüglich Umsatz- und Kostenentwicklung sowie existierender Rabattmodalitäten an die Apotheken für das öffentliche Interesse nicht zugänglich sind. Ein Apothekenbetriebsvergleich, wie er für Präsenzapotheken durch das IFH Köln jährlich erstellt wird und auf dem diese Studie zu großen Teilen aufbaut, wird zwar für den pharmazeutischen Großhandel ebenfalls durch das IFH Köln durchgeführt, allerdings sind diese Daten nicht allgemein zugänglich. ${ }^{133}$

\footnotetext{
132

Hudetz, K. (1999), Electronic Commerce - Chancen und Risiken für den Großhandel, in: Mitteilungen des IFH, Jahrgang 51, Nr. 12, Dezember 1999, S. 251.

133

Der PHAGRO bestătigte, dass eine Verwendung solcher Daten nicht für das öffentliche Interesse bestimmt sei. Siehe Workshop vom 17.09.2001, Berlin.
} 
Es muss infolge dessen verstärkt auf Informationen aus dem Internet (z.B. spezifische Unternehmensinformationen) ${ }^{134}$ und Expertengesprächen zurückgegriffen werden. ${ }^{135}$ Des weiteren kommen Analysen und Berichte zur allgemeinen Entwicklung des Großhandelssegments anderer Branchen zur Anwendung.

Im Sinne einer Vollständigkeit der folgenden Analysen ist es zudem unvermeidlich, aufgrund fehlender Informationen einzelne Annahmen zum Sachverhalt zu treffen.

$\mathrm{Zu}$ den Problemen der Informationsbeschaffung im pharmazeutischen Großhandel tritt hinzu, dass eine Versandstruktur wie in Variante VA III mit Zusammenführung der beiden Handelsstufen derzeit nicht existiert.

Zusammenfassend soll an dieser Stelle herausgestrichen werden, dass der Bezug auf eine einheitliche Informations- und Datenbasis für die Erarbeitung einer Kostenstruktur wünschenswert gewesen wäre, aber wegen der genannten Gründe nicht realisiert werden konnte. Um so mehr liegt das Bestreben in einer umfassenden Gewährleistung von Plausibilität trotz unterschiedlicher Informationsgrundlagen: d.h. wenn möglich, ergehen in Abwägung quantitative Aussagen, die durch qualitative Argumente untermauert werden.

Die Modellentwicklung bedient sich folgender zentraler Thesen:

- Der Brutto-Umsatz des Modells VA III beträgt ca. 487 Mio. DM im Jahr.

- Auch hier gilt die Annahme eines gestiegenen Pro-Kopf-Umsatzes des pharmazeutischen Personals in Versandapotheken (2.4.2.1.).

- Es kommt zudem aufgrund des verstärkten Einsatzes von mittlerem pharmazeutischem Personal (PTA) in Relation zu den Apothekern (siehe 2.4.2.1.) zu Verschiebungen im Verhältnis Apotheker zu mittlerem pharmazeutischem Personal.

- Für die Rabattgewährung des pharmazeutischen Großhandels an die Versandapotheke VA III gelten die selben Argumente wie für VA I unter 2.4.3.

- Die Veränderung der räumlichen Nutzungsmöglichkeiten zu mehr Lagerkapazitäten (2.4.2.1.) gilt in 2.4.5. zudem verstärkt durch die integrierte Großhandelsfunktion.

- Die Telefonberatung wird rund um die Uhr durch zusätzliches pharmazeutisches Personal selbst erstellt.

- Der Vertrieb / Versand der Arzneimittel wird aufgrund des überregionalen bzw. bundesweiten Wirkungskreises der Versandapotheke (siehe Argumente unter 2.4.2.3) durch externe Logistikunternehmen sichergestellt.

\footnotetext{
134 Werden an den jeweiligen Stellen erwăhnt.

Informationen allgemeiner Art liegen bei INIFES vor.

136

Kaapke, A. (1999), Der Jahresbetriebsvergleich des Großhandels im Jahr 1998 in: Mitteilungen des IFH, Jg. 51, Nr. 12, Dezember 1999, S. 258 - 268.
} 
Es ergeben sich folgende Umsatz- und Kostenstrukturen.

\section{UMSATZ}

- Der PHAGRO ${ }^{137}$ veröffentlicht für 1999 Kennzahlen zum Arzneimittelmarkt, die einen Gesamtumsatz des Großhandels (nicht differenziert nach Voll- und Teilsortimentierer) von ca. 32,2 Milliarden DM netto zu AEP ${ }^{138}$ bei einem langfristigen 20Jahres-Trend von etwa $3 \%$ bis 5\% Umsatzzuwachs benennen. ${ }^{139}$ Bei $102 \mathrm{Be}$ triebsstätten im Großhandel ${ }^{140}$ beträgt der durchschnittliche Umsatz je Betriebsstätte ca. 300 Mio. DM.

- Alle 17 vollsortimentierten Großhändler (z.B. Gehe, Anzag, Noweda, Sanacorp, Phoenix) sind Mitglieder des PHAGRO. Ihre Umsätze liegen in Milliardenhöhe. So erwirtschaftete Phoenix mit einem Lagersortiment von ca. 100.000 Artikeln 1999 in 19 Vertriebszentren ca. 8,3 Mrd. DM Umsatz im Inland ${ }^{141}$, was einem durchschnittlichen Umsatz je Vertriebszentrum von ca. 435 Mio. DM entspricht und somit über dem allgemeinen Durchschnitt von 300 Mio. DM liegt.

- Neben dem PHAGRO gibt es Kooperationen, in denen sich vor allem kleinere Vollsortimentierer und Teilsortimentierer, Sortimentsgröße bei ca. $60.000^{142}$ bis 70.000 Artikeln, organisieren. Ein typischer Teilsortierer kann in seinem Sortiment aber auch wesentlich darunter liegen. Die Kooperation PHARMA PRIVAT wird durch 9 Unternehmen mit 18 Betriebsstätten - deren Vertriebsbereich bei ca. zwei Betriebsstätten je Unternehmen zumeist nicht bundesweit flächendeckend, sondern auf bestimmte Regionen fokussiert ist - und einem Umsatz von 3 Milliarden DM Umsatz gebildet. ${ }^{143}$ Damit halten diese ca. $9 \%$ am gesamten deutschen Großhandelsumsatz von 32,2 Mrd. DM. Im Durchschnitt ergeben sich für diese Unternehmen aus den genannten Zahlen ca. 330 Mio. DM Umsatz je Unternehmen bzw. 170 DM Umsatz je Betriebsstätte. Im Vergleich zum Durchschnitt von 300 Mio. DM je Betriebsstätte liegen diese Zahlen fast um die Hälfte darunter.

- Für die Berechnungen der Variante III wird kein marktbeherrschendes Großhandelsunternehmen mit Milliardenumsätzen zugrunde gelegt. Die Orientierung

\footnotetext{
137

Bundesverband des Pharmazeutischen Großhandels.

Apothekeneinkaufspreis ohne Umsatzsteuer.

139

140

PHAGRO (2000), Kennzahlen deutscher Pharmagroßhandel, in: www.phagro.de/Kennzahlen, Zugriff am 05.12.00.

PHAGRO (2000), Kennzahlen deutscher Pharmagroßhandel, in: www.phagro.de/Kennzahlen, Zugriff am 05.12.00.

141

O.V. (2001), Phoenix-Chef sieht Marge durch Pharma-Industrie unter Druck, in: Financial Times Deutschland vom 29.01.2001, www.ftd.de, Zugriff am 29.01.01.

142

143

Krieger - Pharma Großhandlung, siehe unter www.pharma-privat.de, Zugriff am 30.01.01.

Siehe unter Statistik auf der Homepage von PHARMA PRIVAT, www.pharma-privat.de, Zugriff am 30.01.01.
} 
erfolgt an Umsätzen kleinerer und mittlerer Großhandelsunternehmen mit ca. 300 Mio. DM Umsatz netto zu AEP. ${ }^{144}$

- Unter Berechnung der Aufschläge in der Apothekenstufe nach AMPreisV sowie der Mehrwertsteuer ergibt sich ein Bruttoumsatz der Versandapotheke von ca. 487 Mio. DM (vgl. Abbildung II - 9).

Abbildung II - 9: Umsatzberechnung für Versandapotheke mit integriertem Großhandel

\begin{tabular}{|lrc|}
\hline \multicolumn{2}{|c|}{ Versandapotheke mit integriertem Großhandel } \\
Großhandelsstufe & Bezug & Zahlenangaben \\
$\quad$ Wareneinsatz (Hersteller) & in DM & 264.317 .181 \\
$\quad$ Durchschn. Großhandelszuschlag nach AMPREISV & in DM & 35.682 .819 \\
$\quad$ Nettoumsatz Großhandel zu AEP & in DM & 300.000 .000 \\
Apothekenstufe & & \\
$\quad$ Nettoumsatz zu AEP & in DM & 300.000 .000 \\
Durchschn. Apothekenzuschlag nach AMPREISV & in DM & 120.000 .000 \\
$\quad$ Nettoumsatz Apotheke & in DM & 420.000 .000 \\
MwSt & in DM & 67.200 .000 \\
& in \% & $16 \%$ \\
Apothekenumsatz brutto & in DM & 487.200 .000 \\
\hline
\end{tabular}

Quelle: INIFES, eigene Darstellung.

\section{WARENEINSATZ UND HANDELSSPANNE}

- Der Wareneinsatz ist der Umsatz der vorgelagerten Herstellerstufe zu Herstellerabgabepreisen netto. Unter der Rückrechnungsmethode von einem Großhandelsumsatz 300 Mio. netto zu AEP ergeben sich bei Abzug eines durchschnittlich $13,5 \%$ igen $^{145}$ Höchstaufschlages des Großhandels nach AMPreisV auf den Herstellerabgabepreis ca. 264,3 Mio. DM Wareneinsatz.

- Auf der Apothekenstufe wird der Großhandelsumsatz netto zu AEP um die Festzuschläge der AMPreisV erhöht. Diese sind, wie die Großhandelszuschläge auch,

U.a. auch deshalb, da die Leitung der Versandapotheke den derzeitigen Gesetzen zum Apothekenbetrieb entsprechend einem Apotheker als natürlicher Person unterliegen muss (Rechtsform des Einzelunternehmens ist der Regelfall, möglich ist auch eine OHG unter Berücksichtigung des Fremdbesitzverbotes). Dass diese Rechtsvorschriften im Zusammenhang mit dem Modell VA III zukünftig zur Disposition stehen könnten, wurde bereits erwähnt. Deutschland vom 29.01.2001, www.ftd.de, Zugriff am 29.01.01. 
degressiv gestaffelt. Für die Apotheken ergeben sich Zuschläge in einer Spanne von $68 \%$ bis $30 \%$ auf den AEP netto ${ }^{146}$, im Durchschnitt von ca. $40 \%$.

- Der sich daraus ergebende Apothekenumsatz von 420 Mio. DM netto wird um die Mehrwertsteuer von 16\% erhöht. Der resultierende Apothekenumsatz brutto liegt bei 487,2 Mio. DM.

- Die übliche Darstellung der Wertschöpfungsanteile für Hersteller, Großhandel, Apotheken und Staat am Apothekenumsatz ergibt sich aus Abbildung II - 10:

Abbildung II - 10: Wertschöpfungsanteile für Hersteller und Vertrieb

\begin{tabular}{|lccc|}
\hline Wertschöpfung & & Ohne Rabatt & $5 \%$ Rabatt \\
Apothekenumsatz brutto & In DM & 487.200 .000 & 487.200 .000 \\
& in \% AVP brutto & $100,0 \%$ & $100,0 \%$ \\
Wertschöpfung MwSt & In DM & 67.200 .000 & 67.200 .000 \\
Wertschöpfung Hersteller & in \% AVP brutto & $13,8 \%$ & $13,8 \%$ \\
& In DM & 264.317 .181 & 251.101 .322 \\
Handelsspanne Vertrieb & in \% AVP brutto & $54,3 \%^{147}$ & $51,5 \%$ \\
& In DM & 155.682 .819 & 168.898 .678 \\
& in \% AVP brutto & $32,0 \%{ }^{148}$ & $34,7 \%$ \\
\hline
\end{tabular}

Quelle: INIFES, eigene Darstellung

- Durch die Zusammenlegung der Handelsfunktionen Groß- und Einzelhandel in eine einzige Handelsstufe setzt sich die Betriebsspanne für dieses Versandapothekenmodell VA III aus der Addition beider Betriebsspannen, der des Großhandels wie der der Apotheken zusammen. Die Betriebshandelsspanne beträgt demnach $34,7 \%$ des Umsatzes.

- Analog zu den Versandapotheken Variante I und II wird auch hier eine Rabattgewährung von ca. $5 \%$ auf den Herstellerabgabepreis ausgegangen. Wie erwähnt, könnte durch die Konzentration der Einkaufsmacht auf einer gemeinsamen Handelsstufe ein wesentlich höherer Rabatt als realistisch erscheinen.

\section{TATSÄCHLICHE KOSTEN}

Aufgrund der Problematik, dass Daten zur Kostenstruktur des pharmazeutischen Großhandels, z.B. in Form eines Betriebsvergleiches nicht öffentlich zugänglich sind, gestalten sich Angaben über die internen Kosten als schwierig.

\footnotetext{
146

Ab 1063,82 DM AEP netto beträgt der Zuschlag 8,2\% plus 231,25 DM, siehe: Arzneimittelpreisverordnung, Änderung vom 01.07.1998.

Vgl. mit den Zahlen des VFA in Kapitel 1.2.3.3.

148

Vgl. mit den Zahlen des VFA in Kapitel 1.2.3.3.
} 
Zudem soll in dieser Variante III die Zusammenlegung von Apotheken- wie Großhandelsstrukturen erfolgen, da sich davon aufgrund von Synergie- und Größeneffekten positive Wirkungen auf die Kostensenkung erhofft werden. Monetäre Zahlenangaben gestalten sich aus dem Kontext heraus als problematisch, deshalb wird zusätzlich qualitativ argumentiert.

\section{A) Personalkosten für den laufenden Geschäftsbetrieb}

- Das Personal für die Betreuung der Telefonhotline ist in dieser Position nicht eingeschlossen. Es wird in der entsprechenden Positionen „Telefonberatungskosten" extra berücksichtigt.

- Apotheker und mittleres pharmazeutisches Personal übernehmen auch in Variante III die pharmazeutischen Tätigkeiten innerhalb der Apothekenfunktion.

- Die Anzahl des mittleren pharmazeutischen Personals (PTA) entwickelt sich entsprechend gegenüber Versandapotheke VA II: dort waren 10,6 PTA beschäftigt, für das Modell VA III berechnen sich in Abhängigkeit der Umsatzsteigerung gegenüber VA II 256,3 angestellte Personen im Berufsbild einer PTA.

- Das Verhältnis zwischen angestellten approbierten Apothekern zu mittlerem pharmazeutischen Personal wurde für VA I bzw. VA II entsprechend den Strukturen einer Präsenzapotheke mit $1: 1,7$ (bei Teilzeit) entwickelt.

- Für das Modell VA III mit einem fast um das 25fache höheren Umsatz als VA II wird allerdings aufgrund der angedeuteten Größen- und Synergieeffekte nun verstärkt die unter 2.4.2.1. dargestellte Verschiebung der relativen Personalstruktur zwischen angestellten approbierten Apothekern zu mittlerem pharmazeutischen Personal berücksichtigt. Es klang an, dass dieses Verhältnis durch die Verschiebung der Tätigkeitsbereiche bei $1: 2,6$ und mehr liegen kann.

- Entsprechend resultiert in Relation zur Anzahl der benötigten 256 PTA ein Bedarf von ca. 98 angestellten approbierten Apothekern für das Modell VA III.

- Demnach sind 354 pharmazeutische Beschäftigte in der Versandapotheke VA III für den laufenden Apothekengeschäftsbetrieb angestellt.

- Durch die Integration der Großhandelsfunktion in den Geschäftsbetrieb fallen in der Versandapotheke zusätzlich entsprechende Tätigkeiten (Bestellwesen, Kommissionierung, Lagertätigkeiten, Versandvorbereitung, Verpackung) an. Die Automatisierung von Arbeitsabläufen sowie die Implementierung von EDV-Systemen, die Wareneingangs-, Auftragserfassung, Rechnungsstellung, Lagerverwaltung, Chargendokumentation und Bestellmanagement miteinander verbinden, spielen im Großhandel eine wesentliche Rolle. ${ }^{149}$

Ergebnisse von Expertengesprăchen. 
- Entsprechend kann ein Pro-Kopf-Umsatz (in Vollzeit) von ca. 1,5 Mio. DM Großhandelsumsatz netto (identisch mit AEK netto) berechnet werden. ${ }^{150}$ In VA III mit 300 Mio. DM zu AEK netto benötigt man entsprechend einen Mitarbeiterstamm von 200 Vollzeitkräften für die Großhandelsfunktion.

- Diese werden auf Basis des Lohntarifvertrages für den Groß- und Außenhandel nach den Lohngruppen $2 \mathrm{~b}$ und 3 (Kommissionier- und Lagertätigkeiten) mit ca. 3.048 DM Monatsgehalt (bei 12 Monatsgehältern) entlohnt. ${ }^{15}$

- Die zusätzlich zu kalkulierenden sozialen Abgaben belaufen sich auf ca. $20 \%$ der Lohnaufwendungen. ${ }^{152}$

- Entsprechend errechnen sich Personalkosten je Kommissionierer bzw. Lagerfacharbeiter von ca. 43.900 DM p.a. bei Vollzeit.

\section{B) Mietkosten}

- Lageroptimierung durch moderne Lagertechnik, Automatisierung der Lagerabläufe und Lagerverwaltung, die zudem Bestellmanagement und Chargendokumentation miteinander verbindet, sind Voraussetzung für minimale Lagerkosten.

- Allerdings muss aufgrund mangelnder Daten zur Flächennutzung des Pharmagroßhandels sowie zum Einfluss der kombinierten Apotheken- und Großhandelsfunktionen auf die räumliche Nutzung zur Ermittlung der Gesamtfläche und den daraus resultierenden Mietkosten analog zu den Varianten VA I und VA II zurückgegriffen werden.

- Im Durchschnitt werden ca. 12 DM je qm an Mietkosten bzw. Mietwert angesetzt. Auf die Mietkosten werden 28\% für Sach- bzw. Mietnebenkosten aufgeschlagen.

- Die benötigte gesamte Geschäftsfläche berechnet sich aus dem Lagerwert in Kombination mit der räumlichen Aufteilung:

Bei Optimierung des Lagerwertes mit $8 \%$ vom Nettoumsatz (siehe 2.4.2.1.) und einem Umschlag von 8,5 mal p.a. ergibt sich unter Geltung der Annahme eines für Präsenz- wie Versandapotheken einheitlichen Lagerwertes ein Lagerwert von 2,93 TDM $^{153}$ je qm Lager eine notwendige Lagerfläche von ca. 11.500 qm.

Daraus, kombiniert mit der Prozentangabe $72 \%$ anteilige Lagerfläche wie in den zwei vorangegangenen Modellen, ergibt sich eine Geschäftsfläche von ca. 16.000 qm für Versandapothekenmodell VA III.

\footnotetext{
150 Angaben von PHARMA PRIVAT unter www.pharma-privat.de/statistik/html, Zugriff am 30.01.01. Siehe Gewerkschaft Handel, Banken und Versicherungen, Landesbezirksleitung Bayern, Lohntarifvertrag Bayern, §1, Löhne ab 01.07.2001.

152

Errechnet aus der Position „Personalaufwand/Mitarbeiter" des Jahresabschlußberichts von GEHE für 1999, S. 103: Löhne und Gehälter im Verhältnis zu sozialen Abgaben und Altersversorgung. 
Aus unternehmerischer Sicht könnte in Anbetracht der Mietkosten eine Abwägung zwischen Miete bzw. Erwerb von Grundstücken und Gewerbe- bzw. Logistikflächen stehen.

\section{C) Abschreibungen und Zinsen}

- Wie sich die Abschreibungsrate bei Zusammenlegung beider Handelsstufen entwickeln wird, ist nicht vorherzusehen. In Anbetracht einer Funktionserweiterung wird für Variante III im Gegensatz zu den Varianten I und II eine höhere Abschreibungsrate angenommen:

- Für die Abschreibungen des pharmazeutischen Großhandels liegen keine Daten vor.

- Die Abschreibungsquote des Großhandels anderer Branchen beträgt durchschnittlich $1,3 \%$ vom Großhandelsumsatz. ${ }^{154}$

- In Anbetracht fehlender Informationen wird zum einen auf diese Hilfsgröße zurückgegriffen und zum anderen wird diese auf den Umsatz zu AVP bezogen.

- Für die Versandapothekenfunktion wird die Abschreibungsrate analog der Präsenzapotheke kalkuliert.

- Die gesamte Abschreibungsrate für das Modell VA III ergibt sich aus der Addition der Abschreibungsraten für die Großhandels- und die Apothekenfunktion.

- Die Fremdkapitalzinsen werden mangels anderer Informationen in ihrer prozentualen Höhe analog zu den Varianten VA I und VA II übernommen.

\section{D) Laufender Geschäftsbetrieb: Werbung, KFZ, übrige Kosten}

- Genaue Zahlen für den pharmazeutischen Großhandel liegen nicht vor.

- Allerdings unterliegt auch der Pharmagroßhandel den Werbebeschränkungen des $\S 2$ AMG und des Heilmittelwerbegesetzes. Werbung für apothekenpflichtige Arzneimittel ist nur sehr beschränkt möglich (§ 4, II HWG), für zudem rezeptpflichtige Arzneimittel - und die bilden annahmegemäß aufgrund der Zielgruppe das wesentliche Segment der Varianten I bis III - ist jegliche Offentlichkeitswerbung

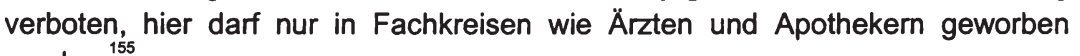
werden.

- Analog zur Argumentation der großen Versandapotheke wird deshalb auch hier von relativen Werbungskosten in \% vom Umsatz in derselben Höhe wie in Präsenzapotheken ausgegangen.

Kaapke, A. (1999), Der Jahresbetriebsvergleich des Großhandels im Jahre 1998, in Mitteilungen des IFH Köln, Jg. 51, Nr. 12, Dezember 1999, S. 267.

Siehe auch EU-Richtlinie 91/26/EWG. 
- Die KFZ-Kosten (darunter sind nicht die Logistikkosten zu verstehen) entwickeln sich gegen null.

- Die "übrigen Kosten" entwickeln sich analog der großen Versandapotheke unterproportional zum Umsatz, den Effekt einer stärkeren Fixkostendegression in Betracht ziehend.

\section{E) Neu: Telefonberatungskosten}

- Die Telefonberatung wird innerhalb wie außerhalb der üblichen Geschäftszeiten der Versandapotheke (siehe Tabelle II - 10) durch eigenes pharmazeutisches Personal sichergestellt.

- Bei analoger Entwicklung der Anruferzahlen zu Variante I und II muss innerhalb der Geschäftszeiten mit ca. 1.300 Anrufen a 5 Minuten je Stunde gerechnet werden (auch wenn sich diese Angaben in der Praxis als möglicherweise zu hoch herausstellen sollten): Dies entspricht 108 Gesprächsstunden je Arbeitsstunde bzw. daraus resultiert die Notwendigkeit von 108 gleichzeitig arbeitenden Telefonkräften je Arbeitsstunde innerhalb der Öffnungszeiten). Außerhalb der Öffnungszeiten ist unter Fortführung der Annahme über die zeitliche Verteilung der Anrufe aus 2.4.2.2. von nur ca. 16 Anrufen a 5 Minuten je Stunde auszugehen.

Wie unter 2.4.1. für Präsenzapotheken ausgewiesen, kann auch hier von 1.710 Arbeitsstunden p.a. je Vollzeitstelle ausgegangen werden: entsprechend sind 161,3 Personen pharmazeutisches Personal bei Vollzeit bzw. 215 bei entsprechender Teilzeit nach Tabelle II - 6 auf die Sicht eines ganzen Jahres zu kalkulieren.

- Die Kosten für die technische Ausstattung je Telefonarbeitsplatz werden analog den Varianten I und II entwickelt. Hinzu kommt eine über vier Jahre linear kalkulierte Abschreibungsrate für eine Call-Distributionsanlage, angenommene Kosten aufgrund des wesentlich erhöhten Anrufvolumens in Variante III ca. 200.000 DM.

- Die Vorhaltung eigenen Telefonberatungspersonals im Vergleich zur Alternative Outsourcing an externe Call-Center außerhalb der Öfnungszeiten (siehe Varianten I und II) ist hier aufgrund der erhöhten Fixkostendegression effizienter.

\section{F) Neu: Logistikstückkosten}

- Die Reichweite des Versandes umsatzstarker Versandapotheken ist als überregional, mindestens bundesweit einzuschätzen (siehe Annahmen unter 2.4.2.3.).

- Die Logistik wird deshalb an externe Logistikdienstleister outgesourct. Die Logistikkosten je Verordnung werden mit 4,79 DM analog der Herleitung unter 2.4.2.3. kalkuliert.

158

Eisinger, H. (1998), So funktioniert der Versandhandel, Wien, S. 163. 


\section{GEWERBESTEUER}

- Die Berechnung erfolgt analog der kleinen und großen Versandapotheke

\section{KALKULATORISCHE KOSTEN}

- Analog der großen Versandapotheke würde sich der kalkulatorische Unternehmerlohn im Bereich der Apothekenfunktion gegen den Grenzwert 0\% entwickeln $^{157}$.

- Allerdings erfolgt durch die Funktionserweiterung ein Blick auf den Unternehmerlohn der Großhandelsfunktion:

- Für den Pharmagroßhandel liegen keine entsprechenden Zahlen vor.

- Ein branchenübergreifender Großhandelsvergleich weist einen kalkulatorischen Unternehmerlohn von ca. 0,5\% des Großhandelsumsatzes (Schwankungsbreite $0,1 \%$ und $1,5 \%$ ) aus.

- Bezogen auf den gesamten Apothekenumsatz zu AVP ergeben sich 0,3\% kalkulatorischer Unternehmerlohn.

- Dieser wäre im Zusammenhang mit einer möglichen Änderung der Gesellschaftsform in eine Kapitalgesellschaft adäquater mit einer Entlohnung der Managementaufgaben des Unternehmens gleichzusetzen.

- Analog der großen Versandapotheken VA II werden die Eigenkapitalzinsen mit $0,4 \%$ übernommen.

\section{BETRIEBSWIRTSCHAFTLICHES ERGEBNIS}

- Das betriebswirtschaftliche Ergebnis fällt aufgrund der Kostendegression insbesondere bei den kalkulatorischen Kosten und den Personalkosten sowie der durch Zusammenlegung der Handelsstufen erzielten höheren Betriebshandelsspanne höher aus als in den Varianten I und II.

Es erfolgt in einem Zwischenschritt die Darstellung der Kostenstruktur ohne Logistikkosten, in einem weiteren Schritt werden diese mit einbezogen. Ursächlich ist dafür deren Kostenstruktur als Stückkosten je Verordnung.

Soweit es sich bei diesem Modell noch um ein Einzelunternehmen mit einem selbständigen Apotheker handelt. Wie bereits angeklungen, ist dieses Modell insbesondere in Form einer Kapitalgesellschaft denkbar, kalkulatorische Kosten für den Unternehmerlohn würden dann überhaupt nicht mehr anfallen, sondern unter die Personalkosten verbucht werden. 
Tabelle II - 18: Umsatz- und Kostenstruktur einer Versandapotheke mit integrierter Großhandelsfunktion vor Berücksichtigung der Logistikstückkosten von 4,79 DM je Verordnung

\begin{tabular}{|c|c|c|}
\hline VORLÄUFIGE UMSATZ- UND KOSTENSTRUKTUR & $\begin{array}{l}\text { in \% des } \\
\text { Umsatz }\end{array}$ & $\begin{array}{c}\text { in \% der } \\
\text { Handelsspanne }\end{array}$ \\
\hline Umsatz brutto in TDM & $100,0 \%$ & \\
\hline Mehrwertsteuer $16 \%$ & $13,8 \%$ & \\
\hline Wareneinsatz & $51,1 \%$ & \\
\hline BETRIEBSHANDELSSPANNE ${ }^{158}$ & $34,7 \%$ & $100,0 \%$ \\
\hline TATSÄCHLICHE KOSTEN OHNE LOGISTIKKOSTEN*) & $13,2 \%$ & $38,1 \%$ \\
\hline Personalaufwand & $5,4 \%$ & $15,6 \%$ \\
\hline Mietaufwand & $0,6 \%$ & $1,7 \%$ \\
\hline Abschreibungen, Zinsen & $2,6 \%$ & $7,5 \%$ \\
\hline Werbung, KfZ, übrige Kosten & $2,6 \%$ & $7,5 \%$ \\
\hline Telefonberatungskosten & $2,0 \%$ & $5,8 \%$ \\
\hline Logistikstückkosten 4,79 DM je Verordnung & s. Tabelle II - & \\
\hline GEWERBESTEUER OHNE LOGISTIKKOSTEN*) & $3,4 \%$ & $9,8 \%$ \\
\hline KALKULATORISCHE KOSTEN & $0,7 \%$ & $2,0 \%$ \\
\hline Kalkulatorischer Unternehmerlohn & $0,3 \%$ & $0,9 \%$ \\
\hline Eigenkapitalzinsen & $0,4 \%$ & $1,1 \%$ \\
\hline $\begin{array}{l}\text { BETRIEBSWIRTSCHAFTLICHES ERGEBNIS OHNE } \\
\text { LOGISTIKKOSTEN*) }\end{array}$ & $17,4 \%$ & $50,1 \%$ \\
\hline
\end{tabular}

") Vorläufige Ergebnisse, mit der folgenden Berechnung der Logistikkosten in Tabelle II - 19 ändern sich diese entsprechend, siehe auch Tabelle II - 22.

Quelle: INIFES, eigene Berechnungen.

Aufgrund der Natur der Logistikkosten als Stückkosten je Verordnung (4,79 DM je Verordnung, unabhängig von der Höhe des Verordnungswertes) können die Logistikkosten der Versandapotheke als prozentuale Ausprägung im Verhältnis zum Umsatz nur auf der Ebene des durchschnittlichen Verordnungswertes bestimmt werden. (siehe Tabelle II - 19).

Aufgrund der Festlegung eines Zielsegments (siehe 2.3.2, Variante A bis C) sind entsprechende durchschnittliche Verordnungswerte je Variante für einen Pharmaversand bekannt. ${ }^{158}$

Entsprechend der Addition beider Betriebsspannen für den Großhandel wie für Apotheken.

Siehe Tabelle II - 3 (Variante A von 66,78 DM), Tabelle II - 4 (Variante B von 64,85 DM), Tabelle II - 5 (Variante C von $61,36 \mathrm{DM})$. 
Diese durchschnittlichen Verordnungswerte müssen jedoch in einem weiteren Schritt aufgrund der Berücksichtigung von Logistikstückkosten differenziert werden. Das heißt, einige der sich im Zielsegment unter epidemiologischen Gesichtspunkten befindlichen Indikationen können aufgrund eines zu niedrigen Verordnungswertes die zusätzlich anfallenden Logistikkosten von 4,79 DM je Verordnung nicht decken. Dies hat zur Konsequenz, dass sie der Umfang des Zielsegments - gemessen in Umsatzzahlen wie in Verordnungszahlen - für jede Zielvariante um diese Indikationsgruppen entsprechend verringert (daraus resultiert entsprechend der Definition in 2.2.1. das Marktpotential). Aus dem Zielsegment können die Indikationsgruppen die Logistikkosten aus ihren durchschnittlichen Verordnungswerten decken, die über einem Minimalverordnungswert von ca. 27 DM liegen. Dieser ergibt sich, indem die Logistikstückkosten von 4,79 ins Verhältnis gesetzt werden zum betriebswirtschaftlichen Ergebnis vor Logistikkosten von 17,4\% (siehe Tabelle II - 12).

Die dieser Restriktion Miniverordnungswert entsprechenden Indikationen bilden im Vergleich zum ursprünglichen Zielsegment jeweils leicht erhöhte durchschnittliche Verordnungswerte: $75,10 \mathrm{DM}$ für Marktpotentialvariante $A^{160}, 71,45 \mathrm{DM}$ für Marktpotentialvariante $B^{161}$ und 72,56 DM für Marktpotentialvariante $C$. $^{162}$

Verringert man diese drei durchschnittlichen Verordnungswerte in einem weiteren Schritt jeweils um den 5\%igen Kassenrabatt (entsprechend für A: 71,35 DM, B: 67,88 DM, C: 68,93 DM) und setzt die Logistikstückkosten von 4,79 DM jeweils ins Verhältnis zu diesen Werten, ergibt sich der prozentuale Anteil der Logistikkosten am Verordnungswert.

Tabelle II - 19: Berechnung der prozentualen Logistikkosten je durchschnittlichen Verordnungswert in Abh. von der Marktpotentialvariante für die Versandapotheke mit integrierter Großhandelsfunktion

\begin{tabular}{|l|c|c|c|}
\hline & $\begin{array}{c}\text { Marktpotential- } \\
\text { variante A }\end{array}$ & $\begin{array}{c}\text { Marktpotential- } \\
\text { variante B }\end{array}$ & $\begin{array}{c}\text { Marktpotential- } \\
\text { variante C }\end{array}$ \\
\hline Durchschnittlicher Verordnungswert & $75,10 \mathrm{DM}$ & $71,45 \mathrm{DM}$ & $72,56 \mathrm{DM}$ \\
\hline Minus 5\% GKV-Rabatt ergibt: & $71,35 \mathrm{DM}$ & $67,88 \mathrm{DM}$ & $68,93 \mathrm{DM}$ \\
\hline Logistikkosten & $4,79 \mathrm{DM}$ & $4,79 \mathrm{DM}$ & $4,79 \mathrm{DM}$ \\
\hline $\begin{array}{l}\text { Logistikkosten in \% vom Verordnungswert } \\
\text { (abzgl. 5\% GKV-Rabatt) }\end{array}$ & $\mathbf{6 , 7} \%$ & $\mathbf{7 , 0} \%$ & $\mathbf{6 , 9} \%$ \\
\hline Korrektur Gewerbesteuer $^{163}$ auf Logistikkosten & $1,1 \%$ & $1,1 \%$ & $1,1 \%$ \\
\hline
\end{tabular}

Quelle: INIFES, eigene Berechnungen.

Siehe Nebenrechnung im Anhang I, Tabelle I - 1.

161 Siehe Nebenrechnung im Anhang I, Tabelle I - 2.

162 Siehe Nebenrechnung im Anhang I, Tabelle I - 3.

163 Die Logistikkosten sind tatsächliche Kosten, welche die Gewerbesteuerzahlungen vermindern. Deshalb muss eine Korrektur der ausgewiesenen Gewerbesteuer erfolgen.
} 
Die prozentualen Logistikkosten wie die Korrektur der Gewerbsteuer können ohne Einschränkungen auf die Ebene des Apothekenumsatzes übertragen werden. Es ergibt sich nun entgültig das betriebswirtschaftliche Ergebnis der Versandapotheke mit integrierter Großhandelsfunktion VA III nach Berücksichtigung der Logistikkosten in Abhängigkeit von der Marktpotentialvariante (Tabelle II - 22).

Tabelle II - 20: Betriebsergebnis Versandapotheke mit integrierter Großhandelsfunktion in Abhängigkeit der Marktpotentialvariante

\begin{tabular}{|l|c|c|c|}
\hline (FORTFÜHRUNG Tabelle II - ) & $\begin{array}{c}\text { Marktpotential- } \\
\text { variante A }\end{array}$ & $\begin{array}{c}\text { Marktpotential- } \\
\text { variante B }\end{array}$ & $\begin{array}{c}\text { Marktpotential- } \\
\text { variante C }\end{array}$ \\
\hline $\begin{array}{l}\text { Betriebshandelsspanne } \\
\text { (siehe Tabelle II - ) }\end{array}$ & \multicolumn{3}{|c|}{$34,7 \%$} \\
\hline $\begin{array}{l}\text { Tatsächliche Kosten ohne Logistikkosten } \\
\text { (siehe Tabelle II - ) }\end{array}$ & $6,7 \%$ & $73,2 \%$ & $6,9 \%$ \\
\hline $\begin{array}{l}\text { Logistikkosten } \\
\text { (siehe Tabelle II - ) }\end{array}$ 164 & $2,3 \%$ & $2,3 \%$ & $2,3 \%$ \\
\hline Gewerbesteuer & & $0,7 \%$ & $11,6 \%$ \\
\hline $\begin{array}{l}\text { Kalkulatorische Kosten } \\
\text { (siehe Tabelle II - ) }\end{array}$ & $11,8 \%$ & $11,5 \%$ & \\
\hline Betriebswirtschaftliches Ergebnis & \multicolumn{3}{|c|}{} \\
\hline
\end{tabular}

Quelle: INIFES, eigene Darstellung.

Nach Berücksichtung der Logistikkosten und der korrigierten Gewerbesteuer ergibt sich ein Betriebsergebnis von ca. 11,6\% (Mittelwert aus den drei Varianten). Dieses stellt den Betrag dar, der von der Betriebsspanne nach Abzug der gesamten Kosten der Versandapotheke VA III als positives Ergebnis verbleibt.

\subsubsection{Zwischenfazit}

Zentrales Element jeder Analyse potentieller monetärer Einsparvolumen einer Ausweitung des Pharmaversandes ist die Simulation der Kostenstrukturen von Versandapotheken. Einerseits lassen sich durch betriebswirtschaftliche Kostenstrukturanalysen die durchschnittlichen Kosten der existierenden Präsenzapotheken mit den zu erwartenden Kosten verschiedener Ausgestaltungsformen von Versandapotheken vergleichen. Andererseits ergeben sich aus den geänderten Betriebsabläufen und Vertriebswegen der Versandapotheke aber auch Differenzen in der Kostenstruktur.

Die Modellrechnungen zur internen Struktur von Versandapotheken umfassen u.a. die Annahmen, dass in den Räumen der Versandapotheke kein oder nur sehr wenig Kundenverkehr stattfindet, Versandapotheken also z.B. auch in Gewerbegebieten mit niedrigeren Mietkosten angesiedelt sind. Annahmegemäß bieten Versandapotheken als Service- und Qualitätsstandard eine 24-Stunden-Telefon-Hotline (an allen Tagen

164

Korrigierter Wert: (Tabelle 17) minus (Tabelle 18). 
des Jahres) an. ${ }^{165}$ In weiteren - jeweils detailliert begründeten - Annahmen wird davon ausgegangen, dass der Lagerumschlag 8,5 mal p.a. beträgt und von einem Lagerwert von $8 \%$ des Nettoumsatzes ausgegangen werden kann, sowie, dass der Umsatz pro pharmazeutischen Mitarbeiter - im Vergleich zur Präsenzapotheke wesentlich höher als in Präsenzapotheken anzusetzen ist.

Ausgangsbasis der in Kapitel 2.4. durchgeführten Untersuchungen ist die interne Struktur einer Präsenzapotheke, die auf Basis zugänglicher Apothekenbetriebsvergleich-Daten berechnet wird und als Referenzmaß der weiteren Simulationen dient. Die durchschnittliche Präsenzapotheke erwirtschaftet dabei mit insgesamt durchschnittlich 6,7 Mitarbeitern einen Bruttoumsatz von 2,53 Mio. DM im Jahr. Das betriebswirtschaftliche Ergebnis liegt bei $0,4 \%$ des Bruttoumsatzes.

Die Strukturmerkmale der Präsenzapotheke werden insbesondere im Modell der "kleinen" Versandapotheke VA I herangezogen. Andererseits dienen die Angaben zur späteren Ermittlung möglicher Kostenersparnisse des Arzneimittelversandes. Unter Zuhilfenahme weiterer Sekundärdaten kann die Kostenstruktur einer zukünftigen Versandapotheke entwickelt werden. Es wurde mehrfach betont, dass mit neuen Vertriebsformen einhergehende neue Kosten (bei Präsenzapotheken fallen aufgrund der vorgeschriebenen Abgabe in den Apothekenräumen keine Logistikkosten an) zusätzlich berücksichtigt werden müssen. Diese schmälern das Betriebsergebnis. Das sich nach Abzug aller Kosten ergebende Betriebsergebnis der Versandapotheke stellt den Betrag dar, der als positives Ergebnis verbleibt. Damit kann diese Überdeckung als Kostenvorteil des Vertriebsweges Versand gegenüber dem Vertriebsweg Präsenzapotheke interpretiert werden.

Die interne Kostenstruktur der Versandapotheken wird an unterschiedliche Umsatzgrößenklassen von Versandapotheken gekoppelt, um Skaleneffekte bei den Kosten und deren Auswirkung auf das Betriebsergebnis zu analysieren. Es ergeben sich drei Varianten von Arzneimittelversendern:

Das Modell der kleinen Versandapotheke VA I beschreibt eine Versandapotheke, die aus den Strukturen der Präsenzapotheke entwickelt wird und einen Bruttoumsatz von ca. DM 5 Mio. - also ca. den doppelten Wert einer durchschnittlichen Präsenzapotheke - erreicht. Die Logistik wird bei dieser regional begrenzten Modellvariante selbst erstellt, die Telefonberatung während der Offnungszeiten durch eigenes pharmazeutisches Personal durchgeführt, außerhalb der Öffnungszeiten an externe Call - Center vergeben. Es zeigt sich, dass mit der Umsatzverdopplung gegenüber Präsenzapotheken trotz neuer Aufwendungen für Auslieferung und Telefonberatung eine wesentliche Verbesserung in der Kostenstruktur erreicht werden kann. Des weiteren ermöglichen hohe Umsätze eine verstärkte Rabattgewährung, die Einfluss auf die Erhöhung der Handelsspanne der Apotheken hat. Die kalkulatorischen Kos-

Vgl. hierzu die Ergebnisse der Bevölkerungsbefragung in Kapitel 3. 
ten halbieren sich aufgrund der Umsatzverdopplung. Für die Mischkalkulation der Apotheken verbleibt ein Betriebsergebnis von ca. $6,2 \%$.

Im Modell der großen Versandapotheke VA II wird - basierend auf den Erfahrungen im europäischen Ausland - von einem Jahresumsatz von 20 Mio. DM ausgegangen. Die Logistik wird bei dieser überregional agierenden Versandapotheke an externe Logistiker vergeben. Die Telefonberatung erfolgt während der Öffnungszeiten durch eigenes pharmazeutisches Personal, außerhalb der Offnungszeiten wird diese an externe Call - Center vergeben. Das Betriebsergebnis beträgt hier ca. $7,7 \%$ vom Umsatz. Insgesamt zeigt sich, dass mit einer Umsatzvervierfachung gegenüber Variante I eine weitere Verbesserungen in der Kostenstruktur erreicht werden konnten, dies trifft hauptsächlich auf die Personalkosten zu. Auch hier ermöglichen hohe Umsätze eine verstärkte Rabattgewährung, die Einfluss auf die Erhöhung der Handelsspanne der Apotheken hat. Mit den Umsatzsteigerungen gehen die kalkulatorischen Kosten (Unternehmerlohn, Eigenkapital) gegen den Grenzwert Null, sie betragen nur noch ein marginales Prozent.

Das Modell der Versandapotheke in Verbund mit Großhandel (VA III) geht von einer Zusammenlegung von Großhandels- und Apothekenfunktion in eine Vertriebsinstanz aus. Obwohl dieses Modell z.T. erheblichen Schwierigkeiten aufgrund fehlenden Datenmaterials einerseits, aber auch der Quantifizierung von Synergieeffekten andererseits unterliegt, haben die Untersuchungen hier eine möglicherweise zukunftsträchtige Struktur aufgezeigt. Die Umsatzzahlen für eine Versandapotheke in Verbund mit Großhandel sind anhand verfügbarer Angaben zu Großhandelsumsätzen für mittelgroße Unternehmen orientiert, wonach sich ein Apothekenumsatz von ca. 487 Mio. DM berechnet. Diese Größe steht allerdings nicht für eine als minimal vorauszusetzende Umsatzschwelle, um beide Handelsstufen miteinander zu verbinden. Hier ist - ohne Abweichungen von der Kostenstruktur - auch ein Gesamtumsatz von 100 Mio. DM bis 200 Mio. DM ohne weiteres vorstellbar. Die Logistik wird bei dieser überregional agierenden Versandapotheke an externe Logistiker vergeben. Die Telefonberatung erfolgt rund um die Uhr durch eigenes pharmazeutisches Personal. Das erzielte betriebswirtschaftliche Ergebnis von ca. $11,6 \%$ liegt im Vergleich zur "großen" Versandapotheke höher. Sicherlich können die hier verwendeten Prozentangaben nicht immer im Maßstab $1: 1$ in die Praxis übernommen werden, dazu sind die aufgezeigten Einschränkungen aufgrund fehlenden Datenmaterials häufig zu groß. Andererseits verdeutlichen sie, dass eine Integration von logistischer und pharmazeutischer Kompetenz für die zukünftige Entwicklung von Versandapotheken eine wesentliche Rolle spielen wird. 
Abbildung II - 11: Verwendung der Apothekenbetriebsspanne - Skaleneffekt der Kosten und des Ergebnisses / der Kostenersparnis in Abhängigkeit der Umsatzgrößenklassen der Apotheke

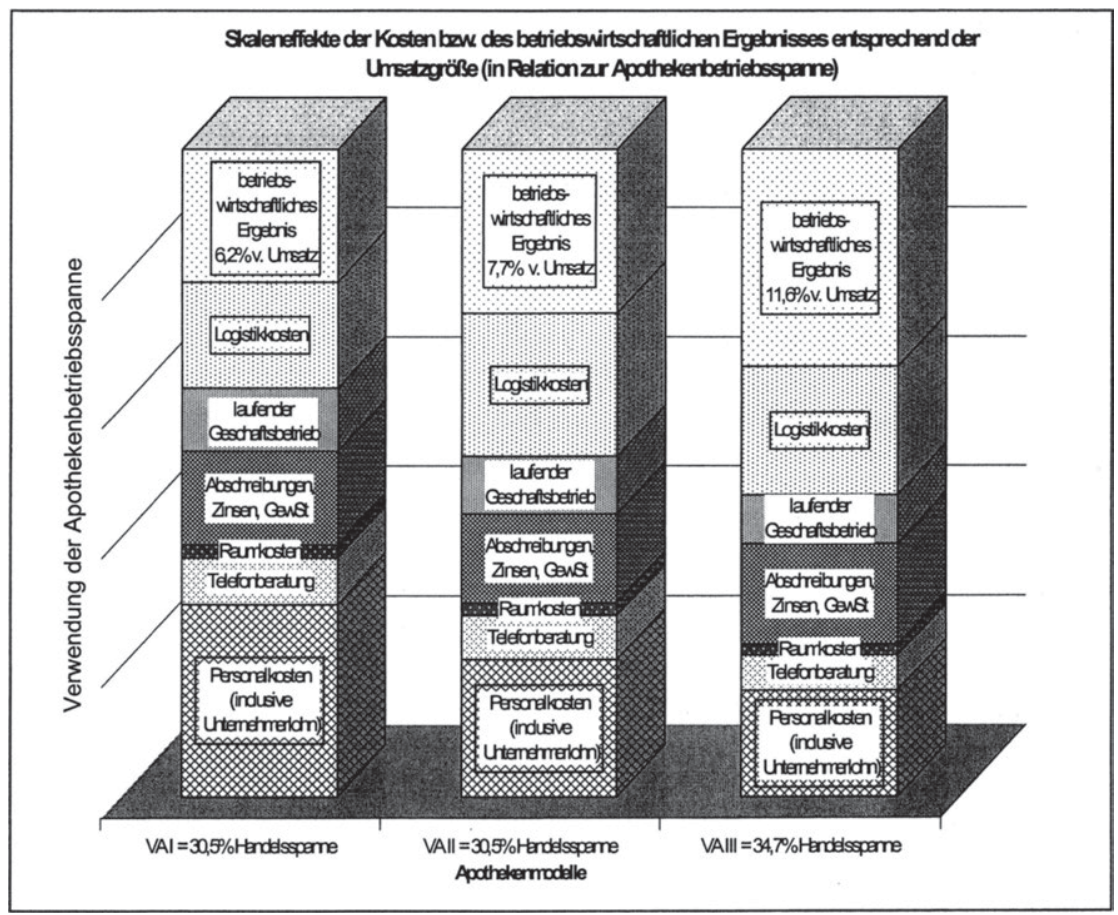

Quelle: INIFES, eigene Darstellung.

\subsection{Markt- und Einsparpotentiale eines Pharmaversandes}

In diesem dritten Modellschritt wird ausführlich auf die Frage eingegangen ${ }^{166}$, welcher Anteil aller nach epidemiologischen Kriterien als versendungsfähig definierten Verordnungen (2.3. Zielsegment des Pharmaversandes) unter Wirkung ökonomischer Restriktionen zu Kosteneinsparungen im Vertrieb beiträgt und welches Einsparpotential für GKV daraus resultieren könnte. Wie bereits dargestellt, erfolgt die Analyse des Marktpotentials in einem mehrstufigen Verfahren.

Das Zielsegment ermittelte sich zunächst aus dem Bereich der Medikation, der als „versandfähig“ mit Blick auf den Endverbraucher Apothekenkunde bezeichnet wer-

166

Nachdem bereits unter 2.4.3., 2.4.4 und 2.4.5. bei der Berechnung der Logistikkosten anhand der durchschnittlichen Verordnungswerte den Marktpotentialvarianten flüchtig vorweggegriffen wurde. 
den kann (Kapitel 2.3). Basierend auf ausländischen Erfahrungen und Expertenmeinungen ist davon auszugehen, dass auch bei optimaler Logistik der Arneimittelversand im Bereich der Akutmedizin nur eine untergeordnete Rolle spielen wird, ${ }^{167}$ während er sich insbesondere für chronische Erkrankungen und Dauerpatienten eher eignet. Das medizinisch und pharmazeutisch sinnvolle Zielsegment wird gemäß der ökonomischen Restriktionen modifiziert, welche sich wiederum aus den Betriebsstrukturen der drei simulierten Versandapothekengrößen errechnen. Daraus resultiert das Marktpotential für einen Pharmaversand.

Aufgrund der Kombination der drei Zielsegmentvarianten A, B und C aus Punkt 2.3. mit den drei Apothekengrößenklassen aus 2.4. ergeben sich neun Marktpotentialausprägungen. Aus diesen neun Marktpotentialausprägungen können in Kombination mit den in Kapitel 2.4. errechneten Kostenvorteilen des Versandhandels neun Varianten an Einsparpotentialen ermittelt werden. Das heißt, je Apothekengrößenklasse ergeben sich drei Varianten an Einsparpotentialen.

In welchem Maß und in welchem Zeitbezug dieses Markt- und Einsparpotential als tatsächliche Marktanteile ${ }^{188}$ bzw. tatsächliche Einsparvolumina durch die potentiellen Anbieter realisiert werden, hängt von weiteren exogenen (betriebsfremden) wie endogenen (betriebsinternen) Restriktionen ab. Denkbar wäre hier insbesondere die Überwindung von Akzeptanzhürden gegenüber Versandapotheken bei Patienten wie verordnenden Ärzten.

\subsubsection{Vorgehensweise der Berechnungen}

Entsprechend der Zielsegmentvarianten $A$ bis $C$ aus 2.3. wird jeweils das maximale Markt- wie Einsparpotential von Versandapotheken unter Beachtung der Umsatzgröße aus 2.4. ermittelt:

1) Dazu wird der indikationsspezifische durchschnittliche Verordnungswert um den Kassenrabatt von $5 \%$ vermindert. Dieser Anteil ist bereits heute bei GKV-Verordnungen durch die Apotheken an die gesetzliche Krankenkasse abzuführen, steht den Präsenzapotheken also nicht zur Kostendeckung zur Verfügung. Entsprechend wird auch bei potentiellen Versendern verfahren.

2) Alle Indikationen, die zusätzlich zu den generellen Handlungskosten die Logistikstückkosten aufgrund eines zu geringen Verordnungswertes aus der Handelsspanne nicht decken und entsprechend mit einem negativen Ergebnis nicht zu Kosteneinsparungen für die GKV beitragen würden, müssen trotz ihrer epidemiologisch begründeten Eignung aus dem Zielsegment entfallen. Diese Verordnun-

167

Dabei wird es Nischenanbieter aufgrund spezialisierter Logistikstrukturen auch in diesen Bereichen, z..B. Impfstoff- und Insulinversand, geben. Hier ist der Endverbraucher allerdings eher eine Institution wie das Gesundheitsamt oder eine Arztpraxis.

Siehe dazu Kapitel 2.8. Überlegungen zu realisierbaren Marktanteilen.

Siehe im Anhang I - Tabelle 1, Tabelle 2 und Tabelle 3. 
gen bilden folglich nicht das Marktpotential, die interne Kostenstruktur wirkt als Markteintrittsbarriere. ${ }^{170}$

Alle Indikationen des Zielsegments, welche die gesamten Handlungskosten inklusive der Logistikstückkosten aus ihrer Handelsspanne decken, können aufgrund des positiven Ergebnisses Kosteneinsparungen für die GKV erwirtschaften. Diese Verordnungen bilden das Marktpotential. ${ }^{171}$

3) Das in Prozent angegebene betriebswirtschaftliche Ergebnis je Versandapothekenmodell entspricht damit dem Kostenvorteil, der erzielt werden kann, wenn unter epidemiologischen wie ökonomischen Kriterien für einen Versand geeignete Indikationen über Versandapotheken vertrieben werden.

4) Der Absolutbetrag für die Ausprägung der Einsparpotentiale ergibt sich, in dem der Kostenvorteil des Versandes auf das Marktpotential (abzüglich 5\% - Kassenrabatt) bezogen wird. Aufgrund der neun Varianten an Marktpotentialen ergeben sich parallel neun Varianten an Einsparpotentialen.

\subsubsection{Ausprägungen der Markt- und Einsparpotentiale}

\subsubsection{Markt- u. Einsparpotential für Zielsegmentvariante A von $41 \%$}

Das epidemiologisch eruierte Zielsegment A (Indikator Packungsgröße ab 50 DDD) mit $41 \%$ vom GKV-Fertigarzneimittelumsatz ist unter Geltung ökonomischer Restriktionen (Deckung aller Handlungskosten inklusive der Logistikkosten) für alle drei Versandapothekenmodelle nur marginal zu modifizieren. Aus dem Zielsegment von 2.3.2.1. entfallen aufgrund eines $z u$ geringen Verordnungswertes die vier Indikationsgruppen (A11, M04, H03, A01).

Das aus dem Zielsegment A resultierende Marktpotential liegt bei 39\%.

Tabelle II - 21: Ausprägungen des Marktpotentials in Abhängigkeit der Umsatzgröße des Zielsegments $A$

\begin{tabular}{|c|c|c|c|}
\hline & VAI & VA II & VA III \\
\hline Marktpotential in Mio. DM & $14.381,50$ & $14.381,50$ & $14.381,50$ \\
\hline $\begin{array}{l}\text { Marktpotential in \% vom GKV-Fertigarzneimittelumsatz } \\
\text { (1999: 36.773,8 Mio. DM) }\end{array}$ & $39 \%$ & $39 \%$ & $39 \%$ \\
\hline (Marktpotential abzüglich 5\% Kassenrabatt in Mio. DM) & $(13.662,43)$ & $(13.662,43)$ & $(13.662,43)$ \\
\hline
\end{tabular}

Quelle: INIFES, eigene Darstellung (siehe dazu ausführlich Tabelle $1 \mathrm{im}$ Anhang I).

\footnotetext{
170

Siehe im Anhang I - Tabelle 1, Tabelle 2 und Tabelle 3.

171 Siehe im Anhang I - Tabelle 1, Tabelle 2 und Tabelle 3.

172 Siehe Nebenrechnung Tabelle 1 im Anhang I.
} 
Zur Berechnung der Einsparpotentiale für dieses Zielsegment A werden die Kostenersparnisse der Versandapotheken - VA I liegt bei ca. $6,3 \%{ }^{173}$, VA II bei ca. $7,8 \%{ }^{174}$ und VA III bei ca. $11,8 \%^{175}$ der Arzneimittelkosten - auf das Marktpotential von 39\% (abzüglich des 5\%igen Kassenrabattes) bezogen.

Tabelle II - 22: Ausprägungen des Einsparpotentials in Abhängigkeit der Umsatzgröße des Zielsegments $A$

\begin{tabular}{|c|c|c|c|}
\hline & VAI & VA II & VA III \\
\hline $\begin{array}{l}\text { Marktpotential am GKV-Fertigarzneimittelumsatz } \\
\text { (Marktpotential abzüglich 5\% Kassenrabatt in Mio. DM) }\end{array}$ & $\begin{array}{c}39 \% \\
(13.662,43)\end{array}$ & $\begin{array}{c}39 \% \\
(13.662,43)\end{array}$ & $\begin{array}{c}39 \% \\
(13.662,43)\end{array}$ \\
\hline Kostenersparnis entsprechend Marktpotentialvariante & $6,3 \%$ & $7,8 \%$ & $11,8 \%$ \\
\hline Einsparpotential d. GKV in Mio. DM ${ }^{176}$ & 861 & 1.060 & 1.607 \\
\hline
\end{tabular}

Quelle: INIFES, eigene Darstellung.

Bei vollständiger Abschöpfung des Marktpotentials wie der Kostenersparnis ergeben sich je nach Umsatzgröße des Versenders 861 Mio. DM (VA I), 1.060 Mio.DM (VA II) bzw. 1.607 Mio. DM (VAIII) Einsparpotential für die GKV durch Versandhandel.

\subsubsection{Markt- u. Einsparpotential für Zielsegmentvariante B von $47 \%$}

Das epidemiologisch eruierte Zielsegment B (Indikator Packungsgröße, gemessen in N3 - Normpackungen des Jahres 1998) mit 47\% vom GKV - Fertigarzneimittelumsatz ist unter Geltung ökonomischer Restriktionen ebenfalls für alle drei Versandapothekenvarianten nur marginale zu modifizieren. Aus dem Zielsegment entfallen einige Indikationsgruppen aufgrund ökonomischer Restriktionen.

Das aus dem Zielsegment B resultierende Marktpotential liegt bei $44 \%$ bzw. $45 \%$.

Tabelle II - 23: Ausprägungen des Marktpotentials in Abhängigkeit der Umsatzgröße des Zielsegments B

\begin{tabular}{|c|c|c|c|}
\hline & VAI & VA II & VA III \\
\hline Marktpotential in Mio. DM & $15.821,32$ & $15.542,48$ & $15.990,23$ \\
\hline $\begin{array}{l}\text { Marktpotential in \% vom GKV-Fertigarzneimittelumsatz } \\
\text { (1998: } 35.661,3 \text { Mio. DM) }\end{array}$ & $44 \%$ & $44 \%$ & $45 \%$ \\
\hline (Marktpotential abzüglich 5\% Kassenrabatt in Mio. DM) & $(15.030,25)$ & $(14.765,36)$ & $(15.190,72)$ \\
\hline
\end{tabular}

Quelle: INIFES, eigene Darstellung (siehe dazu ausführlich Tabelle 2 und Tabelle 4 im Anhang I).

\footnotetext{
173 Siehe Tabelle II - 14 .

174 Siehe Tabelle II - 17.

175 Siehe Tabelle II - 20.
}

176 Die Ergebnisse für das Einsparpotential ergeben sich aus Berechnungen mit ungerundeten Zahlenangaben zur Kostenersparnis bzw. dem Marktpotential, d.h. diese sind auf alle Nachkommastellen exakt ermittelt. Aufgrund der vereinfachten Darstellung in Tabelle II - mit maximal zwei Nachkommastellen ergeben sich marginale Abweichungen. 
Zur Berechnung der Einsparpotentiale für das Zielsegment werden die Kostenersparnisse der Versandapotheken - VA I liegt bei ca. $6,2 \%{ }^{178}$, VA II bei ca. $7,7 \%{ }^{179}$. Und VA III entspricht ca. $11,5 \%{ }^{180}$ der Arzneimittelkosten - auf das Marktpotential von $44 \%$ bzw. $45 \%$ (abzüglich $5 \%$ Kassenrabatt) bezogen.

Tabelle II - 24: Ausprägungen des Einsparpotentials in Abhängigkeit der Umsatzgröße des Zielsegments B

\begin{tabular}{|c|c|c|c|}
\hline & VAI & VA II & VA III \\
\hline $\begin{array}{l}\text { Marktpotential am GKV-Fertigarzneimittelumsatz } \\
\text { (Marktpotential abzüglich 5\% Kassenrabatt in Mio. DM) }\end{array}$ & $\begin{array}{c}44 \% \\
(15.030,25)\end{array}$ & $\begin{array}{c}44 \% \\
(14.765,36)\end{array}$ & $\begin{array}{c}45 \% \\
(15.190,72)\end{array}$ \\
\hline Kostenersparnis entsprechend Marktpotentialvariante & $6,2 \%$ & $7,7 \%$ & $11,5 \%$ \\
\hline Einsparpotential d. GKV in Mio. DM ${ }^{181}$ & 926 & 1.132 & 1.743 \\
\hline
\end{tabular}

Quelle: INIFES, eigene Darstellung.

Um einen vergleichbaren zeitlichen Bezug für die Markt- und Einsparpotentialvarianten des Zielsegments $B$ zu den Zielsegmenten $A$ und $C$ herzustellen, die auf den GKV-Fertigarzneimittelumsatz von 1999 basieren, werden die Marktpotentialausprägungen und die Höhe der prozentualen Kostenersparnis am Marktpotential auf den GKV-Umsatz von 36.773,80 Mio. DM bezogen. Es ergeben sich aus der Hochrechnung die folgenden Werte für Einsparpotentiale:

Tabelle II - 25: Ausprägungen des Einsparpotentials in Abhängigkeit der Umsatzgröße des Zielsegments B Hochrechnung für 1999

\begin{tabular}{|c|c|c|c|}
\hline & VAI & VA II & VA III \\
\hline $\begin{array}{l}\text { Marktpotential am GKV-Fertigarzneimittelumsatz } 1999 \\
\text { (Marktpotential abzüglich 5\% Kassenrabatt in Mio. DM) }\end{array}$ & $\begin{array}{c}44 \% \\
(15.520,90)\end{array}$ & $\begin{array}{c}44 \% \\
(15.247,36)\end{array}$ & $\begin{array}{c}45 \% \\
(15.686,61)\end{array}$ \\
\hline Kostenersparnis entsprechend Marktpotentialvariante & $6,2 \%$ & $7,7 \%$ & $11,5 \%$ \\
\hline Einsparpotential d. GKV in Mio. DM ${ }^{182}$ & 956 & 1.169 & 1.800 \\
\hline
\end{tabular}

Quelle: INIFES, eigene Darstellung.

Siehe Tabelle II - 14 .

Siehe Tabelle II - 17 .

Siehe Tabelle $\|$ - 20 .

181 Die Ergebnisse für das Einsparpotential ergeben sich aus Berechnungen mit ungerundeten Zahlenangaben zur Kostenersparnis bzw. dem Marktpotential, d.h. diese sind auf alle Nachkommastellen exakt ermittelt. Aufgrund der vereinfachten Darstellung in Tabelle II - 23 mit maximal zwei Nachkommastellen ergeben sich marginale Abweichungen.

Die Ergebnisse für das Einsparpotential ergeben sich aus Berechnungen mit ungerundeten Zahlenangaben zur Kostenersparnis bzw. dem Marktpotential, d.h. diese sind auf alle Nachkommastellen exakt ermittelt. Aufgrund der vereinfachten Darstellung in Tabelle II - mit maximal zwei Nachkommastellen ergeben sich marginale Abweichungen.
} 
Bei vollständiger Abschöpfung des Marktpotentials wie der Kostenersparnis ergeben sich je nach Umsatzgröße des Versenders 956 Mio. DM (VA I), 1.168 Mio. DM (VA II) bzw. 1.800 Mio. DM (VAIII) Einsparpotential für die GKV durch Versandhandel.

\subsubsection{Markt- u. Einsparpotential für Zielsegmentvariante $C$ von $57 \%$}

Die bereits betrachtete Zielsegmentvariante $A$ (Packungsgröße ab 50 DDD) ist eine Schnittmenge der Zielsegmentvariante $C$ (Packungsgröße ab 35 DDD). Entsprechend parallel entwickeln sich auch die Markt- und Einsparpotentiale (siehe 2.5.2.1.) für Variante $\mathrm{C}$.

Das epidemiologisch eruierte Zielsegment C mit 57\% vom GKV-Fertigarzneimittelumsatz ist unter Geltung ökonomischer Restriktionen für alle drei Versandapothekenmodelle ebenfalls nur marginal zu modifizieren. Aus dem Zielsegment entfallen aufgrund ökonomischer Restriktionen acht Indikationsgruppen gegenüber dem Zielsegment in 2.3.2.3. ${ }^{183}$

Das aus dem Zielsegment $C$ resultierende Marktpotential liegt bei $52 \%$ bzw. 53\%.

Tabelle II - 26: Ausprägungen des Marktpotentials in Abhängigkeit der Umsatzgröße des Zielsegments $\mathrm{C}$

\begin{tabular}{|c|c|c|c|}
\hline & VAI & VA II & VA III \\
\hline Marktpotential in Mio. DM & $19.519,60$ & $19.248,30$ & $19.519,60$ \\
\hline $\begin{array}{l}\text { Marktpotential in \% vom GKV-Fertigarzneimittelumsatz } \\
\text { (1999: } 36.773,8 \text { Mio. DM) }\end{array}$ & $53 \%$ & $52 \%$ & $53 \%$ \\
\hline (Marktpotential abzüglich 5\% Kassenrabatt in Mio. DM) & $(18.543,60)$ & $(18.285,90)$ & $(18.543,60)$ \\
\hline
\end{tabular}

Quelle: INIFES, eigene Darstellung (siehe dazu ausführlich im Anhang I Tabelle 3).

Zur Berechnung der Einsparpotentiale für das Zielsegment $\mathrm{C}$ werden die Kostenersparnisse der Versandapotheken - VA I liegt bei ca. $6,2 \%{ }^{184}$, VA II bei ca. $7,6 \%{ }^{185}$ und VA III bei ca. $11,6 \%{ }^{186}$ der Arzneimittelkosten - auf das Marktpotential von $52 \%$ bzw. $53 \%$ (abzüglich des $5 \%$ igen Kassenrabattes) bezogen.

\footnotetext{
183 Siehe Nebenrechnung im Anhang I Tabelle 3.

184 Siehe Tabelle II - 14.

185 Siehe Tabelle $\|-17$

186 Siehe Tabelle II -20
} 
Tabelle II-27: Ausprägungen des Einsparpotentials in Abhängigkeit der Umsatzgröße des Zielsegments $\mathrm{C}$

\begin{tabular}{|c|c|c|c|}
\hline & VA I & VA II & VA III \\
\hline $\begin{array}{l}\text { Marktpotential am GKV-Fertigarzneimittelumsatz } \\
\text { (Marktpotential abzüglich 5\% Kassenrabatt in Mio. DM) }\end{array}$ & $\begin{array}{c}53 \% \\
(18.543,6) \\
\end{array}$ & $\begin{array}{c}52 \% \\
(18.285,9) \\
\end{array}$ & $\begin{array}{c}53 \% \\
(18,543,6) \\
\end{array}$ \\
\hline Kostenersparnis entsprechend Marktpotentialvariante & $6,2 \%$ & $7,6 \%$ & $11,6 \%$ \\
\hline Einsparpotential d. GKV in Mio. DM ${ }^{187}$ & 1.142 & 1.398 & 2.144 \\
\hline
\end{tabular}

Quelle: INIFES, eigene Darstellung.

Bei vollständiger Abschöpfung des Marktpotentials wie auch der Kostenersparnis ergeben sich je nach Umsatzgröße des Versenders 1.142 Mio. DM (VA I), 1.398 Mio. DM bzw. 2.144 Mio. DM (VAIII) Einsparpotential für die GKV durch Versandhandel.

\subsubsection{Zwischenfazit zu den Markt- und Einsparpotentialen}

Das epidemiologisch evaluierte Zielsegment aus 2.3. verringert sich gemäß der ökonomischen Restriktionen, die aus den Betriebsstrukturen der drei simulierten Versandapothekenmodelle (2.4.) resultieren. Daraus wird das Marktpotential entwickelt. Aufgrund der Kombination der drei Zielsegmentvarianten A, B und C aus Punkt 2.3. mit den drei Apothekengrößenklassen aus 2.4. ergeben sich neun Marktpotentialausprägungen.

Bei allen drei Ausprägungen des Zielsegments wurde durch die Berücksichtigung einer ökonomischen Restriktion nur eine sehr marginale Modifizierung zwischen $2 \%$ bis $4 \%$ notwendig. Das bedeutet, das aufgrund epidemiologischer Indikatoren erhobene Zielsegment in den Ausprägungen $A, B$ und $C$ findet sich in den entsprechenden Marktpotentialvarianten A, B und C in fast vollem Umfang wider.

Abbildung II - 12: Marktpotentialvarianten

\begin{tabular}{|l|c|c|c|}
\hline Zielsegment - Variante & A & B & C \\
\hline Zielsegment in \% vom GKV-Fertigarzneimittelmarkt 1999 & $41 \%$ & $47 \%$ & $57 \%$ \\
\hline Marktpotential in \% vom GKV-Fertigarzneimittelmarkt 1999 & & & \\
VA I & $39 \%$ & $44 \%$ & $53 \%$ \\
VA II & $39 \%$ & $44 \%$ & $52 \%$ \\
VA III & $39 \%$ & $45 \%$ & $53 \%$ \\
\hline
\end{tabular}

Quelle: INIFES, eigene Darstellung.

187 Die Ergebnisse für das Einsparpotential ergeben sich aus Berechnungen mit ungerundeten Zahlenangaben zur Kostenersparnis bzw. dem Marktpotential, d.h. diese sind auf alle Nachkommastellen exakt ermittelt. Aufgrund der vereinfachten Darstellung in Tabelle II - 26 mit maximal zwei Nachkommastellen ergeben sich marginale Abweichungen. 
Die Skaleneffekte, die sich aufgrund der unterschiedlichen Umsatzgrößen in den Kosten- sowie Ertragsstrukturen der einzelnen Versandapotheken widerspiegeln, haben einen wesentlichen Einfluss auf die Höhe der zu erwartenden Einsparpotentiale für die GKV. Die ausgewiesenen Kosteneinsparungen auf die Arzneimittelpreise gelten unter Annahme der aktuell gültigen Arzneimittelpreisspannenverordnung von 1998. ${ }^{188}$ Entsprechend stellt der Kostenvorteil von ca. 6\% (VA I) ${ }^{189}$ bzw. $8 \%$ (VA II) ${ }^{190}$ bzw. $12 \%$ (VA III) ${ }^{191}$ der Versandapotheken in diesem Segment eine zum derzeitigen 5\%igen GKV-Rabatt zusätzlich mögliche potentielle Preisminderung für solche Arzneimittel, die über den Distributionskanal Versand an die Versicherten gelangen (also nicht für alle Arzneimittel des GKV-Index!). dar. Die Kostenvorteile des Modells VA II liegt in etwa in der von Schweizer Versendern verlautbaren Höhe, wonach die Krankenkassen durch den Direktversand zwischen $10 \%$ bis $15 \%$ der Medikamentenkosten einsparen. ${ }^{192}$ Aufgrund der Reglementierung des Preisniveaus ist ein Vergleich mit den Kosteneinsparungen ausländischer Versender jedoch nur begrenzt auf das deutsche System übertragbar.

Durch die Annahme der Geltung der Arzneimittelpreisverordnung können die entstandenen Kostenvorteile jedoch nicht in Form einer Preisdifferenzierung, d.h. in Form niedrigerer Arzneimittelpreise, der GKV bzw. den Versicherten zugeführt werden. Hier müssen anderen Systeme der Nutzenstiftung des Kostenvorteils in Betracht gezogen werden. Dies könnte z.B. in Form von Rabatten oder Boni erfolgen (siehe folgende Kapitel 2.6. und 2.7.).

Die Ausprägungen des Einsparpotentials ergeben sich jeweils, indem der Kostenvorteil der Versandapotheken auf das Marktpotential (abzüglich 5\% - Kassenrabatt) bezogen wird. Folgende Abbildung gibt einen Überblick über die in 2.5.2 ermittelten Ausprägungen der Einsparpotentiale:

\footnotetext{
188 Effekte, die aus einer möglichen Änderung der Arzneimittelpreisverordnung resultieren, sind nicht Gegenstand der Studie.

Siehe unter 2.4.4.

Siehe unter 2.4.5.

Siehe unter www.mediservice.ch, Zugriff am Juni 2001.

So steht das Schweizer Modell zumindest derzeit durch die Kooperation mit Krankenkassen nicht allen Patienten offen. Zudem findet hier, in der Kooperation zwischen Krankenkasse und "ihrer" Versandapotheke bereits eine Vorauswahl potenzieller Kunden (Versicherte mit Arzneimittel-Ausgaben von mindestens SFr 450 pro Jahr) statt.
} 
Abbildung II - 13: Einsparpotentiale im Überblick

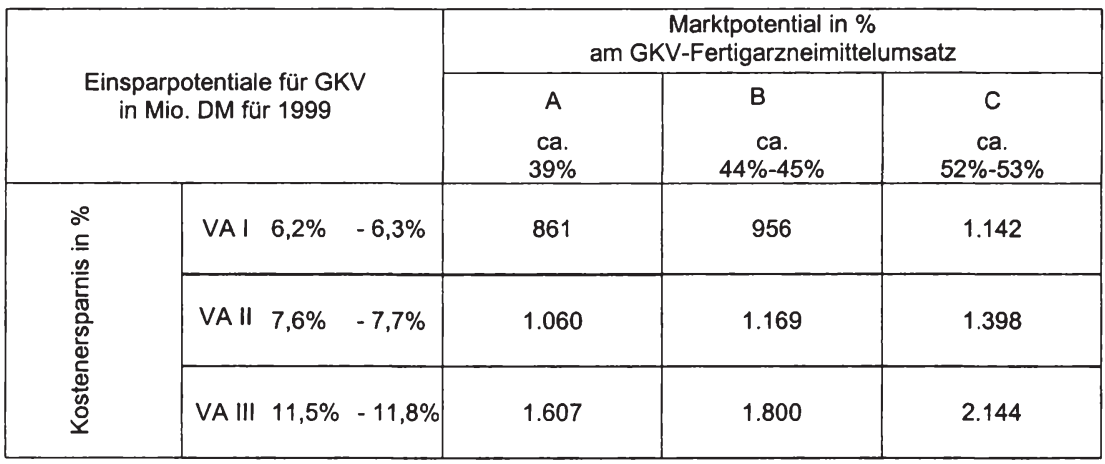

Quelle: INIFES, eigene Darstellung.

Das Intervall möglicher Einsparungen liegt zwischen 861 Mio. DM und 2.144 Mio. DM. Differenziert man diese Betrachtungen nach den Größenklassen der Versandapotheke und den schon angesprochenen Skaleneffekten, zeigt sich, dass je nach Marktpotentialvariante:

- Das Modell der kleinen Versandapotheke mit ca. 5 Mio. DM Umsatz (VA I) mit einer durchschnittlich 6,2\%igen Kosteneinsparung auf die Arzneimittelkosten ein Einsparpotential von 861 Mio. DM bis 1.142 Mio. DM ergibt.

- Das Modell der großen Versandapotheke mit ca. 20 Mio. DM Umsatz (VA II) mit einer durchschnittlich 7,7\%igen Kosteneinsparung ein Einsparpotential von 1.060 Mio. DM bis 1.398 Mio. DM ergibt.

- Das Modell der Versandapotheke mit kombinierter Großhandels- und Apothekenfunktion und einem Umsatz von 487 Mio. DM (VA III) mit einer durchschnittlichen $11,6 \%$ igen Kosteneinsparung ein Einsparpotential von 1.607 Mio. DM bis 2.144 Mio. DM ergibt.

Dies zeigt, dass insbesondere durch umsatzstarke Versender aufgrund deren interner Betriebsstruktur die höchsten Einsparpotentiale innerhalb der entsprechenden Marktpotentiale zu erwarten sind. So liegen die maximal möglichen Einsparvolumina von VA III doppelt so hoch wie die von VA I.

Es wurde bereits darauf hingewiesen, dass die ermittelten Kostenvorteile am Arzneimittelpreis nicht in Form einer Preisdifferenzierung (d.h. in Form geringerer Preise) an die GKV bzw. die Versicherten weitergegeben werden können, da die Annahme der Geltung der Arzneimittelpreisverordnung unterstellt ist. Damit stellt sich die Frage, über welche Erstattungssysteme diese Kostenvorteile den Kostenträgern zugeführt werden können.

Mit den berechneten Kostenvorteilen ist neben den Erstattungsmodalitäten an die GKV auch ein weiterer wichtiger Aspekt verbunden, den es im folgenden ebenfalls zu 
differenzieren gilt. Aus wirtschaftstheoretischen Erwägungen ist davon auszugehen, dass bei einer Deregulierung des Monopols des stationären Vertriebs potentielle Anbieter - seien diese nun neue Akteure oder schon existierende Präsenzapotheken - nur dann einen neuen Vertriebsweg erschließen werden, wenn dazu ein ökonomischer Anreiz besteht. Dieser ist, zumindest in langfristiger Perspektive, mit einer Gewinnerwartung in diesem Markt verbunden.

Beide Aspekte - die Form der Erstattung an die GKV wie die Berücksichtigung ökonomischer Anreize für potentielle Anbieter - bedeuten eine Modifizierung des ermittelten Kostenvorteils dahingehend, dass dieser nicht im vollen Umfang zur Ermittlung von Einsparpotentialen für die GKV zur Verfügung steht. Entsprechend gestaltet sich eine Differenzierung der Kostenvorteil von $6 \%$ bzw. $8 \%$ bzw. $12 \%$ als notwendig:

- Diese kann zum einen erfolgen, in dem der Kostenvorteil durch die Annahme einer durchschnittlichen Gewinnerwartung der potentiellen Anbieter geschmälert wird. Der verbleibende Betrag steht zur Erschließung von Einsparpotentialen der GKV zur Verfügung. (Kapitel 2.6.)

- Zum anderen könnte ein Anteil des Kostenvorteils in Form von einheitlichen Rabatten an die GKV abgeführt werden ${ }^{194}$. Der verbleibende Betrag wäre als Gewinnerwartung für die potentiellen Anbieter zu interpretieren (Kapitel 2.7.)

\subsection{Berücksichtigung einer durchschnittlichen Betriebsrendite in den Modellrechnungen}

Die bisherigen Berechnungen basierten auf der Annahme, dass das gesamte betriebswirtschaftliche Ergebnis der Versandapotheken als Kostenvorteil dem System der GKV zuzurechnen ist.

Eine Modifizierung der Kostenvorteile - und damit auch der entsprechenden Einsparpotentiale - soll jedoch an dieser Stelle erfolgen, denn aufgrund der Wirkung ökonomischer Anreize für individuelles Handeln erscheint eine solche als geboten. Unternehmerische Gewinnüberlegungen sind entsprechend in die weiteren Betrachtungen mit einzubeziehen. Versandapotheken als zusätzliche Marktakteure werden nur dann im Zielsegment agieren, wenn sie zumindest auf mittel- und langfristige Sicht eine positive Unternehmensrendite erwarten können.

Entsprechend muss die Eruierung einer Unternehmensrendite erfolgen. Dies ist allerdings nur als Angabe einer durchschnittlichen Betriebsrendite ${ }^{195}$, wie dies auch für die derzeitigen Präsenzapotheken im Apothekenbetriebsvergleich des IFH Köln bzw. durch die ABDA erfolgt, möglich:

- Für das Jahr 1998 lag die Betriebsrendite der Präsenzapotheken im Durchschnitt bei ca. $0,4 \%$ (siehe Kapitel 2.4.1).

\footnotetext{
194

Diese Rabatte wären zusätzlich zum derzeitigen Kassenrabatt von $5 \%$ zu betrachten.

195

Diese wird im folgenden z.T. auch als Durchschnittliche Betriebsrendite bezeichnet.
} 
- Diese ist jedoch nicht mit einer individuellen Apothekenrendite zu verwechseln, denn wie allgemein existiert auch hier eine positive Korrelation zwischen Umsatzstärke und Rendite. So liegt die Schwankungsbreite einer Präsenzapotheke von 5 Mio. DM Umsatz - diese entspräche aufgrund ihrer Umsatzstärke dem Modell VA I - nach dem IFH Köln zwischen $0 \%$ und $10 \%$.

- Zudem ist eine durchschnittliche Betriebsrendite keine garantierte Rendite. Das heißt, die Möglichkeit eines wirtschaftlichen Misserfolgs in Form eines negativen Ergebnisses der individuellen Apothekenrendite ist auch bei einem positiven branchenbezogenen Durchschnittswert gegeben.

Analog dieser Argumentation ist auch die Einbeziehung einer durchschnittlichen Betriebsrendite für Versandapotheken in die weiteren Modellrechnungen zu verstehen. Es handelt sich dabei weder um eine individuelle noch um eine garantierte Unternehmensrendite.

Eine Modifizierung der Höhe der durchschnittlichen Betriebsrendite von Versandapotheken gegenüber Präsenzapotheken muss allerdings in deren Höhe erfolgen. Wie der zweite Argumentationspunkt verdeutlicht, korrelieren wie allgemein im Wirtschaftssektor auch im Apothekensektor Umsatz und Rendite miteinander. Da in den simulierten Modellen für Versandapotheken von wesentlich umsatzstärkeren Akteuren im Vergleich zur durchschnittlichen Präsenzapotheke mit 2,5 Mio. DM Umsatz ausgegangen wird - dieses Spektrum liegt zwischen einer Verdopplung des Umsatzes bis zu einer 250fachen Umsatzerhöhung - muss sich dieser statistische Zusammenhang auch in der Höhe einer durchschnittlichen Betriebsrendite für Versandapotheken widerspiegeln.

Natürlich gestaltet sich die Ermittlung einer durchschnittlichen Betriebsrendite für Versandapotheken als schwierig. Hier kann nur mit einer Annahme gearbeitet werden. Das Vorgehen gestaltet sich dabei an der Minimaldefinition, dass potentielle Anbieter einen ökonomischen Anreiz verfolgen, um neue Marktstrukturen zu erschließen. Sie erwarten sich also laut Annahme eine Betriebsrendite größer Null. Entsprechend erfolgt die Berechnung einer Rendite für Versandapotheken anhand der Präsenzapotheken, die bereits eine positive Rendite aufweisen. Aus dem gewichteten Durchschnitt ergibt sich ein Wert von ca. 3,5\% Betriebsrendite.

\footnotetext{
196

Müller-Hagedorn, L. (1998), Strategische Optionen für die Apotheke, S. 38, in: Kaapke, A. (Hrsg), Management in Apotheken, Sonderheft 43 des IFH Köln, S. $35-55$.

Würde eine durchschnittliche Betriebsrendite von $0,4 \%$ analog den Präsenzapotheken zugrunde gelegt, hätte dies zur Folge, dass der Anteil des Kostenvorteils für die GKV höher ausfällt als bei einer entsprechend höher angesetzten Durchschnittliche Betriebsrendite. Folglich würden auch die Einsparpotentiale hŏher ausfallen als im folgenden dargestellt.
} 
Tabelle II - 28: Berechnung einer durchschnittlichen Betriebsrendite für Versandapotheken

\begin{tabular}{|c|c|c|c|c|c|}
\hline $\begin{array}{c}\text { Renditespanne von } \\
\text { bis... }\end{array}$ & $\begin{array}{c}\text { relative Häufigkeit } \\
\text { der Apotheken }\end{array}$ & $\begin{array}{c}\text { Durchschnittsrendit } \\
\text { e je Klasse }\end{array}$ & $\begin{array}{c}\text { Gewichtung bzgl. } \\
\text { positive Rendite }\end{array}$ & $\begin{array}{c}\text { Gewichtete } \\
\text { Renditen je } \\
\text { Klasse in \% }\end{array}$ \\
\hline 0,00 & 2,50 & 26,9 & 1,3 & $43 \%$ & 0,5 \\
\hline 2,51 & 5,00 & 20,9 & 3,8 & $33 \%$ & 1,3 \\
\hline 5,01 & 7,50 & 9,4 & 6,3 & $15 \%$ & 0,9 \\
\hline 7,51 & 10,00 & 4,0 & 8,8 & $6 \%$ & 0,6 \\
\hline 10,01 & 12,50 & 1,4 & 11,3 & $2 \%$ & 0,3 \\
\hline & & 62,6 & & $100 \%$ & 3,5 \\
\hline
\end{tabular}

Quelle: INIFES, eigene Darstellung nach Müller-Hagedorn, L., (1998), Strategische Optionen für die Apotheke, S. 37, in: Kaapke, A. (Hrsg), Management in Apotheken, Sonderheft 43 des IFH Köln, S. 35-55.

Es sei noch einmal darauf verwiesen, dass dieser Wert eine Orientierung mangels vorhandener Daten darstellt. Grundsätzlich weisen Unternehmen, und somit auch potentielle Versandapotheken, eine individuelle Rendite auf, die - wie bei Präsenzapotheken auch - im positiven wie negativen Bereich einer bedeutsamen Schwankungsbreite liegen kann und nicht garantiert ist, beeinflusst durch die verschiedensten betrieblichen Faktoren. Auf die Wirkung möglicher economies of scale wurde bereits unter 2.4.3. bis 2.4.5. ausführlich eingegangen.

Zudem spielt die zeitliche Entwicklung einer Rendite eine wesentliche Rolle. Deshalb soll der ermittelte Wert einer 3,5\%igen Rendite zum einen als Durchschnittswert bezüglich einer gesamten Branche, nicht nur für eine einzelne Versandapotheke, und zum anderen als Durchschnittswert aus kurz- und mittelfristiger Perspektive interpretiert und in dieser Interpretation in die Modellrechnungen implementiert werden.

\subsubsection{Einflüsse einer Betriebsrendite auf das Einsparpotential}

Die Berücksichtigung einer Betriebsrendite in den Berechnungen bedeutet nicht, dass von den bisherigen durchschnittlichen Kosteneinsparungen der Versandapotheken von 6,2\% (VAI) bzw. 7,7\% (VA II) bzW. 11,6\% (VA III) ${ }^{198}$ eine entsprechende Durchschnittliche Betriebsrendite von 3,5\% abgezogen werden müsste und sich bei entsprechender Multiplikation mit dem Marktpotential das neue Einsparpotential berechnen ließe. Die Berücksichtigungen einer Durchschnittliche Betriebsrendite ist wesentlich vielschichtiger.

Die Berücksichtigung einer durchschnittlichen Betriebsrendite in den Berechnungen bedeutet, dass diese in die internen Kalkulationen der Versandapotheke als zusätzliche zu deckende Kosten zu behandeln ist. Das heißt, neben den in 2.4.3. bis 2.4.5. ausgewiesenen Kosten inklusive der Logistikkosten muss nun zusätzlich eine Kostenposition von 3,5\% berücksichtigt werden, die es aus den Vertriebsspannen der 
Verordnungswerte des Zielsegments zu decken gilt. Entsprechend weniger Verordnungen werden im Vergleich zu den bisherigen Berechnungen für einen Pharmaversand in Frage kommen.

Folglich wirken zwei wesentliche Effekte auf das Einsparpotential:

- Zum einen fallen die Kostenersparnisse, die an die Krankenkassen weitergeleitet werden könnten, wesentlich geringer aus. $\rightarrow$ geringerer Kostenvorteil im Vergleich zu 2.4.3. bis 2.4.5.

- Zum anderen sinkt die Anzahl der Verordnungen, die neben allen Kosten inklusive der Logistikstückkosten zusätzlich eine durchschnittliche Betriebsrendite von $3,5 \%$ decken könnten. Damit sinkt das jeweilige Marktpotential im Vergleich der Berechnungen ohne durchschnittliche Betriebsrendite unter 2.5.2. erheblich. Entsprechend erhöhen sich die durchschnittlichen Verordnungswerte je Marktpotentialvariante. $\rightarrow$ geringeres Marktpotential im Vergleich zu 2.5.2.

Da das Einsparpotential aus beiden Einflussfaktoren - dem Marktpotential wie der Kostenersparnis - gebildet wird, unterliegt es einer überproportionalen Verringerung durch die Berücksichtigung einer durchschnittlichen Betriebsrendite, wie im folgenden für die drei Zielsegmentvarianten $\mathrm{A}$ bis $\mathrm{C}$ gezeigt wird.

\subsubsection{Ausprägungen der Einsparpotentiale bei Berücksichtigung einer durch- schnittlichen Betriebsrendite}

Durch die Berücksichtigung einer Gewinnüberlegung von $3,5 \%$ in den Kosten fällt zum einen die Komponente der Kostenersparnis geringer aus. Dazu erfolgt ein kurzer Rückgriff auf die Kostenstruktur der Versandapotheken unter 2.4.3. bis 2.4.5:

Die dort ausgewiesenen betriebswirtschaftlichen Ergebnisse vor der Berücksichtigung der Logistikkosten ${ }^{199}$ von ursprünglich $10,4 \%$ (VAI) bzw. 13,4\% (VA II) bzw. $17,4 \%$ (VAIII) müssen um die durchschnittliche Betriebsrendite in Höhe von 3,5\% gekürzt werden, da diese wie eine Kostenposition zu behandeln ist. Entsprechend verringern sich die Spannen auf 6,9\% (VA I), 9,9\% (VA II) bzw. 13,9\% (VA III), aus denen die Logistikkosten je Verordnung von 3,48 DM bzw. 4,79 DM gedeckt werden müssen.

Folglich dezimiert sich gegenüber den Berechnungen ohne durchschnittliche Betriebsrendite in Kapitel 2.5. die Anzahl der Verordnungen, die für einen Versandhandel auch unter den veränderten Bedingungen in Frage kämen. Daraus resultiert zum einen ein geringeres Marktpotential (mit entsprechend gestiegenen durchschnittlichen Verordnungswerten), und zum anderen eine geringerer Kostenvorteil, der den gesetzlichen Kassen für die Erschließung von Einsparpotentialen zufließen würde.

199

Tabelle II - 12 für VA I, Tabelle II - 15 für VA II, Tabelle II - 18 für VA III. 


\subsubsection{Einsparpotential für Zielsegmentvariante $A$ von $41 \%$ (bei durchschnitt- licher Betriebsrendite)}

Auf Zielsegment A (Packungen von mindestens 50 DDD je Verordnung) wirken sich diese Einflussfaktoren auf das Marktpotential sowie einen durch Versandhandel möglichen Kostenvorteil folgendermaßen aus:

Tabelle II - 29: Ausprägungen des Marktpotentials und der Kostenersparnis bei einer durchschnittlichen Betriebsrendite von 3,5\% für Zielsegment $A$

\begin{tabular}{|c|c|c|c|}
\hline & VA I & VA II & VA III \\
\hline Marktpotential in Mio. DM & $11.223,3$ & $11.223,3$ & $14.381,50$ \\
\hline $\begin{array}{l}\text { Marktpotential in \% vom GKV-Fertigarzneimittelumsatz } \\
\text { (1999: } 36.773,8 \text { Mio. DM) }\end{array}$ & $31 \%$ & $31 \%$ & $39 \%$ \\
\hline Durchschnittlicher Verordnungswert in DM & 93,76 & 93,76 & 75,10 \\
\hline Durchschnittlicher Verordnungswert in DM (abzgl. 5\%) & 89,07 & 89,07 & 71,35 \\
\hline Kostenersparnis = gestaffelter Kassenrabatt & $3,6 \%$ & $5,4 \%$ & $8,3 \%$ \\
\hline Kostenersparnis nach Abzug der Betriebsrendite (vor Logistikkosten) ${ }^{201}$ & $6,9 \%$ & $9,9 \%$ & $13,9 \%$ \\
\hline Logistikstückkosten in $\left.\mathrm{DM}^{*}\right)$ & 3,48 & 4,79 & 4,79 \\
\hline Logistikkosten in \% vom Verordnungswert (abzgl. $\left.5 \%)^{*}\right)$ & $3,9 \%$ & $5,4 \%$ & $6,7 \%$ \\
\hline Korrektur Gewerbesteuer auf Logistikkosen*) & $0,6 \%$ & $0,9 \%$ & $1,1 \%$ \\
\hline
\end{tabular}

*) siehe entsprechend Tabelle II - 13, Tabelle II - 16, Tabelle II - 19 ohne Betriebsrendite

Quelle: INIFES, eigene Darstellung.

Das Marktpotential für die Versandapotheken I und II umfasst statt $39 \%$ bei Berechnung ohne durchschnittliche Betriebsrendite nun nur noch maximal $31 \%$ des GKV - Fertigarzneimittelumsatzes. Damit entfällt circa. ein Viertel aller Verordnungen des Zielsegments A aufgrund der internen Umsatz- und Kostenstruktur. Das Marktpotential von Versandapotheke III bleibt im Vergleich zur Berechnung ohne durchschnittliche Betriebsrendite in 2.4.5. unverändert.

Die Kostenersparnis auf die Arzneimittelpreise beträgt nunmehr nur noch $3,6 \%$ (VA 1), 5,4\% (VA II) bzw. 8,3\% (VAIII). Diese könnten in Form eines zusätzlich zum derzeitigen Kassenrabatt von $5 \%$ zu erhebenden Kassenrabatts der GKV zukommen. Allerdings wären diese Kassenrabatte ihrer Natur her entsprechen der Umsätze gestaffelt, und damit nicht wie die bisherige Rabatterhebung der Kassen einheitlich, das heißt unabhängig vom Umsatz.

\footnotetext{
200

Siehe Nebenrechnung im Anhang I, Tabelle 2 bis 5.
201
Tabelle II - 12 für VA I, Tabelle II - 15 für VA II, Tabelle II - 18 für VA III: abzüglich 3,5\% Gewinnkalkulation.
} 
(Exkurs: Aufgrund der für Versandapotheke VA I und VA II gestiegenen Verordnungswerte gegenüber der Berechnung ohne Betriebsrendite ${ }^{202}$ verringert sich bei Konstanz der Logistikstückkosten je Verordnung deren prozentualer Wert.)

Das Einsparpotential ergibt sich - analog den Berechnungen ohne durchschnittliche Betriebsrendite in 2.5.2. - aus der Kombination beider Einflussfaktoren sowie der Annahme einer vollständigen Ausschöpfung:

Tabelle II - 30: Ausprägungen des Einsparpotentials bei einer durchschnittlichen Betriebsrendite von 3,5\% für Zielsegment $A$

\begin{tabular}{|c|c|c|c|}
\hline & VA I & VA II & VA III \\
\hline $\begin{array}{l}\text { Marktpotential am GKV-Fertigarzneimittelumsatz } \\
\text { (Marktpotential abzüglich 5\% Kassenrabatt in Mio. DM) }\end{array}$ & $\begin{array}{c}31 \% \\
(10.662,14)\end{array}$ & $\begin{array}{c}31 \% \\
(10.662,14)\end{array}$ & $\begin{array}{c}39 \% \\
(13.662,43)\end{array}$ \\
\hline Kostenersparnis entsprechend Marktpotentialvariante & $3,6 \%$ & $5,4 \%$ & $8,3 \%$ \\
\hline Einsparpotential d. GKV in Mio. DM ${ }^{203}$ & 386 & 574 & 1.129 \\
\hline
\end{tabular}

Quelle: INIFES, eigene Darstellung.

Bei vollständiger Ausschöpfung des Marktpotentials ergeben sich entsprechend der verringerten Kostenersparnisse je nach Umsatzgröße des Versenders zwischen 386 Mio. DM (VA I) bis 1.129 Mio. DM (VAIII) Einsparpotential für die GKV.

Ein Vergleich des Einsparpotentials ohne und mit durchschnittlicher Betriebsrendite zeigt insbesondere für Versandapotheke I und II erhebliche Unterschiede. Zurückzuführen ist dies auf die zwei vorab genannten Effekte eines gesunkenen Marktpotentials sowie einer modifizierten Kostenersparnis. Für Versandapotheke III ist vor allem der letzte Effekt - also eine Einbuße bei den Kostenersparnissen und damit verringerte Rabattgewährungen - zu beobachten. Deshalb unterliegt das Einsparpotential hier nicht diesen starken Schwankungen.

Siehe Tabelle $\|$ - 13 für VA I und Tabelle $\|$ - 16 für VA II. Nachkommastellen exakt ermittelt. Aufgrund der vereinfachten Darstellung Tabelle II - mit maximal zwei Nachkommastellen ergeben sich marginale Abweichungen. 


\subsubsection{Einsparpotential für Zielsegmentvariante $B$ von $47 \%$ (bei durchschnitt- licher Betriebsrendite)}

Die Vorgehensweise erfolgt analog der unter 2.6.2. genannten, d.h. durch die Berücksichtigung einer durchschnittlichen Betriebsrendite von 3,5\% müssen die Logistikkosten je Verordnung von 3,48 DM bzw. 4,79 DM aus einem niedrigeren betriebswirtschaftlichen Ergebnis vor Logistikkosten von 6,9\% (VA I), 9,9\% (VA II) bzw. 13,9\% (VA III) gedeckt werden.

Auf Zielsegment B (Indikator Packungsgröße, gemessen in N3 - Normpackungen) wirken sich diese Einflussfaktoren auf das Marktpotential sowie eine mögliche Kostenersparnis durch Versandhandel folgendermaßen aus:

Tabelle II - 31: Ausprägungen des Marktpotentials und der Kostenersparnis bei einer durchschnittlichen Betriebsrendite von 3,5\% für Zielsegment B

\begin{tabular}{|c|c|c|c|}
\hline & VA I & VA II & VA III \\
\hline Marktpotential in Mio. DM & $13.044,57$ & $13.044,57$ & $15.821,32$ \\
\hline $\begin{array}{l}\text { Marktpotential in \% vom GKV-Fertigarzneimittelumsatz } \\
\text { (1998: } 35.661,3 \text { Mio. DM) }\end{array}$ & $37 \%$ & $37 \%$ & $44 \%$ \\
\hline Durchschnittlicher Verordnungswert in DM & 83,38 & 83,38 & 72,57 \\
\hline Durchschnittlicher Verordnungswert in DM (abzgl. 5\%) & 79,21 & 79,21 & 68,94 \\
\hline Kostenersparnis = gestaffelter Kassenrabatt & $3,2 \%$ & $4,8 \%$ & $8,1 \%$ \\
\hline Kostenersparnis nach Abzug der Betriebsrendite (vor Logistikkosten) ${ }^{205}$ & $6,9 \%$ & $9,9 \%$ & $13,9 \%$ \\
\hline Logistikstückkosten in DM*) & 3,48 & 4,79 & 4,79 \\
\hline Logistikkosten in \% vom Verordnungswert (abzgl. $\left.5 \%)^{*}\right)$ & $4,4 \%$ & $6,0 \%$ & $6.9 \%$ \\
\hline Korrektur Gewerbesteuer auf Logistikkosen*) & $0,7 \%$ & $0,9 \%$ & $1,1 \%$ \\
\hline
\end{tabular}

*) Siehe entsprechend Tabelle II - 13, Tabelle II - 16, Tabelle II - 19 ohne Betriebsrendite.

Quelle: INIFES, eigene Darstellung.

Das Marktpotential für die Versandapotheken I und II umfasst statt $44 \%$ bei Berechnung ohne durchschnittliche Betriebsrendite nun nur noch maximal $37 \%$ des GKV - Fertigarzneimittelumsatzes. Auch hier kann fast ein Viertel aller Verordnungen des Zielsegments aufgrund der internen Betriebsstruktur nicht in das Marktpotential überführt werden. Für die umsatzstarke Versandapotheke III erschließt sich mit $44 \%$ fast das identische Marktpotential wie unter 2.4.5. ohne Berücksichtigung einer durchschnittlichen Betriebsrendite.

\footnotetext{
204

Siehe Nebenrechnung im Anhang I, Tabelle II - 6 und Tabelle II - 8 . 205 Tabelle II - 12 für VA I, Tabelle II - 15 für VA II, Tabelle II - 18 für VA III: abzüglich 3,5\%
Gewinnkalkulation.
} 
Die Kostenersparnis auf die Arzneimittelpreise beträgt nunmehr nur noch 3,2\% (VA I), 4,8\% (VA II) bzw. 8,1\% (VAIII). Diese könnten in Form eines zusätzlich zum derzeitigen Kassenrabatt von $5 \%$ zu erhebenden Kassenrabatts der GKV zukommen. Allerdings wären diese Kassenrabatte ihrer Natur her entsprechen der Umsätze gestaffelt, und damit nicht wie die bisherige Rabatterhebung der Kassen einheitlich, das heißt unabhängig vom Umsatz.

(Exkurs: Aufgrund der gestiegenen Verordnungswerte gegenüber der Berechnung ohne Durchschnittliche Betriebsrendite ${ }^{206}$ verringert sich bei Konstanz der Logistikstückkosten je Verordnung deren prozentualer Wert.)

Das Einsparpotential ergibt sich - analog den Berechnungen ohne durchschnittliche Betriebsrendite in 2.5.2. - aus der Kombination beider Einflussfaktoren sowie der Annahme einer vollständigen Ausschöpfung:

Tabelle II - 32: Ausprägungen des Einsparpotentials bei einer durchschnittlichen Betriebsrendite von 3,5\% für Zielsegment B

\begin{tabular}{|c|c|c|c|}
\hline & VAI & VA II & VA III \\
\hline $\begin{array}{l}\text { Marktpotential am GKV-Fertigarzneimittelumsatz } \\
\text { (Marktpotential abzüglich } 5 \% \text { Kassenrabatt in Mio. DM) }\end{array}$ & $\begin{array}{c}37 \% \\
(12.392,35)\end{array}$ & $\begin{array}{c}37 \% \\
(12.392,35)\end{array}$ & $\begin{array}{c}44 \% \\
(15.030,25)\end{array}$ \\
\hline Kostenersparnis entsprechend Marktpotentialvariante & $3,2 \%$ & $4,8 \%$ & $8,1 \%$ \\
\hline Einsparpotential d. GKV in Mio. DM ${ }^{207}$ & 398 & 597 & 1.212 \\
\hline
\end{tabular}

Quelle: INIFES, eigene Darstellung.

Bei vollständiger Ausschöpfung des Marktpotentials ergeben sich entsprechend der verringerten Kostenersparnis je nach Umsatzgröße des Versenders zwischen 398 Mio. DM (VA I) bis 1.212 Mio. DM (VAIII) Einsparpotential für die GKV.

Ein Vergleich des Einsparpotentials ohne und mit durchschnittlicher Betriebsrendite zeigt insbesondere für Versandapotheke I und II erhebliche Unterschiede. Zurückzuführen ist dies auf die zwei vorab genannten Effekte eines gesunkenen Marktpotentials sowie einer modifizierten Kostenersparnis. Für Versandapotheke III ist vor allem der letztgenannte Effekt zu beobachten, das Marktpotential blieb fast unverändert. Deshalb unterliegt das Einsparpotential hier nicht diesen starken Schwankungen. Nachkommastellen exakt ermittelt. Aufgrund der vereinfachten Darstellung Tabelle II - mit maximal zwei Nachkommastellen ergeben sich marginale Abweichungen.
} 
Um einen vergleichbaren zeitlichen Bezug für die Markt- und Einsparpotentialvarianten des Zielsegments $B$ zu den Zielsegmenten $A$ und $C$ herzustellen, die auf den GKV-Fertigarzneimittelumsatz von 1999 basieren, werden die Marktpotentialausprägungen und die Höhe der prozentualen Kostenersparnis am Marktpotential auf den GKV-Umsatz von 36.773,80 Mio. DM bezogen. Es ergeben sich folgende Näherungswerte für die Einsparpotentiale:

Tabelle II - 33: Ausprägungen des Einsparpotentials bei einer durchschnittlichen Betriebsrendite von 3,5\% für Zielsegment B Hochrechnung für 1999

\begin{tabular}{|c|c|c|c|}
\hline & VA I & VA II & VA III \\
\hline $\begin{array}{l}\text { Marktpotential am GKV-Fertigarzneimittelumsatz } 1999 \\
\text { (Marktpotential abzüglich 5\% Kassenrabatt in Mio. DM) }\end{array}$ & $\begin{array}{c}37 \% \\
(12.796,88)\end{array}$ & $\begin{array}{c}37 \% \\
12.796,88)\end{array}$ & $\begin{array}{c}44 \% \\
(15.520,90)\end{array}$ \\
\hline Kostenersparnis entsprechend Marktpotentialvariante & $3,2 \%$ & $4,8 \%$ & $8,1 \%$ \\
\hline Einsparpotential d. GKV in Mio. DM ${ }^{20}$ & 411 & 617 & 1.252 \\
\hline
\end{tabular}

Quelle: INIFES, eigene Darstellung.

Bei vollständiger Ausschöpfung des Marktpotentials ergeben sich entsprechend der verringerten Kostenersparnis je nach Umsatzgröße des Versenders zwischen 411 Mio. DM (VA I) bis 1.252 Mio. DM (VAIII) Einsparpotential für die GKV.

\subsubsection{Einsparpotential für Zielsegmentvariante $C$ von $57 \%$ (bei durchschnitt- licher Betriebsrendite)}

Die Vorgehensweise erfolgt analog der unter 2.6.2. genannten, d.h. durch die Berücksichtigung einer durchschnittlichen Betriebsrendite von 3,5\% müssen die Logistikkosten je Verordnung von 3,48 DM bzw. 4,79 DM aus einem niedrigeren betriebswirtschaftlichen Ergebnis vor Logistikkosten von 6,9\% (VA I), 9,9\% (VA II) bzw. 13,9\% (VA III) gedeckt werden.

Auf Zielsegment $C$ (Packungen von mindestens 35 DDD je Verordnung) wirken sich diese Einflussfaktoren auf das Marktpotential sowie eine mögliche Kostenersparnis durch Versandhandel folgendermaßen aus:

Die Ergebnisse für das Einsparpotential ergeben sich aus Berechnungen mit ungerundeten Zahlenangaben zur Kostenersparnis bzw. dem Marktpotential, d.h. diese sind auf alle Nachkommastellen exakt ermittelt. Aufgrund der vereinfachten Tabelle II - 32 mit maximal zwei Nachkommastellen ergeben sich marginale Abweichungen. 
Tabelle II - 34: Ausprägungen des Marktpotentials und der Kostenersparnis bei einer durchschnittlichen Betriebsrendite von 3,5\% für Zielsegment C

\begin{tabular}{|c|c|c|c|}
\hline & VA I & VA II & VA III \\
\hline Marktpotential in Mio. DM & $16.052,6$ & $16.052,6$ & $19.248,3$ \\
\hline $\begin{array}{l}\text { Marktpotential in \% vom GKV-Fertigarzneimittelumsatz } \\
\text { (1999: } 36.773,8 \text { Mio. DM) }\end{array}$ & $44 \%$ & $44 \%$ & $52 \%$ \\
\hline Durchschnittlicher Verordnungswert in DM & 85,07 & 85,07 & 73,61 \\
\hline Durchschnittlicher Verordnungswert in DM (abzgl. 5\%) & 80,82 & 80,82 & 69,93 \\
\hline Kostenersparnis = gestaffelter Kassenrabatt & $3,3 \%$ & $4,9 \%$ & $8,1 \%$ \\
\hline Kostenersparnis nach Abzug der Betriebsrendite (vor Logistikkosten) $^{210}$ & $6,9 \%$ & $9,9 \%$ & $13,9 \%$ \\
\hline Logistikstückkosten in $\left.\mathrm{DM}^{*}\right)$ & 3,48 & 4,79 & 4,79 \\
\hline Logistikkosten in \% vom Verordnungswert (abzgl. $\left.5 \%)^{*}\right)$ & $4,3 \%$ & $5,9 \%$ & $6,9 \%$ \\
\hline Korrektur Gewerbesteuer auf Logistikkosen*) & $0,7 \%$ & $0,9 \%$ & $1,1 \%$ \\
\hline
\end{tabular}

") Siehe entsprechend Tabelle II - 13, Tabelle II - 16, Tabelle II - 19 ohne Betriebsrendite.

Quelle: INIFES, eigene Darstellung.

Das Marktpotential für die Versandapotheken I und II umfasst statt $53 \%$ bzw. $52 \%$ bei Berechnung ohne durchschnittliche Betriebsrendite nun nur noch maximal $44 \%$ des GKV - Fertigarzneimittelumsatzes. Damit entfällt auch in dieser Variante circa. ein Viertel aller Verordnungen des Zielsegments aufgrund der internen Umsatz- und Kostenstruktur. Das Marktpotential von Versandapotheke III bleibt im Vergleich zur Berechnung ohne durchschnittliche Betriebsrendite in 2.4.5. fast unverändert.

Die Kostenersparnis auf die Arzneimittelpreise beträgt nunmehr nur noch $3,3 \%$ (VA I), 4,9\% (VA II) bzw. 8,1\% (VAIII). Diese könnten in Form eines zusätzlich zum derzeitigen Kassenrabatt von 5\% zu erhebenden Kassenrabatts der GKV zukommen. Allerdings wären diese Kassenrabatte ihrer Natur her entsprechen der Umsätze gestaffelt, und damit nicht wie die bisherige Rabatterhebung der Kassen einheitlich, das heißt unabhängig vom Umsatz.

(Exkurs: Aufgrund der für Versandapotheke I und II gestiegenen Verordnungswerte gegenüber der Berechnung ohne durchschnittliche Betriebsrendite ${ }^{211}$ verringert sich bei Konstanz der Logistikstückkosten je Verordnung deren prozentualer Wert.)

Das Einsparpotential ergibt sich - analog den Berechnungen ohne durchschnittliche Betriebsrendite unter 2.5.2. - aus der Kombination beider Einflussfaktoren bei der Annahme vollständiger Ausschöpfung:

210

Tabelle II - 12 für VA I, Tabelle II - 15 für VA II, Tabelle II - 18 für VA III: abzüglich 3,5\% Gewinnkalkulation.

211

Siehe Tabelle II - 13 für VA I und Tabelle $\|$ - 16 für VA II. 
Tabelle II - 35: Ausprägungen des Einsparpotentials bei einer durchschnittlichen Betriebsrendite von 3,5\% für Zielsegment C

\begin{tabular}{|c|c|c|c|}
\hline & VAI & VA II & VA III \\
\hline $\begin{array}{l}\text { Marktpotential am GKV-Fertigarzneimittelumsatz } \\
\text { (Marktpotential abzüglich 5\% Kassenrabatt in Mio. DM) }\end{array}$ & $\begin{array}{c}44 \% \\
(15.249,97)\end{array}$ & $\begin{array}{c}44 \% \\
(15.249,97)\end{array}$ & $\begin{array}{c}52 \% \\
(18.285,89)\end{array}$ \\
\hline Kostenersparnis entsprechend Marktpotentialvariante & $3,3 \%$ & $4,9 \%$ & $8,1 \%$ \\
\hline Einsparpotential d. GKV in Mio. DM ${ }^{212}$ & 501 & 750 & 1.490 \\
\hline
\end{tabular}

Quelle: INIFES, eigene Darstellung.

Bei vollständiger Ausschöpfung des Marktpotentials ergeben sich entsprechend der verringerten Kostenersparnis je nach Umsatzgröße des Versenders zwischen 501 Mio. DM (VA I) bis 1.490 Mio. DM (VA III) Einsparpotential für die GKV

Ein Vergleich des Einsparpotentials ohne und mit durchschnittlicher Betriebsrendite zeigt insbesondere für Versandapotheke I und II erhebliche Unterschiede. Zurückzuführen ist dies auf die zwei vorab genannten Effekte eines gesunkenen Marktpotentials sowie einer modifizierten Kostenersparnis. Für Versandapotheke III ist vor allem der letztgenannte Effekt zu beobachten. Deshalb unterliegt das Einsparpotential hier nicht diesen starken Schwankungen.

\subsubsection{Zwischenfazit zur Berücksichtigung einer durchschnittlichen Betriebs- rendite in den Modellrechnungen}

Das Pro und Contra einer Modellrechnung mit durchschnittlicher Betriebsrendite wurde zu Beginn des Kapitels ausführlich dargestellt. Dabei wird zum einen der Sachverhalt einer im Voraus zu kalkulierenden Betriebsrendite grundsätzlich thematisiert, zum anderen jedoch auch die Ausprägung des Renditewertes. Der in der Studie verwendete Schätzwert von 3,5\% steht im Kontext einer durchschnittlichen Rendite von Versandapotheken, nicht jedoch als Beispiel einer individuellen Unternehmensrendite.

Durch die Berücksichtigung einer durchschnittliche Betriebsrendite kommt es - wie an verschiedenen Stellen angemerkt - zu zwei wesentlichen Effekten innerhalb der Modellrechnungen:

- Zum einen fallen die Kostenersparnisse, die an die Krankenkassen in Form eines zusätzlichen umsatzabhängigen Rabattes weitergeleitet werden könnten, wesentlich geringer aus als in der Berechnung ohne Betriebsrendite:

Die Ergebnisse für das Einsparpotential ergeben sich aus Berechnungen mit ungerundeten Zahlenangaben zur Kostenersparnis bzw. dem Marktpotential, d.h. diese sind auf alle Nachkommastellen exakt ermittelt. Aufgrund der vereinfachten Tabelle II - 34 mit maximal zwei Nachkommastellen ergeben sich marginale Abweichungen. 
Abbildung II - 14: Veränderung der Kostenersparnis / Rabattgewährung durch Berücksichtigung einer durchschnittlichen Betriebsrendite

\begin{tabular}{|c|c|c|c|c|c|c|c|c|}
\hline & \multicolumn{5}{|c|}{ Zielsegmentvariante } & \multicolumn{2}{c|}{ Durchschnitt } \\
\cline { 2 - 9 } & \multicolumn{2}{|c|}{ A } & \multicolumn{2}{|c|}{ B } & \multicolumn{2}{c|}{ C } & \multicolumn{2}{c|}{ - } \\
\hline $\begin{array}{c}\text { Berücksichtigung } \\
\text { einer Betriebsrendite }\end{array}$ & ohne & mit & ohne & mit & ohne & mit & ohne & mit \\
\hline VA I & $6,3 \%$ & $3,6 \%$ & $6,2 \%$ & $3,2 \%$ & $6,2 \%$ & $3,3 \%$ & $6,2 \%$ & $3,4 \%$ \\
\hline VA II & $7,8 \%$ & $5,4 \%$ & $7,7 \%$ & $4,8 \%$ & $7,6 \%$ & $4,9 \%$ & $7,7 \%$ & $5,0 \%$ \\
\hline VA III & $11,8 \%$ & $8,3 \%$ & $11,5 \%$ & $8,1 \%$ & $11,6 \%$ & $8,1 \%$ & $11,6 \%$ & $8,2 \%$ \\
\hline
\end{tabular}

Quelle: INIFES, eigene Darstellung.

Statt durchschnittlich 6,2\% (VA I) bzw. 7,7\% (VA II) bzw. 11,6\% (VA III) Kostenersparnis würden nun mehr bei Kalkulation einer durchschnittlichen Betriebsrendite von 3,5\% durchschnittlich 3,4\% (VA I) bzw. 5,0\% (VA II) bzw. 8,2\% (VA III) Kostenersparnis in Form einer umsatzabhängigen Rabattgewährung an die gesetzlichen Krankenkassen weitergeleitet.

- Zudem verringert sich die Anzahl der Indikationen, die zusätzlich zu den Logistikstückkosten eine Durchschnittliche Betriebsrendite von 3,5\% decken könnten. Damit sinkt das jeweilige Marktpotential gegenüber der Berechnung ohne durchschnittliche Betriebsrendite erheblich. Besonders stark sind davon die umsatzschwächeren Versender VA I und VA II betroffen: deren Marktpotential sinkt im Vergleich zum Zielsegment um ca. ein Viertel. Siehe dazu folgende Abbildung:

Abbildung II - 15: Veränderung der Marktpotentiale durch Berücksichtigung einer durchschnittlichen Betriebsrendite

\begin{tabular}{|c|c|c|c|c|c|c|}
\hline \multirow{3}{*}{\begin{tabular}{|l|} 
\\
$\begin{array}{l}\text { Berücksichtigung } \\
\text { Betriebsrendite }\end{array}$ \\
\end{tabular}} & \multicolumn{6}{|c|}{ Zielsegmentvariante } \\
\hline & \multicolumn{2}{|c|}{$\begin{array}{c}A \\
41 \% \\
\end{array}$} & \multicolumn{2}{|c|}{$\begin{array}{c}\text { B } \\
47 \% \\
\end{array}$} & \multicolumn{2}{|c|}{$\begin{array}{c}C \\
57 \% \\
\end{array}$} \\
\hline & ohne & mit & ohne & mit & ohne & mit \\
\hline VAI & $39 \%$ & $31 \%$ & $44 \%$ & $37 \%$ & $53 \%$ & $44 \%$ \\
\hline VA II & $39 \%$ & $31 \%$ & $44 \%$ & $37 \%$ & $52 \%$ & $44 \%$ \\
\hline VA III & $39 \%$ & $39 \%$ & $45 \%$ & $44 \%$ & $53 \%$ & $52 \%$ \\
\hline
\end{tabular}

Quelle: INIFES, eigene Darstellung. 
Da das Einsparpotential aus beiden Komponenten, also dem Marktpotential wie der Kostenersparnis gebildet wird, unterliegt es folglich insbesondere bei VA I und VA II einer überproportionalen Dezimierung durch die Berücksichtigung einer Betriebsrendite.

Umsatzstarke Versender wie VA III können nach wie vor relativ hohe Einsparpotentiale erzielen. Die Berücksichtigung einer durchschnittlichen Betriebsrendite fällt hier nicht so stark ins Gewicht wie im Vergleich zu VA I und VAll, da hier vor allem der Effekt einer geringeren Kostenersparnis zu beobachten ist, weniger jedoch eines schwindenden Marktpotentials.

Abbildung II - 16: Veränderung der Einsparpotentiale des Pharmaversandes durch Berücksichtigung einer durchschnittlichen Betriebsrendite

\begin{tabular}{|c|c|c|c|c|c|c|c|}
\hline \multirow{3}{*}{\multicolumn{2}{|c|}{$\begin{array}{l}\text { Einsparpotentiale für GKV } \\
\text { in Mio. DM für } 1999\end{array}$}} & \multicolumn{6}{|c|}{$\begin{array}{c}\text { Marktpotential in \% } \\
\text { am GKV-Fertigarzneimittelumsatz } \\
\text { (bei einer durchschnittlichen Betriebsrendite) }\end{array}$} \\
\hline & & \multicolumn{2}{|c|}{$\begin{array}{l}\text { A } \\
\text { ca. }\end{array}$} & \multicolumn{2}{|c|}{$\begin{array}{l}\mathrm{B} \\
\mathrm{ca}\end{array}$} & \multicolumn{2}{|c|}{$\begin{array}{c}\mathrm{C} \\
\mathrm{ca}\end{array}$} \\
\hline & & $31 \%$ & $39 \%$ & $37 \%$ & $44 \%$ & $44 \%$ & $52 \%$ \\
\hline \multirow{3}{*}{ 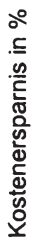 } & VAI $3,2 \%-3,6 \%$ & 386 & - & 411 & - & 501 & - \\
\hline & VA II $4,8 \%-5,4 \%$ & 574 & - & 617 & - & 750 & - \\
\hline & VA III $8,1 \%-8,3 \%$ & - & 1.129 & - & 1.252 & - & 1.490 \\
\hline
\end{tabular}

Quelle: INIFES, eigene Darstellung.

Das Intervall möglicher Einsparungen liegt nunmehr zwischen 386 Mio. DM und 1.490 Mio. DM. Differenziert man diese Betrachtungen nach der Größenklasse der Versandapotheke, zeigt sich, dass je nach Marktpotentialvariante:

- Das Modell der kleinen Versandapotheke (VA I) mit ca. 5 Mio. DM Umsatz und einer zusätzlichen Rabattgewährung von ca. 3,4\% auf die Arzneimittelkosten ein Einsparpotential von 386 Mio. DM bis 501 Mio. DM ergibt.

- Das Modell der großen Versandapotheke mit ca. 20 Mio. DM Umsatz (VA II) und einer zusätzlichen Rabattgewährung von ca. 5,0\% auf die Arzneimittelkosten ein Einsparpotential von 574 Mio. DM bis 750 Mio. DM ergibt.

- Das Modell der Versandapotheken mit kombinierter Großhandels- und Apothekenfunktion mit einem Umsatz von 487 Mio. DM (VA III) und einer zusätzlichen Rabattgewährung von $8,2 \%$ auf die Arzneimittelkosten ein Einsparpotential von 1.129 Mio. DM bis 1.490 Mio. DM ergibt. 
Wie in den Berechnungen ohne durchschnittliche Betriebsrendite zeigt sich auch hier, dass insbesondere durch umsatzstarke Versender aufgrund deren interner Betriebsstruktur die höchsten Einsparpotentiale innerhalb der entsprechenden Marktpotentiale zu erwarten sind. So liegen die maximal möglichen Einsparvolumina von VA III doppelt so hoch wie die von VAI.

Das Modell der Modifizierung der Kostenvorteile in Form der Berücksichtigung einer durchschnittlichen Branchenrendite ergab als Konsequenz nach Umsatzgröße gestaffelte Kassenrabatte.

Wie bereits am Ende des Kapitels 2.5.3. anklang, ist auch eine andere Vorgehensweise bei der Modifizierung der Kostenvorteile eines Versandhandels möglich. Unter der Annahme, dass die AMPreisV gilt und entsprechend keine Preisdifferenzierung für das Zielsegment eines Versandhandels möglich ist, könnte eine Erstattung der Kostenvorteile dieses Vertriebsweges an das derzeit existierende System der Gewährung einheitlicher, d.h. umsatzunabhängiger Rabatte, bei fixen Arzneimittelpreisen orientiert sein. Der Differenzierung der Kostenvorteile in Form der Gewährung zusätzlicher einheitlicher Kassenrabatte ${ }^{213}$ widmet sich die im folgendem Kapitel dargestellte Modellrechnung. Der verbleibende Betrag zwischen Kostenersparnis abzüglich einheitlichem Kassenrabatt wäre als Gewinnerwartung für die potentiellen Anbieter zu interpretieren (Kapitel 2.7.).

\subsection{Berücksichtigung zusätzlicher einheitlicher Kassenrabatte in den Modellrechnungen}

In Kapitel 2.6. wurden alternative Modellrechnungen zur Differenzierung der Kostenersparnisse eines Versandhandels aus Kapitel 2.4. durch die Annahme einer durchschnittlichen Rendite von 3,5\% für Versandapotheken durchgeführt. Aus der Zugrundelegung eines Durchschnitts einer Betriebsrendite resultierte ein von der Umsatzgröße abhängiger Kassenrabatt. ${ }^{214}$

Hintergrund der Überlegungen zur Einbeziehung einer Durchschnittsrendite war, dass der Aufbau neuer Strukturen zumindest auf lange Sicht nur dann durch potentielle Anbieter forciert wird, wenn sich diese einen wirtschaftlichen Anreiz in Form einer Rendite größer Null versprechen. Folglich ist nicht davon auszugehen, dass die Kostenvorteile einer neuen Vertriebsstruktur, wie in den Modellrechnungen ohne durchschnittliche Betriebsrendite postuliert, in ihrem gesamten Umfang einen volkswirtschaftlichen Zusatznutzen - in diesem Fall durch vollständige Abschöpfung durch die Versichertengemeinschaft der GKV - darstellen, sondern dass diese Kostenvorteile zu einem gewissen Teil durch individuelle Gewinnerwartungen der potentiellen Anbieter geschmälert werden.

\footnotetext{
213

Diese Rabatte wären zusătzlich zum derzeitigen Kassenrabatt von $5 \%$ zu betrachten.

214

Siehe Kapitel 2.6.3.: VA I ca. $3,4 \%$, VA II ca. $5,0 \%$, VA III ca. $8,2 \%$. 
Die im folgenden darzustellenden alternativen Modellrechnungen basieren ebenfalls auf der Überlegung, dass die Kostenvorteile eines neuen Vertriebssystems nicht vollständig der GKV zufließen werden, folglich bei den potentiellen Versandapotheken eine Rendite verbleibt. Allerdings ist der Ansatz der Rechnungen in Kapitel 2.7. der eines zusätzlich zum derzeitigen Kassenrabatt von fünf Prozent durch die Versandapotheken anzuführenden einheitlichen Kassenrabatts entsprechend dem derzeitigen Rabattsystem der GKV.

Es sei an dieser Stelle hervorzuhoben, dass das Modell der Gewährung eines zusätzlichen einheitlichen Kassenrabatts durch Versandapotheken an die GKV eine Hilfskonstruktion darstellt. Durch neue Strukturen entstehende Kostenersparnisse, auch ohne eine Preisdifferenzierung (die Geltung der AMPreisV wird in dieser Studie vorausgesetzt), sind der Versichertengemeinschaft der GKV zuzuführen. Unter der Annahme alternativer Rabattgewährungen wird aufgezeigt, welcher Teil der Kostenvorteile den Krankenkassen zur Verfügung stehen könnte.

Ob ein solches Modell auch aus rechtlichen Gründen, insbesondere dem Kartellrecht, möglich ist, soll und kann im Rahmen dieser Untersuchungen nicht beantwortet werden.

\subsubsection{Einflüsse zusätzlicher einheitlicher Kassenrabatte auf das Markt- und Einsparpotential}

In den folgenden Modellrechnungen werden drei alternative einheitliche Kassenrabatte angenommen, die von potentiellen Versandapotheken zusätzlich zum derzeit existierenden Kassenrabatt von fünf Prozent an die GKV zuführen sind.: fünf Prozent, sieben Prozent und zehn Prozent vom Verordnungswert. ${ }^{216}$ Intention der Überlegungen, auf denen dieses Modell basiert, ist eine Orientierung an der derzeitigen Rabattgewährung zwischen Apotheken und GKV, die nicht auf umsatzabhängigen, sondern umsatzunabhängigen einheitlichen Kassenrabatten beruht. Die im Modell alternativ verwendeten Werte von $5 \%$ bzw. $7 \%$ bzw. $10 \%$ zusätzlichem einheitlichen Kassenrabatt sind dabei zum einen an der Höhe des derzeitigen Kassenrabatts bzw. dessen doppelten Wert orientiert, zum anderen erfolgt mit $7 \%$ die Annahme eines Wertes innerhalb dieser Spannweite.

Wie bei den Modellrechnungen unter Berücksichtigung einer durchschnittlichen Betriebsrendite explizit erwähnt, bedeutet auch hier die Einbeziehung der genannten zusätzlichen Kassenrabatte nicht, dass von den durchschnittlichen Kostenvorteilen, die sich bei den Modellrechnungen ohne Berücksichtigung einer durchschnittlichen Betriebsrendite bzw. eines Kassenrabattes ergeben, ein entsprechender zusätzlicher vom Verordnungswert von der GKV erhoben.
} 
Kassenrabatt abgezogen wird und sich bei entsprechender Multiplikation mit dem Marktpotential das neue Einsparpotential ergibt.

Die Berücksichtigung eines zusätzlichen Kassenrabattes bedeutet bzw. führt dazu, dass potentielle Versandapotheken in Kenntnis einer verpflichtenden zusätzlichen Rabattbelastung diese bereits in ihrer Kostenkalkulation berücksichtigen. Das heißt, neben den in 2.4.3. bis 2.4.5. ausgewiesenen Kosten inklusive der Logistikkosten muss nun zusätzliche eine Kostenposition in der Höhe des zusätzlichen Kassenrabattes von $5 \%$ bzw. $7 \%$ bzw. $10 \%$ berücksichtigt werden, die aus den Vertriebsspannen der Verordnungswerte des Zielsegments zu decken sind. Entsprechend weniger Verordnungen werden im Vergleich zu den bisherigen Berechnungen für einen Pharmaversand in Frage kommen.

Die Vorauskalkulation einer zusätzlichen Rabattbelastung als Kostenposition für Versandapotheken führt folglich zu zwei wesentlichen Entwicklungen:

1. Je höher der zusätzlich an die GKV zu gewährende Rabatt festgesetzt wird, umso weniger Verordnungen des Zielsegments werden über den Versandweg vertrieben. Entsprechend sinkt das Marktpotential. So sind bei einem geringeren Kassenrabatt vergleichsweise höhere Marktpotentiale je Versandapotheke zu erwarten als bei höheren Kassenrabatten. Mit steigenden Kassenrabatten wächst die Gefahr, dass tendenziell mehr hochpreisige Indikationsgruppen versandt werden.

2. Zudem werden, da es sich hier um einen umsatzunabhängigen Kassenrabatt handelt, umsatzschwächere Versandapotheken in ihrer Existenz besonders stark von der Höhe des zusätzlichen Einheitsrabatts abhängig sein. So ist prognostizierbar, dass bei höheren zusätzlichen Rabatten umsatzschwächere durch umsatzstärkere Versender aus dem Zielsegment verdrängt werden, da deren Kostenvorteile unter der Höhe des zusätzlich zu gewährenden Kassenrabattes liegen. Entsprechend würde auch der Markteintritt weiterer potentieller Anbieter erschwert. Dies impliziert zudem, dass umsatzschwächere potentielle Versandapotheken aufgrund einer in bezug auf ihren Umsatz relativ höheren Rabattbelastung tendenziell mehr hochpreisige Indikationsgruppen versenden werden als umsatzstarke Versandapotheke, die aufgrund hoher economies of scale eine bessere Kostensituation aufweisen.

\subsubsection{Ausprägungen der Markt- und Einsparpotentiale bei Berücksichtigung zusätzlicher einheitlicher Kassenrabatte}

Es werden die Auswirkungen der Berücksichtigung zusätzlicher einheitlicher Kassenrabatte auf das Markt- und Einsparpotential dargestellt. Dabei wird, im Sinne einer besseren Übersichtlichkeit, in folgenden keine Differenzierung zwischen den einzelnen Zielsegmentvarianten vorgenommen, sondern diese in Abhängigkeit des zusätzlichen Kassenrabatts zusammengefasst. 
Eine detaillierte Aufstellung je Zielsegmentvariante $A, B$ und $C$ ist dem Anhang 1 (Anhang I -Tabelle 9 bis Anhang I -Tabelle 17) zu entnehmen.

Es kommt zu folgenden Veränderungen des Marktpotentials in Abhängigkeit der Höhe eines zusätzlichen einheitlichen Kassenrabattes:

Abbildung II-17: Veränderung der Marktpotentiale bei Berücksichtigung eines zusätzlichen einheitlichen Kassenrabatts (in Abhängigkeit der Höhe des zusätzlichen Kassenrabatts)

\begin{tabular}{|c|c|c|c|c|}
\hline & \multicolumn{4}{|c|}{$\begin{array}{c}\text { Marktpotential in \% des GKV-Fertigarzneimittelumsatzes } \\
\text { (Spannweite zwischen den Zielsegmentvarianten A, B und C) }\end{array}$} \\
\hline $\begin{array}{c}\text { Versand- } \\
\text { apotheke }\end{array}$ & $\begin{array}{c}\text { Ohne Berücksichti- } \\
\text { gung eines einheit- } \\
\text { lichen Kassenra- } \\
\text { battes }\end{array}$ & $\begin{array}{c}\text { Bei Berücksichti- } \\
\text { gung von } \\
\text { zusätzlich } \\
5 \% \text { Kassenrabatt }\end{array}$ & $\begin{array}{c}\text { Bei Berücksichti- } \\
\text { gung von } \\
\text { zusätzlich } \\
7 \% \text { Kassenrabatt }\end{array}$ & $\begin{array}{c}\text { Bei Berücksichti- } \\
\text { gung von } \\
\text { zusätzlich } \\
10 \% \text { Kassenrabatt }\end{array}$ \\
\hline VAI & $39 \%<53 \%$ & $21 \%<25 \%$ & - & - \\
\hline VA II & $39 \%<52 \%$ & $31 \%<40 \%$ & $16 \%<23 \%$ & $21 \%<23 \%$ \\
\hline VA III & $39 \%<53 \%$ & $34 \%<47 \%$ & $31 \%<44 \%$ & 2 \\
\hline
\end{tabular}

Quelle: INIFES, eigene Darstellung.

Es bestätigt sich der prognostizierte Zusammenhang zwischen der Höhe des Kassenrabatts und der Ausprägung des Marktpotentials: Je höher der zusätzliche einheitliche Kassenrabatt ausfällt, umso geringer ist das verbleibende Marktpotential. Diese Tendenz ist insbesondere bei den umsatzschwächeren Versendern zu beobachten.

- Bei Gewährung eines Kassenrabatts von $5 \%$ würde sich das Marktpotential bei allen drei Modellen potentieller Versender gegenüber der Ausgangslange des Kapitels 2.5. verringern - im Modell VA I wäre dies im wesentlich stärken Maße der Fall als in den Modellen VA II und VA III - allerdings wären hier nach wie vor alle drei Modelle einer Versandapotheke denkbar.

- Bei Gewährung eines Kassenrabatts von 7\% verringert sich das Marktpotential gegenüber der vorherigen Alternative stärker. Am geringsten ist davon das mögliche Marktpotential des umsatzstarken Modells VA III betroffen. Eine Halbierung des Marktpotentials gegenüber der Alternativrechnung mit zusätzlich 5\% Kassenrabatt erfährt Modell VA II. Das Modell VA I ist bei dieser angenommenen Rabatthöhe nicht mehr funktionsfähig, d.h. die Kostenvorteile dieses Versandmodells VA I - ca. 6,2\% (siehe Kapitel 2.4.6) - werden durch die Gewährung eines höheren 
Rabattes negiert und die Erzielung von Einsparungen für die GKV nicht mehr möglich.

- Bei Gewährung eines Kassenrabattes von $10 \%$ wären neben dem Modell VA I auch Versender nach dem Modell VA II nicht mehr funktionsfähig. Ausschließlich umsatzstarke Versender nach Modell VA III könnten aufgrund ihrer hohen economies of scale ${ }^{219}$ der erhöhten Rabattgewährung bei einem geringeren Marktpotential entsprechen.

Die Höhe des zusätzlichen einheitlichen Kassenrabatts spielt eine wesentliche Rolle: Je höher der Kassenrabatt, umso stärker ist die Tendenz zu großen Versendern.

Analog zu den vorangegangenen Modellrechnungen erfolgt auch hier die Ermittlung des Einsparpotentials aus der Kombination der beiden Faktoren Marktpotential und zusätzlicher einheitlicher Kassenrabatt.

Abbildung II-18: Veränderung der Einsparpotentiale bei Berücksichtigung eines zusätzlichen einheitlichen Kassenrabatts (in Abhängigkeit der Höhe des zusätzlichen Kassenrabatts)

\begin{tabular}{|c|c|c|c|}
\hline \multirow[b]{2}{*}{$\begin{array}{l}\text { Versand- } \\
\text { apotheke }\end{array}$} & \multicolumn{3}{|c|}{$\begin{array}{c}\text { Einsparpotential für die GKV in Mio. DM für } 1999^{220} \\
\text { (Spannweite in Abhängigkeit der Marktpotentialvarianten A, B und C) }\end{array}$} \\
\hline & $\begin{array}{c}\text { bei Berücksichtigung } \\
\text { von } \\
\text { zusätzlich } \\
5 \% \text { Kassenrabatt }\end{array}$ & $\begin{array}{c}\text { bei Berücksichtigung } \\
\text { von } \\
\text { zusätzlich } \\
7 \% \text { Kassenrabatt }\end{array}$ & $\begin{array}{c}\text { bei Berücksichtigung } \\
\text { von } \\
\text { zusätzlich } \\
10 \% \text { Kassenrabatt }\end{array}$ \\
\hline VA I & $379<467$ & - & - \\
\hline VA II & $561<732$ & $413<604$ & - \\
\hline VA III & $624<864$ & $786<1.124$ & $759<863$ \\
\hline
\end{tabular}

Quelle: INIFES, eigene Darstellung.

Das Intervall möglicher Einsparungen liegt nunmehr zwischen 379 Mio. DM und 1.124 Mio. DM. Die höchsten Einsparungen werden bei $7 \%$ Kassenrabatt durch die umsatzstarken Versender VA III erzielt.

Differenziert nach der Größenklasse der Versandapotheken lässt sich folgendes konstatieren:

- Kleine Versandapotheken mit ca. 5 Mio. DM Umsatz (VA I) können bei einer zusätzlichen Kassenrabattgewährung von 5\% ein Einsparpotential bis zu 467 Mio.

\footnotetext{
219

Siehe Kapitel 2.4.6.

220

Die Ergebnisse für die Einsparpotentiale ergeben sich aus Berechnungen mit ungerundeten Zahlenangaben zum Marktpotential, d.h. diese sind auf alle Nachkommastellen exakt ermittelt. Aufgrund der Angabe ungerundeter Werte in Abbildung II - 17 ergeben sich marginale Abweichungen.
} 
DM realisieren. Eine höhere Rabattgewährung von $7 \%$ und $10 \%$ würde die Kosteneinsparungen von ca. $6,2 \%$ übersteigen und ist somit nicht möglich.

- Große Versandapotheken mit ca. 20 Mio. DM Umsatz (VA II) würden bei einem zusätzlichen Kassenrabatt von 5\% ein Einsparpotential zwischen 561 Mio. DM und 732 Mio. DM realisieren, bei einem höheren zusätzlichen Kassenrabatt von 7\% lägen die Einsparpotentiale zwischen 413 Mio. DM und 604 Mio. DM. Ein höherer Kassenrabatt von $10 \%$ würde die Kosteneinsparungen von ca. $8 \%$ (siehe Kapitel 2.4.6.) übersteigen und ist somit nicht möglich.

- Versandapotheken mit kombinierter Großhandels- und Apothekenfunktion mit einem Umsatz von 487 Mio. DM (VA III) können bei einem zusätzlichen Kassenrabatt von 5\% ein Einsparpotential zwischen 624 Mio. DM und 864 Mio. DM realisieren, bei einem höheren zusätzlichen Kassenrabatt von 7\% lägen die Einsparpotentiale zwischen 786 Mio. DM und 1.124 Mio. DM (Maximalwert). Ein höherer Kassenrabatt von $10 \%$ führt aufgrund des geringeren Marktpotentials zu verringerten Einsparpotentialen zwischen 759 Mio. DM und 863 Mio. DM. Diese sind vergleichbar denen bei einer Rabattgewährung von $5 \%$.

Differenziert nach der Höhe des zusätzlichen einheitlichen Kassenrabatts bleibt festzustellen:

- Eine einheitliche Belastung von zusätzlich 5\% Kassenrabatt unabhängig von der Umsatzgröße würde zwar für VA III nicht das maximale Einsparpotential aufdecken, jedoch wäre die Anbieterstruktur gekennzeichnet durch das Agieren potentieller Versender unterschiedlicher Größe. Die Einsparpotentiale lägen zwischen je nach Umsatzgröße zwischen 379 Mio. DM (VA I) und 864 Mio. DM (VA III).

- $\quad$ Eine einheitliche Belastung von zusätzlich 7\% Kassenrabatt würde den Optimalwert des Einsparpotentials zwischen 786 Mio. DM und 1.124 Mio. DM für VA III realisieren, allerdings ginge diese Rabattregelung zu Lasten der kleinen Versandapotheken des Modells VA I. Deren Funktionsfähigkeit wäre nicht mehr gegeben.

- Eine einheitliche Belastung von zusätzlich $10 \%$ Kassenrabatt würde dazu führen dazu, dass nur das Modell eines umsatzstarken Versenders VA III denkbar wäre. Alle anderen Modelle potentieller Versender würden sich aufgrund der hohen Rabattbelastung, welche die Kostenvorteile des neuen Vertriebsweges übersteigen würde, als nicht funktionsfähig erweisen. Das Einsparpotential von VA III läge zwischen 759 Mio. DM und 863 Mio. DM und damit aufgrund des gesunkenen Marktpotentials unter der maximalen Ausprägung bei 7\% Rabatt.

In langfristiger Perspektive zeigt sich entsprechend, dass für eine Realisierung optimaler Einsparpotentiale das Modell des Versenders VA III bei einer zusätzlichen Rabattgewährung von $7 \%$ zu thematisieren wäre. Die Alternative eines zusätzlichen einheitlichen Kassenrabattes von $10 \%$ würde nicht zu den entsprechenden Einsparpotentialen führen. Im Sinne einer Funktionsfähigkeit aller drei Modelle potentieller Versandapotheken wäre eine Rabattgewährung von $5 \%$ zu favorisieren. 


\section{8. Überlegungen zu realisierbaren Marktanteilen}

Den vorangegangenen Betrachtungen liegt eine wesentliche Einschränkung zugrunde: Die genannten Einsparvolumina können nur erzielt werden, wenn die aufgezeigten Marktpotentiale vollständig ausgeschöpft werden. Davon ist realistischer Weise nicht auszugehen. Entsprechend ist nach der Modifizierung des Kostenvorteils auch das Marktpotential einer Differenzierung hinsichtlich der Höhe der Ausprägungen zu unterziehen.

Im Folgenden soll deshalb mit Szenarien zum kurzfristigen Realisierungsgrad von Marktanteilen gearbeitet werden. Hierzu sind Annahmen zum Realisierungsgrad zu treffen:

- Akzeptanzhürden der Patienten und verordnenden Ärzte bei der Implementierung neuer Vertriebswege könnten dazu führen, dass die Realisierung von Marktpotentialen, zumindest in mittelfristiger Perspektive, nur zögerlich verläuft. Wesentliche Einflüsse auf eine überproportionale Entwicklung des Versandhandels könnten zum einen aus der Gewährleistung einer überzeugenden Qualität und Sicherheit durch die potentiellen Anbieter erwachsen, zum anderen wäre die Implementierung von Anreizsystemen durch die gesetzlichen Krankenkassen für solche Patienten zu überdenken, die diesen Versand nutzen (z.B. in Form von Bonisystemen).

- In der Schweiz geht man von einem Marktpotential von ca. $10 \%$ aller Apothekenkunden aus. Jedoch basiert das derzeitige Schweizer Modell eines Direktversandes auf einer Patientenklientel mit Arzneimittelausgaben über SFr 450 pro Jahr, sodass mit einem Marktpotential von mehr als $10 \%$ der Arzneimittelausgaben gerechnet werden kann.

- US-Quellen sprechen bereits 1997 von einem 12\%igen Umsatzanteil des Arzneimittelversandes am Markt für verschreibungspflichtige Präparate. ${ }^{222}$ Dass es sich dabei um ein stark wachsendes Segment handelt, zeigt die Steigerung des Versandhandels auf derzeit (Stand 2000) ca. 15\% Marktanteil. ${ }^{22}$

Insgesamt ist die Annahme realistisch, dass ein Realisierungsgrad des gesamten Marktpotentials in kurz- und mittelfristiger Perspektive jede dritte zum Versand geeignete Verordnung umfassen könnte: Das würde heißen, dass ca. ein Drittel aller Verordnungen des Marktpotentials über Versandapotheken vertrieben werden könnte, allerdings zwei Drittel nach wie vor über die Präsenzapotheken an den Patienten gelangen.

Laut Expertengespräch Februar 2000.

American Medical Association, Report of the AMA Young Physicians section governing council, 1997, S. 2. 
Überträgt man den Realisierungsgrad von ca. einem Drittel des Marktpotentials (Ausprägungen zwischen $31 \%^{224}$ und $53 \%$ am GKV-Verordnungsmarkt) auf den gesamten GKV-Fertigarzneimittelmarkt, ergäben sich Marktanteile in einer Spanne zwischen $10 \%$ und $18 \%$. Bei einer Durchschnittsbetrachtung kann man entsprechend von $14 \%$ bis $15 \%$ Marktanteil des Arzneimittelversandes am gesamten GKV-Fertigarzneimittelmarkt ausgehen.

\section{Allgemeine Betrachtung ohne Modifizierung der Kostenvorteile}

Das Einsparpotential bei vollständiger Ausschöpfung der Marktpotentiale ergab eine Spannweite von 861 Mio. DM bis 2.144 Mio. DM in Abhängigkeit der Umsatzgröße.

Die realisierbaren Einsparungen bei einem 15\%igen Marktanteil stellt folgende Abbildung dar:

Abbildung II - 19: Einsparvolumina bei einem realisierten Marktanteil von 15\%

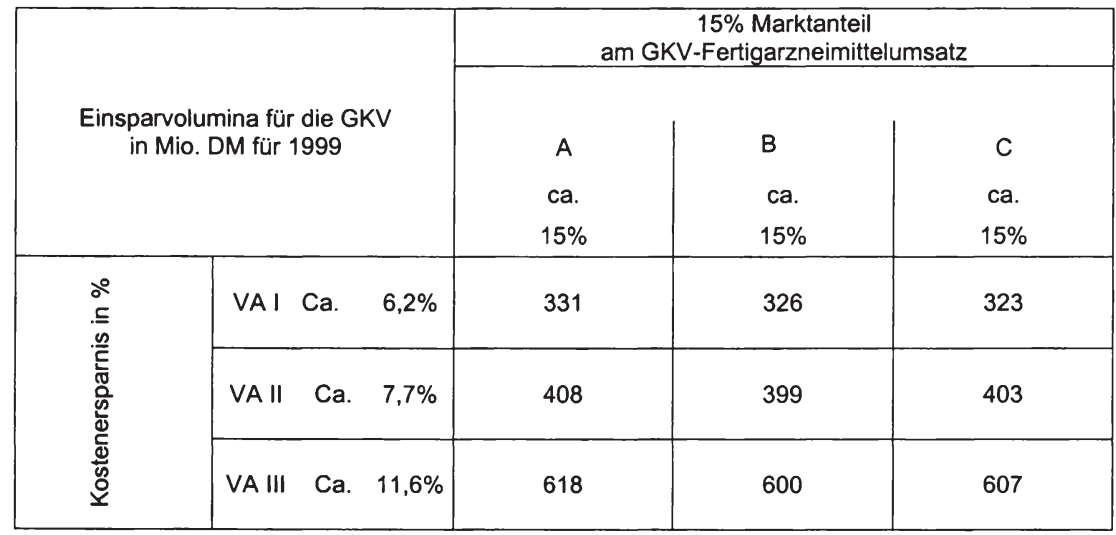

Quelle: INIFES, eigene Berechnungen.

Das Intervall realisierbarer Einsparungsvolumina liegt bei einem 15\%igen Marktanteil des Versandes zwischen 323 Mio. DM (bei ca. 6,2\% Einsparung auf die Arzneimittelkosten) und 618 Mio. DM (bei ca. 11,6\% Kosteneinsparung), differenziert nach Umsatzgröße:

- Das Intervall für VA I erstreckt sich von 323 Mio. DM bis 331 Mio. DM, durchschnittlich würden ca. 327 Mio. DM Einsparungen realisiert.

- Das Intervall für VA II erstreckt sich von 399 Mio. DM bis 408 Mio. DM, durchschnittlich würden ca. 403 Mio. DM Einsparungen realisiert.

- Das Intervall für VA III erstreckt sich von 600 Mio. DM bis 618 Mio. DM, durchschnittlich würden ca. 608 Mio. DM Einsparungen realisiert.

224 Marktpotentiale für VA | und VA || bei Zielsegmentvariante A mit Durchschnittliche Betriebsrendite (2.5.3.2.). 
Bei Betrachtung der Einsparvolumina von 15\% Marktanteil des Versandhandels in Abhängigkeit der Umsatzgröße wie des Marktpotentials zeigen sich folgende Entwicklungen:

1. Konsequenterweise sind die durchschnittlich realisierbaren Einsparungen auch hier beim umsatzstärksten Versender VA III mit ca. 608 Mio. DM aufgrund der Skaleneffekte in der Kosten- und Ertragsstruktur am größten, gefolgt von VA II mit 403 Mio. DM. Am geringsten fallen die Einsparungen bei Modell VA I mit 327 Mio. DM aus. Der Unterschied im Einsparpotential zwischen den Modellvarianten VA III und VA I beträgt also fast $100 \%$.

2. Die Schwankungsbreite zwischen den einzelnen Ausprägungen der verschiedenen Einsparvolumina je Versandapothekenvariante ist relativ gering und spricht damit für eine relativ gute Aussagekraft der gewählten Indikatoren.

3. Die höchsten Einsparungen je Versandapothekentyp werden, betrachtet man die Realisierung eines 15\%igen Marktanteils, in der Marktpotentialvariante A (Indikator Packungsgröße ab 50 DDD), also der Variante mit dem geringsten Potential von ca. $41 \%$, ausgewiesen.

4. Die größten Einsparungen erscheinen dabei durch die umsatzstärkste Versandapotheke VA III realisierbar.

5. Ein Vergleich der Einsparvolumina für Marktanteilsvariante A (operationalisiert durch den Indikator Packungsgröße mit mindestens 50 DDD) und Marktanteilsvariante $C$ (operationalisiert durch den Indikator Packungsgröße mit mindestens 35 DDD) je Versandapothekentyp zeigt, dass die Einsparungen bei Marktanteilsvariante $\mathrm{C}$ marginal unter dem Niveau von $\mathrm{A}$ liegen. Das heißt, Marktpotential Variante C mit einem größeren Umfang von 52\%-53\% am GKV-Arzneimittelumsatz weist bei Realisierung eines 15\% Marktanteils eine geringere Ersparnis aus als die Marktpotentialvariante A mit einem geringen Umfang von 39\%.

$\mathrm{Zu}$ erklären ist dieser Effekt damit, dass Marktpotentialvariante A eine Schnittmenge von Variante $\mathrm{C}$ ist, und deshalb aufgrund der für die Berechnungen zugrunde gelegten größeren Packungsgröße auch einen höheren durchschnittlichen Verordnungswert im Vergleich zu C aufweist ${ }^{226}$.

Damit sind bei Annahme eines - für alle Umsatzgrößen identischen - Realisierungsgrades des Marktanteils (hier gezeigt am Beispiel 15\%) die Einsparungen für die Marktpotentialvariante A größer als die Einsparungen für Marktpotentialvariante C. Dieser Effekt blieb bei der Betrachtung der vollständigen Ausschöpfung der Marktpotentiale und den daraus resultierenden Einsparpotentialen unberücksichtigt. 
Das Einsparpotential bei vollständiger Ausschöpfung der Marktpotentiale ergab eine Spannweite von 386 Mio. DM bis 1.490 Mio. DM in Abhängigkeit der Umsatzgröße.

Die realisierbaren Einsparungen bei einem 15\%igen Marktanteil stellt folgende Abbildung dar:

Abbildung II - 20: Einsparvolumina bei einem realisierten Marktanteil von 15\% (mit Betriebsrendite)

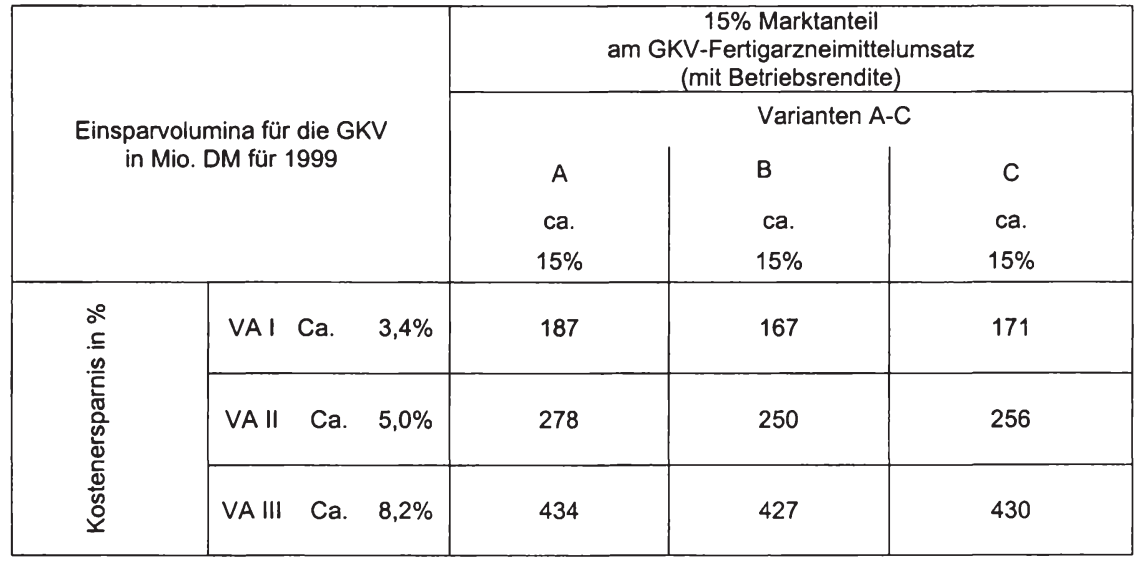

Quelle: INIFES, eigene Berechnungen.

Das Intervall realistischer Einsparungsvolumina liegt bei einem 15\%igen Marktanteil des Versandes zwischen 167 Mio. DM (bei ca. 3,4\% Einsparung auf die Arzneimittelkosten) und 434 Mio. DM (bei ca. 8,2\% Kosteneinsparung), differenziert nach Umsatzgröße:

- Das Intervall für VA I erstreckt sich von 167 Mio. DM bis 187 Mio. DM, durchschnittlich würden ca. 175 Mio. DM Einsparungen realisiert.

- Das Intervall für VA II erstreckt sich von 250 Mio. DM bis 278 Mio. DM, durchschnittlich würden ca. 261 Mio. DM Einsparungen realisiert.

- Das Intervall für VA III erstreckt sich von 427 Mio. DM bis 434 Mio. DM, durchschnittlich würden ca. 430 Mio. DM Einsparungen realisiert.

Es bleibt abschließend festzuhalten, dass die Berücksichtigung einer durchschnittlichen Betriebsrendite einen erheblichen Einfluss auf die Höhe der Einsparvolumina hat. Die größten Auswirkungen zeigen sich bei dem Versandapothekenmodell VA I mit 5 Mio. DM: hier verringert sich das Einsparvolumen um $46 \%$ von durchschnittlich 327 Mio. DM auf 175 Mio. DM. 
VA II mit 20 Mio. DM Umsatz weist bei Berücksichtigung einer durchschnittlichen Betriebsrendite eine Verringerung des Einsparvolumens von ca. $35 \%$ auf, der größte potentielle Anbieter VA III von ca. $30 \%$. Zurückzuführen ist dies auf die wesentlich geringeren Rabatte, die bei einer Berücksichtigung der durchschnittlichen Betriebsrendite an die GKV fließen würden. Siehe dazu die folgende abschließende Abbildung:

Abbildung II - 21: Einsparvolumina und Kostenersparnisse im Vergleich ohne bzw. mit Berücksichtigung einer Betriebsrendite bei $15 \%$ Marktanteil

\begin{tabular}{|c|c|c|c|c|}
\hline & & & \multicolumn{2}{|c|}{ Realisierung eines $15 \%$ Marktanteils } \\
\hline & & & $\begin{array}{l}\text { Vergleich ohne / mit } \\
\text { Betriebsrendite }\end{array}$ & $\begin{array}{c}\text { Durchschnittliche Einsparvolumina für } \\
\text { die GKV in Mio. DM für } 1999\end{array}$ \\
\hline \multirow{6}{*}{ 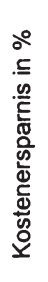 } & \multirow{2}{*}{ VAI Ca. } & & Ohne & 327 \\
\hline & & $3,4 \%$ & Mit & 175 \\
\hline & \multirow[t]{2}{*}{ VA II Ca. } & $7,7 \%$ & Ohne & \\
\hline & & $5,0 \%$ & Mit & 261 \\
\hline & \multirow{2}{*}{ VA III Ca. } & $11,6 \%$ & Ohne & 608 \\
\hline & & $8,2 \%$ & Mit & 430 \\
\hline
\end{tabular}

Quelle: INIFES, eigene Berechnungen.

\section{Betrachtung mit zusätzlichen einheitlichen Kassenrabatten}

Analog zu den vorangegangenen Darstellungen soll in den Modellrechnungen mit einem zusätzlichen einheitlichen Kassenrabatt von einem Realisierungsgrad von $15 \%$ Marktanteil ausgegangen werden. Es ergeben sich folgende realisierbare Einsparvolumen:

Abbildung II - 22: Einsparvolumina bei Berücksichtigung zusätzlicher einheitlicher Kassenrabatte und einem Marktanteil von 15\%

\begin{tabular}{|c|c|c|c|}
\hline & \multicolumn{3}{|c|}{$\begin{array}{c}\text { Einsparvolumina für die GKV in Mio. DM für 1999 } \\
\text { (Spannweite in Abhängigkeit der Marktpotentialvarianten A, B und C) }\end{array}$} \\
\hline $\begin{array}{c}\text { Versand- } \\
\text { apotheke }\end{array}$ & $\begin{array}{c}\text { bei Berücksichtigung } \\
\text { von zusätzlich } \\
5 \% \text { Kassenrabatt }\end{array}$ & $\begin{array}{c}\text { Bei Berücksichtigung } \\
\text { von zusätzlich } \\
7 \% \text { Kassenrabatt }\end{array}$ & $\begin{array}{c}\text { bei Berücksichtigung } \\
\text { von zusätzlich } \\
10 \% \text { Kassenrabatt }\end{array}$ \\
\hline VA I & 276 & - & - \\
\hline VA II & 276 & 386 & - \\
\hline VA III & 276 & 386 & 552 \\
\hline
\end{tabular}

Quelle: INIFES, eigene Darstellung.

227

Die Ergebnisse für die Einsparpotentiale ergeben sich aus Berechnungen mit ungerundeten Zahlenangaben zum Marktpotential, d.h. diese sind auf alle Nachkommastellen exakt ermittelt. Aufgrund der Angabe ungerundeter Werte in Abbildung 1 ergeben sich marginale Abweichungen. 
Das Intervall realisierbarer Einsparvolumina liegt bei einem 15\%igen Marktanteil des Versandes je nach Höhe des zusätzlichen einheitlichen Kassenrabattes zwischen 276 Mio. DM und 552 Mio. DM. Durch die Annahme eines zusätzlichen einheitlichen Kassenrabatts wie fixen Marktanteils ergeben sich innerhalb der drei Alternativen an Kassenrabatten keine Unterschiede in Abhängigkeit von der Umsatzgröße des potentiellen Versenders.

Differenziert nach den Modellen potentieller Versender VA I, VA II und VA III lässt sich folgendes festhalten:

- Kurz- und mittelfristig wären durch das Modell VA I Einsparvolumina von 276 Mio. DM bei einem zusätzlichen Kassenrabatt von $5 \%$ realisierbar.

- Das Intervall realisierbarer Einsparvolumina durch das Modell VA II würde kurzund mittelfristig zwischen 276 Mio. DM (bei einem zusätzlichen Kassenrabatt von 5\%) und 386 Mio. DM (bei 7\% zusätzlichem Kassenrabatt) liegen.

- Kurz- und mittelfristig wären durch das Modell VA III Einsparvolumina von 276 Mio. DM (bei einem zusätzlichen Kassenrabatt von 5\%) bis 552 Mio. DM (bei einem zusätzlichen Kassenrabatt von $10 \%$ ) realisierbar.

Differenziert nach zusätzlichen einheitlichen Kassenrabatts bleibt festzustellen:

- Bei zusätzlicher Rabattgewährung von 5\% könnten 276 Mio. DM durch alle drei Modelle potentieller Versender realisiert werden.

- Bei zusätzlicher Rabattgewährung von 7\% könnten 386 Mio. DM durch die potentiellen Versender VA II und VA III realisiert werden.

- Bei zusätzlicher Rabattgewährung von 10\% könnten 552 Mio. DM durch den potentiellen Versender VA III realisiert werden.

Im Sinne der Realisierung eines maximalen Einsparvolumens von 552 Mio. DM wäre das Versandapothekenmodell VA III bei einer zusätzlichen Rabattgewährung von $10 \%$ die optimale Alternative. Allerdings wären hier keine anderen Modelle potentieller Versender funktionsfähig.

In langfristiger Perspektive (siehe Abbildung II - 18) kristallisierte sich heraus, dass das optimale Einsparvolumen für dieses Modell bei $7 \%$ zusätzlichem Kassenrabatt liegen würde. Unter Berücksichtigung der langfristigen Entwicklungspotentiale erscheinen kurz- und mittelfristig entsprechende Einsparvolumen von 386 Mio. DM bei einem Marktanteil von 15\% (siehe Abbildung II - 22) als realistisch. Neben dem Modell VA III wären hier auch Versender der Umsatzgröße VA III funktionsfähig. Umsatzschwache Versandapotheken nach dem Model VA I würden nicht im Zielsegment agieren. 


\subsection{Zusammenfassung}

Modul 2 hat zum Inhalt, differenzierte Ergebnisse eines potentiellen quantitativen Einsparvolumens einer Ausweitung des Pharmaversandes darzustellen. Grundsätzlich galt es dabei, folgende Fragen zu beantworten:

(1) Welches Medikationssegment, das sogenannte Zielsegment, erschließt sich aus epidemiologischer Sicht dem Versandhandel mit verschreibungspflichtigen Arzneimitteln? (Kapitel 2.3.)

(2) Wie stellen sich die Kosten des neuen Vertriebsweges im Vergleich zur derzeitigen Kostenstruktur der Präsenzapotheke dar? Welche betriebswirtschaftlichen Umsatz-, Kosten- und Ertragsstrukturen bilden sich heraus? (Kapitel 2.4.)

(3) Welcher Anteil der Verordnungen des Versandsegments (2.3.) wird auch unter Geltung ökonomischer Restriktionen (2.4.) durch die potentiellen Anbieter versandt (sogenanntes Marktpotential) und trägt damit zur Realisierung von Einsparungen für das System der GKV bei? (Kapitel 2.5.)

(4) Welches quantitative monetäre Einsparpotential resultiert maximal aus der Zulassung eines Pharmaversands für die GKV? (Kapitel 2.5.)

Zudem werden die Einflüsse einer bereits in der Kostenkalkulation zu berücksichtigenden Durchschnittsrendite auf die Kostenersparnis, das Marktpotential und daraus resultierend - für das Einsparpotential eines Pharmaversandes thematisiert. (Kapitel 2.6.)

Alternativ zur Berücksichtigung einer Durchschnittsrendite und daraus resultierenden umsatzabhängigen Kassenrabatten werden weiterhin Alternativrechnungen mit einheitlichen Kassenrabatten vorgenommen. Auch hier werden die Einflüsse auf die Kostenersparnis, das Markt- und Einsparpotential eines Pharmaversandes analysiert. (Kapitel 2.7.)

In der kurz- bis mittelfristigen Perspektive werden die errechneten Einsparpotentiale für einen Realisierungsgrad des Versandpotentials von $15 \%$ Marktanteil dargestellt (Kapitel 2.8.)

Aufgrund des umfangreichen Bedarfs an Informationen muss auf eine Vielzahl verschiedener Quellen zugrückgegriffen werden. Eine Beeinflussung des Zahlenmaterials durch partikuläre Interessen der jeweiligen Akteure am Arzneimittelmarkt ist nicht auszuschließen. Ferner wirkt erschwerend, dass zum einen eine Differenzierung der Berechnungen aus volkswirtschaftlicher sowie aus betriebswirtschaftlicher Perspektive nötig ist, beide Ebenen zudem zusammengeführt werden müssen. 


\subsubsection{Zielsegment}

Die quantitativen Simulationen basieren auf der Annahme, dass die Medikation für akute Erkrankungen in nächster Zukunft grundsätzlich kein Potential für den Versand von Arzneimitteln darstellt. Dem steht die zeitliche Restriktion einer Lieferfrist von ca. 48 Stunden zwischen Rezepteingang in der Versandapotheke und Empfang beim Patienten entgegen. Es müssen Indikatoren eruiert werden, die zum einen die wesentliche Einschränkung auf nicht chronische Medikation ermöglichen, zum anderen aber auch eine quantitative Messung von Zielsegmenten für einen Versandhandel gewährleisten. Es kristallisiert sich heraus, dass die Packungsgröße, kombiniert mit medizinischen wie pharmakologischen Expertenabschätzungen zur Dringlichkeit einer Medikation, einen adäquaten Indikator bildet: je größer der Packungsinhalt, desto wahrscheinlicher wird diese für eine längerfristige Medikation angewandt, ist damit nicht akut und für den Versandweg potentiell geeignet. Einschränkend sollte hinzugefügt werden, dass die Eruierung der Expertenmeinungen zur Dringlichkeit der Medikation mit Fokussierung auf die Definition der Versendungsfähigkeit nicht für jede Indikationsgruppe ein einheitliches Bild abgibt, sondern dass diese individuellen Einschätzungen nicht unwesentlichen Schwankungen in Abhängigkeit des medizinischen wie pharmakologischen Fachgebietes des Experten unterliegen. Durch die Komplexität der Arzneimitteltherapie und unterschiedlicher Anwendungsmöglichkeiten von Wirkstoffen ist eine Abgrenzung zwischen „versendungsfähig“ und „nicht versendungsfähig" auch vom entsprechenden Krankheitsbild abhängig. Zum anderen wird auch die Einschätzung der Versandfähigkeit von der Entwicklung des Qualitätsniveaus der pharmazeutischen Logistik, d.h. wie sicher und wie schnell eine Zusendung erfolgt, abhängig sein.

Es ergeben sich, basierend auf der Packungsgröße, drei Ausprägungen von Indikatoren (Packungen ab 50 definierten Tagesdosen, Packungen ab 35 definierten Tagesdosen $^{228}$ sowie N3-Normpackungen nach der Zuzahlungsverordnung ${ }^{229}$ ), die zur Operationalisierung dreier Varianten eines Zielsegments für einen potentiellen Pharmaversand führen.

Abbildung II - 23: Darstellung der Varianten A bis C des Zielsegments

\begin{tabular}{|c|c|c|c|}
\hline $\begin{array}{c}\text { Zielseg- } \\
\text { ment- } \\
\text { Variante }\end{array}$ & $\begin{array}{c}\text { Messgröße / } \\
\text { Operationalisierung }\end{array}$ & $\begin{array}{c}\text { Basisparameter: } \\
\text { GKV-Fertigarznei- } \\
\text { Mittelumsatz }\end{array}$ & $\begin{array}{c}\text { Zielsegment: } \\
\text { in \% }\end{array}$ \\
\hline A & Packungsgröße ab 50 DDD je Verordnung & 1999 & $41,0 \%$ \\
\hline B & Packungsgröße N3-Packungen & 1998 & $47,0 \%$ \\
\hline C & Packungsgröße ab 35 DDD je Verordnung & 1999 & $57,0 \%$ \\
\hline
\end{tabular}

Quelle: INIFES, eigene Darstellung.

228

Der Durchschnitt einer allgemeinen GKV-Verordnung lag 1999 bei 35 Tagesdosen. Damit sollen über diesen Indikator die überdurchschnittlich großen Packungen abgebildet werden.

229

Vierte Verordnung zur Änderung der Verordnung über die Zuzahlung bei der Abgabe von Arznei- und Verbandsmitteln in der vertragsärztlichen Versorgung, Bundesgesetzblatt 1997, Teil I, S. 2328. 
Das Zielsegment ist demnach der Teil des GKV-Fertigarzneimittelumsatzes, der durch die Zielfokussierung auf chronische bzw. dauerhafte Medikation abgegrenzt wird, und damit die maximale Obergrenze für eine Option „Versandhandel mit Arzneimitteln“ bildet. Die Herleitung möglicher Zielsegmente basiert dabei auf der Begriffsbestimmung "versandfähig", die sich auf die Ebene der Indikationsgruppe (nach ATC-Kodierung) ${ }^{230}$ bezieht. Die Ebene der Präparate, die für eine Differenzierung nach Länge der Dauermedikation bzw. nicht akuter Medikation sicherlich die optimale Analyseebene wäre, kann aufgrund der immensen Anzahl der Präparate auf dem deutschen Apothekenmarkt - die Zahl wird auf 52.000 und mehr geschätzt aus praktischen Gründen nicht erfolgen. Zudem differieren Präparate oft in ihrer Darreichungsform und Dosierung. Die Berechnungen basierten deshalb auf der Stufe der Indikationsgruppen.

Es ist an dieser Stelle jedoch wiederholt darauf hinzuweisen, dass die ermittelten Ausprägungen von Zielsegmenten für einen Pharmaversand nicht mit Marktanteilen für den Versandhandel zu verwechseln sind. Sie ermöglichen in einem ersten Modellschritt lediglich eine Aussage darüber, welcher Teil des GKV - Fertigarzneimittelumsatzes aus epidemiologischer Herangehensweise ein „versendungsfähiges“ Segment darstellt und somit als Grundlage weiterer Modellentwicklungen gelten kann.

Das Zielsegment fokussiert entsprechend auf die volkswirtschaftliche Ebene der Marktsegmentierung. Ob alle Verordnungen des aus volkswirtschaftlicher Sicht definierten Zielsegments auch unter Einbeziehung betriebswirtschaftlicher Kostenstrukturen potentieller Anbieter für den Versand in Frage kommen, kann hier noch nicht beantwortet werden.

Um also klären zu können, ob unter dem Status quo der Erstattungsfähigkeit der im GKV-Fertigarzneimittelindex erfassten Verordnungen bei Geltung der derzeitigen Arzneimittelpreisverordnung eine Deckung der entstehenden Kosten für den Vertriebsweg Versand aus der Betriebshandelsspanne für Versandapotheken möglich wäre bzw. nicht zu finanziellen Mehrbelastungen der GKV führen würde, muss eine Simulation betriebswirtschaftlicher Kostenstrukturen potentieller Versender erfolgen.

\subsubsection{Betriebs- und Kostenstruktur}

Zentrales Element der Analyse potentieller Einsparvolumen einer Ausweitung des Pharmaversandes ist demnach die Simulation der Kostenstrukturen von Versandapotheken. Einerseits lassen sich durch betriebswirtschaftliche Kostenstrukturanalysen die durchschnittlichen Kosten der existierenden Präsenzapotheken mit den zu enwartenden Kosten verschiedener Ausgestaltungsformen von Versandapotheken vergleichen. Andererseits ergeben sich aus den geänderten Betriebsabläufen und

Variante $\mathrm{B}$ genannten Gründen $\mathrm{ab}$. 
Vertriebswegen der Versandapotheke aber auch Differenzen in der Kostenstruktur. Ausgangsbasis der Untersuchungen in Kapitel 2.4. ist die interne Struktur einer Präsenzapotheke, die auf Basis zugänglicher Apothekenbetriebsvergleich-Daten entwickelt wird und als Referenzmaß weiterer Simulationen dient, insbesondere für das Modell der "kleinen", regional begrenzten Versandapotheke (VA I). Unter Zuhilfenahme weiterer Sekundärdaten können die Kostenstrukturen zukünftiger Versandapotheken entwickelt werden. Die Modellrechnungen zur internen Struktur von Versandapotheken umfassen u.a. die Annahmen, dass:

- in den Räumen der Versandapotheke kein oder nur sehr wenig Kundenverkehr stattfindet, Versandapotheken also auch in Gewerbegebieten mit niedrigeren Mietkosten angesiedelt sein können,

- Versandapotheken als Service- und Qualitätsstandard eine 24-Stunden-TelefonHotline (an allen Tagen des Jahres) anbieten,

- die Lagerwirtschaft anhand der in 2.4.2.1 genannten Argumente weitgehend optimiert gestaltet wird und

- pro pharmazeutischen Mitarbeiter anhand der in 2.4.2.1 genannten Argumente ein wesentlich höherer Umsatz im Vergleich zur Präsenzapotheke erwirtschaftet werden kann.

Die interne Kostenstruktur der Versandapotheken wird an unterschiedliche Umsatzgrößenklassen von Versandapotheken gekoppelt, um Skaleneffekte bei den Kosten und deren Auswirkung auf das Betriebsergebnis zu analysieren. Es werden drei Varianten von Arzneimittelversendern simuliert:

- Das Modell der kleinen Versandapotheke (VA I) beschreibt eine Versandapotheke, die aus den Strukturen der Präsenzapotheke entwickelt wird und einen Bruttoumsatz von ca. 5 Mio. DM - also ca. den doppelten Wert einer durchschnittlichen Präsenzapotheke - erreicht. Die Logistik wird bei dieser regional begrenzten Modellvariante selbst erstellt, die Telefonberatung während der Öffnungszeiten durch eigenes pharmazeutisches Personal durchgeführt, außerhalb der Öfnungszeiten an externe Call - Center vergeben. Es zeigt sich, dass mit der Umsatzverdopplung gegenüber Präsenzapotheken trotz neuer Aufwendungen für Auslieferung und Telefonberatung eine wesentliche Verbesserung in der Kostenstruktur erreicht werden kann. Es verbleibt ein betriebswirtschaftliches Ergebnis von ca. $6,2 \%$.

- Im Modell der großen Versandapotheke (VA II) wird von einem Jahresumsatz von 20 Mio. DM ausgegangen. Die Logistik wird bei dieser überregional agierenden Versandapotheke an externe Logistiker vergeben. Die Telefonberatung erfoigt während der Öffnungszeiten durch eigenes pharmazeutisches Personal, außerhalb der Offnungszeiten durch ein externes Call - Center. Das betriebswirtschaftliche Ergebnis beträgt hier $7,7 \%$ vom Umsatz. Insgesamt zeigt sich, dass mit einer Umsatzvervierfachung gegenüber Versandapothekenvariante I eine Verbesserung in der Kostenstruktur erreicht werden kann, trotzdem die Logistikkos- 
ten gegenüber Versandapotheke I aufgrund höherer Stückkosten bei externen Logistikanbietern steigen.

- Das Modell der Versandapotheke in Verbund mit Großhandel (VA III) geht von einer Zusammenlegung von Großhandels- und Apothekenfunktion in eine Vertriebsinstanz aus. Obwohl dieses Modell z.T. erheblichen Schwierigkeiten aufgrund fehlenden Datenmaterials einerseits, aber auch der Quantifizierung von Synergieeffekten andererseits unterliegt, zeigen die Untersuchungen hier eine möglicherweise zukunftsträchtige Option auf. Der Umsatz wird anhand verfügbarer Angaben zu Großhandelsumsätzen für mittelgroße Unternehmen orientiert, wonach sich ein Apothekenumsatz von ca. 487 Mio. DM ergibt. Diese Größe steht allerdings nicht für eine als minimal vorauszusetzende Umsatzschwelle, um beide Handelsstufen miteinander zu verbinden. Hier ist - ohne Abweichungen von der Kostenstruktur - auch ein Gesamtumsatz von 100 Mio. DM bis 200 Mio. DM ohne weiteres vorstellbar. Die Logistik wird bei dieser überregional agierenden Versandapotheke an externe Logistiker vergeben. Die Telefonberatung erfolgt rund um die Uhr durch eigenes pharmazeutisches Personal. Das erzielte betriebswirtschaftliche Ergebnis von $11,6 \%$ liegt im Vergleich zu den anderen potentiellen Versandapotheken aufgrund von Skaleneffekten wesentlich höher.

\subsubsection{Markt- und Einsparpotentiale}

Nach Kenntnis der betriebswirtschaftlichen Kostenstrukturen potentieller Versender können diese mit den Varianten volkswirtschaftlicher Zielsegmente kombiniert werden, um die Frage zu beantworten, welcher Anteil der unter 2.3. als versendungsfähig definierten Verordnungen bei Geltung der ökonomischer Restriktionen aus 2.4. für den neuen Vertriebsweg Versand in Frage kommen. Daraus resultieren die sogenannte Marktpotentialvarianten.

Abbildung II - 24: Marktpotentialvarianten

\begin{tabular}{|l|c|c|c|}
\hline Zielsegment - Variante & A & B & C \\
\hline Zielsegment in \% vom GKV-Fertigarzneimittelmarkt 1999 & $41 \%$ & $47 \%$ & $57 \%$ \\
\hline Marktpotential in \% vom GKV-Fertigarzneimittelmarkt 1999 & & & \\
VA I & $39 \%$ & $44 \%$ & $53 \%$ \\
VA II & $39 \%$ & $44 \%$ & $52 \%$ \\
VA III & $39 \%$ & $45 \%$ & $53 \%$ \\
\hline
\end{tabular}

Quelle: INIFES, eigene Darstellung.

Weiterhin werden die Betriebsergebnisse der Versandapotheken im Betriebsvergleich als Kostenersparnisse auf die Arzneimittelpreise solcher Verordnungen interpretiert, die für den Versand geeignet sind. Diese gelten unter Annahme der Beibehaltung der aktuell gültigen Arzneimittelpreisspannenverordnung. 
Die Kostenersparnisse der Versandapotheken werden zudem als eine zum derzeitigen 5\%igen GKV-Rabatt zusätzlich mögliche Rabattgewährung an die gesetzlichen Krankenkassen für solche Arzneimittel, die über den Distributionskanal Versand an die Versicherten gelangen könnten, interpretiert. Die Rabattgewährung der VA I liegt demnach bei ca. $6,2 \%$, die der VA II bei ca. $7,7 \%$. Die zusätzlich mögliche Rabatgewährung der VA III mit ca. 11,6\% auf die Arzneimittelkosten liegt in etwa auf dem Niveau Schweizer Versandapotheken, die durch Direktversand zwischen 10\% bis $15 \%$ der Medikamentenkosten einsparen.

Das Einsparpotential für die entsprechende Marktpotentialvariante ergibt sich jeweils, in dem der prozentuale Kostenvorteil der Versandapotheken mit dem maximalen Marktpotential kombiniert wird. Das Intervall möglicher Einsparungen liegt zwischen 861 Mio. DM und 2.144 Mio. DM. Es zeigt sich, dass insbesondere durch umsatzstarke Versender aufgrund deren interner Betriebsstruktur die höchsten Einsparpotentiale innerhalb der entsprechenden Marktpotentiale zu erwarten sind:

- Kleine Versandapotheken mit ca. 5 Mio. DM Umsatz (VA I) können durch eine 6,2\%ige Kosteneinsparung auf die Arzneimittelkosten ein Einsparpotential von 861 Mio. DM (bei 39\% Marktpotential) bis 1.142 Mio. DM (bei 53\% Marktpotential) erzielen.

- Versandapotheken mit ca. 20 Mio. DM Umsatz (VA II) können durch eine 7,7\%ige Kosteneinsparung ein Einsparpotential von 1.060 Mio. DM (bei 39\% Marktpotential) bis 1.398 Mio. DM (bei $52 \%$ Marktpotential) erzielen.

- Versandapotheken mit kombinierter Großhandels- und Apothekenfunktion und einem Umsatz von 487 Mio. DM (VA III) können durch eine 11,6\%ige Kosteneinsparung ein Einsparpotential von 1.607 Mio. DM (bei 39\% Marktpotential) bis 2.144 Mio. DM (bei 53\% Marktpotential) erzielen.

Die genannten Einsparvolumina können jedoch nur erzielt werden, wenn die aufgezeigten maximalen Marktpotentiale von $39 \%$ bis $53 \%$ vollständig ausgeschöpft werden. Davon ist realistischerweise in Zukunft nicht auszugehen. Allerdings, in welchem Maß und in welchem Zeitbezug dieses Marktpotential in Form tatsächlicher Marktanteile zukünftig realisiert werden wird, ist nicht abzuschätzen und hängt von weiteren exogenen (betriebsfremden) wie endogenen (betriebsinternen) Restriktionen, wie z.B. der Überwindung von Akzeptanzhürden gegenüber Versandapotheken bei Patienten wie verordnenden Ärzten, $a b$.

Deshalb wird in einem nächsten Schritt mit Szenarien zum Realisierungsgrad von Marktanteilen gearbeitet. Welche Einsparvolumina für die GKV entstehen und wie diese mit der Größenklasse der Versandapotheke korrespondieren, wird am Beispiel eines Marktanteils des Versandhandels von $15 \%$ am GKV-Fertigarzneimittelumsatz dargestellt. Das Intervall entsprechender Einsparungsvolumina liegt bei einem 15\%igen Marktanteil zwischen 323 Mio. DM (bei ca. 6,2\% Einsparung auf die Arzneimittelkosten) und 618 Mio. DM (bei ca. 11,6\% Kosteneinsparung). 
Die Orientierung an einem 15\%igen Realisierungsgrad basierte auf folgenden Begründungen, insbesondere auf Erfahrungen mit Versandapotheken im Ausland:

- Wonach in der Schweiz mit einem monetären Marktpotential von mehr als $10 \%$ der Arzneimittelausgaben gerechnet werden kann, ${ }^{231}$

- Oder US-Quellen bereits 1997 von einem 12\%igen Umsatzanteil des Arzneimittelversandes am Markt für verschreibungspflichtige Präparate sprachen. ${ }^{232}$ Dass es sich dabei um ein stark wachsendes Segment handelt, zeigt die Steigerung auf derzeit (Stand 2000 / 2001) ca. 15\% Marktanteil des Versandes. ${ }^{233}$

Obwohl alle simulierten Markt- wie Einsparpotentialvarianten gleichermaßen differenziert und fundiert hergeleitet werden, unterliegen sie doch unterschiedlichen Annahmen, auf die hier nochmals kurz kritisch eingegangen wird.

Bei der Ermittlung der Zielsegmente wird davon ausgegangen, dass der Arzneimittelversand an den Endverbraucher nur für solche Indikationsgruppen realistisch ist, die primär Medikamente für nicht akute Erkrankungen bzw. Dauermedikationen beinhalten. Die Auswahl der Indikationsgruppen erfolgt anhand der Kriterien "Packungsgröße", "DDDs" und "Expertenmeinung". Die Abgrenzungen "Umsätze von N3-Packungen" und "50 DDDs und mehr" zielt auf Medikamente aus dem Bereich der mittel- bis langfristigen Medikation ab, beinhaltet, wie dargestellt, aber einige Unwegsamkeiten z.B. bzgl. der mengenmäßigen Abgrenzungen zwischen N2- und N3-Packungen, sowie dahingehend, ob die Grenze von 50 DDDs als "große" Packung allgemeingültig ist. Als "stabilste" Annahme ist deshalb die Abgrenzung anhand des Kriteriums überdurchschnittlicher täglicher Dosierungen - also "35 DDDs und mehr" - zu bewerten, die zu Marktanteilen von ca. 53\% führte. Diese Einschätzung wird auch durch die Expertenmeinung gestützt, wonach $59 \%$ der GKV-Umsätze auf Medikationen für überwiegend chronische Erkrankungen entfallen.

Die Untersuchungen zu den Kosten des Pharmaversandes machen eine Vielzahl von Annahmen notwendig, da hier gänzlich neue Strukturen unter Rückgriff auf sehr spärliches Datenmaterial simuliert werden müssen. Die von INIFES getroffenen Annahmen sind bzgl. der "Chancen" des Pharmaversandes eher konservativ. Damit wird gewährleistet, dass bei der Analyse potentieller Auswirkungen einer Ausweitung des Pharmaversandes in Deutschland ein Versandapotheken-Modell hoher Qualität und solider betriebswirtschaftlicher Struktur das Fundament der Berechnungen bildet. Die INIFES-Berechnungen gehen beispielsweise von einer 24-Stunden-Hotline auch bei "kleinen" Versandapotheken aus. Die Kostenstrukturanalysen gehen zudem z.B. von großzügig gestalteten Lagerflächen und relativ hohen Miet- und Versandkosten aus. Auch die angenommene Rabattgewährung von 5\% durch die Her-

\footnotetext{
231

Siehe Argumentation 2.8.

232

American Medical Association, Report of the AMA Young Physicians section governing council, 1997, 2.

233

Ahrens, H.J., (2001), Interview des Vorstandsvorsitzenden der AOK mit der Zeitschrift impulse, Rubrik Drei Fragen an ..., siehe: impulse / märz 2001, S. 168.
} 
steller stellt, insbesondere unter der Annahme umsatzstarker Versandapotheken, eher eine Untergrenze dar.

Das Modell einer Versandapotheke VA I mit jährlichen Umsätzen in doppelter Höhe des derzeitigen Durchschnitts der Präsenzapotheken (bei weitgehend gleichem Personalbestand wie diese) zeigt, dass der regional begrenzte Versand an den Endverbraucher auch für die existierenden Präsenzapotheken eine attraktive Alternative darstellen kann. Allerdings sind die Einsparpotentiale für die GKV hier relativ gering: sie liegen zwischen ca. 861 und 1.142 Mio. DM bei vollständiger Abschöpfung des Marktpotentials bzw. zwischen 323 Mio. DM und 331 Mio. DM bei einem angenommenen Marktanteil von $15 \%{ }^{234}$ Offen ist auch, in welchem Umfang solche kleinen Versandapotheken in einer Konkurrenzsituation mit großen Versandapotheken oder gar mit Versandapotheken, die im Verbund mit dem Großhandel agieren, langfristig überlebensfähig sind.

Die Erfahrungen aus dem Ausland sowie erste Entwicklungen in Deutschland (z.B. bei Versendern von Impfstoffen etc.) zeigen vielmehr, dass Versandapotheken mit Jahresumsätzen ab 20 Mio. DM eine sehr realistische, betriebswirtschaftlich rentable Unternehmensform in einem liberalisierten Arzneimittel-Distributionssystem darstellen können. In noch stärkerem Maße gilt diese Annahme selbstverständlich für Versandapotheken, die einen direkten Verbund mit dem Pharmagroßhandel eingehen und sozusagen als deren verlängerter Arm für den Vertrieb an den Endverbraucher dienen. Bei diesem letzten beiden Varianten liegen die Einsparpotentiale zwischen ca. 1.060 und 2.144 Mio. DM bei vollständiger Abschöpfung des Marktpotentials bzw. zwischen 399 Mio. DM und 618 Mio. DM bei einem angenommenen Marktanteil von $15 \%$. $^{235}$

Die Betrachtung der Einsparpotentiale wird zusätzlich unter dem Gesichtspunkt der Berücksichtigung einer durchschnittlichen Betriebsrendite durchgeführt. Es wird darauf hingewiesen, dass es Pro- und Contra-Argumente anzuführen gilt, ob in eine volkswirtschaftliche Betrachtung möglicher Markt- und Einsparpotentiale eine betriebswirtschaftliche Gewinnüberlegung von Anfang an mit einbezogen werden sollte. Insbesondere deshalb, da sich bereits die Eruierung einer aus volkswirtschaftlicher Sicht anzunehmenden Durchschnittliche Betriebsrendite für Versandapotheken als problematisch gestaltet, da diese zum einen aufgrund des Geschäftsfeldes im wesentlichen an den heutigen Präsenzapotheken orientiert sein müsste, zum anderen aber aufgrund der zu erwartenden höheren Umsatzstärken potentieller Versender auch deren Renditen über dem heutigen Durchschnitt liegen könnten. Als ProArgument zur Berücksichtigung einer Durchschnittsrendite wird angeführt, dass Versandapotheken als gewinnorientierte Marktakteure eine Unternehmensrendite einkalkulieren und das erzielte betriebswirtschaftliche Ergebnis folglich nicht im vollen

234

235

Ohne Berücksichtigung einer Durchschnittsrendite bzw. zusätzlicher einheitlicher Kassenrabatte.

Ohne Berücksichtigung einer Durchschnittsrendite bzw. zusătzlicher einheitlicher Kassenrabatte. 
Umfang als Kosteneinsparung in Form erhöhter Rabatte der GKV zuführen werden. Dabei handelt es sich bei der hier angenommenen Durchschnittsrendite explizit nicht um eine garantierte individuelle Rendite, sondern um einen angenommenen Branchenwert. Auch im Vertriebssegment der Präsenzapotheken ist es üblich, eine durchschnittliche Rendite - die nach Angaben des IFH Köln wie der ABDA für 1998 bei $0,4 \%$ lag (siehe die Ausführungen in Kapitel 2.4.1.) - zu berechnen. Entsprechendes erfolgt hier für Versandapotheken. Dabei ist natürlich - ebenfalls analog zu den Präsenzapotheken - zu unterstellen, dass die Rendite der jeweiligen Versandapotheke mit deren Umsatz korreliert.

Durch die Berücksichtigung einer durchschnittlichen Betriebsrendite kommt es zu zwei wesentlichen Effekten bei der Berechnung der Kostenersparnisse wie der Marktpotentiale:

- Zum einen fallen die potentiellen zusätzlichen Rabatte der potentiellen Versender an die GKV wesentlich geringer aus,

- Zum anderen verringert sich die Anzahl der Verordnungen, die aus den geringeren Kostenersparnissen die Logistikstückkosten decken könnten (Marktpotential). Besonders stark sind davon die umsatzschwächeren Versender VA I und VA II betroffen, deren Marktpotential im Vergleich zum Zielsegment um ca. ein Viertel sank. Die umsatzstarke Versandapotheke VA III erfährt dagegen durch die Berücksichtigung einer durchschnittlichen Betriebsrendite kaum Verluste im Marktpotential.

Das Einsparpotential als Kombination aus beiden Komponenten Marktpotential wie Kostenersparnis unterliegt folglich insbesondere bei VA I und VA II einer überproportionalen Dezimierung. Das Intervall möglicher Einsparungen liegt nunmehr zwischen 386 Mio. DM und 1.490 Mio. DM. Umsatzstarke Versender wie VA III können nach wie vor relativ hohe Einsparpotentiale erzielen. Die Berücksichtigung einer durchschnittlichen Betriebsrendite fält hier nicht so stark ins Gewicht wie im Vergleich zu VA I und VA II, da hier "nur" der Effekt einer geringen Kostenersparnis zu beobachten ist.

Entsprechend erheblich stellen sich die Veränderungen des Einsparvolumens bei Annahme eines 15\%igen Marktanteils dar: Das Intervall realistischer Einsparungsvolumina liegt nunmehr zwischen 167 Mio. DM (bei ca. 3,4\% Einsparung auf die Arzneimittelkosten für VA I) und 434 Mio. DM (bei ca. 8,2\% Kosteneinsparung VA III).

Es bleibt festzuhalten, dass die Berücksichtigung einer durchschnittlichen Betriebsrendite einen erheblichen Einfluss auf die Höhe der zuvor ausgewiesenen Einsparpotentiale hat. Die größten Auswirkungen zeigen sich bei Versandapotheken mit 5 Mio. DM bzw. 20 Mio. DM Umsatz: hier werden nicht nur wesentlich geringere Rabatte an die GKV abgeführt, sondern es verringert sich auch die Anzahl der Verordnungen, auf die dieser Rabatt gewährt werden könnte (entspricht dem Marktpo- 
tential). Große Versender wie VA III weisen nur den erstgenannten Effekt auf und können deshalb auch hier noch vergleichsweise hohe Einsparungen erzielen.

Die Einsparpotentiale werden alternativ zur Berücksichtigung einer Durchschnittsrendite und umsatzabhängigen Kassenrabatten unter der Annahme der Gewährung zusätzlicher einheitlicher Kassenrabatte an die GKV betrachtet. Rechtliche Aspekte eines solchen Modells bleiben in der Betrachtung außen vor. Intention der Überlegungen, auf denen dieses Modell basiert, ist eine Orientierung an der derzeitigen Rabattgewährung zwischen Apotheken und GKV, die nicht auf umsatzabhängigen, sondern Umsatz unabhängigen einheitlichen Kassenrabatten beruht. Die im Modell alternativ verwendeten Werte von $5 \%$ bzw. $7 \%$ bzw. 10\% zusätzlichem einheitlichen Kassenrabatt sind dabei zum einen an der Höhe des derzeitigen Kassenrabatts bzw. dessen doppelten Wert orientiert, zum anderen erfolgt mit $7 \%$ die Annahme eines Wertes innerhalb dieser Spannweite.

Entsprechend der Höhe der zusätzlichen einheitlichen Kassenrabatte variiert das Marktpotential und die daraus resultierenden Einsparpotentiale erheblich. Bei Berücksichtigung eines einheitlichen Kassenrabatts würden die Einsparungen aufgrund der dargestellten Einflussfaktoren im Vergleich zur Ausgangslage ${ }^{236}$ geringer ausfallen: in Abhängigkeit von der Umsatzgröße des Versenders sowie der Höhe des zusätzlichen einheitlichen Kassenrabatts liegen diese zwischen 379 Mio. DM und 1.124 Mio. DM. Die maximale Ausprägung des Einsparpotentials wird langfristig bei einer zusätzlichen Rabattgewährung von $7 \%$ durch das Modell der umsatzstarken Versandapotheke VA III realisiert.

In kurz- und mittelfristiger Sicht - d.h. bei einem Marktanteil von 15\% - liegen die entsprechenden Einsparvolumina bei 276 Mio. DM (Alternative 5\% zusätzlicher Kassenrabatt) bzw. 386 Mio. DM (Alternative 7\% zusätzlicher Kassenrabatt) bzw. 552 Mio. DM (Alternative 10\% zusätzlicher Kassenrabatt). Unter Berücksichtigung der langfristigen Entwicklungspotentiale liegt der optimale Wert des Einsparpotentials bei $7 \%$ Rabattgewährung. Übertragen auf die kurz- und mittelfristige Perspektive erscheinen entsprechende Einsparvolumen von 386 Mio. DM bei einem Marktanteil von $15 \%$ (siehe Abbildung II - 22) als realisierbar. Neben dem Modell VA III wären hier auch Versender der Umsatzgröße VA III funktionsfähig. Umsatzschwache Versandapotheken nach dem Model VA I würden nicht im Zielsegment agieren.

Der mehrfach erwähnte Zusammenhang zwischen der Höhe des Kassenrabatts und dem Umfang des Marktpotentials soll in diesem Kontext noch einmal thematisiert werden. Wie sich in Abbildung II - 17 zeigt, sinkt generell das Marktpotential mit steigendem zusätzlichen Kassenrabatt, auch wenn davon umsatzschwächere potentielle Versendern stärker betroffen sind. Das aus dem Marktpotential resultierende Einsparpotential zeigt zudem, dass dessen Optimum nicht beim höchsten der drei alternativen Werte zusätzlicher Kassenrabatte liegt (siehe Abbildung II - 18). Dieser Hin-

236

Siehe Kapitel 2.5.2. 
tergrund sollte bei einer Entscheidung bezüglich zusätzlicher einheitlicher Kassenrabatte berücksichtigt werden. In diesem Zusammenhang ist noch einmal auf die Problematik hinzuweisen, dass mit steigendem zusätzlichen Kassenrabatt tendenziell mehr hochpreisige Arzneimittel versandt werden.

Die hier dargestellten Ergebnisse - ob nun in der undifferenzierten Modellrechnung. oder bei Differenzierung der Kosteneinsparung hinsichtlich der Berücksichtigung einer Durchschnittsrendite bzw. zusätzlicher einheitlicher Kassenrabatte - unterliegen dem Vorbehalt, dass eine Bewertung potentieller monetärer Einsparvolumen einer Ausweitung des Pharmaversandes nicht ohne Berücksichtigung struktureller und qualitativer Effekte einer Liberalisierung der Arzneimitteldistribution erfolgen kann. Offen - aber für eine abschließende Bewertung unabdingbar - bleibt an dieser Stelle z.B., wie sich die Akzeptanz des Versandhandels bei allen Beteiligten entwickeln wird oder welche Auswirkungen für den Apothekenmarkt in Deutschland zu erwarten sind. Damit beschäftigt sich Kapitel 4, nachdem im nun folgenden Kapitel 3 die isolierte qualitative Analyse der Einstellungen zum Pharmaversand erfolgt. 


\section{Einstellung zum Pharmaversand}

\subsection{Einleitung}

\subsubsection{Vorbemerkung}

Um eine abschließende Bewertung hinsichtlich potentieller monetärer Einsparpotentiale geben zu können, die sich aus einer Ausweitung des Pharmaversandes für Deutschland ergeben kann, ist es im Rahmen des Forschungsvorhabens notwendig, auch die strukturellen und qualitativen Auswirkungen einer Ausweitung des Pharmaversandes auf Anbieter und Nachfrager anhand von Primärerhebungen und Expertengesprächen abzuschätzen. Dabei ist z.B. darauf abgezielt, welche Akzeptanz des Versandhandels bei Patienten, Ärzten und Apotheken zu erwarten ist, welche Auswirkungen (soziale Kontakte, Beratung, Probleme bei weiteren Verordnungen etc.) der Versandhandel auf die Zielgruppen hat oder auch wie die These, durch den Versandhandel könne ggf. der umfassende Versorgungs- und Sicherstellungsauftrag der Apotheken beeinflusst werden, zu bewerten ist.

\subsubsection{Ergebnisse bisher durchgeführter Befragungen}

Im Fokus bislang durchgeführter Primärerhebungen und Studien ${ }^{1}$ zur Thematik Apotheke und Arzneimitteldistribution standen staatliche Regulierungsmaßnahmen, die Bewertung einer Versandapotheke aus Sicht ihrer Kunden sowie Arzneimittel im Internet. Hintergrund dieser Studien (s. Tabelle III - 1 und III - 2) war zum einen eine Darstellung der Chancen und Risiken bzgl. Arzneimittel im Internet, zum anderen eine Untersuchung der gegenwärtigen und zukünftigen Situation der Apotheken und der Arzneimitteldistribution.

Die Zielgruppen der wissenschaftlichen Befragungen bildeten die Apotheker und die Bevölkerung (Patienten/Kunden). Eine Kombination der Fokusgruppen der Apotheker, Ärzte und der Bevölkerung hat jedoch nie stattgefunden.

Des weiteren sei an dieser Stelle noch einmal auf den Unterschied zwischen Internetapotheke und Versandhandel hingewiesen. Während Internetapotheken nicht den deutschen Qualitätsanforderungen unterliegen und folglich den Bezug von verschreibungspflichtigen Arzneimitteln aus dem Ausland ermöglichen, unterliegt eine Versandapotheke denselben gesetzlichen Anforderungen bezüglich Zulassung, Betriebserlaubnis, Personal, behördlicher Aufsicht, etc., wie die traditionellen Apotheken auch.

Dem Anspruch der von INIFES durchgeführten Studie folgend, die Akzeptanz und generelle Einstellung der von einem Versandhandel Betroffenen zu evaluieren, machte eine eigene Erhebung unabdingbar. Aufgrund der entwickelten Fragebögen ist es möglich, die Ergebnisse einiger Fragen zueinander in Beziehung zu setzen und damit die strukturellen und qualitativen Auswirkungen einer Ausweitung des Pharmaversandes auf Anbieter und Nachfrager abzuschätzen.

Es wird Bezug genommen auf bislang veröffentlichte Studien und Befragungsergebnisse. Die Studien können teilweise beim Verfasser der INIFES eigenen Studie eingesehen werden. 
Tabelle III - 1: Ausgewählte Primärerhebungen zum Arzneimittelvertrieb

\begin{tabular}{|c|c|c|c|c|c|}
\hline Erhebung & Schöffski & $\begin{array}{c}\text { Riegl \& Partner } \\
\text { GmbH }\end{array}$ & $\begin{array}{l}\text { Institut für Sozial- und } \\
\text { Präventivmedizin }\end{array}$ & FORSA & $\begin{array}{c}\text { MediaTransfer } \\
\text { AG }\end{array}$ \\
\hline Befragt wurden... & Apotheker & $\begin{array}{l}\text { Apotheker sowie deren } \\
\text { Mitarbeiter, Konsumen- } \\
\text { ten }\end{array}$ & $\begin{array}{l}\text { Konsumenten (Kunden } \\
\text { einer Versandapotheke) }\end{array}$ & Konsumenten & Internetnutzer \\
\hline Datenbasis & $n=1012$ & $\begin{array}{l}\text { - } \text { Kunden: } n=43.404 \\
\text { - Apotheker und } \\
\text { Mitarbeiter: } \\
n=1.363\end{array}$ & $n=2.500$ & $n=1003$ & $n=1.120$ \\
\hline $\begin{array}{l}\text { Jahr der Erhe- } \\
\text { bung }\end{array}$ & $1993 / 94$ & 1995 & 1998 & 2000 & 2001 \\
\hline Region & $\begin{array}{l}\text { Bundesrepublik } \\
\text { Deutschland }\end{array}$ & $\begin{array}{l}\text { Bundesrepublik } \\
\text { Deutschland }\end{array}$ & Schweiz & $\begin{array}{l}\text { Bundesrepublik } \\
\text { Deutschland }\end{array}$ & $\begin{array}{l}\text { Bundesrepublik Deutsch- } \\
\text { land }\end{array}$ \\
\hline Methode & Schriftliche Befragung & Schriftliche Befragung & Schriftliche Befragung & $\begin{array}{l}\text { Telefonische Befra- } \\
\text { gung }\end{array}$ & Online-Befragung \\
\hline Auftraggeber & Eigenerhebung & $\begin{array}{l}\text { Ratiopharm GmbH \& } \\
\text { Co }\end{array}$ & MediService Apotheke & $\begin{array}{l}\text { Deutsche Angestell- } \\
\text { ten Krankenkasse } \\
\text { (DAK) }\end{array}$ & Eigenerhebung \\
\hline Themen & $\begin{array}{l}\text { Befragung der } \\
\text { Einstellung der } \\
\text { Apotheker zu der } \\
\text { staatlichen Regu- } \\
\text { lierung auf dem } \\
\text { Arzneimittelmarkt } \\
\text { Erfolgsaussichten } \\
\text { für Deregulie- } \\
\text { rungsmaßnah- } \\
\text { men. }\end{array}$ & $\begin{array}{l}\text { Das Fremdbild der } \\
\text { deutschen Apothe- } \\
\text { ke (Kundensicht); } \\
\text { Das Selbstbild der } \\
\text { deutschen Apothe- } \\
\text { ke (Apotheken- } \\
\text { sicht). }\end{array}$ & $\begin{array}{l}\text { Medikamentenkon- } \\
\text { sum; } \\
\text { Aspekte des Medi- } \\
\text { kamentenvertriebs } \\
\text { (z.B. Dauer des Ver- } \\
\text { sandes); } \\
\text { Kundenzufriedenheit } \\
\text { mit einer Versand- } \\
\text { apotheke. }\end{array}$ & \begin{tabular}{|l|} 
Einstellung der \\
Bundesbürger \\
hinsichtlich einer \\
zeitgemäßen \\
Arzneimittelver- \\
sorgung.
\end{tabular} & $\begin{array}{l}\text { - Akzeptanz des Medi- } \\
\text { kamentenhandel im In- } \\
\text { ternet; } \\
\text { - Online-Apotheken; } \\
\text { - Medikamentenhandel } \\
\text { im Internet. }\end{array}$ \\
\hline
\end{tabular}

Quelle: INIFES, eigene Darstellung nach:

Oliver Schöffski 1995; Riegl \& Partner GmbH 1995; Institut für Sozial- und Präventivmedizin 1999; Forsa 2000; MediaTransfer AG 2001 
Tabelle III - 2: Studien zu: Arzneimittel im Internet

\begin{tabular}{|c|c|c|c|c|}
\hline Studie & Autoren & Datum & Inhalt & Zentrale Ergebnisse \\
\hline $\begin{array}{l}\text { Impact of Electronic } \\
\text { Commerce on the } \\
\text { European Pharma- } \\
\text { ceutical Sector }\end{array}$ & $\begin{array}{l}\text { Ashurst Morris Crisp, } \\
\text { Executive Perspecti- } \\
\text { ve, im Auftrag der } \\
\text { Europäischen Kom- } \\
\text { mission, DG III }\end{array}$ & \begin{tabular}{|l|} 
November \\
1998
\end{tabular} & $\begin{array}{l}\text { E-Commerce im Arzneimittel- } \\
\text { sektor in der Europäischen } \\
\text { Union - Überblick über Ent- } \\
\text { wicklungen, rechtliche Rah- } \\
\text { menbedingungen und Emp- } \\
\text { fehlungen }\end{array}$ & $\begin{array}{l}\text { Empfehlungen an die Europäische Kommission: } \\
\text { - Revision der "regulatorischen Barrieren" } \\
\text { - Mindeststandards in den Bereichen Konsumentenschutz und Si- } \\
\text { cherheit } \\
\text { - Bildungsprogramme für die Bevölkerung zur Aufklärung über Risi- } \\
\text { ken des Arzneimittelkaufes im Internet } \\
\text { - Internationale Kooperation, um Verhaltenskodizes und einheitli- } \\
\text { che rechtliche Rahmenbedingungen zu erarbeiten }\end{array}$ \\
\hline $\begin{array}{l}\text { Arzneimittel im In- } \\
\text { ternet. Telefonische } \\
\text { Mehrthemenbefra- } \\
\text { gung }\end{array}$ & \begin{tabular}{|l|} 
EMNID-Institut, \\
Deutschland, im Auf- \\
trag des deutschen \\
Bundesverbandes \\
der Pharmazeuti- \\
schen Industrie
\end{tabular} & \begin{tabular}{|l|} 
März \\
1999
\end{tabular} & $\begin{array}{l}\text { Repräsentative Telefonbefra- } \\
\text { gung in Deutschland zur Er- } \\
\text { hebung der Kenntnis und Ein- } \\
\text { schätzung von Arzneimitteln } \\
\text { im Internet (erste Welle) }\end{array}$ & $\begin{array}{l}\text { - Mehr als } 40 \text { Prozent der Befragten war die Bestellmöglichkeit im } \\
\text { Internet bekannt } \\
\text { - Divergierende Beurteilung der Qualität der Arzneimittel aus dem } \\
\text { Internet } \\
\text { - Unterschiedliche Einschätzung der Preise von Arzneimitteln aus } \\
\text { dem Internet } \\
\text { - Die persönlichen Gesundheitsrisiken beim Kauf von Arzneimitteln } \\
\text { aus dem Internet werden als eher gering eingeschätzt, während } \\
\text { für die Allgemeinheit hohe Gesundheitsrisiken gesehen werden }\end{array}$ \\
\hline
\end{tabular}

Fortsetzung nächste Seite 
$\vec{\Phi} \quad$ Tabelle III - 2/Fortsetzung

\begin{tabular}{|c|c|c|c|c|}
\hline Studie & Autoren & Datum & Inhalt & Zentrale Ergebnisse \\
\hline $\begin{array}{l}\text { Arzneimittel-Kauf im } \\
\text { Internet. Qualitative } \\
\text { Evaluation von re- } \\
\text { zeptpflichtigen oder } \\
\text { in der Schweiz be- } \\
\text { hördlich nicht zuge- } \\
\text { lassenen Angeboten }\end{array}$ & Innovamed, Basel & $\begin{array}{l}\text { Oktober } \\
1999\end{array}$ & $\begin{array}{l}\text { Testbestellungen von Arznei- } \\
\text { mitteln im Internet und deren } \\
\text { pharmazeutische Prüfung }\end{array}$ & $\begin{array}{l}\text { Von } 20 \text { bestellten Arzneimitteln wurden } 17 \text { - ohne Kontrolle beim } \\
\text { Zoll - geliefert, darunter elf nicht in der Schweiz zugelassene und } \\
\text { sechs zugelassene, rezeptpflichtige Arzneimittel } \\
\text { - Vier der sechs rezeptpflichtigen Arzneimittel wurden ohne Vor- } \\
\text { weisung einer ärztlichen Verordnung geliefert, für die beiden übri- } \\
\text { gen war eine Online-Konsultation erforderlich } \\
\text { - Die pharmazeutische Prüfung ergab Mängel in der Qualität und } \\
\text { zum Teil auch in der quantitativen Zusammensetzung der Produk- } \\
\text { te (z.B. nicht identischer bzw. falscher Wirkstoff, zu geringer Wirk- } \\
\text { stoffgehalt,...) } \\
\text { Von den } 17 \text { ausgelieferten Arzneimitteln könnten nur drei mit ei- } \\
\text { nem gesundheitlich kalkulierbaren Risiko eingenommen werden }\end{array}$ \\
\hline $\begin{array}{l}\text { Medikamente aus } \\
\text { dem Netz. Der } \\
\text { schutzlose Patient }\end{array}$ & Arbeiterkammer Wien & $\begin{array}{l}\text { November } \\
1999\end{array}$ & $\begin{array}{l}\text { Untersuchung von deutsch- } \\
\text { sprachigen Webseiten, die } \\
\text { Arzneimittel anbieten }\end{array}$ & $\begin{array}{l}\text { - "Ghost-Anbieter": zwei Drittel der Anbieter konnten nicht bzw. } \\
\text { nicht ausreichend identifiziert werden } \\
\text { - } \quad \text { Kaum Hinweise auf Risiken, Verharmlosung von Nebenwirkungen } \\
\text { - Im Allgemeinen muss der Konsument im Voraus bezahlen } \\
\text { - Geschäftsbedingungen sind selten vorhanden } \\
\text { - Überhöhte Preise }\end{array}$ \\
\hline
\end{tabular}

Fortsetzung nächste Seite 
Tabelle III - 2/Schluss

\begin{tabular}{|c|c|c|c|c|}
\hline Studie & Autoren & Datum & Inhalt & Zentrale Ergebnisse \\
\hline $\begin{array}{l}\text { E-Pharma - Arznei- } \\
\text { mittelvertrieb im In- } \\
\text { ternet }\end{array}$ & $\begin{array}{l}\text { Österreichisches } \\
\text { Bundesinstitut für } \\
\text { Gesundheitswesen } \\
\text { (ÖBIG), im Auftrag } \\
\text { des Bundesministeri- } \\
\text { ums für soziale Si- } \\
\text { cherheit und Genera- } \\
\text { tionen }\end{array}$ & $\begin{array}{l}\text { August } \\
2000\end{array}$ & \begin{tabular}{|l|} 
Analyse des Vertriebs von \\
Arzneimitteln im Internet aus \\
Sicht des Konsumentenschut- \\
zes und unter Berücksichti- \\
gung der für die Arzneimittel- \\
versorgung in Österreich bzw. \\
der EU geltenden Regelungen
\end{tabular} & $\begin{array}{l}\text { - Die Arzneimittelsicherheit wird durch das E-Pharma massiv un- } \\
\text { tergraben } \\
\text { - Über das Internet können Konsumenten Arzneimittel bestellen, } \\
\text { ohne vorher ärztlich untersucht worden zu sein } \\
\text { - Beim Internetkauf von Arzneimitteln werden die Konsumenten } \\
\text { unzureichend betreut und beraten } \\
\text { - Die Globalisierung des Internets fördert internationale Lösungen } \\
\text { - Die Zertifizierung von Internetapotheken ermöglicht eine Kenn- } \\
\text { zeichnung sicherer und seriöser Anbieter }\end{array}$ \\
\hline $\begin{array}{l}\text { Medikamente bald } \\
\text { übers Internet? }\end{array}$ & $\begin{array}{l}\text { EMNID-Institut, } \\
\text { Deutschland, im Auf- } \\
\text { trag des Kohtes Kle- } \\
\text { wes Meinungsbaro- } \\
\text { meters }\end{array}$ & $\begin{array}{l}\text { November } \\
2000\end{array}$ & $\begin{array}{l}\text { Befragung von } 250 \text { Mei- } \\
\text { nungsbildnern aus Politik, } \\
\text { Wirtschaft und Medien zu den } \\
\text { Themen Arzneimittelvertrieb } \\
\text { im Internet und Werbeverbot } \\
\text { für verschreibungspflichtige } \\
\text { Medikamente }\end{array}$ & $\begin{array}{l}\text { - In den nächsten zwei Jahren wird es deutschen Apothekern nicht } \\
\text { erlaubt sein, verschreibungspflichtige Medikamente sowohl über } \\
\text { das Internet als auch den Versandhandel zu vertreiben } \\
\text { Das Werbeverbot für verschreibungspflichtige Medikamente wird } \\
\text { wahrscheinlich in den nächste zwei Jahren nicht aufgehoben } \\
\text { werden }\end{array}$ \\
\hline $\begin{array}{l}\text { Arzneimittel im } \\
\text { Preisvergleich }\end{array}$ & $\begin{array}{l}\text { Stiftung Warentest, } \\
\text { Deutschland }\end{array}$ & April 2001 & $\begin{array}{l}\text { In den USA werden bereits } 13 \\
\text { Prozent aller Arzneimittel über } \\
\text { preisgünstige Internetapothe- } \\
\text { ken vertrieben. Trotz des Ver- } \\
\text { sandhandelsverbotes mit apo- } \\
\text { thekenpflichtigen Medikamen- } \\
\text { ten, sind auch in Deutschland } \\
\text { Einsparungen möglich }\end{array}$ & $\begin{array}{l}\text { - Der Einsatz von Import-Arzneimitteln (Re-/ und Parallel-Importe) } \\
\text { und Generika bietet in Deutschland ein Einsparpotential } \\
\text { - Durch die Verordnung von Generika und Import-Arzneimittel } \\
\text { schonen Ärzte ihr Budget }\end{array}$ \\
\hline
\end{tabular}

Quelle: INIFES, eigene Darstellung nach:

Österreichisches Bundesinstitut für Gesundheitswesen 2000; EMNID-Institut 2000; KOHTES KLEWES 2000; Stiftung Warentest 2001 
Nachfolgend werden einige Studien exemplarisch kurz vorgestellt:

- Eine von Schöffski in Deutschland durchgeführte Apothekerbefragung ${ }^{2}$ untersucht die Einstellung der Apotheker zu der staatlichen Regulierung auf dem Arzneimittelmarkt. Fühlen sich die Apotheker durch die gesetzlichen Vorschriften geknebelt oder geschützt? Wie ist das marktwirtschaftliche Potential überhaupt zu bewerten? Apotheker sind, so zeigte die Befragung, nur zu Änderungen im System bereit, "wenn damit massive Vorteile" verbunden sind. Dies ist beispielsweise bei der Frage der Einstellung zum Substitutionsverbot der Fall. 83,9 Prozent der Apotheker sind für eine Abschaffung des Substitutionsverbotes, vor allem aus wirtschaftlichen Gründen. Sobald es aber um die Einführung von mehr Wettbewerb in das bestehende System geht, verhält sich die überwiegende Zahl der Apotheker ablehnend und möchte weiterhin im Schutz der staatlichen Regulierungen arbeiten. $^{3}$ Die Apotheker befürchten ganz überwiegend negative Auswirkungen durch verstärkten Wettbewerb. Dies zeigt sich besonders bei der Einstellung der Apotheker zum Fremd- und Mehrbesitz: 92,8 Prozent der Apotheker lehnen den Fremd- und Mehrbesitz ab. Es zeigt sich hierbei - so Schöffski - eine „Herr-imHaus"-Mentalität. ${ }^{4}$ Ein Aspekt der Marktorientierung stellt nach Schöffski die direkte Lieferung von Arzneimitteln an den Kunden dar. Während bei einzelnen Apotheken die Hausbelieferung tatsächlich die Ausnahme darstellt, ist bei anderen die Lieferung von Arzneimitteln, die nicht sofort in der Apotheke verfügbar sind, fast schon die Regel. Überhaupt keine Hausbelieferung nehmen 5,4 Prozent vor, 28,1 Prozent bis zu zehnmal, 32,2 Prozent zwischen elf und fünfzigmal. Im Durchschnitt, so zeigt die Untersuchung, beliefern Apotheken 30 Kunden pro Woche. Ob es sich dabei um begründete Einzelfälle handelt, darf bezweifelt werden.

- Riegl \& Partner $\mathrm{GmbH}^{5}$ verfolgt mit der Studie das Ziel, den Stellenwert und die Nutzenstiftungen der Deutschen Apotheke für ihren Kunden "illusionsfrei, selbstkritisch, schonungslos, aber konstruktiv zu diagnostizieren“. Es soll ein Leitfaden für die "Ideale Apotheke" sowohl unter marktwirtschaftlichen Bedingungen als auch der Berücksichtigung der Besonderheiten für eine "verantwortungsvolle Arzneimittelversorgung" entwickelt werden. Das wichtigste Zukunfts- und ChancenKapital der Apotheke steckt daher in den Kunden und nicht nur im Verordnungsverhalten der Ärzte. Die Studie ergibt u.a., dass für 68 Prozent der Kunden eine zuverlässige Beratungstätigkeit durch den Apotheker der wichtigste Vorzug der Apotheke ist. Dies ist jedoch geringer, als die Selbsteinschätzung der Apotheker (82 Prozent) vermuten lässt. Als Gründe für den Besuch einer Apotheke geben 44

Schöffski, O. (1995), Die Regulierung des deutschen Apothekenwesens. Eine ökonomische Analyse, Baden-Baden.

Auf die Frage: "Sollte ein Apotheker mehrere Apotheken besitzen dürfen?", antworteten 7,2 Prozent der teilnehmenden Ärzte mit Ja.

Schöffski, O. (1995), Die Regulierung des deutschen Apothekenwesens. Eine ökonomische Analyse, Baden-Baden, S.231.

Riegl \& Partner $\mathrm{GmbH}$, Institut für Management im Gesundheitsdienst (1995), Das Image der deutschen Apotheke. 
Prozent der Kunden an, dass sie krank sind und direkt vom Arzt kommen, 16 Prozent sind Chroniker und ebenfalls 16 Prozent lösen ein Rezept als Besorger für andere Personen ein. 77 Prozent der befragten Apothekenkunden gehen monatlich mindestens einmal in die Apotheke, 30 Prozent sogar wöchentlich und öfter. Die regelmäßigen Nutzer der Apotheke sind, bezogen auf den monatlichen und öfteren Besuch: 87 Prozent der Chroniker; 84 bis 86 Prozent der Männer über 60 Jahre; 82 bis 84 Prozent der Frauen über 50 Jahre.

- Eine Erhebung des Instituts für Sozial- und Präventivmedizin der Universität Zürich in Kooperation mit der Schweizerischen Patienten-Organisation und der Schweizerischen Patientendienststelle ${ }^{6}$ befasst sich mit der Zufriedenheit der Kunden einer Schweizerischen Versandapotheke (MediService). Die Befragung ergibt eine große Zufriedenheit mit dem Modell der Versandapotheke: 80,4 Prozent der Befragten beurteilten die Dienstleistungen der MediService Apotheke auf einer Skala von 1-10 Punkten (1= sehr gut; $10=$ sehr schlecht) mit sehr gut. Die Studie ergibt zudem, dass 63,9 Prozent der Befragten ihre rezeptpflichtigen Medikamente ausschließlich in der MediService Apotheke beziehen. Nach Aussage von 80,8 Prozent der Kunden erhalten diese ihre Medikamente in der Regel 48 bzw. 72 Stunden nach dem Versand des Rezeptes an die MediService Apotheke.

Die dargestellten Ergebnisse bereits durchgeführter und INIFES vorliegender Studien und Befragungen geben einen umfassenden Überblick über die Thematik des Internethandels mit Arzneimitteln. Da sie jedoch primär vielfach nur den Einfluss auf den Verbraucher und die Arzneimittelversorgung untersuchen, sind sie auf das vorliegende Forschungsvorhaben und dessen relevante Fragestellungen nur bedingt übertragbar. Es ist daher unabdingbar gewesen, eine eigene wissenschaftliche Befragung innerhalb der Zielgruppen durchzuführen, die vor allem von einer Ausweitung des Versandhandels betroffen sind (Apotheker, Ärzte und Bevölkerung). Nur so ist es möglich, in Abhängigkeit der Akzeptanz und der generellen Einstellung aller Beteiligten eine abschließende Bewertung hinsichtlich einer ökonomischen sowie gesundheitspolitischen Vorteilhaftigkeit abzugeben.

Institut für Sozial- und Präventivmedizin der Universität Zürich; Schweizerische PatientenOrganisation (SPO); Schweizerische Patientendienststelle (DVSP) (1999), Zufriedenheit der Kunden der MediService Apotheke - Schlussbericht, Zürich. 


\subsection{Aufbau der Erhebung}

\subsubsection{Ziele}

Um die Einstellungen von Personen zu bestimmten Themenbereichen zu erfragen, existieren in der empirischen Sozialforschung unterschiedliche Methoden. Für die hier relevante Fragestellung gibt es zwei Befragungsmethoden ${ }^{7}$ : Die mündliche Befragung in Form von Interviews und die schriftliche Befragung über Fragebögen. Für die Durchführung von Interviews spricht, dass es sich um eine aktive Interaktion zwischen Interviewer und Befragten handelt. Der Interviewer hat durch gezieltes Nachfragen die Möglichkeit, an Informationen zu gelangen und kann zusätzliche Erläuterungen geben. Gegen persönliche Interviews spricht, dass sie sehr zeit- und personalaufwendig sind. Um die Repräsentativität innerhalb der Gruppe der zu Befragenden zu gewährleisten, müssten finanzielle Mittel eingesetzt werden, die kaum noch getragen werden können.

Die Vorteile der schriftlichen Befragung sind vor allem finanzieller Art; sie sind in der Regel kostengünstiger. Vielfach kann in kürzerer Zeit mit weniger Personalaufwand eine größere Zahl von Befragten erreicht werden. Der Nachteil ist, dass die Fragen zweifelsfrei verständlich und unkompliziert formuliert sein müssen. ${ }^{8}$ Des weiteren muss der Fragebogen den zu Befragenden zum Ausfüllen motivieren. ${ }^{9}$ Denn im Rahmen einer schriftlichen Befragung ist die Zahl der Ausfälle, also jener Befragten, die den Fragebogen nicht beantworten, meist erheblich. ${ }^{10}$ Vor allem - und das gilt für die schriftliche wie mündliche Befragung - sind die Stichprobenprobleme gravierend: Sowohl der Sampling-Error und der Coverage-Error als auch der Nonresponse-Error wirken sich in kaum zu kontrollierender Weise auf die Befragungsergebnisse aus.

Während bislang davon ausgegangen wurde, mündliche Befragungen als face-toface-Interviews durchzuführen, haben heute die Markt- und Meinungsforschungsinstitute Telefoninterviews als schnelle und kostengünstige Erhebungsvariante innerhalb der mündlichen Befragung entdeckt. In Reaktion auf die Nachteile der bisherigen Befragungsmethoden und der mangelnden Kontrolle der Erhebungssituation - sowohl hinsichtlich des Interviewpartners als auch hinsichtlich des Interviewers - hat die telefonische Befragung Einzug in die Umfrageforschung gehalten. Sie ermöglicht es, in stärkerer Weise die Interviewsituation zu standardisieren und die Stichprobenrealisation zu optimieren. ${ }^{11}$ Zu den Vorteilen dieser Methode gehören eine erhöhte Erreichbarkeit und eine fast zeitnahe Verarbeitungsmöglichkeit der erhaltenen Daten. Das

Bortz, J., Döring, N. (1995), Forschungsmethoden und Evaluation, Berlin, S. 216.

Vgl. Atteslander, P. (1995), Methoden der empirischen Sozialforschung, Berlin, S. 167-168.

Schöffski, O. (1995), Die Regulierung des deutschen Apothekenwesens. Eine ökonomische Analyse, Baden-Baden, S. 203.

Atteslander, P. (1995), Methoden der empirischen Sozialforschung, Berlin, S. 168.

Fuchs, M. (1994), Umfrageforschung mit Telefon und Computer - Einführung in die computergestützte telefonische Befragung, Weinheim, S. 35. 
wichtigste Argument für die Durchsetzung der telefonischen Befragung als vermutlich zweiter, zukünftiger Königsweg der empirischen Sozialforschung ist die Verbreitung der elektronischen Datenverarbeitung. ${ }^{12}$ Das telefonische Interview wird daher als ein computergestütztes Telefoninterview ${ }^{13}$ geführt. Von Nachteil ist, dass sich der Interviewer auf relativ einfache Fragegegenstände zu beschränken hat und auf visuelle Hilfsmittel und Tabellen verzichten muss. ${ }^{14}$

Aufgrund der bisherigen Erkenntnisse bzgl. potentieller Befragungsinstrumente, hat sich INIFES für die Anwendung der Computer Assisted Telephone Interviewing (CATI) - Methode entschieden. Die Datenerhebungsmethode CATI verbindet dabei die Vorzüge telefonischer Befragungen mit denjenigen der modernen elektronischen Datenverarbeitung. ${ }^{15}$ Aufgabe der CATI-Methode ist, Daten computergestützt, zentralisiert, standardisiert und qualitätsgesichert telefonisch zu erheben. Die Stichprobe wird automatisch abgearbeitet, der Fragebogen automatisch Frage für Frage präsentiert. Weitere methodenspezifische Vorteile sind, dass mit computergestützten Telefoninterviews eine höhere Teilnahmebereitschaft als bei schriftlichen Befragungen zu erwarten ist, dass heikle Fragen (etwa zum Einkommen) eher beantwortet werden und Berufsgruppen wie Ärzte und Apotheker telefonisch eher erreichbar sind als mit anderen Verfahren.

\subsubsection{Erhebungstechnik}

Nicht um den bisherigen Wissensstand über die Haltung und Einstellung der primär vom Versandhandel Betroffenen (Bundesbürger, Ärzte und vor allem Apotheker) zum Pharmaversand zu konsolidieren, sondern um neue und persönliche Ansichten zu identifizieren, ist es notwendig, eine repräsentative Befragung unter den genannten Betroffenen durchzuführen. Um glaubhaft, also neutral und konsequent, valide und solide (methodisch fundiert) zu sein, ist von einem gewissen Grunddesign auszugehen. ${ }^{16}$ Konzeptioneller Ausgangspunkt ist ein multimethodisches Vorgehen, bei dem eine Kombination quantitativer und qualitativer Verfahren - jeweils durch eine gegenstandsbezogene Auswahl bestimmt - gemeinsam angewendet, und hinsichtlich der Ergebnisse aufeinander bezogen werden. Zwei Stufen der empirischen Erhebung kommen daher zur Anwendung:

\footnotetext{
12 Fuchs, M. (1994), Umfrageforschung mit Telefon und Computer - Einführung in die computergestützte telefonische Befragung, Weinheim, S. 32.

13

15

Vgl. Bortz, J., Döring, N. (1995), Forschungsmethoden und Evaluation, Berlin, S. 219 - 221.

Weitkunat, R., Crispin, A. (2000), Computergestützte Telefoninterviews, in: Zeitschrift für Gesundheitswissenschaften, jg. 8, Heft 2, S. $106-113$.

Vgl. Lamnek, S. (1995), Qualitative Sozialforschung. Bd.1 Methodologie, Weinheim.
} 
1. Eine repräsentative, standardisierte Befragung zur Ermittlung der quantitativen Bedeutsamkeit der einzelnen Sichtweisen

2. Qualitative, problemzentrierte Interviews zur Validierung und Profundierung der unter erstens erhaltenen Ergebnisse sowie zur Vertiefung der Interpretation

$\mathrm{Zu}$ beachten ist, dass die beiden Stufen methodisch aufeinander aufbauen.

In der empirischen Sozialforschung geht es vor allem darum, Aussagen über soziales Handeln, Beziehungen, Einstellungen oder Verhaltensweisen zu erhalten. ${ }^{17}$ Um eine Repräsentativität der Untersuchung zu gewährleisten, sind standardisierte Fragebögen zu entwickeln, die die Grundlage für die Befragungen bilden. Im Anschluss an diese Interviews sind dann, wie bereits dargestellt, problemzentrierte Interviews in Form von Fokusgruppengesprächen mit Experten zu führen. Grundlage dieser Expertengespräche ist die Auswertung der standardisierten Leitfadeninterviews.

Bei der im folgenden dargestellten Befragung wurde die mündliche Form gewählt. Bezogen auf die Zweistufigkeit der empirischen Erhebung wurden in einem ersten Schritt Telefoninterviews mit standardisierten Fragebögen durchgeführt. In einem weiteren Schritt folgten dann leitfadengestützte face-to-face-Interviews. Die telefonische Befragung bei den Apothekern und Ärzten sowie innerhalb der Bevölkerung kann sich, wie bereits angesprochen, die Unterstützung des Computers zunutze machen; daraus resultiert für die vorliegende Studie das computergestützte Telefoninterview (CATI).

Im Vorfeld der Befragung musste zunächst die Frage nach der Grundgesamtheit der zu befragenden Personen beantwortet werden. Da die zu untersuchenden Gruppen der Betroffenen - die Grundgesamtheit - jedoch zu groß war, wurde jeweils eine Stichprobe gezogen, um aus ihr Rückschlüsse auf die Grundgesamtheit ziehen zu können. Sowohl die Auswahl der Stichprobe als auch die technische Durchführung der CATI-Befragungen ist von dem Marktforschungsinstitut TNS EMNID GmbH \& Co, Bielefeld, im Auftrag von INIFES durchgeführt worden. Für die Befragungen wurden folgende Stichproben ermittelt:

- Bevölkerung: Als Grundgesamtheit galt die in Privathaushalten am Ort der Hauptwohnung in der Bundesrepublik Deutschland lebende deutschsprachige Bevölkerung der Bundesrepublik Deutschland mit dem Mindestalter von 14 Jahren. Hierzu gehören in der Bundesrepublik Deutschland 63,25 Mio. (Stand 1999) Männer und Frauen. ${ }^{18}$ Die Medien-Analyse ermittelt die Zahlen auf Basis von Sonderzählungen des Statistischen Bundesamtes und auf Fortschreibung des Microcensus des Statistischen Bundesamtes. Für die Auswahl der Befragungshaushalte

Vgl. Daten der Arbeitsgemeinschaft Media-Analyse (www.agma-mmc.de). 
wurde aus dem ADM-Netz (Arbeitskreis Deutscher Marktforschungsinstitute e.V.) ${ }^{19}$ eine vorher festgelegte Anzahl von Telefonnummern zufällig gezogen. Innerhalb von Sample Points ${ }^{20}$ erfolgte die Auswahl der Zielhaushalte nach dem Prinzip der Zufallsauswahl (Random Verfahren). Von einem Zentral-Computer des dem ADM angeschlossenen Institutes ${ }^{21}$ wurden die $\mathrm{zu}$ befragenden Haushalte aus einem Pool von zufällig gezogenen Telefonnummern (ausgehend von real existierenden Nummern) generiert. Innerhalb der Haushalte wurden die zu befragenden Personen systematisch ausgewählt. Im Rahmen der Auswahl der zu befragenden Person ist diejenige Person befragt worden, die den Anruf annahm. Grundlage für die Auswahl der Befragten waren darüber hinaus Quoten hinsichtlich Geschlecht und Altersgruppen. Die so gewonnene Stichprobe wurde hinsichtlich der Merkmale Bundesland, Ortsgröße, Geschlecht, Alter, Berufstätigkeit, Schulbildung und Haushaltsgröße gewichtet. Nach der Gewichtung war die Stichprobe repräsentativ für Männer und Frauen ab 14 Jahren, d.h. die erhobenen Daten sind im Rahmen der statistischen Schwankungsbreiten auf die Grundgesamtheit hochrechenbar.

- Apotheker: Für die vorliegende Studie waren die Apotheker von Interesse, die primär durch die Einführung des Arzneimittelversandes an die Endverbraucher betroffen sind. Daher sind nicht alle in der Bundesrepublik Deutschland approbierten Apotheker relevant gewesen, sondern nur eine Teilgruppe. Ausgenommen von der Befragung wurden alle unselbständigen Apotheker, d.h. alle Apotheker, die abhängig beschäftigt sind. ${ }^{22}$ In der Bundesrepublik Deutschland sind 46.064 Apotheker in öffentlichen Apotheken tätig. ${ }^{23}$ Es wurden nur die verantwortlichen Apothekenleiter in öffentlichen Apotheken angesprochen - in der Bundesrepublik Deutschland existierten im Jahre 199921.590 Apotheken. $^{24}$ Dabei ist unerheblich, ob diese Apotheker die Apotheke selbst besitzen oder sie gepachtet haben. Aufgrund der Verpflichtung zur persönlichen Leitung stellt der selbständige Apotheker das einzige und damit auch einheitliche Willensbildungszentrum einer öffentlichen Apotheke dar. ${ }^{25}$ Datenquelle für die Stichprobenziehung waren die Gelben Seiten für Deutschland. Grundlage für die Auswahl der Apotheker sind Quoten hinsichtlich der Regionalverteilung (Bundesländer) und des Geschlechtes gewesen. Nach

Aufgrund der Komplexität kann an dieser Stelle nicht näher auf ADM-Netze eingegangen werden. Nähere Informationen sind beim Verfasser erhälttich.

Von den Marktforschungsinstituten verwendete Bundestags-Stimmbezirke - näheres beim Verfasser. In der Bundesrepublik Deutschland existieren derzeit 310 Sample Points (210 West, 100 Ost) über alle Bundesländer und Ortsgrößen. In diesem Fall TNS EMNID.

Hierzu gehören, zum Beispiel, Krankenhausapotheken, Universitäten und die pharmazeutische Industrie.

ABDA - Bundesvereinigung Deutscher Apothekerverbände (2000), Die Apotheke: Zahlen - Daten - Fakten (1999).

ABDA - Bundesvereinigung Deutscher Apothekerverbände (2000), Die Apotheke: Zahlen - Daten - Fakten (1999).

Vgl. Rothe, T. (1980), Möglichkeiten und Grenzen einer marktorientierten Führung öffentlicher Apotheken in der Bundesrepublik Deutschland, Berlin, S. 4. 
diesen Vorgaben wurden mittels Datenbank Telefonnummern gezogen und antelefoniert. Die Befragungsperson wurde entsprechend der vorgegebenen Quoten über die Fragensteuerung im Fragebogen ermittelt (Screening). Die Stichprobe wurde anschließend innerhalb der Zielgruppe hinsichtlich der Merkmale Nielsengebiet ${ }^{26}$ und Geschlecht gewichtet. Nach der Gewichtung war die Stichprobe repräsentativ für die Apotheker in öffentlichen Apotheken, d.h. die erhobenen Daten sind im Rahmen der statistischen Schwankungsbreiten auf die Grundgesamtheit hochrechenbar.

- Ärzte: Wie zuvor bereits schon bei den Apothekern, sind nicht alle in der Bundesrepublik Deutschland tätigen Ärzte für diese Studie relevant, sondern nur eine Teilgruppe. Ausgenommen von der Befragung wurden alle Ärzte ohne Kassenzulassung und unselbständige Ärzte, d.h. alle Ärzte, die abhängig beschäftigt sind. Es sind nur die Ärzte mit einer GKV-Zulassung angesprochen worden. Da das Verordnungsverhalten der Ärzte maßgeblich den Arzneimittelverbrauch bestimmt ${ }^{27}$, wurden folgende Facharztgruppen aufgrund inrer Relevanz für die Fragestellung des Forschungsprojektes zur weiteren Analyse von INIFES ausgewählt: niedergelassene Allgemeinmediziner / Praktiker / Internisten, niedergelassene Gynäkologen, niedergelassene HNO, niedergelassene Urologen und niedergelassene Nervenärzte. Grundlage für diese Selektion bildet Abbildung III - 1, die 14 chronischen Krankheitsbildern typische Arzneimittelgruppen zuordnet, die bei den entsprechenden Krankheiten im Normalfall zu verordnen sind. Daraus ableitend lässt sich die Auswahl der genannten Facharztgruppen begründen. Die zu betrachtende Grundgesamtheit der Vertragsärzte umfasst insgesamt 112.244 (Stand 30.06.1999). ${ }^{23}$ Die Anzahl der niedergelassenen Allgemeinmediziner / Praktiker / Internisten beträgt 58.841 , der Gynäkologen 9.545 , der HNO-Ärzte 3.878, der Urologen 2.502 und der Nervenärzte 4.850. Datenquelle für die Stichprobenziehung waren die Gelben Seiten für Deutschland. Grundlage für die Auswahl der Ärzte sind separate Quoten je Fachrichtung hinsichtlich der Regionalverteilung (Bundesländer), Geschlecht und Altersgruppen gewesen. Nach diesen Vorgaben wurden mittels Datenbank Telefonnummern gezogen und antelefoniert. Die Befragungsperson wurde entsprechend der vorgegebenen Quoten über die Fragesteuerung im Fragebogen ermittelt (Screening). Die Stichprobe ist anschließend innerhalb der Zielgruppe hinsichtlich der Merkmale Nielsengebiet, Geschlecht und Alter gewichtet worden. Nach der Gewichtung ist die Stichprobe repräsentativ für jede niedergelassene Facharztgruppe, d.h. die erhobenen Daten sind im Rahmen der statistischen Schwankungsbreiten auf die Grundgesamtheit hochrechenbar.

In der Bundesrepublik Deutschland existieren sieben Nielsengebiete, denen jeweils Bundesländer zugeordnet sind. Die Einteilung der Nielsengebiete ist dem Anhang zu entnehmen.

Schröder, H., Selke, G. W. (2000), Arzneiverordnungen nach Arztgruppen, in: Schwabe, U., Paffrath, D. (Hrsg.), Arzneiverordnungs-Report 1999, S. 697.

WIdO (2000), An der vertragsärztlichen Versorgung teilnehmende Ärzte nach ihrem Teilnehmestatus, in: www.wido.de/ambulanteVersorgung/Aerzte/Arztzahlen/Dlgesamt.html, Zugriff am 12.07.2000. 
Abbildung III - 1: Chronische Krankheitsbilder und deren pharmazeutische Therapie

\begin{tabular}{|c|c|c|}
\hline Chronische Erkrankungen & ATC-Code & $\begin{array}{l}\text { Wirkstoff / Wirkstoff- } \\
\text { gruppe }\end{array}$ \\
\hline \multicolumn{3}{|c|}{ Stoffwechselerkrankungen } \\
\hline Schilddrüse & $\mathrm{H03}$ & Schilddrüsentherapeutika \\
\hline Diabetes & A10 & Antidiabetika \\
\hline Hyperlipidămie & C10 & Lipidsenkende Mittel \\
\hline Gicht & M04 & Gichtmittel \\
\hline \multicolumn{3}{|c|}{ Psychiatrische Erkrankungen } \\
\hline Psychosen & N05A & Antipsychotika \\
\hline \multicolumn{3}{|c|}{ Nervensystem und Sinnesorgane } \\
\hline Parkinson & N04 & Antiparkinsonmittel \\
\hline $\begin{array}{l}\text { Hereditäre und iodiopathische bzw. } \\
\text { entzündliche und toxische Neuropa- } \\
\text { thien }\end{array}$ & N07XB & Neuropathiepräparate \\
\hline \multicolumn{3}{|c|}{ Kreislauf } \\
\hline $\begin{array}{l}\text { Koronare Herzkrankheit (KHK), Hy- } \\
\text { pertonie, Herzinsuffizienz }\end{array}$ & $\begin{array}{l}\text { C02; C03; C07; } \\
\text { C08; C09; C01D; } \\
\text { B01AC; B01AA; } \\
\text { C01A; C01C }\end{array}$ & $\begin{array}{l}\text { Antihypertonika; Diuretika; } \\
\text { Betarezeptorenblocker; Calcium- } \\
\text { kanalblocker; Hemmstoffe des } \\
\text { Renin-Angiotensin-Systems; } \\
\text { Koronardilatatoren; Throm- } \\
\text { bozytenaggregationshemmer; } \\
\text { Herzglykoside; Inotrope Mittel } \\
\text { (exc. Herzglykoside) }\end{array}$ \\
\hline $\begin{array}{l}\text { Hirnleistungsminderung, zentrale } \\
\text { Durchblutungs- störungen }\end{array}$ & N06BX & $\begin{array}{l}\text { andere Psychostimulantien und } \\
\text { Nootropika }\end{array}$ \\
\hline \multicolumn{3}{|c|}{ Atmungsorgane } \\
\hline Chronische Bronchitis/ Asthma & $\mathrm{R03}$ & Antiasthmatika \\
\hline \multicolumn{3}{|c|}{ Sonstige } \\
\hline Glaukomerkrankungen & S01E & Glaukommittel und Miotika \\
\hline Epilepsie & N03 & Antiepileptika \\
\hline $\begin{array}{l}\text { Periphere Durchblutungsstörungen, } \\
\text { Claudicatio intermittens }\end{array}$ & $\mathrm{C04}$ & Periphere Vasodilatatoren \\
\hline Neubildungen (bösartig und gutartig) & L01; L02; & $\begin{array}{l}\text { Antineoplastische Mittel; endokri- } \\
\text { ne Therapie }\end{array}$ \\
\hline
\end{tabular}

Quelle: Gerste, B., Niemeyer, M., Lauterberg, J. (2000), Wieviel chronisch Kranke gibt es? In: Arnold, M., Litsch. M., Schwartz, F. W. (Hrsg.) (2000), Krankenhaus-Report 1999, Stuttgart, S. 72. 
Für die Untersuchung ist, unter Berücksichtigung des Gesetzes der großen Zahlen ${ }^{29}$, eine Stichprobe von 1006 Bevölkerungsinterviews, 150 Interviews mit Apothekern und 380 Interviews mit Ärzten (100 Allgemeinmediziner / Praktiker / Internisten und je 70 Gynäkologen, HNO-Ärzte, Urologen, Nervenärzte) ermittelt worden. Um die Aussagefähigkeit der zu erhebenden Daten schon bei der Stichprobenziehung zu berücksichtigen, wurde darauf geachtet, dass Prozentzahlen auf der Basis von weniger als 70 Absoluten wegen einer zu geringen Befragtenzahl nur begrenzt aussagefähig sind. ${ }^{30}$

Bezogen auf die bereits erläuterte Zweistufigkeit, schlossen sich im Anschluss an die CATI-Befragungen, u.a. zur Validierung und Profundierung der erhaltenen Ergebnisse sowie zur Vertiefung der Interpretation, 23 Expertengespräche an. Dies war Voraussetzung, um glaubhaft, also neutral und konsequent, valide und solide (methodisch fundiert) zu sein - bei Einhaltung eines gewissen Grunddesigns. ${ }^{31}$ Während die Ergebnisse der Befragung die subjektive Einschätzung der Befragten bzgl. einer Versandapotheke widerspiegelt, wird das Fachwissen von Experten zur Abgabe von Prognosen, die auf mittel- und längerfristigen Entwicklungen ausgerichtet sind, benötigt.

Die Expertengespräche wurden als leitfadengestützte ${ }^{32}$ face-to-face-Interviews geführt. Im Gegensatz zu einer stark strukturierten Befragung bietet diese Form des Interviews, trotz vorbereiteter und vorformulierter Fragen, die Möglichkeit wie bei einem wenig strukturierten Interview, aus dem Gespräch sich ergebende Themen aufzunehmen und sie von den Antworten ausgehend weiter zu verfolgen. ${ }^{33}$

In ca. neunzigminütigen Interviews haben die Experten ihre Einschätzungen der Entwicklung einer Ausweitung des Pharmaversandes in Deutschland dargelegt. Der Gesprächsführung lag sowohl eine Zusammenfassung der Ergebnisse der Primärerhebung, die zwei Wochen vorher zugeschickt wurde, als auch ein strukturierter Fragenkatalog zugrunde, der primär folgende Themen enthielt:

- Stellungnahme zu einigen Aussagen hinsichtlich der Auswirkungen, die eine Legalisierung des Versandhandels bedingen würde.

- Sollte einem bestehenden Kundenbedürfnis nach Bestellung verordneter Arzneimittel über eine Versandapotheke entgegengekommen werden?

- Zu definierende Kriterien und Standards in bezug auf eine qualitative Arzneimittelversorgung.

- Zielgruppendefinition für eine Versandapotheke.

29

30

31

Vgl. Eckey, H.-F. (1992), Statistik: Grundlagen, Methoden, Beispiele, Wiesbaden.
TNS EMNID (2000), Arzneimittel im Internet - Telefonische Mehrthemenbefragung - ,Bielefeld.

Siehe Kapitel 3.2.2.

Der Leitfaden ist im Anhang enthalten.

Atteslander, P. (1995), Methoden der empirischen Sozialforschung, Berlin, S. 162 - 163. 
- Versuch einer Quantifizierung von Arbeitsmarkteffekten, die durch eine Legalisierung des Arzneimittel-Versandhandels zu erwarten sind.

Der Kreis der Befragten setzt sich aus Experten zusammen, die sich in unterschiedlichen Institutionen mit der Thematik des Versandhandels auseinander setzen: Vertreter der Apothekenverbände und der Apothekerkammern, Apotheker, Verbraucherverbände, Juristen, Bundesministerium für Gesundheit, Wissenschaftler, Kostenträger (GKV und PKV), Pharmabranche sowie Versender von Arznei- und Hilfsmitteln. Ebenfalls angefragt wurde bei der Bundesärztekammer und der Kassenärztlichen Bundesvereinigung. Eine Teilnahme wurde aus Gründen, die an dieser Stelle nicht näher erläutert werden sollen, abgelehnt.

Dieses heterogene Spektrum bietet die Möglichkeit, Erwartungen aus unterschiedlichen Perspektiven zu vereinen und in eine Prognose über die zukünftige Entwicklung des Versandhandels mit rezeptpflichtigen Arzneimitteln einfließen zu lassen.

Den Teilnehmern an den Expertengesprächen wurde absolute Anonymität zugesichert. Nachfolgend sind die Experten aufgeführt, die sowohl einer Veröffentlichung ihres Namens als auch ihrer Aussage zugestimmt haben. 


\section{Teilnehmer der Expertengespräche}

\begin{tabular}{|c|c|c|c|}
\hline \multirow{2}{*}{$\begin{array}{l}\text { Martin Ludwi- } \\
\text { chowski }\end{array}$} & \multirow{2}{*}{$\begin{array}{l}\text { Vorsitzender des Fachverban- } \\
\text { des der Versandhändler für } \\
\text { Diabeteshilfsmittel e.V. }\end{array}$} & \multirow[t]{2}{*}{ Stefan Müller } & Rechtsanwalt \\
\hline & & & Linklaters Oppenhoff \& Rădler \\
\hline Jens Apermann & Marketing-Direktor DocMorris & $\begin{array}{l}\text { MR Dr. Gert } \\
\text { Schorn }\end{array}$ & $\begin{array}{l}\text { Zuständig im BMG für E- } \\
\text { Commerce }\end{array}$ \\
\hline \multirow[t]{2}{*}{$\begin{array}{l}\text { Klaus Gritsch- } \\
\text { neder }\end{array}$} & \multirow{2}{*}{$\begin{array}{l}\text { President European Associa- } \\
\text { tion of Mail Service Pharma- } \\
\text { cies }\end{array}$} & \multirow[t]{2}{*}{$\begin{array}{l}\text { Prof. Dr. rer. nat. } \\
\text { Gerd Glaeske }\end{array}$} & $\begin{array}{l}\text { Universităt Bremen - Zentrum } \\
\text { für Sozialpolitik, }\end{array}$ \\
\hline & & & $\begin{array}{l}\text { Forschungseinheit Gesund- } \\
\text { heitspolitik und Evaluation } \\
\text { medizinischer Versorgung } \\
\text { (FEGEM) }\end{array}$ \\
\hline \multirow[t]{3}{*}{ Theresa Kliegl } & $\begin{array}{l}\text { Vice President European As- } \\
\text { sociation of Mail Service } \\
\text { Pharmacies, }\end{array}$ & \multirow[t]{3}{*}{ Christian Weber } & \multirow[t]{3}{*}{$\begin{array}{l}\text { Geschäftsführer des Verban- } \\
\text { des der privaten Krankenver- } \\
\text { sicherung }\end{array}$} \\
\hline & Pharmaceutical Manager & & \\
\hline & DocMorris & & \\
\hline \multirow[t]{2}{*}{ Elke Christmann } & \multirow[t]{2}{*}{ Apothekerin } & \multirow[t]{2}{*}{ Norbert Schleert } & $\begin{array}{l}\text { Leiter der Abteilung Arznei- } \\
\text { mittel, Heil- und Hilfsmittel }\end{array}$ \\
\hline & & & AOK-Bundesverband \\
\hline $\begin{array}{l}\text { Uni.-Prof. Dr. rer. } \\
\text { nat. Rainer } \\
\text { Braun }\end{array}$ & $\begin{array}{l}\text { Hauptgeschäftsführer der } \\
\text { ABDA-Bundesvereinigung } \\
\text { Deutscher Apothekerver- } \\
\text { bände, der Bundesapotheker- } \\
\text { kammer und des Deutschen } \\
\text { Apothekerverbandes e.V. }\end{array}$ & Helmut Schröder & $\begin{array}{l}\text { GKV-Arzneimittelindex - Wis- } \\
\text { senschaftliches Institut der } \\
\text { AOK (WIdO) }\end{array}$ \\
\hline \multirow[t]{3}{*}{ Gerhard Reichert } & $\begin{array}{l}\text { Vorsitzender des Bayerischen } \\
\text { Apothekerverbandes e.V. }\end{array}$ & \multirow[t]{3}{*}{$\begin{array}{l}\text { Wolfgang Kaes- } \\
\text { bach }\end{array}$} & $\begin{array}{l}\text { Apotheker für Arzneimittelin- } \\
\text { formation und Offizinpharma- }\end{array}$ \\
\hline & \multirow[t]{2}{*}{$\begin{array}{l}\text { Mitglied im Vorstand der } \\
\text { ABDA-Bundesvereinigung } \\
\text { Deutscher Apothekerverbände }\end{array}$} & & $\begin{array}{l}\text { zie, } \\
\text { Leiter der Abteilung Arznei- } \\
\text { mittel, Heil- und Hilfsmittel }\end{array}$ \\
\hline & & & BKK Bundesverband \\
\hline \multirow[t]{3}{*}{$\begin{array}{l}\text { Johannes M. } \\
\text { Metzger }\end{array}$} & $\begin{array}{l}\text { Präsident der Bundesapothe- } \\
\text { kerkammer, }\end{array}$ & \multirow[t]{3}{*}{$\begin{array}{l}\text { Wolfgang Hart- } \\
\text { mann-Beesche }\end{array}$} & \multirow{3}{*}{$\begin{array}{l}\text { Apotheker, Versorgungsma- } \\
\text { nagement, BKK Landesver- } \\
\text { band Bayern }\end{array}$} \\
\hline & $\begin{array}{l}\text { Präsident der Bayerischen } \\
\text { Landesapothekerkammer }\end{array}$ & & \\
\hline & $\begin{array}{l}\text { Mitglied im Vorstand der } \\
\text { ABDA-Bundesvereinigung } \\
\text { Deutscher Apothekerverbănde }\end{array}$ & & \\
\hline $\begin{array}{l}\text { Dr. Jens Schnei- } \\
\text { der }\end{array}$ & Apotheker & Ralf Sjuts & $\begin{array}{l}\text { Vorstandsmitglied } \\
\text { BKK Post }\end{array}$ \\
\hline Thomas Isenberg & $\begin{array}{l}\text { Fachbereichsleiter Gesundheit } \\
\text { und Ernăhrung / Bundesver- } \\
\text { band der Verbraucherzentra- } \\
\text { len und Verbraucherverbände }\end{array}$ & Cornelia Yzer & $\begin{array}{l}\text { Hauptgeschäftsführerin, Ver- } \\
\text { band forschender Arznei- } \\
\text { mittelhersteller }\end{array}$ \\
\hline
\end{tabular}

Quelle: INIFES, eigene Darstellung. 


\subsubsection{Ablauf der Erhebung}

Grundlage der CATI-Befragung ist in der vorliegenden Arbeit ein standardisierter Fragebogen. Um den Fragebogen für die jeweils Befragten unmissverständlich zu formulieren, ist eine Zusammenarbeit von Ärten, Apothekern und Ökonomen unabdingbar gewesen. Nur so ließen sich Missinterpretationen und eine negative Beeinflussung der Studienergebnisse vermeiden. Aus diesem Grund wurden vom ersten Entwurf bis zum endgültigen Fragebogen alle Versionen detailliert mit Ärzten, Apothekern und TNS EMNID diskutiert. Es wurden inhaltliche Fehler und Unklarheiten beseitigt und der Umfang des jeweiligen Fragebogens reduziert.

Ein Fragebogen mag zwar diskutiert und gut formuliert worden sein, bei der Befragung unter realistischen Bedingungen können sich jedoch immer noch einige Schwachstellen ergeben. Um dies zu vermeiden, muss vor einer Hauptbefragung ein Pretest durchgeführt werden. Dieser Pretest wird unter gleichen Bedingungen durchgeführt wie die Hauptbefragung, allerdings mit einer geringeren Anzahl von Befragten. Falls sich beim Pretest noch Verständnis- oder Inhaltsprobleme zeigen, ist es möglich, den Fragebogen ohne finanziellen Verlust anzupassen. Daher wurden in der Zeit vom 25. September 2000 bis zum 12. Oktober 2000, auf Basis des jeweiligen Fragebogens, sieben Telefoninterviews mit Apothekern, vierzehn Telefoninterviews mit Ärzten $^{34}$ und vierzehn Telefoninterviews innerhalb der Bevölkerung in einem Pretest geführt. Aufgrund der Brisanz der zu untersuchenden Thematik sind die Teilnehmer an dem Pretest von INIFES ausgesucht worden und um Verschwiegenheit gebeten worden. Nur so war zu vermeiden, dass die Verbände der Apotheker, der Ärzte und der Krankenkassen vor der Hauptbefragung Kenntnis von der Studie erlangten.

Die Fragen des jeweiligen Fragebogens wurden zufriedenstellend beantwortet, d.h. es gab keine Fragen, bei denen ein Großteil der Befragten sich geweigert hätte, eine Antwort zu geben. Auch schienen die Fragen relativ verständlich formuliert worden zu sein. Die Interviews mit den genannten Facharztgruppen ergaben, dass aufgrund des Verordnungsverhaltens und -volumens, von einer weiteren Betrachtung der niedergelassenen Augenärzte abgesehen werden kann. Diese Facharztgruppe wurde daher nicht weiter untersucht.

Der von INIFES durchgeführte Pretest bei der Bevölkerung hat gezeigt, dass einige Personengruppen - vielfach ältere Personen - sich das dargestellte Modell einer Versandapotheke in einigen europäischen Ländern nicht vorstellen können bzw. wollen. Daher hat sich INIFES entschieden, den Fragebogen für die Bevölkerung nochmals zu ändern und die Bevölkerung nach der generellen Akzeptanz des Modells einer Versandapotheke zu befragen.

34

Fünf Interviews mit niedergelassenen Allgemeinmedizinern / Praktikern / Internisten, jeweils zwei Interviews mit niedergelassenen Gynăkologen, niedergelassenen Augenärzten, niedergelassenen Urologen und niedergelassenen Nervenărzten sowie einer niedergelassenen HNOÄrztin. 
Des weiteren wurde der jeweilige Fragebogen nach Auswertung der Antworten um einige Fragen gekürzt bzw. an einigen Stellen umgestellt.

Nach Abschluss und Auswertung aller Tests wurden die Telefoninterviews der Hauptbefragung bei den Ärzten, den Apothekern sowie der Bevölkerung von TNS EMNID in Bielefeld durchgeführt.

Um auswertbare und repräsentative Ergebnisse zu erhalten und die Vorgabe von 380 Interviews zu erfüllen, wurden insgesamt 4867 Ärzte der entsprechenden Facharztgruppen angerufen. In 1805 Fällen kam es zu keinem Interview, da die Zielperson nicht erreichbar bzw. im Urlaub war, die Telefonleitung besetzt, der Anrufbeantworter eingeschaltet war oder ein Informationston / Fax erreicht wurde. Die Teilnahme aus verschiedenen Gründen verweigert haben 1891 Ärzte. Innerhalb des Interviews haben 18 Ärzte die Befragung abgebrochen. Mit 773 Ärzten ist kein Interview zustande gekommen, da zum Zeitpunkt eines Gesprächstermins die Befragung bereits abgeschlossen war oder das Interview aufgrund der Quotenerreichung vom Marktforschungsinstitut nicht fortgesetzt wurde (Stratifikation).

Bei 2113 Bundesbürgern ist ein Interview nicht möglich gewesen, da die Zielperson nicht erreichbar bzw. im Urlaub war, die Telefonleitung besetzt, der Anrufbeantworter eingeschaltet war oder ein Informationston / Fax erreicht wurde. Aus verschiedenen Gründen haben 4140 Personen die Teilnahme am Interview verweigert. Von 178 Personen wurde die Befragung innerhalb des Interviews abgebrochen. Aufgrund der Quotenerreichung oder des Umstandes, dass die Person nicht zur Zielgruppe gehörte, wurde das Interview in 566 Fällen nicht fortgesetzt (Stratifikation).

Die Erstellung eines Ausschöpfungsprotokolls für die Interviews mit Apothekern ist aufgrund technischer Probleme nicht möglich gewesen. Es sei jedoch darauf hingewiesen, dass die Repräsentativität der Befragung bei den Apothekern gewährleistet ist.

Den Teilnehmern an der Befragung wurde die Anonymität des Interviews zugesichert. Die gestellten Fragen wurden repräsentativ auswertbar beantwortet.

Die endgültige Version des Fragebogens für die Ärzte, für die Apotheker und für die Bevölkerung ist im Anhang enthalten.

Die Feldarbeit hat das Marktforschungsinstitut TNS EMNID GmbH \& Co, Bielefeld, im Auftrag von INIFES durchgeführt. Die allgemeinen Arbeitsanweisungen, nach denen alle Interviewer von TNS EMNID verfahren, regeln die einheitliche (in bezug auf Reihenfolge und Wortlaut der Fragen) Durchführung der Interviews. 


\subsection{Inhalte der Befragungen}

In Vorbereitung auf die wissenschaftliche Untersuchung wurden in einem ersten Schritt Arbeitshypothesen aufgestellt, die es empirisch zu überprüfen galt. Die im folgenden aufgeführten Thesen sind das Resultat einer umfangreichen Literaturrecherche.

1. Die gesetzliche Krankenversicherung erhofft sich durch eine Zulassung von Versandapotheken Einsparpotentiale im Bereich der Leistungsausgaben für Arzneimittel aus öffentlichen Apotheken. Nach Ansicht von Experten ließen sich bis zu 800 Mio. DM einsparen. ${ }^{37}$ Ob es tatsächlich zu finanziellen Entlastungen kommen wird, gilt es zunächst bei den Apothekern und Ärzten zu hinterfragen.

Die Arbeitshypothese 1 lautet: Versandapotheken werden zu Kosteneinsparungen im Arzneimittelbereich und zu entsprechenden Auswirkungen für die existierenden Präsenzapotheken führen.

2. Arbeitshypothese 2: Art und Umfang der mit der Einführung des Pharmaversandes potentiell verbundenen (negativen) Auswirkungen für die Präsenzapotheken wird auf jeden Fall vom Umsatz und der Personalstärke einer Apotheke beeinflusst.

3. Sollte die Einführung von Versandapotheken mit negativen Auswirkungen für bestehende Präsenzapotheken verbunden sein, besteht u.U. die Gefahr einer Verschlechterung des umfassenden Versorgungs- und Sicherstellungsauftrages der Apotheken.

Arbeitshypothese 3 lautet demnach: Die Ausweitung des Pharmaversandes ist verbunden mit der Schließung von Apotheken und gefährdet daher den bestehenden Versorgungs- und Sicherstellungsauftrag der Apotheken.

4. Bedingt durch $\S 43$ Arzneimittelgesetz und $\S 17$ Apothekenbetriebsordnung ist sowohl der Vertriebsweg vom Anbieter zum Nachfrager als auch der Ort der Übergabe apothekenpflichtiger Medikamente gesetzlich vorgegeben. Die Ausnahmeregelung des $\S 17$, II Apothekenbetriebsordnung billigt in begründeten Einzelfällen eine Versendung aus der Apotheke oder die Zustellung durch einen Boten an den Arzneimittelempfänger. Nach eigenen Aussagen übernehmen Ärzte in einigen Fällen die Rezeptweiterleitung an eine Apotheke. Dieses Vorgehen legt daher die Vermutung nahe und ist somit Grundlage der Arbeitshypothese 4: Aufgrund des Volumens bereits durchgeführter Heimlieferungen kann nicht mehr vom begründeten Einzelfall gesprochen werden. Die Heimlieferungen entsprechen vielfach schon einer Form des Versandhandels.

U.a. Glaeske, G. (2001), Gefahr für die Verbraucher oder für die Apotheken?, in: die Ersatzkasse $2 / 2001, \mathrm{~S} .45$. 
5. Aufgrund der bestehenden Infrastruktur (u.a. Personalausstattung etc.) und der anfallenden Kosten, wird der Service der Heimlieferungen überwiegend von umsatzstarken Apotheken angeboten. Diese Apotheken streben des weiteren eine Intensivierung der bereits durchgeführten Aktivitäten an.

Die daraus resultierende Arbeitshypothese 5 lautet: Sowohl der von der Apotheke erzielte Umsatz als auch die Personalstärke bestimmen den Umfang der getätigten Heimlieferungen.

6. Die Anzahl der durchgeführten Heimlieferungen lässt weniger auf den begründeten Einzelfall schließen als vielmehr auf ein vorhandenes Kundenbedürfnis. Ist daher davon auszugehen, dass einige Apotheker inre Präsenzapotheke in eine Versandapotheke umwandeln bzw. beides komplementär betreiben werden?

Arbeitshypothese 6 lässt sich wie folgt formulieren: Wer bereits Heimlieferungen durchführt, wird - Änderung der Rechtsgrundlage vorausgesetzt - seine Präsenzapotheke in eine Versandapotheke umwandeln bzw. beides komplementär betreiben.

7. Bislang existieren aufgrund $\S 43$ Arzneimittelgesetz und $\S 17$ Apothekenbetriebsordnung gesetzliche Vorgaben bzgl. der Arzneimitteldistribution. Vor dem Hintergrund sich ändernder Rahmenbedingungen (u.a. Sozio-Demographie, Informations- und Kommunikations-Technologie, Globalisierung) ergibt sich Arbeitshypothese 7: Ärzte und Apotheker räumen komplementären Distributionswegen keine Marktchancen ein.

8. Arbeitshypothese 8: Die Zielgruppe einer Versandapotheke ist nicht dieselbe wie die einer traditionellen Apotheke. Daher ist sie auch nur für eine bestimmte Patientenklientel von Vorteil.

Ob und welche Facharztgruppen ein besonderes Interesse an Kooperationen mit einer Versandapotheke haben und für welche Patienten die Nutzung einer Versandapotheke mit Vorteilen verbunden ist, soll die Befragung klären. Die Bereitschaft, mit Versandapotheken zusammen zu arbeiten, wird von der Facharztgruppe und der Zusammensetzung ihrer Patientenklientel bestimmt.

9. Besonders in Gebieten, in denen die Apothekendichte gering ist, kann eine Versandapotheke Nutzen für die Bevölkerung stiften. Konsumenten, die in ihrer Mobilität eingeschränkt sind und nicht selber das Rezept in der Apotheke einlösen können, sind vielfach auf eine wohnortnahe Apotheke angewiesen. Dies führt zur Arbeitshypothese 9: Die Marktchancen einer Versandapotheke werden vom Versorgungsgrad mit Apotheken beeinflusst.

10. Als Zielgruppe für einen Versandhandel sehen viele Experten Menschen, die unter chronischen Krankheiten leiden und/oder in ihrer Mobilität eingeschränkt sind, 
Berufstätige sowie Patienten mit einer Dauermedikation ${ }^{38}$. Vor allem Chroniker und Patienten mit einer Dauermedikation bedürfen keiner Beratung mehr in der Apotheke. Doch wie sieht die Bereitschaft der Betroffenen aus, Medikamente über eine Versandapotheke zu beziehen?

Arbeitshypothese 10 lautet: Eine konkrete Aussage über potentielle Zielgruppen einer Versandapotheke wird vom Gesundheitszustand, dem Alter und dem Verhältnis der Konsumenten zum Internet beeinflusst.

11. Untersuchungen im Hinblick auf die Nutzung von Internet-Apotheken durch den Verbraucher haben ergeben, dass eine deutliche Diskrepanz zwischen der Akzeptanz von Internet-Apotheken und der Bereitschaft, dort zu kaufen, besteht. ${ }^{39}$ Daraus resultiert die Arbeitshypothese 11: Bestimmte Rahmenbedingungen beeinflussen die Bereitschaft der Bevölkerung, eine Versandapotheke zu nutzen. Hierzu gehören insbesondere Berufstätigkeit, Haushaltsgröße, Internet-User, Alter, Medikamentenkonsum / Gesundheitszustand und Mobilität.

12. Ein häufig von den Apothekern angeführter Kritikpunkt gegenüber dem Versandhandel lautet: Es existiert keine Beratung des Kunden. Viele Apotheker erachten eine umfassende face-to-face Beratung für sehr wichtig, zumal sie im $\S 20$ Apothekenbetriebsordnung gesetzlich festgelegt ist. Wie sieht die Situation in den Präsenzapotheken aus? Wird wirklich jeder Kunde umfassend und unaufgefordert beraten? Beansprucht bzw. wünscht der Kunde überhaupt eine Beratung in der Apotheke?

Diese Punkte gilt es in der Befragung zu untersuchen und führen zur Arbeitshypothese 12: Die Präsenzapotheken bieten ihren Kunden eine umfassende Beratung an; eine Leistung, die Versandapotheken nicht bieten können.

13. Die Verbraucherverbände fordern von Versandapotheken die Aufstellung und Einhaltung gewisser Qualitätsstandards. ${ }^{40}$ Welche Anforderungen die Konsumenten an die Qualität einer Versandapotheke stellen, ist Gegenstand der INIFES-Studie. Die Arbeitshypothese 13 lautet: Die Bereitschaft der Bevölkerung, Medikamente über eine Versandapotheke zu beziehen, ist an hohe Qualitätsanforderungen (z.B. bzgl. Zustellungssicherheit und Beratungsangebot) gebunden.

Die Arbeitshypothesen sind in der folgenden Übersicht III - 1 nochmals zusammenfassend dargestellt.

Ergebnis der von INIFES durchgeführten Expertenbefragungen.

Vgl. MediaTransfer AG (2001), Fragen und Ergebnisse zum Medikamentenhandel im Netz, wuw.b2b.mediatransfer.de - Zugriff am 16.05.2001. 
Übersicht III - 1: Zu überprüfende Arbeitshypothesen, als Grundlage der Analyse der strukturellen und qualitativen Auswirkungen einer Ausweitung des Pharmaversandes auf Anbieter und Nachfrager

1. Versandapotheken werden zu Kosteneinsparungen im Arzneimittelbereich führen.

2. Art und Umfang der mit der Einführung des Pharmaversandes potentiell verbundenen (negativen) Auswirkungen für die Präsenzapotheken sind abhängig von deren Umsatz.

3. Die Ausweitung des Pharmaversandes kann zu einer Gefährdung des umfassenden Versorgungs- und Sicherstellungsauftrages der Apotheken führen (Schließung von Apotheken, Entlassung von Personal).

4. Die von den Apotheken durchgeführten Heimlieferungen entsprechen nicht mehr dem begründeten Einzelfall.

5. Der Umfang bereits durchgeführter Heimlieferungen wird sowohl vom Umsatz als auch von der Personalstärke einer Apotheke beeinflusst.

6. Die Präsenzapotheke in eine Versandapotheke umzuwandeln bzw. beides komplementär zu betreiben, ist abhängig von der Anzahl bereits durchgeführter Heimlieferungen.

7. Für komplementäre Distributionswege existieren keine Marktchancen.

8. Die Bereitschaft, mit Versandapotheken zusammen zu arbeiten, ist von der Facharztgruppe und der Zusammensetzung der Patientenklientel abhängig.

9. Die Marktchancen einer Versandapotheke sind abhängig vom Versorgungsgrad mit Apotheken.

10. Die Zielgruppe einer Versandapotheke wird vom Gesundheitszustand, dem Alter und dem Verhältnis der Konsumenten zum Internet beeinflusst.

11. Die Bereitschaft in der Bevölkerung, eine Versandapotheke zu nutzen, ist abhängig von bestimmten Rahmenbedingungen (z.B. Berufstätigkeit, Haushaltsgröße, Internet-User, Alter, Medikamentenkonsum, Mobilität).

12. Präsenzapotheken beraten ihre Kunden in allen Fällen umfassend und besser, als dies eine Versandapotheke machen könnte.

13. Voraussetzung für die Akzeptanz von Versandapotheken bei der Bevölkerung sind hohe Qualitätsanforderungen (z.B. bzgl. Zustellungssicherheit und Beratungsangebot).

Quelle: INIFES, eigene Darstellung. 
Ziel der Befragungen ist gewesen, die dargestellten Arbeitshypothesen zu überprüfen. Es sollten somit Anhaltspunkte erhalten werden über

- die Einstellung aller Betroffenen zum Vertriebskanal Apotheke

- die potentiellen Zielgruppen für eine Versandapotheke

- die Einstellung aller Betroffenen gegenüber dem Modell des Versandhandels mit Arzneimitteln

- die von den Betroffenen wahrgenommenen Chancen und Risiken einer Versandapotheke

Auf Basis der Arbeitshypothesen wurden, in Vorbereitung auf die Befragungen, in einem zweiten Schritt die Fragebögen entwickelt. Um die Akzeptanz der von einer Ausweitung des Pharmaversandes Betroffenen, unter Berücksichtigung ihrer Position im Gesundheitswesen und den daraus resultierenden Erwartungen, zu eruieren, hat sich INIFES dazu entschieden, sowohl für die Bevölkerung als auch für die Apotheker und Ärzte jeweils einen eigenen Fragebogen aufzustellen. Um jedoch die Vergleichbarkeit der Ergebnisse hinsichtlich einiger Arbeitshypothesen, beispielsweise der Einstellungen zu bestimmten Aussagen bzgl. einer Versandapotheke und der Zielgruppe für einen Versandhandel, zu garantieren, sind einige Fragen Inhalt aller Fragebögen - zumindest aber bei den Apothekern und Ärzten. Der folgende Abschnitt stellt den Aufbau der Fragebögen dar.

\subsubsection{Bevölkerungsbefragung}

Neben den allgemeinen soziodemographischen Daten (Alter, Geschlecht, Haushalts $(\mathrm{HH})$-Größe, HH-Nettoeinkommen, Bundesland, Berufstätigkeit (j/n) und der Anzahl der Kinder unter 14 Jahren) wurden Fragen aus folgenden Bereichen gestellt:

- Gesundheitsversorgung und Gesundheitszustand

Der erste Teil der Befragung ermittelte den Bestand der GKV-Versicherten bzw. Mitversicherten. In Verbindung mit indikationsspezifischen Angaben zum Gesundheitszustand und dem Verbrauch von Arzneimitteln, die vom Arzt verordnet werden, ließ sich ein Grundpotential von Personen für den Versandhandel ermitteln.

\section{- Apothekenwesen}

Die "Besorgerproblematik" ist bei der Eingrenzung potenzieller Kunden einer Versandapotheke ein wichtiger Punkt. Personen, die aus unterschiedlichen Gründen nicht selber ein Rezept einlösen können, bilden daher ein interessantes Klientel. Die Häufigkeit des Apothekenbesuchs zwecks Rezepteinlösung lässt Rückschlüsse über Chroniker bzw. Patienten mit einer Dauermedikation zu. Aufgrund der in einigen Gebieten vorherrschenden Apothekendichte und resultierend daraus eines zunehmenden Wettbewerbs, wurden die Kriterien untersucht, die die Bürger der Auswahl der Apotheke zugrunde legen. Da die Einführung von Ver- 
sandapotheken u.a. unter dem Hinweis einer fehlenden Beratung von den Apothekern abgelehnt wird, war für die Untersuchung von Interesse, inwieweit Kunden derzeit bei der Einlösung eines Rezepts beraten werden.

\section{- Versandapotheke}

Dieser Teil der Befragung befasst sich mit dem Modell einer Versandapotheke. Zu Beginn wurden die Bürger daher gefragt: "Können Sie sich vorstellen, in einigen Fällen die vom Arzt verordneten Arzneimittel von der Apotheke auch per Post zugeschickt zu bekommen?" Da der von INIFES durchgeführte Pretest gezeigt hat, dass dies bei vielen Personen nicht zutrifft, wurden im weiteren Verlauf des Teils der Befragung, der die Meinung der Interviewten zum Versandhandel erfragen soll, nur die Bürger befragt, die sich eine Zusendung von Arzneimitteln vorstellen können. Zunächst wurde den Probanden ein Modell einer Versandapotheke vorgestellt, wie es auch für die Bundesrepublik Deutschland denkbar wäre. Den Interviewten wurden dann Fragen bezüglich der persönlichen Inanspruchnahme einer Versandapotheke und ihrer Anforderungen an eine solche Apotheke gestellt. Obwohl die Versandapotheke von einer Internetapotheke in der Untersuchung zu unterscheiden ist, ließen die Antworten aller Befragten zu ihrem Internetverhalten und der Vorstellbarkeit des Bezugs von Medikamenten über eine Versandapotheke Rückschlüsse auf deren Einstellung gegenüber „neuen Modellen" zu.

\subsubsection{Apothekerbefragung}

Neben den allgemeinen soziodemographischen Daten (Alter, Geschlecht, Berufserfahrung, Umsatzgröße, Bundesland und Gemeindegrößenklasse) wurden Fragen aus folgenden Bereichen gestellt:

\section{- Allgemeine Fragen bzgl. der Apotheke und ihrer Aufgaben}

Zunächst wurden Daten bzgl. des derzeitigen Ist-Zustandes der Apotheken erfasst. Im Vordergrund standen dabei Fragen zur personellen und technischen Ausstattung sowie der Versorgungsdichte. Die ideale Apotheke darf zukünftig keine gewöhnliche Einkaufs- oder Abgabestelle für Arzneimittel sein, sondern hat sich serviceorientiert und kundeninformierend auszurichten. Um sich diesen Anforderungen zu stellen, sind daher zunächst die Kriterien erfragt worden, anhand derer, aus Sicht der Apotheker, die Kunden eine Apotheke auswählen. Des weiteren ist der Anteil derer von Interesse, die Rezepte für andere Personen einlösen. Anhand dieser Daten erhält man einen Ansatzpunkt für künftige Änderungen des Geschäftsfeldes.

- Reformoptionen bzw. Modelle in der Versorgung der Bevölkerung mit Arzneimitteln

Ein möglicher Ansatzpunkt zu mehr Serviceorientierung in der Apotheke stellt eine Aufhebung des $\S 17$, II, Apothekenbetriebsordnung (ApBetrO) - gesetzliche Einzelfallregelung bei der Heimlieferung von Arzneimitteln - dar. Daher wurde nach 
der Einstellung der Apotheker und der derzeitigen Anwendung bzgl. des § 17, II ApBetrO gefragt. Im Ausland existieren bereits unterschiedliche Distributionswege von Arzneimitteln. Vor dem Hintergrund einer Harmonisierung der Märkte in Euro$\mathrm{pa}$, ist die Einstellung der Apotheker zu solchen alternativen Vertriebswegen erfasst worden.

\section{- Versandapotheke}

Dieser Teil der Befragung befasst sich, wie auch bei der Bevölkerung und den Ärzten, mit dem Distributionsmodell "Versandapotheke". Dazu wurde unter unterschiedlichen Gesichtspunkten die Meinung der Apotheker erfragt. Den teilnehmenden Apothekern wurden u.a. Fragen bezüglich ihrer persönlichen Meinung gegenüber einer Versandapotheke und des potenziellen Kundenkreises gestellt. Die Befragung thematisiert überdies die von den Apothekern für sich selbst erwarteten ökonomischen Auswirkungen durch die Einführung einer Versandapotheke. Da die Etablierung eines Versandhandels auch der Reformierung des bisherigen Rezeptes bedarf, wurden den Apothekern abschließend einige Reformoptionen vorgestellt und ihnen die Möglichkeit eingeräumt, ergänzend einige zu nennen.

\subsection{3. Ärztebefragung}

Für die Studie von Interesse sind folgende Facharztgruppen:

- Niedergelassene Allgemeinmediziner / Praktiker / Internisten

- Niedergelassene Gynäkologen

- Niedergelassene HNO-Ärzte

- Niedergelassene Urologen

- Niedergelassene Nervenärzte

Neben den allgemeinen soziodemographischen Daten (Alter, Geschlecht, Niederlassungsjahr, Anzahl Patienten je Quartal, Bundesland und Gemeindegrößenklasse) wurden Fragen aus folgenden Bereichen gestellt:

\section{- Allgemeine Fragen betreffend der Arztpraxis und der Apotheken}

Entsprechend der Annahme, dass eine Versandapotheke vor allem für Chroniker von Interesse ist, wurde zunächst der Anteil von chronisch Kranken am Patientenklientel der Arztpraxis erfragt. Diese Personengruppe, erweitert um die Patienten mit einer Dauermedikation, stellt eine mögliche Hauptzielgruppe für den Versandhandel dar. Um die Abhängigkeit der Apotheken von Arztpraxen zu ermitteln, wurde der Versorgungsgrad mit Apotheken im Umkreis der Praxis ermittelt.

- Reformoptionen in der Versorgung der Bevölkerung mit Arzneimitteln Wie bereits erwähnt, wird der Arzneimittelverbrauch sowohl durch das Verordnungsverhalten als auch die Patientenklientel der Ärzte bestimmt. Die Ärzte sind daher das Bindeglied zwischen Nachfrager und Anbieter von Arzneimitteln und können durch Ihr Verhalten den Vertrieb von Arzneimitteln beeinflussen. Da im Ausland unterschiedliche Distributionswege von Arzneimitteln derzeit bereits An- 
wendung finden, wurde die Einstellung der Ärzte zu solchen alternativen Vertriebswegen erfasst.

\section{- Versandapotheken}

Dieser Teil der Befragung befasst sich, wie auch bei der Bevölkerung und den Apothekern, mit einem neuen, derzeit noch nicht existenten, Apothekenmodell, der Versandapotheke. Dazu wurde unter unterschiedlichen Gesichtspunkten die Meinung der Apotheker erfragt. Den teilnehmenden Apothekern wurden u.a. Fragen bezüglich ihrer persönlichen Meinung gegenüber einer Versandapotheke und des potenziellen Patientenkreises gestellt. Dies ist notwendig, da ohne eine Kooperation der Ärzte das Modell einer Versandapotheke nicht umsetzbar ist. Da die Etablierung einer Versandapotheke der Reformierung des bisherigen Rezeptes bedarf und somit den Zuständigkeitsbereich der Ärzte tangiert, sind den Ärzten einige Reformoptionen zur Stellungnahme vorgestellt worden.

\subsection{Ergebnisse der Befragungen}

\subsubsection{Vorbemerkungen}

Die Auswertung der Daten der Apotheker-, der Ärzte- sowie der Bevölkerungsbefragung wurde mit den Programmen Quantum V.5F und SPSS für Windows, Version 10.0, durchgeführt.

Die Antworten auf offene Fragen wurden bislang bei wissenschaftlichen Untersuchungen für jeden einzelnen Fall sinngemäß in Excel erfasst, kategorisiert und zur Auswertung in eine SPSS-Maske transformiert. Dieses eher mühsame Vorgehen lässt sich durch das CATI-System vereinfachen. ${ }^{41}$ Im Anschluss an das Interview können bereits die Antworten auf die offene Frage vercodet werden. Eine Nachbearbeitung des Datensatzes, z. B. mittels einer Excel-Datei, wird auf diese Weise überflüssig.

Der Vergleich von Zahlenwerten und die Prüfung von Zusammenhängen zwischen einzelnen Daten ist der Schwerpunkt einer empirischen Analyse. Elementares Werkzeug bei dieser Arbeit sind Signifikanzberechnungen, Korrelationskoeffizienten und Regressionsanalysen. Bei der Signifikanzberechnung wird geprüft, ob eine Differenz zwischen zwei Ergebnissen einer Stichprobenerhebung mit einiger oder starker Wahrscheinlichkeit wirklich besteht oder nur zufällig zustande gekommen ist. Der Korrelationskoeffizient ist eine Richtgröße dafür, wie eng, in welchem Grad zwei Merkmale zusammenhängen, oder wie sehr sie sich gegenseitig ausschließen. Eine Regressionsanalyse zeigt die Stärke eines Zusammenhanges für kontinuierlich angeordnete Daten.

\footnotetext{
41 Fuchs, M. (1994), Umfrageforschung mit Telefon und Computer, Weinheim. heitsstörung die Häufigkeit des Apothekenbesuchs zu?
} 
Das Ziel der von INIFES durchgeführten Befragung besteht in einem Vergleich der Befragten hinsichtlich der Akzeptanz und ihrer Einstellung gegenüber einer Versandapotheke. Da die Stichprobengrößen und die inhomogenen Gruppen eine statistische Auswertung erschweren und vielfach unmöglich machen, stellen die folgenden $\mathrm{Er}$ gebnisse Tendenzen im Rahmen der deskriptiven Statistik dar.

\subsubsection{Apothekerbefragung}

\subsubsection{Allgemeine Angaben zur Stichprobe}

Die Gruppe der befragten Apotheker setzte sich aus 63,0 Prozent Frauen und 37,0 Prozent Männer zusammen. 40,0 Prozent waren nicht älter als 39 Jahre (23,0 Prozent Männer, 50,0 Prozent Frauen), 22,0 Prozent sind zwischen 40 und 49 Jahre, 27,0 Prozent zwischen 50 und 59 Jahre (42,0 Prozent Männer, 18,0 Prozent Frauen) und 11,0 Prozent gaben an, älter als 60 Jahre zu sein. In BIK-Ortsgrößen bis 5.000 Einwohner befanden sich 12,0 Prozent der Apotheken, 19,0 Prozent in BIKOrtsgrößen bis 20.000 Einwohner, 15,0 Prozent in Gebieten bis 100.000 Einwohner, 19,0 Prozent der Apotheken hatten sich in Ortsgrößen bis 500.000 Einwohner niedergelassen und in BIK-Ortsgrößen mit 500.000 und mehr Einwohnern existierten 37,0 Prozent Apotheken. Nach Angaben der Apotheker haben 32,0 Prozent bis zu zwei pharmazeutische Fachkräfte - zu Vollzeitkräften zusammengefasst - in ihrer Apotheke angestellt. Zwischen drei und fünf Fachkräfte beschäftigten 45,0 Prozent der Apotheker und 17,0 Prozent mehr als fünf pharmazeutische Angestellte. Der Umsatzklasse unter 1 Mio. DM Umsatz pro Jahr ordneten sich 5,0 Prozent der befragten Apotheker zu. 8,0 Prozent erzielten bis unter 1,25 Mio. DM Umsatz, 8,0 Prozent bis unter 1,5 Mio. DM und 49,0 Prozent gaben an, mehr als 1,5 Mio. DM Umsatz pro Jahr zu erzielen. Immerhin 30,0 Prozent wollten keine Angaben zu ihrem Jahresumsatz machen. Tabelle III - 3 gibt eine Übersicht über die soziodemographischen Angaben der Apotheker. 
Tabelle III - 3: Sozio-Demographie der Apotheker $(n=150)$

\begin{tabular}{|l|c|}
\hline & Prozent \\
\hline Geschlecht & 37 \\
Männer & 63 \\
Frauen & \\
\hline Alter & 40 \\
-39 Jahre & 22 \\
40-49 Jahre & 27 \\
$50-59$ Jahre & 11 \\
60+ Jahre & \\
\hline Beginn der Tätigkeit als Apotheker & 14 \\
bis 1970 & 16 \\
$1971-1975$ & 8 \\
$1976-1980$ & 13 \\
$1981-1985$ & 14 \\
$1986-1990$ & 17 \\
$1991-1995$ & 14 \\
$1996-2000$ & \\
\hline Pharmazeutisches Personal & 32 \\
bis 2 & 45 \\
3 bis 5 & 17 \\
mehr als 5 & 7 \\
Weiß nicht & \\
\hline Umsatzklasse & 5 \\
unter 1 Mio. DM & 8 \\
1 bis 1,25 Mio. DM & 8 \\
1,25 bis 1,5 Mio. DM & 49 \\
über 1,5 Mio. DM & 30 \\
keine Angabe & 12 \\
\hline BIK-Ortsgröße & 19 \\
- u. 5.000 & 15 \\
$5.000-$ u. 20.000 & 19 \\
20.000 - u. 100.000 & 37 \\
100.000 - u. 500.000 & \\
500.000 und mehr & \\
\hline & \\
\hline
\end{tabular}

Quelle: INIFES, eigene Darstellung.

(Ergebnisse einer von TNS EMNID im Auftrag von INIFES im Dezember 2000 durchgeführte CATI-Befragung). 


\subsubsection{Vertriebskanal Apotheke}

Bedingt durch die Klassifikation der Arzneimittel in solche mit und andere ohne Apothekenpflicht nach § 43 Arzneimittelgesetz (AMG) ist der Vertriebsweg vom Anbieter zum Nachfrager gesetzlich vorgegeben. Hinzu kommt mit § 17 Apothekenbetriebsordnung eine weitere Regelung, nach der apothekenpflichtige Medikamente nur in den Betriebsräumen der Apotheken durch pharmazeutisches Personal abgegeben werden darf. Unter die Apothekenpflicht fallen in Deutschland ca. 45.000 Präparate. ${ }^{43}$ Darunter fungieren automatisch alle rezeptpflichtigen Arzneimittel. Der Verkauf apothekenpflichtiger Präparate erfolgt ausschließlich in Apotheken. Die quantitative Arzneimittelversorgung in der Bundesrepublik Deutschland wird von 21.590 Apotheken ${ }^{44}$ sichergestellt. Die Apothekendichte beträgt 3.800 Einwohner pro Apotheke bzw. 17 $\mathrm{km}^{2}$ pro Apotheke.

Vor diesem Hintergrund wird oftmals die These einer existierenden Überversorgung aufgestellt. ${ }^{45}$ Immerhin 27,0 Prozent der Apotheker sprechen von einer Überversorgung in ihrem Einzugsgebiet. ${ }^{48}$ Als gerade angemessen bezeichnen 72,0 Prozent der Apotheker die Apothekendichte in ihrem Umfeld und nur 1,0 Prozent geben an, dass in ihrem Einzugsgebiet eine Unterversorgung existiert. Ein signifikanter Unterschied im Antwortverhalten der befragten Apotheker hinsichtlich der Beurteilung des Versorgungsgrades mit Apotheken ist gegeben. Während bei den ländlichen und stadtrandnahen Apotheken - in Ortsgrößen ${ }^{47}$ bis 100.000 Personen - 22,0 Prozent der Apotheker von einer Überversorgung sprechen, geben 30,0 Prozent der befragten Apotheker in Gebieten mit mehr als 100.000 Personen an, dass in ihrem Einzugsgebiet zu viele Apotheken existieren.

Sterzel, A., Wassener, D. (2001), Arzneimitteldistribution in Deutschland - Versandapotheken als Reformoption?, in: Arbeit und Sozialpolitik 1-2/2001, S. 14-20.

ABDA - Bundesvereinigung Deutscher Apothekerverbände (2000), Die Apotheke: Zahlen - Daten - Fakten (1999).

Ergebnis mehrerer, von INIFES durchgeführter, Experteninterviews. Befragt wurden Vertreter von Standesorganisationen, der Apotheker, der Politik, der Wissenschaft, der Industrie, der Verbraucherverbände und der Krankenkassen. Die Ergebnisse sind anonymisiert wiedergegeben. Die Mitschriften/Bänder der Interviews liegen bei INIFES vor.

Die Frage lautete: Überversorgung mit Apotheken wird heute in manchen Gebieten als Problem angesehen. Wie beurteilen Sie die Situation im Einzugsgebiet Ihrer Apotheke?

47

Die Ortsgrößenklassen werden nach BIK-Stadtregionen ausgewiesen (Quelle: BIK Aschpurwis+Behrens $\mathrm{GmbH}$ ). Der Einsatz der BIK-Stadtregionen-Systematik kann die siedlungsstrukturellen Gegebenheiten in den heutigen Stadtregionen besser erfassen als der bisherige Bourstedt-Ansatz (bzw. als die bisherigen politischen Ortsgrößenklassen in den neuen Bundesländern). Relevante Merkmale der BIK-Stadtregionen sind dabei vor allem die Einwohnerzahl der Kernstadt eines Einzugsbereichs und die Größenordnung sowie Intensităt der Pendleranbindung. 
Aus Gründen des Verbraucherschutzes sieht der Gesetzgeber in der Abgabe von Arzneimitteln und der Beratungsmöglichkeit in der Apotheke eine Einheit, ebenso wie in dem übrigen Netz von gesetzlichen Regelungen zur Gewährleistung der Arzneimittelsicherheit von der Arzneimittelzulassung über die qualifizierte Beratung bis zur Abgabe in der Apotheke.

Apotheker sind nach $\S 20$ ApBetrO aus Gründen der Arzneimittelsicherheit verpflichtet, dem Apothekenkunden informierend und beratend zur Seite zu stehen. Allerdings darf die Beratung nicht die Therapie des verordnenden Arztes beeinträchtigen, eine Kritik der ärztlichen Diagnose oder Verordnung ist dem Apotheker untersagt. D.h., bei Medikamenten auf Rezept - und das ist die große Mehrheit - besteht eine grundsätzliche Beratungspflicht des Apothekers in Bezug auf die vom Arzt verordneten Arzneimittel, z.B. im Kontext der Einnahme, Compliance und Wechselwirkungen mit anderen verordneten Mitteln oder Selbstmedikation; der Apotheker muss sogar kontrollieren und bei Verschreibungsfehlern eingreifen, darf aber nicht die Therapie des Arztes in Frage stellen. Auf die Frage, nach welchen Kriterien die Bürger eine Apotheke auswählen, gaben 91,0 Prozent der Apotheker an, dass für den Kunden bei der Wahl der Apotheke, in der er sein Rezept einlöst, die umfassende Beratung durch den Apotheker sehr wichtig oder wichtig sei. Die Apotheker sehen also die von innen ausgehende Beratung als eine ihrer Kernkompetenzen an. Auf den Beratungsaspekt wirkt jedoch problematisch, dass viele Apothekenkunden "Besorger" für andere Menschen sind, die aufgrund unterschiedlicher Gründe nicht selber eine Apotheke aufsuchen können. 49,0 Prozent der von INIFES befragten Apotheker geben an, dass zwischen 20,0 und 50,0 Prozent ihrer Kundschaft Rezepte für andere Personen als "Besorger" einlösen. Einen "Besorger-Anteil" von mehr als 50,0 Prozent verzeichnen 3,0 Prozent der Apotheker. In diesen Fällen ist daher keine direkte Beratung durch den Apotheker möglich.

Die sog. "Besorger-Problematik" stellt somit den Ausgangspunkt für einen nicht zu unterschätzenden Distributionsweg innerhalb des "Vertriebskanals" Apotheke dar. Viele Arzneimittelbezieher sind in ihrer Mobilität aufgrund einer Krankheit, des Alters oder der Berufstätigkeit stark eingeschränkt. Eine vertraute Person, welche die Rolle eines "Rezeptüberbringers" und "Medikamentenbesorgers" übernimmt, ist oft das nötige Bindeglied zwischen Apotheke und Patienten/Kunden. Um den Kunden jedoch, unter Wahrung der gesetzlichen Bestimmungen, eine optimale Versorgung zu gewährleisten, wenden viele Apotheker die Ausnahmeregelung des $\S 17$, II ApBetrO an. Die Ausnahmeregelung des $\S 17$, II ApBetrO billigt in begründeten Einzelfällen eine Versendung aus der Apotheke oder die Zustellung durch einen Boten an den Arzneimittelempfänger zu. Die Befragungsergebnisse zeigen, dass Heimlieferungen

48

Vgl. ABDA (2001), E-Commerce und Versandhandel mit Arzneimitteln aus Sicht der Apotheker, www.abda.de/ABDAVartikel.htm|?ID=132, Zugriff am 27.04.2001. 
durch Apotheken gängige Praxis sind (s. Abbildung III - 2). Die von INIFES durchgeführten Expertengespräche ${ }^{49}$ bestätigten dies.

Abbildung III - 2: "Heimlieferungen" pro Woche

(Frage: "Im Rahmen der gesetzlichen Ausnahmeregelung ist es Ihnen erlaubt, Kunden nach Hause zu beliefern. Bei wie vielen Kunden trifft dieses pro Woche zu?")

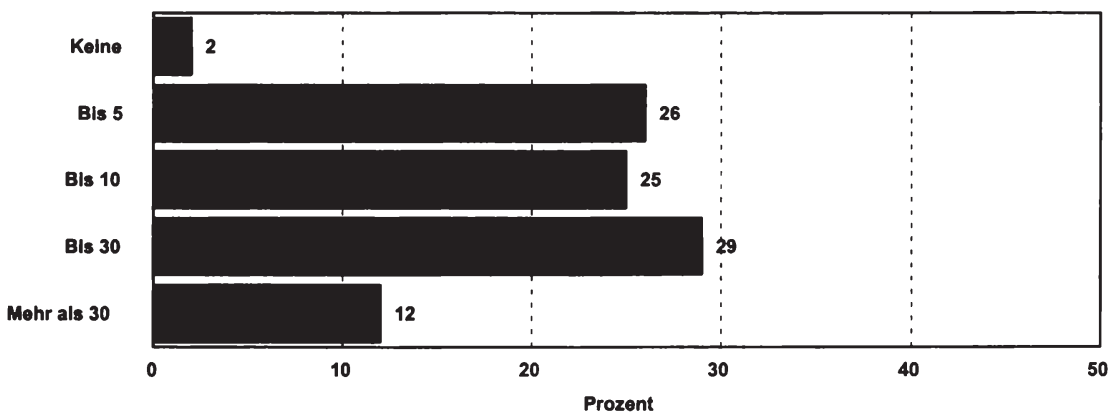

Quelle: INIFES, eigene Darstellung.

(Ergebnisse einer von TNS EMNID im Auftrag von INIFES im Dezember 2000 durchgeführten CATI-Befragung).

Wăhrend einige Apotheker in Expertengesprächen darauf hinwiesen, dass die Belieferung des Kunden nur die Ausnahme darstellt, bestätigt die Befragung die Arbeitshypothese 4, nach der die Lieferung von Arzneimitteln, die nicht sofort in der Apotheke verfügbar sind, vielfach die Regel ist. Von lediglich 2,0 Prozent der Apotheken wird eine Heimlieferung nicht durchgeführt bzw. angeboten, 29,0 Prozent der Apotheken beliefern bis zu 30 Kunden pro Woche direkt und weitere 12,0 Prozent beliefern mehr als 30 Kunden pro Woche. Vor allem umsatzstarke Apotheken mit einem Umsatz von über 1,5 Mio. DM pro Jahr führen zu 16,0 Prozent eine Lieferung bei mehr als 30 Kunden pro Woche durch (in der Umsatzklasse bis 1,5 Mio. DM beliefern nur 6,0 Prozent der Apotheker mehr als 30 Kunden pro Woche).

Die Arbeitshypothese 5, die besagt, dass der Umfang bereits durchgeführter Heimlieferungen sowohl vom Umsatz als auch von der Personalstärke einer Apotheke beeinflusst wird, wird nicht wiederlegt. Der Umfang der Heimlieferungen korreliert neben dem Umsatz auch mit der Personalstärke einer Apotheke. Apotheken, in denen bis zu 2 Vollzeitkräfte als Pharmazeutisches Personal tătig sind, beliefern nur 2,0 Prozent mehr als 30 Kunden pro Woche, bei 3 bis 5 Vollzeitkräften sind es 18,0 Prozent und bei mehr als 5 Vollzeitkräften immerhin noch 17,0 Prozent. Der Heimlieferservice mit Medikamenten durch die Apotheke ist - so die Aussage von Apothekern - somit bereits schon jetzt gängige Praxis und nicht mehr der "begründete Einzelfall".

48

Ergebnis von Expertengesprächen mit Vertretern der Krankenkassen, der Pharmaindustrie und mit Apothekern. 
Dass ein Apotheker einem Kunden auf Wunsch Waren wie beispielsweise Inkontinenz-Produkte liefert, ist eine selbstverständliche Service-Leistung. Auch die Tatsache, dass eine Apotheke nicht alle erhältlichen Medikamente, vor allem Generika, vorhalten kann, ist verständlich.

Die Struktur der Apotheke hat sich in den letzten Jahren dahingehend verändert, dass sie zu einem Just-in-Time Betrieb mutiert ist. Das ganze Lagerhaltungsrisiko wird auf den Großhandel delegiert, der die Apotheke bis zu 6 mal täglich beliefert. Die Kapitalbindung der einzelnen Apotheke wird dadurch minimiert. Aus betriebswirtschaftlicher Sicht ist dies nachvollziehbar. Die Aussage einer Apothekerin, die durch ein Apothekenkammer-Mitglied bestätigt wurde, dass rezeptpflichtige Arzneimittel, die nicht vorrätig sind, selbstverständlich durch einen Boten dem Kunden nach Hause geliefert werden, verdeutlicht, dass der begründete Einzelfall immer häufiger zur Regel wird. Dies zeigt, dass ein Kundenbedürfnis nach Heimlieferungen von Medikamenten vorhanden ist, dem sich auch die Apotheker nicht verschließen können bzw. vielfach nicht verschließen.

Fast ein Viertel der Apotheker (23,0 Prozent) würde eine Lockerung der gesetzlichen Rahmenbedingungen begrüßen, um bestimmte Kundengruppen gezielt durch Heimlieferungen ansprechen zu können. Dies gilt besonders für Apotheker mit einem Umsatz von bis zu 1,5 Millionen DM pro Jahr (28,0 Prozent) und mit höchstens zwei Vollzeitkräften. Dies mag ein Indiz dafür sein, dass vor allem die eher umsatzschwachen Apotheken durchaus an einer Legalisierung und Ausweitung der Heimlieferungen interessiert sind. Gut ein Viertel der Apotheker, die für eine Lockerung der gesetzlichen Vorschriften in bezug auf Heimlieferungen sind (24,0 Prozent), führen schon jetzt mehr als 30 Heimlieferungen pro Woche aus und würden dies auch ausweiten. Die offiziellen Argumente der Apothekerschaft, dass Heimlieferungen nur gesetzeskonform $^{50}$ vorgenommen werden, sehen die Apotheker scheinbar anders. Während es bei Apothekern, die bislang nicht mehr als 30 Heimlieferungen pro Woche durchführen, keine signifikanten Unterschiede bzgl. Zustimmung oder Ablehnung gegenüber einer Lockerung der gesetzlichen Vorschriften zur Belieferung der Kunden gibt, ist die Zustimmung der Apotheken mit einem hohen Anteil an bereits durchgeführten Heimlieferungen überproportional hoch. 24,0 Prozent der Apotheker, die für eine Ausweitung der Heimlieferungen stimmten, beliefern derzeit mehr als 30 Kunden pro Woche, bei den Gegnern sind es nur 8,0 Prozent.

Konsumenten legen bei der Wahl der Apotheke, in der sie ein Rezept einlösen, verschiedene Kriterien zu Grunde. Um den Grad ihrer Bedeutung näher bestimmen zu können, wurden im Rahmen der Befragungen (Apotheker, Ärzte, Bevölkerung) folgende Aspekte untersucht ${ }^{51}$ : Schnelle und gute Erreichbarkeit, Umfassende Beratung, Kostenloser Heimlieferservice von Medikamenten sowie Kunden gehören zur Stammkundschaft.

50

51

Unter Beachtung des $\S 17$, II Apothekenbetriebsordnung.

In Punkt 3.5. sind die Ergebnisse der drei Befragungen gegenübergestellt. 
Anhand einer Skala von 1=sehr wichtig bis 5=völlig unwichtig sind diese Kriterien von den Apothekern bewertet worden. Im Folgenden sind zu diesem Themenbereich die Einschätzungen der Apotheker dargestellt (s. Abbildung III - 3).

Abbildung III - 3: Grad der Wichtigkeit verschiedener Kriterien bei der Auswahl der Apotheke

(Frage: "Welche Kriterien legen Sie bei der Auswahl der Apotheke zugrunde? Wie wichtig sind Innen folgende Aspekte?")

Durchschnitt

Schnelle und gute Erreichbarkeit

Umfassende Beratung

Kunden gehören zur Stammkundschaft

Kostenloser Heimservice von Medikamenten

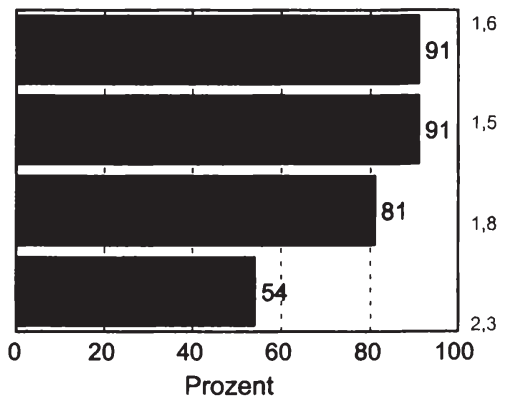

$\mathrm{n}=150$ Apotheker

Top-2-Boxes ("sehr wichtig" und "wichtig"); 1=sehr wichtig, 5=vollig unwichtig

Quelle: INIFES, eigene Darstellung.

(Ergebnisse einer von TNS EMNID im Auftrag von INIFES im Dezember 2000 durchgeführten CATI-Befragung).

Nach Ansicht der Apotheker messen die Kunden bei der Auswahl der Apotheke, in der das Rezept eingelöst wird, besonders den Kriterien einer schnellen und guten Erreichbarkeit (91,0 Prozent) sowie einer umfassenden Beratung (91,0 Prozent) eine große Bedeutung bei. Die schnelle und gute Erreichbarkeit einer Apotheke ist, betrachtet man die Werte bzgl. des Versorgungsgrades und der Apothekendichte, in der Bundesrepublik Deutschland sichergestellt. Untersuchungen zeigen, dass bei 43,2 Prozent der Apotheken bis zu zwei Arztpraxen in einer Entfernung von bis zu drei Gehminuten liegen, weitere 20,9 Prozent haben drei oder vier Praxen in unmittelbarer Nähe.

Bezüglich des Kriteriums einer umfassenden Beratung geben 91,0 Prozent der Apotheker an, dass die Kunden eine Apotheke präferieren, in der sie umfassend beraten werden. Dieser Wert steigt in Abhängigkeit der Personalausstattung einer Apotheke.

Schöffski, O. (1995), Die Regulierung des deutschen Apothekenwesens. Eine ökonomische Analyse, Baden-Baden, S. 229. 
Apotheker, die fünf und mehr Vollzeitkräfte als Pharmazeutisches Personal beschäftigen, schätzen zu 100,0 Prozent ${ }^{53}$ eine umfassende Beratung als bedeutendes Auswahlkriterium ein. Die Angaben der Apotheker lassen daher vermuten, dass sie ihre Kunden gut und umfassend bei der Aushändigung von Arzneimitteln beraten. Vergleicht man die Selbsteinschätzung der Apotheker mit den Antworten der Bevölkerung, werden Diskrepanzen deutlich. 28,0 Prozent der Personen, die ihr Rezept selber einlösen oder die Medikamente von der Apotheke nach Hause geliefert bekommen, werden bei fast keinem Apothekenbesuch beraten, 30,0 Prozent sporadisch bei ca. jedem zweiten oder dritten Apothekenbesuch. Bei ungefähr jedem Apothekenbesuch werden 35,0 Prozent der Befragten bei der Aushändigung von Medikamenten beraten.

Zusammenfassend ergab die Befragung der Apotheker bzgl. des Vertriebskanals Apotheke, dass von der Ausnahmeregelung des § 17, II ApBetrO, die den Apothekern in begründeten Einzelfällen eine Versendung aus der Apotheke oder die Zustellung durch einen Boten an den Arzneimittelempfänger erlaubt, intensiv Gebrauch gemacht wird. 29,0 Prozent der befragten Apotheken beliefern bis zu 30 Kunden pro Woche und weitere 12,0 Prozent mehr als 30 Kunden pro Woche direkt. Dies verdeutlicht, dass der begründete Einzelfall immer häufiger zur Regel wird. Es scheint demnach ein Kundenbedürfnis nach Heimlieferungen von Medikamenten vorhanden zu sein, dem sich viele Apotheker nicht mehr verschließen können bzw. vielfach nicht verschließen. Um bestimmte Kundengruppen gezielt durch Heimlieferungen ansprechen zu können, würden 23,0 Prozent der Apotheker eine Lockerung der bislang bestehenden gesetzlichen Rahmenbedingungen begrüßen. Im Rahmen der Expertengespräche wurde von verschiedenen Stellen darauf hingewiesen, dass viele Apotheken für ihre Botendienste bereits eine Logistik aufgebaut haben, deren Kosten mit einer Ausweitung der Liefertätigkeiten gesenkt werden würden.

Apotheker sind nach $\S 20 \mathrm{ApBetrO}$ aus Gründen der Arzneimittelsicherheit verpflichtet, dem Kunden informierend und beratend zur Seite zu stehen. Die Ergebnisse der Befragung und der Expertengespräche zeigen, dass Apotheker die Beratung des Kunden für einen unverzichtbaren Bestandteil einer qualitativ hochwertigen Arzneimittelversorgung halten. Nach Meinung der Apotheker (91,0 Prozent) stellt für Kunden eine umfassende Beratung durch den Apotheker bei der Auswahl der Apotheke, in der sie ein Rezept einlösen, ein entscheidendes Kriterium dar. Dem stehen jedoch die Antworten der Konsumenten entgegen: 28,0 Prozent werden bei fast keinem Apothekenbesuch beraten, 30,0 Prozent bei circa jedem zweiten oder dritten Apothekenbesuch und nur 35,0 Prozent gaben an, bei jedem Apothekenbesuch umfassend beraten zu werden.

53

Signifikanzniveau aufgrund des Stichprobenumfangs 10 Prozent. 


\subsubsection{Alternative Distributionswege}

Vor dem Hintergrund geforderter Reformen im Gesundheitswesen ist derzeit in der Offentlichkeit eine lebhafte Diskussion u.a. um neue Vertriebsformen und Distributionswege im Arzneimittelsektor entbrannt.

Bevor im Punkt 3.4.2.4. die Vertriebsform der Versandapotheke als ein möglicher Distributionsweg näher untersucht und dargestellt wird, beinhaltet dieses Kapitel eine Bewertung der Marktchancen verschiedener Distributionswege durch Apotheker. Dem derzeit noch streng regulierten Vertriebsweg für Arzneimittel stehen nach Meinung der Betrachter Veränderungen bevor, wie nur selten zuvor in der Geschichte der Apotheken. ${ }^{54}$ Begriffe wie "Apothekenketten", "Versandapotheken", "Internetapotheken ${ }^{{ }^{55}}$ und "Arzneimittelversand" werden derzeit häufiger von Politikern, Interessensverbänden und der Wissenschaft in die Diskussion eingebracht.

Um jedoch zunächst einmal die generelle Einstellung der Apotheker bzgl. der Marktchancen der genannten Distributionswege zu erfragen, wurden sie gebeten, anhand einer Skala von 1=wesentlich bessere Marktchancen bis $5=$ wesentlich schlechtere Marktchancen, die Distributionswege zu bewerten (s. Abbildung III - 4).

Abbildung III - 4: Bewertung der Marktchancen verschiedener Distributionswege

(Frage: "Welchen Distributionswegen für Medikamente auf Rezept räumen Sie zukünftig eher bessere, welchen eher schlechtere Marktchancen ein?")

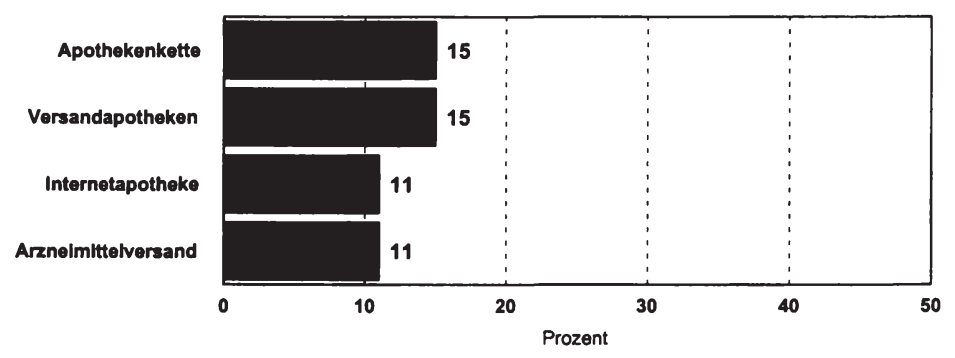

$n=150$ Apotheker

Top-2-Boxes("wesentlich bessere" und "bessere); 1=wesentlich bessere Marktchancen, 5=wesentlich schlechtere Marktchancen

Quelle: INIFES, eigene Darstellung.

(Ergebnisse einer von TNS EMNID im Auftrag von INIFES im Dezember 2000 durchgeführten CATI-Befragung).

54

Schöffski, O. (1995), Apotheker und Industrie, in: ANZAG-Magazin, Oktober/November 1995, S. 27.

55

Eine allgemeingültige Definition bzgl. der Begriffe "Versandapotheke" und "Internetapotheke" existiert bislang noch nicht. 
Die Apotheker wurden wie folgt befragt: Welchen Distributionswegen für Medikamente auf Rezept räumen Sie zukünftig eher bessere, welchen eher schlechtere Marktchancen ein? Arbeitshypothese 7, die besagt, dass für komplementäre Distributionswege keine Marktchancen bestehen, wurde nicht widerlegt. Ein überwiegender Anteil der Apotheker sieht demnach für keinen der alternativen Distributionswege tatsächliche Marktchancen. Nur jeweils 11,0 Prozent der Apotheker räumen Internetapotheken - freie Medikamentenbestellung durch den Verbraucher - und dem Versand von Arzneimitteln durch den Hersteller oder den Großhandel echte Marktchancen ein. 15,0 Prozent sind es bei Apothekenketten und Versandapotheken, also der Medikamentenbestellung in direkter Kooperation mit dem Arzt. Apotheker, die noch nicht sehr lange approbiert sind, bewerten die Marktchancen weitaus positiver als andere. 15,0 Prozent derer, die seit 1981 approbiert sind, sehen für Internetapotheken gute Marktchancen, dagegen nur 5,0 Prozent von denen, die ihre Approbation vor 1981 erlangt haben. Gerade die jüngeren Apotheker sind in ihrer Einstellung liberaler, aber auch pragmatischer. Dies wird auch bei den Bewertungen der Marktchancen für Apothekenketten (Approbation vor 1981: 5,0 Prozent, ab 1981: 22,0 Prozent) deutlich. Ein signifikanter Unterschied zwischen umsatzstarken und umsatzschwächeren Apotheken bzgl. der Bewertung der Marktchancen verschiedener Distributionswege ist nicht gegeben. Das Antwortverhalten ist daher nicht von dem erzielten Umsatz abhängig.

Viele Apotheker befürchten eine starke Konkurrenz und eine Aufgabe ihres freiberuflichen Status durch die Legalisierung neuer Distributionswege. Mit Umsatzeinbußen infolge einer Einführung der Arzneimitteldistribution über Versandapotheken rechnen 58,0 Prozent der Apotheker. Vor allem Apotheken mit mehr als 5 Vollzeitkräften (Pharmazeutisches Personal) geben an, von Umsatzeinbußen betroffen zu sein $(68,0$ Prozent).

\subsubsection{Die Einstellung der Apotheker zum Modell der Versandapotheke}

Der Aufbruch in das Informationszeitalter macht auch vor dem Gesundheitswesen nicht halt. Der innovative Einsatz von Informations- und Kommunikationstechniken eröffnet u.a. für den Arzneimittelsektor neue und alternative Vertriebsformen. In der Öfentlichkeit diskutiert werden derzeit Versand- und Internetapotheken.

Eine allgemeingültige Definition bzgl. der Versandapotheke gibt es bislang nicht. ${ }^{56}$ Vielmehr werden beide Begriffe vielfach synonym verwendet. "Die Internetapotheke ist eine Versandapotheke. Das Internet ist lediglich das Medium zur Geschäftsanbahnung und zum Geschäftsabschluss. Die Abwicklung des Geschäfts erfolgt per In-

56

Siehe Kapitel 1.4. 
ternethandel", so Cornelia Yzer - Hauptgeschäftsführerin des Verbandes forschender Arzneimittelhersteller.

Während es in den USA, Südafrika und auch in europäischen Ländern wie Großbritannien, den Niederlanden, Dänemark und der Schweiz bisher möglich ist, rezeptpflichtige Arzneimittel auch über dem Vertriebsweg einer Versandapotheke zu beziehen, spricht sich die $A B D A^{58}$ gegen diesen Weg des Bezugs von Arzneimitteln aus. "Die Apotheker begrüßen zwar das Internet als exzellentes Medium der Information und Kommunikation, lehnen aber einen Internet-gestützten Versandhandel mit Arzneimitteln ab", so eine offizielle Pressemitteilung. "Für den höchstmöglichen Sicherheitsstandard ist ein Versandhandelsverbot bei Arzneimitteln unverzichtbar", so der ABDA-Präsident Hans-Günter Friese. ${ }^{59}$ Nur so könne eine notwendige Beratung und eine Verbesserung der Compliance der Patienten erfolgen. Nur 78,0 Prozent der Bürger wünschen eine Beratung in der Apotheke und nur ein Drittel der Befragten (35,0 Prozent) werden überhaupt unaufgefordert umfassend in der Apotheke beraten. Die von der ABDA ablehnende Haltung gegenüber der Zulassung des Versandhandels mit Arzneimitteln ist hinlänglich bekannt und soll daher nicht weiter betrachtet werden. Gegenstand der Befragung ist vielmehr die persönliche Einstellung der einzelnen Apotheker gegenüber einer Versandapotheke. Die Apotheker wurden daher im Rahmen einer offenen Frage gebeten, die Assoziationen die sie mit dem Begriff "Versandapotheke" verbinden, wiederzugeben (s. Abbildung III - 5).

Cornelia Yzer, Hauptgeschäftsführerin des Verbandes Forschender Arzneimittelhersteller e.V., auf dem Symposium "E-Commerce mit Arzneimitteln" am 13. Dezember 2000 im Bundesministerium für Gesundheit in Bonn.

ABDA (2001), E-Commerce und Versandhandel mit Arzneimitteln aus Sicht der Apotheker, www.abda.de/ABDAartikel.html?|D=132, Zugriff am 27.04.2001. 
Abbildung III - 5: $\quad$ Assoziationen mit dem Begriff "Versandapotheke"

(Frage:" Was verbinden Sie mit dem Begriff Versandapotheke?")

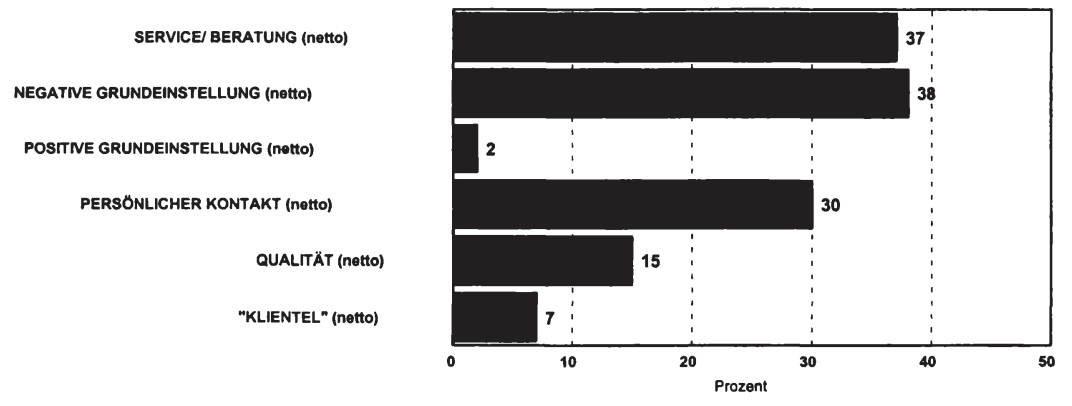

Basis: $n=150$ Apotheker

Quelle: INIFES, eigene Darstellung.

(Ergebnisse einer von TNS EMNID im Auftrag von INIFES im Dezember 2000 durchgeführten CATI-Befragung).

Die Haupteinwände der Apotheker gegenüber dem Modell einer Versandapotheke bezogen sich auf eine zu erwartende Verschlechterung des Services bzw. der Beratung (37,0 Prozent), den persönlichen Kontakt zum Kunden (30,0 Prozent) und die Qualität der Versorgung (15,0 Prozent).

Die negative Grundeinstellung ${ }^{60}$ der Apotheker (38,0 Prozent) wird signifikant von ihrer Berufserfahrung beeinflusst. Während 31,0 Prozent der Apotheker, die seit 1981 tätig sind, eine Versandapotheke ablehnen, beträgt der Wert bei denen, die schon vor 1981 als Apotheker arbeiteten, 47,0 Prozent. Die Gründe für diese negative Grundeinstellung sind vielschichtig. Ein Argument hierbei umfasst die Beratungsleistung der Apotheker. 15,0 Prozent der Apotheker sind der Meinung, dass eine Versandapotheke keine Beratung erbringen kann und 11,0 Prozent vertreten die Ansicht, dass die persönliche Beratung komplett entfallen wird.

Eine Kundenumfrage bei Kunden der schweizerischen Direktserviceapotheke MediService $^{61}$ hat allerdings ergeben, dass 98,6 Prozent der Ratsuchenden die Beantwortung ihrer Fragen als kompetent und ohne Zeitdruck beurteilten und mit dem Service zufrieden waren. Die Beratung bei dieser Versandapotheke scheint, zumindest aus Sicht der Kunden, qualitativ der einer Präsenzapotheke in nichts nachzustehen. Voraussetzung ist die Schaffung von bestimmten Strukturmerkmalen.

60

Hierunter subsumieren sich Aussagen wie: "finde ich nicht gut", "lehne ich ab", "Existenzbedrohend für Apotheken“.

Institut für Sozial- und Präventivmedizin der Universität Zürich; Schweizerische PatientenOrganisation (SPO); Schweizerische Patientendienststelle (DVSP) (1999), Zufriedenheit der Kunden der MediService Apotheke - Schlussbericht, Zürich. 
Nach Aussage eines von INIFES befragten Wissenschaftlers "muss der Kunde [einer Versandapotheke] die Möglichkeit erhalten, 24 Stunden mit der Apotheke in Kontakt zu treten. Von der Apotheke muss ein entsprechender Kontakt aufgebaut sowie ein Arzneimitteldossier angelegt werden. Voraussetzung ist dann jedoch, dass sich der Kunde an eine Versandapotheke bindet“. Mit welchen Modifizierungen zu rechnen ist, sofern mehrere Versandapotheken existieren und der Kunde sich nicht explizit an eine binden möchte, lässt sich zum gegenwärtigen Zeitpunkt schwer sagen, da diesbezügliche Untersuchungen nicht vorliegen.

Ein weiteren Grund für ihre negative Grundeinstellung sei, so die Apotheker, ein möglicher Verlust des persönlichen/sozialen Kontaktes zwischen Apotheker und Kunden. Aber gerade eine Anonymisierung der Beratung kann, so ein Ergebnis der Expertengespräche, von Kunden als positiv bewertet werden. "Arzneimittel, so ein Experte, wie beispielsweise Psychopharmaka werden ungern in der Apotheke abgeholt, wenn damit zu rechnen ist, dass evtl. auch andere Kunden in der Apotheke anwesend sind". Demgegenüber ist aber auch die Ansicht eines Vertreters der Apothekerschaft zu beachten, dass "der Bezug von Arzneimittel über eine Versandapotheke die Hemmschwelle des Kunden senken wird. Kann er bei einer Versandapotheke relativ anonym sein Rezept abgeben, muss er sich in der Präsenzapotheke möglicherweise den lästigen Nachfragen des Apothekers stellen. Für den Apotheker sei der persönliche Kontakt zum Kunden wichtig, um ihn zu einem verantwortungsbewussten Umgang mit den Arzneimitteln zu sensibilisieren".

Lediglich 2,0 Prozent der niedergelassenen Apotheker haben eine positive Grundeinstellung gegenüber dem Modell des Versandhandels. Besonders Apotheken mit einem Umsatz von mehr als DM 1,5 Mio. pro Jahr (2,0 Prozent) bietet ein solches Modell die Möglichkeit, das bereits bestehende Geschäftsfeld auszubauen. Für diese Apotheker stellt eine Versandapotheke eine Alternative dar. Nach Meinung der Befragten ist sie kostengünstiger und unter Beibehaltung der Preisbindung hätte eine solches Vertriebsmodell durchaus eine Chance.

Von den befragten Apothekern gaben 28,0 Prozent an, dass sie sich durchaus vorstellen könnten, beide Vertriebswege komplementär anzuwenden bzw. ihre Präsenzapotheke in eine Versandapotheke umzuwandeln. Diese Angabe wird von der Berufserfahrung des jeweiligen Apothekers, nicht aber von seinem Umsatz beeinflusst. Ein signifikanter Unterschied zwischen den Umsatzklassen "bis zu DM 1,5 Mio. pro Jahr" (31,0 Prozent) und "DM 1,5 Mio. pro Jahr und mehr" (26,0 Prozent) ist nicht zu erkennen. Die Annahme, dass vor allem umsatzstarke Apotheken an einer Umwandlung ihrer Präsenzapotheke in eine Versandapotheke interessiert sind, oder beide Vertriebsformen komplementär anbieten wollen, ist anhand der vorliegenden Daten nicht zu erkennen. Vielmehr würden die umsatzschwachen Apotheken ein solches Modell präferieren. Apotheker, die erst seit 1981 als Pharmazeut arbeiten, könnten sich zu 35,0 Prozent einen solchen Schritt vorstellen, Apotheker, die schon vor 1981 in ihrem Beruf tätig sind, nur zu 19,0 Prozent. Dies lässt erkennen, dass bei einigen 
Apothekern bereits ein Umdenkungsprozess bezüglich einer Öffnung gegenüber alternativer Vertriebsstrukturen begonnen hat.

Das Ergebnis der Apotheker widerlegt teilweise die Arbeitshypothese 6, die besagt, die Präsenzapotheke in eine Versandapotheke umzuwandeln bzw. beides komplementär zu betreiben, sei abhängig von der Anzahl bereits durchgeführter Heimlieferungen. Während 14,4 Prozent der Befragten, die bis zu 10 Heimlieferungen pro Woche durchführen, sich vorstellen können, ihre Präsenz- in eine Versandapotheke umzuwandeln, beträgt die Zustimmung bei denen, die bis zu 30 Heimlieferungen pro Woche vornehmen, 35,7 Prozent. Apotheker, die in der Woche mehr als 30 Heimlieferungen durchführen, können sich zu 19,0 Prozent eine Umwandlung der Präsenzin eine Versandapotheke vorstellen bzw. würden beide Vertriebswege komplementär anbieten.

Zusammenfassend ist eine insgesamt überwiegend negative/ablehnende Grundeinstellung der Apotheker bzgl. einer Versandapotheken zu erkennen. Während es in den USA, Südafrika und auch in einigen europäischen Ländern, wie Großbritannien, den Niederlanden, Dänemark und der Schweiz, bisher möglich ist, rezeptpflichtige Arzneimittel über eine Versandapotheke zu beziehen, ist nach Aussage des ABDAPräsidenten Hans-Günter Friese ${ }^{62}$ zur Aufrechterhaltung des "höchstmöglichen Sicherheitsstandards ein Versandhandelsverbot bei Arzneimitteln unverzichtbar". Doch wie sieht die tatsächliche Einstellung der Apotheker bzgl. einer Versandapotheke aus? Mehr als ein Drittel der Befragten hegen, ohne eine nähere Spezifizierung der Gründe, eine negative Grundeinstellung gegenüber einem Versandhandel. Hierzu zählen u.a. 24,0 Prozent, die eine Versandapotheke ablehnen bzw. die Einführung eines solchen Modells nicht wünschen. Weitere Haupteinwände gegenüber einem solchen Modell der Arzneimitteldistribution stellen eine potentielle Verschlechterung des Services bzw. der Beratung (37,0 Prozent), eine Verschlechterung der qualitativen Arzneimittelversorgung (15,0 Prozent), sowie eine Beeinträchtigung des Kundenkontaktes (30,0 Prozent) dar.

Ein Argument der Apotheker für die negative Grundeinstellung umfasst die Beratungsleistung der Apotheker. Während 11,0 Prozent der Apotheker angeben, dass eine persönliche Beratung von einer Versandapotheke nicht angeboten werden kann, belegt eine Kundenumfrage bei Kunden der schweizerischen Direktserviceapotheke MediService ${ }^{63}$, dass, zumindest aus Sicht der Kunden, die Beratung einer Versandapotheke der einer Präsenzapotheke in nichts nachsteht. 98,6 Prozent der befragten Kunden beurteilten die Beantwortung ihrer Fragen als kompetent und ohne Zeitdruck.

62

In seiner Rede anlässlich des Symposiums "E-Commerce mit Arzneimitteln" am 13. Dezember 2000 im Bundesministerium für Gesundheit in Bonn.

63

Institut für Sozial- und Präventivmedizin der Universităt Zürich; Schweizerische PatientenOrganisation (SPO); Schweizerische Patientendienststelle (DVSP) (1999), Zufriedenheit der Kunden der MediService Apotheke - Schlussbericht, Zürich. 
Trotz der ablehnenden Haltung der Apotheker hinsichtlich einer Legalisierung des Versandhandels kann sich ein Drittel der Befragten durchaus vorstellen, beide Vertriebswege komplementär anzubieten bzw. ihre Präsenzapotheke in eine Versandapotheke umzuwandeln; unabhängig vom derzeitigen Umsatz. Dies lässt erahnen, dass bei einigen Apothekern bereits ein Umdenkungsprozess bezüglich einer Öffnung gegenüber alternativer Vertriebsstrukturen begonnen hat.

\subsubsection{Zielgruppe einer Versandapotheke aus Sicht der Apotheker}

Die Beschaffung von Gütern und Dienstleistungen per Internet bietet den Konsumenten in Deutschland die Möglichkeit, rezeptpflichtige Medikamente über Apotheken außerhalb Deutschlands zu beziehen und somit die Vorteile des Netzes zu nutzen. "Viele Medikamente werden im Internet bis zu 60\% preiswerter angeboten" ${ }^{\text {"64 }}$, so die Arbeitsgemeinschaft der Verbraucherverbände e.V. Erfahrungswerte aus der Schweiz belegen, dass durch die effizientere Abgabe von Arzneimitteln, insbesondere durch eine vorteilhaftere Preisgestaltung durch größere Abgabemengen, Arzneimittel über den Versandweg um ca. 15,0 Prozent preiswerter angeboten werden können. ${ }^{65}$ Um jedoch mögliche Einsparpotentiale näher spezifizieren zu können, ist es zunächst notwendig zu ermitteln, für welche Zielgruppe der Bezug von Arzneimitteln über den Versandhandel überhaupt von Interesse und von Vorteil ist. Bislang wird die Annahme gehegt, dass ein Versandhandel vielfach für Personen von Vorteil ist, die in ihrer Mobilität eingeschränkt, chronisch erkrankt oder berufstätig sind. Nach Ansicht der Verbraucherverbände "sind beispielsweise beim Diabetikerbedarf und auch in anderen Bereichen enorme Preisspannen möglich".

Auf die Frage nach der Bewertung der Vorteilhaftigkeit einer Versandapotheke in bezug auf verschiedene Zielgruppen gaben 49,0 Prozent der Apotheker an, dass eine Versandapotheke für Patienten, die in ihrer Mobilität eingeschränkt sind, von Vorteil ist (s. Abbildung III - 6). Bislang werden diese Patienten, denen es aufgrund der eingeschränkten Mobilität nicht möglich ist, selber in die Apotheke zu gehen, im Rahmen der gesetzlichen Ausnahmeregelung des § 17, II Apothekenbetriebsordnung von den Apotheken beliefert. Wenn die Belieferung dieser Zielgruppe über einen Versand geregelt werden würde, könnte dies, so die Aussagen einiger Experten, mit großen Vorteilen verbunden sein. "Gesetzliche Vorgaben bzgl. der Anforderungen und der Qualitätsnormen eines solchen Versandweges würden eine gewisse Sicherheit generieren und den Betroffenen einen Nutzen stiften".

\footnotetext{
64 Arbeitsgemeinschaft der Verbraucherverbănde e.V. (2000), Medikamentenkauf im Internet, Pressemitteilung (65/00).

65 Hovermann, E. (2001), Versandhandel versus Einzelapotheke, in: Die BKK. Zeitschrift der Betrieblichen Krankenversicherung, Jg. 88, Heft 4, S. 178.

68

Ergebnis der von INIFES durchgeführten Expertengesprăche.
} 
Abbildung III - 6: Bewertung der Vorteilhaftigkeit einer Versandapotheke in bezug auf verschiedene Zielgruppen

(Frage: "Für welche Zielgruppe kann die Etablierung einer Versandapotheke eher von Vorteil, für wen eher von Nachteil sein?")

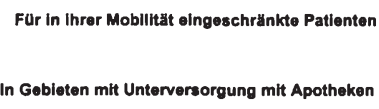

Als Service für Kunden, z.B. Berufstätige

Für Patienten mit chronischen Krankheiten

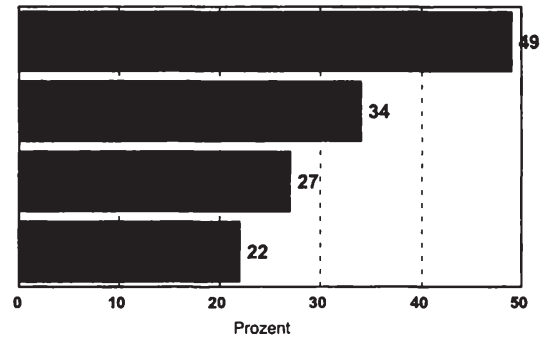

$n=150$ Apotheker

Top-2-Boxes ("von großem Vorteil" und "von Vorteil"); $1=$ von großem Vorteil; 5=von großem Nachteil

Quelle: INIFES, eigene Darstellung.

(Ergebnisse einer von TNS EMNID im Auftrag von INIFES im Dezember 2000 durchgeführten CATI-Befragung).

Die Ergebnisse der von INIFES geführten Experteninterviews haben gezeigt, dass eine Hauptzielgruppe für den Versandhandel Patienten mit chronischen Krankheiten sind. "Bei der Aushändigung ihrer Medikamente bedarf es kaum noch einer Beratung, da sie diese vielfach über Jahre hinweg einnehmen. Des weiteren haben diese Patienten im Regelfall eine gut planbare Medikation, so dass die logistischen Nachteile des Versandhandels, im Sinne von längeren Lieferzeiten, durchaus tolerabel sind".

Bei der Zielgruppe der Chroniker gilt jedoch zu bedenken, dass es auch Indikationen gibt, die einer kontinuierlichen Beratung und Überprüfung der Compliance der Betroffenen bedürfen. Nach Aussage eines Experten aus der Pharmabranche "nimmt, so zeigen Untersuchungen, die Compliance von Asthma-Patienten und Diabetikern im Laufe der Jahre extrem ab". Der Patient erscheint dann häufig nur noch in der Arztpraxis, um sich eine Wiederholungsverordnung ausstellen zu lassen. Als Beispiel nannte der Experte die Cortison-Angst bei Asthma-Patienten. Der Patient muss ständig zur Einnahme des Cortisons angewiesen werden. "Die derzeitige Betreuungskompetenz der Apotheker bedarf", nach Ansicht der Pharma-Industrie, "in diesen Bereichen eine deutliche Verbesserung". Für eine Versandapotheke, aber nicht nur für diese, müssen daher einheitliche Qualitätsstandards bzgl. der Beratung festgelegt werden. Nach Angaben eines Verbandmitgliedes der Apothekerschaft handelt es sich bei den Arzneimitteln für Chroniker um Produkte, die vielfach über der Grenze von DM 100,-- liegen. "Eine Versendung unterhalb dieser Grenze wäre ökonomisch

Aussage eines Experten im Rahmen der Expertengespräche. 
nicht sinnvoll". 22,0 Prozent der von INIFES befragten Apotheker vertreten die Ansicht, dass Patienten mit chronischen Krankheiten eine Zielgruppe für den Versandhandel bilden.

Obwohl aufgrund einer immer mobiler werdenden Gesellschaft viele Bürger häufig nicht mehr in der Lage sind, ein Rezept während der normalen Offnungszeit in einer Apotheke einzulösen, birgt nach Ansicht von nur 27,0 Prozent der Apotheker die Etablierung einer Versandapotheke für Berufstätige und als allgemeiner Service für Kunden Vorteile. Die Apotheker begründen, so das Ergebnis der Expertengespräche, diesen Wert damit, dass eine ordnungsgemäße Übergabe der Medikamente durch einen Zustelldienst nicht gewährleistet ist. Um dies zu umgehen, bieten bestehende Apotheken, die rezeptpflichtige Arzneimittel an Kunden versenden (z.B. MediService und DocMorris), den Service, sich die Medikamente beispielsweise auch an den Arbeitsplatz liefern zu lassen.

Die von den befragten Experten vielfach geäußerte Annahme, dass eine Hauptzielgruppe für den Versandhandel Menschen mit chronischen Krankheiten sind, wird von den Apothekern, so ein Ergebnis der Befragung, nicht geteilt. Die Hälfte der befragten Apotheker sehen vielmehr in Patienten, die in ihrer Mobilität eingeschränkt sind, eine Zielgruppe für eine Versandapotheke. Bislang werden diese Patienten, denen es aufgrund der eingeschränkten Mobilität nicht möglich ist, selber in die Apotheke zu gehen, im Rahmen der gesetzlichen Ausnahmeregelung des $\S 17$, II ApBetrO von den Apotheken beliefert. Der Ansicht, dass Patienten mit chronischen Erkrankungen eine Hauptzielgruppe für einen Versandhandel darstellen, schließt sich nur knapp ein Viertel der Befragten an. So wiesen Apotheker während der Expertengespräche darauf hin, dass einige Indikationsgruppen innerhalb der Chroniker einer kontinuierlichen und persönlichen Beratung bedürfen. Einer Versandapotheke wird diese Beratungskompetenz abgesprochen.

\subsubsection{Potentielle Auswirkungen eines Versandhandels}

Nach Ansicht eines Vertreters eines Apothekerverbandes “... würde eine Legalisierung des Versandhandels nur ein erster Schritt zu weitreichenden strukturellen Veränderungen im deutschen Apothekenwesen bedeuten". Im Rahmen der von INIFES durchgeführten Expertengespräche wurde vielfach darauf hingewiesen, dass "durch eine Offnung des bislang monopolartigen Vertriebs von apothekenpflichtigen (und damit alle rezeptpflichtigen) Präparate nur über Apotheken, in einem nächsten Schritt die Arzneimittelpreisverordnung sowie der Fremd- und Mehrbesitz zur Disposition stünden". Ob eine Wahrung des Besitzstandes allerdings weiterhin durch nationale gesetzliche Regelungen gesichert werden sollte, ist zu hinterfragen. Bei sonst identischen Rahmenbedingungen (Betriebserlaubnis, Beratungspflicht, Arzneimittelsicherheit, behördliche Kontrolle, etc.) dürfte sich sowohl die qualitative als auch die quantitative Arzneimittelversorgung der Bevölkerung durch eine Versandapotheke von der einer Präsenzapotheke unterscheiden. Um jedoch die konkreten Beweggründe der 
Apotheker zu eruieren, warum sie sich vielfach der Einführung einer Versandapotheke als komplementären Distributionsweg für Arzneimittel verschließen, wurden sie gebeten, anhand einer Skala von 1=volle Zustimmung bis 5=totale Ablehnung, in der Öfentlichkeit diskutierte Aussagen zur Versandapotheke zu bewerten (s. Abbildung III - 7).

\section{Abbildung III - 7: Bewertung verschiedener Aussagen zur Versandapotheke}

(Frage: “Bitte bewerten Sie folgende Aussagen auf einer Skala von 1 bis 5 “)

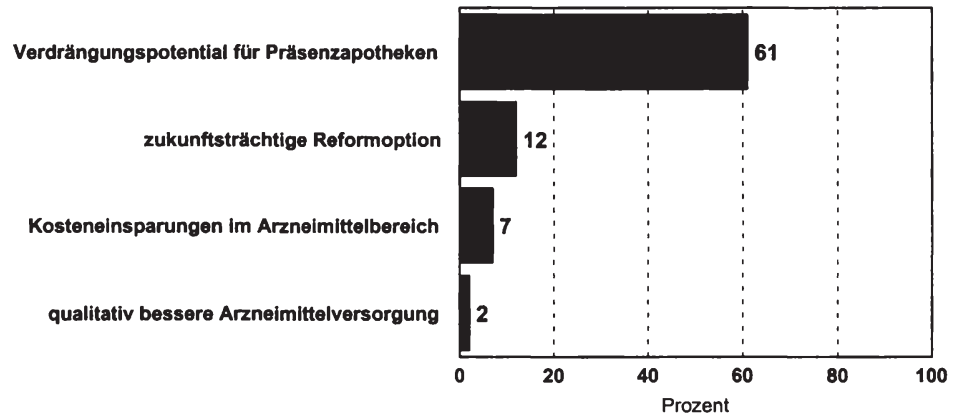

$n=150$ Apotheker

Top-2-Boxes ("volle Zustimmung", "Zustimmung"); 1=volle Zustimmung; 5=totale Ablehnung

Quelle: INIFES, eigene Darstellung.

(Ergebnisse einer von TNS EMNID im Auftrag von INIFES im Dezember 2000 durchgeführten CATI-Befragung).

Der Aussage, dass Versandapotheken zu einer qualitativ besseren Arzneimittelversorgung führen werden, stimmen lediglich 2,0 Prozent der Apotheker zu, 77,0 Prozent lehnen die Aussage total ab. Die Apotheker begründen ihre Ablehnung damit, dass die meisten Menschen eine Stammapotheke haben ${ }^{68}$, in der sie die Beratung und den sozialen Kontakt schätzen. In Verbindung mit der Apotheken-Card kann den Stammkunden, sofern gewünscht, eine umfassende Beratung angeboten werden. Auf dieser Karte werden beispielsweise alle vom Kunden gekauften Präparate (rezeptpflichtige und OTC) gespeichert, so dass Kontraindikationen sofort angezeigt werden können und eine Medikamentenhistorie für jeden Kunden vorliegt. Die Vorteile, die man vielfach einer Versandapotheke zuspricht - bessere Betreuungs-, Beratungs- und Kontrollpflicht - kann eine Präsenzapotheke durch einen gezielten Einsatz der Kundenkarte durchaus auch dem Kunden bieten. Voraussetzung ist jedoch die Definition und Anwendung einheitlicher Qualitätsstandards für den Bereich der Bera- 
tung. Wenn dies konkret umgesetzt wird, werden Versandapotheken nicht unbedingt zu einer qualitativ besseren Arzneimittelversorgung führen.

Der häufig von Vertretern der Krankenkassen geäußerten Aussage, dass Versandapotheken zu Kosteneinsparungen im Arzneimittelbereich führen werden, stimmen 7,0 Prozent der Apotheker zu. Ein signifikanter Unterschied im Antwortverhalten besteht hierbei im Alter der befragten Apotheker. Während Apotheker, die nicht älter als 45 Jahre sind, davon ausgehen, dass Versandapotheken zur Reduzierung der Leistungsausgaben im Arzneimittelbereich beitragen könnten, trauen ihr dies nur 3,0 Prozent der Apotheker zu, die älter als 45 Jahre sind.

Ein generelles Verdrängungspotential für Präsenzapotheken stellt eine Versandapotheke für 61,0 Prozent der Apotheker dar. Diese Aussage wird sowohl vom Alter der Befragten (53,0 Prozent der unter 45jährigen und 70,0 Prozent der über 45jährigen) als auch vom Umsatz der Apotheke beeinflusst. Vor allem Apotheken mit einem Jahresumsatz von bis zu 1,5 Mio. DM sehen zu 75,0 Prozent eine starke Konkurrenz und Existenzbedrohung in einer Versandapotheke. Dem schließen sich aber nur 60,0 Prozent der Apotheken mit einem Jahresumsatz von 1,5 Mio. DM und mehr an. Es handelt sich hierbei vielfach um die Apotheken, die bereits jetzt schon intensiv Patienten nach Hause beliefern. Eine Zulassung des Versandhandels würde es innen dann ermöglichen, die bereits bestehenden Heimlieferungen zu intensivieren und auszuweiten. Sowohl die Bundesapothekerkammer ${ }^{69}$ als auch die ABDA $^{70}$ machen die Bewertung der Aussage, dass Versandapotheken generell ein Verdrängungspotential für Präsenzapotheken darstellen, u.a. von der Ausgestaltung des Umfelds und der Konditionen abhängig. Solange, so die Vertreter beider Organisationen, der Patient ohne Anreizmechanismen der Krankenkassen oder des Arztes eigenverantwortlich entscheiden dürfe, ob er das Rezept bei einer Präsenz- oder Versandapotheke einlöst, stellten Versandapotheken kein Verdrängungspotential dar. Ohne dies, so der Vertreter der Bundesapothekerkammer, "werden Versandapotheken für lediglich zehn Prozent der Warenbewegung verantwortlich sein. Sollten jedoch die Krankenkassen steuernd und lenkend in das System eingreifen, so hätte der Apothekenmarkt mit tiefgreifenden Veränderungen zu rechnen“.

Der Aussage, dass die Einführung des Modells einer Versandapotheke eine zukunftsträchtige Reformoption darstellt, stimmen 12,0 Prozent der befragten Apotheker zu. Ein signifikanter Unterschied im Antwortverhalten ist auch bei dieser Aussage bzgl. des Alters festzustellen (bis 45 Jahre $=17,0$ Prozent, über 45 Jahre $=7,0$ Prozent). Unter Berücksichtigung der bisher gegebenen Bewertungen und Aussagen zu der Thematik des Versandhandels wird ersichtlich, dass sich vor allem die jungen Apotheker viel offener und möglicherweise auch unvoreingenommener mit dem komplementären Distributionsweg auseinandersetzen. Sie sehen in ihm eine Chance, den Problemen des Gesundheitswesens, vor dem Hintergrund wachsender Arz-

Anlässlich eines Expertengespräches.

Anlässlich eines Expertengespräches. 
neimittelausgaben, entgegenzutreten. Die Versandapotheke allein wird nicht zu Kosteneinsparungen führen können, aber sie kann ein Baustein in einer sich ändernden Struktur sein.

Zusammenfassend bleibt zu dem Aspekt der potentiellen Auswirkungen eines Versandhandels festzuhalten, dass viele Apotheker in einer Versandapotheke ein Verdrängungspotential für Präsenzapotheken sehen. Dem stimmen 61,0 Prozent der befragten Apotheker zu. Dieser Wert steigt auf 75,0 Prozent bei denen, die einen Jahresumsatz von bis zu 1,5 Mio. DM erzielen. Sie sehen sich vor allem in ihrer Existenz bedroht.

Die Arbeitshypothese, dass Versandapotheken zu Kosteneinsparungen im Arzneimittelbereich führen werden, ist nach Meinung der Apotheker nicht aufrecht zu erhalten. Lediglich 7,0 Prozent der Befragten gehen davon aus, dass es zu Einsparungen kommen wird. So wiesen Apotheker während der Expertengespräche darauf hin, dass "eine Versandapotheke nur in einem Hochpreis-Segment Kosteneinsparungen realisieren kann. Die Vorhaltung eines Vollsortimentes würde hingegen zu einem Anstieg der Kosten für Arzneimittel führen".

In der Bewertung der Aussagen hinsichtlich Chancen und Risiken gegenüber dem Versandhandel ist ein signifikanter Unterschied in der Altersverteilung zu erkennen. Während die jungen Apotheker einem komplementären Distributionsweg durchaus Chancen einräumen, ist eine Versandapotheke bei den älteren Apothekern überwiegend mit Risiken behaftet. Dies legt die Vermutung nahe, dass sich vor allem die jüngeren Apotheker intensiv mit der Thematik eines Versandhandels auseinander setzen und in ihm evtl. ein potentielles Geschäftsfeld entdecken.

\subsubsection{Wirtschaftliche Folgen einer Legalisierung von Versandapotheken}

Wie bereits dargestellt, besteht nach Ansicht der Apotheker die Gefahr eines Verdrängungswettbewerbes bei einer Legalisierung des Versandhandels. Dies äußert sich vor allem um die Sorge des eigenen Besitzstandes. Während dieser bislang durch gesetzliche Rahmenbedingungen gesichert wurde, gaben 58,0 Prozent der befragten Apotheker an, dass eine Einführung der Arzneimitteldistribution über Versandapotheken den Umsatz der eigenen Apotheke negativ beeinflussen würde (s. Abbildung III - 8). 30,0 Prozent der Apotheker rechnen mit keiner Umsatzbeeinflussung durch eine Versandapotheke und 7,0 Prozent erwarten eine Umsatzsteigerung. 
Abbildung III - 8: Persönliche Umsatzbeeinflussung durch Versandapotheke

(Frage: "Wie, glauben Sie, würde die Einführung der Arzneimitteldistribution über Versandapotheken den Umsatz Ihrer eigenen Apotheke beeinflussen?")

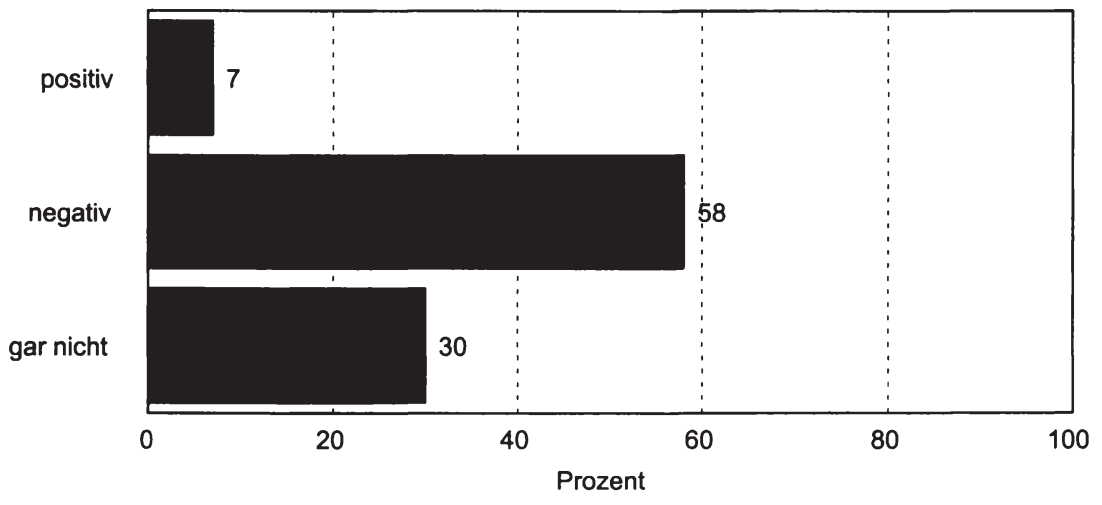

$\mathrm{n}=150$ Apotheker

Quelle : INIFES, eigene Darstellung.

(Ergebnisse einer von TNS EMNID im Auftrag von INIFES im Dezember 2000 durchgeführten CATI-Befragung).

Die Sorge um einen Rückgang des eigenen Umsatzes ist verständlich. Denn die Einführung der Arzneimitteldistribution über Versandapotheken induziert nicht zwangsläufig einen höheren Arzneimittelkonsum der Bevölkerung. Folglich verdrängt jeder neue Player am Markt ein Stück weit alle bisher Agierenden. Das ist aber kein Prädikat einer Versandapotheke, sondern trifft auf jede neue Apotheke ebenso zu. Die vorliegenden Ergebnisse lassen erkennen, dass die Einschätzung der persönlichen Umsatzbeeinflussung nicht vom bestehenden Umsatz der Apotheke abhängig ist. Ein signifikanter Unterschied besteht bei den Apothekern, für die ein Versandhandel hinsichtlich der Personalstärke durchaus positive Umsatzsteigerungen zur Folge hätte. Während nur 2,0 Prozent der Apotheker mit maximal zwei Fachkräften von einer positiven Umsatzsteigerung für die eigene Apotheke ausgehen, gehen immerhin 12,0 Prozent der Apotheker mit mehr als fünf beschäftigten Fachkräften von einer Umsatzsteigerung aus. Da von der Anzahl des in einer Apotheke beschäftigten Pharmazeutischen Personals Rückschlüsse auf den Umsatz einer Apotheke gezogen werden können, ist davon auszugehen, dass vor allem umsatzstarke Apotheken von der Arzneimitteldistribution über Versandapotheken profitieren werden.

Apotheker, die für ihre Apotheke mit Umsatzeinbußen durch eine Zulassung von Versandapotheken rechnen, gehen von unterschiedlichen Konsequenzen aus. Die Apotheke schließen würden 6,0 Prozent der Apotheker, Personal entlassen müssten 69,0 Prozent der Apotheken und 16,0 Prozent rechnen mit keinen Konsequenzen für 
inre Apotheke. Signifikante Unterschiede, hinsichtlich der erwarteten Konsequenzen innerhalb der BIK-Ortsgrößen sind nicht zu erkennen.

Das vorliegende Ergebnis der Apothekerbefragung widerlegt zumindest nicht die Arbeitshypothese 2, nach der eine Ausweitung des Versandhandels mit negativen wirtschaftlichen Auswirkungen für die Präsenzapotheken verbunden sein wird. Die erhobenen Daten lassen jedoch keine konkreten Aussagen darüber zu, ob ein Zusammenhang zwischen Art und Umfang der zu erwartenden Auswirkungen und des Umsatzes der Apotheke existiert.

Von einer grundsätzlichen Gefährdung des umfassenden Versorgungs- und Sicherstellungsauftrages der Apotheken durch Ausweitung des Pharmaversandes, wie in der Arbeitshypothese 3 aufgestellt, kann anhand des Befragungsergebnisses nicht gesprochen werden. Konsequenzen der negativen Umsatzbeeinflussung, zu denen u.a. eine mögliche Schließung von Apotheken zählt, werden von den Apothekern, unabhängig von der BIK-Ortsgröße, gesehen.

\subsubsection{Reformoptionen}

Bei der Suche nach potentiellen, bisher ungenutzten Wirtschaftlichkeitsreserven sowie effizienteren Versorgungsstrukturen in der medizinischen Versorgung, sehen auch die Apotheker durchaus eine Chance, sich aktiv zu beteiligen. Gesetzliche Ånderungen vorausgesetzt, können sich 36,0 Prozent der befragten Apotheker durchaus vorstellen, dass der verordnende Arzt zukünftig das Rezept nach Einwilligung des Patienten per Datenfernübertragung an eine Versandapotheke schickt. Diese stellt dann die Bestellung zusammen und liefert sie an den Patienten aus. Der Patient erscheint nicht mehr persönlich in der Apotheke. In Verbindung mit dem Elektronischen Rezept ließe ein solches Modell Kosteneinsparungen bei den Distributionskosten von Arzneimitteln im Umfang von maximal DM 500 Mio. pro Jahr zu. ${ }^{71}$ Demgegenüber stehen 62,0 Prozent der Apotheker, die ein solches Modell für nicht vorstellbar halten. Für sie ist der persönliche Kontakt im Rahmen einer face-to-faceBeratung unabdingbare Voraussetzung für eine qualitativ hochwertige Arzneimittelversorgung der Bevölkerung.

Eine andere Reformoption stellt folgendes Modell dar: Der Patient erhält das Rezept vom verordnenden Arzt wie bisher ausgehändigt und schickt dieses per Brief an die Versandapotheke. Diesem Modell stimmen 39,0 Prozent der Apotheke zu, 61,0 Prozent lehnen es ab. Die Ablehnung resultiert aus dem fehlenden persönlichen Kontakt, den Sicherheitsrisiken eines solchen Verfahrens sowie der Einschätzung der Apotheker, dass viele Patienten überfordert sein könnten. Nach Ansicht der Apotheker umfassen zukunftsträchtige Reformoptionen vornehmlich die Bereiche: Bestellung und Distribution (s. Abbildung III - 9).

71

Schubert, F. (1999), Das Elektronische Rezept: Chancen, Risiken und Gestaltungsmöglichkeiten, Diplomarbeit der Fachhochschule Heilbronn, S. 9. 
Abbildung III - 9: $\quad$ Sonstige vorstellbare Reformoptionen

(Frage: "Welche anderen Reformoptionen können Sie sich vorstellen?")

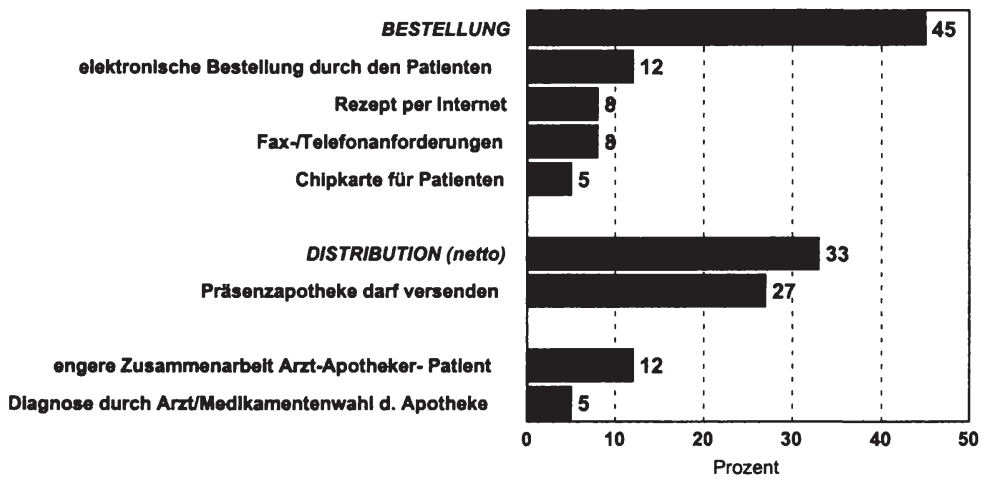

Selektion: Apotheker, die sich noch weitere Reformoptionen vorstellen können ( $n=61)$

Quelle: INIFES, eigene Darstellung.

(Ergebnisse einer von TNS EMNID im Auftrag von INIFES im Dezember 2000 durchgeführten CATI-Befragung).

Im Rahmen einer offenen Frage (Welche anderen Reformoptionen können Sie sich vorstellen?) wurden die Befragten gebeten, weitere Optionen zu nennen. Den größten Reformbedarf sehen die Apotheker im Bereich "Bestellung" (45,0 Prozent). Dieser Bereich setzt sich zusammen aus: elektronische Bestellung durch den Patienten (12,0 Prozent), Rezeptweiterleitung per Internet (8,0 Prozent), Bestellung per Fax oder Telefon (8,0 Prozent) und der Smart-Card (5,0 Prozent). Hierbei handelt es sich um Elemente, die ab Juli 2001 das ABDA-Portal bilden. Dem Patienten wird es dann möglich sein, online (über das Internet) seine Medikamente bei der Apotheke seiner Wahl vorzubestellen. Gegen Vorlage der ärztlichen Verordnung bekommt er dann die Medikamente in der Apotheke ausgehändigt. Die Apotheken wollen auf diese Weise die Vorteile des Internets als Kommunikations- und Informationsplattform nutzen, ohne dabei den persönlichen Kontakt zum Kunden zu verlieren.

Weitere Reformoptionen beziehen sich auf die Distribution von Arzneimitteln (33,0 Prozent). 27,0 Prozent der befragten Apotheker würden es begrüßen, generell bzw. unter bestimmten Voraussetzungen aus der Präsenzapotheke heraus versenden zu dürfen.

Dies trifft vor allem auf Apotheken mit einem Umsatz von mehr als 1,5 Mio. DM (33,0 Prozent) und mit mehr als fünf pharmazeutischen Fachkräften (45,0 Prozent) zu. 


\subsubsection{Fazit}

Aufgrund aktueller Pressemitteilungen ${ }^{72}$ und des in der Öffentlichkeit vielfach synonymen Gebrauchs der Begriffe Versandapotheke und Internetversand zeigte sich im Rahmen der Apotheker-Befragung oftmals eine abwehrende Haltung gegenüber der Durchführung der Telefoninterviews. Die befragten Apotheker sehen zwar den Vorteil des Versandhandels für bestimmte Zielgruppen, z.B. immobile und multimorbide Personen. Sie machen jedoch darauf aufmerksam, dass eine Legalisierung des Versandhandels mit unwägbaren Risiken für den Konsumenten verbunden sein kann. Nach ihrer Meinung würde es sowohl zu einer Verschlechterung der Beratung als auch zu einer Abnahme der Arzneimittelsicherheit kommen. Den Verlust des persönlichen und sozialen Kontaktes zwischen Apotheker und Kunden befürchten 27,0 Prozent. Die befragten Apotheker vertreten zudem die Meinung, dass Versandapotheken weder zu einer qualitativ besseren Arzneimittelversorgung noch zu Kosteneinsparungen im Arzneimittelbereich führen werden. Vielmehr sehen sie in einer Versandapotheke ein mögliches Verdrängungspotential für Präsenzapotheken. Unabhängig von der BlK-Örtsgröße ist die Einführung der Arzneimitteldistribution über Versandapotheken, so das Ergebnis der Befragung, mit einer negativen Umsatzbeeinflussung für die Apotheker verbunden. Als Konsequenz gaben mehr als die Hälfte der Apotheker an, Personal entlassen zu müssen.

\subsection{3. Ärztebefragung}

\subsubsection{Angaben zur Stichprobe}

Die Gruppe der befragten Ärzte setzte sich insgesamt aus 72,0 Prozent Männer und 28,0 Prozent Frauen zusammen. 12,0 Prozent sind nicht älter als 39 Jahre $(11,0$ Prozent Männer, 16,0 Prozent Frauen), 39,0 Prozent sind zwischen 40 und 49 Jahre (35,0 Prozent Männer, 48,0 Prozent Frauen), 37,0 Prozent zwischen 50 und 59 Jahre (41,0 Prozent Männer, 28,0 Prozent Frauen) und 13,0 Prozent gaben an, älter als 60 Jahre zu sein. In BIK-Ortsgrößen bis 5.000 Einwohner befinden sich 6,0 Prozent der Arztpraxen, 13,0 Prozent in BIK-Ortsgrößen bis 20.000 Einwohner, 18,0 Prozent in Gebieten bis 100.000 Einwohner, 16,0 Prozent der Praxen haben sich in Ortsgrößen bis 500.000 Einwohner niedergelassen und in BIK-Ortsgrößen mit 500.000 und mehr Einwohnern befinden sich 47,0 Prozent der Arztpraxen.

Tabelle III - 4 gibt eine Übersicht über die soziodemographischen Angaben der Ärzte.

Z.B.: Arzneien - ein Handel mit Problemen, in: Rheinischer Merkur vom 1.10.2000, S. 34; Internet-Apotheken locken Patienten mit Billig-Angeboten, in: Der Spiegel vom 25.09.2000, S. 88. 
Tabelle III - 4: Sozio-Demographie der Ärzte $(n=380)$

\begin{tabular}{|c|c|c|c|c|c|c|}
\hline & & & Prozent & & & \\
\hline & $\begin{array}{c}\text { Insge- } \\
\text { samt } \\
(n=380)\end{array}$ & $\begin{array}{l}\text { Praktischer Arzt } \\
/ \text { Algemein- } \\
\text { mediziner } / \text { In- } \\
\text { ternist }(n=100) \\
\end{array}$ & $\begin{array}{c}\text { Gynăkologe } \\
(n=70)\end{array}$ & $\begin{array}{c}\text { Neurologe } / \\
\text { Nervenarzt } \\
(n=70)\end{array}$ & $\begin{array}{l}\text { Urologe } \\
(n=70)\end{array}$ & $\begin{array}{c}\text { HNO- } \\
\text { Arzt } \\
(n=70)\end{array}$ \\
\hline \begin{tabular}{|l|} 
Geschlecht \\
Männer \\
Frauen
\end{tabular} & $\begin{array}{l}72 \\
28\end{array}$ & $\begin{array}{l}24 \\
32\end{array}$ & $\begin{array}{l}16 \\
25\end{array}$ & $\begin{array}{l}17 \\
22\end{array}$ & $\begin{array}{c}24 \\
3\end{array}$ & $\begin{array}{l}19 \\
17\end{array}$ \\
\hline \begin{tabular}{|l} 
Alter \\
-39 Jahre \\
$40-49$ Jahre \\
$50-59$ Jahre \\
$60+$ Jahre \\
\end{tabular} & $\begin{array}{l}12 \\
39 \\
37 \\
13\end{array}$ & $\begin{array}{l}13 \\
39 \\
36 \\
13\end{array}$ & $\begin{array}{l}13 \\
37 \\
39 \\
11\end{array}$ & $\begin{array}{c}9 \\
36 \\
39 \\
16\end{array}$ & $\begin{array}{c}15 \\
37 \\
39 \\
9\end{array}$ & $\begin{array}{l}11 \\
44 \\
32 \\
13\end{array}$ \\
\hline 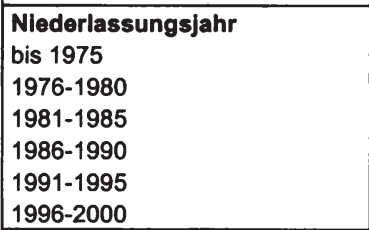 & $\begin{array}{l}10 \\
10 \\
11 \\
14 \\
38 \\
18\end{array}$ & $\begin{array}{c}6 \\
13 \\
15 \\
16 \\
29 \\
19\end{array}$ & $\begin{array}{c}9 \\
13 \\
8 \\
12 \\
39 \\
20\end{array}$ & $\begin{array}{c}10 \\
12 \\
7 \\
7 \\
51 \\
12\end{array}$ & $\begin{array}{c}8 \\
10 \\
13 \\
17 \\
37 \\
15\end{array}$ & $\begin{array}{c}17 \\
1 \\
10 \\
14 \\
36 \\
21\end{array}$ \\
\hline \begin{tabular}{|l} 
Patienten pro Quartal \\
bis unter 150 Patienten \\
150 bis unter 600 Patienten \\
600 bis unter 750 Patienten \\
750 bis unter 900 Patienten \\
900 bis unter 1050 Patienten \\
1050 bis unter 1200 Patienten \\
1200 bis unter 1350 Patienten \\
1350 bis unter 1500 Patienten \\
1500 und mehr Patienten \\
keine Angabe
\end{tabular} & $\begin{array}{c}5 \\
9 \\
6 \\
12 \\
14 \\
11 \\
8 \\
7 \\
20 \\
8\end{array}$ & $\begin{array}{c}4 \\
8 \\
12 \\
14 \\
16 \\
12 \\
6 \\
5 \\
14 \\
9\end{array}$ & $\begin{array}{c}- \\
7 \\
3 \\
16 \\
12 \\
10 \\
8 \\
16 \\
23 \\
6\end{array}$ & $\begin{array}{c}25 \\
26 \\
8 \\
16 \\
6 \\
3 \\
6 \\
1 \\
3 \\
7\end{array}$ & $\begin{array}{c}- \\
3 \\
3 \\
8 \\
24 \\
17 \\
13 \\
9 \\
19 \\
4\end{array}$ & $\begin{array}{c}- \\
- \\
3 \\
7 \\
12 \\
11 \\
7 \\
5 \\
43 \\
12\end{array}$ \\
\hline $\begin{array}{l}\text { Patientenklientel (Durchschn.) } \\
\text { Akut-Kranke } \\
\text { Chronisch-Kranke } \\
\text { zur Vorsorge } \\
\end{array}$ & $\begin{array}{l}38,2 \\
44,0 \\
17,7\end{array}$ & $\begin{array}{c}34,8 \\
55,5 \\
9,8\end{array}$ & $\begin{array}{l}25,1 \\
17,5 \\
57,5\end{array}$ & $\begin{array}{c}44,2 \\
50,7 \\
5,0 \\
\end{array}$ & $\begin{array}{l}30,9 \\
52,3 \\
16,9 \\
\end{array}$ & $\begin{array}{c}56,4 \\
37,3 \\
6,1 \\
\end{array}$ \\
\hline \begin{tabular}{|l|} 
BIK-OrtsgröBe \\
- u. 5.000 \\
$5.000-$ u. 20.000 \\
$20.000-$ u. 100.000 \\
$100.000-$ u. 500.000 \\
500.000 und mehr \\
\end{tabular} & $\begin{array}{c}6 \\
13 \\
18 \\
16 \\
47\end{array}$ & $\begin{array}{l}12 \\
13 \\
19 \\
17 \\
39\end{array}$ & $\begin{array}{c}5 \\
18 \\
14 \\
15 \\
48 \\
\end{array}$ & $\begin{array}{c}1 \\
7 \\
23 \\
9 \\
60\end{array}$ & $\begin{array}{c}3 \\
8 \\
19 \\
26 \\
44\end{array}$ & $\begin{array}{c}5 \\
20 \\
16 \\
10 \\
50\end{array}$ \\
\hline
\end{tabular}

Quelle: INIFES, eigene Darstellung.

(Ergebnisse einer von TNS EMNID im Auftrag von INIFES im Dezember 2000 durchgeführte CATI-Befragung). 


\subsubsection{Die Einstellung der Ärzte zum Vertriebskanal Apotheke}

Das Verfahren der Arzneimittelversorgung über Rezepte umfasst die Auswahl der Medikation durch den Arzt ("Verordnung"), die Überbringung des Arzneiverordnungsblattes ("Rezept") in die Apotheke, die Dispensierung in der Apotheke und die Abrechnung mit den Krankenkassen. Nach Aussagen der befragten Ärzte sorgt eine überdurchschnittliche Infrastruktur für eine optimale Arzneimittelversorgung der Bevölkerung. Zu dieser Infrastruktur ist vor allem die jeweilige Apothekendichte (Erreichbarkeit) im Umfeld der Praxis zu sehen. Als gerade angemessen beurteilen 59,0 Prozent der Ärzte die Apothekendichte im Einzugsgebiet ihrer Praxis, 32,0 Prozent sprechen bereits von einer Überversorgung und lediglich 3,0 Prozent geben an, dass im Einzugsgebiet ihrer Praxis eine Unterversorgung existiert.

Wie bereits bei der Darstellung der Ergebnisse der Apothekerbefragung ausgeführt ${ }^{73}$, unterliegt der Vertriebsweg ( $\$ 43$ Arzneimittelgesetz) und die Abgabe von apothekenpflichtigen Medikamenten ( $\S 17$ ApBetrO) gesetzlichen Bestimmungen. Lediglich der $\S 17$, II Apothekenbetriebsordnung erlaubt in "begründeten Einzelfällen" eine Versendung aus der Apotheke an den Arzneimittelempfänger. Ohne die folgenden Zahlen näher kommentieren zu wollen, übernehmen, gesetzliche Bestimmungen auBer Acht gelassen, 41,0 Prozent der befragten Ärzte für Patienten bei entsprechender Bedürfnislage die Rezeptweiterleitung an die Apotheke (s. Abbildung III - 10).

Abbildung III - 10: Direkte Rezeptweiterleitung an die Apotheke

(Frage: “Übernehmen Sie, gesetzliche Bestimmungen außer Acht gelassen, für Patienten bei entsprechender Bedürfnislage die Rezeptweiterleitung an die Apotheke?")

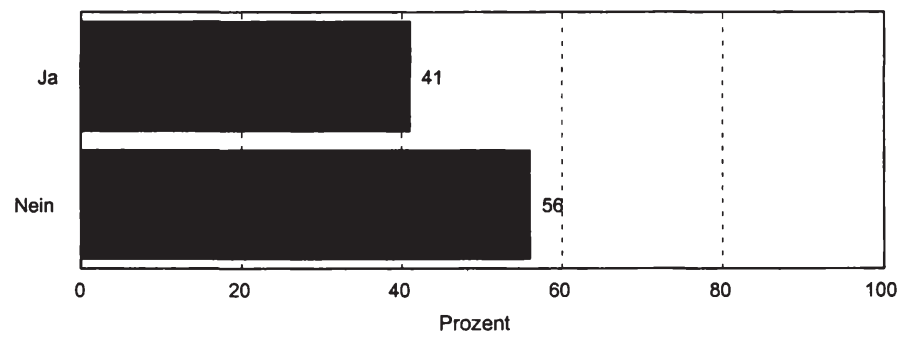

$n=380$ Arzte

Quelle: INIFES, eigene Darstellung.

(Ergebnisse einer von TNS EMNID im Auftrag von INIFES im Dezember 2000 durchgeführten CATI-Befragung). 
Signifikante Unterschiede im Antwortverhalten bestehen zwischen den untersuchten Facharztgruppen. ${ }^{74}$ Während 57,0 Prozent der Ärzte der Facharztgruppe Praktischer Arzt/Allgemeinmediziner/Internist eine direkte Rezeptweiterleitung an die Apotheker anbieten, sind es nur 27,0 Prozent bei den Hals-Nasen-Ohrenärzten. Untersucht man das Patientenklientel beider Facharztgruppen, ist der unterschiedlich hohe Anteil der chronisch Kranken auffällig. Dieser beträgt bei den Hals-Nasen-Ohrenärzten 37,3 Prozent und bei den Praktischen Ärzten/Allgemeinmedizinern/Internisten 55,5 Prozent. Eine Korrelation zwischen der Rezeptweiterleitung und den Variablen "Facharztgruppe" und "Chroniker" ist rechnerisch nicht nachweisbar.

Wie zuvor die Apotheker wurden auch die Ärzte gebeten, anhand einer Skala von $1=$ sehr wichtig bis 5=völlig unwichtig, die Kriterien zu bewerten, die ihrer Meinung nach ihre Patienten bei der Auswahl der Apotheke zu Grunde legen (s. Abbildung III 11).

Abbildung III - 11: Grad der Wichtigkeit verschiedener Kriterien bei der Auswahl der Apotheke

(Frage: "Welche Kriterien legen Sie bei der Auswahl der Apotheke zugrunde? Wie wichtig sind Ihnen folgende Aspekte?")

Schnelle und gute Erreichbarkeit

Umfassende Beratung

Kostenloser Heimlieferservice von Medikamenten

Kunden gehören zur Stammkundschaft

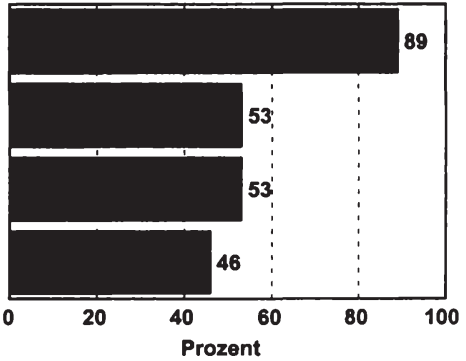

$n=380$ Arzte

Top-2-Boxes ("sehr wichtig" und "wichtig"); 1: sehr wichtig, 5: vollig unwichtig

Quelle: INIFES, eigene Darstellung.

(Ergebnisse einer von TNS EMNID im Auftrag von INIFES im Dezember 2000 durchgeführten CATI-Befragung).

Nach Ansicht der Ärzte messen ihre Patienten bei der Auswahl der Apotheke, in der das Rezept eingelöst wird, besonders den Kriterien einer schnellen und guten $\mathrm{Er}$ reichbarkeit (89,0 Prozent), einer umfassenden Beratung (53,0 Prozent) sowie dem kostenlosen Heimlieferservice von Medikamenten (53,0 Prozent) eine große Bedeutung bei. Das Auswahlkriterium, dass die Patienten Stammkunden einer Apotheke

74

Signifikanzniveau 10 Prozent. 
sind, erachten nur 46,0 Prozent als wichtig. Laut Gesetz besteht sowohl für die Ärzte als auch für die Apotheker eine Beratungspflicht, egal, ob es sich um verschreibungspflichtige oder nicht verschreibungspflichtige Arzneimittel handelt. Allerdings darf die Beratung des Apothekers nicht die Therapie des verordnenden Arztes beeinträchtigen; eine Kritik an der ärztlichen Diagnose oder der Verordnung ist inm untersagt. Daher ist es nicht verwunderlich, dass nur 53,0 Prozent der Ärzte glauben, dass eine umfassende Beratung ausschlaggebend für die Wahl der Apotheke ist. Besonders die Facharztgruppe der Neurologen und Nervenärzte bewertet dieses Kriterium mit nur 45,0 Prozent. Dies mag u.a. an dem Anteil chronisch Kranker an deren Patientenklientel (50,7 Prozent) und daraus folgernd, einem geringeren Beratungsbedarf/Beratungsanspruch durch den Apotheker liegen. Die Beratung erfolgt bei diesen Patienten bereits in der Arztpraxis.

Es bleibt festzuhalten, dass ein überwiegender Teil der befragten Ärzte für Patienten bei entsprechender Bedürfnislage die Rezeptweiterleitung an die Apotheke übernimmt. Vor allem Facharztgruppen mit einem hohen Anteil an zu versorgenden Chronikern bieten diesen "Service" an. Eine Korrelation zwischen der "Weiterleitung" und den Variablen Facharztgruppe und Anteil an chronisch Kranken ist nicht zu ermitteln. Dennoch legt das Vorgehen der Ärzte nahe und bestätigt die Arbeitshypothese 4, dass aufgrund des Volumens bereits durchgeführter Heimlieferungen nicht mehr von einem begründeten Einzelfall gesprochen werden kann. Die Heimlieferungen nehmen vielfach schon die Form des Versandhandels an.

Nach Aussagen der Ärzte legen die Konsumenten bei der Wahl der Apotheke besonders Wert auf eine schnelle und gute Erreichbarkeit. Einer umfassenden Beratung sowie einem kostenlosen Heimlieferservice von Medikamenten messen sie, so das Ergebnis der Befragung, ebenso eine große Bedeutung bei.

\subsubsection{Alternative Distributionswege}

Der bislang streng reglementierte Arzneimittelvertrieb steht seit geraumer Zeit zur Disposition. Dies bedeutet nicht die Aufgabe eines bewährten Systems, sondern die Zulassung komplementärer Vertriebsformen aufgrund einer Globalisierung der Märkte, einhergehend mit der Entwicklung neuer Zielgruppen und Bedürfnisse der Bevölkerung. In der zur Zeit geführten Diskussion bzgl. neuer Vertriebsformen agieren die Bundesärztekammer und die Kassenärztliche Bundesvereinigung eher zurückhaltend. Um jedoch die generelle Einstellung der Ärzte zu verschiedenen Distributionswegen von Medikamenten für die Zukunft zu erfragen, wurden sie gebeten, anhand einer Skala von $1=$ wesentlich bessere Marktchancen bis $5=$ wesentlich schlechtere Marktchancen, die potentiellen Marktchancen der Distributionswege: Versandapotheke, Apothekenkette, Arzneimittelversand und Internetapotheke zu bewerten (s. Abbildung III - 12). 
Abbildung III - 12: Bewertung verschiedener Distributionswege von Medikamenten für die Zukunft

(Frage: "Welchen Distributionswegen für Medikamente auf Rezept răumen Sie zukünftig eher bessere, welchen eher schlechtere Marktchancen ein?")

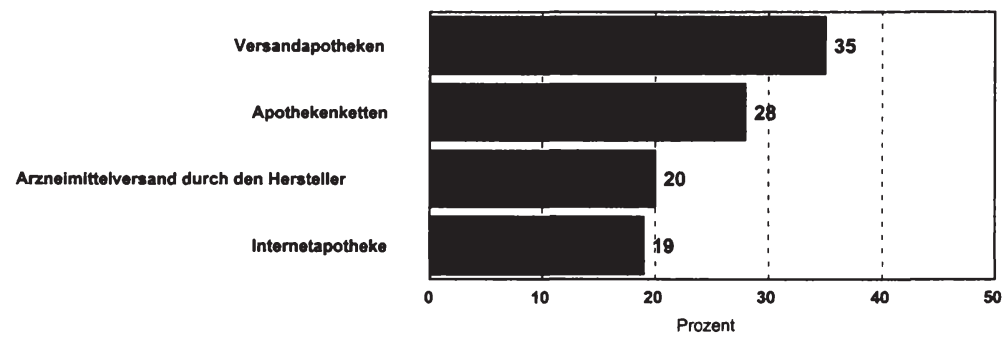

$n=380$ Arzte

Top-2-Boxes ("wesentlich besser" und "besser"); 1= wesentlich bessere Marktchancen, 5=wesentlich schlechtere Marktchancen

Quelle : INIFES, eigene Darstellung.

(Ergebnisse einer von TNS EMNID im Auftrag von INIFES im Dezember 2000 durchgeführten CATI-Befragung).

In der Legalisierung weiterer Vertriebsformen sehen die befragten Ärzte durchaus Kosteneinsparpotentiale für den Arzneimittelsektor. Versandapotheken - Medikamentenbestellung in direkter Kooperation mit dem Arzt - räumen 35,0 Prozent der Ärzte gute Marktchancen ein, 33,0 Prozent hingegen schlechte bis überhaupt keine Marktchancen. Ein signifikanter Unterschied im Antwortverhalten derer, die einer Versandapotheke gute Chancen am Markt einräumen, besteht zwischen den Urologen (44,0 Prozent) und den Hals-Nasen-Ohrenärzten (30,0 Prozent). Dieser Unterschied lässt sich mit der Zusammensetzung des Patientenklientel begründen. Der Anteil der chronisch Kranken beträgt bei den Urologen 52,3 Prozent (Akut=30,9 Prozent) und bei den Hals-Nasen-Ohrenärzten 37,3 Prozent (Akut=56,4 Prozent) ${ }^{75}$ chronisch Kranke Patienten werden vielfach als eine Zielgruppe für eine Versandapotheke gesehen, da ihr Medikamentenbedarf u.a. planbar ist. Während die Praktischen Ärzte / Allgemeinmediziner/Internisten, Gynäkologen, Neurologen / Nervenärzte und Hals-Nasen-Ohrenärzte einer Versandapotheke in Gebieten mit einer bereits bestehenden Überversorgung mit Apotheken wesentlich bessere/bessere Marktchancen einräumen, sehen die Urologen den Vorteil einer Versandapotheke in Gebieten, in denen der Versorgungsgrad eher angemessen ist. Die Annahme, dass eine Versandapotheke besonders für Gebiete mit einer gerade angemessenen Versorgung oder sogar einer bestehenden Unterversorgung mit Apotheken aus Sicht der Ärzte 
Nutzen stiften würde, kann daher aus dem vorliegenden Ergebnis nicht bestätigt werden.

Für Apothekenketten sehen 28,0 Prozent durchaus ein Marktpotential (29,0 Prozent gehen von wesentlich schlechteren/schlechteren Marktchancen aus), während die Chancen für den Arzneimittelversand durch den Hersteller oder den Großhandel (20,0 Prozent) und den Internethandel - freie Medikamentenbestellung durch die Verbraucher (19,0 Prozent) - eher als gering eingeschätzt werden. Vergleicht man jedoch diese Werte mit denen der Apotheker, so kann der Arbeitshypothese 7, die besagt, dass für komplementäre Distributionswege keine Marktchancen existieren, nicht uneingeschränkt zugestimmt werden. Als unabdingbar für die Zulassung komplementärer Distributionswege halten Ärzte eine Diskussion über das Fremd- und Mehrbesitzverbot.

Ebenso wie die Bundesärztekammer und die Kassenärztliche Bundesvereinigung ist die Einstellung der befragten Ärzte bezüglich komplementärer Vertriebsformen von Medikamenten eher zurückhaltend, jedoch keineswegs ablehnend. In einer Legalisierung weiterer Vertriebsformen sehen sie durchaus Kosteneinsparpotentiale für den Arzneimittelsektor. Versandapotheken werden beispielsweise von 35,0 Prozent der Ärzte gute Marktchancen eingeräumt, 33,0 Prozent sehen schlechte bis überhaupt keine Marktchancen. Die Annahme, dass eine Versandapotheke besonders für Gebiete mit einer Unterversorgung mit Apotheken einen Nutzen stiften würde, kann aus den vorliegenden Ergebnissen nicht bestätigt werden.

\subsubsection{Die Einstellung der Ärzte zum Modell der Versandapotheke}

Mehr als ein Drittel der befragten Ärzte räumen, wie bereits gezeigt, einer Versandapotheke als einem komplementären Distributionsweg von Medikamenten für die Zukunft "wesentlich bessere"/"bessere" Marktchancen ein, als beispielsweise einer Internetapotheke oder einem Arzneimittelversand durch den Hersteller. Im Vergleich zu den anderen Vertriebswegen scheint der Versandhandel aus Sicht der Ärzte relativ erfolgversprechend zu sein. Um die Einstellung der Ärzte gegenüber einer Versandapotheke zu erfragen und ein allgemeines Meinungsbild zu erhalten, wurden die Ärzte gebeten, ihre Assoziationen mit dem Begriff "Versandapotheke" im Rahmen einer offenen Frage wiederzugeben (s. Abbildung III - 13).

78

Dies haben persőnliche Gespräche mit Ärzten verschiedener Fachrichtungen gezeigt. 
Abbildung III - 13: Assoziationen mit dem Begriff "Versandapotheke"

(Frage:" Was verbinden Sie mit dem Begriff Versandapotheke?")

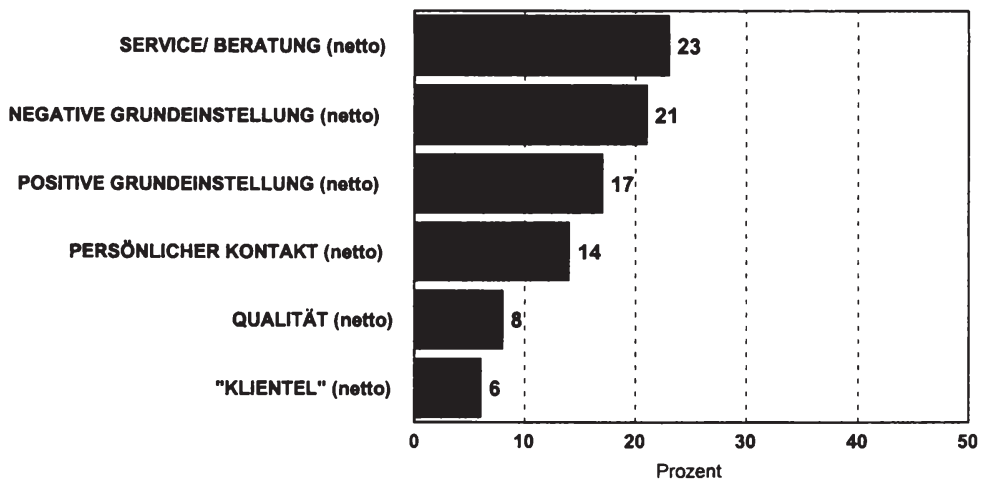

Basis: $n=380$ Arzte

Quelle : INIFES, eigene Darstellung.

(Ergebnisse einer von TNS EMNID im Auftrag von INIFES im Dezember 2000 durchgeführten CATI-Befragung).

Negative Auswirkungen auf den Service bzw. die Beratung erwarten 23,0 Prozent, eine Aufhebung des persönlichen Kontaktes zwischen Patienten und Apotheker befürchten 14,0 Prozent und an Qualitätseinbußen in der Versorgung glauben 8,0 Prozent. Dem Bereich Service/Beratung zu zurechnen ist die Einschätzung, dass der Patient mit einer wesentlich längeren Bezugsdauer zu rechnen hat (10,0 Prozent). Erfahrungen aus dem Ausland zeigen, dass zwischen der Übertragung des Rezeptes und der Zusendung des Medikaments häufig mehr als 24 Stunden liegen, die Präsenzapotheke das Präparat binnen weniger Stunden liefern kann. Demgegenüber verbinden jedoch 17,0 Prozent der niedergelassenen Ärzte eine positive Grundeinstellung mit dem Begriff der Versandapotheke. Die Gründe dafür sind vielfältig und reichen von: "gute Einrichtung" (5,0 Prozent) über "ist kostengünstiger" (4,0 Prozent) bis "ist bequem" - z.B. für Behinderte - (3,0 Prozent).

Bezüglich der negativen Merkmale existieren zwischen den befragten Facharztgruppen kaum Unterschiede. Ein signifikanter Unterschied zwischen den Urologen und den Hals-Nasen-Ohrenärzten besteht hinsichtlich der Beurteilung des Items "Qualität". Während 13,0 Prozent der Urologen mit Qualitätseinbußen rechnen, trifft dies bei den Hals-Nasen-Ohrenärzten nur zu 3,0 Prozent zu. Ob die befragten Ärzte vor dem Hintergrund ihres Patientenklientel geantwortet haben oder die Werte ihre eigene Meinung widerspiegeln, lässt sich nicht eruieren. Auffällig ist jedoch, der Anteil chronisch erkrankter Personen bei den Urologen umfasst durchschnittlich 52,3 Prozent der Patientenklientel, bei den Hals-Nasen-Ohrenärzten nur 37,3 Prozent. 
Der Unterschied wird noch deutlicher, betrachtet man den Anteil der über 60jährigen an den chronisch Kranken. Dieser beträgt bei den Hals-Nasen-Ohrenärzten 44,3 Prozent, bei den Urologen immerhin 71,6 Prozent.

Hinsichtlich der "positive Grundeinstellung" gegenüber einer Versandapotheke bestehen signifikante Unterschiede zwischen den untersuchten Facharztgruppen. Einer Versandapotheke gegenüber positiv und offen stehen 27,0 Prozent der Gynäkologen und 22,0 Prozent der Hals-Nasen-Ohrenärzte, während es nur 11,0 Prozent der Praktischen Ärzte / Allgemeinmediziner / Internisten sind. Der Anteil der chronisch Kranken und der über 60jährigen an den chronisch Kranken beträgt bei den Praktischen Ärzten / Allgemeinmedizinern / Internisten durchschnittlich 55,5 Prozent bzw. 58,6 Prozent und bei den Gynäkologen 17,5 Prozent und 42,5 Prozent. Vor allem bei den Gynäkologen ist die positive Grundeinstellung gegenüber einer Versandapotheke verständlich, da sich ihr Patientenklientel vor allem aus Patientinnen zusammensetzt, die zur Vorsorgeuntersuchung (durchschnittlich 57,5 Prozent) kommen und u.a. die verordneten Kontrazeptiva selber bezahlen müssen.

Gesetzt den Fall, es gäbe eine Versandapotheke, könnten sich 51,0 Prozent der befragten Ärzte persönlich vorstellen, mit einer solchen Apotheke zu kooperieren, 47,0 Prozent lehnen dies ab.

Dieser Anteil der Befürworter für eine Kooperation ist bei den Gynäkologen (51,0 Prozent), Neurologen/Nervenärzte (52,0 Prozent) und den Praktischen Ärzten/Allgemeinmedizinern/Internisten (51,0 Prozent) fast identisch. Wenig überraschend sind die Werte für die Urologen und die Hals-Nasen-Ohrenärzte, wenngleich die Antworten zu der offenen Frage vermuten ließen, dass die Bereitschaft, mit einer Versandapotheke zusammen zu arbeiten, bei den Urologen wesentlich geringer ausgeprägt ist als bei den Hals-Nasen-Ohrenärzten. Eine Kooperation mit einer Versandapotheke einzugehen, könnten sich 60,0 Prozent der Urologen (37,0 Prozent lehnen dies $a b$ ) und 44,0 Prozent der Hals-Nasen-Ohrenärzte vorstellen (dem widersprechen 55,0 Prozent). Verglichen mit dem Anteil der Chroniker je Facharztgruppe kann darauf geschlossen werden, dass die Bereitschaft zur Kooperation mit einer Versandapotheke bei den Ärzten in Beziehung steht zu dem Anteil der zu behandelnden Chroniker.

Die Ergebnisse der Ärztebefragung lassen eine insgesamt positive, wenn auch etwas zurückhaltende Grundeinstellung gegenüber einer Versandapotheke erkennen. Negative Auswirkungen werden in den Bereichen Service bzw. Beratung sowie dem persönlichen Kontakt zwischen Arzt, Apotheker und dem Patienten erwartet.

Vor allem Ärzte mit einem hohen Anteil an zu behandelnden Chronikern können sich eine Zusammenarbeit mit einer Versandapotheke vorstellen. Dies bestätigt die Arbeitshypothese 8, wonach die Bereitschaft, mit einer Versandapotheke zusammen zu arbeiten, sowohl von der Facharztgruppe als auch der Zusammensetzung der Patientenklientel abhängig ist. 


\subsubsection{Zielgruppe einer Versandapotheke}

Aufgrund der bereits beschriebenen strukturellen und logistischen Gegebenheiten einer Versandapotheke ist davon auszugehen, dass die Zielgruppe einer Versandapotheke nicht dieselbe sein kann wie die einer öffentlichen Apotheke. Bislang wird die Annahme gehegt, dass ein Versandhandel vielfach für Personen von Vorteil ist, die in ihrer Mobilität eingeschränkt, chronisch erkrankt oder berufstätig sind.

Auf die Frage nach der Bewertung der Vorteilhaftigkeit einer Versandapotheke in bezug auf verschiedene Zielgruppen gaben 64,0 Prozent der Ärzte an, dass eine Versandapotheke für Patienten, die in ihrer Mobilität eingeschränkt sind, von Vorteil sein kann (s. Abbildung III - 14).

Abbildung III - 14: Bewertung der Vorteilhaftigkeit einer Versandapotheke in bezug auf verschiedene Patientengruppen

(Frage: "Für welche Ihrer Patienten könnte die Etablierung einer Versandapotheke eher von Vorteil, für wen eher von Nachteil sein?"

In Gebleten mit Unterversorgung mit Apotheken

Fur In Ihrer Mobilitat oingeschränkte Patienten

Fur Patlenten mit chronischen Krankheiten

Als Service fur Kunden, z.B. Berufstatige

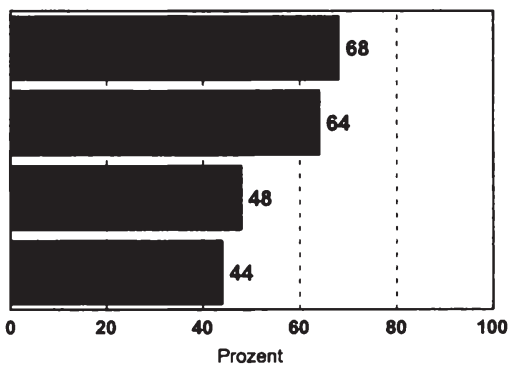

$n=380$ Arzte

Top-2-Boxes ("von großem Vorteil" und "von Vorteil"); $1=$ von großem Vorteil, $5=$ von großem Nachteil

Quelle: INIFES, eigene Darstellung.

(Ergebnisse einer von TNS EMNID im Auftrag von INIFES im Dezember 2000 durchgeführten CATI-Befragung). 
Aus dem Antwortverhalten der Ärzte wird deutlich, dass vor allem Fachrichtungen mit einem hohen Anteil an zu versorgenden Chronikern den Vorteil einer Versandapotheke für die Versorgung in ihrer Mobilität eingeschränkter Patienten sehen. Bei den Urologen beträgt die Zustimmung immerhin 75,0 Prozent. Patienten, die in ihrer Mobilität eingeschränkt sind, können sich ohnehin nicht die Arzneimittel selber besorgen und benötigen daher einen Botendienst. Da ein solcher, so die Auskunft eines Vertreters einer Krankenkasse, "möglicherweise jedes Mal neu organisiert werden müsste, wäre es sicherlich einfacher, dies auf dem Wege des Versandhandels zu erledigen“. ${ }^{77}$ Der Annahme, dass die Etablierung einer Versandapotheke generell für Patienten mit chronischen Krankheiten von Vorteil ist, schließen sich 48,0 Prozent der Ärzte an, 25,0 Prozent lehnen sie ab. Vor allem die Neurologen/Nervenärzte $(35,0$ Prozent) und die Praktischen Ärzte/Allgemeinmediziner/Internisten (31,0 Prozent) vertreten die Ansicht, dass eine Versandapotheke durchaus mit Nachteilen für bestimmte Personen verbunden sein kann. Die Patientenstruktur beider Facharztgruppen bedarf vielfach einen persönlichen Ansprechpartner bei der Abgabe von Arzneimitteln. Viele Präparate, wie zum Beispiel die Gruppe der Psychopharmaka, sollten niemals, um einen Missbrauch zu vermeiden, über eine Versandapotheke erhältlich sein.

Zusammenfassend bleibt zu sagen, dass nach Ansicht der befragten Ärzte besonders Patienten, die in ihrer Mobilität eingeschränkt sind, eine Zielgruppe für eine Versandapotheke darstellen. Hierbei handelt es sich um die Patientengruppe, die schon heute vielfach von der Apotheke nach Hause beliefert wird. Die bislang bestehende Annahme, dass die Chroniker eine Hauptzielgruppe bilden, muss differenzierter betrachtet werden. Die Facharztgruppen der Neurologen/Nervenärzte sowie der Praktischen Ärzte / Allgemeinmediziner / Internisten, die einen überdurchschnittlich hohen Anteil zu behandelnder Chroniker betreuen, vertreten die Ansicht, dass eine Versandapotheke durchaus mit Risiken behaftet sein kann. Die Patientenstruktur beider Fachrichtungen bedarf vielfach einen persönlichen Ansprechpartner bei der Abgabe von Arzneimitteln und kann, trotz gegenteiliger Meinung einiger Experten, nicht auf eine persönliche Beratung verzichten.

\subsubsection{Erwartete potentielle Auswirkungen eines Versandhandels}

Während derzeit von Seiten der Apotheker, der Politik, der GKV, der Verbraucherverbände und der Pharmaindustrie eine sehr kontroverse Diskussion zu dem Thema Versandhandel mit rezeptpflichtigen Arzneimitteln geführt wird, wollen sich die Vertreter der Ärzte zu Versandapotheken und deren potentiellen Auswirkungen auf das bestehende System oftmals erst dann äußern, wenn von der Politik diesbezügliche Vorgaben und Entscheidungen vorliegen. Um jedoch ein erstes Meinungsbild der Ärzte zu erhalten, wurden sie gebeten, anhand einer Skala von $1=$ volle Zustimmung bis $5=$ totale Ablehnung, die in der Öffentlichkeit diskutierten

Anlässlich eines Expertengespräches. 
bis 5=totale Ablehnung, die in der Offentlichkeit diskutierten Aussagen zur Versandapotheke zu bewerten (s. Abbildung III - 15).

Abbildung III - 15: Bewertung verschiedener Aussagen zur Versandapotheke

(Frage: "Stellen Sich sich nun vor, es würde eine solche Versandapotheke auch in Deutschland existieren. Stimmen Sie folgenden Aussagen zu?")

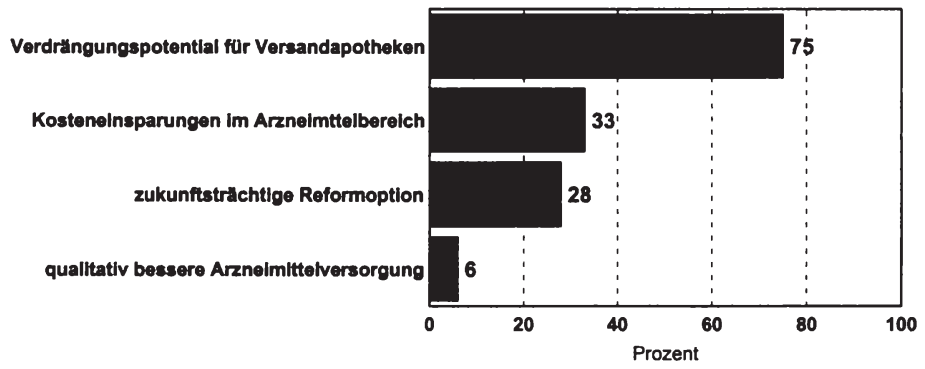

$n=380$ Arzte

Top-2-Boxes ("volle Zustimmung" und "Zustimmung"); $1=$ volle Zustimmung, 5= totale Ablehnung

Quelle : INIFES, eigene Darstellung.

(Ergebnisse einer von TNS EMNID im Auftrag von INIFES im Dezember 2000 durchgeführten CATI-Befragung).

Der Aussage, dass Versandapotheken zu einer qualitativ besseren Arzneimittelversorgung führen werden, stimmen lediglich 6,0 Prozent der Ärzte zu, 48,0 Prozent lehnen die Aussage total ab. Die Zustimmung der Neurologen/Nervenärzte ist mit 13,0 Prozent signifikant höher als bei den Urologen, die dieser Aussage nur zu 2,0 Prozent zustimmen.

33,0 Prozent der befragten Ärzte stimmen der Aussage - Versandapotheken werden zu Kosteneinsparungen im Arzneimittelbereich führen - zu, während 28,0 Prozent dies total ablehnen. Besonders die Neurologen/Nervenärzte rechnen mit massiven Kosteneinsparungen (46,0 Prozent), diese Ansicht teilen aber nur 22,0 Prozent der Gynäkologen. Dies ist damit zu begründen, dass ein Großteil der Präparate, die ein Gynäkologe verordnet, nicht erstattungsfähig sind.

Nach Ansicht der Ärzte (75,0 Prozent) stellen Versandapotheken generell ein Verdrängungspotential für traditionelle Apotheken dar. Diese Zustimmung ist unabhängig von der befragten Facharztgruppe.

Der Annahme, dass die Einführung dieses Modells einer Versandapotheke eine zukunftsträchtige Reformoption darstellt, erteilen 28,0 Prozent der Ärzte ihre Zustimmung, während 22,0 Prozent sie total ablehnen. 48,0 Prozent der Ärzte, die sich per- 
sönlich eine Kooperation mit einer Versandapotheke vorstellen könnten, halten die Einführung des Modells einer Versandapotheke für eine zukunftsträchtige Reformoption. Signifikante Unterschiede hinsichtlich der untersuchten Facharztgruppen und dem Anteil der Chroniker bestehen nicht.

\subsubsection{Die Einstellung der Ärzte zu Reformoptionen}

Neben den Apothekern versuchen auch die Ärzte auf Grundlage der neuen Medien Wirtschaftlichkeitsreserven im Gesundheitswesen und vor allem in der Arzneimittelversorgung zu identifizieren. Sie erhoffen sich durch besser aufeinander abgestimmte Versorgungsketten, effizientere Informationswege und schnittstellenübergreifende Kommunikation die Versorgungsqualität für ihre Patienten zu steigern und die Kosten der GKV zu reduzieren. ${ }^{78}$ Für den Bereich der Arzneimittelversorgung sei folgendes angesprochen: Mit der Dokumentation der Arzneimittelversorgung verbindet sich das Ziel der Erhöhung der medizinisch-pharmakologischen Qualität der Arzneimittelversorgung. Die Arzneimitteldokumentation liefert dem Arzt wichtige Hinweise zur Vermeidung von Doppeluntersuchungen und unerwünschten Arzneimittelinteraktionen. Zur Zeit erfolgt diese Dokumentation für die vom Arzt verordneten Arzneimittel in der traditionellen papierbasierten oder elektronischen Patientenakte des jeweiligen Arztes. Nicht erfasst werden aber Präparate, die von anderen Ärzten verordnet werden, in der Apotheke gekaufte OTC-Präparate und Arzneimittel, die nicht der Apothekenpflicht unterliegen.

Gesetzliche Veränderungen vorausgesetzt, können sich 56,0 Prozent der befragten Ärzte zukünftig vorstellen, dass der verordnende Arzt das Rezept nach Einwilligung des Patienten per Datenfernübertragung an eine Versandapotheke schickt. Diese stellt dann die Bestellung zusammen und liefert sie an den Patienten aus. Der Patient erscheint nicht mehr persönlich in der Apotheke. Abgelehnt wird ein solches Vorgehen von 44,0 Prozent der Ärzte.

Eine andere Reformoption stellt folgendes Modell dar: Der Patient erhält das Rezept vom verordnenden Arzt wie bisher ausgehändigt und schickt dieses per Brief an die Versandapotheke. Eine solche Option erachten 53,0 Prozent der Ärzte für sinnvoll, 46,0 Prozent für nicht erstrebenswert.

Des weiteren für durchaus vorstellbar halten 66,0 Prozent der Ärzte eine dritte Reformoption: Das Rezept wird durch den verordnenden Arzt auf einer Smart-Card gespeichert, die der Patient danach in der Apotheke zur Einlösung vorlegt. 31,0 Prozent der befragten Ärzte können sich dies nicht vorstellen. Nach Ansicht der Apotheker umfassen zukunftsträchtige Reformoptionen vornehmlich die Bereiche: Bestellung und Distribution (s. Abbildung III - 16).

Vgl. Hovermann, E. (2001), Versandhandel versus Einzelapotheke, in: Die BKK. Zeitschrift der betrieblichen Krankenversicherung, Jg. 88, Heft 4, S. 175. 
Abbildung III - 16: Sonstige vorstellbare Reformoptionen (offene Frage)

(Frage: "Können Sie sich noch weitere Reformoptionen vorstellen?")

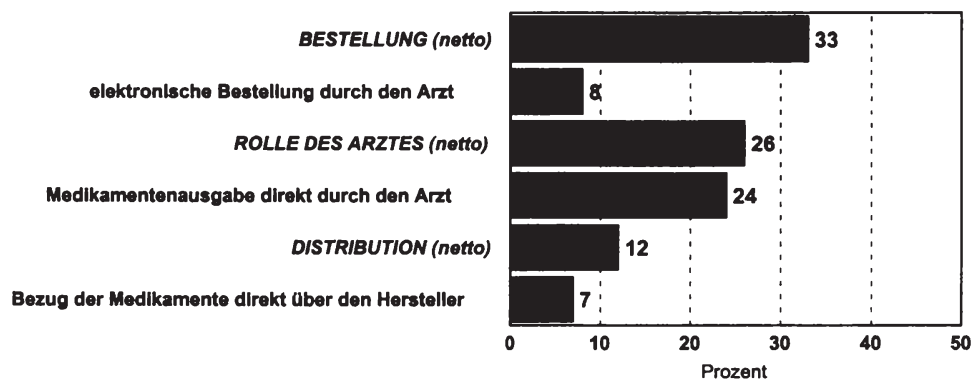

Selektion: Azzte, die sich noch weitere Reformoptionen vorstellen können ( $n=112)$

Quelle : INIFES, eigene Darstellung.

(Ergebnisse einer von TNS EMNID im Auftrag von INIFES im Dezember 2000 durchgeführten CATI-Befragung).

Den größten Reformbedarf sehen die Ärzte im Bereich "Bestellung" (33,0 Prozent). Dieser Bereich setzt sich u.a. zusammen aus: elektronische Bestellung durch den Arzt (8,0 Prozent), Rezept per Internet "versenden" (5,0 Prozent) und der Einführung des Elektronischen Rezeptes (5,0 Prozent).

Ein weiterer Bereich, der von den Ärzten in der Befragung angesprochen wurde, bezieht sich auf die Stärkung der Rolle des Arztes bzgl. der Medikamentenabgabe direkt durch den Arzt (26,0 Prozent). Der Arzt besitzt in Deutschland kein Dispensierrecht, immerhin 24,0 Prozent der befragten Ärzte würden eine Aufhebung dieses Verbotes begrüßen. 12,0 Prozent der Ärzte, die sich weitere Reformoptionen vorstellen können, gaben an, dass die derzeitige Distribution ein Reformpotential bildet. Zur Reduzierung des Vertriebskostenanteils an Arzneimittelpreisen können sich 7,0 Prozent der Ärzte vorstellen, dass der Bezug der Medikamente zukünftig direkt über den Hersteller möglich sein wird. 


\subsubsection{Fazit}

Die Einstellung der Ärzte gegenüber dem Modell einer Versandapotheke ist zum gegenwärtigen Zeitpunkt als abwartend bzw. zurückhaltend zu bewerten. Dies mag zum einen mit einem bestehenden Informationsdefizit hinsichtlich eines Versandhandels verbunden sein. Zum anderen ist den Ärzten die von innen, innerhalb eines solchen Modells, zu übernehmende Aufgabe noch nicht ersichtlich.

Vielen Ärzten ist besonders die persönliche Rücksprache zwischen Arzt und Apotheker bei der Aushändigung der Medikamente in der Apotheke wichtig. Ein solcher Kontakt ist für sie vor allem daher wichtig, um Unklarheiten bzgl. des Rezeptes und möglicher Wechselwirkungen unverzüglich zu klären. Einen solchen Kontakt sehen die befragten Ärzte allerdings durch den Versandhandel gefährdet. Während 75,0 Prozent der Ärzte in einer Versandapotheke ein Verdrängungspotential für Präsenzapotheken sehen, erachten 33,0 Prozent Kosteneinsparungen im Arzneimittelbereich durch Versandapotheken für möglich.

Nach Ansicht der befragten Ärzte stellen besonders Patienten, die in ihrer Mobilität eingeschränkt sind, eine Zielgruppe für eine Versandapotheke dar. Die bislang bestehende Annahme, dass die Chroniker eine Hauptzielgruppe bilden, kann anhand der Befragungsergebnisse nicht bestätigt werden. Facharztgruppen mit einem überdurchschnittlich hohen Anteil zu behandelnder Chroniker vertreten die Ansicht, dass eine Versandapotheke durchaus mit Risiken behaftet sein kann. Trotz gegenteiliger Meinung einiger Experten, dass vor allem chronisch erkrankte Personen keiner Beratung bei der Abgabe von Medikamenten in der Apotheke bedürfen, kann und darf nach Angaben der befragten Fachärzte bei dieser Patientenklientel nicht auf eine persönliche Beratung verzichtet werden.

\subsubsection{Bevölkerungsbefragung}

\subsubsection{Allgemeine Angaben zur Stichprobe}

Das Kollektiv der antwortenden Bevölkerung setzte sich insgesamt aus 48,0 Prozent Männer und 52,0 Prozent Frauen zusammen. Eine detaillierte Altersverteilung ist der Tabelle III - 5 zu entnehmen. 52,0 Prozent gaben an, berufstätig bzw. vorübergehend arbeitslos zu sein, 48,0 Prozent waren nicht berufstätig (Rentner, Auszubildende, Schüler, Studenten usw.). Die Haushaltsgröße im Rahmen der Befragung umfasste 19,0 Einpersonen-, 35,0 Zweipersonen-, 21,0 Dreipersonen- und 25,0 Prozent Vierund Mehrpersonenhaushalte. Während 89,0 Prozent der über 60jährigen angaben, in Ein- bzw. Zweipersonenhaushalten zu leben, beträgt der Anteil der 30 bis 39jährigen, die in einer vergleichbaren Haushaltsgröße leben, 41,0 Prozent. In BIK-Ortsgrößen bis 5.000 Einwohner leben 14,0 Prozent der befragten Personen, 16,0 Prozent in BlK-Ortsgrößen bis 20.000 Einwohner, 15,0 Prozent in Gebieten bis 100.000 Einwohner, 17,0 Prozent der Bevölkerung lebt in Ortsgrößen bis 500.000 Einwohner und 
in BIK-Ortsgrößen mit 500.000 und mehr Einwohnern wohnen 38,0 Prozent der Bundesbürger.

Tabelle III - 5: Sozio-Demographie der Bevölkerung ( $n=1006)$

\begin{tabular}{|c|c|c|c|c|c|c|}
\hline & \multicolumn{6}{|c|}{ Prozent } \\
\hline & $\begin{array}{c}\text { Insgesamt } \\
(n=1006)\end{array}$ & $\begin{array}{c}14-29 \\
\text { Jahre }\end{array}$ & $\begin{array}{c}30-39 \\
\text { Jahre }\end{array}$ & $\begin{array}{c}40-49 \\
\text { Jahre }\end{array}$ & $\begin{array}{c}50-59 \\
\text { Jahre }\end{array}$ & $\begin{array}{c}60+ \\
\text { Jahre }\end{array}$ \\
\hline $\begin{array}{l}\text { Geschlecht } \\
\text { Männer } \\
\text { Frauen }\end{array}$ & $\begin{array}{l}48 \\
52\end{array}$ & $\begin{array}{l}51 \\
49\end{array}$ & $\begin{array}{l}55 \\
45\end{array}$ & $\begin{array}{l}40 \\
60\end{array}$ & $\begin{array}{l}51 \\
49\end{array}$ & $\begin{array}{l}43 \\
57\end{array}$ \\
\hline $\begin{array}{l}\text { Alter } \\
\text { 14-29 Jahre } \\
\text { 30-39 Jahre } \\
\text { 40-49 Jahre } \\
\text { 50-59 Jahre } \\
60+\text { Jahre }\end{array}$ & $\begin{array}{l}22 \\
19 \\
16 \\
16 \\
28\end{array}$ & $\begin{array}{c}100 \\
- \\
- \\
- \\
-\end{array}$ & $\begin{array}{c}- \\
100 \\
- \\
- \\
-\end{array}$ & $\begin{array}{c}- \\
- \\
100 \\
- \\
-\end{array}$ & $\begin{array}{c}- \\
- \\
- \\
100 \\
-\end{array}$ & $\begin{array}{c}- \\
- \\
- \\
- \\
100\end{array}$ \\
\hline $\begin{array}{l}\text { Beruf́stätigkeit } \\
\text { berufstătig einschließlich vorübergehend } \\
\text { arbeitslos } \\
\text { nicht berufstătig (Rentner, Schüler, Stu- } \\
\text { denten usw.) }\end{array}$ & $\begin{array}{l}52 \\
48\end{array}$ & $\begin{array}{l}48 \\
52\end{array}$ & $\begin{array}{l}87 \\
13\end{array}$ & $\begin{array}{l}79 \\
21\end{array}$ & $\begin{array}{l}70 \\
30\end{array}$ & $\begin{array}{c}7 \\
93\end{array}$ \\
\hline $\begin{array}{l}\text { Haushaltsgröße } \\
\text { Ein Person } \\
\text { Zwei Personen } \\
\text { Drei Personen } \\
\text { Vier Personen und mehr }\end{array}$ & $\begin{array}{l}19 \\
35 \\
21 \\
25 \\
\end{array}$ & $\begin{array}{c}8 \\
20 \\
30 \\
42 \\
\end{array}$ & $\begin{array}{l}16 \\
25 \\
22 \\
37 \\
\end{array}$ & $\begin{array}{l}11 \\
21 \\
29 \\
39 \\
\end{array}$ & $\begin{array}{l}15 \\
51 \\
22 \\
11 \\
\end{array}$ & $\begin{array}{c}37 \\
52 \\
7 \\
4 \\
\end{array}$ \\
\hline $\begin{array}{l}\text { Haushaltsnettoeinkommen } \\
\text { - unter 2.000 DM } \\
2.000 \text { - unter } 3.000 \mathrm{DM} \\
3.000 \text { - unter } 4.000 \mathrm{DM} \\
4.000 \text { - unter 5.000 DM } \\
5.000 \text { - unter } 6.000 \mathrm{DM} \\
6.000 \text { + } \\
\text { keine Angabe }\end{array}$ & $\begin{array}{c}12 \\
18 \\
16 \\
14 \\
9 \\
13 \\
20 \\
\end{array}$ & $\begin{array}{c}7 \\
12 \\
14 \\
14 \\
8 \\
20 \\
25 \\
\end{array}$ & $\begin{array}{c}8 \\
18 \\
24 \\
14 \\
12 \\
11 \\
13 \\
\end{array}$ & $\begin{array}{c}7 \\
12 \\
9 \\
26 \\
10 \\
21 \\
15 \\
\end{array}$ & $\begin{array}{l}11 \\
13 \\
13 \\
14 \\
14 \\
13 \\
23 \\
\end{array}$ & $\begin{array}{c}21 \\
27 \\
16 \\
7 \\
4 \\
4 \\
22 \\
\end{array}$ \\
\hline $\begin{array}{l}\text { BIK-Stadtregion } \\
- \text { u. } 5.000 \\
5.000-\text { u. } 20.000 \\
20.000-\text { u. } 100.000 \\
100.000-\text { u. } 500.000 \\
500.000 \text { und mehr } \\
\end{array}$ & $\begin{array}{l}14 \\
16 \\
15 \\
17 \\
38 \\
\end{array}$ & $\begin{array}{l}15 \\
13 \\
12 \\
19 \\
41 \\
\end{array}$ & $\begin{array}{l}15 \\
19 \\
16 \\
19 \\
32\end{array}$ & $\begin{array}{l}20 \\
17 \\
10 \\
20 \\
33 \\
\end{array}$ & $\begin{array}{l}13 \\
15 \\
21 \\
10 \\
42 \\
\end{array}$ & $\begin{array}{l}11 \\
16 \\
15 \\
18 \\
40 \\
\end{array}$ \\
\hline
\end{tabular}

Quelle: INIFES, eigene Darstellung.

(Ergebnisse einer von TNS EMNID im Auftrag von INIFES im Dezember 2000 durchgeführte CATI-Befragung). 


\subsubsection{Gesundheitszustand der Bevölkerung}

Erfahrungen mit Versandapotheken im Ausland zeigen, dass aufgrund epidemiologischer und demographischer Entwicklungen nachfrageseitige Potentiale in Form neuer Zielgruppen für Versandapotheken existieren.

Um die Akzeptanz des Versandhandels bei den Patienten zu eruieren und die bisherigen Annahmen bzgl. der Zielgruppen für eine Versandapotheke zu untersuchen, hat die INIFES-Befragung in einem ersten Schritt den Gesundheitszustand der in der Bundesrepublik Deutschland lebenden Männer und Frauen im Alter ab 14 Jahren untersucht.

Von den befragten Personen sind 91,0 Prozent gesetzlich krankenversichert bzw. mitversichert. 9,0 Prozent gaben an, nicht in einer gesetzlichen Krankenkasse versichert bzw. mitversichert zu sein.

Eine präzise, allgemeingültige Definition für "chronisch krank" existiert in der Gesundheitsökonomie nicht. In der Literatur ${ }^{80}$ werden Patienten aufgrund ihrer Medikation als "chronisch krank" bezeichnet, wenn sie eine kontinuierliche Arzneiversorgung aus ein und derselben Arzneimittelgruppe über mindestens drei Quartale aufweisen. Das SGB V (§ 62) spricht von chronischer Erkrankung bei Versicherten, die "... wegen derselben Erkrankung in Dauerbehandlung sind...". Dieser Definition folgend, leiden als Ergebnis der durchgeführten Bevölkerungsbefragung 27,0 Prozent der Bundesbürger unter einer chronischen Erkrankung. ${ }^{81}$ Eine geschlechtsspezifische Untersuchung zeigt, dass der Anteil der chronisch Kranken Frauen in den Altersklassen 50-59 Jahre (31,0 Prozent) und über 60 Jahre (55,0 Prozent) den der Männer in denselben Altersklassen (30,0 Prozent bzw. 44,0 Prozent) übersteigt. Dies belegt, dass vor allem Personen über 60 Jahre, hierbei vor allem die Frauen, unter dauerhaften Gesundheitsstörungen leiden, die von einem Arzt behandelt werden. Hinzu kommt, dass 54,0 Prozent der Personen über 60 Jahre in einem Einpersonenhaushalt leben, wobei der Anteil der Frauen 60,0 Prozent beträgt (Männer $=40,0$ Prozent). Nach Angaben der Befragten, die unter dauerhaften Gesundheitsstörungen leiden, die vom Arzt behandelt werden, lassen sich diese bestimmten Indikationsgruppen zuordnen (s. Abbildung III - 17).

\footnotetext{
79

Sterzel, A., Wassener, D. (2001), Arzneimitteldistribution in Deutschland - Versandapotheken als Reformoption?, in: Arbeit und Sozialpolitik 1-2/2001, S. 20.

Gerste, B., Niemeyer, M., Lauterberg, J. (2000), Wie viel chronisch Kranke gibt es?, in: Arnold, M., Litsch, M., Schwartz, F. W. (Hrsg.), Krankenhaus-Report 1999, Stuttgart, S. 69.

81

Die von INIFES gestellte Frage lautete: Leiden Sie unter dauerhaften Gesundheitsstörungen, die vom Arzt behandelt werden?
} 
Abbildung III - 17: Art der dauerhaften, vom Arzt behandelten Gesundheitsstörungen

(Frage: "In welche der folgenden Kategorien lässt sich Ihre dauerhafte gesundheitliche Beeintrăchtigung einordnen?")

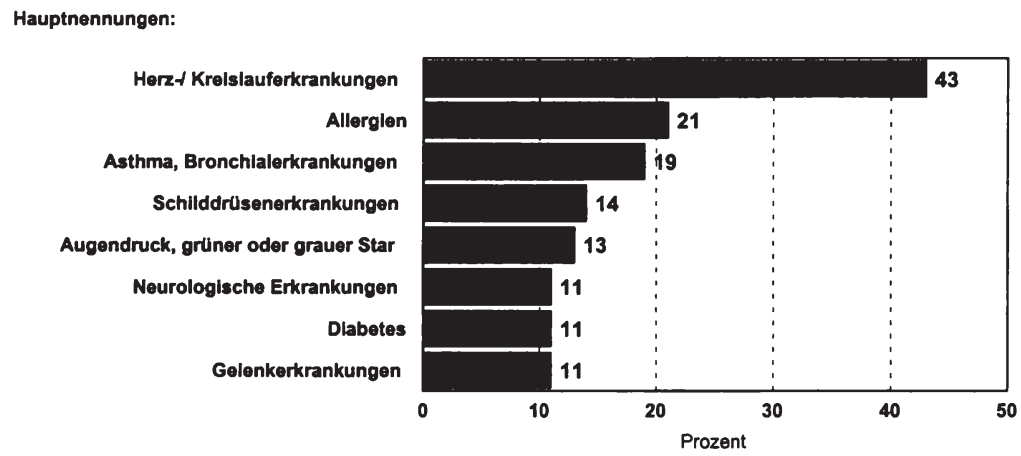

Selektion: Personen mit dauerhaften, vom Arzt behandelten Gesundheitsstörungen $(n=268)$

Quelle : INIFES, Eigene Darstellung.

(Ergebnisse einer von TNS EMNID im Auftrag von INIFES im Dezember 2000 durchgeführten CATI-Befragung).

Unter Herz-/Kreislauferkrankungen leiden 43,0 Prozent (61,0 Prozent der über 60jährigen), unter Allergien leiden 21,0 Prozent (64,0 Prozent der 14-29jährigen) und von Asthma betroffen sind 19,0 Prozent. 14,0 Prozent der befragten Personen gaben an, an der Schilddrüse behandelt zu werden, wobei sich der Anteil der Frauen $(23,0$ Prozent) signifikant von dem der Männer (4,0 Prozent) unterscheidet. Während nur 14,0 Prozent der Bürger wegen des Augendrucks oder eines grünen bzw. grauen Stars in augenärztlicher Behandlung sind, sind es in der Altersklasse der über 60jährigen immerhin 22,0 Prozent. 11,0 Prozent der Personen mit einer vom Arzt behandelten dauerhaften Gesundheitsstörung werden aufgrund einer Neurologischen Erkrankung behandelt, ebenso viele wegen Diabetes. Von den Diabetikern sind 17,0 Prozent ălter als 60 Jahre.

Die Befragung ergab, dass 41,0 Prozent der Personen mit dauerhaften, vom Arzt behandelten Gesundheitsstörungen regelmäßig 2-4 Medikamente ${ }^{82}$ pro Tag, 17,0 Prozent sogar täglich mehr als 5 Arzneimittel einnehmen. 21,0 Prozent der Befragten konsumieren täglich ein rezeptpflichtiges Medikament. Mit zunehmenden Alter ist ein signifikanter Anstieg des Konsums von verschiedenen rezeptpflichtigen Medikamenten zu erkennen.

82 Es handelt sich hierbei um die vom Arzt verordneten Arzneimittel und nicht die Anzahl der Tabletten. 
Mehr Männer geben an, nicht regelmäßig rezeptpflichtige Medikamente pro Tag einzunehmen (24,0 Prozent Männer versus 16,0 Prozent Frauen).

Die Befragungsergebnisse belegen, dass vermehrt ältere Personen angeben, unter chronischen Erkrankungen zu leiden. Des weiteren kommt es bei dieser Patientenklientel mit zunehmendem Alter zu einem signifikanten Anstieg des Konsums von verschiedenen rezeptpflichtigen Medikamenten. Unabhängig von bereits bestehenden Bezugsgewohnheiten von Medikamenten und der Einstellung zum Versandhandel, stellt diese Bevölkerungsgruppe ein besonderes Potential bzw. eine Hauptzielgruppe für eine Versandapotheke dar.

\subsubsection{Die Einstellung der Bevölkerung zum Vertriebskanal Apotheke}

Bislang lösen Patienten aufgrund des $§ 43$ Arzneimittelgesetz (AMG) die Rezepte mit verschreibungspflichtigen Arzneimitteln in der Apotheke ihrer Wahl ein, wo sie die vom Arzt verordneten Medikamente ausgehändigt bekommen. Auf diese Weise lösen 91,0 Prozent der Bürger ein Rezept selber ein. Wobei ein Anteil von 94,0 Prozent bei den Frauen darauf hinweist, dass diese häufiger für ihren Partner die Einlösung des Rezeptes übernehmen (Anteil der Männer ist 88,0 Prozent). Auch Personen über 60 Jahre erledigen die Rezepteinlösung in der Apotheke noch zu 88,0 Prozent selber. Viele Arzneimittelbezieher sind in ihrer Mobilität aufgrund einer Krankheit, des Alters oder der Berufstätigkeit stark eingeschränkt. Eine vertraute Person, welche die Rolle eines "Rezeptüberbringers" und "Medikamentenbesorgers" übernimmt, ist dann das nötige Bindeglied zwischen Apotheke und Patienten/Kunden. Für 5,0 Prozent der Bundesbürger ist es nach eigenen Angaben üblich, dass Angehörige, Vertrauenspersonen oder der Arzt die Rezeptübergabe und die Aushändigung des Medikamentes übernehmen. So erhalten vor allem Männer (8,0 Prozent) und Personen über 60 Jahren (8,0 Prozent) die vom Arzt verordneten Medikamente.

Die Ausnahmeregelung des $\S 17, \|$ ApBetrO billigt in begründeten Einzelfällen eine Versendung aus der Apotheke oder die Zustellung durch einen Boten an den Arzneimittelempfänger zu. In der Regel erhalten 2,0 Prozent der befragten Personen die Medikamente von der Apotheke nach Hause geliefert. Bei den Altersklassen 30-39 Jahre und über 60 Jahre beträgt der Anteil 4,0 Prozent. 5,0 Prozent der Personen, die unter einer dauerhaften, vom Arzt behandelten Gesundheitsstörung leiden ${ }^{83}$, bekommen ihre Medikamente von der Apotheke häufiger nach Hause geliefert. Dies zeigt, dass Chroniker nicht automatisch auf Heimlieferungen angewiesen sind, sondern durchaus selber eine Apotheke aufsuchen. Die vorliegenden Daten lassen jedoch keine weitere Differenzierung nach der Art der chronischen Erkrankung und dem Anteil der entsprechenden Abhängigkeit von Heimlieferungen zu. Es ist aber selbstredend, dass nicht jede chronische Krankheit verbunden ist mit einem Bedarf an Lieferung der Medikamente aus der Apotheke an den Arzneimittelbezieher. Es ist

83

Also die chronisch Kranken nach Annahme des Verfassers. 
jedoch durchaus annehmbar, dass wesentlich mehr Patienten, die unter dauerhaften, vom Arzt zu behandelnden Gesundheitsstörungen leiden, auf eine Heimlieferung der Arzneimittel angewiesen wären. Diese Patienten beauftragen dann vielfach andere Personen mit der Einlösung des Rezeptes.

Um ein vom Arzt ausgestelltes Rezept einzulösen, gehen 22,0 Prozent der Bundesbürger, die ihr Rezept selber einlösen oder die Medikamente von der Apotheke nach Hause geliefert bekommen, durchschnittlich ein- bis zweimal im Monat in die Apotheke (s. Abbildung III - 18).

Abbildung III - 18: Häufigkeit der Einlösung von Rezepten in der Apotheke (Frage: "Wie oft gehen Sie durchschnittlich selbst in eine Apotheke, um ein Rezept einzulösen?")

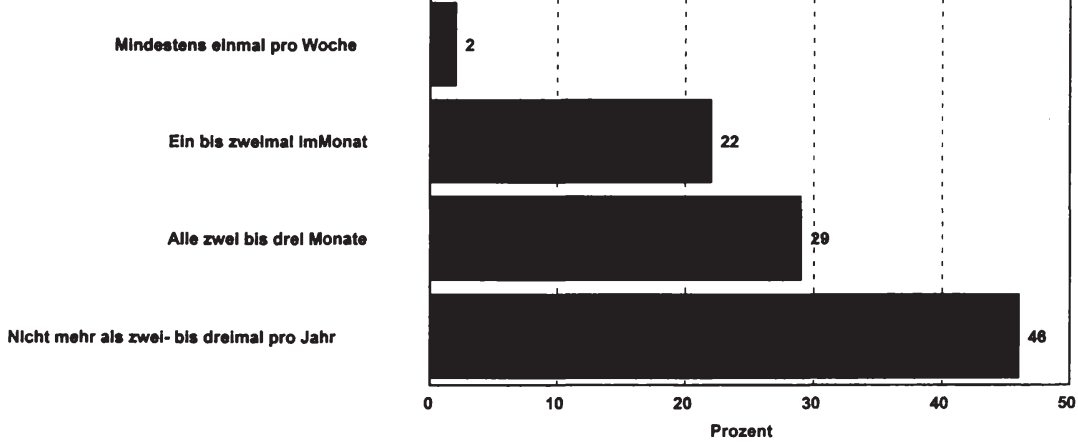

Selektion: Personen, die ihr Rezept selber einlÖsen oder die Medikamente von der Apotheke nach Hause geliefert bekommen ( $n=941$ )

Quelle : INIFES, eigene Darstellung.

(Ergebnisse einer von TNS EMNID im Auftrag von INIFES im Dezember 2000 durchgeführten CATI-Befragung).

Mit zunehmenden Alter ist ein signifikanter Anstieg der Häufigkeit der Einlösung von Rezepten in der Apotheke zu erkennen. 51,0 Prozent der Patienten mit einer dauerhaften, vom Arzt behandelten Gesundheitsstörung müssen durchschnittlich ein- bis zweimal im Monat eine Apotheke aufsuchen, um die verordneten Medikamente zu erhalten, bei den Patienten ohne eine dauerhaften Gesundheitsstörung sind dies 12,0 Prozent. Durchschnittlich mindestens einmal pro Woche gehen 2,0 Prozent der Befragten selber in eine Apotheke, um ein Rezept einzulösen. Alle zwei bis drei Monate gehen 29,0 Prozent in eine Apotheke und 46,0 Prozent der Personen, die ihr Rezept selber einlösen oder die ihre Medikamente von der Apotheke nach Hause geliefert bekommen, gehen nicht mehr als zwei- bis dreimal pro Jahr in die Apotheke.

Vor allem die Männer (56,0 Prozent) und Personen im Alter zwischen 14 und 49 Jahren (58,0 Prozent) lösen höchstens zwei- bis dreimal pro Jahr ein Rezept in einer Apotheke ein. 
Ein signifikanter Unterschied besteht hinsichtlich des Gesundheitszustandes. Während 57,0 Prozent der Befragten eher selten in eine Apotheke gehen, um ein Rezept einzulösen, lösen nur 14,0 Prozent der Chroniker nicht öfter als zwei- bis dreimal pro Jahr ein Rezept in einer Apotheke ein.

Die Bundesbürger legen bei der Wahl der Apotheke, in der sie ein Rezept einlösen, verschiedene Kriterien zu Grunde. Dazu gehören u.a. eine schnelle und gute Erreichbarkeit, eine umfassende Beratung, kostenloser Heimlieferservice von Medikamenten sowie Stammkunde einer Apotheke. Anhand einer Skala von 1=sehr wichtig bis $5=$ völlig unwichtig wurden diese Kriterien von den Befragten bewertet (s. Abbildung III - 19).

Abbildung III - 19: Grad der Wichtigkeit verschiedener Kriterien bei der Auswahl der Apotheke

(Frage: "Welche Kriterien legen Sie bei der Auswahl der Apotheke zugrunde? Wie wichtig sind Ihnen folgende Aspekte?")

Schnelle und gute Erreichbarkeit

Umfassende Beratung

Bin Stammkunde einer bestimmten Apotheke

Kostenloser Heimlieferservice von Medikamenten

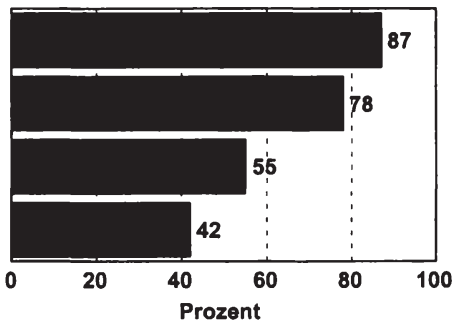

Selektion: Personen, die ihr Rezept selber einlösen oder die Medikamente von der Apotheke nach Hause

geliefert bekommen $(n=941)$

Top-2-Boxes ("sehr wichtig" und "wichtig"); 1=sehr wichtig, 5=völlig unwichtig

Quelle: INIFES, eigene Darstellung.

(Ergebnisse einer von TNS EMNID im Auftrag von INIFES im Dezember 2000 durchgeführten CATI-Befragung).

Bei der Auswahl der Apotheke, in der das Rezept eingelöst wird, werden von der Bevölkerung vor allem Kriterien einer schnellen und guten Erreichbarkeit (87,0 Prozent) sowie einer umfassenden Beratung (78,0 Prozent) zu Grunde gelegt. Bezüglich der schnellen und guten Erreichbarkeit erwarten vielfach Frauen (90,0 Prozent) und Berufstätige (90,0 Prozent), die ihr Rezept selber in der Apotheke einlösen, dass die Apotheke schnell und gut erreichbar ist. Eine umfassende Beratung ist vor allem Frauen ab 30 Jahre wichtig (83,0 Prozent), während Männern ab 60 Jahre (72,0 Prozent) das Kriterium der umfassenden Beratung bei der Wahl der Apotheke nicht mehr ganz so wichtig ist. Entgegen der bisherigen Annahmen legen die Chroniker viel Wert auf eine Beratung in der Apotheke (83,0 Prozent). Obwohl sie vielfach über Jahre 
dieselben Medikamente bekommen, enwarten sie von der Apotheke, in der sie ihr Rezept einlösen, umfassend beraten zu werden. Vertiefend zu den Auswahlkriterien der Apotheke wurden die Personen, die ihr Rezept selber einlösen, gefragt, wie oft sie in der Apotheke bei der Aushändigung von Medikamenten beraten werden (s. Abbildung III - 20).

Abbildung III - 20: Beratung bei der Aushändigung von Medikamenten

(Frage: "Wie oft berăt man Sie in der Apotheke bei der Aushändigung von Medikamenten?")

Bel ungefahr jedem Apothekenbesuch

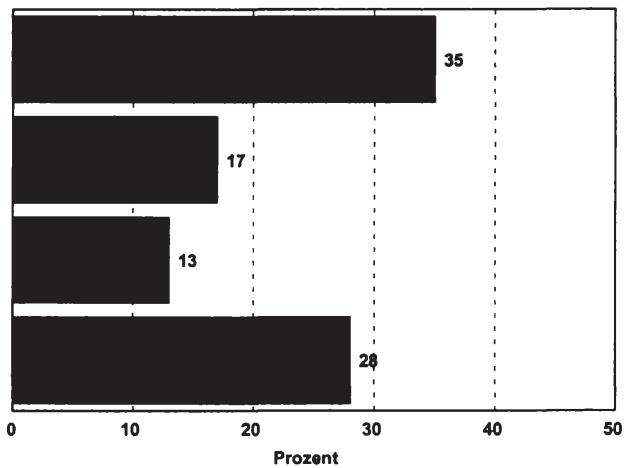

Selektion: Personen, die ihr Rezept selber einlosen oder die Medikamente von der Apotheke nach Hause geliefert bekommen $(n=941)$

Quelle: INIFES, eigene Darstellung.

(Ergebnisse einer von TNS EMNID im Auftrag von INIFES im Dezember 2000 durchgeführten CATI-Befragung).

Immerhin ein Drittel der Kunden (28,0 Prozent) wird bei fast keinem Apothekenbesuch beraten, 30,0 Prozent werden sporadisch bei ca. jedem zweiten oder dritten Apothekenbesuch und nur 35,0 Prozent werden bei jedem Apothekenbesuch beraten. Obwohl gerade die Chroniker angaben, besonders viel Wert darauf zu legen, in der Apotheke beraten zu werden, werden 26,0 Prozent von ihnen bei fast keinem Apothekenbesuch beraten. 33,0 Prozent der Personen, die unter einer dauerhaften, vom Arzt behandelten Gesundheitsstörung leiden, erhalten bei fast jedem Apothekenbesuch eine Beratung. Ein Zusammenhang ist zwischen der tatsächlichen und der erwarteten Beratung bei der Aushändigung von Medikamenten zu erkennen. Kunden, die angeben, immer oder öfters bei der Medikamentenübergabe beraten zu werden, ist das Kriterium der umfassenden Beratung bei der Auswahl der Apotheke meist wichtig bis sehr wichtig (89,0 Prozent bzw. 84,0 Prozent). Personen, die fast nie in der Apotheke beraten werden, achten zu 61,0 Prozent auf eine umfassende Beratung bei der Wahl ihrer Apotheke. 
Das Kriterium eines kostenlosen Heimlieferservices von Medikamenten bei der Auswahl ihrer Apotheke ist 42,0 Prozent der Befragten wichtig bis sehr wichtig. Diesen Service schätzen vor allem Chroniker (50,0 Prozent), Menschen, die alleine in einer Wohnung leben (50,0 Prozent) und Frauen der Altersklassen 50-59 Jahre (50,0 Prozent) sowie Personen über 60 Jahre (57,0 Prozent). Die Sozio-Demographie der Befragung hat ergeben, dass vor allem Frauen über 60 Jahre in einem Einpersonenhaushalt leben (46,0 Prozent).

Laut der Befragung ist es 55,0 Prozent der Bundesbürger wichtig bis sehr wichtig, eine Stammapotheke zu haben, in der sie ihre Rezepte einlösen. Mit zunehmenden Alter ist ein signifikanter Anstieg der Wichtigkeit dieses Kriteriums zu erkennen. Für die älteren Menschen ist eine Stammapotheke mehr als nur ein Ort, um das Rezept einzulösen. Sie legen, dies ergaben die Expertengespräche, vor allem Wert auf den persönlichen Kontakt und eine Beratung. Die Stammapotheke liegen vielfach alle relevanten Daten und Informationen bzgl. des Patienten und seiner Medikamentenhistorie vor.

Zusammenfassend ergab die Befragung der Bevölkerung bzgl. ihrer Einstellung zum Vertriebskanal Apotheke, dass sie die Rezepte mit verschreibungspflichtigen Arzneimitteln überwiegend in der Offizinapotheke einlöst. Aufgrund einer eingeschränkten Mobilität sind einige Menschen darauf angewiesen, dass eine vertraute Person die Rolle des "Rezeptüberbringers" und "Medikamentenbesorgers" für sie übernimmt. Überdies billigt die Ausnahmeregelung des $\S 17$, II ApBetrO in begründeten Einzelfällen eine Versendung aus der Apotheke oder die Zustellung durch einen Boten an den Arzneimittelempfänger zu. 2,0 Prozent der Befragten erhalten bislang auf diesem Wege ihre Medikamente. Bei den Chronikern beträgt der Anteil 5,0 Prozent.

Bei der Auswahl der Apotheke, in der das Rezept eingelöst wird, werden von der Bevölkerung vor allem Kriterien einer schnellen und guten Erreichbarkeit sowie einer umfassenden Beratung zu Grunde gelegt. Entgegen der bisherigen Annahmen legen besonders die Chroniker viel Wert auf die Beratung in der Apotheke (83,0 Prozent). Obwohl sie vielfach über Jahre dieselben Medikamente bekommen, erwarten sie von der Apotheke, in der sie ihr Rezept einlösen, umfassend beraten zu werden.

19,0 Prozent aller Befragten gaben an, in einem Einpersonenhaushalt zu leben. 


\subsubsection{Einstellung zu alternativen Distributionswegen}

Das Verfahren der Arzneimittelversorgung über Rezept erfolgt für die Konsumenten bislang nach gesetzlichen Vorgaben. Der Arzt bestimmt das Medikament und die Medikation und überreicht das papiergebundene Rezept dem Patienten zur Vorlage und Einlösung in der Apotheke.

Auf die Frage, ob die Auskunftspersonen es sich vorstellen können, in einigen Fällen die vom Arzt verordneten Arzneimittel von der Apotheke auch per Post zugeschickt zu bekommen, antworteten 42,0 Prozent aller Befragten mit Ja, 57,0 Prozent lehnen einen solchen Weg des Arzneimittelbezugs ab. Eine solch ergänzende Form des Arzneimittelbezugs begrüßen die Berufstätigen (47,0 Prozent) und Personen zwischen 14 und 49 Jahre, wobei die Zustimmung der Männer zwischen 30 und 39 Jahre mit 60,0 Prozent einen signifikanten Unterschied zur Gesamtheit der befragten Personen aufweist. Eine zurückhaltende und eher skeptische Grundeinstellung gegenüber alternativer Vertriebswege zeichnete sich innerhalb der Altersklassen 50-59 Jahre und über 60 Jahre bereits bei dem von INIFES durchgeführten Pretest ab. Die Hauptbefragung ergab, dass sich 37,0 Prozent im Alter von 50-59 Jahre und 31,0 Prozent der über 60jährigen vorstellen können, in einigen Fällen die vom Arzt verordneten Arzneimittel über den Postweg zugeschickt zu bekommen.

Während Patienten, wie bereits gezeigt, durchaus den kostenlosen Heimlieferservice von Medikamenten durch die Apotheke in Anspruch nehmen, können bzw. wollen es sich vielfach ältere Menschen nicht vorstellen, Arzneimittel auch auf einem anderen Vertriebsweg zu erhalten als bisher. Die Besonderheit des Gutes "Arzneimittel" widerstrebt einem postalischen Bezug. Es überwiegt in vielen Fällen Angst und Unsicherheit. Der von INIFES durchgeführte Pretest bei der Bevölkerung hat gezeigt, dass der Öffentlichkeit bislang noch keine objektiven Informationen bezüglich eines Versandhandels mit Medikamenten vorliegen. Vielmehr wurden bislang nur negative Ergebnisse durchgeführter Testkäufe bei Internetapotheken veröffentlicht. Zudem können sich viele Befragte nichts unter dem Begriff "Versandapotheke" vorstellen. Die angesprochenen Probleme haben INIFES dazu veranlasst, eine "Filterfrage" in den Befragungsbogen der Bevölkerung aufzunehmen. Bevor die Grundgesamtheit ( $n=1006$ ) gefragt wurde, ob sie sich den Medikamentenbezug über eine Versandapotheke vorstellen kann, wurde folgende Frage gestellt: Können Sie sich vorstellen, in einigen Fällen die vom Arzt verordneten Arzneimittel von der Apotheke auch per Post zugeschickt zu bekommen? Ausschließlich die Personen, die sich einen solchen Weg des Arzneimittelbezugs vorstellen können $(n=418)$, wurden im Folgenden zu ihrer grundsätzlichen Einstellung bezüglich einer Versandapotheke befragt.

In einigen europäischen Ländern, wie der Schweiz und den Niederlanden, haben sich Versandapotheken bereits etabliert. Der Bezug der Arzneimittel läuft folgendermaßen ab: Das Rezept wird vom Arzt oder dem Patienten an eine Versandapotheke geschickt. Diese stellt die Medikamente zusammen und liefert sie - meist per Post 
und binnen 48 Stunden - nach Hause. Die Apotheke bietet eine telefonische Beratung rund um die Uhr an.

Deutschen Patienten ist es bislang erlaubt, Arzneimittel aus anderen EU-Staaten nur zum Eigenbedarf auch auf dem Wege des Versandhandels zu beziehen. ${ }^{86}$

Im Folgenden wurden die Personen, die sich vorstellen können, Arzneimittel direkt per Post zugeschickt zu bekommen, gebeten, zu verschiedenen Aussagen bzgl. einer Versandapotheke Stellung zu beziehen (s. Tabelle III - 6).

Tabelle III - 6: Bewertung verschiedener Aussagen zur Versandapotheke

(Frage: "Stellen Sich sich nun vor, es würde eine solche Versandapotheke auch in Deutschland existieren. Stimmen Sie folgenden Aussagen zu?")

\begin{tabular}{|c|c|c|}
\hline Aussagen & $\begin{array}{l}\text { stimme zu } \\
\text { Befragte, die sich den } \\
\text { Versand per Post vor- } \\
\text { stellen können } \\
\quad(n=418)\end{array}$ & $\begin{array}{l}\text { stimme zu } \\
\text { entspricht dem } \\
\text { Wert aller Be- } \\
\text { fragten } \\
(n=1006)\end{array}$ \\
\hline $\begin{array}{l}\text { Sofern kein Risiko besteht, wäre eine Versandapotheke } \\
\text { eine sinnvolle Alternative für mich. }\end{array}$ & 70,0 Prozent & 29,1 Prozent \\
\hline $\begin{array}{l}\text { Wegen der schlechten Erreichbarkeit der nächsten Apo- } \\
\text { theke wäre eine Versandapotheke eine sinnvolle Alterna- } \\
\text { tive für mich. }\end{array}$ & 50,0 Prozent & 20,7 Prozent \\
\hline $\begin{array}{l}\text { Bei eingeschränkter Mobilität wäre eine Versandapotheke } \\
\text { eine sinnvolle Alternative für mich. }\end{array}$ & 86,0 Prozent & 35,7 Prozent \\
\hline $\begin{array}{l}\text { Bei dauerhaften gesundheitlichen Störungen und planba- } \\
\text { rem Medikamentenkonsum wäre eine Versandapotheke } \\
\text { eine sinnvolle Alternative für mich. }\end{array}$ & 85,0 Prozent & 35,3 Prozent \\
\hline $\begin{array}{l}\text { Bei akuten Erkrankungen wäre eine Versandapotheke ei- } \\
\text { ne sinnvolle Alternative für mich. }\end{array}$ & 62,0 Prozent & 25,8 Prozent \\
\hline $\begin{array}{l}\text { Da mir die Beratung und der soziale Kontakt in der Apo- } \\
\text { theke sehr wichtig sind, wäre eine Versandapotheke keine } \\
\text { sinnvolle Alternative für mich. }\end{array}$ & 39,0 Prozent & 16,2 Prozent \\
\hline
\end{tabular}

Quelle: INIFES, eigene Darstellung.

(Ergebnisse einer von TNS EMNID im Auftrag von INIFES im Dezember 2000 durchgeführten CATI-Befragung).

Die Befragung ergab, dass eine Versandapotheke für 70,0 Prozent derjenigen, die sich den Versand von Arzneimitteln per Post vorstellen können, durchaus eine Alternative sein kann, sofern kein Risiko besteht. Bezogen auf die Grundgesamtheit be-

85

Auskunft anlässlich eines Expertengesprächs.

86

Korzilius, H. (1999), "Surfen" in gefährlichen Gewässern - Medikamente im Internet -, in: Deutsches Ärzteblatt, Nr. 6, S. A-314. 
trägt die Zustimmung 29,0 Prozent. Es gilt daher für einheitliche Qualitätsstandards nach ISO 9002, für eine transparente Arzneimittelsicherheit sowie für gleiche Rahmenbedingungen zu sorgen. Frauen über 60 Jahre (44,0 Prozent) sehen, selbst wenn es keine Risiken gäbe, in einer Versandapotheke keine Alternative. Menschen, die häufiger im Monat ein Rezept in der Apotheke einlösen, präferieren eine Versandapotheke (76,0 Prozent).

Wie die Ergebnisse der Apotheker- und Ärztebefragung gezeigt haben ${ }^{87}$, existiert in der Bundesrepublik Deutschland ein angemessener Versorgungsgrad mit Apotheken, vielfach sogar eine Überversorgung. Wegen der schlechten Erreichbarkeit der nächsten Apotheke wäre für 20,7 Prozent der Bürger eine Versandapotheke eine sinnvolle Alternative. Dies trifft vor allem für BIK-Stadtregionen mit bis zu 5.000 Einwohnern zu (65,0 Prozent). Hinsichtlich sonstiger Kriterien, wie Alter, Geschlecht oder dem Gesundheitszustand, existieren keine Unterschiede bei der Bewertung dieser Frage.

Der Annahme entsprechend, dass Personen mit eingeschränkter Mobilität eine potentielle Zielgruppe für eine Versandapotheke sind, gaben 86,0 Prozent der Befragten, die sich eine Zusendung per Post vorstellen können, an, dass bei eingeschränkter Mobilität eine Versandapotheke eine sinnvolle Alternative für sie sei $(35,7$ Prozent der Gesamtbevölkerung). Dem stimmen besonders Personen zwischen 14 und 29 Jahren (36,9 Prozent) und 30-39 Jahren (39,1 Prozent) zu. Gut ein Viertel der über 60jährigen (25,0 Prozent) teilt diese Ansicht nicht. Sollten die chronisch Kranken in ihrer Mobilität zukünftig eingeschränkt sein, würden sie durchaus die Dienste einer Versandapotheke in Anspruch nehmen (33,6 Prozent).

Bei dauerhaften gesundheitlichen Störungen und planbarem Medikamentenkonsum ist für 85,0 Prozent der befragten Personen eine Versandapotheke eine sinnvolle Alternative. Mit zunehmenden Alter ist ein signifikanter Anstieg der Ablehnung dieser Aussage zu erkennen. Bei den über 60jährigen wächst die Zustimmung wieder an (76,0 Prozent). Chroniker, deren Situation durch diese Aussage beschrieben wird, sehen in einer Versandapotheke zu 72,0 Prozent eine Alternative, 27,0 Prozent könnten auf sie verzichten.

Akute Erkrankungen erfordern häufig einen sofortigen Beginn der medikamentösen Therapie. Eine Versandapotheke mit Lieferzeiten von oftmals mehr als 24 Stunden ist unter diesen Bedingungen keine sinnvolle Alternative zu der Präsenzapotheke. Diese Ansicht vertreten 37,0 Prozent der Befragten. Eine Versandapotheke sei bei akuten Erkrankungen eine sinnvolle Alternative, sagten 62,0 Prozent. Bei den 1429jährigen stimmen dem 74,0 Prozent zu.

Für viele Menschen ist die Apotheke mehr als nur ein Ort, an dem sie ihr Rezept einlösen, sondern sie bietet auch den Raum für soziale Kontakte und Kommunikation.

Vgl. 3.4.2.2 und 3.4.3.2. 
Oft sind diese Menschen Stammkunde einer Apotheke (48,0 Prozent) und haben einen persönlichen Kontakt zu "ihrem" Apotheker hergestellt. Aus diesem Grund und wegen der Beratung in der Apotheke sehen 39,0 Prozent in einer Versandapotheke keine sinnvolle Alternative für sich. Dieser Wert steigt mit zunehmenden Alter an und beträgt in der Altersklasse über 60 Jahre 58,0 Prozent. Chroniker gaben an, dass sie Wert auf den sozialen Kontakt legen und Beratung sehr wichtig ist und sie dies nicht missen wollen (51,0 Prozent). Demgegenüber können 56,0 Prozent auf die Beratung und den sozialen Kontakt in der Apotheke verzichten (bei den chronisch Kranken 40,0 Prozent). Besonders Personen, die nicht mehr als zwei- bis dreimal pro Jahr ein Rezept in der Apotheke einlösen (64,0 Prozent) und Kunden, die bei fast keinem Apothekenbesuch beraten werden (70,0 Prozent), erachten die Beratung und den sozialen Kontakt in der Apotheke für nicht wichtig bzw. für nicht existent.

Die Einstellung der Bevölkerung zu alternativen Distributionswegen wird vielfach dadurch beeinflusst, dass der Öfentlichkeit bislang noch keine objektiven Informationen bezüglich eines Versandhandels mit Medikamenten vorliegen. Vielmehr wurden nur negative Ergebnisse durchgeführter Testkäufe bei Internetapotheken veröffentlicht. Zudem können sich viele Befragte nichts unter dem Begriff "Versandapotheke" vorstellen.

Fast die Hälfte der Bevölkerung kann sich vorstellen, die vom Arzt verordneten Arzneimittel von der Apotheke auch per Post zugeschickt zu bekommen. Von den Befragten, die sich persönlich die direkte Zustellung von Arzneimitteln vorstellen können ( $n=418)$, gaben 70,0 Prozent an, dass eine Versandapotheke durchaus eine Alternative für sie sein könne, sofern kein Risiko besteht. Es gilt daher für einheitliche Qualitätsstandards nach ISO 9002 für eine transparente Arzneimittelsicherheit sowie für gleiche Rahmenbedingungen zu sorgen.

Der Annahme entsprechend, dass Personen mit eingeschränkter Mobilität eine potentielle Zielgruppe für eine Versandapotheke sind (Arbeitshypothese 11), gaben 86,0 Prozent der Befragten an, dass bei eingeschränkter Mobilität eine Versandapotheke eine sinnvolle Alternative für sie sei. Sollten die chronisch Kranken in ihrer Mobilität zukünftig eingeschränkt sein, würden sie durchaus die Dienste einer Versandapotheke in Anspruch nehmen.

Arbeitshypothese 13 besagt, dass Voraussetzung für die Akzeptanz von Versandapotheken in der Bevölkerung hohe Qualitätsanforderungen (z.B. bzgl. Zustellungssicherheit und Beratungsangebot) sind - Qualitätsansprüche, die von reinen Internetapotheken $^{88}$ oftmals nicht garantiert werden können. Diese Arbeitshypothese wird durch die Ergebnisse der Befragung gestützt: Um dem Kunden überhaupt einen Anreiz zu geben, seine Medikamente bei einer Versandapotheke zu bestellen, muss die Zustellung sicher und kostenlos sein. Für 85,0 Prozent der Befragten, die sich

88

Zur Differenzierung zwischen Versandapotheken und Internetapotheken vgl. Sterzel, A., Wassener, D., (2001), Arzneimitteldistribution in Deutschland - Versandapotheken als Reformoption?, in: Arbeit und Sozialpolitik 1-2/2001, S. 17 - 18. 
den Versand vorstellen können, ist es daher wichtig bis sehr wichtig, dass sie die bestellten Medikamente sicher und kostenlos zugestellt bekommen. Ein weiteres zu bewertendes Kriterium stellt die Medikamentenbelieferung binnen 48 Stunden dar. 86,0 Prozent halten dies für wichtig bis sehr wichtig, während 71 Prozent dieser Befragten eine 24-Stunden Telefonhotline als Beratungsservice für sehr wichtig oder wichtig halten.

\subsubsection{Einstellung zum Modell der Versandapotheke}

Während im vorherigen Abschnitt die Vorstellbarkeit der Zusendung von verordneten Arzneimitteln per Post erfragt wurde, wird im Folgenden explizit die Vorstellbarkeit des Bezugs von Medikamenten über eine Versandapotheke untersucht. 39,0 Prozent der Bundesbürger können sich durchaus vorstellen, Medikamente über eine Versandapotheke zu beziehen; 60,0 Prozent lehnen dies ab (s. Abbildung III - 21).

Abbildung III - 21: Vorstellbarkeit des Bezugs von Medikamenten über eine Versandapotheke

(Frage: “Könnten Sie sich vorstellen, Medikamente über eine Versandapotheke zu beziehen?“)

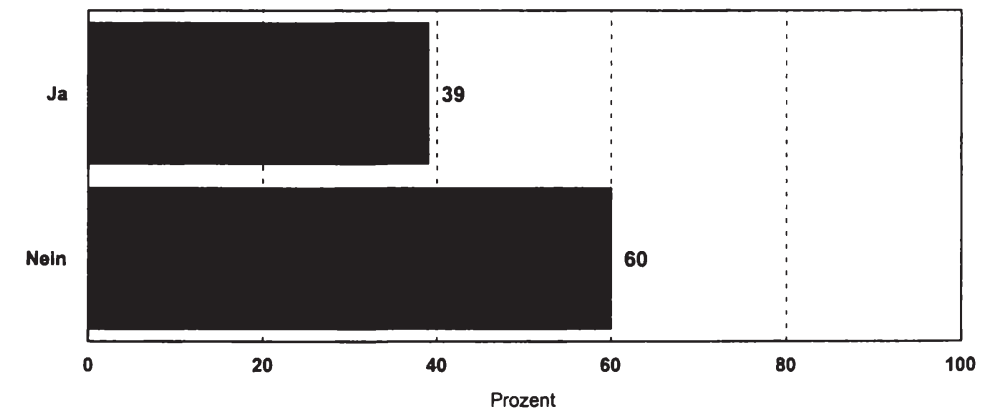

$n=1006$ Befragte

Quelle: INIFES, eigene Darstellung.

(Ergebnisse einer von TNS EMNID im Auftrag von INIFES im Dezember 2000 durchgeführten CATI-Befragung).

Ein signifikanter Unterschied besteht zwischen Männern (45,0 Prozent) und Frauen (34,0 Prozent). Dies mag daran liegen, dass auch der Anteil der Internet-User in dieser Altersklasse bei den Männern (36,0 Prozent) höher ist als bei den Frauen (27,0 Prozent) und Männer den neuen Medien und dem neuen Vertriebsweg gegenüber aufgeschlossener sind. Die Ergebnisse zeigen, dass keine Korrelation zwischen der Nutzung des Internets und des Bezugs von Arzneimitteln über eine Versandapotheke besteht. Die Bereitschaft, eine Versandapotheke in Anspruch zu nehmen, ist mit zu- 
nehmendem Alter rückläufig. Während der Anteil der 30-39jährigen 53,0 Prozent beträgt, liegt er bei den über 60jährigen nur noch bei 20,0 Prozent.

Bislang wird die Annahme gehegt, dass ein Versandhandel vielfach für Personen von Vorteil ist, die in ihrer Mobilität eingeschränkt, chronisch erkrankt oder berufstätig sind (Arbeitshypothese 10). Die Bevölkerungsbefragung unterstützt diese Arbeitshypothese nur teilweise. Während 43,0 Prozent der Befragten ohne eine dauerhafte, vom Arzt zu behandelnde Gesundheitsstörung sich - trotz des derzeit noch geringen Wissensstands über die Möglichkeiten und Gefahren des Pharmaversandes - vorstellen können, "... Medikamente über eine Versandapotheke zu beziehen", liegt dieser Anteil bei chronisch Kranken bei 30 \% (vgl. Abbildung III - 22).

Bei dem Wert für die Chroniker ist jedoch zu berücksichtigen, dass er sich auf die gesamte betrachtete Grundgesamtheit bezieht. Bei gesonderter Betrachtung der Vorteilhaftigkeit des Versandhandels für chronisch erkrankte Personen zeigt sich, dass diese Patientenklientel regelmäßig mehrere vom Arzt verordnete Arzneimittel benötigt $^{89}$ und öfters in die Apotheke geht, um ein Rezept einzulösen.

Abbildung III - 22: Vorstellbarkeit des Bezugs von Medikamenten über eine Versandapotheke - in Abhängigkeit vom Gesundheitszustand

ohne dauerhafte Erkrankung

chronisch Kranke

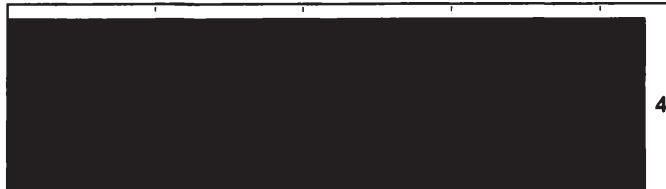

43

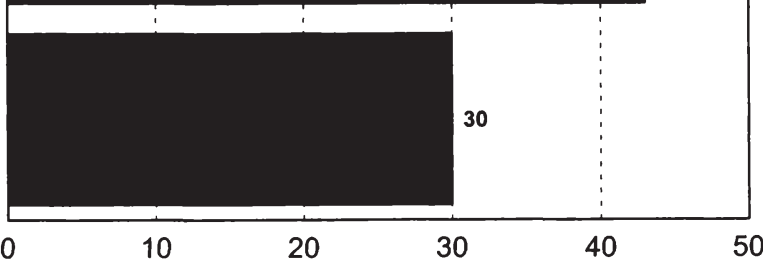

Prozent

$n=1006$

Quelle: INIFES, eigene Darstellung.

(Ergebnisse einer von TNS EMNID im Auftrag von INIFES im Dezember 2000 durchgeführten CATI-Befragung).

44,3 Prozent der Chroniker nehmen regelmäßig 2-4 verschiedene, vom Arzt verordnete, Arzneimittel pro Tag ein.

90

49,3 Prozent der Chroniker I̋sen ein- bis zweimal im Monat ein Rezept ein. 
Die Annahme, dass Patienten, die viele verschiedene vom Arzt verordnete Arzneimittel einnehmen, ein besonderes Interesse an einer Versandapotheke hätten, lässt sich anhand der vorliegenden Ergebnisse nicht bestätigen. Die Akzeptanz gegenüber der Versandapotheke beträgt bei Patienten, die 2-4 Medikamente pro Tag einnehmen 36,0 Prozent, bei einem Medikament pro Tag 23,0 Prozent und 11,0 Prozent derer, die sich den Bezug ihrer Medikamente über die Versandapotheke vorstellen können, nehmen aufgrund gesundheitlicher Beeinträchtigungen regelmäßig 5 oder mehr Präparate pro Tag ein.

Die Befragung ergab, dass 90,0 Prozent der Befragten, die eine Versandapotheke akzeptieren, das Rezept selber einlösen, 3,0 Prozent die Medikamente häufiger von der Apotheke nach Hause geliefert bekommen. Des weiteren ist den Ergebnissen zu entnehmen, dass Personen, bei denen die Vorstellbarkeit des Bezugs von Medikamenten über eine Versandapotheke gegeben ist, relativ selten in die Apotheke gehen, um ein Rezept einzulösen (50,0 Prozent gehen nicht mehr als zwei- bis dreimal pro Jahr).

Überdies sind für Personen, die sich vorstellen können, Medikamente über eine Versandapotheke zu beziehen, folgende Punkte bzw. Voraussetzungen von Interesse (s. Tabelle III - 7):

Tabelle III - 7: Rahmenbedingungen für die Akzeptanz einer Versandapotheke

(Frage: "Stellen Sie sich nun vor, es würde eine solche Versandapotheke auch in Deutschland existieren. Stimmen Sie folgenden Aussagen zu?" "Welche der folgenden Anforderungen müsste eine solche Versandapotheke Ihrer Meinung nach mindestens erfüllen?")

\begin{tabular}{|c|c|c|}
\hline Voraussetzungen & $\begin{array}{c}\text { "sehr wichtig" } \\
\text { und } \\
\text { "wichtig" } \\
\text { Befragte, die sich den Be- } \\
\text { zug über eine Versand- } \\
\text { apotheke vorstellen kön- } \\
\text { nen ( } n=298)\end{array}$ & $\begin{array}{l}\text { "sehr wichtig" } \\
\text { und } \\
\text { "wichtig" } \\
\text { entspricht dem Wert aller } \\
\text { Befragten }(n=1006)\end{array}$ \\
\hline Risikoloser Bezug & 85,0 Prozent & 25,2 Prozent \\
\hline Vorteil bei eingeschränkter Mobilität & 94,0 Prozent & 27,8 Prozent \\
\hline $\begin{array}{l}\text { Vorteil bei dauerhaften gesundheitlichen Stö- } \\
\text { rungen und planbarem Medikamentenbezug }\end{array}$ & 93,0 Prozent & 27,5 Prozent \\
\hline $\begin{array}{l}\text { Kostenlose und sichere Zustellung der Medi- } \\
\text { kamente }\end{array}$ & 88,0 Prozent & 26,1 Prozent \\
\hline $\begin{array}{l}\text { Medikamentenbelieferung binnen } 48 \text { Stun- } \\
\text { den }\end{array}$ & 90,0 Prozent & 26,7 Prozent \\
\hline 24 Stunden Telefon-Hotline & 74,0 Prozent & 21,9 Prozent \\
\hline
\end{tabular}

Quelle: INIFES, eigene Darstellung.

(Ergebnisse einer von TNS EMNID im Auftrag von INIFES im Dezember 2000 durchgeführten CATI-Befragung). 


\subsubsection{Anforderungen an eine Versandapotheke}

Gesetzt den Fall, dass das Versandhandelsverbot - $§ 43$ AMG - aufgehoben wird und die Bürger ihre Rezepte auch bei einer Versandapotheke einlösen könnten, müsste eine solche Apotheke bestimmte Mindestanforderungen erfüllen. Personen, die sich vorstellen können, Arzneimittel direkt per Post zugeschickt zu bekommen, wurden gebeten, Anforderungen, die eine Versandapotheke ihrer Meinung nach mindestens erfüllen müssen, zu bewerten (s. Abbildung III - 23).

\section{Abbildung III - 23: Anforderungen an eine Versandapotheke}

(Frage: "Welche der folgenden Anforderungen müsste eine solche Versandapotheke Ihrer Meinung nach mindestens erfüllen?")

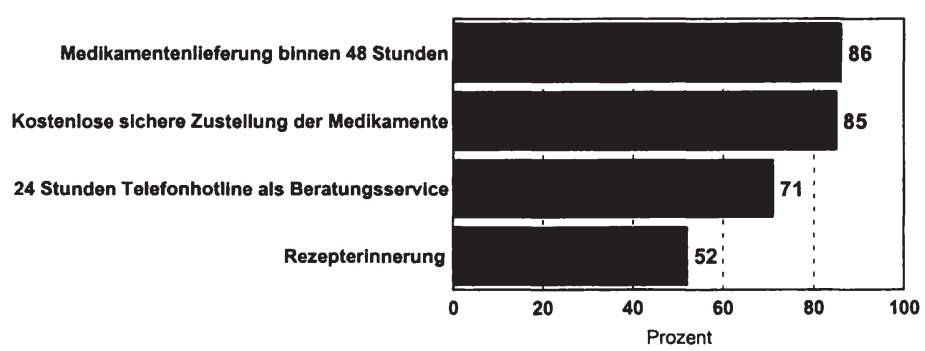

Selektion: Personen, die sich vorstellen können, Arzneimittel direkt per Post zugeschickt zu bekommen ( $n=418$ ) Top-2-Boxes ("sehr wichtig" und "wichtig"); 1=sehr wichtig, 5=völlig unwichtig

Quelle: INIFES, eigene Darstellung.

(Ergebnisse einer von TNS EMNID im Auftrag von INIFES im Dezember 2000 durchgeführten CATI-Befragung).

Um dem Konsumenten überhaupt einen Anreiz zu geben, seine Medikamente bei einer Versandapotheke zu bestellen, muss die Zustellung sicher und kostenlos sein. Für 85,0 Prozent der Befragten, die sich den Versand per Post vorstellen können, ist es daher wichtig bis sehr wichtig, dass sie die bestellten Medikamente sicher und kostenlos zugestellt bekommen. Für Personen zwischen 30 und 39 Jahren ist dies zu 93,0 Prozent unabdingbare Voraussetzung.

Ein weiteres zu bewertendes Kriterium stellt die Medikamentenlieferung binnen 48 Stunden dar. 86,0 Prozent derer, die sich den Versand per Post vorstellen können, halten dies für wichtig bis sehr wichtig. Dieser Wert ist bei allen untersuchten Faktoren nahezu konstant. Personen, die unter einer dauerhaften, vom Arzt behandelten Gesundheitsstörung leiden und deren Medikamentenbedarf in der Regel planbar ist, ist dieses Kriterium in 81,0 Prozent der untersuchten Fälle wichtig bis sehr wichtig. 
Dem von den Apothekern geäußerten Kritikpunkt einer nicht vorhandenen Beratung, entgegnen bereits im Ausland bestehende Versandapotheken mit einer 24 Stunden Telefon-Hotline als Beratungsservice. Die Befragung hat ergeben, dass 71,0 Prozent der Befragten, die sich den Versand per Post vorstellen können, einen solchen Service für wichtig bis sehr wichtig einschätzen. Der Anteil der 30-39jährigen (85,0 Prozent) an diesem Ergebnis ist signifikant höher als bei den über 60jährigen (60 Prozent). Personen, die bereits in der Präsenzapotheke großen Wert auf eine umfassende Beratung gelegt haben, ist auch eine 24 Stunden Telefon-Hotline als Beratungsservice wichtiger (79,0 Prozent) als den Personen, die ganz auf eine Beratung verzichten können (40,0 Prozent).

Versandapotheken im Ausland ${ }^{91}$ bieten ihren Kunden den Service der Rezepterinnerung an. Dies kann vor allem für Chroniker und Patienten mit einer Dauermedikation eine große Erleichterung sein. Als wichtig bis sehr wichtig bewerten 52,0 Prozent der Befragten dieses Kriterium. Während die jüngeren Altersklassen dem eher zustimmen, beträgt der Wert bei den über 60jährigen 44,0 Prozent (13,0 Prozent ist eine Rezepterinnerung völlig unwichtig). Die Zustimmung bei den Chronikern liegt bei 42,0 Prozent. Personen, die bei einer traditionellen Apotheke Wert auf einen kostenlosen Heimlieferservice von Medikamenten gelegt haben, bewerten das Kriterium der Rezepterinnerung zu 62,0 Prozent für wichtig bis sehr wichtig.

Dass eine Versandapotheke für eine Verbesserung bei der Versorgung mit Arzneimitteln auf Rezept für sie persönlich sorgen würde, glauben 44,0 Prozent der Befragten, 53,0 Prozent gehen davon aus, dass ihre persönliche Versorgung durch eine Versandapotheke nicht verbessert wird. Mit zunehmenden Alter ist eine signifikante Abnahme der Zustimmung bzgl. dieser Aussage zu erkennen. Vor allem Personen über 60 Jahre (31,0 Prozent) und chronisch Kranke (34,0 Prozent) glauben nicht an eine Verbesserung der persönlichen Versorgung mit Arzneimitteln auf Rezept durch Versandapotheken.

\subsubsection{Fazit}

Die Einstellung der Bevölkerung zu alternativen Distributionswegen wird vielfach dadurch beeinflusst, dass der Öfentlichkeit bislang noch keine objektiven Informationen bezüglich eines Versandhandels mit Medikamenten vorliegen. Vielmehr wurden nur negative Ergebnisse durchgeführter Testkäufe bei Internetapotheken veröffentlicht. Zudem können sich viele Befragte nichts unter dem Begriff "Versandapotheke" vorstellen.

Die Auswertung der vorliegenden Daten hat ergeben, dass sich zum gegenwärtigen Zeitpunkt mehr als die Hälfte der Konsumenten nicht vorstellen können, in einigen Fällen die vom Arzt verordneten Arzneimittel von der Apotheke auch per Post zugeschickt zu bekommen und 60,0 Prozent sich nicht vorstellen können, Medikamente

Z.B. MediService in der Schweiz und DocMorris in den Niederlanden. 
über eine Versandapotheke zu beziehen. Die von INIFES im Vorfeld der Befragung durchgeführten Pretests und die im Rahmen der Studie durchgeführten Expertengespräche verdeutlichten aber, dass trotz eines geringen Kenntnisstandes der Bevölkerung bzgl. einer Versandapotheke ein großes Interesse an dieser Thematik besteht. Die bislang bestehenden Annahmen über die Vorteilhaftigkeit einer Versandapotheke für bestimmte Zielgruppen konnten aufgrund der Aussagen der Bevölkerung nicht widerlegt werden. 30,0 Prozent der chronisch erkrankten Personen können sich vorstellen, Medikamente über eine Versandapotheke zu beziehen (bei nicht chronisch Kranken liegt der Wert bei 42 Prozent). Vor allem die Patientenklientel der Chroniker benötigt aufgrund der gesundheitlichen Beeinträchtigungen vielfach mehrere vom Arzt verordnete Arzneimittel und geht mindestens ein- bis zweimal im Monat in die Apotheke, um ein Rezept einzulösen. Des weiteren legt diese Patientenklientel legt mit 83,0 Prozent bei Auswahl der Apotheke, in der sie ihr Rezept einlöst, einen signifikant höheren Wert auf eine umfassende Beratung, als Personen ohne eine dauerhafte, vom Arzt zu behandelnde Gesundheitsstörung. Eine Versandapotheke mit den entsprechenden Service-Angeboten, wie zum Beispiel einer automatischen Rezepterinnerung und einem umfassenden Beratungsangebot, stellt für diese Patientenklientel durchaus eine alternative Bezugsquelle dar.

\subsection{Zusammenfassung}

Zur Evaluation der Akzeptanz und der generellen Einstellung niedergelassener Apotheker und Ärzte sowie der Bevölkerung gegenüber einer Versandapotheke hat das Internationale Institut für empirische Sozialökonomie (INIFES) im Dezember 2000 eine repräsentative CATI-Befragung durchgeführt. Ziel der Befragung war es, Anhaltspunkte zu erhalten über

- die Einstellung zum Vertriebskanal Apotheke

- die potentiellen Zielgruppen für eine Versandapotheke

- die Akzeptanz eines Versandhandels

- die Chancen und Risiken einer Versandapotheke

\section{Vertriebskanal Apotheke}

Bislang lösen Patienten aufgrund des $§ 43$ Arzneimittelgesetz (AMG) die Rezepte mit verschreibungspflichtigen Arzneimitteln in der Apotheke ein, wo sie die vom Arzt verordneten Medikamente ausgehändigt bekommen. Die quantitative Arzneimittelversorgung in der Bundesrepublik Deutschland wird von 21.590 Apotheken $^{92}$ sicherge- $^{2}$ stellt. Von einer Überversorgung sprechen 32,0 Prozent der Ärzte und immerhin 27,0 Prozent der Apotheker. Als gerade angemessen bezeichnen 72,0 Prozent der Apotheker die Apothekendichte (bei den Ärzten liegt dieser Wert bei 59,0 Prozent). Eine Unterversorgung wird als kaum existent betrachtet. Die Versorgung der Bevölkerung mit Arzneimitteln ist somit sichergestellt (s. Abbildung III - 24).

92 ABDA - Bundesvereinigung Deutscher Apothekerverbände (2000), Die Apotheke: Zahlen - Daten - Fakten (1999). 
Abbildung III - 24: Beurteilung der Apothekendichte

(Frage: "Überversorgung mit Apotheken wird heute in manchen Gebieten als Problem angesehen. Wie beurteilen Sie die Situation im Einzugsgebiet Ihrer Apotheke/Praxis?")

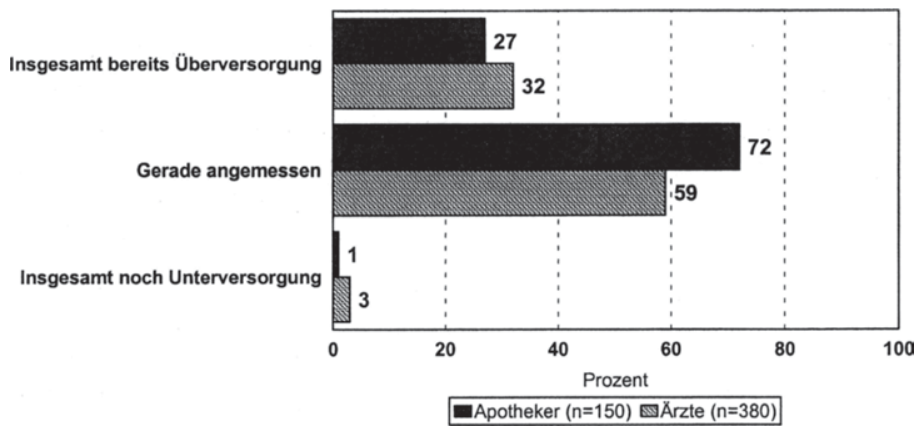

Quelle : INIFES, eigene Darstellung.

(Ergebnisse einer von TNS EMNID im Auftrag von INIFES im Dezember 2000 durchgeführten CATI-Befragung).

Die überwiegende Anzahl der befragten Bürger lösen ein Rezept selber ein, wobei der Wert im Alter rückläufig ist. Dies steht jedoch im Widerspruch zu den Angaben der befragten Apotheker. 49,0 Prozent gaben an, dass zwischen 20,0 Prozent und 50,0 Prozent der Kunden Rezepte nicht für sich selber, sondern für eine andere Person als "Besorger" einlösen. Der Anteil der "Rezeptbesorger" beträgt bei 3,0 Prozent der Apotheken sogar mehr als 50,0 Prozent. Die sog. "Besorger-Problematik" beschreibt somit eine nicht zu unterschätzende Distributionsstufe innerhalb des "Vertriebskanals" Apotheke. Viele Arzneimittelbezieher sind in ihrer Mobilität aufgrund einer Krankheit, des Alters oder der Berufstätigkeit stark eingeschränkt. Eine vertraute Person, welche die Rolle eines "Rezeptüberbringers" und "Medikamentenbesorgers" übernimmt, ist oft das nötige Bindeglied zwischen Apotheke und Patienten/Kunden. Für 7,0 Prozent der Bundesbürger ist es nach eigenen Angaben üblich, Medikamente häufiger von der Apotheke nach Hause geliefert zu bekommen bzw. das Rezept von Angehörigen, Vertrauenspersonen oder dem Arzt einlösen zu lassen. Vor allem Personen der Altersklasse 30 - 39 Jahre (8,0 Prozent) und der über 60jährigen (12,0 Prozent) erhalten auf diesem Wege die verordneten Medikamente. Aufgrund der bisher vorliegenden Ergebnisse, dass 41,0 Prozent der befragten Ärte - gesetzliche Bestimmungen außer Acht gelassen - für Patienten bei entsprechender Bedürfnislage die Rezeptweiterleitung an die Apotheke übernehmen, den Aussagen der Experten $^{93}$ sowie des $\S 17$, II ApBetrO, existiert in der Bundesrepublik Deutschland bereits ein Versandhandel. Die Ausnahmeregelung des $\S 17$, II ApBetrO billigt in begründe-

93

Im Anschluss an die Befragungen wurden Experteninterviews mit Vertretern von Standesorganisationen, der Apotheker, der Politik, der Wissenschaft, der Industrie, der Verbraucherverbände und der Krankenkassen geführt. 
ten Einzelfällen eine Versendung aus der Apotheke oder die Zustellung durch einen Boten an den Arzneimittelempfänger zu.

Die INIFES-Befragung ergab, dass 2,0 Prozent der Bundesbürger Medikamente häufiger von der Apotheke nach Hause geliefert bekommen. Von den Personen, die ihr Rezept selber einlösen oder die Medikamente von der Apotheke nach Hause geliefert bekommen, gaben 42,0 Prozent an, dass sie bei der Auswahl der Apotheke das Kriterium des kostenlosen Heimlieferservices von Medikamenten für sehr wichtig bzw. wichtig empfinden (s. Abbildung III - 19). ${ }^{94}$ Besonders die Altersklassen der 3039jährigen (44,0 Prozent), der 50-59jährigen (48,0 Prozent) und der über 60jährigen (51,0 Prozent) schätzen einen kostenlosen Heimlieferservice von Medikamenten. Aus ihrer Erfahrung heraus halten 54,0 Prozent der befragten Apotheker den Heimlieferservice für einen wichtigen Aspekt bei der Wahl der Apotheke. Dies zeigt, dass ein Kundenbedürfnis nach Heimlieferungen von Medikamenten vorhanden ist, dem sich auch die Apotheker nicht verschließen können bzw. vielfach nicht verschließen.

Während einige Apotheker in Expertengesprächen darauf hinwiesen, dass die Belieferung des Kunden nur die Ausnahme darstellt, ist die Lieferung von Arzneimitteln, die nicht sofort in der Apotheke verfügbar sind, vielfach die Regel. Von lediglich 2,0 Prozent der Apotheker wird eine Heimlieferung nicht durchgeführt bzw. angeboten, 29,0 Prozent der Apotheker beliefern bis zu 30 Kunden pro Woche direkt und weitere 12,0 Prozent beliefern mehr als 30 Kunden pro Woche. Vor allem umsatzstarke Apotheken führen eine Lieferung bei mehr als 30 Kunden pro Woche durch. Der Umfang der Heimlieferungen korreliert neben dem Umsatz auch mit der Personalstärke einer Apotheke. Der Heimlieferservice mit Medikamenten durch die Apotheke ist somit bereits schon jetzt gängige Praxis und nicht mehr der "begründete Einzelfall". Fast ein Viertel der Apotheker würde eine Lockerung der gesetzlichen Rahmenbedingungen begrüßen, um bestimmte Kundengruppen gezielt durch Heimlieferungen ansprechen zu können. Dies gilt besonders für Apotheker mit einem Umsatz bis zu 1,5 Mio. DM pro Jahr und mit höchstens zwei Vollzeitkräften. Gut ein Viertel der Apotheker, die für eine Lockerung der gesetzlichen Vorschriften in bezug auf Heimlieferungen sind, führen schon jetzt mehr als 30 Heimlieferungen pro Woche aus und würden dies auch ausweiten Dem stimmen auch 26,0 Prozent der Apotheker zu, die bislang bis zu 30 Heimlieferungen pro Woche durchführen.

Personen legen bei der Wahl der Apotheke, in der sie ein Rezept einlösen, verschiedene Kriterien zu Grunde. Für den Inhalt der Studie von Interesse sind: Schnelle und gute Erreichbarkeit, Umfassende Beratung, Kostenloser Heimlieferservice von Medikamenten sowie Kunden gehören zur Stammkundschaft. Anhand einer Skala von $1=$ sehr wichtig bis 5=völlig unwichtig sind diese Kriterien von den Apothekern, den Ärzten und der Bevölkerung bewertet worden (s. Abbildung III - 25).

94

Die Kriterien wurden anhand einer Skala von $1=$ sehr wichtig bis $5=$ völlig unwichtig bewertet. 
Abbildung III - 25: Grad der Wichtigkeit verschiedener Kriterien bei der Auswahl der Apotheke

(Frage: "Welche Kriterien legen Sie bei der Auswahl der Apotheke zugrunde? Wie wichtig sind Innen folgende Aspekte?")

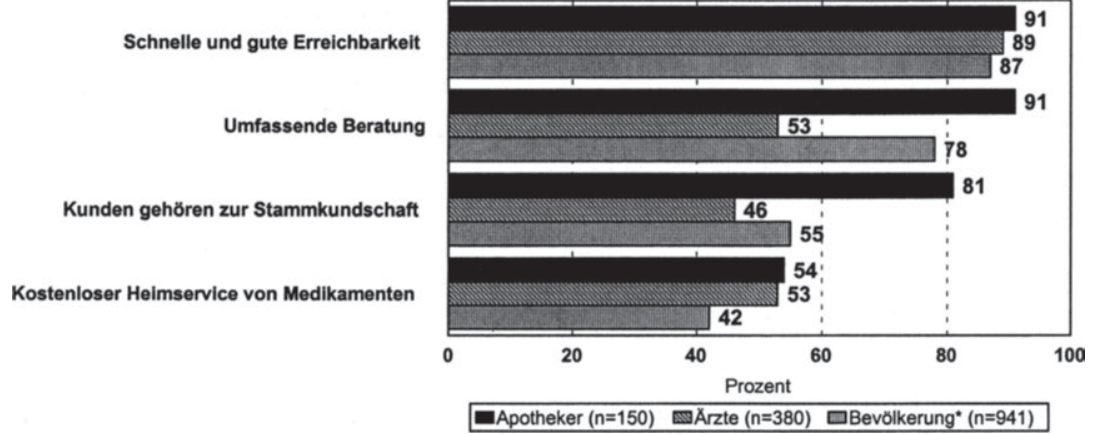

": Selektion Personen, die ihr Rezept selbst einlరsen oder die Medikamente nach Hause geliefert bekommen Top-2-Boxes ("sehr wichtig" und "wichtig"); 1=sehr wichtig, 5=vollig unwichtig

Quelle : INIFES, eigene Darstellung.

(Ergebnisse einer von TNS EMNID im Auftrag von INIFES im Dezember 2000 durchgeführten CATI-Befragung).

Bei der Auswahl der Apotheke, in der das Rezept eingelöst wird, werden von der Bevölkerung vor allem Kriterien einer schnellen und guten Erreichbarkeit sowie einer umfassenden Beratung zu Grunde gelegt. Bezüglich der schnellen und guten Erreichbarkeit stimmt dieser Wert mit der Selbsteinschätzung der Apotheker und der der Ärzte überein. Bei dem Kriterium einer umfassenden Beratung liegt jedoch ein Missverhältnis vor. Vertiefend zu den Auswahlkriterien der Apotheke wurden die Personen, die ihr Rezept selber einlösen gefragt, wie oft sie in der Apotheke bei der Aushändigung von Medikamenten beraten werden. Immerhin ein Drittel der Kunden wird bei fast keinem Apothekenbesuch beraten, 30,0 Prozent werden sporadisch bei ca. jedem zweiten oder dritten Apothekenbesuch und nur 35,0 Prozent wird bei jedem Apothekenbesuch beraten.

Nach $\S 20$ ApBetrO obliegt dem Apotheker aus Gründen der Arzneimittelsicherheit die Pflicht, dem Apothekenkunden informierend und beratend zur Seite zu stehen. Die Apotheker gaben an, dass für 91,0 Prozent der Kunden eine umfassende Beratung bei ihrer Wahl der Apotheke besonders wichtig ist. Dieser Wert steigt in Abhängigkeit der Personalausstattung einer Apotheke. Apotheker, die fünf und mehr Vollzeitkräfte als Pharmazeutisches Personal beschäftigen, schätzen zu 100,0 Prozent ${ }^{95}$

95

Signifikanzniveau aufgrund des Stichprobenumfangs 10 Prozent. 
eine umfassende Beratung als bedeutendes Auswahlkriterium ein. Laut Gesetz besteht sowohl für die Ärzte als auch für die Apotheker eine Beratungspflicht, egal, ob es sich um verschreibungspflichtige oder nicht verschreibungspflichtige Arzneimittel handelt. Allerdings darf die Beratung des Apothekers nicht die Therapie des verordnenden Arztes beeinträchtigen; eine Kritik an der ärtlichen Diagnose oder der Verordnung ist ihm untersagt. Daher ist es nicht verwunderlich, dass nur 53,0 Prozent der Ärzte glauben, dass eine umfassende Beratung ausschlaggebend für die Wahl der Apotheke ist. Besonders die Facharztgruppe der Neurologen und Nervenärzte bewertet diese Kriterium mit nur 45,0 Prozent. ${ }^{96}$ Dies mag u.a. an dem Anteil chronisch Kranker an deren Patientenklientel (50,7 Prozent) und daraus folgernd, einem geringeren Beratungsbedarf/Beratungsanspruch durch den Apotheker liegen.

\section{Potentielle Zielgruppen für eine Versandapotheke}

Die Beschaffung von Gütern und Dienstleistungen per Internet bietet den Menschen auch in Deutschland die Chance, rezeptpflichtige Medikamente über Apotheken außerhalb Deutschlands zu beziehen und somit die Vorteile des Netzes zu nutzen. Bislang wird die Annahme gehegt, dass ein Versandhandel vielfach für Personen von Vorteil ist, die in ihrer Mobilität eingeschränkt, chronisch erkrankt oder berufstätig sind. Nach Ansicht eines Vertreters der Verbraucherverbände sind beispielsweise beim Diabetikerbedarf und auch in anderen Bereichen enorme Preisspannen möglich. Auf die Frage nach der Bewertung der Vorteilhaftigkeit einer Versandapotheke in bezug auf verschiedene Zielgruppen gaben 64,0 Prozent der Ärzte an, dass eine Versandapotheke für Patienten, die in ihrer Mobilität eingeschränkt sind, von Vorteil ist (diese Meinung teilen 49,0 Prozent der befragten Apotheker) (s. Abbildung III - 26). 
Abbildung III - 26: Bewertung der Vorteilhaftigkeit einer Versandapotheke in bezug auf verschiedene Zielgruppen

(Frage: “Für welche Zielgruppe kann die Etablierung einer Versandapotheke eher von Vorteil, für wen eher von Nachteil sein?")

Für in ihrer Mobilität eingeschränkte Patienten

Für Patienten in Gebieten mit Unterversorgung

Als Service für Kunden, z.B. Berufstätige

Für Patienten mit chronischen Krankheiten

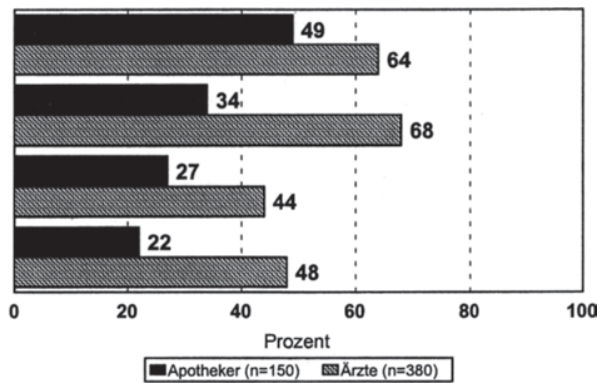

Top-2-Boxes ("von großem Vorteil" und "von Vorteil"); 1=von großem Vorteil; 5=von großem Nachteil

Quelle : INIFES, eigene Darstellung.

(Ergebnisse einer von TNS EMNID im Auftrag von INIFES im Dezember 2000 durchgeführten CATI-Befragung).

Aus dem Antwortverhalten der Ärzte wird deutlich, dass vor allem Fachrichtungen mit einem hohen Anteil an zu versorgenden Chronikern den Vorteil einer Versandapotheke in der Versorgung in ihrer Mobilität eingeschränkter Patienten sehen. Bei den Urologen beträgt die Zustimmung immerhin 75,0 Prozent. Patienten, die in ihrer Mobilität eingeschränkt sind, können sich ohnehin die Arzneimittel nicht selber besorgen und benötigen daher einen Botendienst. Da ein solcher, so die Auskunft eines Krankenkassenvertreters, möglicherweise jedes Mal neu organisiert werden müsste, wäre es sicherlich einfacher, dies auf dem Wege des Versandhandels zu erledigen. 86,0 Prozent der Personen, die sich vorstellen können, Arzneimittel direkt per Post zugeschickt zu bekommen, gaben an, dass bei eingeschränkter Mobilität eine Versandapotheke durchaus eine sinnvolle Alternative für sie wäre. Die Annahme, dass vor allem chronisch Kranke eine Zielgruppe für den Versandhandel bilden, bestätigen 22,0 Prozent der Apotheker. Aus Sicht der Ärzte sind es 48,0 Prozent. Bei diesem Wert zeigt sich, dass vor allem die Fachrichtungen mit einem hohen Anteil chronisch erkrankter Patienten der Überzeugung sind, dass die Etablierung einer Versandapotheke für dieses Patientenklientel von Vorteil ist. 30,0 Prozent der Bundesbürger, die unter einer chronischen Erkrankung leiden, können sich vorstellen, Medikamente über eine Versandapotheke zu beziehen. Dieses lehnen hingegen 70,0 Prozent ab. Anzumerken ist, dass vor allem die Klientel der Chroniker aufgrund der gesundheitlichen Beeinträchtigungen vielfach mehrere vom Arzt verordnete Arzneimittel $(41,0$ 
Prozent benötigen 2-4 verschiedene Präparate pro Tag) benötigt, mindestens ein- bis zweimal im Monat in der Apotheke ein Rezept einlöst (51,0 Prozent) und mit 83,0 Prozent bei Auswahl der Apotheke, in der sie ihr Rezept einlösen, einen signifikant höheren Wert auf eine umfassende Beratung legt, als Personen ohne eine dauerhafte, vom Arzt zu behandelnde Gesundheitsstörung.

44,0 Prozent der Ärzte sehen die Versandapotheke als Dienstleister für Berufstätige und Personen, die aus den unterschiedlichsten Gründen gerne die Möglichkeit hätten, ihre Rezepte bei einer Versandapotheke einzulösen. Nach Ansicht der Apotheker (22,0 Prozent) bietet eine Versandapotheke kaum Vorteile für diese Zielgruppe. Die Einstellung der Berufstätigen hinsichtlich der Vorteilhaftigkeit, auf diese Art Medikamente zu beziehen, ist geteilt. Sowohl für als auch gegen einen solchen Service sprechen sich 50,0 Prozent der Befragten aus.

\section{Akzeptanz eines Versandhandels}

Um die Akzeptanz bei den Apothekern und Ärzten bzgl. eines Versandhandels zu eruieren, wurde ihnen im Rahmen einer offenen Frage die Möglichkeit gegeben, ihre Assoziationen zu dieser Thematik wiederzugeben (s. Abbildung III - 27).

Abbildung III - 27: Assoziationen mit dem Begriff "Versandapotheke"

(Frage:" Was verbinden Sie mit dem Begriff Versandapotheke?")

NEGATIVE GRUNDEINSTELLUNG

SCHLECHTERER SERVICE/ BERATUNG (netto)

KEIN PERSÖNLICHER KONTAKT (netto)

SCHLECHTERE QUALITÄT (netto)

POSITIVE GRUNDEINSTELLUNG (netto)

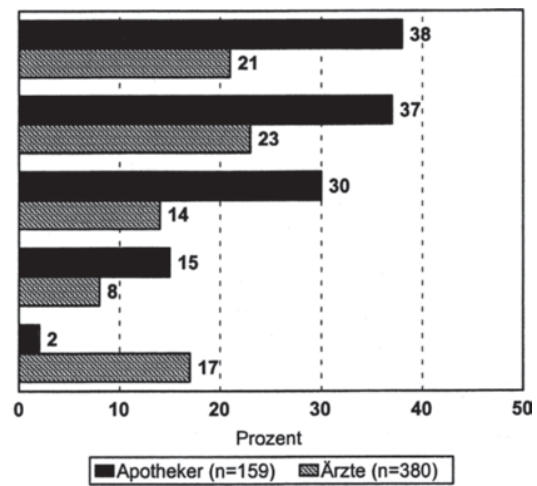

Quelle : INIFES, eigene Darstellung.

(Ergebnisse einer von TNS EMNID im Auftrag von INIFES im Dezember 2000 durchgeführten CATI-Befragung). 
Die Haupteinwände der Apotheker gegenüber dem Modell einer Versandapotheke beziehen sich auf eine zu erwartende Verschlechterung des Services bzw. der Beratung, den persönlichen Kontakt zum Kunden und die Qualität der Versorgung. Die Gründe für diese negative Grundeinstellung sind vielschichtig. Ein Argument umfasst wiederum die Beratungsleistung der Apotheker. 15,0 Prozent der Apotheker sind der Meinung, dass Versandapotheken keine Beratung erbringen werden und 11,0 Prozent sind der Ansicht, dass die persönliche Beratung wegfallen wird. Vergleicht man diese Werte mit der derzeit tatsächlich durchgeführten Beratung in den Apotheken, bleibt zu hinterfragen, warum in der Präsenzapotheke nicht mehr Wert auf eine umfassende und kundenorientierte Beratung gelegt wird, während einer Versandapotheke diese Kompetenz abgesprochen wird.

Eine Kundenumfrage bei Kunden der schweizerischen Direktserviceapotheke MediService ${ }^{97}$ hat ergeben, dass 98,6 Prozent der Ratsuchenden die Beantwortung ihrer Fragen als kompetent und ohne Zeitdruck beurteilten und mit dem Service zufrieden waren. Als einen weiteren Grund für ihre negative Grundeinstellung gaben die Apotheker einen möglichen Verlust des persönlichen/sozialen Kontaktes zwischen Apotheker und Kunden an. Aber gerade eine Anonymisierung der Beratung kann von Kunden als positiv bewertet werden. Arzneimittel wie beispielsweise Psychopharmaka werden ungern in der Apotheke abgeholt, wenn damit zu rechnen ist, dass evtl. auch andere Kunden in der Apotheke anwesend sind.

Lediglich 2,0 Prozent der niedergelassenen Apotheker haben eine positive Grundeinstellung gegenüber dem Modell einer Versandapotheke. Für diese Apotheker kann eine Versandapotheke eine Alternative zu einer Präsenzapotheke sein, sie ist kostengünstiger und unter Beibehaltung der Preisbindung hätte eine Versandapotheke durchaus eine Chance.

Bei der Diskussion über das Für und Wider einer Versandapotheke gilt es zu bedenken, dass diese primär nur ergänzend zu den traditionellen Apotheken tätig sein kann. Auf die Tätigkeit von Präsenzapotheken kann vor allem im Akut-Bereich nicht verzichtet werden. Von den befragten Apothekern gaben 28,0 Prozent an, dass sie sich durchaus vorstellen könnten, beide Vertriebswege komplementär anzuwenden bzw. ihre Präsenzapotheke in eine Versandapotheke umzuwandeln.

Die Ergebnisse der Ärzte-Befragung lassen eine insgesamt positivere Grundeinstellung gegenüber einer Versandapotheke erkennen (s. Abbildung III - 13). Negative Auswirkungen auf den Service bzw. die Beratung erwarten 23,0 Prozent. Eine Einschränkung des persönlichen Kontaktes zwischen Patienten und Apotheker befürchten 14,0 Prozent und an Qualitätseinbußen in der Versorgung glauben 8,0 Prozent. Demgegenüber verbinden jedoch 17,0 Prozent der niedergelassenen Ärzte eine positive Grundeinstellung mit dem Begriff der Versandapotheke. Bezüglich der negati-

97

Institut für Sozial- und Präventivmedizin der Universităt Zürich; Schweizerische PatientenOrganisation (SPO); Schweizerische Patientendienststelle (DVSP) (1999), Zufriedenheit der Kunden der MediService Apotheke - Schlussbericht, Zürich. 
ven Merkmale existieren zwischen den befragten Facharztgruppen kaum Unterschiede. Ein signifikanter Unterschied zwischen den Urologen und den Hals-NasenOhrenärzten besteht hinsichtlich der Beurteilung des Items "Qualität". Während 13,0 Prozent der Urologen mit Qualitätseinbußen rechnen, trifft dies bei den Hals-NasenOhrenärzten nur zu 3,0 Prozent zu. Ob die befragten Ärzte vor dem Hintergrund ihrer Patientenklientel geantwortet haben oder die Werte ihre eigene Meinung widerspiegeln, lässt sich nicht eruieren. Auffällig ist jedoch, dass der Anteil chronisch erkrankter Personen bei den Urologen durchschnittlich 52,3 Prozent der Patientenklientel, bei den Hals-Nasen-Ohrenärzten nur 37,3 Prozent umfasst. Der Unterschied wird noch deutlicher, betrachtet man den Anteil der über 60jährigen an den chronisch Kranken. Dieser beträgt bei den Hals-Nasen-Ohrenärzten 44,3 Prozent, bei den Urologen immerhin 71,6 Prozent. Dies führt zu der Annahme, dass Urologen aufgrund der Zusammensetzung ihrer Patienten in einer Versandapotheke die Gefahr der Verringerung der Arzneimittelsicherheit und eine fehlende Kontrolle der Rezeptierung durch den Apotheker sehen. Auch der Bereich "positive Grundeinstellung" ist beeinflusst durch die verschiedenen Facharztgruppen. Das Item "positive Grundeinstellung" subsummiert u.a. die Antworten: gute Einrichtung, kann eine Alternative sein, ist kostengünstiger, bequem (z.B. für Behinderte). Einer Versandapotheke stehen 27, 0 Prozent der Gynäkologen und 22,0 Prozent der Hals-Nasen-Ohrenärzte offen gegenüber, während es nur 11,0 Prozent der Praktischen Ärzte / Allgemeinmediziner / Internisten sind.

Der Anteil der chronisch Kranken und der über 60jährigen an den chronisch Kranken beträgt bei den Praktischen Ärzten/Allgemeinmedizinern/Internisten durchschnittlich 55,5 Prozent bzw. 58,6 Prozent und bei den Gynäkologen 17,5 Prozent und 42,5 Prozent. Vor allem bei den Gynäkologen ist die positive Grundeinstellung gegenüber einer Versandapotheke verständlich, da sich ihr Patientenklientel vor allem aus Patientinnen zusammensetzt, die zur Vorsorgeuntersuchung (durchschnittlich 57,5 Prozent) kommen und u.a. die verordneten Kontrazeptiva selber bezahlen müssen. Gesetzt den Fall, es gäbe eine Versandapotheke in Deutschland, könnten sich 51,0 Prozent der befragten Ärzte persönlich vorstellen, mit einer solchen Apotheke zu kooperieren. Dieser Anteil ist bei den Gynäkologen, Neurologen / Nervenärzte und den Praktischen Ärzten / Allgemeinmedizinern / Internisten fast identisch. Überraschend sind jedoch die Werte der Urologen und der Hals-Nasen-Ohrenärzte. Die Antworten zu der offenen Frage hätten vermuten lassen, dass die Bereitschaft, mit einer Versandapotheke zusammenzuarbeiten, bei den Urologen wesentlich geringer ausgeprägt ist als bei den Hals-Nasen-Ohrenärzten. Verglichen mit dem Anteil der Chroniker je Facharztgruppe kann darauf geschlossen werden, dass die Bereitschaft zur Kooperation mit einer Versandapotheke bei den Ärzten in Beziehung steht zu dem Anteil der zu behandelnden Chroniker.

Die Einstellung der Bevölkerung gegenüber einer Versandapotheke wurde mit folgender Frage näher untersucht: Können Sie sich vorstellen, Medikamente über eine Versandapotheke zu beziehen? 39,0 Prozent der befragten Personen können sich durchaus den Bezug von Medikamenten auf diesem Wege vorstellen; 60,0 Prozent 
lehnen dies ab. Ein signifikanter Unterschied besteht zwischen Männern und Frauen. Dies mag daran liegen, dass auch der Anteil der Internet-User in dieser Altersklasse bei den Männern (36,0 Prozent) höher ist als bei den Frauen (27,0 Prozent) und die Männer den neuen Medien und dem neuen Vertriebsweg gegenüber aufgeschlossener sind. Eine Korrelation zwischen der Nutzung des Internets und des Bezugs von Arzneimitteln über eine Versandapotheke besteht nicht. Die Bereitschaft, eine Versandapotheke in Anspruch zu nehmen, ist mit zunehmenden Alter rückläufig. Während der Anteil der 30-39jährigen 53,0 Prozent beträgt, liegt er bei den über $60 j a ̈ h r i g e n$ nur noch bei 20,0 Prozent. Es besteht ein signifikanter Unterschied zwischen Personen, die angeben, gesund zu sein und denen, die unter einer dauerhaften, vom Arzt behandelten Gesundheitsstörung leiden bzgl. der Akzeptanz einer Versandapotheke. Während sich 43,0 Prozent der Befragten ohne eine dauerhafte, vom Arzt zu behandelnde Gesundheitsstörung den Bezug von Medikamenten über eine Versandapotheke vorstellen können, sind es bei den Chronikern 30,0 Prozent.

\section{Chancen und Risiken einer Versandapotheke}

Um die Chancen und Risiken einer Versandapotheke aus Sicht der Ärzte und Apotheker erfragen, wurden diese gebeten, anhand einer Skala von 1=volle Zustimmung bis $5=$ totale Ablehnung die in der Offentlichkeit diskutierten Aussagen zur Versandapotheke zu bewerten (s. Abbildung III - 28).

Abbildung III - 28: Bewertung verschiedener Aussagen zur Versandapotheke

(Frage: "Bitte bewerten Sie folgende Aussagen auf einer Skala von 1 bis 5")

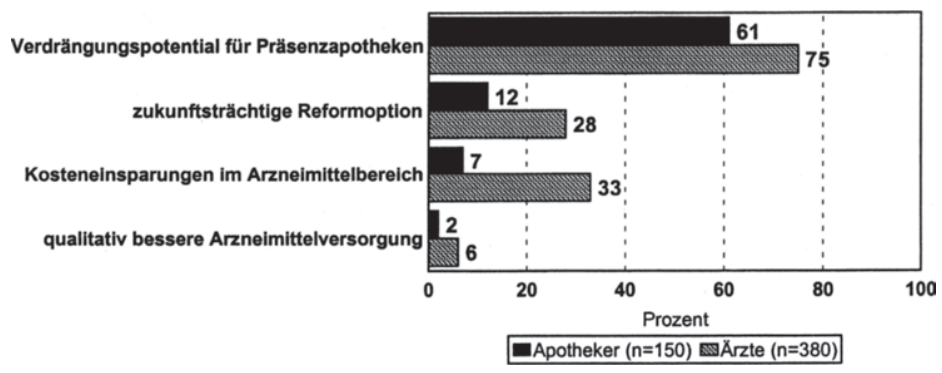

Top-2-Boxes ("volle Zustimmung" und "Zustimmung"); 1=volle Zustimmung; 5=totale Ablehnung

Quelle: INIFES, eigene Darstellung.

(Ergebnisse einer von TNS EMNID im Auftrag von INIFES im Dezember 2000 durchgeführten CATI-Befragung). 
Aus Sicht der Apotheker stellen Versandapotheken generell ein Verdrängungspotential für Präsenzapotheken dar. Vor allem Apotheken mit einem Umsatz bis zu 1,5 Mio. DM geben an, von dieser Konkurrenz betroffen zu sein. Die Konsequenz für die Apotheker ist, dass die Einführung der Arzneimitteldistribution über Versandapotheken ihren Umsatz beeinflussen kann. Von negativen Umsatzeinbußen gehen 58,0 Prozent der befragten Apotheker aus, bei 30,0 Prozent wird es zu keiner Beeinflussung des Umsatzes kommen. Die Folgen der negativen Umsatzbeeinflussung wären die Schließung von Apotheken und die Entlassung von Personal. Von den befragten Ärzten sind 75,0 Prozent der Meinung, dass Versandapotheken generell ein Verdrängungspotential für Präsenzapotheken darstellen.

Dass die Einführung des Modells einer Versandapotheke eine zukunftsträchtige Reformoption darstellt, glauben 28,0 Prozent der Ärzte, bei den Apothekern sind es 12,0 Prozent.

Die Antworten bzgl. der Annahmen, dass Versandapotheken zu Kosteneinsparungen im Arzneimittelbereich führen und für eine qualitativ bessere Arzneimittelversorgung sorgen, zeigen, dass sowohl die Apotheker als auch die Ärzte sehr zurückhaltend sind, wenn es darum geht, Chancen und Risiken einer Versandapotheke unvoreingenommen zu bewerten.

Vor dem Hintergrund der raschen - und nicht aufzuhaltenden - Verbreitung neuer Medien und des elektronischen Handels muss die konstruktive öffentliche Debatte, die sich sowohl mit möglichen Einsparungen in Millionenhöhe bei den Distributionskosten, als auch mit der Gewährleistung von Sicherheit und Qualität der Arzneimittelversorgung befasst, konsequent fortgeführt werden.

Abschließend gilt zu sagen, dass die Einführung neuer Vertriebswege im Arzneimittelsektor nur dann volkswirtschaftlich und sozialpolitisch akzeptabel sein kann, wenn auch die Bedenken der Betroffenen ernst genommen und konstruktiv diskutiert werden. Wenn es gelingt, dies zu berücksichtigen und gleichzeitig Wirtschaftlichkeitsreserven auszuschöpfen ohne das bestehend hohe Niveau der Arzneimittelversorgung zu gefährden, sind Versandapotheken als Vertriebsform in Zukunft verstärkt in eine kritische und ergebnisoffene Diskussion um liberalisierte Wege in der Arzneimitteldistribution einzubeziehen. 


\section{Zusammenfassung und Bewertung der Projektergeb- nisse}

\subsection{Zusammenfassung der Projektergebnisse}

In Deutschland wurden im Jahr 2000 im Rahmen des Versorgungsauftrages der gesetzlichen Krankenkassen ca. 15,3\% der gesamten GKV-Leistungsausgaben für Arzneimittel aus öffentlichen Apotheken aufgewendet. Dies entspricht einem Betrag von 37,8 Mrd. DM. 1999 waren es noch 36,15 Mrd. DM, 1998 ca. 33,6 Mrd. DM. Diese Entwicklung bedeutet einen Anstieg von ca. 4,4\% im Jahr 2000 bzw. 7,4\% im Jahr 1999 im Vergleich zum Vorjahr. Damit verzeichnet der Arzneimittelbereich „...die mit Abstand problematischste Entwicklung unter allen Ausgabenbereichen..." der GKV. Um diesem Ausgabenanstieg entgegenzuwirken, wurden in der Vergangenheit Kostendämpfungsmaßnahmen in Form von Arzneimittelbudgets, Negativlisten oder Zuzahlungsregelungen eingeführt. Trotzdem konnte, abgesehen von kurzfristigen Dämpfungseffekten, eine Konsolidierung der Arzneimittelausgaben bislang nicht erreicht werden. Zudem sind nach der Ankündigung der Abschaffung der Arzneimittelbudgets durch die Politik vom Februar 2001 die Arzneimittelausgaben der GKV um $9,7 \%$ im ersten Quartal gegenüber dem Vorjahr angewachsen. ${ }^{2}$ Damit ist das Ziel der Beitragssatzstabilität im System der GKV stark gefährdet. Jüngste Ankündigungen von Beitragssatzerhöhungen durch verschiedene Kassen sind Ausdruck der angespannten Situation.

Soll die Finanzierbarkeit einer qualitativ hochwertigen Arzneimittelversorgung der Versicherten nicht gefährdet werden, müssen zukünftige Reformmaßnahmen auch auf eine effizientere Nutzung der bereits vorhandenen Ressourcen ausgerichtet werden. Dabei darf die Diskussion um Reformen im Arzneimittelsektor keine Bereiche aussparen. Der Arzneimittelvertrieb gehört deshalb ohne Zweifel mit in die Agenda der zu prüfenden Reformoptionen. Die Versicherten haben einen berechtigten Anspruch auf eine qualitativ hochwertige und kosteneffiziente Arzneimittelversorgung. ${ }^{3}$

Der Vertrieb von Medikamenten ist bisher streng von den Pharmaherstellern über den pharmazeutischen Großhandel bis zu den Apotheken reguliert. Unter die Apothekenpflicht fallende Präparate - ca. 45.000 - dürfen nur in den Räumen einer öffentlichen Apotheke abgegeben werden. Versandhandel, wie er für freiverkäufliche Arzneimittel, Verband- und Heilmittel oder Medizinprodukte auch in Deutschland legal und üblich ist, wird für apothekenpflichtige Medikamente gesetzlich ausgeschlossen.

\footnotetext{
Bundesministerium für Gesundheit (2000), Knapp eine Milliarde Überschuss und stabiles Beitragsniveau: Finanzentwicklung der gesetzlichen Krankenversicherung in 1999 überaus positiv! In: www.bmgesundheit.de, Pressemitteilung Nr. 20, Zugriff am 15.03.2000.

BKK Bundesverband, (2001), Pressemitteilung vom 27.06.2001 GKV: Krankenkassen gegen die Abschaffung von Arznei- und Heilmittelbudgets - Ausgaben explodieren - wachsende Beitragssätze, siehe unter www.bkk.de, Zugriff am 27.06.01.
} 
Die Regulierung der Distributionsrichtung über mehrere vertikale Handelsstufen umfasst zudem gesetzlich festgelegte Höchst- und Festzuschläge der Vertriebsinstanzen Großhandel und Apotheke auf den Herstellerpreis. Letztendlich resultiert daraus ein Vertriebskostenanteil von fast einem Drittel an den Medikamentenkosten. Allein für öffentliche Apotheken, die im Jahre 1999 insgesamt 50,3 Mrd. DM (ohne MwSt.) bzw. ca. 58 Mrd. DM brutto ${ }^{4}$ umsetzten, beläuft sich der Vertriebskostenanteil in Form der Apothekenbetriebsspanne auf ca. 27,3\% des Bruttoumsatzes ${ }^{5}$. Inzwischen wird manche ärztliche Behandlung des Patienten einschließlich der Verordnung einer Arznei geringer vergütet als die Abgabe des Präparates in der Apotheke. ${ }^{6}$

Eine Ausweitung des Pharmaversandes auf verschreibungspflichtige Medikamente könnte demnach die Erschließung von Wirtschaftlichkeitsreserven fördern. Zudem erscheinen durch eine Modifizierung des Apothekenzuschlages sowie die Möglichkeit erheblicher Rabatte beim direkten Bezug vom Pharmahersteller bedeutsame Preisvergünstigungen pro Medikament möglich. Die Kostenträger veröffentlichten bisher Schätzungen zur finanziellen Entlastung des sozialen Krankenversicherungssystems zwischen 800 Mio. $\mathrm{DM}^{7}$ bis $1.700 \mathrm{Mio}^{\mathrm{D}} \mathrm{DM}^{8}$, die durch den Versandhandel möglich wären. Diese Zahlen stützen sich insbesondere auf Erfahrungen aus Ländern, die den Distributionskanal Versand legalisiert haben und ca. $10 \%$ bis $15 \%$ aller Verordnungen auf diese Weise vertreiben, bei Kosteneinsparungen von $10 \%$ bis $30 \%$.

Dazu sei vermerkt, dass aufgrund differierender Gesundheits- und Sozialsysteme bilaterale Vergleiche dieser Art Verzerrungen aufweisen können. So wird eine Diskussion um eine Liberalisierung der Vertriebswege nicht die derzeitige Preisregulierung ausschließen können. Andererseits ist eine Änderung der Arzneimittelpreisverordnung nicht allein ursächlich mit der Freigabe des Versandhandels verbunden. Zudem induzieren neue Vertriebswege neue betriebswirtschaftliche Kosten.

Aus volkswirtschaftlicher Sicht sind potentielle Wirtschaftlichkeitsreserven deshalb nicht ohne weiteres durch Übertragung ausländischer Erfahrungen auf Deutschland monetär zu eruieren. Als Orientierungs- und Anhaltspunkte können sie gleichwohl fungieren. Neben monetären Aspekten belegen Erfahrungen mit Versandapotheken im Ausland allerdings, dass aufgrund epidemiologischer und demografischer Ent-

$16 \%$ Mehrwertsteuersatz auf Nettoumsatz.

ABDA, (1999), Die Apotheke Zahlen, Daten, Fakten, Abbildung 13, sowie Wirtz, K., (2000), Bericht über die Ergebnisse des Betriebsvergleiches der Apotheken im Jahre 1998, in: Mitteilungen des IFH Köln, Jahrgang 52, S. 25.

Schleert, D., Kaesbach, W. (1997), Arzneimitteldistribution - value for money? Konsequenz aus der Reformdiskussion um die Arzneimittelpreisverordnung, in: Forum für Gesellschaftspolitik, Februar 1997, S. 53.

Schleert, D.(2000), zitiert in: Zander, B., Invasion der @-Pillen, in: STERN 24/2000, S 198.

Wissenschaftliches Institut der AOK, (2001), Versandapotheke - eine preiswerte Alternative, Aktuelle Modelirechnung des WidO berechnet Einsparpotential, siehe unter www.wido.de, Zugriff am 25.06.2001.

Berechnungen dieser Art fokussieren insbesondere auf Versender in der Schweiz, den Niederlanden oder den USA. 
wicklungen nachfrageseitig Potentiale in Form neuer Zielgruppen für Versandapotheken zu existieren scheinen. Dies sind v.a. Menschen mit Dauermedikation.

Die Diskussion über neue Vertriebswege ausschließlich unter Wirtschaftlichkeits- und Kostenargumenten zu führen, würde den Anspruch der Gewährleistung einer qualitativ hochwertigen Arzneimittelversorgung demnach untergraben. So betont das Bundesministerium für Gesundheit, die oberste Priorität der Regierung sei der Schutz des Verbrauchers und die Sicherstellung einer ordnungsgemäßen Arzneimittelversorgung.

Die Apotheker argumentieren, dass bei Medikamentenbezug via Versand keine oder nur kaum Beratung stattfinden könnte und führen in diesem Zusammenhang die Beratung des Kunden in der Apotheke als Wettbewerbsvorteil gegenüber anderen potentiellen Anbietern, so auch Versandapotheken, an. ${ }^{10}$ Allerdings führen schon heute demografische wie epidemiologische Entwicklungen die persönliche Beratung in der Apotheke an gewisse Grenzen: Der wachsende Bevölkerungsanteil an älteren Menschen, chronisch Kranken oder Multimorbiden - im Jahr 2000 ist fast jeder vierte Deutsche über 60 Jahre alt, 2040 wird es mehr als ein Drittel sein - belastet in Zukunft nicht nur verstärkt die Arzneimittelausgaben, sondern bedingt aufgrund des notwendigen Medikamentenkonsums ebenfalls die Notwendigkeit häufiger Apothekenbesuche. ${ }^{11}$ Diese sind jedoch aufgrund alters- wie epidemiologisch bedingter Beeinträchtigungen der Mobilität des Patienten für diesen persönlich nur schwerlich oder gar nicht möglich. Andere Personen (Familienmitglieder, externe Hilfspotentiale, etc.), die das Rezept in die Apotheke bringen und dort die Medikamente besorgen, werden nötig. Eine direkte persönliche Beratung des Patienten durch den Apotheker ist in solchen Fällen nicht gegeben. Die gesetzliche Regulierung der Medikamentenabgabe in den Apothekenräumen, die auf der Erklärungsbedürftigkeit apothekenpflichtiger Medikamente und der daraus abzuleitenden Beratungspflicht des Apothekers beruht, verfehlt hier ihre Intention.

Vor diesem Hintergrund wird argumentiert, die Vertriebswege der Arzneimittel vom Hersteller über den Großhandel bis hin zur Apotheke seien zum Teil ineffizient. Nicht nur die Kostenträger, auch immer mehr politische Verantwortungsträger werben dafür, Alternativen zur öffentlichen Apotheke unter Berücksichtigung von Lieferbereitschaft und Service zuzulassen. ${ }^{12}$ Befürworter sehen insbesondere für chronisch Kranke und pflegebedürftige Personen die Möglichkeit der Inanspruchnahme von Versandapotheken als vorteilhaft an.

Aussage im Rahmen der von INIFES durchgeführten Expertengespräche.

Drei Viertel der Apothekenkunden sind kranke Menschen, mehr als jeder sechste leidet an einer chronischen Krankheit mit stetigem Arzneimittelbedarf. Zwei Drittel der Apothekenkunden sind Dauerpatienten, die regelmäßig mindestens einmal monatlich Medikamente aus der Apotheke beziehen müssen, davon sind fast alle mehr als 60 Jahre alt., Siehe: Riegl \& Partner GmbH, Institut für Management im Gesundheitsdienst, Das Image der deutschen Apotheke, Augsburg 1995. jetzt Unterstützung, in: DIE WELT vom 12.06.2001, S.16. 
Volkswirtschaftlich und sozialpolitisch akzeptabel können neue Vertriebswege im Arzneimittelsektor nur dann sein, wenn Wirtschaftlichkeitsreserven ausgeschöpft werden können, ohne das bestehende hohe Niveau der Arzneimittelversorgung zu gefährden. Für Deutschland lagen bisher keine umfassenden wissenschaftlichen Untersuchungen zu den potentiellen Auswirkungen der Einführung des Arzneimittelversandes vor, die als Grundlage für eine sachliche Diskussion um Pro und Contra dieser Vertriebsform dienen konnten.

Die vorliegende INIFES -Studie beschäftigt sich deshalb mit den zwei wesentlichen Aspekten, die in der Diskussion um die Implementierung eines Arzneimittelversandes in Deutschland eine Rolle spielen:

- Zum einen geht es um die Frage der Ausschöpfung von Wirtschaftlichkeitspotentialen. Dazu werden monetäre Einsparpotentiale für die GKV ermittelt.

- Zum anderen sind für die Akzeptanz neuer Vertriebswege nicht nur monetäre Aspekte entscheidend, sondern auch qualitative Einflüsse auf das System der Arzneimittelversorgung. Dazu werden Ergebnisse von Expertengesprächen sowie einer Befragung der Bevölkerung, der Ärzte und Apotheker zum Thema Versandapotheken in Deutschland präsentiert und bewertet.

Erst auf Basis dieser beiden Bausteine kann eine sachliche Diskussion um die Auswirkungen einer Einführung eines Versandhandels - positiv wie negativ - geführt werden.

Des weiteren haben die Verfasser der Studie in den vorliegenden Bericht Anregungen mit aufgenommen, die an Sie während der Präsentation der Projektergebnisse im Rahmen eines Workshops herangetragen wurden.

\subsubsection{Modell einer Versandapotheke, das der Studie zugrunde liegt}

An dieser Stelle ist hervorzuheben, dass sich die Studie auf Versandapotheken im nationalen Kontext, nicht auf Internetapotheken mit grenzüberschreitendem Pharmaversand bezieht. Die Aufhebung des Versandhandelsverbotes nach § 43, I AMG wird also unterstellt. Ansonsten sind Versandapotheken im Sinne dieser Studie als Apotheken wie öffentliche Präsenzapotheken auch zu definieren, mit allen Anforderungen an die Arzneimittelsicherheit und Arzneimittelqualität. Im Gegensatz zu den Präsenzapotheken wird der neue Vertriebsweg des Versandes eingeführt. Der wesentliche Unterschied besteht in der Kommunikationsform zwischen Arzt, Apotheke und Patient sowie in der Art der Medikamentenabgabe an den Patienten. ${ }^{13}$

Gegenstand dieser Studie sind explizit nicht, wie bereits oben vermerkt, die aus dem Ausland - unter Umgehung des deutschen Versandhandelsverbotes wie der deut-

13

Vgl. hierzu: Sterzel, A. Wassener, D., Arzneimitteldistribution in Deutschland - Versandapotheken als Reformoption?, in: Arbeit und Sozialpolitik, (2001)1/2, Jg. 55, S. $14 \mathrm{ff}$. 
schen Preisspannenverordnung - nach Deutschland versendenden Internetapotheken. Wie der Name schon sagt, ist das Merkmal von Internetapotheken die Nutzung des Internets als Bestell- und Kommunikationsplattform zwischen Apotheke und $\mathrm{Pa}$ tienten. Eine konventionelle Versandapotheke mit einer Ausrichtung auf ältere und kranke Menschen bedient sich im Gegensatz dazu hauptsächlich der Kommunikation mit dem Telefon. Vorteil ist, dass alle Bevölkerungsteile zeitlich wie räumlich unumschränkten Zugang zu diesem Medium haben sowie im Umgang damit vertraut sind. Das Internet ist zwar zukünftig aufgrund der technischen Entwicklungen als Serviceund Informationsmedium auch für konventionelle Versandapotheken nicht auszuschließen, es bildet aber keinesfalls eine Voraussetzung für generelle Funktionsabläufe. Würde ein Versandhandelsverbot innerhalb Deutschlands aufgehoben, wäre sogar denkbar, dass auch technisch versierte Nachfrager, die den "Umweg" des Arzneimittelversands via Internet aus dem Ausland nur solange als Alternative nutzen, wie Versandapotheken in Deutschland nicht zugelassen sind, sich dieser Form des Arzneimittelbezuges neben den bisher genannten Zielgruppen bedienen.

Die mit einer Legalisierung des Versandhandels und somit mit der Aufhebung des Versandhandelsverbotes nach § 43, I AMG zu erwartenden Sekundäreffekte ${ }^{14}$ sowie deren langfristigen Folgewirkungen werden, unter Berücksichtigung des Forschungsvertrages, nicht explizit thematisiert.

Mit einer Legalisierung des Versandhandels wird primär das Mehr- und Fremdbesitzverbot zur Diskussion stehen. Die Aufhebung des Mehrbesitzverbots hat zur Konsequenz, dass die persönliche Leitung der Apotheke durch einen approbierten Apotheker nicht mehr Voraussetzung für den Betrieb sein darf, da sonst die Möglichkeit des Mehrbesitzes unterlaufen würde. ${ }^{15}$ Daraus folgernd ist zu beachten, warum der Besitzer einer Apotheke überhaupt ein Apotheker sein soll, wenn ein angestellter Apotheker im Angestelltenverhältnis ausreicht. Der sich ergebenden Möglichkeit, dass durch die Abschaffung des Fremdbesitzverbots auch die Krankenkassen im Sinne von $\$$ 140 SGB V eröffnen dürften, wurde von Vertretern der Kostenträger widersprochen. ${ }^{16}$

Während sich das der Studie zu Grunde liegende Modell auf Versandapotheken im nationalen Kontext bezieht, sind den Verfassern durchaus die Europa-Rechtlichen Dimensionen einer Legalisierung des Versandhandels bewusst. Einen Arzneimittelversand ausschließlich in den Grenzen Deutschlands zu organisieren ist unter Berücksichtigung des EG-Vertrages nicht möglich. Ausländische EU-Apotheken werden sich in Kooperation oder Konkurrenz zu deutschen Apotheken am Versandhandel beteiligen. Daher ist es eminent wichtig, bei einer Öffnung der Vertriebswege den Versandhandel an Bedingungen hinsichtlich der Patienten- und Arzneimittelsicherheit

Z.B.: eine Änderung der AMPreisV, des Fremd- und Mehrbesitzverbotes, des Aut-IdemVerbotes, des Heilmittelwerbegesetzes, der $\S \S 43$ und 73 AMG sowie $\S 17$ ApBetrO sowie Europa-Rechtliche Regelungen.

15 Schöffski, O. (1995), Die Regulierung des deutschen Apothekenwesens. Eine ökonomische Analyse, Baden-Baden, S. 295.

Aussage auf dem Workshop am 17.09.2001 in Berlin. 
zu knüpfen und ggf. nur im Einzelfall zu erlauben. Die Europäische Arzneimittelrichtlinie, die den Versandhandel verschreibungspflichtiger Medikamente erlaubt, sofern der Absender eine zugelassene Apotheke ist, würde bei einer Aufhebung des $\S 43$ AMG in deutsches Recht umgesetzt werden.

Geht man vom Szenario „Versandapotheken für verordnete Medikamente“ aus, bieten sich die folgenden Schritte einer Modellentwicklung an.

(1) Es wird von einem Versichertenverhältnis Patient - GKV ausgegangen (in Abbildung IV - 1 nicht explizit erwähnt).

(2) Während eines Arztbesuches werden dem Patienten Arzneimittel verordnet.

(3) Das Rezept erstellt der Arzt bei Einverständnis des Patienten elektronisch und schickt es per Datenfernübertragung an eine Versandapotheke.

(4) Diese wiederum prüft die Rezeptangaben und wendet sich bei Rückfragen an den verordnenden Arzt.

(5) Danach stellt die Versandapotheke die Medikamente dem Rezept entsprechend zusammen und versendet diese umgehend an den Patienten.

(6) Die Versandapotheke verrechnet die Arzneimittelkosten mit den Spitzenverbänden der Krankenkassen.

(7) Die Krankenkassen rechnen die ärztlichen Leistungen nach wie vor mit den Kassenärztlichen Vereinigungen ab; hierbei gibt es also keinerlei Veränderungen.

Die Arzneimittelzuzahlungen, die angesichts der derzeitigen Rechtslage explizit nicht nur als Mittel zur Kosteneinsparung für die Krankenkassen, sondern auch als Instrument zur Nachfragesteuerung vorgesehen sind, könnten, ähnlich der Krankenhauszuzahlung, dem Patienten von der entsprechenden Kasse in Form einer Rechnung belastet werden. ${ }^{18}$ Es ist davon auszugehen, dass die Mehrzahl der potentiellen Nutzer von Versandapotheken aufgrund chronischer Erkrankungen von Zuzahlungen nach § 61 ff SGB V befreit sein werden (durchschnittlich ist bereits jede zweite Verordnungen zuzahlungsbefreit) ${ }^{19}$. Eine Übernahme der Zuzahlungen, wie zeitweise von Internetapotheken angekündigt, wäre derzeit rechtlich gemäss $§ 31$ Abs. 3 SGB V nicht zulässig.

(8) Für Deutschland entsteht das in Abbildung IV - 1 dargestellte Gesamtmodell eines Arzneimittelversandes verordneter Medikamente. Dabei ist hervorzuheben, dass die Versandapotheke selbständig, d.h. nicht im Verbund mit den Krankenkassen agiert. Die Unabhängigkeit der Versandapotheke ermöglicht den Zugang

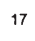

18

Näheres unter: www.vzbv.de.

In Expertengesprächen wurde von Seiten der Kostenträger diese Lösungsmöglichkeit vorgeschlagen.

O. V. (2001), Treiben Kassen Missbrauch mit der Rezeptgebühr?, in: www.medical-tribune, Zugriff am 09.07.01. 
aller Patienten, egal bei welcher Krankenkasse das Versicherungsverhältnis besteht.

Welchen Weg das Rezept einschlägt, ob in die Offizin einer Präsenzapotheke oder in eine Versandapotheke, hängt (weiter) von den einzelnen Krankheitsbildern, der Dringlichkeit des Medikamentenbedarfes sowie den Kosten des jeweiligen Vertriebsweges ab. Denn es eignen sich nicht alle Arzneimittel zum Vertrieb über Versandapotheken. Nur wenn die Medikamente aufgrund ihrer Eigenschaften versandfähig sind sowie keine akute Dringlichkeit der Medikation vorliegt, wird die Versandapotheke unter Abwägung der Kosten als Alternative zur Präsenzapotheke fungieren können.

Abbildung IV - 1: Gesamtmodell einer unabhängigen Versandapotheke

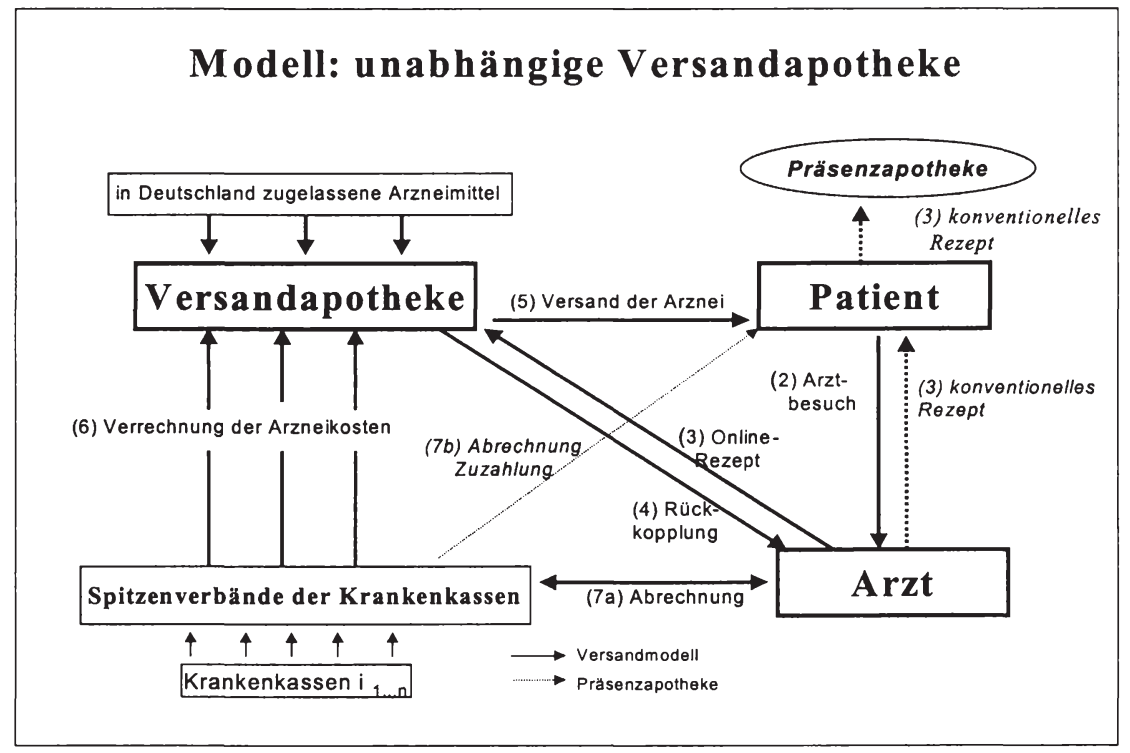

Quelle: INIFES, eigene Darstellung.

Die elektronische Übertragung der Rezeptdaten direkt vom verschreibenden Arzt an die Versandapotheke stellt - insbesondere aus Sicht der Verbraucher - einen besonderen Vorteil dar, da sich so die Transaktionskosten (postalische Versendung der Rezepte durch die Patienten oder den Arzt; persönliche Weitergabe der Rezepte an die Apotheke) erheblich reduzieren. Folgerichtig wird im Zusammenhang mit Versandapotheken nahezu immer von einer elektronischen Rezeptdatenübertragung ausgegangen. Eine effizientere Netztechnik in Form des elektronischen Rezeptes als übergreifendes Übermittlungsinstrument zwischen Arzt, Patient, Apotheker und Krankenkasse erfüllt die Schaffung effizienter Versorgungsstrukturen, ermöglicht Kosteneinsparungen und erhöht Qualität und Aktualität des gegenwärtigen Verordnungsablaufes. 


\subsubsection{Zusammenfassung der quantitativen Projektergebnisse}

Im Folgenden werden die Ergebnisse der Berechnungen eines potentiellen monetären Einsparvolumens einer Ausweitung des Pharmaversandes dargestellt. Grundsätzlich gilt es dabei, folgende Fragen zu beantworten:

1) Für welche Patienten eignen sich Versandapotheken (Zielsegment)?

2) Wie stellen sich die Kosten des neuen Vertriebsweges im Vergleich zur derzeitigen Kostenstruktur der Präsenzapotheke dar?

3) Welches Marktpotential ergibt sich aus dem Medikationssegment und den jeweiligen betriebsinternen Umsatz-, Kosten- und Ertragsstrukturen?

4) Welches Einsparpotential resultiert aus der Zulassung eines Pharmaversands für die gesetzliche Krankenversicherung?

5) Wie verringert sich das Einsparpotential bei Modifizierung der Kostenvorteile in Form

a) der Berücksichtigung einer durchschnittlichen Betriebsrendite (und daraus resultierender umsatzabhängiger Kassenrabatte)?

b) der Berücksichtigung zusätzlicher einheitlicher Kassenrabatte?

6) Welche Marktanteile und Einsparvolumina der GKV sind kurz- und mittelfristig realisierbar (d.h. hier erfolgt eine Modifizierung der Marktpotentiale)?

7) Abschließende Anmerkungen zu den getroffenen Annahmen. 
Abbildung IV - 2: Begriffsbestimmung und Darstellung der Vorgehensweise bei den Modellrechnungen

\section{GKV-Fertigarzneimittelmarkt}

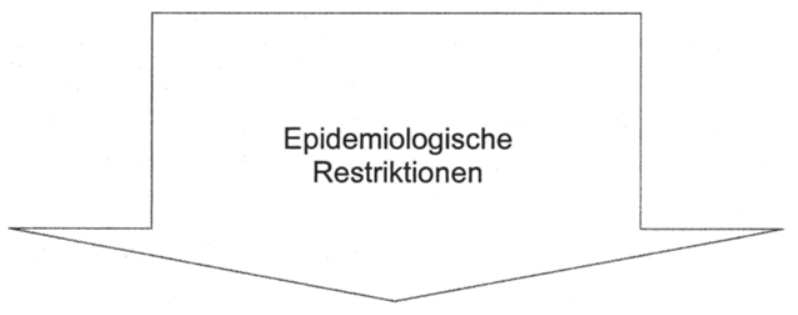

\section{Zielsegment von Versandapotheken (auch Versandpotential genannt)}

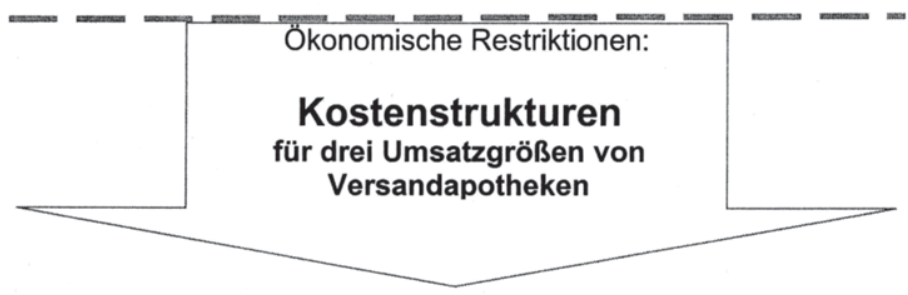

\section{Marktpotentiale \\ Einsparpotentiale}

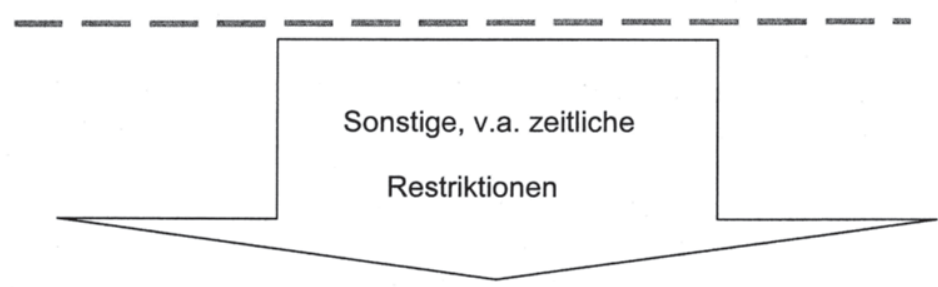

Realisierung von

Marktanteilen

Realisierung von

Einsparvolumina

Quelle: INIFES, eigene Darstellung. 
Einleitend ergehen einige wesentliche Anmerkungen zur Problematik der Datenbeschaffung und -verwendung, bevor eine Zusammenfassung der einzelnen Punkte (1) bis (7) erfolgt:

- Aufgrund des umfangreichen Bedarfs an Informationen muss auf eine Vielzahl verschiedener Quellen zugrückgegriffen werden. Eine Beeinflussung des Zahlenmaterials durch partikuläre Interessen der jeweiligen Akteure am Arzneimittelmarkt ist nicht auszuschließen.

- Einige wichtige Daten sind nicht für das allgemeine öffentliche Interesse zugänglich und konnten entsprechend in dieser Studie nicht berücksichtigt werden. Dies betrifft vor allem den Bereich des pharmazeutischen Großhandels. Alternativ muss mit anderen verfügbaren Datenquellen bzw. mit Annahmen gearbeitet werden.

- Ferner wirkt erschwerend, dass zum einen eine Differenzierung der Berechnungen aus volkswirtschaftlicher sowie aus betriebswirtschaftlicher Perspektive nötig ist, zum anderen beide Ebenen zusammengeführt werden müssen.

Es schließt sich nun eine Betrachtung der einzelnen aufgeführten Punkt an.

\section{(1) Für welche Patienten eignen sich Versandapotheken (Zielsegment)?}

Die quantitative Simulation basiert auf der Annahme, dass Medikation für akute Erkrankungen in nächster Zukunft grundsätzlich kein Potential für den Versand von Arzneimitteln darstellt. Dem steht die zeitliche Restriktion einer Lieferfrist von ca. 48 Stunden zwischen Rezepteingang in der Versandapotheke und Empfang beim Patienten entgegen.

Es müssen entsprechend Indikatoren eruiert werden, die zum einen die wesentliche Einschränkung auf nicht chronische Medikation ermöglichen, zum anderen aber auch eine quantitative Messung von Zielsegmenten für einen Versandhandel gewährleisten.

Es kristallisiert sich heraus, dass die Packungsgröße, kombiniert mit medizinischen wie pharmakologischen Expertenabschätzungen zur Dringlichkeit einer Medikation, einen adäquaten Indikator für die Versendungsfähigkeit einer Verordnung bildet: Je größer der Packungsinhalt der Verordnung, desto wahrscheinlicher wird diese für eine längerfristige Medikation angewandt, ist damit nicht akut und für den Versandweg potentiell geeignet. Zusätzlich eingeholte Expertenmeinungen von Ärzten wie Apothekern zur Abschätzung der Dringlichkeit der Medikation je Indikationsgruppe ermöglichen eine Kontrolle des Indikators Packungsgröße. Durch die Komplexität der Arzneimitteltherapie und unterschiedlicher Anwendungsmöglichkeiten von Wirkstoffen ist eine Abgrenzung zwischen „versendungsfähig“ und „nicht versendungsfähig“ auch vom entsprechenden Krankheitsbild abhängig. Schließlich ist die Einschätzung der Versandfähigkeit von der Entwicklung des Qualitätsniveaus der pharmazeuti- 
schen Logistik, d.h. wie sicher und wie schnell eine Zusendung in Zukunft erfolgen kann, abhängig.

Es ergeben sich basierend auf der Einheit der Packungsgröße drei Ausprägungen von Indikatoren

- Packungen mit mindestens 50 definierten Tagesdosen (zudem modifiziert nach Expertenmeinungen)

- Packungen mit mindestens 35 definierten Tagesdosen ${ }^{20}$ (zudem modifiziert nach Expertenmeinungen)

- N3-Normpackungen nach der Zuzahlungsverordnung ${ }^{21}$ (zudem modifiziert nach Expertenmeinungen),

die zur Operationalisierung dreier Varianten eines Zielsegments führen.

Abbildung IV - 3: Darstellung der Varianten A bis $\mathrm{C}$ des Zielsegments

\begin{tabular}{|c|c|c|c|}
\hline $\begin{array}{c}\text { Zielseg- } \\
\text { ment- } \\
\text { variante }\end{array}$ & $\begin{array}{c}\text { Messgröße / } \\
\text { Operationalisierung }\end{array}$ & Basisparameter: & $\begin{array}{l}\text { Zielsegment: } \\
\text { in } \%\end{array}$ \\
\hline A & Packungsgröße ab 50 DDD je Verordnung & \multirow{3}{*}{$\begin{array}{c}\text { GKV- } \\
\text { Fertigarznei- } \\
\text { mittelumsatz } \\
1999\end{array}$} & $41,0 \%$ \\
\hline B & Packungsgröße N3-Packungen & & $47,0 \%$ \\
\hline C & Packungsgröße ab 35 DDD je Verordnung & & $57,0 \%$ \\
\hline
\end{tabular}

Quelle: INIFES, eigene Darstellung.

Das Zielsegment ist demnach der Teil des GKV-Fertigarzneimittelumsatzes, der durch die Zielfokussierung auf chronische bzw. dauerhafte Medikation abgegrenzt wird, und damit die maximale Obergrenze für eine Option „Versandhandel mit Arzneimitteln" bildet.

Die Herleitung möglicher Zielsegmente basiert dabei auf der Begriffsbestimmung "versandfähig", die sich auf die Ebene der Indikationsgruppe (nach ATC-Kodierung) bezieht. Diese bietet den Vorteil einer Operationlisierung / Quantifizierung von messbaren Zielsegmenten für den Versand.

Entsprechend wird auf den durchschnittlichen Verordnungswert je Indikationsgruppe fokussiert, nicht auf das einzelne Präparat. Dabei umfasst eine Indikationsgruppe eine Vielzahl von Präparaten, in einigen Fällen einige hundert und mehr. Der Verord-

Der Durchschnitt einer allgemeinen GKV-Verordnung lag 1999 bei 35 Tagesdosen. Damit sollen über diesen Indikator die überdurchschnittlich großen Packungen abgebildet werden.

Vierte Verordnung zur Änderung der Verordnung über die Zuzahlung bei der Abgabe von Arznei- und Verbandsmitteln in der vertragsärztlichen Versorgung, Bundesgesetzblatt 1997, Teil I, S. 2328.

Ausnahme bildet Variante B, diese weicht von der ATC-Kodifizierung aus den unter 4.2. Variante $B$ genannten Gründen $a b$. 
nungswert je Indikationsgruppe stellt entsprechend den Durchschnittswert über alle verordneten Präparate dieser Gruppe dar. ${ }^{23}$ In diesem Zusammenhang ist zu betonen, das eine Operationalisierung der Zielsegmente auf der Ebene der einzelnen Präparate unbestritten wünschenswert gewesen wäre, jedoch aufgrund der unüberschaubaren Präparateanzahl von über 45.000 bis 50.000 Stück bei einem zudem kontinuierlichen pharmazeutischen Fortschritt sich als nicht umsetzbar gestaltet.

Zudem ist an dieser Stelle darauf hinzuweisen, dass die ermittelten Ausprägungen von Zielsegmenten für einen Pharmaversand nicht mit Marktanteilen für den Versandhandel zu verwechseln sind. Sie ermöglichen in einem ersten Modellschritt lediglich eine Aussage darüber, welcher Teil des GKV - Fertigarzneimittelumsatzes aus epidemiologischer Herangehensweise ein „versendungsfähiges" Segment darstellt und somit als Grundlage weiterer Modellentwicklungen gelten kann. Damit unterscheidet sich diese Herangehensweise gegenüber den oftmals in Diskussionen geäußerten verkürzten Berechnungen zum möglichen Umfang eines Arzneimittelversandes, welche die gesamten GKV-Ausgaben als Grundlage für Marktberechnungen heranziehen (z.B. 15\% erwarteter Marktanteil des Versandes an den GKV-Ausgaben aufgrund der Erfahrungen in den USA), ohne dabei eine Zielgruppenbildung anhand epidemiologischer Kriterien vorzunehmen. Das würde bedeuten, dass alle Verordnungen unabhängig von ihrer jeweiligen Art und Zusammensetzung als versendungsfähig angesehen werden. Dem widersprechen die angeführten Literaturquellen sowie Erfahrungen im Ausland.

Die hier gewählte zielgruppenorientierte Differenzierung des GKV-Marktes nach epidemiologischen Kriterien zeigt, dass die jeweiligen Umsätze je Verordnung (in Abhängigkeit der Zielsegmentvariante ergeben sich 61,36 DM bzw. 64,85 DM bzw. 66,78 DM) über dem GKV-Durchschnitt von 46,99 DM liegen: Dem stehen jedoch überproportional größere Packungsinhalte gegenüber: So umfassen die Verordnungen des Zielsegments mindestens 60 Tagesdosen Packungsinhalt statt 35 Tagesdosen im gesamten Durchschnitt der GV-Verordnungen. Das heißt, in Relation zum Umsatz einer Tagesdosis liegen die versendungsfähigen Verordnungen unter dem Durchschnitt aller GKV-Verordnungen. Zudem fallen unter das Zielsegment des Pharmaversandes aufgrund der gewählten Indikatoren auch solche Indikationsgruppen, die mit ihrem Verordnungswert unter dem GKV-Durchschnitt liegen.

Die bisherigen Ausführungen zum Zielsegment fokussieren auf die ausschließlich volkswirtschaftliche Ebene der Marktsegmentierung. Um zu klären, in welchem Umfang dieses Zielsegment durch die potentiellen Versandapotheken unter Einbeziehung betriebswirtschaftlichen Kostenstrukturen als Markteintrittsschranke genutzt

23

Der höchste durchschnittliche Verordnungswert je Indikationsgruppe im GKV-Segment liegt im übrigen zählt dieser nicht zum erhobenen Zielsegment eines Versandes - im Jahre 1999 bei ca. 1005 DM (H01) AVK brutto. Entsprechend der Staffelung der Arzneimittelpreisverordnung liegt dieser zudem nicht in der höchsten Preisstufe (die Kappung der Arzneimittelpreisverordnung 1998 ist entsprechend ohne Einfluss). Der höchste durchschnittliche Verordnungswert einer als versendungsfähig eingestuften Indikation liegt bei ca. 550 DM (L02) AVK brutto. 
wird, muss eine Simulation betriebswirtschaftlicher Kostenstrukturen potentieller Versender erfolgen.

(2) Wie stellen sich die Kosten des neuen Vertriebsweges im Vergleich zur derzeitigen Kostenstruktur der Präsenzapotheke dar?

Zentrales Element der Analyse potentieller Einsparvolumen einer Ausweitung des Pharmaversandes ist die Simulation der Kostenstrukturen von Versandapotheken. Einerseits lassen sich durch betriebswirtschaftliche Kostenstrukturanalysen die durchschnittlichen Kosten der existierenden Präsenzapotheken mit den zu erwartenden Kosten verschiedener Ausgestaltungsformen von Versandapotheken vergleichen. Andererseits ergeben sich aus den geänderten Betriebsabläufen und Vertriebswegen der Versandapotheke aber auch Differenzen in der Kostenstruktur. Ausgangsbasis der Berechnungen ist die interne Struktur einer Präsenzapotheke, die auf dem Fundament zugänglicher Apothekenbetriebsvergleich-Daten entwickelt wird und als Referenzmaß weiterer Simulationen dient. Die durchschnittliche Präsenzapotheke erwirtschaftet mit insgesamt durchschnittlich 6,7 Mitarbeitern ${ }^{24}$ einen Bruttoumsatz von 2,53 Mio. DM im Jahr. Die durchschnittliche Betriebsrendite wird bei 0,4\% des Bruttoumsatzes angegeben.

Unter Zuhilfenahme weiterer Sekundärdaten können die Kostenstrukturen zukünftigen Versandapotheke entwickelt werden. Die Kostenstrukturen von Versandapotheken umfassen u.a. die folgenden Punkte:

- In den Räumen der Versandapotheke findet kein oder nur sehr wenig Kundenverkehr statt. Versandapotheken können also auch in Gewerbegebieten mit niedrigeren Mietkosten angesiedelt sein.

- Nach $\S 20$ ApBetrO muss aus Gründen der Arzneimittelsicherheit der Apothekenkunde bei der Abgabe des Arzneimittels pharmazeutisch beraten werden. Die Pflicht zur Sicherstellung einer qualifizierten Beratung als Bestandteil der pharmazeutischen Tätigkeit gilt demzufolge für alle Apotheken, unabhängig von der Vertriebsart. Da die Medikamentenabgabe, und damit die Kommunikation, zwischen der Versandapotheke und dem Kunden nicht wie in einer Präsenzapotheke face to - face bei persönlicher Anwesenheit erfolgt, muss die Beratung durch eine andere Art der Kommunikation garantiert werden.

Wesentliches Kommunikationsmedium von Versandapotheken, so zeigen Erfahrungen im Ausland, ist das Telefon. Als Dienstleistung werden Telefon-Hotlines mit Kundendienst und pharmazeutischer Beratung angeboten, die in den meisten Fällen rund um die Uhr an 365 Tagen im Jahr erreichbar sind. Dieser Service ist i.d.R. für den Anrufer kostenlos. Deshalb wird auch in den Kostenstrukturen der Modelle von Versandapotheken ausgegangen, die als Qualitätsstandard eine kostenlose 24-Stunden-Telefon-Hotline (an allen Tagen des Jahres) anbieten. Während der Öffnungszeiten wird diese Leistung durch das pharmazeutische Personal

24

Inklusive Apothekenleiter. 
der Versandapotheken selbst erbracht. Dazu muss zusätzliches pharmazeutisches Personal in den Versandapotheken eingestellt werden. Außerhalb der Öffnungszeiten wird eine Abwägung zwischen Selbsterstellung und Outsourcing erfolgen. Dafür ursächlich ist vor allem die zeitliche Verteilung der Anrufe: Mehr als 95\% der Anrufe erfolgen während der Geschäftszeit, während es außerhalb der Geschäftszeit z.T. weniger als $5 \%$ sind.

Eine Abwägung dieser beiden Alternativen wird, so zeigt sich, nur bei sehr umsatzstarken Versandapotheken zur Entscheidung der Selbsterbringung führen, Versandapotheken im unteren und mittleren Umsatzsegment werden außerhalb der Dienstzeiten ihre Telefon-Hotline an externe Call-Center vergeben oder diese im Kooperationsverbund mit anderen (Versand)Apotheken erbringen.

- Insbesondere für das pharmazeutische Personal der Versandapotheken ist entsprechend der Verschiebung der Tätigkeitsbereiche (es findet kein persönlicher Kontakt mehr mit dem Patienten statt, der Kostenanteil des Verkaufs, der bei Präsenzapotheken den höchsten Ausgabenanteil an den Personalkosten bildet, entfällt) sowie einer technischen wie organisatorischen Optimierung der Arbeitsabläufe eine höheren Produktivität zu erwarten. Gestützt wird die Annahme einer erhöhten Pro-Kopf-Produktivität durch internationale Erfahrungen bezüglich Versandapotheken.

- Die Logistik ist neben der pharmazeutischen Kernkompetenz eine Leistung, die für Versandapotheken einen strategischen Parameter darstellt. Darunter ist die Sicherstellung der Aufgaben zu verstehen, die garantieren, dass das richtige Arzneimittel für den richtigen Patienten rechtzeitig am richtigen Ort und in einwandfreier Qualität unter Einhaltung aller fachlichen und gesetzlichen Anforderungen kostenoptimal zur Verfügung steht.

Im Falle von Versandapotheken handelt es sich um eine Business-to-CustomerLogistik (B-2-C): Adressat der Lieferung ist der Patient, der das Arzneimittel an einem bestimmten Aufenthaltsort (zu Hause, am Arbeitsplatz, am Urlaubsort) erhalten möchte. Die Logistik potentieller Versandapotheken wird aufgrund der konsumentenorientierten Fokussierung im Vergleich zum pharmazeutischen Großhandel mit Business-Orientierung komplexere Liefer- und Zustellmodalitäten sowie vergleichsweise kleinere Auftrags- bzw. Versandwerte aufweisen.

Eine Make-or-Buy-Entscheidung hängt hier u.a. von der angestrebten räumlichen Reichweite des Versandes ab. Daraus ist zu schließen, dass mit dem Anspruch der Erschließung eines bundesweiten Kundenkreises (was wiederum eine entsprechende Umsatzgrößeklasse induziert) die Logistik einer potentiellen Versandapotheke tendenziell an externe Anbieter ausgelagert wird. Zudem wird die Entwicklung des Logistik-Marktes durch E-Commerce oder E-Logistics forciert, weil die stark gestiegene Nachfrage nach Logistikleistungen insbesondere aus dem Konsumentenbereich umfassende Dienstleistungsangebote erfordert.

- Mit der Zunahme der Umsatzstärke der Apotheken ist allgemein, d.h. auch analog zu den Einkaufsmodalitäten der Präsenzapotheken, eine Rabattgewährung des 
pharmazeutischen Großhandels an die Versandapotheken anzunehmen. Der Einkauf größerer Warenmengen führt zu einer Stärkung der Einkaufsmacht und entsprechend zu einer Gewährung von Rabatten und Skonti durch den Großhandel bzw. die Hersteller.

Allerdings sind bezüglich der Rabattgewährung keine öffentlichen Daten von Seiten des Großhandels wie der Hersteller verfügbar. Deshalb fokussieren sich die Annahmen einerseits auf vertrauliche Expertengespräche sowie andererseits auf die wenigen zu dieser Thematik verfügbaren Literaturquellen:

- Allgemein ist von einer regionalen Abhängigkeit der Höhe der Großhandelsrabatte auszugehen. In Regionen mit einer stärkeren Wettbewerbsstruktur des Großhandels werden höhere Rabatte an Apotheken gewährt als Regionen mit einigen wenigen marktdominierenden Großhändlern.

- Laut Expertenmeinung erhalten Präsenzapotheken mit einem unterdurchschnittlichen jährlichen Umsatz von ca. 1,5 Mio. DM einen Großhandelsrabatt von $11 \%$ auf den Apothekeneinkaufspreis plus Skonto. ${ }^{25}$

- Diese Rabattgewährung wird zudem von der Literatur gestützt, wonach durchschnittlich ca. $15 \%$ des Apothekeneinkaufspreises als Rabattgewährung des Großhandels an die Apotheken fließen. ${ }^{26}$

- Eine andere Literaturquelle geht von ca. 1,5 Mrd. DM bis ca. 2 Mrd. DM aus, die vom Großhandel wie den Herstellern jährlich den Apotheken gewährt werden: „Da nach der Arzneimittelpreisverordnung apothekenpflichtige Arzneimittel Medikamente überall den gleichen Endverkaufspreis haben, können die Rabatte nicht im Preiswettbewerb an Kassen und Versicherten weitergegeben werden. Im Reingewinn, der 1998 nach ABDA - Angaben für alle öffentlichen Apotheken zusammen nur 0,4 Mrd. DM betrug, können sich die Rabatte aber ebenso wenig niederschlagen. Bleibt somit nur noch die Vermutung, dass damit der "Schlupf“ auf der Apothekenstufe finanziert wird ..... ${ }^{{ }^{27}}$

- Zudem erhalten die Apotheken mehr und mehr sogenannte Naturalrabatte, insbesondere bei Generika, von den Herstellern. Insider rechnen mit ca. 750 Mio. DM jährlich, die den Apotheken in dieser Form als stiller Gewinn zufließen „Der Hersteller ... stellt dem Apotheker eine bestimmte Menge von Packungen in Rechnung, liefert dann aber schließlich mehr. Dieses Mehr ist der Naturalrabatt. ... Dass der Apotheker die geschenkte Packung zu Lasten der Krankenkassen verkauft, versteht sich von selbst. ${ }^{28}$

25

Diese Informationen liegen INIFES vertraulich vor.

Schöffski, O. (1995), Die Regulierung des deutschen Apothekenwesens. Eine ökonomische Analyse, Baden-Baden, S. 168.

Cassel, D. (2000), Effiziente Distributionswege, in: Wille, E., Albring, M. (Hrsg.), Rationalisierungsreser-ven im gesundheitswesen, Peter Lang Verlag, Frankfurt / Main, S. 308.

Laschet, $H$. (2001), Kasse machen trotz knapper Kassen - die heimlichen Apothekergewinne, S. 6, in: ÄRZTE ZEITUNG, Nr. 163, Donnerstag, 13.09.2001, S. 6 - 7. 
Die interne Kostenstruktur der Versandapotheken wird in den Modellrechnungen für unterschiedliche Umsatzgrößenklassen von Versandapotheken entwickelt, um Skaleneffekte bei den Kosten und deren Auswirkung auf das Betriebsergebnis zu analysieren. Es werden drei Varianten von Arzneimittelversendern simuliert:

- Das Modell der kleinen Versandapotheke (VA I) beschreibt eine Versandapotheke, die aus den Strukturen der Präsenzapotheke entwickelt wird und einen Bruttoumsatz von ca. 5 Mio. DM - also ca. den doppelten Wert einer durchschnittlichen Präsenzapotheke - erreicht. (Dazu sei angemerkt, dass auch heute eine Vielzahl von Präsenzapotheken mit Umsatzwerten um ca. 5 Mio. DM existiert.) ${ }^{29}$

Aufgrund des gestiegenen Umsatzvolumens ist eine Rabattgewährung durch die vorgelagerten Hersteller- bzw. Handelsstufen wahrscheinlich. Auf Grundlage der oben zitierten Quellen wird in der Modellrechnung eine Rabattgewährung des Großhandels, allerdings in einem sehr moderaten Maß von $5 \%$ auf den Apothekeneinkaufspreis (AEK), angenommen. Entsprechend dem Grundsatz bundesweit einheitlicher Apothekenabgabepreise können Rabatte nicht an den Kunden weitergegeben werden und erhöhen so ausschließlich die Handelsspanne.

Die Logistikleistung, d.h. die Auslieferung bzw. Versendung der Arzneimittel, wird bei dieser regional begrenzt tätigen Versandapotheke selbst erbracht.

Die Telefonberatung der Apothekenkunden erfolgt während der Öffnungszeiten durch eigenes pharmazeutisches Personal, außerhalb der Öfnungszeiten erfolgt eine Fremdvergabe an qualitativ adäquate externe Call-Center. Auch kommt die Alternative von Kooperationsverbünden mit anderen (Versand)Apotheken während dieser Zeit in Frage.

Es zeigt sich, dass mit der Umsatzverdopplung gegenüber Präsenzapotheken trotz neuer Aufwendungen für Auslieferung und Telefonberatung eine wesentliche Verbesserungen in der Kostenstruktur erreicht werden kann. Es verbleibt ein betriebswirtschaftliches Ergebnis von ca. 6,2\%.

- Im Modell der großen Versandapotheke (VA II) wird von einem Jahresumsatz von 20 Mio. DM ausgegangen. (Dazu sei angemerkt, dass auch einige wenige Präsenzapotheken mit Umsatzwerten um ca. 20 Mio. DM existieren.) ${ }^{30}$

Auch beim Modell der großen Versandapotheke mit dem vierfachen Umsatzvolumen der Variante VA I gilt die Annahme einer 5\%-igen Rabattgewährung auf den Apothekeneinkaufspreis durch den Großhandel.

Die Logistik wird bei dieser überregional bis bundesweit agierenden Versandapotheke durch externe Logistiker (z.B. durch die Post oder andere Zustelldienste) sichergestellt.

29

Statistisches Bundesamt, zitiert in: Hackenbroch, V. (2000), Schwindel auf Rezept, S. 164, in: DER SPIEGEL 33/2000, S. 164 - 168

Statistisches Bundesamt, zitiert in: Hackenbroch, V. (2000), Schwindel auf Rezept, S. 164, in: DER SPIEGEL 33/2000, S. 164 - 168. 
Die Telefonberatung erfolgt während der Dienstzeiten durch eigenes pharmazeutisches Personal, außerhalb der Dienstzeiten durch ein externes Call-Center oder im Kooperationsverbund mit anderen (Versand)Apotheken.

Das betriebswirtschaftliche Ergebnis beträgt hier entsprechend den Modellrechnungen ca. $7,7 \%$ vom Umsatz.

Insgesamt zeigt sich, dass mit einer Umsatzvervierfachung gegenüber Versandapothekenvariante I eine Verbesserung in der Kostenstruktur erreicht werden kann, obwohl die Logistikkosten gegenüber Versandapotheke I steigen.

- Das Modell der Versandapotheke in Verbund mit Großhandel (VA III) geht von einer Zusammenlegung von Großhandels- und Apothekenfunktion in eine Vertriebsinstanz aus. Obwohl dieses Modell z.T. erheblichen Schwierigkeiten aufgrund nicht zugänglichen Datenmaterials - insbesondere bezüglich des Großhandels - unterliegt, und zudem die Quantifizierung von Synergieeffekten sich als schwierig erweist, zeigen die Untersuchungen hier eine möglicherweise zukunftsträchtige Option auf.

Im Rahmen dieser Modellvariante einer Versandapotheke könnte allerdings auch wenn dies im Rahmen dieser Studie nicht weiter thematisiert werden kann das Fremd- und Mehrbesitzverbot der $\S \S 2$ und 7 Apothekengesetz zur Disposition stehen.

Der Umsatz wird anhand verfügbarer Angaben zu Großhandelsumsätzen für mittelgroße Unternehmen orientiert, wonach sich ein Apothekenumsatz von ca. 487 Mio. DM ergibt. Diese Größe steht allerdings nicht für eine als minimal vorauszusetzende Umsatzschwelle, um beide Handelsstufen miteinander zu verbinden. Hier ist - ohne Abweichungen von der Kostenstruktur - auch ein Gesamtumsatz von 100 Mio. DM bis 200 Mio. DM ohne weiteres vorstellbar. Analog zu den Versandapotheken Variante I und II wird auch hier eine Rabattgewährung von ca. $5 \%$ auf den Apothekeneinkaufspreis angenommen. Allerdings erscheint durch die Konzentration der Einkaufsmacht auf einer gemeinsamen Handelsstufe auch ein wesentlich höherer Rabatt realistisch. Zudem wird die Betriebshandelsspanne beider Vertriebsinstanzen - Apotheke und Großhandel - durch die Zusammenlegung in eine Handelsstufe miteinander addiert.

Die Logistik wird bei dieser überregional bis bundesweit agierenden Versandapotheke an externe Logistiker vergeben.

Die Telefonberatung wird rund um die Uhr durch eigenes pharmazeutisches Personal erbracht.

Das nach den Modellrechnungen erzielbare betriebswirtschaftliche Ergebnis von $11,6 \%$ liegt im Vergleich zu den anderen potentiellen Versandapotheken aufgrund der Skaleneffekte wesentlich höher. 
Mit der Darstellung unterschiedlicher Umsatzgrößenklassen von Versandapotheken wurden neben den Effekten auf mögliche Rabattnutzungen durch die Konzentration der Einkaufsmacht auch erhebliche Skaleneffekte in der Kostenstruktur deutlich. Unter Abzug aller Kosten verbleiben von der Apothekenbetriebsspanne in Abhängigkeit der Apothekengröße ca. 6,2\% bzw. 7,7\% bzw. 11,6\% als betriebswirtschaftliches Ergebnis.

Das aus der Handelsspanne nach Abzug aller Kosten resultierende Betriebsergebnisse stellt die maximal mögliche Kostenersparnis des jeweiligen Versandapothekenmodells gegenüber Präsenzapotheken dar. Die Skaleneffekte, die sich aufgrund der unterschiedlichen Umsatzgrößen in den Kosten- sowie Ertragsstrukturen der einzelnen Versandapotheken widerspiegeln, haben einen wesentlichen Einfluss auf die Höhe der zu erwartenden Einsparpotentiale für die GKV.

Abbildung IV - 4: Verwendung der Handelsspanne - Skaleneffekt der Kosten und des Betriebsergebnisses (= Kostenersparnis) in Abhängigkeit der Umsatzgrößenklassen der Versandapotheke

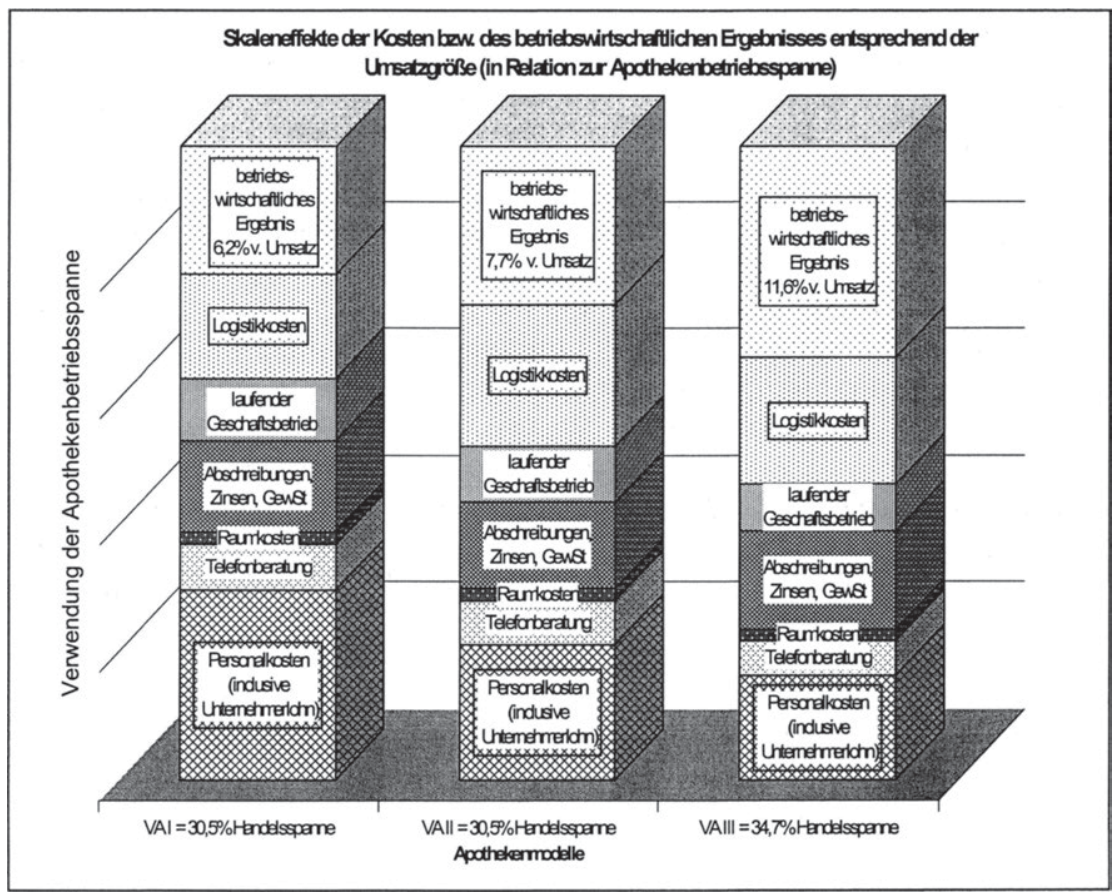

Quelle: INIFES, eigene Darstellung. 
(3) Welches Marktpotential ergibt sich?

Nach Kenntnis der betriebswirtschaftlichen Kostenstrukturen potentieller Versender werden diese mit den Varianten volkswirtschaftlicher Zielsegmente kombiniert. Daraus resultiert das Marktpotential.

Es zeigt sich, dass das Zielsegment (aus epidemiologischen Kriterien für den Versand in Frage kommende Indikationen) auch unter Geltung ökonomischer Restriktionen nur gering modifiziert werden muss. Das heißt, das Marktpotential weicht vom Zielsegment jeweils nur marginal um maximal $4 \%$ ab. Es entfallen die Indikationen, die aus der Apothekenbetriebsspanne nicht alle Handlungskosten (inklusive Kosten für Logistik und Telefonberatung) je durchschnittliche Verordnung decken und damit nicht zu Kosteneinsparungen beitragen. (Abbildung IV - 5)

Abbildung IV - 5: Marktpotentialvarianten

\begin{tabular}{|c|c|c|c|}
\hline Zielsegment - Variante & A & B & $\mathrm{C}$ \\
\hline Zielsegment in \% vom GKV-Fertigarzneimittelmarkt 1999 & $41 \%$ & $47 \%$ & $57 \%$ \\
\hline Marktpotential in \% vom GKV-Fertigarzneimittelmarkt 1999 & & & \\
\hline VAI & $39 \%$ & $44 \%$ & $53 \%$ \\
\hline VA II & $39 \%$ & $44 \%$ & $52 \%$ \\
\hline VA III & $39 \%$ & $45 \%$ & $53 \%$ \\
\hline
\end{tabular}

Quelle: INIFES, eigene Darstellung

(4) Welches Einsparpotential resultiert aus der Zulassung eines Pharmaversands für die gesetzliche Krankenversicherung?

Die Kosteneinsparungen auf die Arzneimittelpreise von ca. 6,2\% (VA I ), 7,7\% (VA II) bzw. 11,6\% (VA III) gelten unter der Annahme der Beibehaltung der aktuell gültigen Arzneimittelpreisverordnung.

Die Ausprägungen des Einsparpotentials ergeben sich jeweils, indem der prozentuale Kostenvorteil der Versandapotheken auf das Marktpotential (abzüglich 5\% - Kassenrabatt) ${ }^{31}$ bezogen wird. Folgende Abbildung gibt einen Überblick über die erzielbaren Ausprägungen der Einsparpotentiale:

In der folgenden Abbildung IV-6 enthalten die ausgewiesenen Prozentangaben für das Marktpotential den Kassenrabatt. Die dargestellten neun Ausprägungen des Einsparpotentials sind um den $5 \%$-Kassenrabatt bereits gekürzt. 
Abbildung IV - 6: Einsparpotentiale im Überblick

\begin{tabular}{|c|c|c|c|c|}
\hline \multirow{2}{*}{\multicolumn{2}{|c|}{$\begin{array}{l}\text { Einsparpotentiale für GKV } \\
\text { in Mio. DM für } 1999^{32}\end{array}$}} & \multicolumn{3}{|c|}{$\begin{array}{c}\text { Marktpotential in \% } \\
\text { am GKV-Fertigarzneimittelumsatz } \\
\text { (ohne Durchschnittliche Betriebsrendite) }\end{array}$} \\
\hline & & $\mathrm{A}:$ & & C \\
\hline \multirow{3}{*}{ 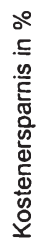 } & VAI $6,2 \%-6,3 \%^{33}$ & 861 & 956 & 1.142 \\
\hline & VA II $7,6 \% \cdot-7,7 \%^{34}$ & 1.060 & 1.169 & 1.398 \\
\hline & VA III $11,5 \%-11,8 \%{ }^{35}$ & 1.607 & 1.800 & 2.144 \\
\hline
\end{tabular}

Quelle: INIFES, eigene Darstellung.

Das Intervall möglicher Einsparungen liegt zwischen 861 Mio. DM und 2.144 Mio. DM. Differenziert man diese Betrachtungen nach den Größenklassen der Versandapotheke und den schon angesprochenen Skaleneffekten, zeigt sich, dass:

- das Modell der kleinen Versandapotheke mit ca. 5 Mio. DM Umsatz (VA I) durch eine 6,2\%ige Kosteneinsparung auf die Arzneimittelkosten ein Einsparpotential von 861 Mio. DM bis 1.142 Mio. DM ergibt,

- das Modell der großen Versandapotheke mit ca. 20 Mio. DM Umsatz (VA II) durch eine 7,7\%ige Kosteneinsparung ein Einsparpotential von 1.060 Mio. DM bis 1.398 Mio. DM ergibt,

- das Modell der Versandapotheke mit kombinierter Großhandels- und Apothekenfunktion und einem Umsatz von 487 Mio. DM (VA III) durch eine 11,6\%ige Kosteneinsparung ein Einsparpotential von 1.607 Mio. DM bis 2.144 Mio. DM ergibt.

Dies zeigt, dass insbesondere durch umsatzstarke Versender aufgrund deren interner Betriebsstruktur die höchsten Einsparpotentiale innerhalb der entsprechenden Marktpotentiale zu erwarten sind. So liegen die maximal möglichen Einsparvolumina von VA III doppelt so hoch wie die von VA I.

Die Ergebnisse für das Einsparpotential ergeben sich aus Berechnungen mit ungerundeten Zahlenangaben zur Kostenersparnis bzw. dem Marktpotential, d.h. diese sind auf alle Nachkommastellen exakt ermittelt. Aufgrund der Angabe gerundeter Werte in Abbildung IV - 6 ergeben sich marginale Abweichungen.

Durchschnittlich $6,2 \%$.

Durchschnittlich $7,7 \%$.

Durchschnittlich $11,6 \%$. 
Die ermittelten Kostenvorteile am Arzneimittelpreis können jedoch durch die Annahme der Geltung der Arzneimittelpreisverordnung nicht in Form einer Preisdifferenzierung (d.h. in Form geringerer Preise) an die GKV bzw. die Versicherten weitergegeben werden. Damit stellt sich die Frage, über welche Erstattungssysteme diese Kostenvorteile den Kostenträgern zugeführt werden können.

Mit den berechneten Kostenvorteilen ist neben den Erstattungsmodalitäten an die GKV auch ein weiterer wichtiger Aspekt verbunden, den es im folgenden ebenfalls zu differenzieren gilt. Aus wirtschaftstheoretischen Erwägungen ist davon auszugehen, dass bei einer Deregulierung des Monopols des stationären Vertriebs potentielle Anbieter - seien diese nun neue Akteure oder schon existierende Präsenzapotheken nur dann einen neuen Vertriebsweg erschließen werden, wenn dazu ein ökonomischer Anreiz besteht. Dieser ist, zumindest in langfristiger Perspektive, mit einer Gewinnerwartung in diesem Markt verbunden.

Beide Aspekte - die Form der Erstattung an die GKV wie die Berücksichtigung ökonomischer Anreize für potentielle Anbieter - bedeuten eine Modifizierung des ermittelten Kostenvorteils dahingehend, dass dieser nicht im vollen Umfang zur Ermittlung von Einsparpotentialen für die GKV zur Verfügung steht. Entsprechend gestaltet sich eine Differenzierung der Kostenvorteile von $6 \%$ bzw. $8 \%$ bzw. $12 \%$ als notwendig:

- Diese kann zum einen erfolgen, in dem der Kostenvorteil durch die Annahme einer durchschnittlichen Gewinnerwartung der potentiellen Anbieter geschmälert wird. Der verbleibende Betrag steht zur Erschließung von Einsparpotentialen der GKV zur Verfügung.

- Zum anderen könnte ein Anteil des Kostenvorteils in Form von einheitlichen Rabatten an die GKV abgeführt werden ${ }^{36}$. Der verbleibende Betrag wäre als Gewinnerwartung für die potentiellen Anbieter zu interpretieren.

(5a) Wie verringert sich das Einsparpotential bei Berücksichtigung einer durchschnittlichen Betriebsrendite für Versandapotheken?

Im Folgenden wird die Berücksichtigung einer durchschnittlichen Betriebsrendite und ihre Auswirkungen auf die Einsparvolumina der GKV thematisiert. Versandapotheken als zusätzliche Marktakteure werden nur dann im Zielsegment agieren, wenn sie zumindest auf mittel- und langfristige Sicht eine positive Unternehmensrendite erwarten können.

Entsprechend muss die Eruierung einer Unternehmensrendite erfolgen. Dies ist allerdings nur als Angabe einer durchschnittlichen Betriebsrendite ${ }^{37}$, wie dies auch für die derzeitigen Präsenzapotheken im Apothekenbetriebsvergleich des IFH Köln bzW. durch die ABDA erfolgt, möglich:

Diese Rabatte wären zusätzlich zum derzeitigen Kassenrabatt von $5 \%$ zu betrachten.

Diese wird im folgenden z.T. auch als Durchschnittliche Betriebsrendite bezeichnet. 
- Für das Jahr 1998 lag die Betriebsrendite der Präsenzapotheken im Durchschnitt bei ca. $0,4 \%$ (siehe Kapitel 2.4.1).

- Diese ist jedoch nicht mit einer individuellen Apothekenrendite zu verwechseln, denn wie allgemein existiert auch hier eine positive Korrelation zwischen Umsatzstärke und Rendite. So liegt die Schwankungsbreite einer Präsenzapotheke von 5 Mio. DM Umsatz, diese entspräche aufgrund ihrer Umsatzstärke dem Modell VA I, nach dem IFH Köln zwischen $0 \%$ und $10 \%{ }^{38}$

- Zudem ist eine durchschnittliche Betriebsrendite keine garantierte Rendite. Das heißt, die Möglichkeit eines wirtschaftlichen Misserfolgs in Form eines negativen Ergebnisses der individuellen Apothekenrendite ist auch bei einem positiven branchenbezogenen Durchschnittswert gegeben.

Analog dieser Argumentation ist auch die Einbeziehung einer durchschnittlichen Betriebsrendite für Versandapotheken in die weiteren Modellrechnungen zu verstehen. Es handelt sich dabei weder um eine individuelle noch um eine garantierte Unternehmensrendite.

Wie der zweite Argumentationspunkt verdeutlicht, korrelieren wie allgemein im Wirtschaftssektor auch im Apothekensektor Umsatz und Rendite miteinander. Da in den simulierten Modellen für Versandapotheken von wesentlich umsatzstärkeren Akteuren im Vergleich zur durchschnittlichen Präsenzapotheke mit 2,5 Mio. DM Umsatz ausgegangen wird - dieses Spektrum liegt zwischen einer Verdopplung des Umsatzes bis zu einer 250fachen Umsatzerhöhung - muss sich dieser statistische Zusammenhang auch in der Höhe einer durchschnittlichen Betriebsrendite für Versandapotheken widerspiegeln. ${ }^{39}$ Die Orientierung erfolgt dabei an der Minimaldefinition, dass potentielle Anbieter einen ökonomischen Anreiz verfolgen, um neue Marktstrukturen zu erschließen. Sie erwarten sich also laut Annahme eine Betriebsrendite gröBer Null. Entsprechend erfolgt die Berechnung einer Rendite für Versandapotheken anhand der Präsenzapotheken, die eine positive Rendite aufweisen. Aus dem gewichteten Durchschnitt ergibt sich ein Wert von ca. 3,5\% Betriebsrendite.

Die Berücksichtigung einer durchschnittlichen Betriebsrendite in den Berechnungen bedeutet nicht, dass von den bisherigen durchschnittlichen Kosteneinsparungen der Versandapotheken von 6,2\% (VAI) bzw. 7,7\% (VA II) bzw. 11,6\% (VA III) eine entsprechende Durchschnittliche Betriebsrendite von 3,5\% abgezogen werden müsste und sich bei entsprechender Multiplikation mit dem Marktpotential das neue Einsparpotential berechnen ließe. Die Berücksichtigung einer durchschnittlichen Betriebs-

38

Müller-Hagedorn, L. (1998), Strategische Optionen für die Apotheke, S. 38, in: Kaapke, A. (Hrsg.), Management in Apotheken, Sonderheft 43 des IFH Köln, S. 35 - 55.

39

Würde eine durchschnittliche Betriebsrendite von $0,4 \%$ analog den Präsenzapotheken zugrunde gelegt, hătte dies zur Folge, dass der Anteil des Kostenvorteils für die GKV höher ausfällt als bei einer entsprechend höher angesetzten Durchschnittliche Betriebsrendite. Folglich würden auch die Einsparpotentiale höher ausfallen als im folgenden dargestellt.

Siehe weiter oben unter 4.1.2. 
rendite in den Berechnungen bedeutet, dass diese in die internen Kalkulationen der Versandapotheke wie zusätzliche zu deckende Kosten einfließen. Das heißt, neben allen Kosten müsste nun zusätzlich eine Kostenposition für die Durchschnittliche Betriebsrendite als Gewinnvorauskalkulation berücksichtigt werden, die es aus den Vertriebsspannen der Verordnungswerte des Zielsegments zu decken gilt.

Durch die Vorauskalkulation einer durchschnittlichen Betriebsrendite kommt es zu zwei wesentlichen Effekten bei der Berechnung der Kostenersparnisse wie der Marktpotentiale:

- Zum einen fallen die Kostenersparnisse, wesentlich geringer aus, nämlich ca. $3,4 \%$ (VA I), ca. 5,0\% (VA II), bzw. 8,2\% (VA III). D.h. nur diese würden nach Abzug einer durchschnittlichen Betriebsrendite in Form von zusätzlichen umsatzabhängigen Rabatten an die Krankenkassen weitergeleitet werden.

- Zum anderen sinkt die Anzahl der Indikationen, die neben allen Kosten inklusive der Logistikkosten zusätzlich eine durchschnittliche Betriebsrendite aus der Handelsspanne decken. Damit sinkt das jeweilige Marktpotential im Gegensatz zur bisherigen Betrachtung erheblich. Besonders stark sind davon die umsatzschwächeren Versender VA I und VA II betroffen: Deren Marktpotential sinkt im Vergleich zum Zielsegment um ca. ein Viertel. Siehe dazu folgende Abbildung:

Abbildung IV - 7: Veränderung der Marktpotentiale durch Berücksichtigung einer Betriebsrendite

\begin{tabular}{|c|c|c|c|c|c|c|}
\hline \multirow{2}{*}{} & \multicolumn{4}{|c|}{ Zielsegmentvariante } \\
\cline { 2 - 7 } & \multicolumn{2}{|c|}{ A } & \multicolumn{2}{c|}{ B } & \multicolumn{2}{c|}{ C } \\
\hline $\begin{array}{c}\text { Berücksichtigung Be- } \\
\text { triebsrendite }\end{array}$ & ohne & mit & ohne & mit & ohne & mit \\
\hline VAI & $39 \%$ & $31 \%$ & $44 \%$ & $37 \%$ & $53 \%$ & $44 \%$ \\
\hline VA II & $39 \%$ & $31 \%$ & $44 \%$ & $37 \%$ & $52 \%$ & $44 \%$ \\
\hline VA III & $39 \%$ & $39 \%$ & $45 \%$ & $44 \%$ & $53 \%$ & $52 \%$ \\
\hline
\end{tabular}

Quelle: INIFES, eigene Darstellung.

Da das Einsparpotential aus beiden Einflussfaktoren - dem Marktpotential wie der Kostenersparnis - gebildet wird, unterliegt es folglich insbesondere bei VA I und VA II einer überproportionalen Dezimierung durch die Berücksichtigung einer durchschnittlichen Betriebsrendite. Umsatzstarke Versender wie VA III können nach wie vor relativ hohe Einsparpotentiale erzielen. 
Folgende Abbildung ${ }^{41}$ gibt einen Überblick über die Ausprägungen der Einsparpotentiale bei Berücksichtigung einer durchschnittlichen Betriebsrendite wieder:

Abbildung IV - 8: Veränderung der Einsparpotentiale durch Berücksichtigung einer Betriebsrendite

\begin{tabular}{|c|c|c|c|c|c|c|c|}
\hline \multirow{4}{*}{\multicolumn{2}{|c|}{$\begin{array}{c}\text { Einsparpotentiale für GKV } \\
\text { in Mio. DM für } 1999^{42}\end{array}$}} & \multicolumn{6}{|c|}{$\begin{array}{c}\text { Marktpotential in \% } \\
\text { am GKV-Fertigarzneimittelumsatz } \\
\text { (bei durchschnittlicher Betriebsrendite) }\end{array}$} \\
\hline & & \multirow{2}{*}{\multicolumn{2}{|c|}{$\begin{array}{l}\text { A } \\
\text { ca. }\end{array}$}} & \multirow{2}{*}{\multicolumn{2}{|c|}{$\begin{array}{l}\text { B } \\
\text { ca. }\end{array}$}} & \multirow{2}{*}{\multicolumn{2}{|c|}{$\begin{array}{l}\text { C } \\
\text { ca. }\end{array}$}} \\
\hline & & & & & & & \\
\hline & & $31 \%$ & $39 \%$ & $37 \%$ & $44 \%$ & $44 \%$ & $52 \%$ \\
\hline \multirow{3}{*}{ 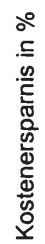 } & VAI $3,2 \%-3,6 \%{ }^{43}$ & 386 & - & 411 & - & 501 & - \\
\hline & VA II $4,8 \%-5,4 \%^{44}$ & 574 & - & 617 & - & 750 & - \\
\hline & VA III $8,1 \%-8,3 \%{ }^{45}$ & - & 1.129 & - & 1.252 & - & 1.490 \\
\hline
\end{tabular}

Quelle: INIFES, eigene Darstellung.

Das Intervall möglicher Einsparungen liegt nunmehr zwischen 386 Mio. DM und 1.490 Mio. DM. Differenziert man diese Betrachtungen nach der Größenklasse der Versandapotheke, zeigt sich, dass:

- das Modell der kleinen Versandapotheken (VA I) mit ca. 5 Mio. DM Umsatz und einer durchschnittlichen zusätzlichen umsatzabhängigen Rabattgewährung von 3,4\% ein Einsparpotential von 386 Mio. DM bis 501 Mio. DM ergibt,

- das Modell einer großen Versandapotheke mit ca. 20 Mio. DM Umsatz (VA II) und einer durchschnittlichen zusätzlichen umsatzabhängigen Rabattgewährung von 5,0\% ein Einsparpotential von 574 Mio. DM bis 750 Mio. DM ergibt,

- das Modell einer Versandapotheken mit kombinierter Großhandels- und Apothekenfunktion mit einem Umsatz von 487 Mio. DM (VA III) und einer durch-

In der folgenden Abbildung IV - 8 enthalten die ausgewiesenen Prozentangaben für das Marktpotential den Kassenrabatt. Die dargestellten neun Ausprägungen des Einsparpotentials sind um den $5 \%$-Kassenrabatt bereits gekürzt.

Die Ergebnisse für das Einsparpotential ergeben sich aus Berechnungen mit ungerundeten Zahlenangaben zur Kostenersparnis bzw. dem Marktpotential, d.h. diese sind auf alle Nachkommastellen exakt ermittelt. Aufgrund der Angabe gerundeter Werte in Abbildung IV - 8 ergeben sich marginale Abweichungen.

Durchschnittlich $3,4 \%$.

Durchschnittlich $5,0 \%$.

45

Durchschnittlich $8,2 \%$. 
schnittlichen zusätzlichen umsatzabhängigen Rabattgewährung von 8,2\% ein Einsparpotential von 1.129 Mio. DM bis 1.490 Mio. DM ergibt.

(5b) Wie verringert sich das Einsparpotential bei Berücksichtigung einer zusätzlicher einheitlicher Kassenrabatte für Versandapotheken?

Die im folgenden dargestellten alternativen Modellrechnungen basieren ebenfalls auf der Überlegung, dass die Kostenvorteile eines neuen Vertriebssystems nicht vollständig der GKV zufließen werden, folglich bei den potentiellen Versandapotheken eine Rendite verbleibt. Allerdings ist der Ansatz der Rechnungen hier ein (zusätzlich zum derzeitigen Kassenrabatt von fünf Prozent) durch die Versandapotheken abzuführender einheitlicher Kassenrabatt entsprechend dem derzeitigen Rabattsystem der GKV.

Es sei an dieser Stelle hervorgehoben, dass das Modell der Gewährung eines zusätzlichen einheitlichen Kassenrabatts durch Versandapotheken an die GKV eine Hilfskonstruktion darstellt, um durch neue Strukturen entstehende Kostenersparnisse auch ohne eine Preisdifferenzierung (die Geltung der AMPreisV wird in dieser Studie vorausgesetzt) der Versichertengemeinschaft der GKV zuzuführen. Ob ein solches Modell auch aus rechtlichen Gründen, insbesondere dem Kartellrecht, möglich ist, soll und kann im Rahmen dieser Untersuchungen nicht beantwortet werden.

Es werden drei alternative zusätzliche einheitliche Kassenrabatte angenommen: fünf Prozent, sieben Prozent und zehn Prozent vom Verordnungswert. ${ }^{47}$ Diese sind dabei zum einen an der Höhe des derzeitigen Kassenrabatts bzw. dessen doppeltem Wert orientiert, zum anderen erfolgt mit 7\% die Annahme eines Wertes innerhalb dieser Spannweite.

Wie bei den Modellrechnungen unter Berücksichtigung einer durchschnittlichen $\mathrm{Be}$ triebsrendite explizit erwähnt, bedeutet auch hier die Einbeziehung der genannten zusätzlichen Kassenrabatte nicht, dass von den durchschnittlichen Kostenvorteilen, die sich bei den Modellrechnungen ohne Berücksichtigung einer durchschnittlichen Betriebsrendite bzw. eines Kassenrabattes ergeben, ein entsprechender zusätzlicher Kassenrabatt abgezogen wird und sich bei entsprechender Multiplikation mit dem Marktpotential das neue Einsparpotential ergibt. Die Berücksichtigung eines zusätzlichen Kassenrabattes bedeutet vielmehr, dass potentielle Versandapotheken in Kenntnis einer verpflichtenden zusätzlichen Rabattbelastung diese bereits in ihrer Kostenkalkulation berücksichtigen. Das heißt, neben allen Kosten muss nun zusätzliche eine Kostenposition in der Höhe des zusätzlichen Kassenrabattes von $5 \%$ bzw. $7 \%$ bzw. $10 \%$ berücksichtigt werden, die aus den Vertriebsspannen der Verordnungswerte des Zielsegments zu decken ist.

Die kartellrechtliche Legitimation eines solchen Modells wird durch die ABDA hinterfragt.

47

Entsprechend würden inklusive der derzeitigen Rabattgewährung $10 \%$ bzw. $12 \%$ bzw. $15 \%$ vom Verordnungswert von der GKV erhoben. 
Die Vorauskalkulation einer zusätzlichen Rabattbelastung als Kostenposition für Versandapotheken führt folglich zu wesentlichen Entwicklungen:

- Je höher der zusätzliche einheitliche Kassenrabatt ausfällt, umso geringer ist das verbleibende Marktpotential. Diese Tendenz ist insbesondere bei den umsatzschwächeren Versendern zu beobachten. Dies impliziert zudem, dass umsatzschwächere potentielle Versandapotheken aufgrund einer in bezug auf ihren Umsatz relativ höheren Rabattbelastung tendenziell mehr hochpreisige Indikationsgruppen versenden werden als umsatzstarke Versandapotheke, die aufgrund hoher economies of scale eine bessere Kostensituation aufweisen.

Abbildung IV - 9: Veränderung der Marktpotentiale bei Berücksichtigung eines zusätzlichen einheitlichen Kassenrabatts (in Abhängigkeit der Höhe des zusätzlichen Kassenrabatts)

\begin{tabular}{|c|c|c|c|c|}
\hline & \multicolumn{4}{|c|}{$\begin{array}{c}\text { Marktpotential in \% des GKV-Fertigarzneimittelumsatzes } \\
\text { (Spannweite zwischen den Zieisegmentvarianten A, B und C) }\end{array}$} \\
\hline $\begin{array}{c}\text { Versand- } \\
\text { apotheke }\end{array}$ & $\begin{array}{c}\text { Ohne Berücksichti- } \\
\text { gung eines einheit- } \\
\text { lichen Kassenra- } \\
\text { battes }\end{array}$ & $\begin{array}{c}\text { Bei Berücksichti- } \\
\text { gung von } \\
\text { zusätzlich } \\
5 \% \text { Kassenrabatt }\end{array}$ & $\begin{array}{c}\text { Bei Berücksichti- } \\
\text { gung von } \\
\text { zusätzlich } \\
7 \% \text { Kassenrabatt }\end{array}$ & $\begin{array}{c}\text { Bei Berücksichti- } \\
\text { gung von } \\
\text { zusätzlich } \\
10 \% \text { Kassenrabatt }\end{array}$ \\
\hline VAI & $39 \%-53 \%$ & $21 \%-25 \%$ & - & - \\
\hline VA II & $39 \%-52 \%$ & $31 \%-40 \%$ & $16 \%-23 \%$ & - \\
\hline VA III & $39 \%-53 \%$ & $34 \%-47 \%$ & $31 \%-44 \%$ & $21 \%-23 \%$ \\
\hline
\end{tabular}

Quelle: INIFES, eigene Darstellung.

- Bei Gewährung eines zusätzlichen Kassenrabatts von 5\% würde sich das Marktpotential bei allen drei Modellen potentieller Versender verringern - im Modell VA I wäre dies im wesentlich stärken Maße der Fall als in den Modellen VA II und VA III - allerdings wären hier nach wie vor alle drei Modelle einer Versandapotheke denkbar.

- Bei Gewährung eines zusätzlichen Kassenrabatts von 7\% verringert sich das Marktpotential gegenüber der vorherigen Alternative stärker. Am geringsten ist davon das mögliche Marktpotential des umsatzstarken Modells VA III betroffen. Eine Halbierung des Marktpotentials gegenüber der Alternativrechnung mit zusätzlich 5\% Kassenrabatt erfährt Modell VA II. Das Modell VA I ist bei dieser angenommenen Rabatthöhe nicht mehr funktionsfähig, d.h. die Kostenvorteile dieses Modells VA I - ca. 6,2\% (siehe Kapitel 2.4.6) - werden durch die Gewährung 
eines höheren Rabattes negiert und die Erzielung von Einsparungen für die GKV nicht mehr möglich.

- Bei Gewährung eines zusätzlichen Kassenrabattes von $10 \%$ wären neben dem Modell VA I auch Versender nach dem Modell VA II nicht mehr funktionsfähig. Ausschließlich umsatzstarke Versender nach Modell VA III könnten aufgrund ihrer hohen economies of scale ${ }^{50}$ der erhöhten Rabattgewährung bei einem geringeren Marktpotential entsprechen.

Analog zu den vorangegangenen Modellrechnungen erfolgt auch hier die Ermittlung des Einsparpotentials aus der Kombination der beiden Faktoren Marktpotential und zusätzlicher einheitlicher Kassenrabatt.

Abbildung IV-10: Veränderung der Einsparpotentiale bei Berücksichtigung eines zusätzlichen einheitlichen Kassenrabatts (in Abhängigkeit der Höhe des zusätzlichen Kassenrabatts)

\begin{tabular}{|c|c|c|c|}
\hline & \multicolumn{3}{|c|}{$\begin{array}{c}\text { Einsparpotential für die GKV in Mio. DM für 1999 } \\
\text { (Spannweite in Abhängigkeit der Marktpotentialvarianten A, B und C) }\end{array}$} \\
\hline $\begin{array}{c}\text { Versand- } \\
\text { apotheke }\end{array}$ & $\begin{array}{c}\text { Bei Berücksichtigung } \\
\text { von } \\
\text { zusätzlich } \\
5 \% \text { Kassenrabatt }\end{array}$ & $\begin{array}{c}\text { Bei Berücksichtigung } \\
\text { von } \\
\text { zusätzlich } \\
7 \% \text { Kassenrabatt }\end{array}$ & $\begin{array}{c}\text { Bei Berücksichtigung } \\
\text { von } \\
\text { zusätzlich } \\
10 \% \text { Kassenrabatt }\end{array}$ \\
\hline VA I & $379<467$ & - & - \\
\hline VA II & $561<732$ & $413<604$ & - \\
\hline VA III & $624<864$ & $786<1.124$ & $759<863$ \\
\hline
\end{tabular}

Quelle: INIFES, eigene Darstellung.

Das Intervall möglicher Einsparungen liegt nunmehr zwischen 379 Mio. DM und 1.124 Mio. DM. Die höchsten Einsparungen werden bei $7 \%$ zusätzlichem Kassenrabatt durch die umsatzstarken Versender VA III erzielt. Differenziert nach der Größenklasse der Versandapotheken lässt sich folgendes konstatieren:

- Kleine Versandapotheken mit ca. 5 Mio. DM Umsatz (VA I) können bei einer zusätzlichen Kassenrabattgewährung von 5\% ein Einsparpotential bis zu 467 Mio. DM realisieren. Eine höhere Rabattgewährung von $7 \%$ und $10 \%$ würde die Kosteneinsparungen von ca. $6,2 \%$ übersteigen und ist somit nicht möglich.

- Große Versandapotheken mit ca. 20 Mio. DM Umsatz (VA II) würden bei einem zusätzlichen Kassenrabatt von $5 \%$ ein Einsparpotential zwischen 561 Mio. DM

50 51

Siehe Kapitel 2.4.6.

Die Ergebnisse für die Einsparpotentiale ergeben sich aus Berechnungen mit ungerundeten Zahlenangaben zum Marktpotential, d.h. diese sind auf alle Nachkommastellen exakt ermittelt. Aufgrund der Angabe ungerundeter Werte in Abbildung II - 17 ergeben sich marginale Abweichungen. 
und 732 Mio. DM realisieren, bei einem höheren zusätzlichen Kassenrabatt von 7\% lägen die Einsparpotentiale zwischen 413 Mio. DM und 604 Mio. DM. Ein höherer Kassenrabatt von $10 \%$ würde die Kosteneinsparungen von ca. $8 \%$ (siehe Kapitel 2.4.6.) übersteigen und ist somit nicht möglich.

- Versandapotheken mit kombinierter Großhandels- und Apothekenfunktion mit einem Umsatz von 487 Mio. DM (VA III) können bei einem zusätzlichen Kassenrabatt von 5\% ein Einsparpotential zwischen 624 Mio. DM und 864 Mio. DM realisieren, bei einem höheren zusätzlichen Kassenrabatt von $7 \%$ lägen die Einsparpotentiale zwischen 786 Mio. DM und 1.124 Mio. DM (Maximalwert). Ein höherer Kassenrabatt von $10 \%$ führt aufgrund des geringeren Marktpotentials zu verringerten Einsparpotentialen zwischen 759 Mio. DM und 863 Mio. DM. Diese sind vergleichbar denen bei einer Rabattgewährung von $5 \%$.

Differenziert nach der Höhe des zusätzlichen einheitlichen Kassenrabatts bleibt festzustellen:

- Eine Belastung von zusätzlich 5\% Kassenrabatt unabhängig von der Umsatzgröße würde zwar für VA III nicht das maximale Einsparpotential aufdecken, jedoch wäre die Anbieterstruktur gekennzeichnet durch das Agieren potentieller Versender unterschiedlicher Größe. Die Einsparpotentiale lägen je nach durchschnittlicher Umsatzgröße zwischen 379 Mio. DM (VA I) und 864 Mio. DM (VA III).

- Eine Belastung von zusätzlich 7\% Kassenrabatt würde den Optimalwert des Einsparpotentials zwischen 786 Mio. DM und 1.124 Mio. DM für VA III realisieren, allerdings ginge diese Rabattregelung zu Lasten der kleinen Versandapotheken des Modells VA I. Deren Funktionsfähigkeit wäre nicht mehr gegeben.

- Eine Belastung von zusätzlich $10 \%$ Kassenrabatt würde dazu führen dazu, dass nur das Modell eines umsatzstarken Versenders VA III denkbar wäre. Alle anderen Modelle potentieller Versender würden sich aufgrund der hohen Rabattbelastung, welche die Kostenvorteile des neuen Vertriebsweges übersteigen würde, als nicht funktionsfähig erweisen. Das Einsparpotential von VA III läge zwischen 759 Mio. DM und 863 Mio. DM und damit aufgrund des gesunkenen Marktpotentials unter der maximalen Ausprägung bei $7 \%$ Rabatt.

(6) Welche Marktanteile und Einsparvolumina der GKV sind kurz- und mittelfristig realisierbar?

Den vorangegangenen Betrachtungen liegt eine wesentliche Voraussetzung zugrunde: Die genannten Einsparvolumina können nur erzielt werden, wenn die aufgezeigten Marktpotentiale jeweils vollständig ausgeschöpft werden. Davon ist realistischer Weise bestenfalls langfristig, nicht jedoch in kurz- oder mittelfristiger Zukunft auszugehen.

Für Szenarien zum kurzfristigen Realisierungsgrad von Marktanteilen sind Annahmen zu treffen: 
- Akzeptanzhürden der Patienten und verordnenden Ärzte bei der Implementierung neuer Vertriebswege könnten dazu führen, dass die Realisierung von Marktpotentialen, zumindest in mittelfristiger Perspektive, nur zögerlich verläuft.

- In der Schweiz geht man von einem Marktpotential von ca. $10 \%$ aller Apothekenkunden aus. Jedoch basiert das derzeitige Schweizer Modell eines Direktversandes auf einer Patientenklientel mit Arzneimittelausgaben über SFr 450 pro Jahr, sodass mit einem Marktpotential von mehr als $10 \%$ der Arzneimittelausgaben gerechnet werden kann. ${ }^{52}$

- US-Quellen sprachen bereits 1997 von einem 12\%igen Umsatzanteil des Arneimittelversandes am Markt für verschreibungspflichtige Präparate. ${ }^{53}$ Dass es sich dabei um ein stark wachsendes Segment handelt, zeigt die Steigerung des Versandhandels auf derzeit (Stand 2000) ca. 15\% Marktanteil.

Bei einer Durchschnittsbetrachtung für Deutschland gestaltet es sich entsprechend als sinnvoll, ebenfalls von ca. $15 \%$ Marktanteil des Arzneimittelversandes am gesamten GKV-Fertigarzneimittelmarkt ausgehen.

Das langfristige Einsparpotential bei vollständiger Ausschöpfung der Marktpotentiale sowie vollständiger Kostenersparnis ergab eine Spannweite von 861 Mio. DM bis 2.144 Mio. DM in Abhängigkeit der Umsatzgröße. Die kurzfristig realisierbaren Einsparungen bei einem $15 \%$ igen Marktanteil stellt folgende Abbildung $^{55}$ dar:

52

Laut Expertengespräch Februar 2000.

American Medical Association, Report of the AMA Young Physicians section governing council, 1997, S. 2.

Ahrens, H.J., (2001), Interview des Vorstandsvorsitzenden der AOK mit der Zeitschrift impulse, Rubrik Drei Fragen an ..., siehe: impulse / mărz 2001, S. 168.

In der folgenden Abbildung IV-9 enthalten die ausgewiesenen Prozentangaben für den Marktanteil den Kassenrabatt. Die dargestellten neun Ausprägungen der Einsparvolumina sind um den $5 \%-$ Kassenrabatt bereits gekürzt. 
Abbildung IV -11: Einsparvolumina bei einem realisierten Marktanteil von 15\% (ohne Betriebsrendite)

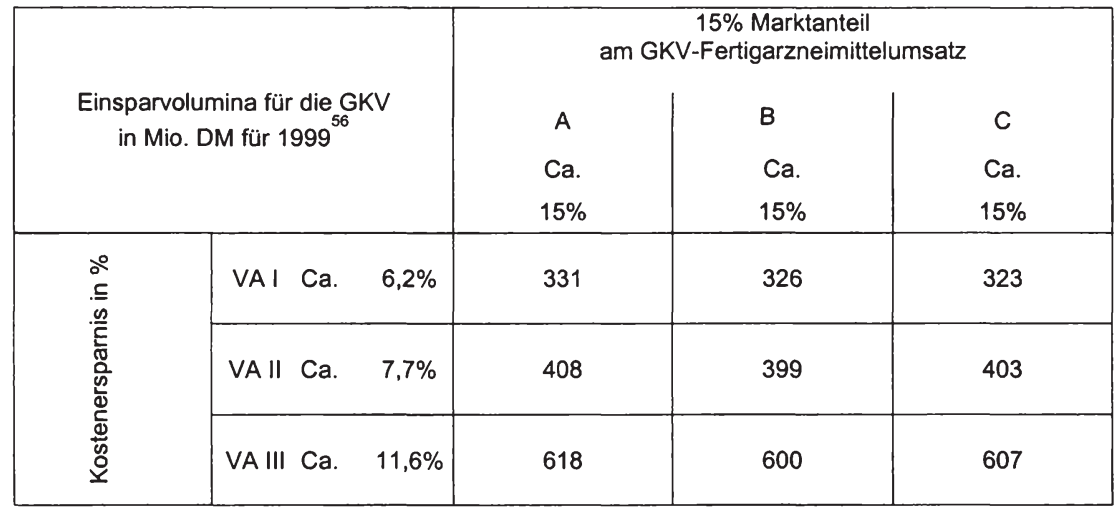

Quelle: INIFES, eigene Berechnungen.

Das Intervall realisierbarer Einsparungsvolumina liegt bei einem 15\%igen Marktanteil des Versandes zwischen 323 Mio. DM (bei ca. 6,2\% Einsparung auf die Arzneimittelkosten) und 618 Mio. DM (bei ca. 11,6\% Kosteneinsparung), differenziert nach Umsatzgröße:

- Das Intervall für VA I erstreckt sich von 323 Mio. DM bis 331 Mio. DM.

- Das Intervall für VA II erstreckt sich von 399 Mio. DM bis 408 Mio. DM.

- Das Intervall für VA III erstreckt sich von 600 Mio. DM bis 618 Mio. DM.

Unter Berücksichtigung einer durchschnittlichen Betriebsrendite von 3,5\% verringern sich diese Einsparungen erheblich.

Die Ergebnisse für die Einsparvolumina ergeben sich aus Berechnungen mit ungerundeten Zahlenangaben zur Kostenersparnis bzw. dem Marktanteil, d.h. diese sind auf alle Nachkommastellen exakt ermittelt. Aufgrund der Angabe gerundeter Werte in Abbildung IV - 9 ergeben sich marginale Abweichungen. 
Abbildung IV -12: Einsparvolumina bei einem realisierten Marktanteil von 15\% (mit Betriebsrendite)

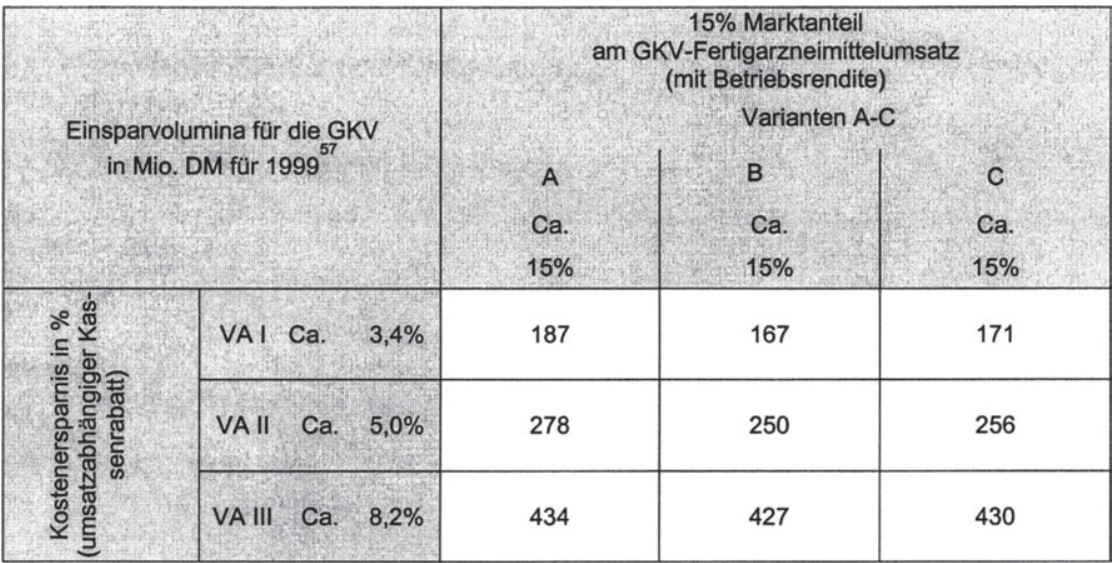

Quelle: INIFES, eigene Berechnungen.

Das Intervall realistischer Einsparungsvolumina liegt bei einem 15\%igen Marktanteil des Versandes zwischen 167 Mio. DM (bei ca. 3,4\% Einsparung auf die Arzneimittelkosten) und 434 Mio. DM (bei ca. 8,2\% Kosteneinsparung), differenziert nach Umsatzgröße:

- Das Intervall für VA l erstreckt sich von 167 Mio. DM bis 187 Mio. DM.

- Das Intervall für VA II erstreckt sich von 250 Mio. DM bis 278 Mio. DM.

- Das Intervall für VA III erstreckt sich von 427 Mio. DM bis 434 Mio. DM.

Es zeigt sich, dass die Berücksichtigung einer durchschnittlichen Betriebsrendite einen erheblichen Einfluss auf die Höhe der Einsparvolumina hat. Die größten negativen Auswirkungen zeigen sich bei dem Versandapothekenmodell VA I mit 5 Mio. DM: hier verringert sich das Einsparvolumen um 46\% von durchschnittlich 327 Mio. DM auf 175 Mio. DM ${ }^{58}$. VA II mit 20 Mio. DM Umsatz weist bei Berücksichtigung einer durchschnittlichen Betriebsrendite eine Verringerung des Einsparvolumens von ca. $35 \%$ auf, der größte potentielle Anbieter VA III von ca. $30 \%$. Zurückzuführen ist dies auf die wesentlich geringeren Rabatte, die bei einer Berücksichtigung der durchschnittlichen Betriebsrendite an die GKV fließen würden.

In den Modellrechnungen mit einem zusätzlichen einheitlichen Kassenrabatt ergeben sich folgende realisierbare Einsparvolumen bei 15\% Marktanteil:

57

Die Ergebnisse für die Einsparvolumina ergeben sich aus Berechnungen mit ungerundeten Zahlenangaben zur Kostenersparnis bzw. dem Marktanteil, d.h. diese sind auf alle Nachkommastellen exakt ermittelt. Aufgrund der Angabe gerundeter Werte in Abbildung IV - 10 ergeben sich marginale Abweichungen.

Durchschnitt über die jeweiligen drei Ausprägungen an Marktanteilen für VA I. 
Abbildung IV - 13: Einsparvolumina bei Berücksichtigung zusätzlicher einheitlicher Kassenrabatte und einem Marktanteil von $15 \%$

\begin{tabular}{|c|c|c|c|}
\hline & \multicolumn{3}{|c|}{ Einsparvolumina für die GKV in Mio. DM für 1999 } \\
Versand- \\
apotheke & $\begin{array}{c}\text { Bei Berücksichtigung } \\
\text { von } \\
\text { zusätzlich } \\
\text { \% Kassenrabatt }\end{array}$ & $\begin{array}{c}\text { Bei Berücksichtigung } \\
\text { von } \\
\text { zusätzlich } \\
7 \% \text { Kassenrabatt }\end{array}$ & $\begin{array}{c}\text { Bei Berücksichtigung } \\
\text { von } \\
\text { zusätzlich } \\
10 \% \text { Kassenrabatt }\end{array}$ \\
\hline VA I & 276 & - & - \\
\hline VA II & 276 & 386 & 552 \\
\hline VA III & 276 & 386 & - \\
\hline
\end{tabular}

Quelle: INIFES, eigene Darstellung.

Das Intervall realisierbarer Einsparvolumina liegt bei einem 15\%igen Marktanteil des Versandes je nach Höhe des zusätzlichen einheitlichen Kassenrabattes zwischen 276 Mio. DM und 552 Mio. DM. Durch die Annahme eines zusätzlichen einheitlichen Kassenrabatts wie fixen Marktanteils ergeben sich innerhalb der drei Alternativen an Kassenrabatten keine Unterschiede in Abhängigkeit von der Umsatzgröße des potentiellen Versenders.

Differenziert nach den Modellen potentieller Versender VA I, VA II und VA III lässt sich folgendes festhalten:

- Kurz- und mittelfristig wären durch das Modell VA I Einsparvolumina von 276 Mio. DM bei einem zusätzlichen Kassenrabatt von $5 \%$ realisierbar.

- Das Intervall realisierbarer Einsparvolumina durch das Modell VA II würde kurzund mittelfristig zwischen 276 Mio. DM (bei einem zusätzlichen Kassenrabatt von 5\%) und 386 Mio. DM (bei 7\% zusätzlichem Kassenrabatt) liegen.

- Kurz- und mittelfristig wären durch das Modell VA III Einsparvolumina von 276 Mio. DM (bei einem zusätzlichen Kassenrabatt von $5 \%$ ) bis 552 Mio. DM (bei einem zusätzlichen Kassenrabatt von 10\%) realisierbar.

Differenziert nach zusätzlichen einheitlichen Kassenrabatts bleibt festzustellen:

- Bei zusätzlicher Rabattgewährung von 5\% könnten 276 Mio. DM durch alle drei Modelle potentieller Versender realisiert werden.

59

Die Ergebnisse für die Einsparpotentiale ergeben sich aus Berechnungen mit ungerundeten Zahlenangaben zum Marktpotential, d.h. diese sind auf alle Nachkommastellen exakt ermittelt. Aufgrund der Angabe ungerundeter Werte in Abbildung 1 ergeben sich marginale Abweichungen. 
- Bei zusätzlicher Rabattgewährung von 7\% könnten 386 Mio. DM durch die potentiellen Versender VA II und VA III realisiert werden.

- Bei zusätzlicher Rabattgewährung von 10\% könnten 552 Mio. DM durch den potentiellen Versender VA III realisiert werden.

\section{(7) Abschließende Anmerkungen zu den getroffenen Annahmen}

Obwohl alle simulierten Markt- wie Einsparpotentialvarianten gleichermaßen differenziert und fundiert hergeleitet werden, unterliegen sie doch unterschiedlichen Annahmen, auf die hier nochmals kurz kritisch eingegangen wird.

Bei der Ermittlung der Zielsegmente wird davon ausgegangen, dass der Arzneimittelversand an den Endverbraucher nur für solche Indikationsgruppen realistisch ist, die primär Medikamente für nicht akute Erkrankungen bzw. Dauermedikationen beinhalten. Die Auswahl der Indikationsgruppen erfolgt anhand der Kriterien "Packungsgröße", "DDDs" und "Expertenmeinung". Die Abgrenzungen "Umsätze von N3-Packungen" und "50 DDDs und mehr" zielt auf Medikamente aus dem Bereich der mittel- bis langfristigen Medikation ab, beinhaltet, wie dargestellt, aber einige Unwegsamkeiten z.B. bzgl. der mengenmäßigen Abgrenzungen zwischen N2- und N3-Packungen, sowie dahingehend, ob die Grenze von 50 DDDs als "große" Packung allgemeingültig ist. Als "stabilste" Annahme ist deshalb die Abgrenzung anhand des Kriteriums überdurchschnittlicher täglicher Dosierungen - also "35 DDDs und mehr" - zu bewerten, die zu Marktanteilen von ca. 53\% führte. Diese Einschätzung wird auch durch die Expertenmeinung gestützt, wonach $59 \%$ der GKV-Umsätze auf Medikationen für überwiegend chronische Erkrankungen entfallen.

Die Untersuchungen zu den Kosten des Pharmaversandes machen eine Vielzahl von Annahmen notwendig, da hier gänzlich neue Strukturen unter Rückgriff auf sehr spärtiches Datenmaterial simuliert werden müssen. Die von INIFES getroffenen Annahmen sind bzgl. der "Chancen" des Pharmaversandes eher konservativ. Damit wird gewährleistet, dass bei der Analyse potentieller Auswirkungen einer Ausweitung des Pharmaversandes in Deutschland ein Versandapotheken-Modell hoher Qualität und solider betriebswirtschaftlicher Struktur das Fundament der Berechnungen bildet. Die INIFES-Berechnungen gehen beispielsweise von einer 24-StundenTelefonhotline auch bei "kleinen" Versandapotheken aus. Die Kostenstrukturanalysen gehen zudem z.B. von großzügig gestalteten Lagerflächen und relativ hohen Miet- und Versandkosten aus. Auch die angenommene Rabattgewährung von 5\% durch die Hersteller stellt, insbesondere unter der Annahme umsatzstarker Versandapotheken, eher eine Untergrenze dar.

In den Berechnungen werden drei verschiedene Typen von Versandapotheken modelliert: VA I, VA II und VA III.

Das Modell einer Versandapotheke VA I mit jährlichen Umsätzen in doppelter Höhe des derzeitigen Durchschnitts der Präsenzapotheken (bei weitgehend gleichem Per- 
sonalbestand wie diese) zeigt, dass der regional begrenzte Versand an den Endverbraucher auch für die existierenden Präsenzapotheken eine attraktive Alternative darstellen kann.

Offen ist aber, in welchem Umfang solche kleinen Versandapotheken in einer Konkurrenzsituation mit großen Versandapotheken oder gar mit Versandapotheken, die im Verbund mit dem Großhandel agieren, langfristig überlebensfähig sind. Die Erfahrungen aus dem Ausland sowie erste Entwicklungen in Deutschland (z.B. bei Versendern von Impfstoffen etc.) zeigen vielmehr, dass Versandapotheken mit Jahresumsätzen ab 20 Mio. DM eine sehr realistische, betriebswirtschaftlich rentable Unternehmensform in einem liberalisierten Arzneimittel-Distributionssystem darstellen können. In noch stärkerem Maße gilt diese Annahme selbstverständlich für Versandapotheken, die einen direkten Verbund mit dem Pharmagroßhandel eingehen und sozusagen als deren verlängerter Arm für den Vertrieb an den Endverbraucher dienen.

Bei diesen letzten beiden Varianten liegen die Einsparpotentiale zwischen ca. 1.060 Mio. DM und 2.144 Mio. DM bei vollständiger Abschöpfung des Marktpotentials bzw. zwischen 323 Mio. DM und 618 Mio. DM

Durch eine Differenzierung der Kostenvorteile eines Versandes zum einen durch eine durchschnittliche Betriebsrendite wie durch zusätzliche einheitliche Kassenrabatte reduzieren sich die Einsparpotentiale für die GKV.

Das Einsparpotential als Kombination aus beiden Komponenten "Marktpotential" wie "Kostenersparnis" unterliegt insbesondere bei VA I und VA II einer überproportionalen Dezimierung:

- Bei Berücksichtigung einer durchschnittlichen Branchenrendite liegt das Intervall möglicher Einsparungen nunmehr zwischen 386 Mio. DM (VA I) und 1.490 Mio. DM (VA III).

- Bei Berücksichtigung zusätzlicher einheitlicher Kassenrabatte liegt das Intervall möglicher Einsparpotentiale zwischen 379 Mio. DM (VA I) und 1.124 Mio. DM (VA III).

Entsprechend erheblich stellen sich die Veränderungen des Einsparvolumens bei Annahme eines 15\%igen Marktanteils dar:

- Bei Berücksichtigung einer durchschnittlichen Betriebsrendite liegt das Intervall der Einsparungsvolumina nunmehr zwischen 167 Mio. DM (bei ca. 3,4\% zusätzlichen Kassenrabatt für VA I) und 434 Mio. DM (bei ca. 8,2\% zusätzlichen Kassenrabatt durch VA III).

- Bei Berücksichtigung zusätzlicher einheitlicher Kassenrabatte liegt das Intervall der Einsparvolumina nunmehr zwischen 276 Mio. DM (bei 5\% zusätzlichen Kassenrabatt für alle Modelle VA I, VA II und VA III) und 552 Mio. DM (bei 10\% Kassenrabatt für Modell VA III). 
Die INIFES-Modellrechnungen simulieren die unmittelbaren monetären Auswirkungen der Ausweitung des Versandhandels in Deutschland. Sogenannte Sekundäreffekte - also die im weiteren Verlauf der Ausweitung des Arzneimittelversandes ggf. zu enwartenden strukturellen Effekte - sind nicht berücksichtigt. Sie würden den Rahmen der Modellrechnungen sprengen, sind aber sicherlich ein wichtiger Gegenstand zukünftiger Forschung.

Die hier dargestellten Ergebnisse unterliegen weiterhin dem Vorbehalt, dass eine Bewertung potentieller monetärer Einsparvolumen einer Ausweitung des Pharmaversandes nicht ohne Berücksichtigung struktureller und qualitativer Effekte einer Liberalisierung der Arzneimitteldistribution erfolgen kann.

Deshalb werden im folgenden die Ergebnisse der qualitativen Untersuchung dargestellt, bevor in Kapitel 4.2. eine Bewertung des Arzneimittelversandes - basierend auf quantitativen wie qualitativen Studienergebnissen - anhand von Pro- und ContraArgumenten erfolgt.

\subsubsection{Zusammenfassung der qualitativen Projektergebnisse}

Zur Evaluation der Akzeptanz und der generellen Einstellung niedergelassener Apotheker und Ärzte sowie der Bevölkerung gegenüber einer Versandapotheke hat das Internationale Institut für empirische Sozialökonomie (INIFES) im Dezember 2000 eine repräsentative CATI-Befragung durchgeführt. Ziele der Befragung waren, Anhaltspunkte zu erhalten über

- die Einstellung zum Vertriebskanal Apotheke

- die potentiellen Zielgruppen für eine Versandapotheke

- die Akzeptanz eines Versandhandels

- die Chancen und Risiken einer Versandapotheke

\section{Vertriebskanal Apotheke}

Bislang lösen Patienten aufgrund des $\S 43$ Arzneimittelgesetz (AMG) die Rezepte mit verschreibungspflichtigen Arzneimitteln in der Apotheke ein, wo sie die vom Arzt verordneten Medikamente ausgehändigt bekommen. Die quantitative Arzneimittelversorgung in der Bundesrepublik Deutschland wird von 21.590 Apotheken ${ }^{60}$ sicherge- $^{2}$ stellt. Von einer Überversorgung sprechen 32,0 Prozent der Ärzte und immerhin 27,0 Prozent der Apotheker. Als gerade angemessen bezeichnen 72,0 Prozent der Apotheker die Apothekendichte (bei den Ärzten liegt dieser Wert bei 59,0 Prozent). Eine Unterversorgung wird als kaum existent betrachtet. Die Versorgung der Bevölkerung mit Arzneimitteln ist somit sichergestellt (s. Abbildung IV - 11). 
Abbildung IV - 14: Beurteilung der Apothekendichte

(Frage: "Überversorgung mit Apotheken wird heute in manchen Gebieten als Problem angesehen. Wie beurteilen Sie die Situation im Einzugsgebiet Ihrer Apotheke/Praxis?")

Insgesamt bereits Öberversorgung

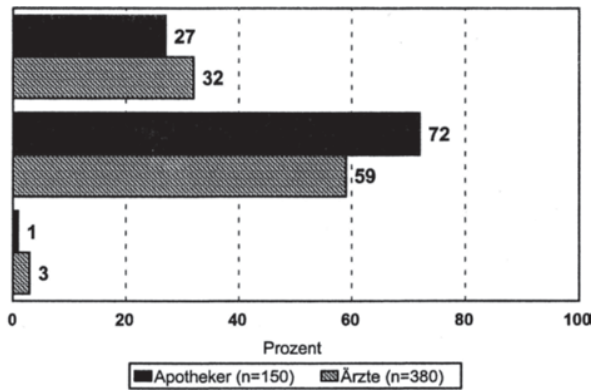

Quelle : INIFES, eigene Darstellung.

(Ergebnisse einer von TNS EMNID im Auftrag von INIFES im Dezember 2000 durchgeführten CATI-Befragung).

Die überwiegende Anzahl der befragten Bürger gab an, das Rezept selber einzulösen, wobei der Wert im Alter rückläufig ist. Dies steht jedoch im Widerspruch zu den Angaben der befragten Apotheker. 49,0 Prozent gaben an, dass zwischen 20,0 Prozent und 50,0 Prozent der Kunden Rezepte nicht für sich selber, sondern für eine andere Person als "Besorger" einlösen. Der Anteil der "Rezeptbesorger" beträgt bei 3,0 Prozent der Apotheken sogar mehr als 50,0 Prozent. Die sog. "BesorgerProblematik" beschreibt somit eine nicht zu unterschätzende Distributionsstufe innerhalb des "Vertriebskanals" Apotheke. Viele Arzneimittelbezieher sind in ihrer Mobilität aufgrund einer Krankheit, des Alters oder der Berufstätigkeit stark eingeschränkt. Eine vertraute Person, welche die Rolle eines "Rezeptüberbringers" und "Medikamentenbesorgers" übernimmt, ist oft das nötige Bindeglied zwischen Apotheke und Patienten/Kunden. Für 7,0 Prozent der Bundesbürger ist es nach eigenen Angaben üblich, Medikamente häufiger von der Apotheke nach Hause geliefert zu bekommen bzw. das Rezept von Angehörigen, Vertrauenspersonen oder dem Arzt einlösen zu lassen. Vor allem Personen der Altersklasse 30 - 39 Jahre (8,0 Prozent) und der über 60jährigen (12,0 Prozent) erhalten auf diesem Wege die verordneten Medikamente. Aufgrund der bisher vorliegenden Ergebnisse ,dass 41,0 Prozent der befragten Ärzte - gesetzliche Bestimmungen außer Acht gelassen - für Patienten bei entsprechender Bedürfnislage die Rezeptweiterleitung an die Apotheke übernehmen, den Aussagen der Experten ${ }^{81}$ sowie des $\S 17$, II ApoBetrO, existiert in der Bundesrepublik Deutschland bereits ein Versandhandel. Die Ausnahmeregelung des $\S 17,11$

61

Im Anschluss an die Befragungen wurden Experteninterviews mit Vertretern von Standesorganisationen, der Apotheker, der Politik, der Wissenschaft, der Industrie, der Verbraucherverbände und der Krankenkassen geführt. 
ApoBetrO billigt in begründeten Einzelfällen eine Versendung aus der Apotheke oder die Zustellung durch einen Boten an den Arzneimittelempfänger zu.

Die INIFES-Befragung ergab, dass 2,0 Prozent der Bundesbürger Medikamente häufiger von der Apotheke nach Hause geliefert bekommen. Von den Personen, die ihr Rezept selber einlösen oder die Medikamente von der Apotheke nach Hause geliefert bekommen, gaben 42,0 Prozent an, dass sie bei der Auswahl der Apotheke das Kriterium des kostenlosen Heimlieferservices von Medikamenten für sehr wichtig bzw. wichtig empfinden (s. Abbildung III - 19). ${ }^{62}$ Besonders die Altersklassen der $30-$ 39jährigen (44,0 Prozent), der 50-59jährigen (48,0 Prozent) und der über 60jährigen (51,0 Prozent) schätzen einen kostenlosen Heimlieferservice von Medikamenten. Aus ihrer Erfahrung heraus halten 54,0 Prozent der befragten Apotheker den Heimlieferservice für einen wichtigen Aspekt bei der Wahl der Apotheke. Dies zeigt, dass ein Kundenbedürfnis nach Heimlieferungen von Medikamenten vorhanden ist, dem sich auch die Apotheker nicht verschließen können bzw. vielfach nicht verschließen.

Während einige Apotheker in Expertengesprächen darauf hinwiesen, dass die Belieferung des Kunden nur die Ausnahme darstellt, ist die Lieferung von Arzneimitteln, die nicht sofort in der Apotheke verfügbar sind, vielfach die Regel. Von lediglich 2,0 Prozent der Apotheker wird eine Heimlieferung nicht durchgeführt bzw. angeboten, 29,0 Prozent der Apotheker beliefern bis zu 30 Kunden pro Woche direkt und weitere 12,0 Prozent beliefern mehr als 30 Kunden pro Woche. Vor allem umsatzstarke Apotheken führen eine Lieferung bei mehr als 30 Kunden pro Woche durch. Der Umfang der Heimlieferungen korreliert neben dem Umsatz auch mit der Personalstärke einer Apotheke. Der Heimlieferservice mit Medikamenten durch die Apotheke ist somit bereits schon jetzt gängige Praxis und nicht mehr der "begründete Einzelfall". Fast ein Viertel der Apotheker würde eine Lockerung der gesetzlichen Rahmenbedingungen begrüßen, um bestimmte Kundengruppen gezielt durch Heimlieferungen ansprechen zu können. Dies gilt besonders für Apotheker mit einem Umsatz bis zu 1,5 Mio. DM pro Jahr und mit höchstens zwei Vollzeitkräften. Gut ein Viertel der Apotheker, die für eine Lockerung der gesetzlichen Vorschriften in bezug auf Heimlieferungen sind, führen schon jetzt mehr als 30 Heimlieferungen pro Woche aus und würden dies auch ausweiten Dem stimmen auch 26,0 Prozent der Apotheker zu, die bislang bis zu 30 Heimlieferungen pro Woche durchführen.

Personen legen bei der Wahl der Apotheke, in der sie ein Rezept einlösen, verschiedene Kriterien zu Grunde. Für den Inhalt der Studie von Interesse sind: Schnelle und gute Erreichbarkeit, Umfassende Beratung, Kostenloser Heimlieferservice von Medikamenten sowie Kunden gehören zur Stammkundschaft. Anhand einer Skala von $1=$ sehr wichtig bis $5=$ völlig unwichtig sind diese Kriterien von den Apothekern, den Ärzten und der Bevölkerung bewertet worden (s. Abbildung IV - 12).

62

Die Kriterien wurden anhand einer Skala von $1=$ sehr wichtig bis $5=$ völlig unwichtig bewertet. 

Abbildung IV - 15: Grad der Wichtigkeit verschiedener Kriterien bei der Auswahl der
Apotheke

(Frage: "Welche Kriterien legen Sie bei der Auswahl der Apotheke zugrunde? Wie wichtig sind Innen folgende Aspekte?")

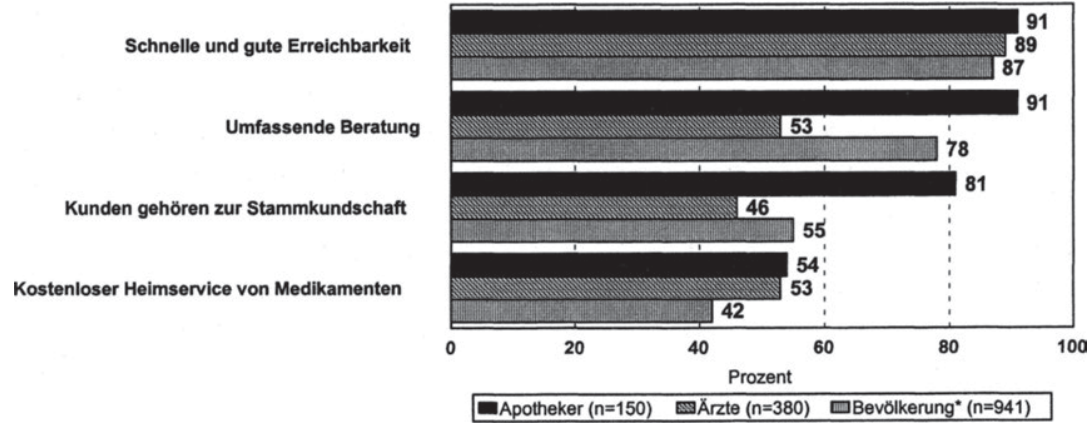

*: Selektion Personen, die ihr Rezept selbst einlosen oder die Medikamente nach Hause geliefert bekommen Top-2-Boxes ("sehr wichtig" und "wichtig"); 1=sehr wichtig, 5=völlig unwichtig

Quelle : INIFES, eigene Darstellung.

(Ergebnisse einer von TNS EMNID im Auftrag von INIFES im Dezember 2000 durchgeführten CATI-Befragung).

Bei der Auswahl der Apotheke, in der das Rezept eingelöst wird, werden von der Bevölkerung vor allem Kriterien einer schnellen und guten Erreichbarkeit sowie einer umfassenden Beratung zu Grunde gelegt. Bezüglich der schnellen und guten $\mathrm{Er}$ reichbarkeit stimmt dieser Wert mit der Selbsteinschätzung der Apotheker und der der Ärzte überein. Bei dem Kriterium einer umfassenden Beratung liegt jedoch ein Missverhältnis vor. Vertiefend zu den Auswahlkriterien der Apotheke wurden die Personen, die ihr Rezept selbst einlösen, gefragt, wie oft sie in der Apotheke bei der Aushändigung von Medikamenten beraten werden. Immerhin ein Drittel der Kunden wird bei fast keinem Apothekenbesuch beraten, 30,0 Prozent werden sporadisch bei ca. jedem zweiten oder dritten Apothekenbesuch und nur 35,0 Prozent wird bei jedem Apothekenbesuch beraten.

Nach § 20 ApBetrO obliegt dem Apotheker aus Gründen der Arzneimittelsicherheit die Pflicht, dem Apothekenkunden informierend und beratend zur Seite zu stehen. Die Apotheker gaben an, dass für 91,0 Prozent der Kunden eine umfassende Beratung bei ihrer Wahl der Apotheke besonders wichtig ist. Dieser Wert steigt in Abhängigkeit der Personalausstattung einer Apotheke. Apotheker, die fünf und mehr Vollzeitkräfte als Pharmazeutisches Personal beschäftigen, schätzen zu 100,0 Prozent ${ }^{63}$

63

Signifikanzniveau aufgrund des Stichprobenumfangs 10 Prozent. 
eine umfassende Beratung als bedeutendes Auswahlkriterium ein. Laut Gesetz besteht sowohl für die Ärzte als auch für die Apotheker eine Beratungspflicht, egal, ob es sich um verschreibungspflichtige oder nicht verschreibungspflichtige Arzneimittel handelt. Allerdings darf die Beratung des Apothekers nicht die Therapie des verordnenden Arztes beeinträchtigen; eine Kritik an der ärztlichen Diagnose oder der Verordnung ist ihm untersagt. Daher ist es nicht verwunderlich, dass nur 53,0 Prozent der Ärzte glauben, dass eine umfassende Beratung ausschlaggebend für die Wahl der Apotheke ist. Besonders die Facharztgruppe der Neurologen und Nervenärzte bewertet diese Kriterium mit nur 45,0 Prozent. ${ }^{84}$ Dies mag u.a. an dem Anteil chronisch Kranker an deren Patientenklientel (50,7 Prozent) und daraus folgernd, einem geringeren Beratungsbedarf/Beratungsanspruch durch den Apotheker liegen.

\section{Potentielle Zielgruppen für eine Versandapotheke}

Die Beschaffung von Gütern und Dienstleistungen per Internet bietet den Menschen auch in Deutschland die Chance, rezeptpflichtige Medikamente über Apotheken außerhalb Deutschlands zu beziehen und somit die Vorteile des Netzes zu nutzen. Bislang wird die Annahme gehegt, dass ein Versandhandel vielfach für Personen von Vorteil ist, die in ihrer Mobilität eingeschränkt, chronisch erkrankt oder berufstätig sind. Nach Ansicht eines Vertreters der Verbraucherverbände sind beispielsweise beim Diabetikerbedarf und auch in anderen Bereichen enorme Preisspannen möglich. Auf die Frage nach der Bewertung der Vorteilhaftigkeit einer Versandapotheke in bezug auf verschiedene Zielgruppen gaben 64,0 Prozent der Ärte an, dass eine Versandapotheke für Patienten, die in ihrer Mobilität eingeschränkt sind, von Vorteil ist (diese Meinung teilen 49,0 Prozent der befragten Apotheker) (s. Abbildung IV - 13). 

Abbildung IV - 16: Bewertung der Vorteilhaftigkeit einer Versandapotheke in bezug
auf verschiedene Zielgruppen

(Frage: "Für welche Zielgruppe kann die Etablierung einer Versandapotheke eher von Vorteil, für wen eher von Nachteil sein?")

Für in ihrer Mobilität eingeschränkte Patienten

Für Patienten in Gebieten mit Unterversorgung

Als Service für Kunden, z.B. Berufstätige

Für Patienten mit chronischen Krankheiten

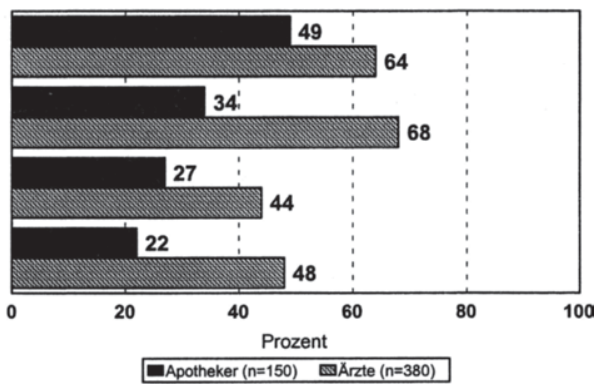

Top-2-Boxes ("von großem Vorteil" und "von Vorteil"); 1=von großem Vorteil; 5=von großem Nachteil

Quelle : INIFES, eigene Darstellung.

(Ergebnisse einer von TNS EMNID im Auftrag von INIFES im Dezember 2000 durchgeführten CATI-Befragung).

Aus dem Antwortverhalten der Ärzte wird deutlich, dass vor allem Fachrichtungen mit einem hohen Anteil an zu versorgenden Chronikern den Vorteil einer Versandapotheke in der Versorgung in ihrer Mobilität eingeschränkter Patienten sehen. Bei den Urologen beträgt die Zustimmung immerhin 75,0 Prozent. Patienten, die in ihrer Mobilität eingeschränkt sind, können sich ohnehin die Arzneimittel nicht selber besorgen und benötigen daher einen Botendienst. Da ein solcher, so die Auskunft eines Krankenkassenvertreters, möglicherweise jedes Mal neu organisiert werden müsste, wäre es sicherlich einfacher, dies auf dem Wege des Versandhandels zu erledigen. 86,0 Prozent der Personen, die sich vorstellen können, Arzneimittel direkt per Post zugeschickt zu bekommen, gaben an, dass bei eingeschränkter Mobilität eine Versandapotheke durchaus eine sinnvolle Alternative für sie wäre. Die Annahme, dass vor allem chronisch Kranke eine Zielgruppe für den Versandhandel bilden, bestätigen 22,0 Prozent. Aus Sicht der Ärzte sind es 48,0 Prozent. Bei diesem Wert zeigt sich, dass vor allem die Fachrichtungen mit einem hohen Anteil chronisch erkrankter Patienten der Überzeugung sind, dass die Etablierung einer Versandapotheke für dieses Patientenklientel von Vorteil ist. 30,0 Prozent der Bundesbürger, die unter einer chronischen Erkrankung leiden, können sich vorstellen, Medikamente über eine Versandapotheke zu beziehen. Dieses lehnen hingegen 70,0 Prozent ab. Anzumerken ist, dass vor allem die Klientel der Chroniker aufgrund der gesundheitlichen Beeinträchtigungen vielfach mehrere vom Arzt verordnete Arzneimittel (41,0 Prozent benötigen 
2-4 verschiedene Präparate pro Tag) benötigt, mindestens ein- bis zweimal im Monat in der Apotheke ein Rezept einlöst (51,0 Prozent) und mit 83,0 Prozent bei Auswahl der Apotheke, in der sie ihr Rezept einlösen, einen signifikant höheren Wert auf eine umfassende Beratung legt, als Personen ohne eine dauerhafte, vom Arzt zu behandelnde Gesundheitsstörung.

44,0 Prozent der Årzte sehen die Versandapotheke als Dienstleister für Berufstätige und Personen, die aus den unterschiedlichsten Gründen gerne die Möglichkeit hätten, ihre Rezepte bei einer Versandapotheke einzulösen. Nach Ansicht der Apotheker (22,0 Prozent) bietet eine Versandapotheke kaum Vorteile für diese Zielgruppe. Die Einstellung der Berufstätigen hinsichtlich der Vorteilhaftigkeit, auf diese Art Medikamente zu beziehen, ist geteilt. Sowohl für als auch gegen einen solchen Service sprechen sich 50,0 Prozent der Befragten aus.

\section{Akzeptanz eines Versandhandels}

Um die Akzeptanz bei den Apothekern und Ärzten bzgl. eines Versandhandels zu eruieren, wurde ihnen im Rahmen einer offenen Frage die Möglichkeit gegeben, ihre Assoziationen zu dieser Thematik wiederzugeben (s. Abbildung IV - 14).

Abbildung IV - 17: Assoziationen mit dem Begriff "Versandapotheke" (Frage:" Was verbinden Sie mit dem Begriff Versandapotheke?")

NEGATIVE GRUNDEINSTELLUNG

SCHLECHTERER SERVICE/ BERATUNG (netto)

KEIN PERSÖNLICHER KONTAKT (netto)

SCHLECHTERE QUALITÄT (netto)

POSITIVE GRUNDEINSTELLUNG (netto)

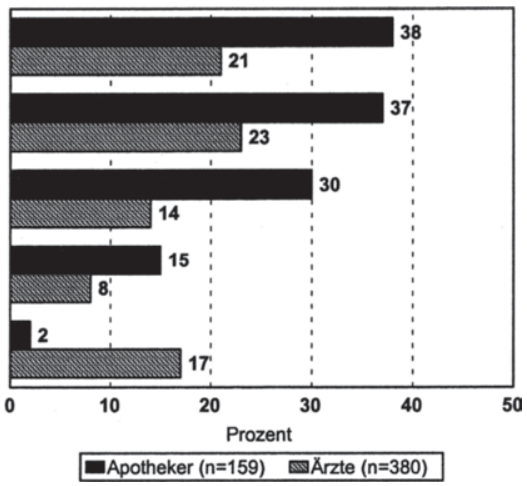

Quelle : INIFES, eigene Darstellung.

(Ergebnisse einer von TNS EMNID im Auftrag von INIFES im Dezember 2000 durchgeführten CATI-Befragung).

Die Haupteinwände der Apotheker gegenüber dem Modell einer Versandapotheke beziehen sich auf eine zu enwartende Verschlechterung des Services bzw. der Beratung, den persönlichen Kontakt zum Kunden und die Qualität der Versorgung. Die 
Gründe für diese negative Grundeinstellung sind vielschichtig. Ein Argument umfasst wiederum die Beratungsleistung der Apotheker. 15,0 Prozent der Apotheker sind der Meinung, dass Versandapotheken keine Beratung erbringen werden und 11,0 Prozent sind der Ansicht, dass die persönliche Beratung wegfallen wird. Vergleicht man diese Werte mit der derzeit tatsächlich durchgeführten Beratung in den Apotheken, bleibt zu hinterfragen, warum in der Präsenzapotheke nicht mehr Wert auf eine umfassende und kundenorientierte Beratung gelegt wird, während einer Versandapotheke diese Kompetenz abgesprochen wird.

Eine Kundenumfrage bei Kunden der schweizerischen Direktserviceapotheke MediService $^{65}$ hat ergeben, dass 98,6 Prozent der Ratsuchenden die Beantwortung inrer Fragen als kompetent und ohne Zeitdruck beurteilten und mit dem Service zufrieden waren. Als einen weiteren Grund für ihre negative Grundeinstellung gaben die Apotheker einen möglichen Verlust des persönlichen/sozialen Kontaktes zwischen Apotheker und Kunden an. Aber gerade eine Anonymisierung der Beratung kann von Kunden als positiv bewertet werden. Arzneimittel wie beispielsweise Psychopharmaka werden ungern in der Apotheke abgeholt, wenn damit zu rechnen ist, dass evtl. auch andere Kunden in der Apotheke anwesend sind.

Lediglich 2,0 Prozent der niedergelassenen Apotheker haben eine positive Grundeinstellung gegenüber dem Modell einer Versandapotheke. Für diese Apotheker kann eine Versandapotheke eine Alternative zu einer Präsenzapotheke sein, sie ist kostengünstiger und unter Beibehaltung der Preisbindung hätte eine Versandapotheke durchaus eine Chance.

Bei der Diskussion über das Für und Wider einer Versandapotheke gilt es zu bedenken, dass diese primär nur ergänzend zu den traditionellen Apotheken tätig sein kann. Auf die Tätigkeit von Präsenzapotheken kann vor allem im Akut-Bereich nicht verzichtet werden. Von den befragten Apothekern gaben 28,0 Prozent an, dass sie sich durchaus vorstellen könnten, beide Vertriebswege komplementär anzuwenden bzw. ihre Präsenzapotheke in eine Versandapotheke umzuwandeln.

Die Ergebnisse der Ärzte-Befragung lassen eine insgesamt positivere Grundeinstellung gegenüber einer Versandapotheke erkennen (s. Abbildung III - 13). Negative Auswirkungen auf den Service bzw. die Beratung erwarten 23,0 Prozent. Eine Einschränkung des persönlichen Kontaktes zwischen Patienten und Apotheker befürchten 14,0 Prozent und an Qualitätseinbußen in der Versorgung glauben 8,0 Prozent. Demgegenüber verbinden jedoch 17,0 Prozent der niedergelassenen Ärzte eine positive Grundeinstellung mit dem Begriff der Versandapotheke. Bezüglich der negativen Merkmale existieren zwischen den befragten Facharztgruppen kaum Unterschiede. Ein signifikanter Unterschied zwischen den Urologen und den Hals-NasenOhrenärzten besteht hinsichtlich der Beurteilung des Items "Qualität". Während 13,0

65

Institut für Sozial- und Präventivmedizin der Universität Zürich; Schweizerische PatientenOrganisation (SPO); Schweizerische Patientendienststelle (DVSP) (1999), Zufriedenheit der Kunden der MediService Apotheke - Schlussbericht, Zürich. 
Prozent der Urologen mit Qualitätseinbußen rechnen, trifft dies bei den Hals-NasenOhrenärzten nur zu 3,0 Prozent zu. Ob die befragten Ärzte vor dem Hintergrund ihrer Patientenklientel geantwortet haben oder die Werte ihre eigene Meinung widerspiegeln, lässt sich nicht eruieren. Auffällig ist jedoch, dass der Anteil chronisch erkrankter Personen bei den Urologen durchschnittlich 52,3 Prozent der Patientenklientel, bei den Hals-Nasen-Ohrenärzten nur 37,3 Prozent umfasst. Der Unterschied wird noch deutlicher, betrachtet man den Anteil der über 60jährigen an den chronisch Kranken. Dieser beträgt bei den Hals-Nasen-Ohrenärzten 44,3 Prozent, bei den Urologen immerhin 71,6 Prozent. Dies führt zu der Annahme, dass Urologen aufgrund der Zusammensetzung ihrer Patienten in einer Versandapotheke die Gefahr der Verringerung der Arzneimittelsicherheit und eine fehlende Kontrolle der Rezeptierung durch den Apotheker sehen. Auch der Bereich "positive Grundeinstellung" ist beeinflusst durch die verschiedenen Facharztgruppen. Das Item "positive Grundeinstellung" subsumiert u.a. die Antworten: gute Einrichtung, kann eine Alternative sein, ist kostengünstiger, bequem (z.B. für Behinderte). Einer Versandapotheke stehen 27, 0 Prozent der Gynäkologen und 22,0 Prozent der Hals-Nasen-Ohrenärzte offen gegenüber, während es nur 11,0 Prozent der Praktischen Ärzte / Allgemeinmediziner / Internisten sind.

Der Anteil der chronisch Kranken und der über 60jährigen an den chronisch Kranken beträgt bei den Praktischen Ärzten/Allgemeinmedizinern/Internisten durchschnittlich 55,5 Prozent bzw. 58,6 Prozent und bei den Gynäkologen 17,5 Prozent und 42,5 Prozent. Vor allem bei den Gynäkologen ist die positive Grundeinstellung gegenüber einer Versandapotheke verständlich, da sich ihr Patientenklientel vor allem aus Patientinnen zusammensetzt, die zur Vorsorgeuntersuchung (durchschnittlich 57,5 Prozent) kommen und u.a. die verordneten Kontrazeptiva selber bezahlen müssen. Gesetzt dem Fall, es gäbe eine Versandapotheke in Deutschland, könnten sich 51,0 Prozent der befragten Ärzte persönlich vorstellen, mit einer solchen Apotheke zu kooperieren. Dieser Anteil ist bei den Gynäkologen, Neurologen / Nervenärzte und den Praktischen Ärzten / Allgemeinmedizinern / Internisten fast identisch. Überraschend sind jedoch die Werte der Urologen und der Hals-Nasen-Ohrenärzte. Die Antworten zu der offenen Frage hätten vermuten lassen, dass die Bereitschaft, mit einer Versandapotheke zusammenzuarbeiten, bei den Urologen wesentlich geringer ausgeprägt ist als bei den Hals-Nasen-Ohrenärzten. Verglichen mit dem Anteil der Chroniker je Facharztgruppe kann darauf geschlossen werden, dass die Bereitschaft zur Kooperation mit einer Versandapotheke bei den Ärzten in Beziehung steht zu dem Anteil der zu behandelnden Chroniker.

Die Einstellung der Bevölkerung gegenüber einer Versandapotheke wurde mit folgender Frage näher untersucht: Können Sie sich vorstellen, Medikamente über eine Versandapotheke zu beziehen? 39,0 Prozent der befragten Personen können sich durchaus den Bezug von Medikamenten auf diesem Wege vorstellen; 60,0 Prozent lehnen dies ab. Ein signifikanter Unterschied besteht zwischen Männern und Frauen. Dies mag daran liegen, dass auch der Anteil der Internet-User in dieser Altersklasse bei den Männern (36,0 Prozent) höher ist als bei den Frauen (27,0 Prozent) und die 
Männer den neuen Medien und dem neuen Vertriebsweg gegenüber aufgeschlossener sind. Eine Korrelation zwischen der Nutzung des Internets und des Bezugs von Arzneimitteln über eine Versandapotheke besteht nicht. Die Bereitschaft, eine Versandapotheke in Anspruch zu nehmen, ist mit zunehmendem Alter rückläufig. Während der Anteil der 30-39jährigen 53,0 Prozent beträgt, liegt er bei den über $60 j a ̈ h r i g e n$ nur noch bei 20,0 Prozent. Es besteht ein signifikanter Unterschied zwischen Personen, die angeben, gesund zu sein und denen, die unter einer dauerhaften, vom Arzt behandelten Gesundheitsstörung leiden bzgl. der Akzeptanz einer Versandapotheke. Während sich 43,0 Prozent der Befragten ohne eine dauerhafte, vom Arzt zu behandelnde Gesundheitsstörung den Bezug von Medikamenten über eine Versandapotheke vorstellen können, sind es bei den Chronikern 30,0 Prozent.

\section{Chancen und Risiken einer Versandapotheke}

Um die Chancen und Risiken einer Versandapotheke aus Sicht der Ärzte und Apotheker zu erfragen, wurden diese gebeten, anhand einer Skala von 1=volle Zustimmung bis $5=$ totale Ablehnung die in der Offentlichkeit diskutierten Aussagen zur Versandapotheke zu bewerten (s. Abbildung IV - 15).

\section{Abbildung IV - 18: Bewertung verschiedener Aussagen zur Versandapotheke}

(Frage: "Bitte bewerten Sie folgende Aussagen auf einer Skala von 1 bis 5")

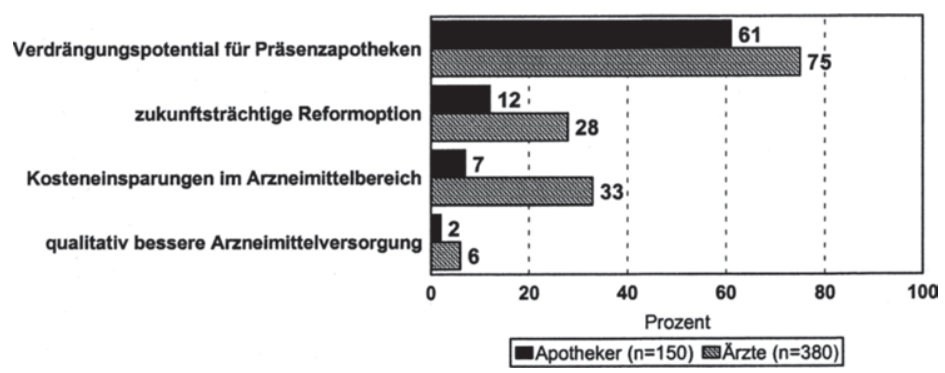

Top-2-Boxes ("volle Zustimmung" und "Zustimmung"); 1=volle Zustimmung; 5=totale Ablehnung

Quelle: INIFES, eigene Darstellung.

(Ergebnisse einer von TNS EMNID im Auftrag von INIFES im Dezember 2000 durchgeführten CATI-Befragung).

Aus Sicht der Apotheker stellen Versandapotheken generell ein Verdrängungspotential für Präsenzapotheken dar. Vor allem Apotheken mit einem Umsatz bis zu 1,5 Mio. DM geben an, von dieser Konkurrenz betroffen zu sein. Die Konsequenz für die Apotheker ist, dass die Einführung der Arzneimitteldistribution über Versandapo- 
theken ihren Umsatz beeinflussen kann. Von negativen Umsatzeinbußen gehen 58,0 Prozent der befragten Apotheker aus, bei 30,0 Prozent wird es zu keiner Beeinflussung des Umsatzes kommen. Die Folgen der negativen Umsatzbeeinflussung wären die Schließung von Apotheken und die Entlassung von Personal. Von den befragten Ärzten sind 75,0 Prozent der Meinung, dass Versandapotheken generell ein Verdrängungspotential für Präsenzapotheken darstellen.

Dass die Einführung des Modells einer Versandapotheke eine zukunftsträchtige Reformoption darstellt, glauben 28,0 Prozent der Ärzte, bei den Apothekern sind es 12,0 Prozent.

Die Antworten bzgl. der Annahmen, dass Versandapotheken zu Kosteneinsparungen im Arzneimittelbereich führen und für eine qualitativ bessere Arzneimittelversorgung sorgen, zeigen, dass sowohl die Apotheker als auch die Ärzte sehr zurückhaltend sind, wenn es darum geht, Chancen und Risiken einer Versandapotheke unvoreingenommen zu bewerten.

Vor dem Hintergrund der raschen - und nicht aufzuhaltenden - Verbreitung neuer Medien und des elektronischen Handels muss die konstruktive öffentliche Debatte, die sich sowohl mit möglichen Einsparungen in Millionenhöhe bei den Distributionskosten, als auch mit der Gewährleistung von Sicherheit und Qualität der Arzneimittelversorgung befasst, konsequent fortgeführt werden.

Abschließend gilt zu sagen, dass die Einführung neuer Vertriebswege im Arzneimittelsektor nur dann volkswirtschaftlich und sozialpolitisch akzeptabel sein kann, wenn auch die Bedenken der Betroffenen ernst genommen und konstruktiv diskutiert werden. Wenn es gelingt, dies zu berücksichtigen und gleichzeitig Wirtschaftlichkeitsreserven auszuschöpfen ohne das bestehend hohe Niveau der Arzneimittelversorgung zu gefährden, sind Versandapotheken als Vertriebsform in Zukunft verstärkt in eine kritische und ergebnisoffene Diskussion um liberalisierte Wege in der Arzneimitteldistribution einzubeziehen.

\subsection{Bewertung der Projektergebnisse}

Im Jahre 1999 betrugen die GKV - Ausgaben für apothekenpflichtige Arzneimittel auf Rezept 36,15 Mrd. DM. Der Apothekenumsatz für Arzneimittel zu Endpreisen belief sich auf ca. 58 Mrd. DM, wovon 27,3\% auf Apothekenzuschläge entfielen ${ }^{66}$.

Vor diesem Hintergrund ist von verschiedenen Seiten immer wieder argumentiert worden, dass der stationäre Vertriebsweg für Arzneimittel vom Hersteller über den Großhandel bis hin zur Apotheke zum Teil ineffizient sei, durch einen Versandhandel Kosteneinsparungen bei den Medikamentenkosten entsprechend erzielbar erschei-

68

ABDA (1999), Die Apotheke Zahlen Daten Fakten, Tabelle 10 (Angabe des Apothekenumsatzes netto mit 50,3 Mrd. DM, Berechnung des Bruttoumsatzes erfolgte entsprechend mit 16\% Mwst.) sowie Tabelle 13. 
nen ohne dass dabei die Qualität der Versorgung beeinträchtigt werden würde. Für einige Zielgruppen seien sogar Verbesserungen in der Versorgungs- und Servicequalität vorstellbar.

Diese grundsätzliche These wird in der INIFES-Studie empirisch hinterfragt. Dabei werden die wichtigsten Argumente anhand der folgenden, aus dem SGB $V^{67}$ sowie dem AMG entnommenen und in der gesundheitspolitischen Diskussion üblichen Kriterien für Effizienz und Versorgungsqualität, bewertet:

1. Kostenvorteile und Einsparpotentiale;

2. Warensortiment und Preisvergleich;

3. Versorgungsdichte und Arbeitsmarkteffekte;

4. Nutzerfreundlichkeit, telefonische Erreichbarkeit und Zustellsicherheit;

5. Beratungssicherheit, Service und Information;

6. Soziale Funktion von Arzt und Apotheke.

Im folgenden sollen die wesentlichen Studienergebnisse eine ausführliche Bewertung erfahren und mit Pro- und Contra-Argumenten unterlegt werden. Es gilt, einerseits die Vorteile eines Versandes verschreibungspflichtiger Arzneimittel über Versandapotheken aufzuzeigen, und andererseits die Nachteile dieses Vertriebsweges ausdrücklich zu thematisieren. Beide Komponenten sind Teil einer sachorientierten Diskussion über eine Vertriebspluralität, in der dem Patienten die Wahl zwischen dem traditionellen Vertriebskanal Präsenzapotheke und neuen Alternativen wie der Versandapotheke ermöglicht wird.

\subsubsection{Kostenvorteile und Einsparpotentiale durch Versandhandel}

Die Zulassung zusätzlicher Anbieter im Arzneimittelvertrieb - in diesem Fall die Legitimation von Versandapotheken - ist im wesentlichen mit dem Ziel verbunden, Wirtschaftlichkeitspotentiale nutzbar zu machen. Nach dem Minimalprinzip der Wirtschaftstheorie ist entsprechend nach Wegen zu suchen, die bei Sicherung der derzeit geltenden Qualitätsstandards der Arzneimittelversorgung der Bevölkerung mit geringeren Kosten einhergehen als der derzeitige Arzneimittelvertrieb über Präsenzapotheken.

Damit ist zum Ausdruck gebracht, dass sich die Legitimation von Versandapotheken auch auf deren Potential zur Ausschöpfung von Ineffizienzen im Vertriebssystem begründet. 


\begin{tabular}{|c|c|}
\hline Positive Aspekte & Negative Aspekte \\
\hline $\begin{array}{l}\text { Kosteneinsparung } \\
\text { - Korrelation zwischen Umsatzstärke und } \\
\text { Kosteneinsparung (Economies of Scale), } \\
\text { kurz- und mittelfristige Prognose: } \\
\text { VAl: } 6 \% \quad \text { VAll: } 8 \% \text { VA III: } 12 \% \\
\text { hier liegen langfristig weitere Potentiale } \\
\text { Geltung der AMPreisV, d.h. keine Verzer- } \\
\text { rungen durch die Ausschöpfung interna- } \\
\text { tionaler Preisniveaus } \\
\text { basiert auf konservativen Annahmen un- } \\
\text { ter Wahrung höchster Sicherheits- und } \\
\text { Qualitätsstandards }\end{array}$ & $\begin{array}{l}\text { Kosteneinsparung } \\
\text { - entsprechend der Umsatzstärke kann } \\
\text { der Einfluss der Versandapotheken auf } \\
\text { das Marktgeschehen zunehmen } \\
\text { Kosteneinsparungen können aufgrund } \\
\text { der Geltung einheitlicher Preise nach } \\
\text { AMPreisV nicht über eine Preisdifferen- } \\
\text { zierung an die GKV weitergeleitet wer- } \\
\text { den. Entsprechend müssen andere Er- } \\
\text { stattungsmodalitäten zum Einsatz kom- } \\
\text { men. }\end{array}$ \\
\hline
\end{tabular}

Quelle: INIFES, eigene Darstellung.

Es ist davon auszugehen, dass Versandapotheken im Gegensatz zu den heutigen Präsenzapotheken einen wesentlich höheren Umsatz je Apotheke aufweisen werden. Wie in den Präsenzapotheken auch, werden diese Umsätze in den Versandapotheken durch unterschiedliche Kostenträger (GKV, PKV, Selbstzahler) finanziert. Hier ergeben sich keinerlei Unterschiede zu den jetzigen Abläufen.

Umsatzstarke Anbieter, wie in Modell VA III (Versandapotheke mit ca. 500 Mio. DM $U_{m s a t z}{ }^{88}$ ) weisen geringere Grenzkosten aufgrund einer optimierten Betriebsstruktur auf. Zudem trägt eine mit der Umsatzstärke korrelierende Einkaufsmacht nicht unwesentlich zu geringeren Kosten des Wareneinsatzes bei. Economies of Scale sind ein wesentlicher Punkt in der Betrachtung von Kostenpotentialen.

Der Vorteil großer Betriebsstrukturen auf das Ergebnis wird im Modell VA III durch die Konzentration der Großhandels- und Einzelhandelsstufe auf eine gemeinsame Handelsstufe um so deutlicher. ${ }^{69}$ So werden im Modell der VA III ca. $12 \%$ Kosteneinsparungen am Umsatz im Vergleich zur Präsenzapotheke erreicht. Insbesondere in dieser Modellvariante kann allerdings langfristig das Fremd- und Mehrbesitzverbot der $\S \S 2$ und 7 Apothekengesetz zur Disposition stehen. Die von Seiten der Apothekerschaft diesbezüglich befürchteten potentiellen "strukturellen Verwerfungen" müssen bei der weiteren die mögliche Einführung von Versandapotheken begleitenden, Bewertung berücksichtigt werden.

Wie in Kapitel 4.1.2. beschrieben.

69

So treibt bereits die GEHE AG als größter europäischer Pharmagroßhändler die Expansion im Einzelhandel voran (Italien, Großbritannien, Niederlande, Norwegen). Die im Einzelhandel erzielbaren Renditen seien deutlich interessanter als im Großhandel: „Wir werden jede Möglichkeit nutzen, die sich uns bietet" (Vorstandsvorsitzender Fritz Oesterle), siehe in: o.V. (2001), Gehe plant Wachstum in Europa, Apothekerzeitung vom 30.4.2001, 17. Jg. Nr. 18, S. 2. 
Versandapotheken mit einem regionalen Wirkungskreis, wie das Modell VA I mit ca. 5 Mio. DM Umsatz, sind in ihrem Umsatzpotential schon deshalb begrenzt; Größenersparnisse wie im Modell der VA III nicht erzielbar. Auch die Ausübung von Einkaufsmacht auf die Hersteller oder den Großhandel ist eingeschränkt. Trotzdem können aufgrund der allgemeinen Kostenpotentiale eines Versandhandels, wie geringer Lager- und Raumkosten oder höherer Produktivitätserwartungen, durchaus Kostenverbesserungen gegenüber Präsenzapotheken erzielt werden, hier sind es ca. $6 \%$. Für eine kleinräumige Betrachtung ist dies nicht zu vernachlässigen. Aus volkswirtschaftlicher Perspektive eröffnet sich allerdings nur noch eine halb so großes Kosteneinsparpotential wie bei umsatzstarken Versendern. Entsprechend ist das Modell der VA I für eine volkswirtschaftliche Betrachtung, die den weiteren Ausführungen zugrunde liegt, weniger relevant.

In kurz- und mittelfristiger Perspektive ist das Modell VA II mit einem Umsatz von ca. 20 Mio. DM durchaus realistisch ${ }^{70}$. Kosteneinsparungen von ca. $8 \%$, die hier zu erwarten wären, liegen im Wert entsprechend zwischen den Modellen eines regional begrenzten Versenders VA I und dem Modell eines landesweiten Versenders mit integriertem Großhandel VA III.

Die in den INIFES - Modellrechnungen ermittelten Kosteneinsparungen (Modell VA II und VA III) von $8 \%$ und $12 \%$ sind im Vergleich zur Schweiz bzw. den Erwartungen der Kostenträger (von ca. 15\%) geringer. Diese Abweichungen sind vor allem im Vorgehen der Studie begründet, die auf sehr konservativen Annahmen und Berechnungen bezüglich der Kostenstruktur aufbaut. Qualitätsstandards wie eine telefonische Erreichbarkeit an allen Tagen des Jahres rund um die Uhr, zudem sichergestellt durch pharmazeutisches Personal und für den Anrufer kostenlos, verursachen vergleichsweise hohe Betriebskosten. Ähnliches gilt für eine funktionierende B-2-C Logistik.

Allerdings zeigen die qualitativen Ergebnisse, dass ein Kostensparen an der Qualität des Angebotes negative Konsequenzen bezüglich der Akzeptanz durch die potentiellen Nutzer hätte. In deren Augen ist das Vorhalten dieser Servicestandards, die sicherlich über das Angebot einer Präsenzapotheke hinausgehen, eine wesentliche Voraussetzung für eine zukünftige Nutzung des Versandhandels. Deshalb sollte die telefonische Verfügbarkeit sowie deren Umfang nicht in die Entscheidungsgewalt der einzelnen Versandapotheke gestellt werden, sondern im rechtlichen Rahmen vorgegeben sein. So verlangen z.B. die meisten US-Bundesstaaten von Versandapotheken eine kostenfreie Telefonnummer, unter der jederzeit oder in einem bestimmten Stundenumfang eine telefonische Beratung zur Verfügung steht. ${ }^{71}$

70

In diesen Umsatzgrößen bewegen sich derzeit im Ausland bereits existierenden Versandapotheken.

71

Blankenberg, K., (2000), www.zukunft.de? - Einblicke in Amerikas legale Online-Apotheken, S. 26, in: Pharmazeutische Zeitung Nr. 49, 145. Jahrgang vom 07.12.2000, S. $25-26$. 
Neben der Abschätzung von Kosteneinsparungen tritt im Rahmen einer quantitativen Analyse die Überlegung zu möglichen Marktpotentialen des Versandhandels. Hier kommt es wesentlich auf die Beachtung epidemiologischer Restriktionen an. Nicht der gesamte Arzneimittelmarkt mit seinen unterschiedlichsten medizinischen Indikationen ist für die Abschätzung von Einsparpotentialen für den Versandhandel relevant, sondern nur solche Arzneimittel, die für diesen Vertriebsweg geeignet sind. Wesentliches Kriterium ist eine nicht akut bestehende Nachfrage nach Arzneimitteln. Daraus lässt sich analog die Zielgruppe eines Versandes ableiten: Insbesondere Patienten mit Dauermedikation und chronischen Krankheiten können von einem Versandhandel profitieren. Für einen akuten Medikationsbedarf ist der Versandhandel bei Lieferfristen von bis zu 48 Stunden (teilweise auch länger) nicht geeignet. Auch wären die Kosten des Versandes so hoch, dass die modellierten Kosteneinsparungen unerreicht blieben.

US-Studien sprechen davon, dass bereits jede zweite Verordnung keine akute Medikation darstellt. ${ }^{72}$ Die durchgeführten Untersuchungen zum Marktpotential anhand epidemiologischer Indikatoren ergeben Zahlen zwischen $40 \%$ und $50 \%$ des GKVFertigarzneimittelumsatzes. Anhand epidemiologischer Kriterien stellt sich das Marktpotential eines Versandhandels somit als erheblich dar. Für die Zukunft kann zudem mit einem hohen Wachstumspotential gerechnet werden, vorausgesetzt, die Akzeptanz von Seiten der Patienten und der Ärzte steht dabei nicht entgegen.

In kurz- und mittelfristiger Sicht kann dieses Potential objektiv nicht vollständig ausgeschöpft werden. Ansatzpunkte, welcher Realisierungsgrad in absehbarer Zeit möglich sein wird, können nur schwer erhoben werden, entsprechend den internationalen Erfahrungen erscheinen $15 \%$ auch für Deutschland nicht realistisch.

Übersicht IV - 2: Marktpotential und Marktanteil

\begin{tabular}{|c|c|}
\hline Positive Aspekte & Negative Aspekte \\
\hline $\begin{array}{l}\text { Marktanteil } \\
\text { - Marktanteil von 15\% kurz- und mittelfristig } \\
\text { realistisch } \\
\text { - in langfristiger Perspektive Steigerung } \\
\text { vorstellbar: das epidemiologisch begrün- } \\
\text { dete Marktpotential umfasst bis zu } 50 \% \\
\text { des GKV-Umsatzes }\end{array}$ & $\begin{array}{l}\text { Marktanteil } \\
\text { - Akzeptanz in Bevölkerung, Kooperation } \\
\text { mit Ärzten als wesentlicher Faktor für } \\
\text { Marktanteile, noch keine quantitativen } \\
\text { Einflüsse ableitbar } \\
\text { - in regionaler Perspektive ist Modell VA I } \\
\text { vorstellbar (insbesondere in Verbindung } \\
\text { mit Präsenzapotheke); in volkswirtschaft- } \\
\text { licher Perspektive sind die Modelle VA II } \\
\text { und VA III relevant }\end{array}$ \\
\hline
\end{tabular}

Quelle: INIFES, eigene Darstellung.

72

Buhle, L. (1999), Potential for Profit, Potential for Real Change, in: www.managedcaremag.com, 07/1999. 
Entsprechend einem angenommenen Marktanteil von 15\% am GKV-Fertigarzneimittelumsatz ergeben sich für 1999 Einsparungen von ca. 400 Mio. DM bis 620 Mio. DM.

Bei Vorauskalkulation einer durchschnittlichen Betriebsrendite würden die Einsparungen aufgrund der dargestellten Einflussfaktoren geringer ausfallen. Die Berechnungen ergeben zwischen 250 Mio. DM und 430 Mio. DM.

Bei Vorauskalkulation zusätzlicher einheitlicher Kassenrabatte würden die Einsparpotentiale allgemein geringer ausfallen und zudem in Abhängigkeit der Höhe des gewählten Kassenrabattes zwischen 276 Mio. DM und 552 Mio. DM differieren.

Diese Einsparvolumina liegen aufgrund der schon angesprochenen konservativen Kostenschätzungen möglicherweise hinter den Erwartungen der Kostenträger zurück, wohlgemerkt sind diese Zahlen allerdings Mindestangaben in kurz- und mittelfristiger Perspektive. Es ist deshalb eher wahrscheinlich, dass Einsparungen in absehbarer Zeit diesen Umfang übertreffen werden. Aufgrund des hohen Marktpotentials kann bei einem stetig steigenden Realisierungsgrad langfristig mindestens von einer Verdopplung der Wirtschaftlichkeitspotentiale ausgegangen werden.

Übersicht IV - 3: Einsparvolumen für die GKV

\begin{tabular}{|c|c|}
\hline Positive Aspekte & Negative Aspekte \\
\hline $\begin{array}{l}\text { Einsparvolumen für GKV } \\
\text { - Einsparung bei } 15 \% \text { Marktanteil (1999): } \\
\text { VA II: } 400 \text { Mio.DM } \\
\text { VA III: } 620 \text { Mio.DM } \\
\text { Bei durchschnittlicher Betriebsrendite: } \\
\text { VA II: ca. } 260 \text { Mio. DM } \\
\text { VA III: ca. } 430 \text { Mio. DM } \\
\text { Bei zusätzlichen einheitlichen Kassenra- } \\
\text { batten }{ }^{73} \text { : } \\
\text { VA II: ca. } 276 \text { Mio. DM - } 386 \text { Mio. DM } \\
\text { VA III: ca. } 276 \text { Mio. DM - } 552 \text { Mio. DM } \\
\text { Langfristige Perspektive eröffnet Einspa- } \\
\text { rungen von weit über } 1.000 \text { Mio. DM }\end{array}$ & $\begin{array}{l}\text { Einsparvolumen für GKV } \\
\text { - Einsparungen gelten unter Beibehaltung } \\
\text { der AMPreisV } \\
\text { - Ergebnisoffener Wettbewerbsprozess, } \\
\text { d.h. Berücksichtigung von Gegenreaktio- } \\
\text { nen auf Versandhandel in den Einsparpo- } \\
\text { tentialen nicht darstellbar } \\
\text { Die Aufhebung des Fremd- und Mehrbe- } \\
\text { sitzverbotes steht bei Modell VA III zur } \\
\text { Disposition }\end{array}$ \\
\hline
\end{tabular}

Quelle: INIFES, eigene Darstellung.

Modellrechnungen zu zukünftigen Einsparpotentialen bleiben der Kritik ausgesetzt, dass sie keinen ergebnisoffenen Wettbewerb widerspiegeln. Gegenreaktionen auf den Versandhandel von Seiten der Präsenzapotheken wie möglicherweise des

73

Je nach Höhe der angenommenen Kassenrabatte. 
Großhandels können aber quantitativ in Modellrechnungen nicht wissenschaftlich valide den Einsparpotentialen gegengerechnet werden.

Zudem ist die Arzneimittelpreisverordnung in ihrer jetzigen Gültigkeit (inklusive der Kappung 1998) unterstellt. Eine Änderung der Arzneimittelpreisverordnung wird in der Gesundheitspolitik zwar wiederholt diskutiert, jedoch liegen zum aktuellen Zeitpunkt (Herbst 2001) keine verlässlichen Details zu möglichen Modifizierungen vor. Ein Abweichen von der derzeitigen Struktur der Arzneimittelpreisverordnung würde sich folglich im Rahmen der Spekulation bewegen.

Ein Abweichen von der derzeitigen Regulierung der Besitzverhältnisse im Apothekensektor ist durch das Modell VA III - einer Versandapotheke mit kombinierter Großhandelsfunktion - nicht auszuschließen. Die Umsatzgröße eines solchen potentiellen Versenders könnte zur Bildung von Kapitalgesellschaften führen. Ein Aufweichen des Fremd- und Mehrbesitzverbotes ist folglich in der Zukunft vorstellbar.

Dabei ist nochmals zu betonen, dass es nicht Untersuchungsgegenstand dieser Studie ist, die Auswirkungen einer möglichen Abweichung von anderen derzeit bestehenden Regulierungen wie der Arzneimittelpreisverordnung, des Fremd- und Mehrbesitzverbotes oder anderer Vorschriften bzw. Verbote zu untersuchen. Im Rahmen der Ausführungen wird auf absehbare Entwicklungen hingewiesen, diese können jedoch nicht Gegenstand quantitativer Analysen sein.

Schließlich ist kritisch anzumerken, dass die genannten Einsparungen nur realisiert werden können, wenn die Bereitschaft von Seiten der Zielgruppe existiert, diesen Vertriebsweg in Anspruch zu nehmen. Eine wesentliche Rolle spielen in diesem Zusammenhang Anreizsysteme, die neben den Krankenkassen als Kostenträgern auch die Patienten berücksichtigen.

Übersicht IV - 4: Anreizsysteme für Krankenkassen und Versicherte

\begin{tabular}{|l|l|}
\hline \multicolumn{1}{|c|}{ Positive Aspekte } & \multicolumn{1}{|c|}{ Negative Aspekte } \\
\hline $\begin{array}{l}\text { Anreizsysteme für Versicherte } \\
\text { - Einsparungen könnten an GKV über Ra- } \\
\text { battsysteme weitergeleitet werden }\end{array}$ & $\begin{array}{l}\text { Anreizsysteme für Versicherte } \\
\text { Fehlsteuernde Anreize bei mangelnder } \\
\text { Konsumentensouveränität (Preis statt } \\
\text { Qualität als Kriterium) }\end{array}$ \\
$\begin{array}{l}\text { Anreizsysteme für Versicherte implemen- } \\
\text { tieren, z.B über Boni- oder Rückerstat- } \\
\text { tungsregelungen (Dies wären Anreizsys- } \\
\text { teme, welche aufgrund der Zielgruppe } \\
\text { des Versandes auf kranke, und nicht auf } \\
\text { gesunde Versicherte fokussiert sind) }\end{array}$ & \\
\hline
\end{tabular}

Quelle: INIFES, eigene Darstellung. 
Aufgrund des existierenden Festpreissystems auf der Apothekenstufe und den daraus resultierenden bundesweiten Apothekeneinheitspreisen können Einsparungen nicht über geringere Medikamentenpreise an die Krankenkassen weitergegeben werden. Entsprechend ist an zusätzliche Rabatte ähnlich dem derzeitigen Kassenrabatt von $5 \%$ zu denken. Zum einen ergäbe sich dabei die Möglichkeit der Erhebung zusätzlicher umsatzabhängiger Kassenrabatte, zum anderen könnten zusätzliche einheitliche Kassenrabatte entsprechend der aktuellen Rabattgewährungsmodalitäten erhoben werden. Beide Aspekte wurden in den Modellrechnungen berücksichtigt. Generell ist anzumerken, dass umsatzgestaffelte Rabatte nicht nur für Versandapotheken eine Reformoption wären. Dies würde im Umkehrschluss natürlich ein Abweichen von der derzeitigen einheitlichen Staffelung der Kassenrabatte bedeuten.

Um die dargestellten Einsparpotentiale für das Gesamtsystem (also vor allem für Versicherte und Patienten) realisieren zu können, ist es sinnvoll Anreize zur Nutzung des neuen Versandweges auch für Patienten zu schaffen. So sind neben den zusätzlichen Rabatten an die Krankenkassen und den damit für die Versicherten verbundenen Beitragssatzeffekte auch Bonisysteme oder Rückerstattungen denkbar. Mit Blick auf die Zielgruppe der Versandhandelsnutzer, Patienten mit chronischen Krankheiten oder dauerhafter Medikation, sind diese Art der Anreizschaffung insbesondere deshalb zu begrüßen, da hier offensichtlich nicht die gesunden, sondern hauptsächlich die dauerhaft kranken und gesundheitlich eingeschränkten Versicherten profitieren.

\subsubsection{Warensortiment, Preisvergleich und Vorwurf des „Rosinenpickens“}

Eine Zulassung von Versandapotheken ist ohne Ausnahme an die Gewährleistung der Arzneimittel- und Patientensicherheit zu knüpfen. So sind Versandapotheken wie Präsenzapotheken auch von den zuständigen deutschen Behörden zuzulassen und zu überwachen. Entsprechend sind an Versandapotheken dieselben Qualitätsstandards zur Erfüllung räumlicher, personeller und fachlicher Mindestvoraussetzungen zu stellen wie in Präsenzapotheken.

Das heißt, ein Versandhandel in Deutschland ist nur mit in Deutschland zugelassenen Arzneimitteln möglich. Im Segment der verschreibungspflichtigen Arzneimittel muss zudem das Rezept in schriftlicher oder elektronischer Form der Versandapotheke vorliegen. Die Apotheker in den Versandapotheken haben sich, wie in Präsenzapotheken auch, entsprechend des Substitutionsverbotes an die Anweisungen des Arztes zu halten, sofern dieser nicht „aut-idem“ vermerkt hat. ${ }^{74}$ Ein Beipackzettel in Deutsch muss den zum Versand bestimmten Arzneimitteln stets beiliegen. Entsprechend der Forderung nach einer Überwachung durch die zuständigen Behörden ist nicht a priori davon auszugehen, dass die Produktsicherheit durch den Vertrieb über Versandapotheken gefährdet wäre.

74

Eine Diskussion des Aut-Idem-Verbotes kann im Rahmen dieser Studie nicht erfolgen, auch wenn diese Regulierung ohne Zweifel in eine Agenda möglicher Reformoptionen gehört. 
Übersicht IV - 5: Arzneimittelsicherheit

\begin{tabular}{|l|l|}
\hline \multicolumn{1}{|c|}{ Positive Aspekte } & Negative Aspekte \\
\hline Arzneimittelsicherheit & Arzneimittelsicherheit \\
- Sitz der Versandapotheke ist Deutsch- \\
land (EU)
\end{tabular}

Quelle: INIFES, eigene Darstellung.

Versandapotheken wird häufig vorgeworfen, nur ein Teilsortiment statt eines Vollsortiments vorzuhalten. Wie bereits an anderen Stellen erwähnt, stellt die Zulassung von Versandapotheken - insbesondere mit dem Fokus auf Patienten mit Dauermedikation bzw. mit Medikation zur Behandlung chronischer Erkrankungen - eine verbraucherfreundliche Alternative zu den Präsenzapotheken dar, die keinesfalls darauf ausgerichtet ist, eine flächendeckende Versorgung der Bevölkerung zu garantieren. Entsprechend dem Zielsegment und der Restriktion einer Lieferzeit, welche bei noch so optimierter Logistik im Versandhandel, wie das Wort schon ausdrückt, nicht entfallen wird, umfasst das Versandsegment hauptsächlich die entsprechenden Arzneimittel, weniger jedoch Medikation für den Akutbedarf. Daraus leitet sich ab, dass eine Versandapotheke den größten Nutzen stiftet, wenn sie ein „zielgruppenorientiertes Vollsortiment" vorhält.

Grundsätzlich ist auch die Vorhaltung eines Vollsortiments vorstellbar bzw. mit der Zulassung regelbar. Nach wie vor aber wäre damit nicht das Problem gelöst, dass die benötigten Arzneimittel nur mit einer Verzögerung aufgrund der Lieferzeit beim Patienten ankämen. Diese Restriktion ist dem System eines Versandhandels immanent. Selbst Expressversendungen könnten dieses Problem nicht lösen, würden zudem aber erhebliche Logistikkosten induzieren, die mögliche Einsparpotentiale in Frage stellen würden. Bei akutem Medikamentenbedarf ist nach wie vor die Präsenzapotheke die einzig adäquate Vertriebsform.

Die Teilsortimentierung der Versandapotheken schließt jedoch nicht aus, dass sich diese an der regionalen Notversorgung, z.B. im Verbund eines regionalen Apothekennetzes, beteiligen müssen. Dazu bedarf es keiner Vorhaltung einer Offizin. Zudem wirkt der Qualitätsstandard der Versandapotheken, eine kostenlose 24-Stun- 
den-Telefonhotline anzubieten, auf die Integration in ein Notfallversorgungsnetzwerk förderlich.

Übersicht IV - 6: Notversorgung

\begin{tabular}{|l|l|}
\hline \multicolumn{1}{|c|}{ Positive Aspekte } & \multicolumn{1}{c|}{ Negative Aspekte } \\
\hline $\begin{array}{l}\text { Notversorgung } \\
\text { Versandapotheken in Notfallversorgung } \\
\text { integrierbar (z.B. über Notrufnummer ei- } \\
\text { nes regionalen Apothekennetzes) }\end{array}$ & $\begin{array}{l}\text { Notversorgung } \\
\text { Sortiment der Versandapotheken nicht } \\
\text { auf akute Krankheiten ausgerichtet } \\
\text { - Notfallversorgung bedarf nicht der Vorhal- } \\
\text { tung einer Offizin, } \\
\text { 24 - Stunden -Telefonhotline kommt der } \\
\text { Nutzung für Notdienst entgegen }\end{array}$ \\
\hline
\end{tabular}

Quelle: INIFES, eigene Darstellung.

Bleibt der Vorwurf des „Rosinenpickens“, also eine mögliche Fokussierung auf hochpreisige Arzneimittel. Anhand der Modellrechnungen ist erkennbar, dass ein Preisvergleich einer durchschnittlichen Verordnung des Zielsegments des Pharmaversandes mit dem Durchschnitt aller GKV-Verordnungen auf der Ebene der Tagesdosen (defined daily dose - DDD) zugunsten der Versandapotheken ausfällt: Mit einem Preis von ca. 1,00 DM sind die Tagesdosen der über Versandapotheken vertriebenen Verordnungen durchschnittlich billiger als im GKV-Durchschnitt mit 1,33 DM je Tagesdosis. Der Vergleich der Preise auf der Ebene der DDD ist dabei nicht zufällig gewählt. Nach Expertenansicht ist diese Form des Vergleiches am besten geeignet, den Arzneimittelverbrauch direkt - ohne Verzerrungen durch Einflüsse wie die Packungsgröße oder Dosisstärken - zu messen. Entsprechend beinhalten auch die Preise keine Verzerrungen durch die genanten Einflüsse. ${ }^{75}$ Ein Preisvergleich auf der Verordnungsebene enthält Verzerrungen insbesondere bezüglich der Packungsgröße. Da die gewählte Segmentierungsstrategie aufgrund der Zielgruppen den Indikator "große Packungen“ nutzt, ergeben sich entsprechend höhere durchschnittliche Verordnungswerte der Versandapotheken mit ca. 61 bis 67 DM gegenüber dem GKV-Durchschnitt von ca. 47 DM je Verordnung. Jedoch muss man bedenken, dass der Packungsinhalt der entsprechenden Verordnung des Zielsegments der Versandapotheke ca. 60 bis 70 Tagesdosen umfasst, während eine durchschnittliche GKVVerordnung nur 35 Tagesdosen, also nur den halben Packungsinhalt, vorhält.

Die Diskussion um eine mögliche Orientierung des Versandhandels auf höherpreisige Arzneimittel ist insbesondere unter dem Aspekt der Ausgestaltung von Erstattungssystemen an die GKV zu betrachten: Aufgrund der Geltung der Arzneimittelpreisverordnung können die Kostenvorteile eines Versandhandels nicht direkt über

75

Schröder, H., Selke, W., Ergänzende statistische Übersicht, S. 796f, in: Schwabe., U., Paffrath, D., (Hrsg.), (2001), Arzneiverordnungsreport 2000, S. $796-881$. 
niedrigere Preise zwischen den Versandapotheken und der GKV verrechnet werden. Entsprechend muss die Implementierung von Erstattungssystemen in Betracht gezogen werden.

Zeigte die Ebene der Marktpotentialbetrachtung bei vollständiger Abschöpfung der Kostenvorteile (ökonomische Restriktion) nur eine marginale Abweichung der Marktpotentiale von den Zielsegmenten (und damit von der Höhe des durchschnittlichen Verordnungswertes), so wurde bei der Modifizierung der Kostenvorteile bezüglich möglicher zusätzlicher Rabattgewährungen offensichtlich, dass die Höhe der zusätzlichen Rabattbelastung der Versandapotheke auf deren Marktpotential und entsprechend auch das Einsparpotential einen erheblichen Einfluss hat. Werden zu hohe Rabatte erhoben, sinkt das Markt- und entsprechend auch das Einsparpotential: Die von den Versandapotheken zusätzlich zum derzeit existierenden Kassenrabatt abzuführenden Rabatte schmälern die jeweiligen Kostenvorteile. Je höher die Rabattbelastung ausfällt, desto geringer ist der Anteil, der dem potentiellen Versender als Rendite verbleibt. In Fällen, in denen die zusätzliche Rabattbelastung den Kostenvorteil übersteigt, ist ein Ausscheiden der potentiellen Versender (insbesondere der umsatzschwächeren Versandapotheke VA I) aus dem Zielsegment Versand prognostizierbar.

Dieser Zusammenhang zwischen Höhe der Rabattbelastung und Markt- bzw. Einsparpotential impliziert zudem, dass umsatzschwächere potentielle Versandapotheken aufgrund einer in Bezug auf ihren Umsatz relativ höheren Rabattbelastung tendenziell mehr hochpreisige Indikationsgruppen versenden werden als umsatzstarke Versandapotheke, die aufgrund hoher economies of scale eine bessere Kostensituation aufweisen. 
Übersicht IV - 7: Warensortiment und Preisvergleich

\begin{tabular}{|c|c|}
\hline Positive Aspekte & Negative Aspekte \\
\hline $\begin{array}{l}\text { Warensortiment } \\
\text { - „Zielgruppenorientiertes Vollsortiment“, } \\
\text { d.h. Vollsortiment für Indikationen der } \\
\text { Dauermedikation oder chronisch Kranker } \\
\text { - Im Ausland (USA) fördern Versandapo- } \\
\text { theken die Abgabe preiswerter Generika } \\
\text { - Verordnungen des Zielsegments des Ver- } \\
\text { sandhandels im Preisvergleich der } \\
\text { Tagesdosen durchschnittlich billiger: ca. } 1 \\
\text { DM je Tagesdosis [DDD] im Vergleich } \\
\text { zum GKV-Durchschnitt mit 1,33 DM je } \\
\text { DDD } \\
\text { Es gelten auch Indikationen als versen- } \\
\text { dungsfähig, deren Verordnungswert unter } \\
\text { dem GKV-Durchschnitt liegt }\end{array}$ & $\begin{array}{l}\text { Warensortiment } \\
\text { - Kein Vollsortiment, da akute Medikation } \\
\text { nicht als Zielsegment definiert wird (Re- } \\
\text { striktion der 48-Stunden-Lieferzeit) } \\
\text { - Bei Preisvergleich auf Verordnungsebene } \\
\text { liegen die durchschnittlichen Verord- } \\
\text { nungswerte des Zielsegments des Ver- } \\
\text { sandhandels mit ca. } 61 \text { bis } 67 \text { DM über } \\
\text { dem GKV-Durchschnitt mit } 47 \text { DM } \\
\text { Die Ausgestaltung der Rabattsysteme hat } \\
\text { einen wesentlichen Einfluss auf die Höhe } \\
\text { der Markt- und Einsparpotentiale. Wird } \\
\text { die Rabattbelastung zu groß, hat dies ne- } \\
\text { gative Auswirkungen auf die Höhe des } \\
\text { Einsparpotentials. Zudem steigt die Ten- } \\
\text { denz zu höherpreisigen Indikationen, ins- } \\
\text { besondere bei umsatzschwachen Ver- } \\
\text { sendern. }\end{array}$ \\
\hline
\end{tabular}

Quelle: INIFES, eigene Darstellung.

Dabei kann im Rahmen dieser Studie keine weiterführende juristische Betrachtung der Erhebung zusätzlicher Kassenrabatte in der Gestalt vorgenommen werden, ob diese aus kartellrechtlicher Sicht auch möglich wären. Das Konstrukt zusätzlicher Kassenrabatte dient hier lediglich zur Modifizierung der quantitativen Simulation von Einsparpotentialen.

\subsubsection{Einflüsse auf die Versorgungsdichte und Arbeitsmarkteffekte}

Mit ca. 3.800 Einwohnern je Apotheke liegt die Apothekendichte in Deutschland weit über dem europäischen Durchschnitt. Vielfach wird deshalb von einer Überversorgung gesprochen.

Das Vorhalten von Überkapazitäten widerspricht dem Wirtschaftlichkeitsgebot des $\S 12$ SGB V, wenn davon eine kostentreibende Wirkung auf die Solidargemeinschaft der GKV ausgeht. Mit der Einführung von Versandapotheken ist durchaus intendiert, durch das wettbewerbliche Nebeneinander von Vertriebswegen Überkapazitäten im Apothekensektor abzubauen. Es ist allerdings nicht davon auszugehen, dass bei einem kurz- bis mittelfristig erreichbaren Marktanteil der Versandapotheken von 15\% entsprechend $15 \%$ der Präsenzapotheken schließen müssten. Zur genauen Abschätzung, welche und wie viele Präsenzapotheken von einer Schließung betroffen 
wären, sind kleinräumige Untersuchungen notwendig, die allerdings im Rahmen dieser Studie aufgrund fehlenden Datenmaterials nicht erfolgen konnten.

Übersicht IV - 8: Versorgungsdichte

\begin{tabular}{|c|c|}
\hline Positive Aspekte & Negative Aspekte \\
\hline $\begin{array}{l}\text { Versorgungsdichte } \\
\text { - Apothekendichte in Deutschland im euro- } \\
\text { päischen Vergleich sehr hoch, Deutsch- } \\
\text { land: 3.800 Einw. I Apotheke Europa: } \\
4.625 \text { Einw. IApotheke } \\
\text { - Überversorgung mit Apotheken wird kos- } \\
\text { tentreibende Funktion unterstellt } \\
\text { - Ziel der "angemessenen“ Versorgung ist } \\
\text { quantitativ nicht definiert, starke regionale } \\
\text { Unterschiede } \\
\text { Deutschland West: } 3.640 \\
\text { Deutschland Ost: } 4.890 \text { (1997) } \\
\text { - Bei } 15 \% \text { Marktanteil Versand ist nicht da- } \\
\text { von auszugehen, dass } 15 \% \text { der Prä- } \\
\text { senzpotheken schließen müssten } \\
\text { Ausreichende Versorgungsdichte mit Prä- } \\
\text { senzapotheken ist nicht gefährdet } \\
\text { Flächendeckende Versorgung mit Ver- } \\
\text { sandapotheken weder möglich noch an- } \\
\text { gestrebt }\end{array}$ & $\begin{array}{l}\text { Versorgungsdichte } \\
\text { - Zur Abschätzung, welche und wie viele } \\
\text { Präsenzapotheken von einer Schließung } \\
\text { betroffen wären, sind kleinräumige Un- } \\
\text { tersungen notwendig (erfolgten im Rah- } \\
\text { men dieser Studie nicht) }\end{array}$ \\
\hline
\end{tabular}

Quelle: INIFES, eigene Darstellung.

Eine flächendeckende Versorgung mit Versandapotheken ist trotz des Hinweises auf ein wettbewerbliches Nebeneinander der Vertriebswege weder intendiert noch möglich. Von einer nach wie vor ausreichenden Versorgungsdichte durch Präsenzapotheken ist auch nach einer beabsichtigten Marktbereinigung ohne Zweifel auszugehen, das Ziel der Sicherung einer hochwertigen Versorgung der Bevölkerung mit Arzneimitteln ist garantiert. Bereits heute ist eine innerhalb Deutschlands regional stark schwankende Apothekendichte allgemein als „ausreichend" akzeptiert, obwohl z.B. in den neuen Bundesländern ca. 4.890 Einwohner pro Apotheke versorgt werden müssen, während es im Vergleich zu den alten Bundesländern nur ca. 3.640 sind. Gebiete mit einer geringeren Apothekendichte könnten folglich überdurchschnittlich von einer Zulassung weiterer Vertriebswege profitieren.

ÖBIG, (1998), Arzneimittel Vertrieb in Europa, S. 332. 
Übersicht IV - 9: Arbeitsmarkteffekte

\begin{tabular}{|c|c|}
\hline Positive Aspekte & Negative Aspekte \\
\hline $\begin{array}{l}\text { Arbeitsmarkteffekte } \\
\text { - Hohe Arbeitskräftenachfrage nach phar- } \\
\text { mazeutischem Personal (auf } 10 \text { Angebo- } \\
\text { te kommt nur eine Bewerbung) } \\
\text { - Versandapotheken benötigen Personal, } \\
\text { wenn auch aufgrund einer erhöhten Pro- } \\
\text { duktivität nicht in dem Maße, wie es } \\
\text { durch Präsenzapotheken freigesetzt wer- } \\
\text { den würde }\end{array}$ & $\begin{array}{l}\text { Arbeitsmarkteffekte } \\
\text { - Keine Abschätzung möglich, wie viele } \\
\text { Präsenzapotheken von einer Schließung } \\
\text { betroffen sein werden, folglich sind Ar- } \\
\text { beitsmarkteffekte nicht quantifizierbar } \\
\text { - Negative Arbeitsmarkteffekte sind auf- } \\
\text { grund der Personalnachfrage der Ver- } \\
\text { sandapotheken sowie des Arbeitskräf- } \\
\text { temangels als marginal einzustufen }\end{array}$ \\
\hline
\end{tabular}

Quelle: INIFES, eigene Darstellung.

Natürlich ist, wie bereits erwähnt, von einer Schließung einzelner Präsenzapotheken und entsprechend mit einer Freisetzung von Arbeitskräften auszugehen, allerdings dürften sich negative Arbeitsplatzeffekte in kurzfristiger Perspektive nur marginal auswirken. Zum einen deshalb, da am Markt bereits heute eine starke Nachfrage, insbesondere nach pharmazeutischem Personal, existiert, zum anderen, da durch den Markteintritt von neuen Anbietern, als solche fungieren die Versandapotheken, eine zusätzliche Nachfrage nach Arbeitskräften ausgelöst wird.

Abschließend ist festzuhalten, dass entsprechend der Intention des Abbaus von Überkapazitäten auf der Angebotsseite im Hinblick auf mögliche Arbeitsmarkteffekte im Apothekensektor gelten muss, dass das Ziel von Reformmaßnahmen die Ausschöpfung von Wirtschaftlichkeitspotentialen, nicht jedoch einen Bestandsschutz von Apothekerinteressen sowie das Gewähren von Beschäftigungsgarantien ist.

\subsubsection{Nutzerfreundlichkeit, Erreichbarkeit und Zustellsicherheit}

Bislang wird der Weg eines Medikaments vom Pharmahersteller zum Patienten in Deutschland streng reguliert. Eine Öffnung des strukturierten Vertriebsweges bietet nicht nur finanzielle Entlastungen für die Kostenträger und die Beitragszahler, sondern eröffnet auch neue Serviceangebote für die Verbraucher. Viele Arzneimittelbezieher sind in ihrer Mobilität aufgrund einer Krankheit, des Alters oder der Berufstätigkeit stark eingeschränkt. Diese könnten sich den Weg in die Apotheke oder die Abhängigkeit von anderen Personen ersparen, wenn der Versandhandel legalisiert würde.

Heyde, Insa, (2001), Auch öffentliche Apotheken leiden unter Mangel an Arbeitskräften, S. 7, in: apv- news, 02/01, S. $7-8$. 
Übersicht IV - 10: Nutzerfreundlichkeit

\begin{tabular}{|c|c|}
\hline Posi & kte \\
\hline $\begin{array}{l}\text { Heimlieferservice, Zielgruppe, } \\
\text { § 17,II ApBetrO }\end{array}$ & $\begin{array}{l}\text { Heimlieferservice, Zielgruppe, } \\
\S 17 \text {,II ApBetro }\end{array}$ \\
\hline $\begin{array}{l}\text { Bestimmte Patientengruppen könnten } \\
\text { sich durch die Legalisierung des Ver- } \\
\text { sandhandels den Weg in die Apotheke } \\
\text { oder die Abhängigkeit von anderen er- } \\
\text { sparen (Besorgerproblematik). }\end{array}$ & $\begin{array}{l}\text { - } \$ 17, \text { II ApBetrO garantiert bereits heute } \\
\text { eine umfassende Versorgung mit Arz- } \\
\text { neimitteln. } \\
\text { - Versandapotheken können keine Versor- } \\
\text { gung mit Akut-Therapeutika anbieten. }\end{array}$ \\
\hline $\begin{array}{l}\text { Die Inanspruchnahme des } \S 17 \text {, II } \\
\text { ApBetrO zeigt, dass ein Versandhandel } \\
\text { bereits existiert. }\end{array}$ & $\begin{array}{l}\text { Ein Heimlieferservice setzt die Anwesen- } \\
\text { heit des Empfängers voraus. Ansonsten } \\
\text { Zweitzustellung. }\end{array}$ \\
\hline $\begin{array}{l}\text { Bequeme Art des Warenbezugs nach } \\
\text { Hause oder an einen anderen Aufent- } \\
\text { haltsort. }\end{array}$ & $\begin{array}{l}\text { - Apotheker und bestimmte Facharztgrup- } \\
\text { pen sehen in den Chronikern keine Ziel- } \\
\text { gruppe für den Versandhandel. }\end{array}$ \\
\hline $\begin{array}{l}\text { Zielgruppe einer Versandapotheke: Pati- } \\
\text { enten mit einer planbaren Dauermedika- } \\
\text { tion, Chroniker, immobile Patienten }\end{array}$ & $\begin{array}{l}\text { - Chroniker sind vielfach multimorbide und } \\
\text { erhalten mehrere Verordnungen pro Re- } \\
\text { zept (sowohl Akut- als auch Dauer- } \\
\text { medikation). }\end{array}$ \\
\hline
\end{tabular}

Quelle: INIFES, eigene Darstellung.

Zur Sicherstellung einer umfassenden Arzneimittelversorgung der Bevölkerung erlaubt $\S 17, \|$ ApBetrO die Versendung aus der Apotheke oder die Zustellung durch Boten in "begründeten Einzelfällen" an den Arzneimittelempfänger. Sowohl die von INIFES durchgeführte Primärerhebung als auch Expertengespräche mit Apothekem zeigen, dass das derzeitige Ausmaß der Inanspruchnahme dieser Ausnahmeregelung nicht mehr dem "begründeten Einzelfall" entspricht, sondern vielfach die Ausmaße eines Versandhandels annimmt. Die Struktur der Apotheke hat sich in den letzten Jahren dahingehend verändert, dass sie zu einem Just-in-Time Betrieb mutiert ist. Das gesamte Lagerhaltungsrisiko übernimmt der Großhandel, der die Apotheke bis zu 6 mal täglich beliefert. Rezeptpflichtige Arzneimittel, die nicht in der Apotheke vorrätig sind, werden dem Kunden im regionalen Umkreis der Apotheke durch einen Boten kostenlos nach Hause geliefert. ${ }^{78}$ Auf diesem Weg wird eine umfassende Arzneimittelversorgung und gleichzeitig eine Kundenbindung garantiert.

"Anstatt einen Botendienst jedes Mal neu zu organisieren", so die Aussage eines Experten, "wäre ein Versandhandel mit Vorteilen verbunden." Der Kunde bekäme die Möglichkeit, sich die Arzneimittel nach Hause oder einen anderen Aufenthaltsort lie-

Nach Aussage von Apothekern übernehmen vielfach Schüler, Studenten und Rentner diesen Botendienst. Hans-Günter Friese, Präsident der Bundesvereinigung deutscher Apothekerverbände (ABDA) wies in seinem Vortrag auf dem Workshop am 17.09.2001 in Berlin darauf hin, dass im Rahmen des $\S 17$, II ApBetrO das Apothekenpersonal die Auslieferungen übernimmt. 
fern zu lassen. Dieser Service setzt jedoch stets die Anwesenheit des Empfängers oder einer autorisierten Vertrauensperson voraus.

Unter Abwägung der positiven und negativen Aspekte hinsichtlich der Vorteilhaftigkeit einer Versandapotheke für bestimmte Zielgruppen, lassen sowohl die Ergebnisse der Primärerhebungen als auch die Expertengespräche eine übereinstimmende Bewertung zu: Experten der Kostenträger, der Pharmabranche und Wissenschaftler sehen vor allem in Patienten mit einer planbaren Dauermedikation, Chronikern und in ihrer Mobilität eingeschränkte Personen eine Zielgruppe für den Versandhandel. Ältere und immobile Patienten könnten sich den Weg in die Apotheke oder die Abhängigkeit von anderen ersparen. Vorraussetzung ist, dass der Arzt das Rezept direkt auf elektronischem Weg an die vom Patienten zu bestimmende Versandapotheke sendet und diese den Heimlieferservice vornimmt. ${ }^{79}$ Aufgrund einer planbaren und dauerhaften Medikation, verbinden mit einer Legalisierung des Versandhandels viele Experten ${ }^{80}$ Vorteile für chronisch Kranke. Als Begründung wird vielfach angegeben, dass gerade diese Klientel ihre Arzneimittel gut kenne und sie dauernd einnehmen müsse.

Demgegenüber sehen die Apotheker und bestimmte Facharztgruppen in den Chronikern keine Zielgruppe für den Versandhandel. Nach Aussage eines Experten sind Chroniker vielfach multimorbid und erhalten mehrere Verordnungen pro Rezept (sowohl Akut- als auch Dauermedikation). Aufgrund der strukturellen Gegebenheiten einer Versandapotheke ist eine Versorgung mit Akut-Therapeutika nicht möglich. Der Versandhandel kann daher auch nur ein komplementärer verbraucherfreundlicher Vertriebsweg zu Präsenzapotheke sein, die nach wie vor flächendeckend präsent sein wird.

Während der Verbraucher beim Gang in die Apotheke bestimmten zeitlichen Restriktionen unterliegt, bietet eine Versandapotheke eine - an 365 Tagen im Jahr - 24stündige Erreichbarkeit für den Patienten. Wesentliches Kommunikationsmedium von Versandapotheken, so zeigen Erfahrungen im Ausland, ist das Telefon. Die bislang im Ausland zugelassenen seriösen Versandapotheken bieten ihren Kunden TelefonHotlines mit Kundendienst und pharmazeutischer Beratung an. Fragen können, wann immer sie auftreten, umgehend und kompetent beantwortet werden. Zusätzlich wird aktive Unterstützung im Hinblick auf die richtige Einnahme der Arznei angeboten. Dieser Service ist i.d.R. für den Anrufer kostenlos.

Kirschner, K. (2001), Versandhandel für apothekenpflichtige Arzneimittel freigeben, in: Die Ersatzkasse 06/2001, S. 217.

80

81

82

334

Im Rahmen der von INIFES geführten Expertengespräche.

Vgl. Glaeske, G. (2001), Gefahr für die Verbraucher oder für die Kranken?, in: Die Ersatzkasse 02/2001, S. 45.

Glaeske, G. (2001), Gefahr für die Verbraucher oder für die Apotheken?, in: Die Ersatzkasse 02/2001, S. 45. 
Übersicht IV - 11: Telefonische Erreichbarkeit

\begin{tabular}{|c|c|}
\hline Positive Aspekte & Negative Aspekte \\
\hline $\begin{array}{l}\text { Telefonische Erreichbarkeit } \\
\text { - Rund um die Uhr - ermöglicht orts- und } \\
\text { zeitunabhängige Erreichbarkeit. } \\
\text { - Für Patienten kostenloser Telefonservice. } \\
\text { - Telefonservice wird durch pharmazeuti- } \\
\text { sches Personal sichergestellt. } \\
\text { - Vorhalten einer Telefonhotline muss in den } \\
\text { Qualitätsstandards einer Versandapotheke } \\
\text { vorgeschrieben sein - begründet sich auf } \\
\text { die Beratungspflicht des § } 20 \text { ApBetrO. } \\
\text { - Anstelle des Outsourcens außerhalb der } \\
\text { Dienstzeit könnten Apotheken-Koopera- } \\
\text { tionsverbünde zur Übernahme der telefo- } \\
\text { nischen Beratung organisiert werden - hier } \\
\text { können Versand- wie Präsenzapotheken } \\
\text { partizipieren. } \\
\text { - Umsatzstarke Anbieter (im dreistelligen } \\
\text { Mio. Bereich) können kostenoptimal inner- } \\
\text { halb wie außerhalb der Öfnungszeiten } \\
\text { diesen Service mit eigenen personellen } \\
\text { wie technischen Ressourcen erstellen. } \\
\text { Kleinere und mittlere Anbieter werden } \\
\text { während der Öfnungszeiten selber er- } \\
\text { reichbar sein; feiertags und nachts an ex- } \\
\text { terne Call-Center vergeben. }\end{array}$ & $\begin{array}{l}\text { Telefonische Erreichbarkeit } \\
\text { - Telefonservice induziert zusätzliche Be- } \\
\text { triebskosten. } \\
\text { - Pharmazeutische Call-Center existieren } \\
\text { bislang nicht. } \\
\text { - Eine Beratung per Telefon ersetzt kei- } \\
\text { ne, in vielen Fällen erforderliche, face- } \\
\text { to-face Beratung. } \\
\text { Die Betriebskosten für ein Call-Center } \\
\text { führen zu Wettbewerbsverzerrungen } \\
\text { zwischen "kleinen" und "großen" Ver- } \\
\text { sendern. }\end{array}$ \\
\hline
\end{tabular}

Quelle: INIFES, eigene Darstellung.

Im Sinne des Verbraucherschutzes und der Akzeptanz durch den Patienten ${ }^{84}$ wird es daher unabdingbar sein, dass eine in Deutschland zugelassene Versandapotheke eine Telefon-Hotline mit pharmazeutischen Personal, unter Einhaltung bestimmter Qualitätsstandards, für den Verbraucher anbieten muss.

Bei der Implementierung einer telefonischen Beratung ist jedoch zu berücksichtigen, dass dies mit zusätzlichen Betriebskosten verbunden ist. Der Erstellung einer für den

83

Kooperationsverbünde werden durch den BZVZ in dem Papier "Internethandel mit Medikamenten: In Europa Qualităt sichern und Chancen für Verbraucher nutzen“, S.11, năher erläutert.

84

Die Bevölkerungsbefragung hat gezeigt, dass 74,0 Prozent der Befragten, die sich einen Bezug über eine Versandapotheke vorstellen können, die 24-Stunden Verfügbarkeit als Qualitätskriterium fordern. 
Kunden kostenlosen Telefon-Hotline setzt voraus, dass sowohl eine technische Infrastruktur als auch qualifiziertes pharmazeutisches und ggf. medizinisches Personal zusätzlich zum üblichen Apothekenbetrieb vorgehalten wird. Da derzeit keine pharmazeutischen Telefonberatungsdienste existieren, wird eine Einschätzung hinsichtlich der Fragestellung, ob eine telefonische Beratung eigen oder extern erstellt wird, erschwert. Eine Gegenüberstellung der entstehenden Kosten hat gezeigt, dass es sowohl für umsatzstarke als auch für kleinere und mittlere Versender kostenoptimal wäre, während der Offnungszeiten eine eigene Telefonberatung anzubieten. Außerhalb der Dienstzeiten einer Versandapotheke kann der Telefonservice jedoch den Wettbewerb zwischen den Anbietern verzerren. Um dies zu vermeiden ist es für Versender mit geringeren Umsätzen kostenoptimierend, die telefonische Beratung an externe Call-Center zu vergeben.

In Expertengesprächen betonten viele Pharmazeuten, dass bei der Abgabe von Medikamenten eine umfassende face-to-face Beratung unabdingbar ist. Dies könne eine telefonische Beratung nicht ersetzen, so die Pharmazeuten. Eine Kundenumfrage bei Kunden der schweizerischen Direktserviceapotheke MediService zeigt, dass die Beratung einer Versandapotheke qualitativ der einer Präsenzapotheke in nichts nachsteht.

Wie bereits erwähnt, kann eine Versandapotheke aufgrund der strukturellen Gegebenheiten im Vergleich zu einer Präsenzapotheke keine umgehende Lieferung der Arzneimittel gewährleisten. Erfahrungen aus dem Ausland sowie die geführten Expertengespräche zeigen, dass von einer Lieferfrist von ca. 48 Stunden zwischen Rezepteingang in der Apotheke und der Zustellung beim Patienten auszugehen ist.

Übersicht IV - 12: Zustellsicherheit

\begin{tabular}{|c|c|}
\hline Positive Aspekte & Negative Aspekte \\
\hline $\begin{array}{l}\text { Logistische Zustellsicherheit } \\
\text { - Eine Zustellung der Medikamente wie in } \\
\text { der Schweiz, garantiert eine ausschließli- } \\
\text { che Lieferung an den Besteller oder eine } \\
\text { autorisierte Person (bereits bei der Be- } \\
\text { stellung angegeben). Der frei zu bestim- } \\
\text { mende Ort der Zustellung ermöglicht eine } \\
\text { gewisse Flexibilität. }\end{array}$ & $\begin{array}{l}\text { Logistische Zustellsicherheit } \\
\text { - Lieferzeiten von maximal } 48 \text { Stunden } \\
\text { können nicht immer garantiert werden } \\
\text { (Wochenende und Feiertage). } \\
\text { - Verlässlichkeit externer Logistiker be- } \\
\text { züglich Qualität und Zuverlässigkeit } \\
\text { (Zeit) ist durch die Versandapotheke } \\
\text { schwer zu sanktionieren (Vertragsgestal- } \\
\text { tung). } \\
\text { Verlust der Sendung kann nicht ausge- } \\
\text { schlossen werden. }\end{array}$ \\
\hline
\end{tabular}

Quelle: INIFES, eigene Darstellung. 
Die Gegenüberstellung der positiven und negativen Aspekte bzgl. der Zustellsicherheit zeigt, dass die Zustellung von Arzneimitteln mit gewissen Unabwägbarkeiten verbunden ist. Allerdings zeigen die Erfahrungen der Schweizer Direktserviceapotheke MediService, dass es möglich und durchführbar ist, die Lieferung ausschließlich an den Besteller oder einer von ihm autorisierten Person gegen Unterschrift auszuhändigen. Der frei zu bestimmende Ort der Zustellung ermöglicht vor allem Berufstätigen eine gewisse Flexibilität. Ist der Empfänger nicht erreichbar, ist eine Zweitzustellung zu vereinbaren.

Die Logistik wird für den Versandhandel einen strategischen Parameter darstellen. Versandapotheken müssen garantieren können, dass das bestellte Medikament für den richtigen Patienten rechtzeitig am richtigen Ort und in einwandfreier Qualität unter Einhaltung der gesetzlichen Anforderungen zur Verfügung steht. ${ }^{85}$ Die strategische Positionierung und Effizienz-Kriterien des jeweiligen Anbieters entscheiden darüber, ob er die Logistik selber übernimmt oder diese an externe Logistikunternehmen auslagert. Die Nachteile eines Outsourcing der Logistik sind in der begrenzten Kontrolle der Auslieferung zu sehen. So lässt sich der Verlust von einzelnen Sendungen nicht ausschließen. Übernimmt der Versender selber die Logistik, so erreichen die Arzneimittel den Patienten eventuell am selben, in aller Regel jedoch am nächsten bzw. übernächsten Werktag nach Rezepteingang. Im Falle der Nichtabnahme von Lieferungen durch den Adressaten können die Retouren durch den Apothekenzustelldienst wieder mitgenommen werden. Eine Auslieferung durch einen externen Logistiker kann hingegen erst nach einigen Werktagen erfolgen.

\subsubsection{Sicherstellung von Beratung, Service und Information}

Bei der Bestellung apothekenpflichtiger Arzneimittel sind, unter Berücksichtigung des $\S 20 \mathrm{ApBetrO}$, von einer Versandapotheke Instrumentarien vorzuhalten, die eine Beratung nach pharmazeutisch-fachlichen Standards erlauben. Erfahrungen aus dem Ausland und bereits in Deutschland aktiver Versandhändler von Heil- und Hilfsmitteln zeigen, dass das Telefon aufgrund seiner Verbreitung das wesentliche Kommunikationsmedium ist. Wie bereits erläutert, ermöglicht eine Versandapotheke ein Serviceangebot der orts- und zeitunabhängigen Telefonberatung. 
Übersicht IV - 13: Sicherstellung der Beratung

\begin{tabular}{|c|c|}
\hline Positive Aspekte & Negative Aspekte \\
\hline $\begin{array}{l}\text { Telefonberatung, Interaktion mit Arzt } \\
\text { - Telefonische Beratung senkt die Hemm- } \\
\text { schwelle bestimmte Fragen zu stellen. } \\
\text { - Telefonberatung ist umfassender (siehe } \\
\text { MerckMedco), wenn die Beratung von inter- } \\
\text { disziplinären-Teams durchgeführt wird. } \\
\text { Beratung unabhängig von Ort und Zeit. } \\
\text { Elektronisch gestützte Dokumentationssys- } \\
\text { teme (u.a. Elektronisches-Rezept) können } \\
\text { im Versandhandel eine Analyse der Arznei- } \\
\text { mittelverordnungen und des Arzneimittel- } \\
\text { verbrauchs ermöglichen und Grundlage für } \\
\text { individuelle Beratungsaussagen seitens des } \\
\text { Apothekers der Versandapotheke sein, so- } \\
\text { fern der Verbraucher dieser Option aus- } \\
\text { drücklich zustimmt und der Datenschutz und } \\
\text { die Datensicherheit umfassend gewährleistet } \\
\text { sind. Somit kann sowohl die Dienstleis- } \\
\text { tungsqualität als auch der Bereich "Pharma- } \\
\text { ceutical Care“ über denjenigen einer durch- } \\
\text { schnittlichen Präsenzapotheke liegen. Die } \\
\text { Compliance des Patienten kann überprüft } \\
\text { und der Patient bei Problemen besser bera- } \\
\text { ten werden. } \\
\text { Die Zusammenstellung der bestellten Medi- } \\
\text { kamente ist sicher, da die Versandapotheke } \\
\text { bei Unklarheiten direkten Kontakt mit den } \\
\text { verordnenden Ärten und / oder den Patien- } \\
\text { ten aufnimmt und zudem eine Endkontrolle } \\
\text { durch einen Apotheker erfolgt }\end{array}$ & $\begin{array}{l}\text { Telefonberatung, Interaktion mit Arzt } \\
\text { - Eine fehlende persönliche face-to- } \\
\text { face-Beratung unterstützt die Anony- } \\
\text { misierung, eine Herabsetzung der } \\
\text { Hemmschwelle bei Bezug und Kon- } \\
\text { sum von Arzneimitteln und erleichtert } \\
\text { einen Arzneimittelmissbrauch. } \\
\text { - Die Ärzte befürchten eine mangelnde } \\
\text { Interaktion zwischen Apotheker-Arzt- } \\
\text { Patient. } \\
\text { Um die Vorteile einer Versandapothe- } \\
\text { ke hinsichtlich Beratung und Service } \\
\text { nutzen zu können, ist der Kunde ver- } \\
\text { pflichtet, seine Medikamente (sowohl } \\
\text { rezeptpflichtige als auch nicht rezept- } \\
\text { pflichtige) über eine Versandapotheke } \\
\text { zu beziehen. }\end{array}$ \\
\hline
\end{tabular}

Quelle: INIFES, eigene Darstellung.

Vorteil der telefonischen Beratung einer Versandapotheke ist, dass der Verbraucher relativ anonym eine kompetente Beratung und Betreuung erhält. Diese Form der Beratung senkt tendenziell die Hemmschwelle, bestimmte Fragen zu stellen. Während in einer Präsenzapotheke aufgrund der räumlichen Gegebenheiten ein persönliches Gespräch vielfach nicht geführt werden kann, kann eine Versandapotheke diesbezügliche Vorteile bieten. 
Zu beachten sind jedoch die von Seiten der Apotheker geäußerten Einwände gegenüber einer solchen Form der Beratung. "Die Gefahr einer fehlenden persönlichen face-to-face Beratung, so ein Vertreter der Apothekerschaft, wird den Arzneimittelmissbrauch erleichtern“. Um diesen Bedenken Rechnung zu tragen müssen Versandapotheken sowohl durch die Zusammensetzung der Beratungs-Teams als auch durch technische Voraussetzungen dafür Sorge tragen, dass ein Missbrauch so gut wie möglich ausgeschlossen wird.

Auch Ärzte befürchten durch die Einführung des Versandhandels eine mangelnde Interaktion zwischen Arzt und Apotheker. Vielen Ärzten ist es wichtig, bei auftretenden Unklarheiten hinsichtlich der Verordnung, vom Apotheker kontaktiert zu werden. Bereits existierende Versandapotheken / Internetapotheken weisen darauf hin, dass bei der Zusammenstellung der bestellten Medikamente darauf geachtet wird, dass bei auftretenden Unklarheiten hinsichtlich der Verordnung direkt Kontakt mit dem verordneten Arzt aufgenommen wird und zudem eine Endkontrolle durch einen Apotheker erfolgt.

Elektronisch gestützte Dokumentationssysteme (u.a. elektronisches Rezept) können im Versandhandel eine Analyse der Arzneimittelverordnungen sowie des Arzneimittelverbrauchs ermöglichen. Gleichzeitig bilden sie die Grundlage für individuelle Beratungsaussagen seitens des Versandapothekers, sofern der Verbraucher dieser Option ausdrücklich zustimmt und der Datenschutz sowie die Datensicherheit gewährleistet sind. Somit kann sowohl die Dienstleistungsqualität als auch der Bereich "Pharmaceutical Care" über denjenigen einer durchschnittlichen Präsenzapotheke liegen. Die "Compliance" des Patienten wird überprüft und bei Bedarf erfolgt eine Beratung. Diese Form der Beratung und der Betreuung kann jedoch nur dann erfolgen, wenn der Verbraucher seine Medikamente (sowohl rezeptpflichtige als auch nicht rezeptpflichtige) ausschließlich über eine Versandapotheke bezieht.

Eine solche Bindung an eine Versandapotheke kann vor allem für chronisch Kranke von Vorteil sein, bedeutet dies doch eine zusätzliche Erleichterung für sie, wenn sie rechtzeitig von ihrer Versandapotheke benachrichtigt werden, dass sie ein neues Rezept benötigen. Der Gang zum Arzt kann entfallen, wenn dieser das Rezept per Datenfernübertragung an die Versandapotheke weiterleitet.

Übersicht IV - 14: Rezepterinnerung

\begin{tabular}{|l|l|}
\hline \multicolumn{1}{|c|}{ Positive Aspekte } & \multicolumn{1}{c|}{ Negative Aspekte } \\
\hline $\begin{array}{l}\text { Servlce der Rezepterinnerung } \\
\text { - Versandapotheken bieten den Service } \\
\text { der Rezepterinnerung an. }\end{array}$ & $\begin{array}{l}\text { Service der Rezepterinnerung } \\
\begin{array}{l}\text { Setzt die Bindung an eine Versandapothe- } \\
\text { ke voraus. }\end{array}\end{array}$ \\
\hline
\end{tabular}

Quelle: INIFES, eigene Darstellung.

86

Im Rahmen der von INIFES geführten Expertengespräche. 
Insgesamt 900 Millionen Verordnungen auf ca. 600 Millionen Rezepten werden jedes Jahr in deutschen Apotheken abgewickelt. Diese Menge kann kostengünstiger erledigt werden, wenn man ein elektronisches Rezept implementiert. Während das Konzept der Schweizer Versandapotheke MediService auf einer postalischen Rezeptübermittlung durch die Versicherten an die Versandapotheke beruht, stellt die elektronische Übertragung der Rezeptdaten direkt vom verschreibenden Arzt an die Versandapotheke einen Vorteil dar, da sich so die Transaktionskosten (postalische Versendung der Rezepte durch die Patienten oder den Arzt; persönliche Weitergabe der Rezepte an die Apotheke) erheblich reduzieren.

Übersicht IV - 15: Elektronisches Rezept

\begin{tabular}{|c|c|}
\hline Positive Aspekte & Negative Aspekte \\
\hline $\begin{array}{l}\text { Elektronisches Rezept: Dokumentation } \\
\text { und Kontrolle der Compliance } \\
\text { - Elektronisch gestützte Dokumentations- } \\
\text { systeme (u.a. Elektronisches-Rezept) } \\
\text { können eine Analyse der Arzneimittelver- } \\
\text { ordnungen und des Arzneimittelver- } \\
\text { brauchs ermöglichen und Grundlage für } \\
\text { individuelle Beratungsaussagen seitens } \\
\text { des Apothekers der Versandapotheke } \\
\text { sein, sofern der Verbraucher dieser Opti- } \\
\text { on ausdrücklich zustimmt und der Daten- } \\
\text { schutz und die Datensicherheit umfas- } \\
\text { send gewährleistet sind. Somit kann so- } \\
\text { wohl die Dienstleistungsqualität als auch } \\
\text { der Bereich “Pharmaceutical Care“ über } \\
\text { denjenigen einer durchschnittlichen Prä- } \\
\text { senzapotheke liegen. Die Compliance } \\
\text { des Patienten kann überprüft und der Pa- } \\
\text { tient besser beraten werden. } \\
\text { - Elektronische Übertragung der Rezepte } \\
\text { reduziert die Transaktionskosten. } \\
\text { Die GKV-Aufwendungen für die manuelle } \\
\text { Nachbereitung und das Einlesen der kon- } \\
\text { ventionellen Rezepte betragen } 60 \text { Mio. } \\
\text { DM pro Jahr. }\end{array}$ & $\begin{array}{l}\text { Elektronisches Rezept: Dokumentation } \\
\text { und Kontrolle der Compliance } \\
\text { - Die Vertraulichkeit der zu speichernden } \\
\text { Daten kann nicht gewährleistet werden. } \\
\text { - Es existieren noch keine separaten } \\
\text { Kommunikationsplattformen (wer hat auf } \\
\text { welche Daten Zugriff und kann diese bei } \\
\text { Bedarf löschen). }\end{array}$ \\
\hline
\end{tabular}

Quelle: INIFES, eigene Darstellung.

Eine Umstellung auf durchgängig elektronische Kommunikation ermöglicht mittel- bis langfristig sowohl eine Reduktion der Kosten und eine lückenlose und übergangslose Abbildung des Datenflusses im Gesundheitswesen als auch eine umfassende Informationsplattform. Nach Einschätzung von Experten können durch den Einsatz von IT-Standards die Datenströme im Gesundheitswesen effizienter gestaltet werden. Die Informationsströme lassen sich über eine Vernetzung zeitnah, valide und bedarfsgerechter darstellen. 
Das Konzept des elektronischen Rezepts muss allerdings strengen Sicherheitsansprüchen genügen. ${ }^{87}$ Von besonderem Interesse hierbei ist, dass die Vertraulichkeit der Daten gewährleistet wird. D.h., nur die definierten Kommunikationspartner (z.B. verschreibender Arzt, Patient und die im Vorfeld definierte Versandapotheke) dürfen Zugriff auf die für sie bestimmten Informationen haben und diese ggf. löschen.

\subsubsection{Bewertung der sozialen Funktion von Arzt und Apotheke}

Das Verfahren der Arzneimittelversorgung über Rezept umfasst die Auswahl der Medikation durch den Arzt ("Verordnung"), die Überbringung des Arzneiverordnungsblattes ("Rezept") in die Apotheke, die Dispensierung in der Apotheke und die Abrechnung mit den Krankenkassen.

Laut Gesetz besteht sowohl für die Ärzte als auch für die Apotheker eine Beratungspflicht, sowohl bei verschreibungspflichtigen als auch bei nicht verschreibungspflichtigen Arzneimitteln. Allerdings darf die Beratung des Apothekers nicht die Therapie des verordnenden Arztes beeinträchtigen; eine Kritik an der ärztlichen Diagnose oder der Verordnung ist inm untersagt.

Übersicht IV - 16: Soziale Funktionen

\begin{tabular}{|c|c|}
\hline Positive Aspekte & Negative Aspekte \\
\hline $\begin{array}{l}\text { Soziale Funktionen } \\
\text { - Aufgrund der strukturellen Gegebenhei- } \\
\text { ten, stellt ein Versandhandel nur einen } \\
\text { komplementären, nicht aber einen substi- } \\
\text { tutiven Vertriebsweg dar. } \\
\text { - Die Befragung ergab, dass ein Drittel der } \\
\text { Verbraucher nicht in der Apotheke bera- } \\
\text { ten wird. }\end{array}$ & $\begin{array}{l}\text { Soziale Funktionen } \\
\text { - Der Arzt nimmt eine zentrale Rolle im } \\
\text { Arzneimittelversand ein. Ärzte sprechen } \\
\text { von einer Zunahme der Korruption zwi- } \\
\text { schen Arzt und (Versand-)Apotheker. } \\
\text { - Die fehlende persönliche Inaugenschein- } \\
\text { nahme des Kunden durch den Apotheker } \\
\text { ist mit einer Verschlechterung der } \\
\text { Compliance verbunden und kann eine } \\
\text { Verschlechterung der Therapie bedin- } \\
\text { gen. } \\
\text { Die Apotheker argumentieren, dass eine } \\
\text { Versandapotheke keine Beratung statt- } \\
\text { finden könnte und verweisen auf die von } \\
\text { innen durchgeführte Beratung des Kun- } \\
\text { den in der Apotheke. } \\
\text { Die INIFES-Befragung ergab, dass } 55,0 \\
\text { Prozent der Kunden Stammkunden einer } \\
\text { bestimmten Apotheke sind (nach Anga- } \\
\text { ben der Bayr. Apothekenkammer sind es } \\
80,0 \text { Prozent). }\end{array}$ \\
\hline
\end{tabular}

Quelle: INIFES, eigene Darstellung.

87

Vgl. Aktionsforum "Telematik im Gesundheitswesen" (2001), Management-Papier zur Sicherheitsinfrastruktur, Köln 2001. 
Unter Abwägung der positiven und negativen Aspekte ist hier festzuhalten, dass die soziale Funktion von Arzt und Apotheker nicht zu unterschätzen ist. Der Arzt wird in dem Modell des Versandhandels eine zentrale Rolle einnehmen. Seine Aufgabe wird es sein, das Rezept per Datenfernübertragung an die vom Patienten zu bestimmende Versandapotheke zu schicken. Sofern der Patient nicht angibt, an welchen Anbieter die Verordnung weitergeleitet werden soll, obliegt es dem Arzt, dies zu entscheiden. Dieses Vorgehen birgt, so die Auskunft einzelner Ärzte und der Grundtenor der Teilnehmer des von INIFES durchgeführten Workshops, die Gefahr, dass das Verordnungsverhalten der Ärzte durch bestimmte Faktoren beeinflussbar ist. Solche Fehlanreize der finanziellen Entlohnung gilt es bereits im Vorfeld des Versandhandels einzugrenzen bzw. zu unterbinden.

Die Vertreter der Apothekerschaft wiesen im Rahmen der Expertengespräch wiederholt darauf hin, dass die von den Apothekern durchgeführte Beratung ein strategischer Wettbewerbsvorteil gegenüber anderen potentiellen Anbietern sei. Ihrer Meinung nach ist ein Versandhandel mit einem Qualitätsverlust in der Beratung verbunden. Kundenbefragungen in der Schweiz zeigen jedoch, dass eine Versandapotheke mit einer 24 Stunden Telefon-Hotline durchaus eine, aus Sicht des Kunden, umfassende Beratung und ggf. Hilfeleistung gewährleisten kann.

Während die INIFES-Befragung ergab, dass ein Drittel der Verbraucher in der Apotheke nicht beraten wird, haben Versandapotheken aufgrund der Dokumentationssysteme einen höheren Informationsstand als die meisten Apotheken, was bei guter Betreuung einen sinnvolleren Arzneimitteleinsatz gewährleisten kann.

Nachteilig für Versandapotheken ist der fehlende persönliche Kontakt zum Kunden. Wird die soziale Funktion des Arztes in dem Modell des Versandhandels gestärkt, da er der einzige Ansprechpartner bei direkten Rückfragen ist, so kommt es zu einer Abnahme des sozialen Bezugs zwischen Apotheker und Kunden. Ob die Ärzte jedoch die innen zukommende zentrale Position innerhalb eines Versandhandels übernehmen wollen und können, ist mit den Beteiligten zu klären.

Unter Berücksichtigung qualitativer Einwände der Apotheker gegen das Modell einer Versandapotheke ist zu überprüfen, ob ein Versandhandelsverbot gesetzlich verankert bleiben soll. 


\subsection{Abschließende Thesen zur Implementierung eines Arzneimittel- versandes in Deutschland}

Zielsetzung der gesetzlichen Krankenversicherung ist es, "... eine gute Versorgung der Versicherten im Krankheitsfall auf qualitativ hohem Niveau zu zumutbaren Beiträgen sicherzustellen. [...] Diese hohen Anforderungen an den Versorgungsstandard müssen durch einen effizienten und zielorientierten Einsatz der Finanzmittel bei einer dauerhaften Stabilisierung der Beitragsätze erreicht werden. ${ }^{.88}$

Im Rahmen des Versorgungsauftrages der gesetzlichen Krankenkassen wurden im Jahr 2000 für Arzneimittel aus öffentlichen Apotheken 37,8 Mrd. DM aufgewendet. Dieses bedeutet im Vergleich zum Jahr 1999 ein Anstieg von ca. 4,4\%. Damit verzeichnet der Arzneimittelbereich "... die mit Abstand problematischste Entwicklung unter allen Ausgabenbereichen..." der GKV. ${ }^{89}$ Hinzu kommt, dass sich der Vertriebskostenanteil in Form der Apothekenbetriebsspanne im Jahre 1999 auf ca. 27,3\% des Bruttoumsatzes ${ }^{90}$ belief.

Vor diesem Hintergrund führt die derzeitige Diskussion um Reformoptionen im Arzneimittelsektor auch in die Richtung des Distributionskanals Apotheke. Unter Berücksichtigung des $\S 70,1$ SGB V: "[...] Die Versorgung der Versicherten muss ausreichend und zweckmäßig sein, das Maß des Notwendigen nicht überschreiten und muss wirtschaftlich erbracht werden" und der in der vorliegenden Studie gewonnenen Erkenntnisse, stellt der Versandhandel unter Grundlage der Wirtschaftlichkeit einen zusätzlichen Vertriebsweg dar.

Innerhalb des durch den Versandhandel zu bedienenden Segmentes kann eine Versandapotheke durchaus zu erheblichen Kosteneinsparungen gegenüber der derzeitigen Medikamentendistribution führen. Dies resultiert aus der Annahme, dass Versandapotheken in aller Regel einen wesentlich höheren Umsatz je Apotheke vereinen werden, als dies bisher den Präsenzapotheken möglich ist. In kurz- bis mittelfristiger Sicht erscheinen Einsparpotentiale von mindestens 400 bis 600 Mio. DM als realisierbar, in langfristiger Perspektive wären durchaus höhere Wirtschaftlichkeitspotentiale von weit mehr als 1.000 Mio. DM möglich.

Unter Abwägung der positiven und negativen Aspekte hinsichtlich der Vorteilhaftigkeit einer Versandapotheke sind vor allem Patienten mit einer planbaren Dauermedikation, Chroniker und in ihrer Mobilität eingeschränkte Personen als eine Zielgruppe für den Versandhandel geeignet. Das Beispiel der Schweizer Direktservice Apotheke

88

89

80

Vgl. www.Dialog-Gesundheit.de/AU...k/Gesetzentwurf/SGBGesetz1806.html, Zugriff am 07.10.1999; §§ 70 und 71 , I SGB V.

Bundesministerium für Gesundheit (2000), Knapp eine Milliarde Überschuss und stabiles Beitragsniveau: Finanzentwicklung der gesetzlichen Krankenversicherung in 1999 überaus positiv! In: www.bmgesundheit.de, Pressemitteilung Nr. 20, Zugriff am 15.03.2000.

ABDA (1999), Die Apotheke Zahlen, Daten, Fakten, Abbildung 13, sowie Wirtz, K. (2000), Bericht über die Ergebnisse des Betriebsvergleiches der Apotheken im Jahre 1998, in: Mitteilungen des IFH Köln, Jg. 52, S. 25. 
MediService zeigt, dass von realisierten Einsparpotentialen einer Versandapotheke vor allem kranke Patienten und nicht die gesunden Versicherten profitieren. So erhält in der Schweiz jeder Kunde der Versandapotheke rund die Hälfte seines jährlichen Selbstbehaltes für Medikamente erstattet. ${ }^{9}$

Die durch den Versandhandel erwarteten Einsparvolumina können nur dann erzielt werden, wenn die maximalen Marktpotentiale vollständig ausgeschöpft werden. Davon ist realistischerweise in Zukunft nicht auszugehen. Allerdings, in welchem Maß und in welchem Zeitbezug dieses Marktpotential in Form tatsächlicher Marktanteile zukünftig realisiert werden wird, ist nicht abzuschätzen und hängt von exogenen (betriebsfremden) wie endogenen (betriebsinternen) Restriktionen, wie z.B. der Überwindung von Akzeptanzhürden gegenüber Versandapotheken bei Patienten wie den Ärzten, ab. Aufgrund der geringeren Grenzkosten (economies of scale), einer Optimierung der Betriebsabläufe sowie einer mit der Umsatzstärke korrelierenden Einkaufsmacht der Versandapotheken werden Kostensenkungen induziert. Und dies trotz neu entstehender Betriebsaufwendungen (z.B. für Logistik oder telefonische Beratungsdienste).

Unter Güterabwägungskriterien zeigt sich eindeutig, dass der Versandhandel positive Wirkungen hat, wenn bestimmte Rahmenbedingungen berücksichtigt werden:

Die Zulassung von Versandapotheken ist ohne Ausnahme an die Gewährleistung der Arzneimittel- und Patientensicherheit zu knüpfen.

Versandapotheken als verbraucherfreundliche Alternative, insbesondere für Patienten mit Dauermedikation bzw. mit Medikation zur Behandlung chronischer Erkrankungen, können nicht darauf ausgerichtet sein, eine flächendeckende Versorgung der Bevölkerung zu garantieren. Daraus leitet sich ab, dass eine Versandapotheke den größten Nutzen stiftet, wenn sie ein "zielgruppenorientiertes Vollsortiment" vorhäit.

Die Logistik wird für den Versandhandel einen strategischen Parameter darstellen. Darunter ist die Sicherstellung der Aufgaben zu verstehen, die garantieren, dass das bestellte Medikament für den richtigen Patienten rechtzeitig am richtigen Ort und in einwandfreier Qualität unter Einhaltung der gesetzlichen Anforderungen zur Verfügung steht. Die Kosten für den Versand werden von den Anbietern übernommen.

Nachteilig für Versandapotheken ist der fehlende persönliche Kontakt zum Kunden. Hier müssen verstärkte Anstrengungen unternommen werden, um die Beratung auf anderem Wege sicherzustellen und sowohl durch die Zusammensetzung der Beratungsteams als auch durch technische Voraussetzungen dafür Sorge zu tragen, dass ein Missbrauch ausgeschlossen wird. Eine telefonische Erreichbarkeit, für den Patienten kostenlos sowie rund um die Uhr, ist ein wesentlicher Standard, der von den

91

www.mediservice.ch/de home/services/services/31400.html, Zugriff am 08.08.2001. 
Patienten gewünscht wird und zudem eine orts- und zeitunabhängige Beratung ermöglicht. Dies entspricht dem Patienten- und Verbraucherschutz.

Die qualitativen Rahmenbedingungen zeigen, dass die Sicherung eines qualitativ hochwertigen Versandhandels für die Akzeptanz der Beteiligten förderlich ist und sich auf Dauer als vorteilhaft - auch in Anbetracht der erzielbaren Kosteneinsparungen - erweisen wird.

Die Patienten sollten an erzielbaren Einsparungen partizipieren. Anreizsysteme zur Nutzung des neuen Versandweges wären mit Blick auf die Zielgruppe - Patienten mit chronischen Krankheiten oder dauerhafter Medikation - insbesondere deshalb zu begrüßen, da hier offensichtlich die dauerhaft kranken und gesundheitlich eingeschränkten Versicherten profitieren würden. 


\section{Anhang I: Nebenrechnungen zu den Marktpotentialen}

\section{Ohne Berücksichtigung anderer Faktoren}

Entsprechend der Zielsegmentvarianten A bis $C$ aus346 2.3. wird jeweils das maximale Markt- wie Einsparpotential von Versandapotheken unter Beachtung der Umsatzgröße aus 2.4. ermittelt:

1) Dazu wird der indikationsspezifische durchschnittliche Verordnungswert um den Kassenrabatt von 5\% vermindert. Dieser Anteil ist bereits heute bei GKVVerordnungen durch die Apotheken an die gesetzliche Krankenkasse abzuführen, steht den Präsenzapotheken also nicht zur Kostendeckung zur Verfügung. Entsprechend wird auch bei potentiellen Versendern verfahren.

2) In einem weiteren Schritt werden die in 2.4. hergeleiteten betriebswirtschaftlichen Ergebnisse vor Logistikkosten als Kosteneinsparungen vor Logistikkosten in Höhe von 10,4\% (VAI), 13,4\% (VA II) bzw. 17,4\% (VA III) interpretiert und auf den Verordnungswert je Indikationsgruppe des Zielsegments bezogen.

3) Daraus sind jeweils die Logistikstückkosten zu decken. Diese sind unabhängig von der Höhe des Verordnungswertes und entsprechen mindestens 3,48 DM je Verordnung für VA I bzw. 4,79 DM für VA II und VA III.

Alle Verordnungen, die aus dem Ergebnis vor Logistikkosten die Logistikstückkosten nicht decken können, also ein negatives Ergebnis aufweisen würden, werden trotz ihrer epidemiologisch begründeten Eignung nicht über den Versandweg abgegeben. Aufgrund des negativen Ergebnisses wären keine Kosteneinsparungen für die GKV zu erwarten. Diese Verordnungen fallen folglich nicht unter das Marktpotential, die interne Kostenstruktur wirkt als Markteintrittsbarriere.

Alle Verordnungen des Zielsegments, die aus dem Ergebnis vor Logistikkosten die Logistikstückkosten decken können, also ein positives Ergebnis aufweisen würden, werden potentiell über den Versandweg abgegeben. Aufgrund des positiven Ergebnisses wären Kosteneinsparungen für die GKV zu erwarten. Diese Verordnungen bilden das Marktpotential. 
Für das Zielsegment A (Indikator Packungsgröße ab 50 DDD) ergeben sich in Abhängigkeit von der Umsatzgröße der Versender folgende Marktpotentiale:

Anhang I - Tabelle 1: Ausprägungen der Marktpotentiale für Zielsegmentvariante A

\begin{tabular}{|c|c|c|c|c|c|c|c|c|c|}
\hline \multirow{2}{*}{\multicolumn{4}{|c|}{ Arzneimittelverbrauch 1999}} & \multicolumn{2}{|c|}{ VAI } & \multicolumn{2}{|c|}{ VAII } & \multicolumn{2}{|c|}{ VAIII } \\
\hline & & & & \multirow{2}{*}{$\begin{array}{c}\text { Ergebris va } \\
\text { Logistikosten } \\
10,40 \%\end{array}$} & \multirow{2}{*}{$\begin{array}{l}\begin{array}{l}\text { Logistik } \\
\text { kosten }\end{array} \\
3,48 \mathrm{CM}\end{array}$} & \multirow{2}{*}{\begin{tabular}{|c|}
$\begin{array}{c}\text { Egebris vor } \\
\text { Logistikosten }\end{array}$ \\
$13,40 \%$ \\
\end{tabular}} & \multirow{2}{*}{$\begin{array}{l}\begin{array}{c}\text { Logistik- } \\
\text { kosten }\end{array} \\
4,79 \mathrm{CM}\end{array}$} & \multirow{2}{*}{\begin{tabular}{|c|}
$\begin{array}{c}\text { Eggebris var } \\
\text { Logistikosten }\end{array}$ \\
$17,40 \%$ \\
\end{tabular}} & \multirow{2}{*}{$\frac{\begin{array}{l}\text { Logistik } \\
\text { kosten }\end{array}}{4,79 \mathrm{dM}}$} \\
\hline ATC & $\begin{array}{c}\text { Umsatz Mio } \\
\text { DM }\end{array}$ & VOin Mio & $\begin{array}{l}\text { OM/VO } \\
(-5 / A\end{array}$ & & & & & & \\
\hline ம2 & 604,50 & 1,10 & 522,07 & 54,30 & 3,48 & $\varpi, 96$ & 4,79 & 90,84 & 4,79 \\
\hline M05 & 285,70 & 0,80 & 339,27 & 35,28 & 3,48 & 45,46 & 4,79 & 59,03 & 4,79 \\
\hline C10 & $1.69,50$ & 9,40 & 171,76 & 17,86 & 3,48 & 23,02 & 4,79 & 29,89 & 4,79 \\
\hline 005 & $\approx 6,40$ & 0,80 & 102,60 & 10,67 & 3,48 & 13,75 & 4,79 & 17,85 & 4,79 \\
\hline$\infty 9$ & 2490,80 & 25,80 & 91,72 & 9,54 & 3,48 & 12,29 & 4,79 & 15,96 & 4,79 \\
\hline 803 & 477,70 & 5,20 & 87,27 & 9,08 & 3,48 & $11, \boldsymbol{\theta}$ & 4,79 & 15,19 & 4,79 \\
\hline cos & 453,10 & 5,00 & 86,09 & 8,96 & 3,48 & 11,54 & 4,79 & 14,98 & 4,79 \\
\hline $\mathrm{A} 10$ & $1.772,80$ & 20,6 & 81,76 & 8,50 & 3,48 & 10,96 & 4,79 & 14,23 & 4,79 \\
\hline 801 & 671,30 & 9,60 & 6,43 & 6,91 & 3,48 & 8,90 & 4,79 & 11,56 & 4,79 \\
\hline$\infty 8$ & $1.282,00$ & 19,70 & 61,82 & 6,43 & 3,48 & 8,28 & 4,79 & 10,76 & 4,79 \\
\hline$\infty 7$ & $1.399,50$ & 21,70 & 61,27 & 6,37 & 3,48 & 8,21 & 4,79 & 10,66 & 4,79 \\
\hline 93 & $1.253,40$ & 24,70 & 48,21 & 5,01 & 3,48 & 6,46 & 4,79 & 8,39 & 4,79 \\
\hline$\infty 1$ & $1.247,50$ & 30,40 & 38,98 & 4,05 & 3,48 & 5,22 & 4,79 & 6,78 & 4,79 \\
\hline $\mathfrak{3}_{3}$ & 65,30 & 16,70 & 37,39 & 3,89 & 3,48 & 5,01 & 4,79 & 6,51 & 4,79 \\
\hline A11 & 165,60 & 5,80 & 27,12 & \multirow{4}{*}{\multicolumn{2}{|c|}{$\begin{array}{c}\text { Ergebris vor Logistikosten< } \\
\text { Logistikosten }\end{array}$}} & \multirow{4}{*}{\multicolumn{2}{|c|}{$\begin{array}{c}\text { Ergebnis vor Logistikkosten< } \\
\text { Logistikosten }\end{array}$}} & \multirow{4}{*}{\multicolumn{2}{|c|}{$\begin{array}{c}\text { Ergebris vor Logistikosten < } \\
\text { Logistikosten }\end{array}$}} \\
\hline $\mathrm{MO4}$ & 112,20 & 5,70 & 18,70 & & & & & & \\
\hline $\mathrm{HDO}$ & 311,00 & 16,80 & 17,59 & & & & & & \\
\hline $\mathrm{A} 01$ & 81,70 & 5,60 & 13,86 & & & & & & \\
\hline $\begin{array}{l}\text { Zel- } \\
\text { segment }\end{array}$ & 15052,00 & 225,40 & 66,78 & \multicolumn{2}{|c|}{14381,50} & \multicolumn{2}{|c|}{14381,50} & \multicolumn{2}{|c|}{$14.381,50$} \\
\hline GN-Markt & 36773,80 & 782,60 & 46,9 & & & & & & \\
\hline in $\%$ & $41 \%$ & $29 \%$ & & \multicolumn{2}{|c|}{$39 \%$} & \multicolumn{2}{|c|}{$39 \%$} & \multicolumn{2}{|c|}{$39 \%$} \\
\hline \multicolumn{4}{|c|}{ durchschnittlicher Wert je Verordnung in DM } & & 75,10 & \multicolumn{2}{|r|}{75,10} & \multicolumn{2}{|r|}{75,10} \\
\hline \multicolumn{4}{|c|}{$\begin{array}{l}\text { durchschnittlicher Wert je Verordnung in DM } \\
\text { (abzg. } 5 \% \text { Kassenrabatt) }\end{array}$} & & 71,34 & \multicolumn{2}{|r|}{71,34} & \multicolumn{2}{|r|}{71,34} \\
\hline
\end{tabular}

Quelle: INIFES, eigene Berechnung. 
Für das Zielsegment B (Indikator Packungsgröße, gemessen an den verordneten N3-Normpackungen des Jahre 1998) ergeben sich in Abhängigkeit von der Umsatzgröße der Versender die folgenden Marktpotentiale:

Anhang I - Tabelle 2: Ausprägung der Marktpotentiale für Zielsegmentvariante B

\begin{tabular}{|c|c|c|c|c|c|c|c|c|}
\hline $\begin{array}{c}\text { Indikationsgruppe } \\
\text { nach Roter Liste } \\
\text { bzw. Zuzahlungs- } \\
\text { verordnung }\end{array}$ & $\begin{array}{l}\text { Umsatz } \\
\text { gesamt in } \\
\text { Mio. DM }\end{array}$ & $\begin{array}{l}\text { Umsatz N3 } \\
\text { in Mio. DM }\end{array}$ & $\begin{array}{c}\text { Anteil N3- } \\
\text { Umsatz am } \\
\text { Gesamtum- } \\
\text { satz }\end{array}$ & $\begin{array}{l}\text { N3-VO } \\
\text { in Mio. } \\
\text { Stück }\end{array}$ & $\begin{array}{l}\text { DMNO } \\
\text { je N3 - } \\
\text { Packung } \\
\text { (-5\% Ra- } \\
\text { batt) }\end{array}$ & $\begin{array}{l}\text { Ergebni } \\
\text { VAI } \\
10,4 \%\end{array}$ & is vor Logistikko & $\begin{array}{l}\text { osten } \\
\text { VA III } \\
17,4 \%\end{array}$ \\
\hline Diätetika & 5,56 & 1,13 & $20,28 \%$ & 0,004 & 308,61 & 32,10 & 41,35 & 53,70 \\
\hline Lipidsenker & $1.495,58$ & 1085,14 & $72,56 \%$ & 5,43 & 199,92 & 19,75 & 25,45 & 33,05 \\
\hline ... bis & \multicolumn{8}{|c|}{ Vollständige Darstellung siehe Anhang I - Tabelle 4} \\
\hline Laxantia & 92,65 & 44,64 & $48,19 \%$ & 1,17 & 36,10 & 3,75 & 4,84 & 6,28 \\
\hline $\begin{array}{l}\text { Mineralstoff- } \\
\text { präparate }\end{array}$ & 390,28 & 278,84 & $71,45 \%$ & 7,60 & 34,85 & 3,62 & \multirow{3}{*}{$\begin{array}{c}\text { Ergebnis vor } \\
\text { Logistik- } \\
\text { kosten } \\
< \\
\text { Logistik- } \\
\text { kosten }\end{array}$} & 6,06 \\
\hline Ophtalmika & 555,59 & 168,91 & $30,40 \%$ & 5,77 & 27,81 & \multirow[b]{2}{*}{$\begin{array}{c}\text { Ergebnis vor } \\
\text { Logistik- } \\
\text { kosten } \\
< \\
\text { Logistik- } \\
\text { kosten }\end{array}$} & & 4,84 \\
\hline$\cdots$ & & & & & & & & 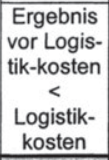 \\
\hline Zielsegment & & $16.698,5$ & & 257,49 & 64,85 & $15.821,32$ & $15.542,48$ & $15.990,23$ \\
\hline $\begin{array}{l}\text { Gesamt GKV- } \\
\text { Umsatz } 1998\end{array}$ & $35.611,3$ & & & 801,10 & 44,45 & & & ias. \\
\hline $\begin{array}{l}\text { In \% des } \\
\text { GKV-Marktes }\end{array}$ & & $47 \%$ & & $32 \%$ & $146 \%$ & $44 \%$ & $44 \%$ & $45 \%$ \\
\hline \multicolumn{6}{|c|}{ Durchschnittlicher Verordnungswert in DM } & 72,57 & 73,87 & 71,45 \\
\hline \multicolumn{6}{|c|}{ Durchschnittlicher Verordnungswert in DM (abzgl. 5\% Kassenrabatt) } & 68,94 & 70,18 & 67,88 \\
\hline
\end{tabular}

Quelle: INIFES, eigene Berechnung. 
Es ergeben sich unter Berücksichtigung verschiedener Umsatz- und Betriebsstrukturen folgende drei Marktpotentialvarianten für Zielsegment $C$ (überdurchschnittliche Packungsgröße ab 35 DDD):

Anhang I-Tabelle 3: Ausprägungen der Marktpotentiale für Zielsegmentvariante C

\begin{tabular}{|c|c|c|c|c|c|c|c|c|c|}
\hline \multirow{2}{*}{\multicolumn{4}{|c|}{ Arzneimittelverbrauch 1999}} & \multicolumn{2}{|c|}{ VAI } & \multicolumn{2}{|c|}{ VAII } & \multicolumn{2}{|c|}{ VAIII } \\
\hline & & & & \multirow{3}{*}{$\begin{array}{c}\begin{array}{c}\text { Eggebris vor } \\
\text { Logistiklosten }\end{array} \\
10,40 \% \\
\end{array}$} & \multirow{3}{*}{$\begin{array}{l}\text { Logistik- } \\
\text { kosten } \\
3,48 \mathrm{CM}\end{array}$} & \multirow{3}{*}{\begin{tabular}{|c|}
$\begin{array}{c}\text { Ergebris vor } \\
\text { Logistikkosten }\end{array}$ \\
$13,40 \%$ \\
\end{tabular}} & \multirow{3}{*}{$\begin{array}{c}\begin{array}{c}\text { Logistik } \\
\text { kosten }\end{array} \\
4,79 \mathrm{CM}\end{array}$} & \multirow{3}{*}{$\begin{array}{c}\begin{array}{c}\text { Eggebris vor } \\
\text { Logistiklosten }\end{array} \\
17,40 \%\end{array}$} & \multirow{3}{*}{$\begin{array}{l}\text { Logistik } \\
\text { kosten }\end{array}$} \\
\hline & Umsatz & & LuIVO & & & & & & \\
\hline ATC & Mio DM & Vo in Mio & $(-5 \%)$ & & & & & & \\
\hline L2 & 604,50 & 1,10 & 522,07 & 54,30 & 3,48 & 69,96 & 4,79 & 90,84 & 4,79 \\
\hline MD5 & 285,70 & 0,80 & 339,27 & 35,28 & 3,48 & 45,46 & 4,79 & 59,03 & 4,79 \\
\hline $\mathrm{C10}$ & $1.699,50$ & 9,40 & 171,76 & 17,86 & 3,48 & 23,02 & 4,79 & 29,89 & 4,79 \\
\hline D05 & 86,40 & 0,80 & 102,60 & 10,67 & 3,48 & 13,75 & 4,79 & 17,85 & 4,79 \\
\hline$\infty 9$ & 2490,80 & 25,80 & 91,72 & 9,54 & 3,48 & 12,29 & 4,79 & 15,96 & 4,79 \\
\hline $\mathrm{BO3}$ & 477,70 & 5,20 & 87,27 & 9,08 & 3,48 & $11, \oplus$ & 4,79 & 15,19 & 4,79 \\
\hline$\infty 2$ & 453,10 & 5,00 & 86,09 & 8,96 & 3,48 & 11,54 & 4,79 & 14,98 & 4,79 \\
\hline $\mathrm{ND3}$ & 434,50 & 4,90 & 84,24 & 8,76 & 3,48 & 11,29 & 4,79 & $14, \infty 6$ & 4,79 \\
\hline ND7 & 325,90 & 3,70 & 83,68 & 8,70 & 3,48 & 11,21 & 4,79 & 14,56 & 4,79 \\
\hline A10 & $1.772,80$ & 20,60 & 81,76 & 8,50 & 3,48 & 10,96 & 4,79 & 14,23 & 4,79 \\
\hline GO4 & 712,40 & 9,60 & 70,50 & 7,33 & 3,48 & 9,45 & 4,79 & 12,27 & 4,79 \\
\hline $\mathrm{ROB}$ & $1.935,50$ & 27,60 & $\varpi, \infty 2$ & 6,93 & 3,48 & 8,93 & 4,79 & 11,59 & 4,79 \\
\hline $\mathrm{B} 01$ & 671,30 & 9,60 & 6,43 & 6,91 & 3,48 & 8,90 & 4,79 & 11,56 & 4,79 \\
\hline$\infty 8$ & $1.282,00$ & 19,70 & 61,82 & 6,43 & 3,48 & 8,28 & 4,79 & 10,76 & 4,79 \\
\hline$\infty 7$ & $1.399,50$ & 21,70 & 61,27 & 6,37 & 3,48 & 8,21 & 4,79 & 10,66 & 4,79 \\
\hline ND6 & $1.421,00$ & 23,20 & 58,19 & 6,05 & 3,48 & 7,80 & 4,79 & 10,12 & 4,79 \\
\hline GO3 & $1.253,40$ & 24,70 & 48,21 & 5,01 & 3,48 & 6,46 & 4,79 & 8,39 & 4,79 \\
\hline$\infty 1$ & $1.247,50$ & 30,40 & 38,98 & 4,05 & 3,48 & 5,22 & 4,79 & 6,78 & 4,79 \\
\hline$\infty 3$ & 657,30 & 16,70 & 37,39 & 3,89 & 3,48 & 5,01 & 4,79 & 6,51 & 4,79 \\
\hline 066 & 37,50 & $1, \infty$ & 35,63 & 3,71 & 3,48 & 4,77 & 4,79 & 6,20 & 4,79 \\
\hline $\mathrm{HO2}$ & 271,30 & 7,50 & 34,36 & 3,57 & 3,48 & \multirow{9}{*}{\multicolumn{2}{|c|}{$\begin{array}{c}\text { Ergebris vor Logistikosten } \\
\text { < Logistikosten }\end{array}$}} & 5,98 & 4,79 \\
\hline A11 & 165,60 & 5,80 & 27,12 & \multirow{8}{*}{\multicolumn{2}{|c|}{$\begin{array}{l}\text { Ergebris vor Logistikosten } \\
<\text { Logistikosten }\end{array}$}} & & & \multirow{8}{*}{\multicolumn{2}{|c|}{$\begin{array}{c}\text { Ergebris vor Logistikosten } \\
\text { <Logistikosten }\end{array}$}} \\
\hline $\mathrm{A} 06$ & 108,70 & 4,30 & 24,02 & & & & & & \\
\hline D11 & 74,00 & 3,30 & 21,30 & & & & & & \\
\hline $\mathbf{S 0 1}$ & 624,50 & 28,70 & 20,67 & & & & & & \\
\hline DO2 & 87,30 & 4,40 & 18,85 & & & & & & \\
\hline MO4 & 112,20 & 5,70 & 18,70 & & & & & & \\
\hline $\mathrm{HD3}$ & 311,00 & 16,80 & 17,59 & & & & & & \\
\hline $\mathrm{A} 01$ & 81,70 & 5,60 & 13,86 & & & & & & \\
\hline $\begin{array}{l}\text { Ziel- } \\
\text { segment }\end{array}$ & $21.084,60$ & 343,60 & 61,36 & \multicolumn{2}{|c|}{$19.519,60$} & \multicolumn{2}{|c|}{$19.248,30$} & \multicolumn{2}{|c|}{$19.519,60$} \\
\hline $\begin{array}{l}\text { GN. } \\
\text { Markt }\end{array}$ & $36.773,80$ & 782,60 & 46,99 & & & & & & \\
\hline in $\%$ & $57 \%$ & $44 \%$ & & \multicolumn{2}{|c|}{$53 \%$} & \multicolumn{2}{|c|}{$52 \%$} & \multicolumn{2}{|c|}{$53 \%$} \\
\hline \multicolumn{4}{|c|}{ durchschnittlicher Verordnungswert in DM } & 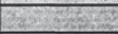 & 7256 & 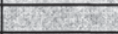 & 73,61 & 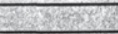 & 72,56 \\
\hline \multicolumn{4}{|c|}{$\begin{array}{l}\text { durchschnittlicher Verordnungswert in DM } \\
\text { (abzgl. } 5 \% \text { Kassenrabatt) }\end{array}$} & & 68,94 & \multicolumn{2}{|r|}{ E,93 } & \multicolumn{2}{|r|}{68,94} \\
\hline
\end{tabular}

Quelle: INIFES, eigene Berechnung. 
W Anhang I-Tabelle 4: Ausprägungen der Marktpotentiale für Zielsegmentvariante B (vollständige Darstellung)

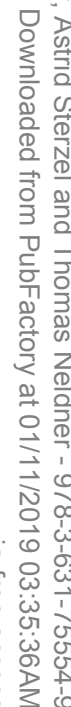

\begin{tabular}{|c|c|c|c|c|c|c|c|c|c|c|c|}
\hline \multicolumn{6}{|c|}{ Arzneimittelverbrauch 1998} & \multicolumn{2}{|c|}{ VAI } & \multicolumn{2}{|c|}{ VA II } & \multicolumn{2}{|c|}{ VAIII } \\
\hline Indikation nach Roter Liste & $\begin{array}{l}\text { Umsatz gesamt } \\
\text { in Mio. DM }\end{array}$ & $\begin{array}{l}\text { Umsatz N3 in } \\
\text { Mio DM }\end{array}$ & $\begin{array}{l}\text { Anteil des N3. } \\
\text { Umsatzes am } \\
\text { Gesamtum-satz }\end{array}$ & $\begin{array}{l}\text { N3-Vo in } \\
\text { Mio }\end{array}$ & $\begin{array}{l}\text { DMNN3-Vo } \\
\text { (-5\% Rabatt) }\end{array}$ & $\begin{array}{l}\text { Ergebnis vor } \\
\text { Logistik- } \\
\text { kosten }\end{array}$ & $\begin{array}{l}\text { Logistik- } \\
\text { kosten }\end{array}$ & $\begin{array}{l}\text { Ergebnis vor } \\
\text { Logistik- } \\
\text { kosten }\end{array}$ & $\begin{array}{l}\text { Logistik- } \\
\text { kosten }\end{array}$ & $\begin{array}{l}\text { Ergebnis vor } \\
\text { Logistik- } \\
\text { kosten }\end{array}$ & $\begin{array}{l}\text { Logistik- } \\
\text { kosten }\end{array}$ \\
\hline $\begin{array}{l}\text { Hypophysen-, } \\
\text { Hypothalamushormone }\end{array}$ & kein Versand & & & & & & & & & & \\
\hline Anthelminthika & kein Versand & & & & & & & & & & \\
\hline Immunmodulatoren & kein Versand & $=$ & & & & & & & & & \\
\hline Zytostatika usw. & kein Versand & & & & & & & & & & \\
\hline Diătetika/Ernăhrungstherapeutika & 5,56 & 1,13 & $20,28 \%$ & 0,00 & 308,61 & 32,10 & 3,48 & 41,35 & 4,79 & 53,70 & 4,79 \\
\hline Antibiotika/Antiinfektiva & kein Versand & & & & & & & & & & \\
\hline Lipidsenker & $1.495,58$ & $1.085,14$ & $72,56 \%$ & 5,43 & 189,92 & 19,75 & 3,48 & 25,45 & 4,79 & 33,05 & 4.79 \\
\hline Entwöhnungsmittel & kein Versand & & & & & & & & & & \\
\hline Abmagerungsmittel/Appetitzūgler & 0,99 & 0,27 & $27,45 \%$ & 0,00 & 138,49 & 14,40 & 3,48 & 18,56 & 4,79 & 24,10 & 4,79 \\
\hline Tuberkulosemittel & kein Versand & & & & & & & & & & \\
\hline Antihypertonika & $2.406,53$ & $1.933,79$ & $80,36 \%$ & 14,94 & 122,99 & 12,79 & 3,48 & 16,48 & 4,79 & 21,40 & 4,79 \\
\hline Enzyminhibitoren usw. & 16,53 & 5,94 & $35,92 \%$ & 0,05 & 117,06 & 12,17 & 3,48 & 15,69 & 4,79 & 20,37 & 4,79 \\
\hline Antiepileptika & 387,61 & 180,18 & $46,48 \%$ & 1,50 & 114,37 & 11,89 & 3,48 & 15,33 & 4,79 & 19,90 & 4,79 \\
\hline Osteoporosemittel/Ca-Stoffw.reg. & 349,59 & 194,46 & $55,63 \%$ & 1,69 & 109,06 & 11,34 & 3,48 & 14,61 & 4,79 & 18,98 & 4,79 \\
\hline Magen-Darm-Mittel & $2.324,78$ & 973,45 & $41,87 \%$ & 8,49 & 108,91 & 11,33 & 3,48 & 14,59 & 4,79 & 18,95 & 4,79 \\
\hline Urologika & 738,38 & 391,14 & $52,97 \%$ & 3,58 & 103,69 & 10,78 & 3,48 & 13,89 & 4,79 & 18,04 & 4,79 \\
\hline Parkinsonmittel usw. & 440,06 & 369,06 & $83,87 \%$ & 3,41 & 102,76 & 10,69 & 3,48 & 13,77 & 4,79 & 17.88 & 4,79 \\
\hline Neuropathiepräparate usw. & 331,62 & 226,93 & $68.43 \%$ & 2,33 & 92,45 & 9.62 & 3.48 & 12,39 & 4,79 & 16,09 & 4,79 \\
\hline Antiallergika & kein Versand & & & & & & & & & & \\
\hline Antiarrhythmika & 349,18 & 312,82 & $89,59 \%$ & 3,34 & 88,95 & 9,25 & 3,48 & 11,92 & 4,79 & 15,48 & 4,79 \\
\hline Antiphlogistika & 124,18 & 32,28 & $25,99 \%$ & 0,36 & & & & & & & \\
\hline Hepatika & 96,61 & 75,56 & $78,21 \%$ & 0,92 & 77,72 & 8,08 & 3,48 & 10.41 & 4.79 & 13.52 & 4,79 \\
\hline Antidiabetika & $1.612,54$ & $1.566,71$ & $97,16 \%$ & 19,20 & 77.52 & 8,06 & 3,48 & 10,39 & 4,79 & 13,49 & 4,79 \\
\hline Muskelrelaxantia & kein Versand & & & & & & & & & & \\
\hline Broncholytika/Antiasthmatika & $1.874,64$ & 532,21 & $28,39 \%$ & 7,64 & 66,14 & 6,88 & 3,48 & 8,86 & 4,79 & 11,51 & 4,79 \\
\hline
\end{tabular}

Fortsetzung nächste Seite 
Anhang I - Tabelle 4: Ausprägungen der Marktpotentiale für Zielsegmentvariante B (vollständige Darstellung)

\begin{tabular}{|c|c|c|c|c|c|c|c|c|c|c|c|}
\hline \multicolumn{6}{|c|}{ Arzneimittelverbrauch 1998} & \multicolumn{2}{|c|}{ VAI } & \multicolumn{2}{|c|}{ VAII } & \multicolumn{2}{|c|}{ VAIII } \\
\hline Indikation nach Roter Liste & $\begin{array}{c}\text { Umsatz gesamt } \\
\text { in Mio. DM }\end{array}$ & $\begin{array}{c}\text { Umsatz N3 in } \\
\text { Mio DM }\end{array}$ & $\begin{array}{l}\text { Anteil des N3- } \\
\text { Umsatzes am } \\
\text { Gesamtum-satz }\end{array}$ & $\begin{array}{l}\mathrm{N} 3-\mathrm{VO} \text { in } \\
\text { Mio }\end{array}$ & $\begin{array}{l}\text { DM/N3-VO } \\
(-5 \% \text { Rabatt })\end{array}$ & $\begin{array}{c}\text { Ergebnis vor } \\
\text { Logistik- } \\
\text { kosten }\end{array}$ & $\begin{array}{l}\text { Logistik- } \\
\text { kosten }\end{array}$ & $\begin{array}{c}\text { Ergebnis vor } \\
\text { Logistik- } \\
\text { kosten }\end{array}$ & $\begin{array}{l}\text { Logistik- } \\
\text { kosten }\end{array}$ & $\begin{array}{l}\text { Ergebnis vor } \\
\text { Logistik- } \\
\text { kosten }\end{array}$ & $\begin{array}{l}\text { Logistik- } \\
\text { kosten }\end{array}$ \\
\hline Antidementiva (Nootropika) & 574,62 & 433,59 & $75,46 \%$ & 6,24 & 65,97 & 6,86 & 3,48 & 8,84 & 4,79 & 11,48 & 4,79 \\
\hline Cholinergika & \begin{tabular}{|l} 
kein Versand \\
\end{tabular} & & & & & & & & & & \\
\hline Cholagoga und Gallenwegstherap. & 71,15 & 61,00 & $85,74 \%$ & 0,90 & 64,74 & 6,73 & 3,48 & 8,67 & 4,79 & 11,26 & 4,79 \\
\hline Venentherapeutika & 276,60 & 183,50 & $66,34 \%$ & 2,69 & 64,70 & 6,73 & 3,48 & 8,67 & 4,79 & 11,26 & 4,79 \\
\hline Antimykotika & kein Versand & & & & & & & & & & \\
\hline Durchblutungsfördernde Mittel & 327,81 & 261,54 & $79,78 \%$ & 3,92 & 63,42 & 6,60 & 3,48 & 8,50 & 4,79 & 11,04 & 4,79 \\
\hline Dermatika & 885,98 & 151,55 & $17,11 \%$ & 2,33 & 61,87 & 6,43 & 3,48 & 8,29 & 4,79 & 10,77 & 4,79 \\
\hline Beta-,Ca-BI.,Angiotensin-Hemmst. & $2.833,99$ & $2.431,35$ & $85,79 \%$ & 37,82 & 61,07 & 6,35 & 3,48 & 8,18 & 4,79 & 10,63 & 4,79 \\
\hline Wundbehandlungsmittel & \begin{tabular}{|l|} 
kein Versand \\
\end{tabular} & & & & & & & & & & \\
\hline Spasmolytika & kein Versand & & & & & & & & & & \\
\hline Sexualhormone & $1.144,62$ & 859,11 & $75,06 \%$ & 15,37 & 53,12 & 5,52 & 3,48 & 7,12 & 4,79 & 9,24 & 4,79 \\
\hline Antihypotonika & 137,56 & 74,91 & $54,46 \%$ & 1,35 & 52,84 & 5,50 & 3,48 & 7,08 & 4,79 & 9,19 & 4,79 \\
\hline Koronarmittel & 837,09 & 706,96 & $84,45 \%$ & 12,95 & 51,87 & 5,39 & 3,48 & 6,95 & 4,79 & 9,03 & 4,79 \\
\hline Corticoide (Interna) & kein Versand & & & & & & & & & & \\
\hline Antiemetika/Antivertiginosa & kein Versand & & & & & & & & & & \\
\hline Migränemittel & 162,30 & 8,78 & $5,41 \%$ & 0,17 & 48,16 & 5,01 & 3,48 & 6,45 & 4,79 & 8,38 & 4,79 \\
\hline Hämorrhoidenmittel & 67,21 & 9,68 & $14,41 \%$ & 0,19 & 48,16 & 5,01 & 3,48 & 6,45 & 4,79 & 8,38 & 4,79 \\
\hline Acidosetherapeutika & 7,37 & 6,44 & $87,46 \%$ & 0,13 & 48,14 & 5,01 & 3,48 & 6,45 & 4,79 & 8,38 & 4,79 \\
\hline Antihämorrhagika & 53,78 & 6,99 & $13,00 \%$ & 0,14 & 47,82 & 4,97 & 3,48 & 6,41 & 4,79 & 8,32 & 4,79 \\
\hline Psychopharmaka & $1.782,76$ & $1.020,11$ & $57,22 \%$ & 20,29 & 47,77 & 4,97 & 3,48 & 6,40 & 4,79 & 8,31 & 4,79 \\
\hline Infusionslösungen usw. & kein Versand & & & & & & & & & & \\
\hline Thrombozytenaggregationshemmer & 185,74 & 157,62 & $84,86 \%$ & 3,31 & 45,25 & 4,71 & 3,48 & 6,06 & 4,79 & 7,87 & 4,79 \\
\hline Diuretika & 628,74 & 424,89 & $67,58 \%$ & 9,02 & 44,74 & 4,65 & 3,48 & 5,99 & 4,79 & 7,78 & 4,79 \\
\hline Diagnostika usw. & kein Versand & & & & & & & & & & \\
\hline Analgetika/Antirheumatika & $1.822,67$ & 626,95 & $34,40 \%$ & 14,85 & 40,12 & 4,17 & 3,48 & 5,38 & 4,79 & 6,98 & 4,79 \\
\hline Balneotherapeutika usw. & 42,17 & 10,24 & $24,29 \%$ & 0,24 & 39,85 & 4,14 & 3,48 & 5,34 & 4,79 & 6.93 & 4,79 \\
\hline Gynäkologika & 213,11 & 67,88 & $31,85 \%$ & 1,63 & 39,62 & 4,12 & 3,48 & 5,31 & 4,79 & 6,89 & 4,79 \\
\hline
\end{tabular}

Fortsetzung nächste Seite 
Anhang 1 - Tabelle 4: Ausprägungen der Marktpotentiale für Zielsegmentvariante B (vollständige Darstellung)

\begin{tabular}{|c|c|c|c|c|c|c|c|c|c|c|c|}
\hline \multicolumn{6}{|c|}{ Arzneimittelverbrauch 1998} & \multicolumn{2}{|c|}{ VAI } & \multicolumn{2}{|c|}{ VAII } & \multicolumn{2}{|c|}{ VAIII } \\
\hline Indikation nach Roter LIste & $\begin{array}{c}\text { Umsatz gesamt } \\
\text { in Mio. DM }\end{array}$ & $\begin{array}{l}\text { Umsatz N3 in } \\
\text { Mio DM }\end{array}$ & $\begin{array}{l}\text { Anteil des N3- } \\
\text { Umsatzes am } \\
\text { Gesamtum-satz }\end{array}$ & $\begin{array}{l}\mathrm{N} 3-\mathrm{VO} \text { in } \\
\mathrm{MiO}\end{array}$ & $\begin{array}{l}\text { DM/N3-VO } \\
\text { (-5\% Rabatt) }\end{array}$ & $\begin{array}{l}\text { Ergebnis vor } \\
\text { Logistik- } \\
\text { kosten }\end{array}$ & $\begin{array}{l}\text { Logistik- } \\
\text { kosten }\end{array}$ & $\begin{array}{c}\text { Ergebnis vor } \\
\text { Logistik- } \\
\text { kosten }\end{array}$ & $\begin{array}{l}\text { Logistik- } \\
\text { kosten }\end{array}$ & $\begin{array}{c}\text { Ergebnis vor } \\
\text { Logistik- } \\
\text { kosten }\end{array}$ & $\begin{array}{l}\text { Logistik- } \\
\text { kosten }\end{array}$ \\
\hline Antianämika & 428,11 & 59,98 & $14,01 \%$ & 1,45 & 39,35 & 4,09 & 3,48 & 5,27 & 4,79 & 6,85 & 4,79 \\
\hline Antikoagulantia & 319,66 & 53,59 & $16,76 \%$ & 1,37 & 37,29 & 3,88 & 3,48 & 5,00 & 4,79 & 6,49 & 4,79 \\
\hline Roborantia/Tonika & 0,17 & 0,09 & $57,04 \%$ & 0,00 & 37,21 & 3,87 & 3,48 & 4,99 & 4,79 & 6,48 & 4,79 \\
\hline \begin{tabular}{|l|} 
Laxantia \\
\end{tabular} & 92,65 & 44,64 & $48,19 \%$ & 1,17 & 36,10 & 3,75 & 3,48 & 4,84 & 4,79 & 6,28 & 4,79 \\
\hline Mineralstoffpräparate & 390,28 & 278,84 & $71,45 \%$ & 7,60 & 34,85 & 3,62 & 3,48 & \multirow{16}{*}{\multicolumn{2}{|c|}{$\begin{array}{l}\text { Ergebnis vor } \\
\text { Logistikkosten }< \\
\text { Logistikkosten }\end{array}$}} & 6,06 & 4,79 \\
\hline Antitussiva/Expektorantia & kein Versand & & & & & \multirow{15}{*}{\multicolumn{2}{|c|}{$\begin{array}{l}\text { Ergebnis vor } \\
\text { Logistikkosten < } \\
\text { Logistikkosten }\end{array}$}} & & & & \\
\hline Nicht in Roter Liste & kein Versand & & & & & & & & & & \\
\hline Ophthalmika & 555,59 & 168,91 & $30,40 \%$ & 5,77 & 27,81 & & & & & 4,84 & 4,79 \\
\hline \begin{tabular}{|l|} 
Vitamine \\
\end{tabular} & 178,81 & 126,34 & $70,66 \%$ & 4,49 & 26,75 & & & & & \multirow{12}{*}{\multicolumn{2}{|c|}{$\begin{array}{l}\text { Ergebnis vor } \\
\text { Logistikkosten }< \\
\text { Logistikkosten }\end{array}$}} \\
\hline Desinfizientia/Antiseptika & kein Versand & & & & & & & & & & \\
\hline \begin{tabular}{|l|} 
Arteriosklerosemittel \\
\end{tabular} & 0,30 & 0,08 & $25,37 \%$ & 0,00 & 24,78 & & & & & & \\
\hline Hypnotika/Sedativa & 287,64 & 9,18 & $3,19 \%$ & 0,35 & 24,57 & & & & & & \\
\hline Rhinologika/Sinusitismittel & kein Versand & & & & & & & & & & \\
\hline \begin{tabular}{|l|} 
Lokalanästhetika/Neuraltherap. \\
\end{tabular} & kein Versand & & & & & & & & & & \\
\hline \begin{tabular}{|l|} 
Gichtmittel \\
\end{tabular} & 110,63 & 91,55 & $82,76 \%$ & 4,12 & 21,10 & & & & & & \\
\hline Kardiaka & 217,66 & 187,71 & $86,24 \%$ & 9,18 & 19,42 & & & & & & \\
\hline \begin{tabular}{|l|} 
Mund- und Rachentherapeutika \\
\end{tabular} & kein Versand & & & & & & & & & & \\
\hline \begin{tabular}{|l|} 
Schilddrüsentherapeutika \\
\end{tabular} & 304,09 & 277,40 & $91,22 \%$ & 14,28 & 18,45 & & & & & & \\
\hline \begin{tabular}{|l|} 
Umstimmungsmittel \\
\end{tabular} & kein Versand & & & & & & & & & & \\
\hline Karies- und Parodontosemittel & 23,08 & 16,02 & $69,43 \%$ & 1,27 & 11,94 & & & & & & \\
\hline Zielsegment (A10 N2 als N3) & & $16.698,5$ & & 257,49 & 64,85 & \multicolumn{2}{|c|}{15821,32} & \multicolumn{2}{|c|}{15542,48} & \multicolumn{2}{|c|}{15990,23} \\
\hline Gesamt GKV 1998 & $35.611,3$ & & & 801,10 & 44,45 & & & & & & \\
\hline in \% vom GKV-Umsatz & . & $47 \%$ & & $32 \%$ & $146 \%$ & \multicolumn{2}{|c|}{$44 \%$} & 449 & & \multicolumn{2}{|c|}{$45 \%$} \\
\hline \multirow{2}{*}{\multicolumn{6}{|c|}{$\begin{array}{l}\text { durchschnittlicher Verordnungswert in DM } \\
\text { durchschnittlicher Verordnungswert in DM (abzgl. 5\% Kassenrabatt) }\end{array}$}} & & 72,57 & & 73,87 & & 71,45 \\
\hline & & & & & & & 68,94 & & 70,18 & & 67,88 \\
\hline
\end{tabular}


Das Vorgehen folgt dem Schema aus der Berechnung der Marktpotentiale ohne Durchschnittliche Betriebsrendite, zusätzlich muss hier allerdings der Zwischenschritt 2b) durchgeführt werden:

1) Der indikationsspezifische durchschnittliche Verordnungswert wird um den Kassenrabatt von 5\% vermindert. Dieser Anteil ist bereits heute bei GKVVerordnungen durch die Apotheken an die gesetzliche Krankenkasse abzuführen, steht den Präsenzapotheken also nicht zur Kostendeckung zur Verfügung. Entsprechend wird auch bei potentiellen Versendern verfahren.

2a) In einem weiteren Schritt werden die in 2.4. hergeleiteten betriebswirtschaftlichen Ergebnisse vor Logistikkosten als Kostenersparnisse vor Logistikkosten in Höhe von 10,4\% (VAI), 13,4\% (VA II) bzw. 17,4\% (VA III) interpretiert.

2b) Durch die Berücksichtigung einer durchschnittlichen Betriebsrendite ist dieses allerdings um die Durchschnittliche Betriebsrendite von 3,5\% zu kürzen. Es ergeben sich 6,9\% (VAI), 9,9\% (VAII) bzW. 13,9\% (VA III).

3) Daraus sind jeweils die Logistikkosten zu decken. Diese sind unabhängig von der Höhe des Verordnungswertes und entsprechen mindestens 3,48 DM je Verordnung für VA I bzW. 4,79 DM für VA II und III.

Alle Verordnungen, die aus dem Ergebnis vor Logistikkosten die Logistikstückkosten nicht decken können, also ein negatives Ergebnis aufweisen würden, werden trotz ihrer epidemiologisch begründeten Eignung nicht über den Versandweg abgegeben. Aufgrund des negativen Ergebnisses wären keine Kosteneinsparungen für die GKV zu erwarten. Diese Verordnungen fallen folglich nicht unter das Marktpotential, die interne Kostenstruktur wirkt als Markteintrittsbarriere.

Alle Verordnungen des Zielsegments, die aus dem Ergebnis vor Logistikkosten die Logistikstückkosten decken können, also ein positives Ergebnis aufweisen würden, werden potentiell über den Versandweg abgegeben. Aufgrund des positiven Ergebnisses wären Kosteneinsparungen für die GKV zu enwarten. Diese Verordnungen bilden das Marktpotential. 
Für das Zielsegment A (Indikator Packungsgröße ab 50 DDD) ergeben sich in Abhängigkeit von der Umsatzgröße der Versender folgende Marktpotentiale:

Anhang I - Tabelle 5: Marktpotentiale für Zielsegmentvariante A (mit durchschnittlicher Betriebsrendite)

\begin{tabular}{|c|c|c|c|c|c|c|c|c|c|}
\hline \multirow{2}{*}{\multicolumn{4}{|c|}{ Arzneimittelverbrauch 1999}} & \multicolumn{2}{|c|}{ VAI } & \multicolumn{2}{|c|}{ VAII } & \multicolumn{2}{|c|}{ VAIII } \\
\hline & & & & \multirow{2}{*}{\begin{tabular}{|c|}
$\begin{array}{c}\text { Ergebris vor } \\
\text { Logistikosten }\end{array}$ \\
$6,90 \%$ \\
\end{tabular}} & \multirow{2}{*}{$\begin{array}{l}\text { Logistik } \\
\text { kosten } \\
3,48 \mathrm{DM}\end{array}$} & \multirow{2}{*}{\begin{tabular}{|c|}
$\begin{array}{c}\text { Ergebris vơ } \\
\text { Logistikkosten }\end{array}$ \\
$9,90 \%$ \\
\end{tabular}} & \multirow{2}{*}{$\begin{array}{l}\text { Logistik } \\
\text { kosten } \\
4,79 \mathrm{DM}\end{array}$} & \multirow{2}{*}{\begin{tabular}{|c|}
$\begin{array}{c}\text { Ergebris vor } \\
\text { Logistikosten }\end{array}$ \\
$13,90 \%$ \\
\end{tabular}} & \multirow{2}{*}{$\begin{array}{l}\text { Logistike } \\
\text { kosten } \\
4,79 \mathrm{CM}\end{array}$} \\
\hline ATC & $\begin{array}{c}\text { Unsatz Mio } \\
\text { DM }\end{array}$ & VOin Mio & $\begin{array}{l}\text { DM/VO } \\
(-5 \% / 9\end{array}$ & & & & & & \\
\hline LR & 604,50 & 1,10 & 522,07 & 36,02 & 3,48 & 51,68 & 4,79 & 72,57 & 4,79 \\
\hline M05 & 285,70 & 0,80 & 339,27 & 23,41 & 3,48 & 33,59 & 4,79 & 47,16 & 4,79 \\
\hline C10 & $1.69,50$ & 9,40 & 171,76 & 11,85 & 3,48 & 17,00 & 4,79 & 23,87 & 4,79 \\
\hline 005 & 86,40 & 0,80 & 102,60 & 7,08 & 3,48 & 10,16 & 4,79 & 14,26 & 4,79 \\
\hline$\infty 9$ & 2490,80 & 25,80 & 91,72 & 6,33 & 3,48 & 9,08 & 4,79 & 12,75 & 4,79 \\
\hline 803 & 477,70 & 5,20 & 87,27 & 6,02 & 3,48 & 8,64 & 4,79 & 12,13 & 4,79 \\
\hline co2 & 453,10 & 5,00 & 86,09 & 5,94 & 3,48 & 8,52 & 4,79 & 11,97 & 4,79 \\
\hline A10 & $1.772,80$ & 20,60 & 81,76 & 5,64 & 3,48 & 8,09 & 4,79 & 11,36 & 4,79 \\
\hline B01 & 671,30 & 9,60 & 66,43 & 4,58 & 3,48 & 6,58 & 4,79 & 9,23 & 4,79 \\
\hline$\infty 8$ & $1.282,00$ & 19,70 & 61,82 & 4,27 & 3,48 & 6,12 & 4,79 & 8,59 & 4,79 \\
\hline$\infty 7$ & $1.399,50$ & 21,70 & 61,27 & 4,23 & 3,48 & 6,07 & 4,79 & 8,52 & 4,79 \\
\hline $\mathrm{GO3}$ & $1.253,40$ & 24,70 & 48,21 & \multirow{7}{*}{\multicolumn{2}{|c|}{$\begin{array}{c}\text { Ergebris vor Logistikosten < } \\
\text { Logistikosten }\end{array}$}} & \multirow{7}{*}{\multicolumn{2}{|c|}{$\begin{array}{c}\text { Ergebris vor Logistiklosten < } \\
\text { Logistiklosten }\end{array}$}} & 6,70 & 4,79 \\
\hline$\infty 1$ & $1.247,50$ & 30,40 & 38,98 & & & & & 5,42 & 4,79 \\
\hline$\infty 3$ & 687,30 & 16,70 & 37,39 & & & & & 5,20 & 4,79 \\
\hline A11 & 165,60 & 5,80 & 27,12 & & & & & \multirow{4}{*}{\multicolumn{2}{|c|}{$\begin{array}{l}\text { Ergebris vor Logistikesten < } \\
\text { Logistikosten }\end{array}$}} \\
\hline $\mathrm{MO4}$ & 112,20 & 5,70 & 18,70 & & & & & & \\
\hline $\mathrm{HDO}$ & $311, \infty$ & 16,80 & 17,59 & & & & & & \\
\hline $\mathrm{A} 01$ & 81,70 & 5,60 & 13,86 & & & & & & \\
\hline $\begin{array}{l}\text { Ziel- } \\
\text { segment }\end{array}$ & $15.052,00$ & 225,40 & 66,78 & \multicolumn{2}{|c|}{$11.223,30$} & \multicolumn{2}{|r|}{$11.223,30$} & \multicolumn{2}{|c|}{$14.381,50$} \\
\hline GNV-Markt & $36.773,80$ & 782,60 & 46,99 & & & & 57 & - & +3 \\
\hline in $\%$ & $41 \%$ & $29 \%$ & & \multicolumn{2}{|c|}{$31 \%$} & \multicolumn{2}{|c|}{$31 \%$} & \multicolumn{2}{|c|}{$39 \%$} \\
\hline \multicolumn{4}{|c|}{ durchschnittlicher Verordnungswert in DM } & & 93,76 & & 93,76 & & 75,10 \\
\hline \multicolumn{4}{|c|}{$\begin{array}{l}\text { durchschnittlicher Verorchungswert in DM } \\
\text { (abzgl. } 5 \% \text { Kassenrabatt) }\end{array}$} & & 89,07 & \multicolumn{2}{|r|}{89,07} & & 71,34 \\
\hline
\end{tabular}

Quelle: INIFES, eigene Berechnung. 
Für das Zielsegment B (Indikator Packungsgröße, gemessen an den verordneten N3-Normpackungen des Jahre 1998) ergeben sich in Abhängigkeit von der Umsatzgröße der Versender die folgenden Marktpotentiale:

Anhang 1-Tabelle 6: Ausprägung der Marktpotentiale für Zielsegmentvariante B (mit Betriebsrendite)

\begin{tabular}{|c|c|c|c|c|c|c|c|c|}
\hline $\begin{array}{l}\text { Indlikationsgruppe } \\
\text { hach Roter Liste } \\
\text { bzw. Zuzahlungs- } \\
\text { verordnung }\end{array}$ & $\begin{array}{c}\text { Umsatz } \\
\text { gesamt } \\
\text { in Miס. DM }\end{array}$ & $\begin{array}{l}\text { Umsatz N3 } \\
\text { in Mio. DM }\end{array}$ & $\begin{array}{c}\text { Anteil N3- } \\
\text { Umsatz am } \\
\text { Gesamt- } \\
\text { umsatz }\end{array}$ & $\begin{array}{l}\text { N3-VO } \\
\text { in Mio. } \\
\text { Stúck }\end{array}$ & $\begin{array}{l}\text { DMNO } \\
\text { Je N3 - } \\
\text { Packung } \\
\text { (-5\% Ra- } \\
\text { batt) }\end{array}$ & \multicolumn{3}{|c|}{ Ergebnis vor Logistikkosten } \\
\hline Diătetika & 5,56 & 1,13 & $20,28 \%$ & 0,004 & 308,61 & 32,10 & 41,35 & 53,70 \\
\hline Lipidsenker & $1.495,58$ & 1085,14 & $72,56 \%$ & 5,43 & 199,92 & 19,75 & 25,45 & 33,05 \\
\hline .... bis & \multicolumn{8}{|c|}{ Vollständige Darstellung siehe Anhang I- Tabelle 8} \\
\hline Koronarmittel & 837,09 & 706,96 & $84,45 \%$ & 12,95 & 51,87 & 3,58 & 4,84 & 6,28 \\
\hline$\cdots$ & $\cdots$ & $\cdots$ & $\cdots$ & $\cdots$ & $\cdots$ & \multirow{3}{*}{$\begin{array}{c}\text { Ergebnis } \\
\text { vor Logistik- } \\
\text { kosten } \\
< \\
\text { Logistik- } \\
\text { kosten }\end{array}$} & \multirow{3}{*}{$\begin{array}{c}\text { Ergebnis } \\
\text { vor Logistik- } \\
\text { kosten } \\
< \\
\text { Logistik- } \\
\text { kosten }\end{array}$} & $\cdots$ \\
\hline $\begin{array}{l}\text { Mineralstoffprä- } \\
\text { parate }\end{array}$ & 390,28 & 278,84 & $71,45 \%$ & 7,60 & 34,85 & & & 4,84 \\
\hline$\cdots$ & & & & & & & & $\begin{array}{c}\text { Ergebnis } \\
\text { vor Logistik- } \\
\text { kosten } \\
< \\
\text { Logistik- } \\
\text { kosten } \\
\end{array}$ \\
\hline Zielsegment & & $16.698,5$ & & 257,49 & 64,85 & $13.044,57$ & $13.044,47$ & $15.821,32$ \\
\hline $\begin{array}{l}\text { Gesamt GKV- } \\
\text { Umsatz } 1998\end{array}$ & $35.611,3$ & & & 801,10 & 44,45 & & & \\
\hline $\begin{array}{l}\text { In } \% \text { des } \\
\text { GKV-Marktes }\end{array}$ & & $47 \%$ & & $32 \%$ & $146 \%$ & $37 \%$ & $37 \%$ & $44 \%$ \\
\hline \multicolumn{6}{|c|}{ Durchschnittlicher Verordnungswert in DM } & 83,38 & 83,38 & 72,57 \\
\hline $\begin{array}{l}\text { Durchschnittliche } \\
\text { (abzüglich 5\% Ka }\end{array}$ & $\begin{array}{l}\text { Verordnı } \\
\text { issenrabatt }\end{array}$ & ungswer & M & & & 79,21 & 79,21 & 68,49 \\
\hline
\end{tabular}

Quelle: INIFES, eigene Berechnung. 
Es ergeben sich unter Berücksichtigung verschiedener Umsatz- und Betriebsstrukturen folgende drei Marktpotentialvarianten für Zielsegment $C$ (überdurchschnittliche Packungsgröße ab 35 DDD):

Anhang 1 - Tabelle 7: Marktpotentiale für Zielsegmentvariante C (mit durchschnittlicher Betriebsrendite)

\begin{tabular}{|c|c|c|c|c|c|c|c|c|c|}
\hline \multirow{2}{*}{\multicolumn{2}{|c|}{ Arzimittelverbrauch 1999}} & & & \multicolumn{2}{|c|}{ VAI } & \multicolumn{2}{|c|}{ VAII } & \multicolumn{2}{|c|}{ VAIII } \\
\hline & & & & $\begin{array}{l}\text { Ergebris vor } \\
\text { Logistikkosten }\end{array}$ & $\begin{array}{l}\text { Logistik- } \\
\text { kosten }\end{array}$ & $\begin{array}{l}\text { Ergebris vor } \\
\text { Logistikkosten }\end{array}$ & $\begin{array}{l}\text { Logistik- } \\
\text { kosten }\end{array}$ & $\begin{array}{l}\text { Ergebris var } \\
\text { Logistikkosten } \\
\end{array}$ & $\begin{array}{l}\text { Logistik- } \\
\text { kosten }\end{array}$ \\
\hline ATC & Umsatz Mo DM & Voin Mo & $\begin{array}{l}\text { DinTVo } \\
(-5 \% 9\end{array}$ & $6,9 \%$ & $3,48 \mathrm{CM}$ & $9,90 \%$ & $4,79 \mathrm{DM}$ & $13,90 \%$ & $4,79 \mathrm{DM}$ \\
\hline LOR & 604,50 & 1,10 & 522,07 & 36,02 & 3,48 & 51,68 & 4,79 & 72,57 & 4,79 \\
\hline MD5 & 285,70 & 0,80 & 339,27 & 23,41 & 3,48 & 33,59 & 4,79 & 47,16 & 4,79 \\
\hline $\mathrm{C} 10$ & $1.699,50$ & 9,40 & 171,76 & 11,85 & 3,48 & 17,00 & 4,79 & 23,87 & 4,79 \\
\hline D05 & 86,40 & 0,80 & 102,60 & 7,08 & 3,48 & 10,16 & 4,79 & 14,26 & 4,79 \\
\hline$\infty$ & 2490,80 & 25,80 & 91,72 & 6,33 & 3,48 & 9,08 & 4,79 & 12,75 & 4,79 \\
\hline $\mathrm{BO} 3$ & 477,70 & 5,20 & 87,27 & 6,02 & 3,48 & 8,64 & 4,79 & 12,13 & 4,79 \\
\hline$\infty 2$ & 453,10 & 5,00 & 86,09 & 5,94 & 3,48 & 8,52 & 4,79 & 11,97 & 4,79 \\
\hline $\mathrm{ND3}$ & 434,50 & 4,90 & 84,24 & 5,81 & 3,48 & 8,34 & 4,79 & 11,71 & 4,79 \\
\hline $\mathrm{ND7}$ & 325,90 & 3,70 & 83,68 & 5,77 & 3,48 & 8,28 & 4,79 & 11,63 & 4,79 \\
\hline $\mathrm{A} 10$ & $1.772,80$ & 20,60 & 81,76 & 5,64 & 3,48 & 8,09 & 4,79 & 11,36 & 4,79 \\
\hline GO4 & 712,40 & 9,60 & 70,50 & 4,86 & 3,48 & 6,98 & 4,79 & 9,80 & 4,79 \\
\hline $\mathrm{R} 03$ & $1.935,50$ & 27,60 & 66,62 & 4,60 & 3,48 & 6,60 & 4,79 & 9,26 & 4,79 \\
\hline B01 & 671,30 & 9,60 & 66,43 & 4,58 & 3,48 & 6,58 & 4,79 & 9,23 & 4,79 \\
\hline$\infty$ & $1.282,00$ & 19,70 & 61,82 & 4,27 & 3,48 & 6,12 & 4,79 & 8,59 & 4,79 \\
\hline$\infty 7$ & $1.399,50$ & 21,70 & 61,27 & 4,23 & 3,48 & 6,07 & 4,79 & 8,52 & 4,79 \\
\hline ND6 & $1.421,00$ & 23,20 & 58,19 & 4,01 & 3,48 & 5,76 & 4,79 & 8,09 & 4,79 \\
\hline $\mathrm{GO3}_{3}$ & $1.253,40$ & 24,70 & 48,21 & \multirow{13}{*}{\multicolumn{2}{|c|}{$\begin{array}{c}\text { Ergebris vor Logistikkosten < } \\
\text { Logistikkosten }\end{array}$}} & \multirow{13}{*}{\multicolumn{2}{|c|}{$\begin{array}{c}\text { Ergebnis vor Logistikkostn < } \\
\text { Logistikkosten }\end{array}$}} & 6,70 & 4,79 \\
\hline$\infty 1$ & $1.247,50$ & 30,40 & 38,98 & & & & & 5,42 & 4,79 \\
\hline$\infty$ & 657,30 & 16,70 & 37,39 & & & & & 5,20 & 4,79 \\
\hline$\infty$ & 37,50 & $1, \infty$ & 35,63 & & & & & 4,95 & 4,79 \\
\hline $\mathrm{HOQ}$ & 271,30 & 7,50 & 34,36 & & & & & \multirow{9}{*}{\multicolumn{2}{|c|}{$\begin{array}{c}\text { Ergebnis vor Logistikkosten < } \\
\text { Logistikkosten }\end{array}$}} \\
\hline A11 & 165,60 & 5,80 & 27,12 & & & & & & \\
\hline$A 06$ & 108,70 & 4,30 & 24,02 & & & & & & \\
\hline D11 & 74,00 & 3,30 & 21,30 & & & & & & \\
\hline SO1 & 624,50 & 28,70 & 20,67 & & & & & & \\
\hline DO2 & 87,30 & 4,40 & 18,85 & & & & & & \\
\hline MD4 & 112,20 & 5,70 & 18,70 & & & & & & \\
\hline $\mathrm{HD3}$ & 311,00 & 16,80 & 17,59 & & & & & & \\
\hline A01 & 81,70 & 5,60 & 13,86 & & & & & & \\
\hline Ziel-segment & $21.084,60$ & 343,60 & 61,36 & \multicolumn{2}{|c|}{$16.052,60$} & \multicolumn{2}{|c|}{$16.052,60$} & \multicolumn{2}{|c|}{$19.248,30$} \\
\hline GKV-Markt & $36 \pi 73,80$ & 782,60 & 46,99 & & & & & & \\
\hline in $\%$ & $57 \%$ & $44 \%$ & & \multicolumn{2}{|c|}{$44 \%$} & \multicolumn{2}{|c|}{$44 \%$} & \multicolumn{2}{|c|}{$52 \%$} \\
\hline \multicolumn{4}{|c|}{ durchschnittlicher Verordinungswert in DM } & & 85,07 & & 85,07 & & 73,61 \\
\hline $\begin{array}{l}\text { durchschnittlic } \\
\text { (abregl. 5\% Kas }\end{array}$ & $\begin{array}{l}\text { icher Verordn } \\
\text { sserrabatt) }\end{array}$ & $\operatorname{tin} D M$ & & & 80,82 & & 80,82 & & $\Leftrightarrow, 93$. \\
\hline
\end{tabular}

Quelle: INIFES, eigene Berechnung. 
Anhang 1 - Tabelle 8: $\quad$ Ausprägungen der Marktpotentiale für Zielsegmentvariante B (vollständige Darstellung), (mit Durchschnittsrendite)

\begin{tabular}{|c|c|c|c|c|c|c|c|c|c|c|c|}
\hline \multicolumn{6}{|c|}{ Arzneimittelverbrauch 1998} & \multicolumn{2}{|c|}{ VAI } & \multicolumn{2}{|c|}{ VAll } & \multicolumn{2}{|c|}{ VAIII } \\
\hline Indikation nach Roter Liste & $\begin{array}{l}\text { Umsatz gesamt } \\
\text { in Mio. DM }\end{array}$ & $\begin{array}{l}\text { Umsatz N3 in } \\
\text { Mio DM }\end{array}$ & $\begin{array}{l}\text { Anteil des N3- } \\
\text { Umsatzes am } \\
\text { Gesamtumsatz }\end{array}$ & $\begin{array}{l}\mathrm{N3}-\mathrm{VO} \text { in } \\
\mathrm{Mio}\end{array}$ & $\begin{array}{l}\text { DMNN3-VO } \\
\text { (-5\% Rabatt) }\end{array}$ & $\begin{array}{c}\text { Ergebnis vor } \\
\text { Logistik- } \\
\text { kosten }\end{array}$ & $\begin{array}{l}\text { Logistik- } \\
\text { kosten }\end{array}$ & $\begin{array}{c}\text { Ergebnis vor } \\
\text { Logistik- } \\
\text { kosten }\end{array}$ & $\begin{array}{l}\text { Logistik- } \\
\text { kosten }\end{array}$ & $\begin{array}{c}\text { Ergebnis vor } \\
\text { Logistik- } \\
\text { kosten }\end{array}$ & $\begin{array}{l}\text { Logistik- } \\
\text { kosten }\end{array}$ \\
\hline $\begin{array}{l}\text { Hypophysen-, } \\
\text { Hypothalamushormone }\end{array}$ & kein Versand & & & & & & & & & & \\
\hline Anthelminthika & kein Versand & & & & & & & & & & \\
\hline Immunmodulatoren & kein Versand & & & & & & & & & & \\
\hline Zytostatika usw. & kein Versand & & & & & & & & & & \\
\hline Diătetika/Ernährungstherapeutika & 5,56 & 1,13 & $20,28 \%$ & 0,00 & 308,61 & 21,29 & 3,48 & 30,55 & 4,79 & 42,90 & 4,79 \\
\hline Antibiotika/Antiinfektiva & kein Versand & & & & & & & & & & \\
\hline Lipidsenker & $1.495,58$ & $1.085,14$ & $72,56 \%$ & 5,43 & 189,92 & 13,10 & 3,48 & 18,80 & 4,79 & 26,40 & 4,79 \\
\hline Entwöhnungsmittel & kein Versand & & & & & & & & & & \\
\hline Abmagerungsmittel/Appetitzügler & 0,99 & 0,27 & $27,45 \%$ & 0,00 & 138,49 & 9,56 & 3,48 & 13,71 & 4,79 & 19,25 & 4,79 \\
\hline Tuberkulosemittel & kein Versand & & & & & & & & & & \\
\hline Antihypertonika & $2.406,53$ & $1.933,79$ & $80,36 \%$ & 14,94 & 122,99 & 8,49 & 3,48 & 12,18 & 4,79 & 17,10 & 4,79 \\
\hline Enzyminhibitoren usw. & 16,53 & 5,94 & $35,92 \%$ & 0,05 & 117,06 & 8,08 & 3,48 & 11,59 & 4,79 & 16,27 & 4,79 \\
\hline Antiepileptika & 387,61 & 180,18 & $46,48 \%$ & 1,50 & 114,37 & 7,89 & 3,48 & 11,32 & 4,79 & 15,90 & 4,79 \\
\hline Osteoporosemittel/Ca-Stoffw.reg. & 349,59 & 194,46 & $55,63 \%$ & 1,69 & 109,06 & 7,53 & 3,48 & 10,80 & 4,79 & 15,16 & 4,79 \\
\hline Magen-Darm-Mittel & $2.324,78$ & 973,45 & $41,87 \%$ & 8,49 & 108,91 & 7,51 & 3,48 & 10,78 & 4,79 & 15,14 & 4,79 \\
\hline Urologika & 738,38 & 391,14 & $52,97 \%$ & 3,58 & 103,69 & 7,15 & 3,48 & 10,27 & 4,79 & 14,41 & 4,79 \\
\hline Parkinsonmittel usw. & 440,06 & 369,06 & $83,87 \%$ & 3,41 & 102,76 & 7,09 & 3,48 & 10,17 & 4,79 & 14,28 & 4,79 \\
\hline Neuropathiepräparate usw. & 331,62 & 226,93 & $68,43 \%$ & 2,33 & 92,45 & 6,38 & 3,48 & 9,15 & 4,79 & 12,85 & 4,79 \\
\hline Antiallergika & kein Versand & & & & & & & & & & \\
\hline Antiarrhythmika & 349,18 & 312,82 & $89,59 \%$ & 3,34 & 88,95 & 6,14 & 3,48 & 8,81 & 4,79 & 12,36 & 4,79 \\
\hline Antiphlogistika & 124,18 & 32,28 & $25,99 \%$ & 0,36 & & & & & & & \\
\hline Hepatika & 96,61 & 75,56 & $78,21 \%$ & 0,92 & 77,72 & 5,36 & 3,48 & 7,69 & 4,79 & 10,80 & 4,79 \\
\hline Antidiabetika & $1.612,54$ & $1.566,71$ & $97,16 \%$ & 19,20 & 77,52 & 5,35 & 3,48 & 7,67 & 4,79 & 10,78 & 4,79 \\
\hline Muskelrelaxantia & kein Versand & & & & & & & & & & \\
\hline Broncholytika/Antiasthmatika & $1.874,64$ & 532,21 & $28,39 \%$ & 7,64 & 66,14 & 4,56 & 3,48 & 6,55 & 4,79 & 9,19 & 4,79 \\
\hline Antidementiva (Nootropika) & 574,62 & 433,59 & $75,46 \%$ & 6,24 & 65,97 & 4,55 & 3,48 & 6,53 & 4,79 & 9,17 & 4,79 \\
\hline
\end{tabular}

Fortsetzung nächste Seite 


\begin{tabular}{|c|c|c|c|c|c|c|c|c|c|c|c|}
\hline \multicolumn{6}{|c|}{ Arzneimittelverbrauch 1998} & \multicolumn{2}{|c|}{ VAI } & \multicolumn{2}{|c|}{ VAII } & \multicolumn{2}{|c|}{ VA III } \\
\hline Wilikation nach Roler Liste & $\begin{array}{l}\text { Um satz gesamt } \\
\text { in Mio, oM }\end{array}$ & $\begin{array}{c}\text { Umsatz N3 in } \\
\text { Mio OM }\end{array}$ & $\begin{array}{l}\text { Anteil des } N_{3} \\
\text { Umsatzes am } \\
\text { Gesamiumsatz } \\
\end{array}$ & $\begin{array}{l}\mathrm{N} 3-\mathrm{vo} \text { in } \\
\mathrm{MiO}\end{array}$ & $\begin{array}{l}\text { DMIN3-vo } \\
\text { (. } 5 \% \text { Rabati) }\end{array}$ & $\begin{array}{c}\text { Ergebnis vor } \\
\text { Logistik- } \\
\text { Kosten }\end{array}$ & $\begin{array}{l}\text { Logistik- } \\
\text { kosten }\end{array}$ & $\begin{array}{l}\text { Ergebnis vor } \\
\text { Logistik. } \\
\text { Kosten }\end{array}$ & $\begin{array}{l}\text { Logistik- } \\
\text { kosten }\end{array}$ & $\begin{array}{c}\text { Ergebnis vor } \\
\text { Logistik- } \\
\text { Kosten }\end{array}$ & $\begin{array}{l}\text { Logistik- } \\
\text { kosten }\end{array}$ \\
\hline Cholinergika & kein Versand & & & & & & & & & & \\
\hline Cholagoga und Gallenwegstherap. & 71,15 & 61,00 & $85,74 \%$ & 0,90 & 64,74 & 4,47 & 3,48 & 6,41 & 4,79 & 9,00 & 4,79 \\
\hline Venentherapeutika & 276,60 & 183,50 & $66,34 \%$ & 2,69 & 64,70 & 4,46 & 3,48 & 6,41 & 4,79 & 8,99 & 4,79 \\
\hline Antimykotika & kein Versand & & & & & & & & & & \\
\hline Durchblutungsfördernde Mittel & 327,81 & 261,54 & $79,78 \%$ & 3,92 & 63,42 & 4,38 & 3,48 & 6,28 & 4,79 & 8,82 & 4,79 \\
\hline Dermatika & 885,98 & 151,55 & $17,11 \%$ & 2,33 & 61,87 & 4,27 & 3,48 & 6,13 & 4,79 & 8,60 & 4,79 \\
\hline Beta-.Ca-BI.,Angiotensin-Hemmst. & $2.833,99$ & $2.431,35$ & $85,79 \%$ & 37,82 & 61,07 & 4,21 & 3,48 & 6,05 & 4,79 & 8,49 & 4,79 \\
\hline Wundbehandlungsmittel & kein Versand & & & & & & & & & & \\
\hline Spasmolytika & kein Versand & & & & & & & & & & \\
\hline Sexualhormone & $1.144,62$ & 859,11 & $75,06 \%$ & 15,37 & 53,12 & 3,66 & 3,48 & 5,26 & 4,79 & 7,38 & 4,79 \\
\hline Antihypotonika & 137,56 & 74,91 & $54,46 \%$ & 1,35 & 52,84 & 3,65 & 3,48 & 5,23 & 4,79 & 7,35 & 4,79 \\
\hline Koronarmittel & 837,09 & 706,96 & $84,45 \%$ & 12,95 & 51,87 & 3,58 & 3,48 & 5,14 & 4,79 & 7,21 & 4,79 \\
\hline Corticoide (Interna) & kein Versand & & & & & & & & & & \\
\hline Antiemetika/Antivertiginosa & kein Versand & & & & & & & & & & \\
\hline Migrănemittel & 162,30 & 8,78 & $5,41 \%$ & 0,17 & 48,16 & & & & & 6,69 & 4,79 \\
\hline Hămorrhoidenmittel & 67,21 & 9,68 & $14,41 \%$ & 0,19 & 48,16 & & & & & 6,69 & 4,79 \\
\hline Acidosetherapeutika & 7,37 & 6,44 & $87,46 \%$ & 0,13 & 48,14 & & & & & 6,69 & 4,79 \\
\hline Antihämorrhagika & 53,78 & 6,99 & $13,00 \%$ & 0,14 & 47,82 & & & & & 6,65 & 4,79 \\
\hline Psychopharmaka & $1.782,76$ & $1.020,11$ & $57,22 \%$ & 20,29 & 47,77 & & & & & 6,64 & 4,79 \\
\hline Infusionslösungen usw. & kein Versand & & & & & & & & & & \\
\hline Thrombozytenaggregationshemmer & 185,74 & 157,62 & $84,86 \%$ & 3,31 & 45,25 & & & & & 6,29 & 4,79 \\
\hline Diuretika & 628,74 & 424,89 & $67,58 \%$ & 9,02 & 44,74 & Vorläufiges E & rgebnis < & Vorlăufiges & rgebnis < & 6,22 & 4,79 \\
\hline Diagnostika usw. & kein Versand & & & & & Logistikk & & & & & \\
\hline Analgetika/Antirheumatika & $1.822,67$ & 626,95 & $34,40 \%$ & 14,85 & 40,12 & & & & & 5,58 & 4,79 \\
\hline Balneotherapeutika usw. & 42,17 & 10,24 & $24,29 \%$ & 0,24 & 39,85 & & & & & 5,54 & 4,79 \\
\hline Gynäkologika & 213,11 & 67,88 & $31,85 \%$ & 1,63 & 39,62 & & & & & 5,51 & 4,79 \\
\hline Antianämika & 428,11 & 59,98 & $14,01 \%$ & 1,45 & 39,35 & & & & & 5,47 & 4,79 \\
\hline Antikoagulantia & 319,66 & 53,59 & $16,76 \%$ & 1,37 & 37,29 & & & & & 5,18 & 4,79 \\
\hline Roborantia/Tonika & 0,17 & 0,09 & $57,04 \%$ & 0,00 & 37,21 & & & & & 5,17 & 4,79 \\
\hline Laxantia & 92,65 & 44,64 & $48,19 \%$ & 1,17 & 36,10 & & & & & 5,02 & 4,79 \\
\hline Mineralstoffpräparate & 390,28 & 278,84 & $71,45 \%$ & 7,60 & 34,85 & & & & & 4,84 & 4,79 \\
\hline Antitussiva/Expektorantia & kein Versand & & & & & & & & & & \\
\hline
\end{tabular}

Fortsetzung nächste Seite 
Anhang I-Tabelle 8: Ausprägungen der Marktpotentiale für Zielsegmentvariante B (vollständige Darstellung), (mit Durchschnittsrendite)

\begin{tabular}{|c|c|c|c|c|c|c|c|c|c|c|c|}
\hline \multicolumn{6}{|c|}{ Arzneimittelverbrauch 1998} & \multicolumn{2}{|c|}{ VAI } & \multicolumn{2}{|c|}{ VAII } & \multicolumn{2}{|c|}{ VAIII } \\
\hline Indikation nach Roter Liste & $\begin{array}{l}\text { Umsatz gesamt } \\
\text { in Mio. DM }\end{array}$ & $\begin{array}{c}\text { Umsatz N3 in } \\
\text { Mio DM }\end{array}$ & $\begin{array}{l}\text { Anteil des N3- } \\
\text { Umsatzes am } \\
\text { Gesamtumsatz }\end{array}$ & $\begin{array}{l}\text { N3-VO in } \\
\text { Mio }\end{array}$ & $\begin{array}{l}\text { DMN3-VO } \\
\text { (-5\% Rabatt) }\end{array}$ & $\begin{array}{c}\text { Ergebnis vor } \\
\text { Logistik- } \\
\text { kosten }\end{array}$ & $\begin{array}{l}\text { Logistik- } \\
\text { Kosten }\end{array}$ & $\begin{array}{l}\text { Ergebnis vor } \\
\text { Logistik- } \\
\text { kosten }\end{array}$ & $\begin{array}{l}\text { Logistik- } \\
\text { kosten }\end{array}$ & $\begin{array}{c}\text { Ergebnis vor } \\
\text { Logistik- } \\
\text { kosten }\end{array}$ & $\begin{array}{l}\text { Logistik- } \\
\text { kosten }\end{array}$ \\
\hline Nicht in Roter Liste & kein Versand & & & & & \multirow{14}{*}{\multicolumn{2}{|c|}{$\begin{array}{l}\text { Ergebnis vor } \\
\text { Logistikkosten< } \\
\text { Logistikkosten }\end{array}$}} & \multirow{14}{*}{\multicolumn{2}{|c|}{$\begin{array}{l}\text { Ergebnis vor } \\
\text { Logistikkosten < } \\
\text { Logistikkosten }\end{array}$}} & \multirow{14}{*}{\multicolumn{2}{|c|}{$\begin{array}{l}\text { Ergebnis vor } \\
\text { Logistikkosten< } \\
\text { Logistikkosten }\end{array}$}} \\
\hline Ophthalmika & 555,59 & 168,91 & $30,40 \%$ & 5,77 & 27,81 & & & & & & \\
\hline \begin{tabular}{|l|} 
Vitamine \\
\end{tabular} & 178,81 & 126,34 & $70,66 \%$ & 4,49 & 26,75 & & & & & & \\
\hline Desinfizientia/Antiseptika & kein Versand & & & & & & & & & & \\
\hline Arteriosklerosemittel & 0,30 & 0,08 & $25,37 \%$ & 0,00 & 24,78 & & & & & & \\
\hline Hypnotika/Sedativa & 287,64 & 9,18 & $3,19 \%$ & 0,35 & 24,57 & & & & & & \\
\hline Rhinologika/Sinusitismittel & kein Versand & & & & & & & & & & \\
\hline Lokalanästhetika/Neuraltherap. & kein Versand & & & & & & & & & & \\
\hline Gichtmittel & 110,63 & 91,55 & $82,76 \%$ & 4,12 & 21,10 & & & & & & \\
\hline Kardiaka & 217,66 & 187,71 & $86,24 \%$ & 9,18 & 19,42 & & & & & & \\
\hline Mund- und Rachentherapeutika & kein Versand & & & & & & & & & & \\
\hline Schilddrüsentherapeutika & 304,09 & 277,40 & $91,22 \%$ & 14,28 & 18,45 & & & & & & \\
\hline Umstimmungsmittel & kein Versand & & & & & & & & & & \\
\hline Karies- und Parodontosemittel & 23,08 & 16,02 & $69,43 \%$ & 1,27 & 11,94 & & & & & & \\
\hline Zielsegment (A10 N2 als N3) & & $16.698,5$ & & 257,49 & 64,85 & \multicolumn{2}{|c|}{$13.044,57$} & \multicolumn{2}{|c|}{$13.044,47$} & \multicolumn{2}{|c|}{$15.821,32$} \\
\hline Gesamt GKV 1998 & $35.611,3$ & & & 801,10 & 44,45 & \multicolumn{2}{|l|}{3} & 2 & & & \\
\hline in \% vom GKV-Umsatz & & $47 \%$ & & $32 \%$ & $146 \%$ & \multicolumn{2}{|c|}{$37 \%$} & \multicolumn{2}{|c|}{$37 \%$} & \multicolumn{2}{|c|}{$44 \%$} \\
\hline durchschnittlicher Verordnungs & $t$ in DM & & & & & 83,3 & & 83, & & \multirow{2}{*}{\multicolumn{2}{|c|}{$\begin{array}{l}72,57 \\
68,49\end{array}$}} \\
\hline durchschnittlicher Verordnungs & t in DM (abzgl. 5\% & Kassenrabatt) & & & & 79,2 & & 79,2 & & & \\
\hline
\end{tabular}


Das Vorgehen folgt bei dieser Modellrechnung dem folgenden Schema:

1. Der indikationsspezifische durchschnittliche Verordnungswert wird um den aktuellen Kassenrabatt von $5 \%^{1}$ plus einem einheitlichen zusätzlichen Kassenrabatt von $5 \%, 7 \%$ und $10 \%$ (alternativ) vermindert. Es ergeben sich entsprechend Kasenrabatte von insgesamt 10\%, 12\% bzw. 15\% auf den Verordnungswert je Indikationsgruppe.

2. In einem weiteren Schritt werden die in 2.4. hergeleiteten betriebswirtschaftlichen Ergebnisse vor Logistikkosten in Höhe von 10,4\% (VAI), 13,4\% (VA II) bzw. $17,4 \%$ (VA III) aufgrund der Berücksichtigung eines einheitlichen zusätzlichen Kassenrabatts um die unter 1. genannten Beträge gekürtt. Es ergeben sich - in Abhängigkeit der Höhe des zusätzlichen einheitlichen Kassenrabatts - folgende Ergebnisse vor Logistikkosten:

Anhang I-Abbildung 1: Auswirkungen eines einheitlichen Kassenrabatts auf das Ergebnis vor Logistikkosten

\begin{tabular}{|c|c|c|c|}
\hline \multirow{2}{*}{} & \multicolumn{3}{|c|}{ Ergebnis vor Logistikkosten } \\
\cline { 2 - 4 } & VAI & VA II & VA III \\
\hline Ergebnis vor Logistikkosten ( Kapitel 2.4) & $10,4 \%$ & $13,4 \%$ & $17,4 \%$ \\
\hline Bei Berücksichtigung eines einheitlichen Kassenrabatts: & Minus a) $5 \%$ bzw. b) $7 \%$ bzw. c) $10 \%$ \\
\hline a) $5 \%$ zusätzlicher Kassenrabatt & $5,4 \%$ & $8,4 \%$ & $12,4 \%$ \\
\hline b) $7 \%$ zusätzlicher Kassenrabatt & $3,4 \%$ & $6,4 \%$ & $10,4 \%$ \\
\hline c) $10 \%$ zusätzlicher Kassenrabatt & $0,4 \%$ & $3,4 \%$ & $7,4 \%$ \\
\hline
\end{tabular}

Quelle: INIFES, eigene Berechnung.

4) Daraus sind jeweils die Logistikkosten zu decken. Diese sind unabhängig von der Höhe des Verordnungswertes und entsprechen mindestens 3,48 DM je Verordnung für VA I bzw. 4,79 DM für VA II und III.

Alle Verordnungen des Zielsegments, die aus dem Ergebnis vor Logistikkosten die Logistikstückkosten decken, also ein positives Ergebnis aufweisen, werden potentiell über den Versandweg abgegeben. Aufgrund des positiven Ergebnisses sind Kosteneinsparungen für die GKV zu erwarten. Diese Verordnungen bilden das Marktpotential. 
5) Das Einsparpotential ergibt sich, indem der zusätzliche Kassenrabatt von 5\%, 7\% bzw. $10 \%$ auf das Marktpotential bezogen wird.

Für die Zielsegmentvariante A (Indikator Packungsgröße ab 50 DDD) ergeben sich in Abhängigkeit der Umsatzgröße folgende Markt- und Einsparpotentiale in Abhängigkeit des zusätzlichen einheitlichen Kassenrabatts.

\section{$\rightarrow$ Bei einem zusätzlichen einheitlichen Kassenrabatt von 5\%:}

Anhang I - Tabelle 9: Markt- und Einsparpotentiale für Zielsegmentvariante A (bei einem zusätzlichen Kassenrabatt von 5\%)

\begin{tabular}{|c|c|c|c|c|c|c|c|c|c|}
\hline \multirow{2}{*}{\multicolumn{4}{|c|}{ Arzneimittelverbrauch 1999}} & \multicolumn{2}{|c|}{ VAI } & \multicolumn{2}{|c|}{ VAII } & \multicolumn{2}{|c|}{ VAIII } \\
\hline & & & & \multirow{2}{*}{\begin{tabular}{|c|}
$\begin{array}{c}\text { Ergebnis vor } \\
\text { Logistikkosten }\end{array}$ \\
$5,40 \%$ \\
\end{tabular}} & \multirow{2}{*}{$\begin{array}{l}\text { Logistik- } \\
\text { kosten } \\
3,48 \mathrm{DM}\end{array}$} & \multirow{2}{*}{\begin{tabular}{|c|}
$\begin{array}{c}\text { Ergebnis vor } \\
\text { Logistikkosten }\end{array}$ \\
$8,40 \%$ \\
\end{tabular}} & \multirow{2}{*}{$\begin{array}{l}\text { Logistik- } \\
\text { kosten } \\
4,79 \mathrm{DM}\end{array}$} & \multirow{2}{*}{\begin{tabular}{|c|}
$\begin{array}{c}\text { Ergebnis vor } \\
\text { Logistikkosten }\end{array}$ \\
$12,40 \%$ \\
\end{tabular}} & \multirow{2}{*}{$\begin{array}{c}\begin{array}{c}\text { Logistik- } \\
\text { kosten }\end{array} \\
4,79 \mathrm{DM}\end{array}$} \\
\hline ATC & $\begin{array}{c}\text { Umsatz Mio } \\
\text { DM }\end{array}$ & Voln Mio & $\begin{array}{l}\text { DM/VO } \\
(-10 \%)\end{array}$ & & & & & & \\
\hline L02 & 604,50 & 1,10 & 494,59 & 26,71 & 3,48 & 41,55 & 4,79 & 61,33 & 4,79 \\
\hline MD5 & 285,70 & 0,80 & 321,41 & 17,36 & 3,48 & 27,00 & 4,79 & 39,86 & 4,79 \\
\hline $\mathrm{C10}$ & $1.699,50$ & 9,40 & 162,72 & 8,79 & 3,48 & 13,67 & 4,79 & 20,18 & 4,79 \\
\hline D05 & 86,40 & 0,80 & 97,20 & 5,25 & 3,48 & 8,16 & 4,79 & 12,05 & 4,79 \\
\hline$\infty 99$ & $2.490,80$ & 25,80 & 86,89 & 4,69 & 3,48 & 7,30 & 4,79 & 10,77 & 4,79 \\
\hline $\mathrm{B} 03$ & 477,70 & 5,20 & 82,68 & 4,46 & 3,48 & 6,95 & 4,79 & 10,25 & 4,79 \\
\hline$\infty 2$ & 453,10 & 5,00 & 81,56 & 4,40 & 3,48 & 6,85 & 4,79 & 10,11 & 4,79 \\
\hline $\mathrm{A} 10$ & $1.772,80$ & 20,60 & 77,45 & 4,18 & 3,48 & 6,51 & 4,79 & 9,60 & 4,79 \\
\hline $\mathrm{B} 01$ & 671,30 & 9,60 & 62,93 & \multirow{10}{*}{\multicolumn{2}{|c|}{$\begin{array}{c}\text { Ergebnis vor Logistikkosten } \\
<\text { Logistikkosten }\end{array}$}} & 5,29 & 4,79 & 7,80 & 4,79 \\
\hline$\infty 8$ & $1.282,00$ & 19,70 & 58,57 & & & 4,92 & 4,79 & 7,26 & 4,79 \\
\hline$\infty 7$ & $1.399,50$ & 21,70 & 58,04 & & & 4,88 & 4,79 & 7,20 & 4,79 \\
\hline $\mathrm{GO3}$ & $1.253,40$ & 24,70 & 45,67 & & & \multirow{7}{*}{\multicolumn{2}{|c|}{$\begin{array}{l}\text { Ergebnis vor } \\
\text { Logistikkosten < } \\
\text { Logistikkosten }\end{array}$}} & 5,66 & 4,79 \\
\hline$\infty 1$ & $1.247,50$ & 30,40 & 36,93 & & & & & \multirow{6}{*}{\multicolumn{2}{|c|}{$\begin{array}{l}\text { Ergebnis vor } \\
\text { Logistikkosten < } \\
\text { Logistikkosten }\end{array}$}} \\
\hline$\infty 03$ & 657,30 & 16,70 & 35,42 & & & & & & \\
\hline A11 & 165,60 & 5,80 & 25,70 & & & & & & \\
\hline MD4 & 112,20 & 5,70 & 17,72 & & & & & & \\
\hline $\mathrm{HD3}$ & 311,00 & 16,80 & 16,66 & & & & & & \\
\hline $\mathrm{A} 01$ & 81,70 & 5,60 & 13,13 & & & & & & \\
\hline \begin{tabular}{l|} 
Zlel- \\
segment \\
\end{tabular} & $15.052,00$ & 225,40 & 66,78 & \multicolumn{2}{|c|}{$7.870,50$} & \multicolumn{2}{|c|}{$11.223,30$} & \multicolumn{2}{|c|}{$12.476,70$} \\
\hline $\begin{array}{l}\text { GWV- } \\
\text { Markt } \\
\end{array}$ & $36.773,80$ & 782,60 & 46,99 & & & & & & \\
\hline in $\%$ & $41 \%$ & $29 \%$ & 85 & $21 \%$ & & $31 \%$ & & $34 \%$ & \\
\hline \multicolumn{4}{|c|}{ durchschnittlicher Verordnungswert in DM } & \multicolumn{2}{|r|}{114,56} & \multicolumn{2}{|r|}{93,76} & \multirow{2}{*}{\multicolumn{2}{|c|}{77,76}} \\
\hline \multicolumn{4}{|c|}{$\begin{array}{l}\text { durchschnittlicher Verordnungswert in DM } \\
\text { (abzgl. 10\% Kassenrabatt) }\end{array}$} & \multicolumn{2}{|r|}{103,11} & \multicolumn{2}{|r|}{84,39} & & \\
\hline \multicolumn{4}{|c|}{ Ergebnis vor Logistikosten (nach 24.) } & \multicolumn{2}{|c|}{$10,4 \%$} & \multicolumn{2}{|c|}{$13,4 \%$} & \multicolumn{2}{|c|}{$17,4 \%$} \\
\hline \multicolumn{4}{|c|}{ zusätzlicher Kassenrabatt } & $5,0 \%$ & & $5,0 \%$ & & $5,0 \%$ & \\
\hline $\begin{array}{l}\text { Ergebnis vo } \\
\text { Kassenraba }\end{array}$ & $\begin{array}{l}\text { Logistikkosten } \\
\text { tt von } 5 \%)\end{array}$ & abzgl. zusätz & lichem & $5,4 \%$ & & $8,4 \%$ & & $12,4 \%$ & \\
\hline Logistikkos & en in \% des vo- & Wertes & & $3,4 \%$ & & $5,7 \%$ & & $6,2 \%$ & \\
\hline korrigierte & Senerbestever ac & fLogistikkost & & $0,5 \%$ & & $0,9 \%$ & & $1,0 \%$ & \\
\hline $\begin{array}{l}\text { Ensparpot } \\
\text { Ausschöpf }\end{array}$ & $\begin{array}{l}\text { ential GKV in Mi } \\
\text { ung des Markty }\end{array}$ & $\begin{array}{l}\text { O. DM bel vol } \\
\text { otentlals }\end{array}$ & Ist. & & 394 & & 561 & & 624 \\
\hline $\begin{array}{l}\text { Ensparpot } \\
\text { von } 15 \%\end{array}$ & ential GKV In MI & DM bel Mar & ktantell & & 276 & & 276 & & 276 \\
\hline
\end{tabular}

Quelle: INIFES, eigene Berechnung. 


\section{$\rightarrow$ Bei einem zusätzlichen einheitlichen Kassenrabatt von 7\%:}

Anhang I - Tabelle 10: Markt- und Einsparpotentiale für Zielsegmentvariante A (bei einem zusätzlichen Kassenrabatt von 7\%)

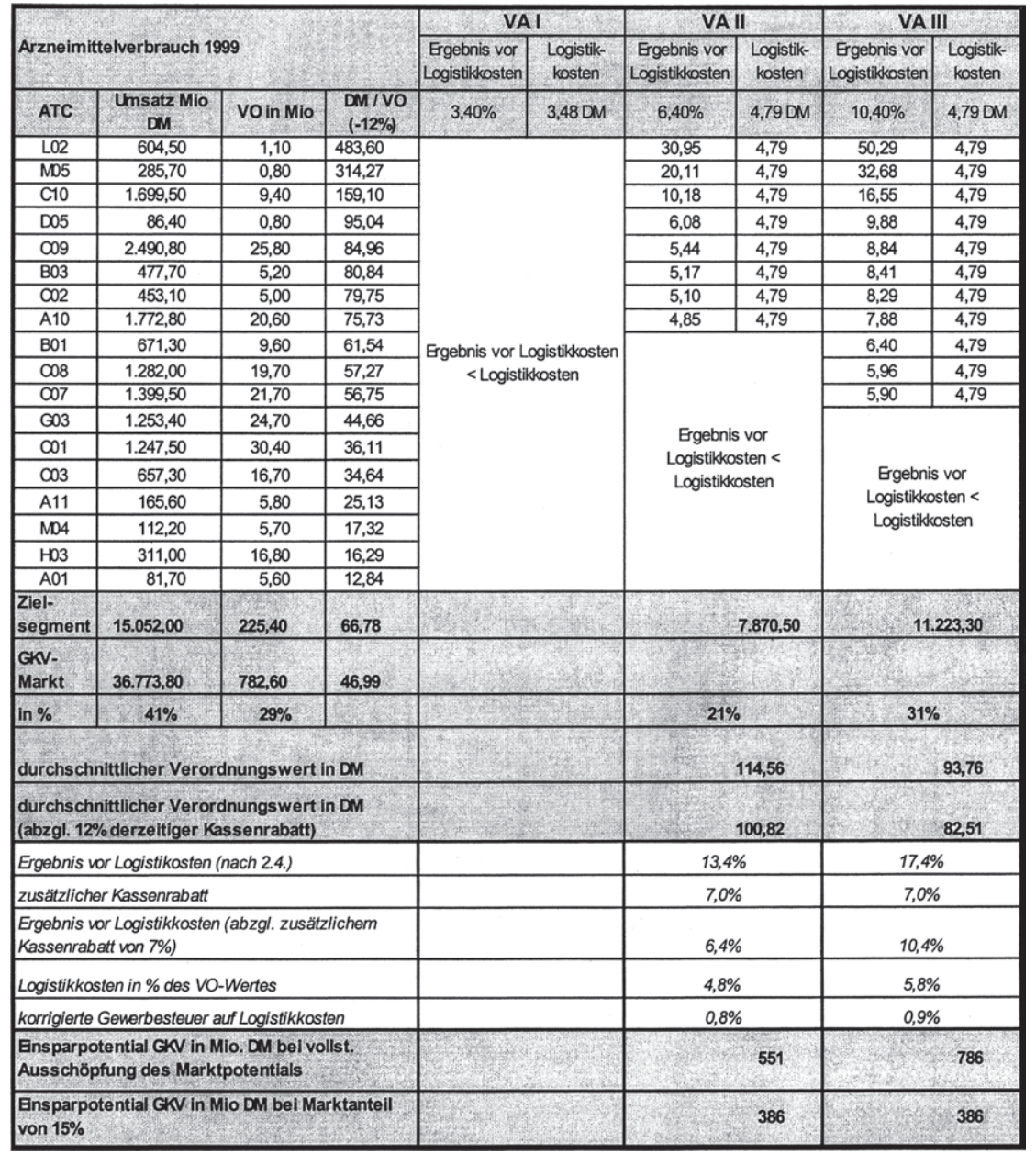

Quelle: INIFES, eigene Berechnung.

Anmerkung: Im Modell der Versandapotheke VA I könnten aus modelltheoretischer Sicht die Indikationen L02, M05 und C10 alle Kosten inklusive eines zusätzlichen Rabattes aus der Betriebsspanne decken, im Sinne einer allgemeingültigen Betrachtung ist dieses Marktpotential jedoch zu vernachlässigen. 
$\rightarrow$ Bei einem zusätzlichen einheitlichen Kassenrabatt von 10\%:

Anhang 1 - Tabelle 11: Markt- und Einsparpotentiale für Zielsegmentvariante A (bei einem zusätzlichen Kassenrabatt von 10\%)

\begin{tabular}{|c|c|c|c|c|c|c|c|c|c|}
\hline \multirow{2}{*}{\multicolumn{4}{|c|}{ Arzneimittelverbrauch 1999}} & \multicolumn{2}{|c|}{ VAI } & \multicolumn{2}{|c|}{ VAII } & \multicolumn{2}{|c|}{ VAIII } \\
\hline & & & & \multirow{2}{*}{\begin{tabular}{|c|}
$\begin{array}{c}\text { Ergebnis vor } \\
\text { Logistikkosten }\end{array}$ \\
$0,40 \%$ \\
\end{tabular}} & \multirow{2}{*}{$\begin{array}{c}\begin{array}{c}\text { Logistik- } \\
\text { kosten }\end{array} \\
3,48 \mathrm{DM}\end{array}$} & \multirow{2}{*}{\begin{tabular}{|c|}
$\begin{array}{c}\text { Ergebnis vor } \\
\text { Logistikkosten }\end{array}$ \\
$3,40 \%$ \\
\end{tabular}} & \multirow{2}{*}{$\begin{array}{l}\text { Logistik- } \\
\text { kosten } \\
4,79 \mathrm{DM}\end{array}$} & \multirow{2}{*}{\begin{tabular}{|c|}
$\begin{array}{c}\text { Ergebnis vor } \\
\text { Logistikkosten }\end{array}$ \\
$7.40 \%$ \\
\end{tabular}} & \multirow{2}{*}{$\frac{\begin{array}{c}\text { Logistik- } \\
\text { kosten }\end{array}}{4,79 \mathrm{DM}}$} \\
\hline ATC & $\begin{array}{c}\text { Umsatz Mio } \\
\text { DM }\end{array}$ & Vo in Mio & $\begin{array}{l}\text { DM/VO } \\
(-15 \%)\end{array}$ & & & & & & \\
\hline $\mathrm{LO2}$ & 604,50 & 1,10 & 467,11 & \multirow{18}{*}{\multicolumn{2}{|c|}{$\begin{array}{l}\text { Ergebnis vor Logistikkosten } \\
\quad<\text { Logistikkosten }\end{array}$}} & \multirow{18}{*}{\multicolumn{2}{|c|}{$\begin{array}{l}\text { Ergebnis vor } \\
\text { Logistikkosten < } \\
\text { Logistikkosten }\end{array}$}} & 34,57 & 4,79 \\
\hline MD5 & 285,70 & 0,80 & 303,56 & & & & & 22,46 & 4,79 \\
\hline $\mathrm{C} 10$ & $1.699,50$ & 9,40 & 153,68 & & & & & 11,37 & 4,79 \\
\hline D05 & 86,40 & 0,80 & 91,80 & & & & & 6,79 & 4,79 \\
\hline$\infty 09$ & $2.490,80$ & 25,80 & 82,06 & & & & & 6,07 & 4,79 \\
\hline $\mathrm{BO3}$ & 477,70 & 5,20 & 78,09 & & & & & 5,78 & 4,79 \\
\hline$\infty 2$ & 453,10 & 5,00 & 77,03 & & & & & 5,70 & 4,79 \\
\hline A10 & $1.772,80$ & 20,60 & 73,15 & & & & & 5,41 & 4,79 \\
\hline $\mathrm{B} 01$ & 671,30 & 9,60 & 59,44 & & & & & \multirow{10}{*}{\multicolumn{2}{|c|}{$\begin{array}{l}\text { Ergebnis vor } \\
\text { Logistikkosten < } \\
\text { Logistikkosten }\end{array}$}} \\
\hline$\infty 88$ & $1.282,00$ & 19,70 & 55,31 & & & & & & \\
\hline$\infty 7$ & $1.399,50$ & 21,70 & 54,82 & & & & & & \\
\hline $\mathrm{GO3}$ & $1.253,40$ & 24,70 & 43,13 & & & & & & \\
\hline$\infty 1$ & $1.247,50$ & 30,40 & 34,88 & & & & & & \\
\hline$\infty 23$ & 657,30 & 16,70 & 33,46 & & & & & & \\
\hline A11 & 165,60 & 5,80 & 24,27 & & & & & & \\
\hline MD4 & 112,20 & 5,70 & 16,73 & & & & & & \\
\hline $\mathrm{H} 03$ & 311,00 & 16,80 & 15,74 & & & & & & \\
\hline $\mathrm{A} 01$ & 81,70 & 5,60 & 12,40 & & & & & & \\
\hline \begin{tabular}{l|} 
Zlel- \\
segment
\end{tabular} & $15.052,00$ & 225,40 & 66,78 & & & & & 7.8 & 870,50 \\
\hline $\begin{array}{l}\text { GKV. } \\
\text { Markt } \\
\end{array}$ & $36.773,80$ & 782,60 & 46,99 & & & & & & \\
\hline in $\%$ & $41 \%$ & $29 \%$ & 695 & & & & & $21 \%$ & 6 \\
\hline \multicolumn{4}{|c|}{ durchschnittlicher Verordnungswert in DM } & & & & & \multicolumn{2}{|r|}{114,56} \\
\hline \multicolumn{4}{|c|}{$\begin{array}{l}\text { durchschnittllcher Verordnungswert in DM } \\
\text { (abzgl. 15\%Kassenrabatt) }\end{array}$} & & & & & \multicolumn{2}{|r|}{97,38} \\
\hline \multicolumn{4}{|c|}{ Ergebnis vor Logistikosten (nach 2.4.) } & & & & & \multicolumn{2}{|c|}{$17,4 \%$} \\
\hline \multicolumn{4}{|c|}{ zusätzlicher Kassenrabatt } & & & & & \multicolumn{2}{|c|}{$10,0 \%$} \\
\hline \multicolumn{4}{|c|}{$\begin{array}{l}\text { Ergebnis vor Logistikkosten (abzgl. zusätzlichem } \\
\text { Kassenrabatt von 10\%) }\end{array}$} & & & & & \multicolumn{2}{|c|}{$7,4 \%$} \\
\hline \multicolumn{4}{|c|}{ Logistikkosten in \% des vO-Wertes } & & & & & \multicolumn{2}{|c|}{$4,9 \%$} \\
\hline \multicolumn{4}{|c|}{ korrigierte Generbestever auf Logistikkosten } & & & & & \multicolumn{2}{|c|}{$0,8 \%$} \\
\hline \multicolumn{4}{|c|}{$\begin{array}{l}\text { Ensparpotential GKV in Mio. DM bel vollst. } \\
\text { Ausschöpfung des Marktpotentlals }\end{array}$} & & & & & & 787 \\
\hline $\begin{array}{l}\text { Ensparpo } \\
\text { von } 15 \%\end{array}$ & ential GKV in Mi & o DM bel Mar & ktantell & & & & & & 552 \\
\hline
\end{tabular}

Quelle: INIFES, eigene Berechnung.

Anmerkung: Im Modell der Versandapotheke VA II könnten aus modelltheoretischer Sicht die Indikationen L02, M05 und C10 alle Kosten inklusive eines zusätzlichen Rabattes aus der Betriebsspanne decken, im Sinne einer allgemeingültigen Betrachtung ist dieses Marktpotential jedoch zu vernachlässigen. 
Für die Zielsegmentvariante B (Indikator Packungsgröße N3) ergeben sich in Abhängigkeit der Umsatzgröße folgende Markt- und Einsparpotentiale in Abhängigkeit des zusätzlichen einheitlichen Kassenrabatts.

\section{$\rightarrow$ Bei einem zusätzlichen einheitlichen Kassenrabatt von 5\%:}

Anhang I - Tabelle 12: Markt- und Einsparpotentiale für Zielsegmentvariante B (bei einem zusätzlichen Kassenrabatt von 5\%)

\begin{tabular}{|c|c|c|c|c|c|c|c|c|c|c|}
\hline \multicolumn{5}{|c|}{ A rzne im itte Iverbrauch 1998} & \multicolumn{2}{|c|}{ VA 1} & \multicolumn{2}{|c|}{ VA II } & \multicolumn{2}{|c|}{ VA Hil } \\
\hline $\begin{array}{l}\text { Indikation } \\
\text { (Rote Liste) } \\
\text { Sith? }\end{array}$ & $\begin{array}{c}\text { Umsalzgesamt } \\
\text { in Mio.o M }\end{array}$ & $\begin{array}{c}\text { Umsatz } \mathrm{N}_{3} \text { in } \\
\mathrm{Mia}=\mathrm{OM}\end{array}$ & $\begin{array}{c}\text { N3.vo in } \\
\text { Mio }\end{array}$ & $\begin{array}{r}\text { DMie.N3.Vo } \\
\text { (-10\%Rabatt) }\end{array}$ & $\begin{array}{c}\begin{array}{c}\text { Ergobnis vor } \\
\text { Logistik. } \\
\text { kosten }\end{array} \\
5,40 \%\end{array}$ & $\begin{array}{l}\text { Logistik: } \\
\text { kosten: } \\
3.480 \mathrm{M}\end{array}$ & $\begin{array}{l}\text { Ergobnis vor } \\
\text { Logistik } \\
\text { kosten } \\
8, A 0 \% \\
\end{array}$ & $\begin{array}{l}\begin{array}{l}\text { Logistik: } \\
\text { kosten }\end{array} \\
\frac{4.790 \mathrm{M}}{}\end{array}$ & $\begin{array}{c}\text { Ergebrisvor } \\
\begin{array}{c}\text { Logistikg } \\
\text { Kosten }\end{array} \\
1240 \%\end{array}$ & $\begin{array}{l}\begin{array}{c}\text { Logstix } \\
\text { kosten }\end{array} \\
4,790 M \\
\end{array}$ \\
\hline D iatetika usw. & 5,56 & 1,13 & 0,00 & 292,37 & 15,79 & 3,48 & 24,56 & 4,79 & 36,25 & 4,79 \\
\hline Lipidsenker & $1.495,58$ & $1.085,14$ & 5,43 & 179,92 & 9,72 & 3,48 & 15,11 & 4,79 & 22,31 & 4,79 \\
\hline A ppetitzugier usw. & 0,99 & 0,27 & 0,00 & 131,20 & 7,08 & 3,48 & 11,02 & 4,79 & 16,27 & 4,79 \\
\hline A ntihyperto nika & $2.406,53$ & $1.933,79$ & 14,94 & 116,51 & 6,29 & 3,48 & 9,79 & 4,79 & 14,45 & 4,79 \\
\hline Enzyminhibito ren & 16,53 & 5,94 & 0,05 & 110,89 & 5,99 & 3,48 & 9,32 & 4,79 & 13,75 & 4,79 \\
\hline A ntiepileptika & 387,61 & 180,18 & 1,50 & 108,35 & 5,85 & 3,48 & 9,10 & 4,79 & 13,44 & 4,79 \\
\hline Osteoporosemittel & 349,59 & 194,46 & 1,69 & 103,32 & 5,58 & 3,48 & 8,68 & 4,79 & 12,81 & 4,79 \\
\hline$M$ agen $D$ arm. $M$ ittel & $2.324,78$ & 973,45 & 8,49 & 103,18 & 5,57 & 3,48 & 8,67 & 4,79 & 12,79 & 4,79 \\
\hline \begin{tabular}{|l} 
Urologika \\
\end{tabular} & 738,38 & 391,14 & 3,58 & 98,23 & 5,30 & 3,48 & 8,25 & 4,79 & 12,18 & 4,79 \\
\hline Parkinsonmittel & 440,06 & 369,06 & 3,41 & 97,35 & 5,26 & 3,48 & 8,18 & 4,79 & 12,07 & 4,79 \\
\hline Neuro pathieprap. & 331,62 & 226,93 & 2,33 & 87,59 & 4,73 & 3,48 & 7,36 & 4,79 & 10,86 & 4,79 \\
\hline A ntiarrhythmika & 349,18 & 312,82 & 3,34 & 84,27 & 4,55 & 3,48 & 7,08 & 4,79 & 10,45 & 4,79 \\
\hline A ntiphlo gis tika & 124,18 & 32,28 & 0,36 & 80,69 & 4,36 & 3,48 & 6.78 & 4,79 & 10,01 & 4,79 \\
\hline Hepatika & 96,61 & 75,56 & 0,92 & 73,63 & 3,98 & 3,48 & 6,18 & 4,79 & 9,13 & 4,79 \\
\hline A ntidia betika & $1.612,54$ & $1.566,71$ & 19,20 & 73,44 & 3,97 & 3,48 & 6,17 & 4,79 & 9,11 & 4,79 \\
\hline $\begin{array}{l}\text { Broncho lytika / } \\
\text { A ntias thmatika }\end{array}$ & $1.874,64$ & 532,21 & 7,64 & 62,66 & \multirow{20}{*}{\multicolumn{2}{|c|}{$\begin{array}{l}\text { Vorlaufiges Ergebnis < } \\
\text { Logistikkosten }\end{array}$}} & 5,26 & 4,79 & 7,77 & 4,79 \\
\hline $\begin{array}{l}\text { A ntidementiva } \\
\text { (Noootro pika) } \\
\end{array}$ & 574,62 & 433,59 & 6,24 & 62,50 & & & 5.25 & 4,79 & 7,75 & 4,79 \\
\hline $\begin{array}{l}\text { Cho lagoga und } \\
\text { Gallenwegstherap. }\end{array}$ & 71,15 & 61,00 & 0,90 & 61,33 & & & 5,15 & 4,79 & 7,60 & 4,79 \\
\hline Venentherapeutika & 276,60 & 183,50 & 2,69 & 61,30 & & & 5,15 & 4,79 & 7,60 & 4,79 \\
\hline Durchblutungs f. $M$. & 327,81 & 261,54 & 3,92 & 60,08 & & & 5,05 & 4,79 & 7,45 & 4,79 \\
\hline Dermatika & 885,98 & 151,55 & 2,33 & 58,61 & & & 4,92 & 4,79 & 7,27 & 4,79 \\
\hline $\begin{array}{l}\text { Beta-,Ca-BL, A ngio- } \\
\text { tensin.Hemmstoffe }\end{array}$ & $2.833,99$ & $2.431,35$ & 37,82 & 57,86 & & & 4,86 & 4,79 & 7,17 & 4,79 \\
\hline Sexualthormone & $1.144,62$ & 859,11 & 15,37 & 50,32 & & & \multirow{13}{*}{\multicolumn{2}{|c|}{$\begin{array}{l}\text { Voriaufiges Ergebnis < } \\
\text { Logistikkosten }\end{array}$}} & 6,24 & 4,79 \\
\hline A ntihypo to nika & 137,56 & 74,91 & 1,35 & 50,06 & & & & & 6.21 & 4,79 \\
\hline Koronarmittel & 837,09 & 706,96 & 12,95 & 49,14 & & & & & 6.09 & 4,79 \\
\hline M igranemittel & 162,30 & 8,78 & 0,17 & 45,63 & & & & & 5,66 & 4,79 \\
\hline Hamorrho idenmittel & 67,21 & 9,68 & 0,19 & 45,62 & & & & & 5,66 & 4,79 \\
\hline A cido se the ra peutika & 7,37 & 6,44 & 0,13 & 45,60 & & & & & 5,65 & 4,79 \\
\hline A ntihamo rrhagika & 53,78 & 6,99 & 0,14 & 45,30 & & & & & 5,62 & 4,79 \\
\hline Psycho pharmaka & $1.782,76$ & $1.020,11$ & 20,29 & 45,25 & & & & & 5,61 & 4,79 \\
\hline $\begin{array}{l}\text { Thro mbo zyten: } \\
\text { aggregationshemmer }\end{array}$ & 185,74 & 157,62 & 3,31 & 42,87 & & & & & 5,32 & 4.79 \\
\hline Diuretika & 628,74 & 424,89 & 9,02 & 42,38 & & & & & 5,26 & 4,79 \\
\hline $\begin{array}{l}\text { A naigetika / } \\
\text { Antirheumatika }\end{array}$ & $1.822,67$ & 626,95 & 14,85 & 38,01 & & & & & \multirow{3}{*}{\multicolumn{2}{|c|}{$\begin{array}{l}\text { Ergebnis vor } \\
\text { Logistikkosten } \\
\text { Logistikkosten }\end{array}$}} \\
\hline \begin{tabular}{|c}
$\ldots$ \\
Kariest
\end{tabular} & - &.- & $\ldots$ & $=$ & & & & & & \\
\hline $\begin{array}{l}\text { Karies } \text {-, } \\
\text { Parodonto semittel }\end{array}$ & 23,08 & 16,02 & 1,27 & 11,31 & & & & & & \\
\hline $\begin{array}{l}\text { Zielsegment } \\
\text { (A } 10 \text { N2 als N 3) }\end{array}$ & $5 y$ & $16.698,5$ & 257,49 & 64,85 & 3. & $.348,84$ & & $.403,59$ & & 679,10 \\
\hline GKV-M arkt & $35.611,3$ & 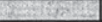 & 801,10 & & 35 & 8 & 85 & 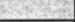 & 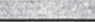 & $4055=2$ \\
\hline in $\%$ & 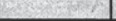 & $47 \%$ & $32 \%$ & 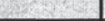 & $21 \%$ & anc & $32 \%$ & 进 & $41 \%$ & 2 \\
\hline Durchs chnittliche & Verordnungsw & vert in D M & $3 x^{2}-2 \times 2$ & 8 & & 112,62 & & 89,94 & $C_{i}$ & 77,38 \\
\hline Durchschnittliche & Verordnungsw & vert in DM (at & $\mathrm{ggI.} 10 \% \mathrm{Ka}$ & ssenrabatt) & 8 & 101,36 & & 80,94 & $\omega_{2}$ & 69,64 \\
\hline Ergebnis vor Logistik & sten (nach 2.4.) & & & & $10,4 \%$ & & $13,4 \%$ & & $17,4 \%$ & \\
\hline zus atzlicher Kas s enrat & & & & & $5,0 \%$ & & $5,0 \%$ & & $5,0 \%$ & \\
\hline Ergebnis vor Lo gis tikk & osten nach Kasser & nrabatt & & & $5,4 \%$ & & $8,4 \%$ & & $12.4 \%$ & \\
\hline Logistikkosten in \% de & vo.Wertes & & & & $3,4 \%$ & & $5.9 \%$ & & $6.9 \%$ & \\
\hline korrigierte Gewerbes te & ver auf Lo gis tikko & sten & & & $0,5 \%$ & & $0,9 \%$ & & $1.1 \%$ & \\
\hline $\begin{array}{l}\text { Einsparpotentiald } \\
\text { Marktpotentia is }\end{array}$ & er GKV in $M$ io. & DM BeIA USS & hopfungd. & & & 367 & & 570 & & 734 \\
\hline Einsparpotential G & $\mathrm{KV}$ in $\mathrm{M}$ io $\mathrm{DM}$ & bei $M$ arktant & i) von $15 \%$ & & & 267 & & 267 & & 267 \\
\hline 1999 & & & & & & & & & & \\
\hline $\begin{array}{l}\text { Eins parpotentiald } \\
\text { Marktpotentials }\end{array}$ & T GKV In MTO. & OM belAuss & Toplungd. & & & 379 & & 589 & & 758 \\
\hline Einsparpotential G & $\mathrm{KV}$ in $\mathrm{M} 10 \mathrm{DM}$ & bel $M$ arktant & II von $15 \%$ & & & 276 & & 276 & & 276 . \\
\hline
\end{tabular}

Quelle: INIFES, eigene Berechnung. 


\section{$\rightarrow$ Bei einem zusätzlichen einheitlichen Kassenrabatt von 7\%:}

Anhang I - Tabelle 13: Markt- und Einsparpotentiale für Zielsegmentvariante B (bei einem zusätzlichen Kassenrabatt von $7 \%$ )

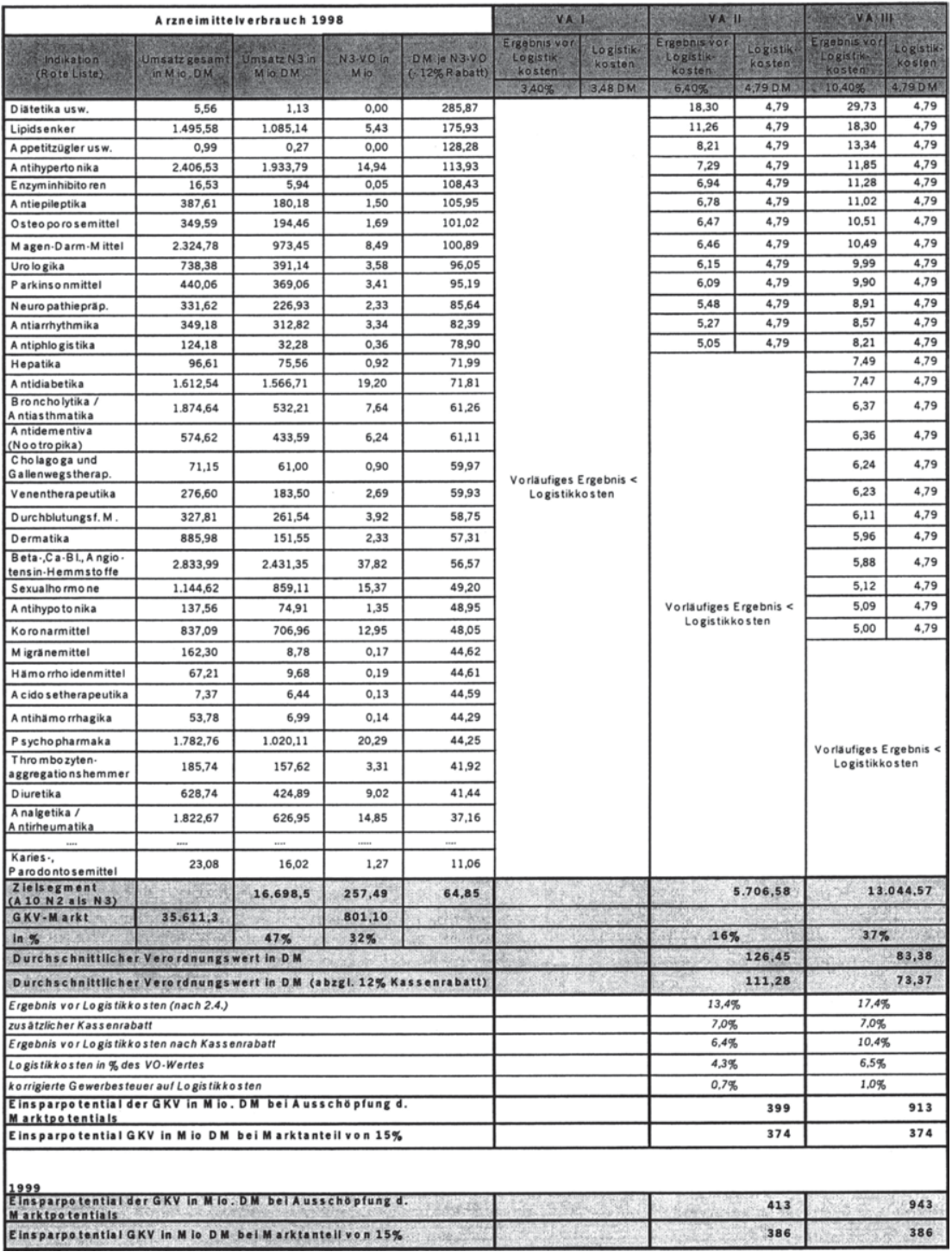

Quelle: INIFES, eigene Berechnung.

Anmerkung: Im Modell der Versandapotheke VA I könnten aus modelltheoretischer Sicht die ersten sechs Indikationen alle Kosten inklusive eines zusätzlichen Rabattes aus der Betriebsspanne decken, im Sinne einer allgemeingültigen Betrachtung ist dieses Marktpotential jedoch zu vernachlässigen. 


\section{$\rightarrow$ Bei einem zusätzlichen einheitlichen Kassenrabatt von 10\%:}

Anhang I - Tabelle 14: Markt- und Einsparpotentiale für Zielsegmentvariante B (bei einem zusätzlichen Kassenrabatt von 10\%)

\begin{tabular}{|c|c|c|c|c|c|c|c|c|c|c|}
\hline \multicolumn{5}{|c|}{ A rzne im ittelverbrauch 1998} & \multicolumn{2}{|c|}{ VAI } & \multicolumn{2}{|c|}{ VAII } & \multicolumn{2}{|c|}{ VA IIIS } \\
\hline \multirow{2}{*}{$\begin{array}{l}\text { Incikation } \\
\text { (Rote Liste) }\end{array}$} & \multirow{2}{*}{$\begin{array}{c}\text { Umsatz gesamt } \\
\text { in } \mathrm{M} \text { io.DM }\end{array}$} & \multirow{2}{*}{$\begin{array}{l}\text { Umsaz } N 3 \text { in } \\
\text { Mio DM }\end{array}$} & \multirow[t]{2}{*}{$\begin{array}{l}\text { N3.vo in } \\
\text { Mio }\end{array}$} & \multirow{2}{*}{$\begin{array}{l}\text { DM Je N3vo } \\
\text { (15\%Rabatt) }\end{array}$} & \multirow{2}{*}{\begin{tabular}{|c|}
$\begin{array}{c}\text { Ergebris vor } \\
\text { Logistik } \\
\text { kosten }\end{array}$ \\
$0,40 \%$ \\
\end{tabular}} & \multirow{2}{*}{$\begin{array}{l}\text { Logistik. } \\
\text { kosten } \\
3.480 \mathrm{M}\end{array}$} & \multirow{2}{*}{\begin{tabular}{|c|}
$\begin{array}{c}\text { Ergebris var } \\
\text { Loistik- } \\
\text { kosten }\end{array}$ \\
$3.40 \%$ \\
\end{tabular}} & \multirow{2}{*}{$\begin{array}{l}\text { Logistik } \\
\text { kosten } \\
4.79 \mathrm{DM}\end{array}$} & \multirow{2}{*}{$\begin{array}{l}\text { Ergebnis vor } \\
\text { oogistik } \\
\text { kosten } \\
7.40 \%\end{array}$} & \multirow{2}{*}{$\begin{array}{l}\text { Koristik } \\
\text { kosten } \\
4.79 \text { DM } \\
\end{array}$} \\
\hline & & & & & & & & & & \\
\hline Diatetika usw. & 5,56 & 1,13 & 0,00 & 276,12 & \multirow{35}{*}{\multicolumn{2}{|c|}{$\begin{array}{l}\text { Vorlaufiges Ergebnis < } \\
\text { Logistikkosten }\end{array}$}} & \multirow{35}{*}{\multicolumn{2}{|c|}{$\begin{array}{l}\text { Voriaufiges Ergebnis < } \\
\text { Logistikkosten }\end{array}$}} & 20,43 & 4,79 \\
\hline Lipidsenker & $1.495,58$ & $1.085,14$ & 5,43 & 169,93 & & & & & 12,57 & 4,79 \\
\hline A ppetitzugler usw. & 0,99 & 0,27 & 0,00 & 123,91 & & & & & 9,17 & 4,79 \\
\hline A ntihyperto nika & $2,406,53$ & $1.933,79$ & 14,94 & 110,04 & & & & & 8,14 & 4,79 \\
\hline Enzyminhibito ren & 16,53 & 5,94 & 0.05 & 104,73 & & & & & 7,75 & 4,79 \\
\hline A ntiepileptika & 387,61 & 180,18 & 1,50 & 102,33 & & & & & 7,57 & 4,79 \\
\hline Osteoporosemittel & 349,59 & 194,46 & 1,69 & 97,58 & & & & & 7,22 & 4,79 \\
\hline M agen-Darm-M ittel & $2.324,78$ & 973,45 & 8,49 & 97,45 & & & & & 7,21 & 4,79 \\
\hline Urologika & 738,38 & 391,14 & 3,58 & 92,78 & & & & & 6.87 & 4,79 \\
\hline Parkinsonmittel & 440,06 & 369,06 & 3,41 & 91,94 & & & & & 6,80 & 4.79 \\
\hline Neuro pathieprap. & 331,62 & 226,93 & 2,33 & 82,72 & & & & & 6,12 & 4,79 \\
\hline A ntiarmythmika & 349,18 & 312,82 & 3,34 & 79,59 & & & & & 5,89 & 4,79 \\
\hline A ntiphlo gistika & 124,18 & 32,28 & 0,36 & 76,21 & & & & & 5,64 & 4,79 \\
\hline Hepatika & 96,61 & 75,56 & 0,92 & 69,54 & & & & & 5,15 & 4,79 \\
\hline A ntidiabetika & $1.612,54$ & $1.566,71$ & 19,20 & 69,36 & & & & & 5,13 & 4,79 \\
\hline $\begin{array}{l}\text { Broncholytika / } \\
\text { A ntias thmatika }\end{array}$ & 1874,64 & 532,21 & 7,64 & 59,18 & & & & & & \\
\hline $\begin{array}{l}\text { A ntidementiva } \\
\text { (Nootro pika) }\end{array}$ & 574,62 & 433,59 & 6,24 & 59.03 & & & & & & \\
\hline $\begin{array}{l}\text { Cholagoga und } \\
\text { Gallenwegstherap. }\end{array}$ & 71,15 & 61,00 & 0,90 & 57,92 & & & & & & \\
\hline Venentherapeutika & 276,60 & 183,50 & 2,69 & 57,89 & & & & & & \\
\hline Durchblutungst. $M$. & 327,81 & 261,54 & 3,92 & 56,75 & & & & & & \\
\hline Dermatika & 885,98 & 151,55 & 2,33 & 55,36 & & & & & & \\
\hline $\begin{array}{l}\text { Beta.,Ca.BL, Angio- } \\
\text { tensin-Hemmstoffe }\end{array}$ & $2.833,99$ & $2.431,35$ & 37,82 & 54,64 & & & & & & \\
\hline Sexualhormone & $1.144,62$ & 859,11 & 15,37 & 47,52 & & & & & & \\
\hline A ntihypo to nika & 137,56 & 74,91 & 1,35 & 47,28 & & & & & & \\
\hline Koronarmittel & 837,09 & 706,96 & 12,95 & 46,41 & & & & & Vorlaufiges E & Ergebnis < \\
\hline M igranemittel & 162,30 & 8,78 & 0,17 & 43,09 & & & & & & \\
\hline Hamo mho idenmittel & 67,21 & 9,68 & 0,19 & 43,09 & & & & & & \\
\hline A cido setherapeutika & 7,37 & 6,44 & 0,13 & 43,07 & & & & & & \\
\hline A ntihamorrhagika & 53,78 & 6,99 & 0,14 & 42,78 & & & & & & \\
\hline Psycho pharmaka & $1.782,76$ & $1.020,11$ & 20,29 & 42,74 & & & & & & \\
\hline $\begin{array}{l}\text { Thro mbo zyten. } \\
\text { aggregationshemmer }\end{array}$ & 185,74 & 157,62 & 3,31 & 40,49 & & & & & & \\
\hline Diuretika & 628,74 & 424,89 & 9,02 & 40,03 & & & & & & \\
\hline $\begin{array}{l}\text { Analgetika / } \\
\text { Antirheumatika }\end{array}$ & 1822,67 & 626,95 & 14,85 & 35,89 & & & & & & \\
\hline$\ldots$ & $\ldots$ & - & $\ldots$ & $\ldots$ & & & & & & \\
\hline $\begin{array}{l}\text { Karies :, } \\
\text { Parodontosemittel }\end{array}$ & 23,08 & 16,02 & 1,27 & 10,68 & & & & & & \\
\hline $\begin{array}{l}\text { Zielsegment } \\
\text { (A } 10 \mathrm{N2} \text { als N } 3)\end{array}$ & 28: & $16.698,5$ & 257,49 & 64,85 & & & & & 7. & $.348,84$ \\
\hline GKV,M arkt & $35.611,3$ & & 801,10 & 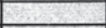 & & & & & B & 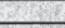 \\
\hline in $\%$ & 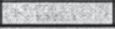 & $47 \%$ & $32 \%$ & 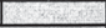 & & 62 & 203048 & +8 & $21 \%$ & 3 \\
\hline Durchschnittliche & Verordnungs & vert in DM & 80 & & & & 2 & 5 & 5 & 212,62 \\
\hline Durchschnittliche & Verordnungs & vert in DM (a & $2 \mathrm{gl} .15 \% \mathrm{~K}$ & assenrabatt) & & & & & 83 & 95,73 \\
\hline Ergebnis vor Logis tikk & osten (nach 2.4.) & & & & & & & & $17,4 \%$ & \\
\hline zus atzlicher Kass enrab & & & & & & & & & $10,0 \%$ & \\
\hline Ergebnis vor Lo gistikk & osten nach Kasse & nrabatt & & & & & & & $7,4 \%$ & \\
\hline Logistikkosten in \% de & s vo.Wertes & & & & & & & & $5,0 \%$ & \\
\hline korrigierte Gewerbeste & uer auf Lo gis tikko & sten & & & & & & & $0,8 \%$ & \\
\hline $\begin{array}{l}\text { Einsparpotentiald } \\
\text { Marktootentials }\end{array}$ & er GKV in $M$ io. & DMbeIAUSS & chopfung d & & & & & & & 735 \\
\hline Einsparpotential G & $\mathrm{KV}$ in $\mathrm{M}$ io $\mathrm{DM}$ & bei M arktant & il von $15 \%$ & & & & & & & 534 \\
\hline 1999 & & & & & & & & & & \\
\hline $\begin{array}{l}\text { Eins parpotentlal } \\
\text { Markteotentials }\end{array}$ & er GKV In MTo, & DM BeIAuss & chopfung a & & & & & & & 759 \\
\hline Einsparpotential G & $\mathrm{KV}$ in $\mathrm{M}$ io D M & bei M arktant & il von $15 \%$ & 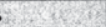 & & & & & & 552 \\
\hline
\end{tabular}

Quelle: INIFES, eigene Berechnung.

Anmerkung: Im Modell der Versandapotheke VA II könnten aus modelltheoretischer Sicht die ersten zwei Indikationen alle Kosten inklusive eines zusätzlichen Rabattes aus der Betriebsspanne decken, im Sinne einer allgemeingültigen Betrachtung ist dieses Marktpotential jedoch zu vernachlässigen. 
Für die Zielsegmentvariante $C$ (Indikator Packungsgröße ab 35 DDD) ergeben sich in Abhängigkeit der Umsatzgröße folgende Markt- und Einsparpotentiale in Abhängigkeit des zusätzlichen einheitlichen Kassenrabatts.

\section{$\rightarrow$ Bei einem zusätzlichen einheitlichen Kassenrabatt von 5\%:}

Anhang I - Tabelle 15: Markt- und Einsparpotentiale für Zielsegmentvariante $C$ (bei einem zusätzlichen Kassenrabatt von $5 \%$ )

\begin{tabular}{|c|c|c|c|c|c|c|c|c|c|}
\hline \multirow{2}{*}{\multicolumn{4}{|c|}{ Arzneimittelverbrauch 1999}} & \multicolumn{2}{|c|}{ VAI } & \multicolumn{2}{|c|}{ VAII } & \multicolumn{2}{|c|}{ VA III } \\
\hline & & & & \multirow{2}{*}{\begin{tabular}{|c|}
$\begin{array}{c}\text { Ergebnis vor } \\
\text { Logistikkosten }\end{array}$ \\
$5,4 \%$ \\
\end{tabular}} & \multirow{2}{*}{$\begin{array}{l}\text { Logistik- } \\
\text { kosten } \\
3,48 \mathrm{DM}\end{array}$} & \multirow{2}{*}{\begin{tabular}{|c|}
$\begin{array}{c}\text { Ergebnis vor } \\
\text { Logistikkosten }\end{array}$ \\
$8,40 \%$ \\
\end{tabular}} & \multirow{2}{*}{$\begin{array}{l}\begin{array}{c}\text { Logistik- } \\
\text { kosten }\end{array} \\
4,79 \mathrm{DM}\end{array}$} & \multirow{2}{*}{\begin{tabular}{|c|}
$\begin{array}{c}\text { Ergebnis vor } \\
\text { Logistikkosten }\end{array}$ \\
$12,40 \%$ \\
\end{tabular}} & \multirow{2}{*}{$\begin{array}{l}\text { Logistik- } \\
\text { kosten } \\
4,79 \mathrm{DM}\end{array}$} \\
\hline ATC & $\begin{array}{c}\text { Umsatz Mlo } \\
\text { DM }\end{array}$ & Vo in Mio & $\begin{array}{l}\text { DMIVO } \\
(-10 \%)\end{array}$ & & & & & & \\
\hline L02 & 604,50 & 1,10 & 494,59 & 26,71 & 3,48 & 41,55 & 4,79 & 61,33 & 4,79 \\
\hline M05 & 285,70 & 0,80 & 321,41 & 17,36 & 3,48 & 27,00 & 4,79 & 39,86 & 4,79 \\
\hline C10 & $1.699,50$ & 9,40 & 162,72 & 8,79 & 3,48 & 13,67 & 4,79 & 20,18 & 4,79 \\
\hline D05 & 86,40 & 0,80 & 97,20 & 5,25 & 3,48 & 8,16 & 4,79 & 12,05 & 4,79 \\
\hline$\infty 09$ & $2.490,80$ & 25,80 & 86,89 & 4,69 & 3,48 & 7,30 & 4,79 & 10,77 & 4,79 \\
\hline $\mathrm{B} 03$ & 477,70 & 5,20 & 82,68 & 4,46 & 3,48 & 6,95 & 4,79 & 10,25 & 4,79 \\
\hline C22 & 453,10 & 5,00 & 81,56 & 4,40 & 3,48 & 6,85 & 4,79 & 10,11 & 4,79 \\
\hline $\mathrm{NO3}$ & 434,50 & 4,90 & 79,81 & 4,31 & 3,48 & 6,70 & 4,79 & 9,90 & 4,79 \\
\hline N07 & 325,90 & 3,70 & 79,27 & 4,28 & 3,48 & 6,66 & 4,79 & 9,83 & 4,79 \\
\hline A10 & $1.772,80$ & 20,60 & 77,45 & 4,18 & 3,48 & 6,51 & 4,79 & 9,60 & 4,79 \\
\hline G04 & 712,40 & 9,60 & 66,79 & 3,61 & 3,48 & 5,61 & 4,79 & 8,28 & 4,79 \\
\hline R03 & $1.935,50$ & 27,60 & 63,11 & \multirow{18}{*}{\multicolumn{2}{|c|}{$\begin{array}{c}\text { Ergebnis vor Logistikkosten } \\
<\text { Logistikkosten }\end{array}$}} & 5,30 & 4,79 & 7,83 & 4,79 \\
\hline B01 & 671,30 & 9,60 & 62,93 & & & 5,29 & 4,79 & 7,80 & 4,79 \\
\hline$\infty 88$ & $1.282,00$ & 19,70 & 58,57 & & & 4,92 & 4,79 & 7,26 & 4,79 \\
\hline$\infty 7$ & $1.399,50$ & 21,70 & 58,04 & & & 4,88 & 4,79 & 7,20 & 4,79 \\
\hline N06 & $1.421,00$ & 23,20 & 55,13 & & & \multirow{14}{*}{\multicolumn{2}{|c|}{$\begin{array}{c}\text { Ergebnis vor Logistikkosten < } \\
\text { Logistikkosten }\end{array}$}} & 6,84 & 4,79 \\
\hline G03 & $1.253,40$ & 24,70 & 45,67 & & & & & 5,66 & 4,79 \\
\hline$\infty 01$ & $1.247,50$ & 30,40 & 36,93 & & & & & \multirow{12}{*}{\multicolumn{2}{|c|}{$\begin{array}{c}\text { Ergebnis vor Logistikkosten } \\
<\text { Logistikkosten }\end{array}$}} \\
\hline$\infty 3$ & 657,30 & 16,70 & 35,42 & & & & & & \\
\hline$\infty 06$ & 37,50 & 1,00 & 33,75 & & & & & & \\
\hline $\mathrm{HO2}$ & 271,30 & 7,50 & 32,56 & & & & & & \\
\hline A11 & 165,60 & 5,80 & 25,70 & & & & & & \\
\hline A06 & 108,70 & 4,30 & 22,75 & & & & & & \\
\hline D11 & 74,00 & 3,30 & 20,18 & & & & & & \\
\hline S01 & 624,50 & 28,70 & 19,58 & & & & & & \\
\hline D02 & 87,30 & 4,40 & 17,86 & & & & & & \\
\hline M04 & 112,20 & 5,70 & 17,72 & & & & & & \\
\hline $\mathrm{H} 03$ & 311,00 & 16,80 & 16,66 & & & & & & \\
\hline $\mathrm{A} 01$ & 81,70 & 5,60 & 13,13 & & & & & & \\
\hline $\begin{array}{l}\text { Zlel- } \\
\text { segment }\end{array}$ & $21.084,60$ & 343,60 & 61,36 & \multicolumn{2}{|c|}{$9.343,30$} & \multicolumn{2}{|c|}{$14.631,60$} & \multicolumn{2}{|c|}{$17.306,00$} \\
\hline $\begin{array}{l}\text { GKV- } \\
\text { Markt }\end{array}$ & $36.773,80$ & 782,60 & 46,99 & & & & & & \\
\hline in $\%$ & $57 \%$ & $44 \%$ & & $25 \%$ & & $40 \%$ & & $47 \%$ & es \\
\hline \multicolumn{4}{|c|}{ durchschnittlicher Verordnungsw ert in DM } & \multicolumn{2}{|c|}{107,52} & & 88,41 & \multicolumn{2}{|r|}{81,10} \\
\hline \multicolumn{4}{|c|}{$\begin{array}{l}\text { durchs chnittlicher Verordnungswert in DM } \\
\text { (abzgl. } 10 \% \text { Kassenrabatt) }\end{array}$} & \multicolumn{2}{|r|}{96,77} & \multicolumn{2}{|r|}{79,57} & & 72,99 \\
\hline Ergebnis ve & r Logistikkosten & (nach 2.4.) & & $10,4 \%$ & & $13,4 \%$ & & $17,4 \%$ & \\
\hline zusä́tzliche & Kassenrabatt 5 & & & $5,0 \%$ & & $5,0 \%$ & & $5,0 \%$ & \\
\hline Ergebnis ve & r Logistikkosten & nach Kassent & & $5,4 \%$ & & $8,4 \%$ & & $12,4 \%$ & \\
\hline Logistikkos & ten in \% des VO- & Wertes & & $3,6 \%$ & & $6,0 \%$ & & $6,6 \%$ & \\
\hline korrigierte & Gewerbestever a & If Logistikkos & & $0,6 \%$ & & $1,0 \%$ & & $1,1 \%$ & \\
\hline $\begin{array}{l}\text { Ensparpo: } \\
\text { Ausschöp }\end{array}$ & $\begin{array}{l}\text { ential GKV In M } \\
\text { fung des Markt }\end{array}$ & $\begin{array}{l}\text { I0 DM beivo } \\
\text { potentials }\end{array}$ & & & 467 & & 732 & & 865 \\
\hline $\begin{array}{l}\text { Ens parpo } \\
15 \%\end{array}$ & ential GKV in M & lo DM bel Ma & stantell von & & 276 & & 276 & & 276 \\
\hline
\end{tabular}

Quelle: INIFES, eigene Berechnung. 


\section{$\rightarrow$ Bei einem zusätzlichen einheitlichen Kassenrabatt von 7\%:}

Anhang I - Tabelle 16: Markt- und Einsparpotentiale für Zielsegmentvariante C (bei einem zusätzlichen Kassenrabatt von $7 \%$ )

\begin{tabular}{|c|c|c|c|c|c|c|c|c|c|}
\hline \multirow{2}{*}{\multicolumn{4}{|c|}{ Arzneimittelverbrauch 1999}} & \multicolumn{2}{|l|}{ VAI } & \multicolumn{2}{|c|}{ VAII } & \multicolumn{2}{|c|}{ VAIII } \\
\hline & & & & \multirow{2}{*}{\begin{tabular}{|c|}
$\begin{array}{c}\text { Ergebnis vor } \\
\text { Logistikkosten }\end{array}$ \\
$3,4 \%$ \\
\end{tabular}} & \multirow{2}{*}{$\begin{array}{l}\begin{array}{l}\text { Logistik- } \\
\text { kosten }\end{array} \\
3,48 \mathrm{DM}\end{array}$} & \multirow{2}{*}{\begin{tabular}{|c|}
$\begin{array}{c}\text { Ergebnis vor } \\
\text { Logistikkosten }\end{array}$ \\
$6,40 \%$ \\
\end{tabular}} & \multirow{2}{*}{$\begin{array}{l}\text { Logistik- } \\
\text { kosten }\end{array}$} & \multirow{2}{*}{$\begin{array}{c}\begin{array}{c}\text { Ergebnis vor } \\
\text { Logistikkosten }\end{array} \\
10.40 \%\end{array}$} & \multirow{2}{*}{$\begin{array}{l}\text { Logistik- } \\
\text { kosten } \\
4.79 \mathrm{DM}\end{array}$} \\
\hline ATC & $\begin{array}{l}\text { Umsatz Mio } \\
\text { DM }\end{array}$ & Vo in Mio & $\begin{array}{l}\text { DM /VO } \\
(-12 \%)\end{array}$ & & & & & & \\
\hline L02 & 604,50 & 1,10 & 483,60 & \multirow{29}{*}{\multicolumn{2}{|c|}{$\begin{array}{c}\text { Ergebnis vor Logistikkosten } \\
<\text { Logistikkosten }\end{array}$}} & 30,95 & 4,79 & 50,29 & 4,79 \\
\hline MO5 & 285,70 & 0,80 & 314,27 & & & 20,11 & 4,79 & 32,68 & 4,79 \\
\hline $\mathrm{C} 10$ & $1.699,50$ & 9,40 & 159,10 & & & 10,18 & 4,79 & 16,55 & 4,79 \\
\hline D05 & 86,40 & 0,80 & 95,04 & & & 6,08 & 4,79 & 9,88 & 4,79 \\
\hline$\infty 09$ & $2.490,80$ & 25,80 & 84,96 & & & 5,44 & 4,79 & 8,84 & 4,79 \\
\hline $\mathrm{B} 03$ & 477,70 & 5,20 & 80,84 & & & 5,17 & 4,79 & 8,41 & 4,79 \\
\hline$\infty 2$ & 453,10 & 5,00 & 79,75 & & & 5,10 & 4,79 & 8,29 & 4,79 \\
\hline $\mathrm{NO3}$ & 434,50 & 4,90 & 78,03 & & & 4,99 & 4,79 & 8,12 & 4,79 \\
\hline N07 & 325,90 & 3,70 & 77,51 & & & 4,96 & 4,79 & 8,06 & 4,79 \\
\hline $\mathrm{A} 10$ & $1.772,80$ & 20,60 & 75,73 & & & 4,85 & 4,79 & 7,88 & 4,79 \\
\hline G04 & 712,40 & 9,60 & 65,30 & & & \multirow{19}{*}{\multicolumn{2}{|c|}{$\begin{array}{c}\text { Ergebnis vor Logistikkostn } \\
<\text { Logistikkosten }\end{array}$}} & 6,79 & 4,79 \\
\hline R03 & $1.935,50$ & 27,60 & 61,71 & & & & & 6,42 & 4,79 \\
\hline B01 & 671,30 & 9,60 & 61,54 & & & & & 6,40 & 4,79 \\
\hline 008 & $1.282,00$ & 19,70 & 57,27 & & & & & 5,96 & 4,79 \\
\hline$\infty 07$ & $1.399,50$ & 21,70 & 56,75 & & & & & 5,90 & 4,79 \\
\hline N06 & $1.421,00$ & 23,20 & 53,90 & & & & & 5,61 & 4,79 \\
\hline G03 & $1.253,40$ & 24,70 & 44,66 & & & & & \multirow{13}{*}{\multicolumn{2}{|c|}{$\begin{array}{l}\text { Ergebnis vor Logistikkosten } \\
<\text { Logistikkosten }\end{array}$}} \\
\hline$\infty 1$ & $1.247,50$ & 30,40 & 36,11 & & & & & & \\
\hline $\mathrm{CO3}_{3}$ & 657,30 & 16,70 & 34,64 & & & & & & \\
\hline 006 & 37,50 & 1,00 & 33,00 & & & & & & \\
\hline $\mathrm{H} 02$ & 271,30 & 7,50 & 31,83 & & & & & & \\
\hline A11 & 165,60 & 5,80 & 25,13 & & & & & & \\
\hline $\mathrm{A} 06$ & 108,70 & 4,30 & 22,25 & & & & & & \\
\hline D11 & 74,00 & 3,30 & 19,73 & & & & & & \\
\hline S01 & 624,50 & 28,70 & 19,15 & & & & & & \\
\hline $\mathrm{D} 02$ & 87,30 & 4,40 & 17,46 & & & & & & \\
\hline MO4 & 112,20 & 5,70 & 17,32 & & & & & & \\
\hline $\mathrm{H} 03$ & 311,00 & 16,80 & 16,29 & & & & & & \\
\hline A01 & 81,70 & 5,60 & 12,84 & & & & & & \\
\hline $\begin{array}{l}\text { Ziel- } \\
\text { segment }\end{array}$ & $21.084,60$ & 343,60 & 61,36 & & & \multicolumn{2}{|c|}{$8.630,90$} & \multicolumn{2}{|c|}{$16.052,60$} \\
\hline $\begin{array}{l}\text { GKV- } \\
\text { Markt }\end{array}$ & $36.773,80$ & 782,60 & 46,99 & & & & & & \\
\hline in $\%$ & $57 \%$ & $44 \%$ & & & & $23 \%$ & & $44 \%$ & \\
\hline \multicolumn{4}{|c|}{ durchschnittlicher Verordnungswert in DM } & & & \multicolumn{2}{|c|}{111,65} & \multicolumn{2}{|r|}{85,07} \\
\hline \multicolumn{4}{|c|}{$\begin{array}{l}\text { durchschnittlicher Verordnungs wert in DM } \\
\text { (abzgl. 12\% Kassenrabatt) }\end{array}$} & & & \multicolumn{2}{|r|}{98,26} & \multicolumn{2}{|r|}{74,86} \\
\hline \multicolumn{4}{|c|}{ Ergebnis vor Logistikkosten (nach 2.4.) } & & & $13,4 \%$ & & $17,4 \%$ & \\
\hline zusätzliche & Kassenrabatt $7{ }^{\circ}$ & & & & & $7,0 \%$ & & $7,0 \%$ & \\
\hline Ergebnis vo & Logistikkosten & ach Kassenre & & & & $6,4 \%$ & & $10,4 \%$ & \\
\hline Logistikkos & en in \% des VO- & Vertes & & & & $4,9 \%$ & & $6,4 \%$ & \\
\hline korrigierte & ewerbesteuer au & Logistikkost & & & & $0,8 \%$ & & $1,0 \%$ & \\
\hline $\begin{array}{l}\text { Ensparpo } \\
\text { Ausschōp }\end{array}$ & $\begin{array}{l}\text { ntial GKV in MI } \\
\text { ung des Markte }\end{array}$ & $\begin{array}{l}\text { DM bei vol } \\
\text { otentials }\end{array}$ & & & & & 604 & & 1.124 \\
\hline $\begin{array}{l}\text { Ensparpo } \\
15 \%\end{array}$ & ntial GKV in Mi & DM bei Ma & antell von & & & & 386 & & 386. \\
\hline
\end{tabular}

Quelle: INIFES, eigene Berechnung.

Anmerkung: Im Modell der Versandapotheke VA I könnten aus modelltheoretischer Sicht die ersten drei Indikationen alle Kosten inklusive eines zusätzlichen Rabattes aus der Betriebsspanne decken, im Sinne einer allgemeingültigen Betrachtung ist dieses Marktpotential jedoch zu vernachlässigen. (siehe dazu auch Variante A). 


\section{$\rightarrow$ Bei einem zusätzlichen einheitlichen Kassenrabatt von 10\%:}

Anhang I - Tabelle 17: Markt- und Einsparpotentiale für Zielsegmentvariante C (bei einem zusätzlichen Kassenrabatt von $10 \%$ )

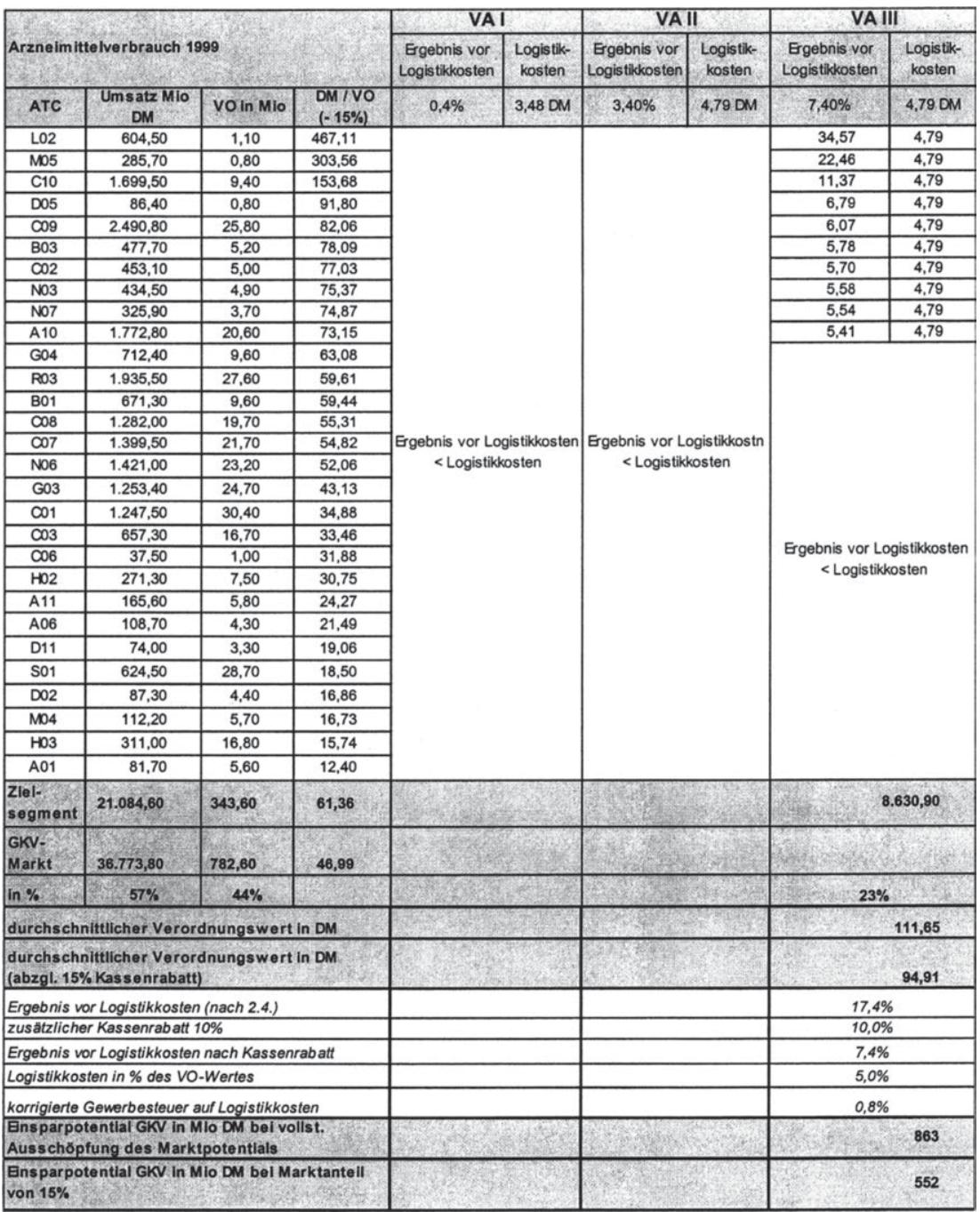

Quelle: INIFES, eigene Berechnung.

Anmerkung: Im Modell der Versandapotheke VA II könnten aus modelltheoretischer Sicht die ersten drei Indikationen alle Kosten inklusive eines zusätzlichen Rabattes aus der Betriebsspanne decken, im Sinne einer allgemeingültigen Betrachtung ist dieses Marktpotential jedoch zu vernachlässigen. (siehe dazu auch Variante A). 


\section{Fragebogen der Telefonbefragung: Ärzte}

Int.: Guten Tag, mein Name ist vom EMNID-Institut in Bielefeld. Wir führen derzeit eine repräsentative Befragung bei niedergelassenen Allgemeinmedizinern, Praktikern und Internisten (bzw. andere Facharztgruppe nennen) bezüglich der Zukunft des Arzneimittelvertriebs in Deutschland durch. Dazu würde ich Ihnen gerne ein paar Fragen stellen. Seien Sie versichert, dass alle Ihre Angaben vertraulich behandelt werden.

1) Wie setzt sich das Patientenklientel Ihrer Praxis prozentual zusammen? (Geschlossene Frage bzw. Offene Frage)

\{Int.: Antwortalternativen vorlesen, Mehrfachnennungen sind zulässig\}
a) Chronisch Kranke
... Prozent
$\rightarrow$ davon sind älter als 60 Jahre
... Prozent
b) Akut-Kranke
... Prozent
c) Patienten zur Vorsorgeuntersuchung
... Prozent
d) Weiß nicht

2) Überversorgung mit Apotheken wird heute in manchen Gebieten als Problem angesehen. Wie beurteilen Sie die Situation im Einzugsgebiet Ihrer Praxis? (Geschlossene Frage)

\{Int.: Antwortalternativen vorlesen, Mehrfachnennungen sind zulässig, zusätzliche Alternativen nicht aufnehmen \}
a) $\quad \square \quad$ Insgesamt bereits Überversorgung
b) $\square \quad$ Gerade angemessen
c) Insgesamt noch Unterversorgung
d) $\square \quad$ Weiß nicht 
3) Übernehmen Sie, gesetzliche Bestimmungen außer Acht gelassen, für Patienten bei entsprechender Bedürfnislage die Rezeptweiterleitung an die Apotheke? (Alternativfrage)
a) $\quad \square \quad \mathrm{Ja}$
b) $\quad$ Nein

4) Kunden wählen die Apotheke, in der sie Rezepte einlösen, nach bestimmten Kriterien aus. Wie wichtig sind Ihrer Erfahrung nach folgende Aspekte. Bewerten Sie jedes Kriterium auf einer Skala von 1 bis 5 , wobei 1 „sehr wichtig“ und 5 „völlig unwichtig“" ist. (Geschlossene Frage mit Skalierung und einer Antwortalternative als Offene Frage)

\{Int: Antwortalternativen vorlesen, Mehrfachnennungen sind zulässig\}

a) Schnelle und gute Erreichbarkeit

b) Umfassende Beratung

c) Kostenloser Heimlieferservice von Medikamenten

d) Kunden gehören zur Stammkundschaft

5) Welchen Distributionswegen für Medikamente auf Rezept räumen Sie zukünftig eher bessere, welchen eher schlechtere Marktchancen ein? Bitte bewerten Sie jedes Kriterium auf einer Skala von 1 bis 5 , wobel 1 „wesentlich bessere“ und 5 "wesentlich schlechtere" ist. (Geschlossene Frage mit Skalierung)

\{lnt: Antwortalternativen vorlesen, Mehrfachnennungen sind zulässig, zusätzliche Alternativen nicht aufnehmen \}

a) Apothekenketten

b) Arzneimittelversand durch den Hersteller oder den Großhandel

c) Versandapotheken - Medikamentenbestellung in direkter Kooperation mit dem Arzt

d) Internetapotheke - freie Medikamentenbestellung durch die Verbraucher 
Int.: In einigen europäischen Ländern gibt es zusätzlich zu den Präsenz-Apotheken auch solche, die Medikamente auf Rezept landesweit per Post an den Bürger versenden. Dazu möchten wir gerne Ihre Meinung erfragen. Das Modell sieht folgenden Ablauf vor: Das Rezept wird vom Arzt oder dem Patienten an die Versandapotheke geschickt. Diese stellt entsprechend der Verordnung die Medikamente zusammen und liefert sie an den Patienten - im allgemeinen per Post binnen 48 Stunden - nach Hause. Die Apotheke bietet eine telefonische Beratung rund um die Uhr an. Damit sind diese Apotheken in ihrer Arbeitsweise von den sogenannten Internetapotheken konsequent zu unterscheiden.

6) Was verbinden Sie mit dem Begriff Versandapotheken? (Offene Frage)

7) Für welche Ihrer Patienten könnte die Etablierung einer Versandapotheke eher von Vorteil, für wen eher von Nachteil sein? Bitte bewerten Sie jedes Kriterium auf einer Skala von 1 bis 5 , wobei 1 „von großem Vorteil“ und 5 „von großem Nachteil“ ist. (Geschlossene Frage mit Skalierung)

\{lnt.: Antwortalternativen vorlesen, Mehrfachnennungen sind zulässig, zusätzliche Alternativen nicht aufnehmen \}
a) Für Patienten, die in Ihrer Mobilität eingeschränkt sind
b) Für Patienten mit chronischen Krankheiten
c) Als Service für Kunden, z. B. Berufstätige
d) Für Patienten in Gebieten mit einer Unterversorgung mit Apotheken

8) Gesetzt den Fall es gäbe Versandapotheken, könnten Sie sich persönlich vorstellen mit einer solchen Apotheke zu kooperieren? (Alternativfrage)
a) $\quad \mathrm{Ja}$
b) $\quad$ Nein
c) Weiß nicht 
9) Bitte bewerten Sie folgende Aussagen auf einer Skala von 1 bis 5, wobei 1 „volle Zustimmung“ und 5 „totale Ablehnung“ ist. (Geschlossene Frage Skalierung) \{lnt.: Antwortalternativen vorlesen, Mehrfachnennungen sind zulässig, zusätzliche Alternativen nicht aufnehmen \}

a) Versandapotheken werden zu einer qualitativ besseren Arzneimittelversorgung führen.

b) Versandapotheken werden zu Kosteneinsparungen im Arzneimittelbereich führen.

c) Versandapotheken stellen generell ein Verdrăngungspotential für Präsenzapotheken dar

d) Die Einführung dieses Modells der Versandapotheke stellt eine zukunftsträchtige Reformoption dar.

10) Welche der folgenden Reformoptionen - gesetzliche Änderungen vorausgesetzt können Sie sich vorstellen? (Geschlossene Frage und einer Antwortalternative als Offene Frage)

\{lnt: Antwortaltemativen vorlesen, Mehrfachnennungen sind zulässig, zusätzliche Altemativen nicht aufnehmen \}

a) Der verordnende Arzt schickt das Rezept nach Einwilligung des Patienten per Datenfernübertragung an die Versandapotheke. Der Patient erscheint nicht mehr persönlich in der Apotheke.
$\square$ Ja
Nein

b) Das Rezept wird durch den verordnenden Arzt auf einer Smart-Card gespeichert, die der Patient danach in der Apotheke zur Einlösung vorlegt.
$\square \mathrm{Ja}$
Nein

c) Der Patient erhält das Rezept vom verordnenden Arzt und schickt dies per Brief an die Versandapotheke.
$\square \mathrm{Ja}$
Nein

d) andere Reformoptionen:

\{Int: bitte Antwortalternative aufnehmen\}

Int: Ich bedanke mich für Ihre Teilnahme an der Befragung und wünsche Ihnen noch einen angenehmen Tag. 


\section{Fragebogen der Telefonbefragung: Apotheker}

Int.: Guten Tag, mein Name ist .......vom EMNID-Institut in Bielefeld. Wir führen zur Zeit eine bundesweite repräsentative Befragung bei Apothekern bezüglich der Zukunft des Arzneimittelvertriebs in Deutschland durch, für die Ihre Apotheke zufällig ausgewählt wurde. Dazu würde ich auch Ihnen gerne ein paar Fragen stellen. Seien Sie versichert, dass alle Ihre Angaben vertraulich behandelt werden.

1) Wie viel pharmazeutisches Personal - zu Vollzeitkräften zusammengefasst - ist außer Ihnen in Ihrer Apotheke beschäftigt? (Geschlossene Frage)
a) $\quad$ bis 2
b) $\quad \square \quad 3$ bis 5
c) $\quad$ mehr als 5
d) $\square \quad$ Weiß nicht

2) Überversorgung mit Apotheken wird heute in manchen Gebieten als Problem angesehen. Wie beurteilen Sie die Situation im Einzugsgebiet Ihrer Apotheke? (Geschlossene Frage)

\{lnt.: Antwortalternativen vorlesen, zusätzliche Alternativen nicht aufnehmen\}
a) $\quad \square \quad$ Insgesamt bereits Überversorgung
b) $\quad \square \quad$ Gerade angemessen
c) $\quad$ Insgesamt noch Unterversorgung
d) $\quad$ Weiß nicht 
3) Kunden wählen die Apotheke, in der sie Rezepte einlösen, nach bestimmten Kriterien aus. Wie wichtig sind nach Ihrer Erfahrung folgende Aspekte. Bewerten Sie jedes Kriterium auf einer Skala von 1 bis 5 , wobei 1 „sehr wichtig“ und 5 „völlig unwichtig" ist. (Geschlossene Frage mit Skalierung)

\{lnt: Antwortaltemativen vorlesen, Mehrfachnennungen sind zulässig, zusätzliche Alternativen nicht aufnehmen \}
a) Schnelle und gute Erreichbarkeit
b) Umfassende Beratung
c) Kostenloser Heimlieferservice von Medikamenten
d) Kunden gehören zur Stammkundschaft

4) Welcher Prozentsatz Ihrer Kundschaft löst Rezepte nicht für sich selbst, sondern für eine andere Person als „Besorger" ein? (Geschlossene Frage)
a) $\quad \square \quad$ weniger als $20 \%$
b) $\quad$ zwischen $20 \%$ und $50 \%$
c) $\quad$ mehr als $50 \%$
d) Keine Angaben möglich

5) Im Rahmen der gesetzlichen Ausnahmeregelung ist es Ihnen erlaubt, Kunden nach Hause zu beliefern. Bei wie vielen Kunden trifft dieses pro Woche zu? (Geschlossene Frage)
a) $\square \quad$ Keine
b) $\quad$ Bis 5
c) $\quad$ Bis 10
d) $\square$ Bis 30
e) $\square \quad$ Mehr als 30
f) Weiß nicht 
6) Würden Sie eine Lockerung der gesetzlichen Vorschriften begrüßen, um bestimmte Kundengruppen gezielt durch Heimlieferungen ansprechen zu können? (Alternativfrage)
a) $\quad \square \quad \mathrm{Ja}$
b) $\quad$ Nein
c) Weiß nicht

7) Welchen Distributionswegen für Medikamente auf Rezept räumen Sie zukünftig eher bessere, welchen eher schlechtere Marktchancen ein? Bitte bewerten Sie jedes Kriterium auf einer Skala von 1 bis 5 , wobei 1 "wesentlich bessere" und 5 „wesentlich schlechtere“ ist. (Geschlossene Frage mit Skalierung) \{Int.: Antwortalternativen vorlesen, Mehrfachnennungen sind zulässig zusätzliche Alternativen nicht aufnehmen

a) Apothekenketten

b) Arzneimittelversand durch den Hersteller oder den Großhandel

c) Versandapotheken - Medikamentenbestellung in direkter Kooperation mit dem Arzt

d) Internetapotheke - freie Medikamentenbestellung durch die Verbraucher

Int: In einigen europäischen Ländern gibt es zusätzlich zu den Präsenz-Apotheken auch solche, die Medikamente auf Rezept landesweit per Post an den Bürger versenden. Dazu möchten wir gerne Ihre Meinung erfragen. Das Modell sieht folgenden Ablauf vor: Das Rezept wird vom Arzt oder dem Patienten an die Versandapotheke geschickt. Diese stellt entsprechend der Verordnung die Medikamente zusammen und liefert sie an den Patienten - im allgemeinen per Post binnen 48 Stunden - nach Hause. Die Apotheke bietet eine telefonische Beratung rund um die Uhr an. Damit sind diese Apotheken in ihrer Arbeitsweise von den sogenannten Internetapotheken konsequent zu unterscheiden. 
8) Was verbinden Sie mit dem Begriff Versandapotheke? (Offene Frage)

9) Könnten Sie sich grundsätzlich vorstellen Ihre Präsenzapotheke in eine Versandapotheke umzuwandeln bzw. beide Vertriebswege komplementär anzubieten? (Alternativfrage)
a) $\square \quad \mathrm{Ja}$
b) $\quad$ Nein
c) $\quad$ Weiß nicht

10) Für welche Zielgruppe kann die Etablierung einer Versandapotheke eher von Vorteil, für wen eher von Nachteil sein? Bitte bewerten Sie jedes folgende Kriterium auf einer Skala von 1 bis 5, wobei 1 „von großem Vorteil“ und 5 „von großem Nachteil" ist. (Geschlossene Frage mit Skalierung)

\{Int: Antwortalternativen vorlesen, Mehrfachnennungen sind zulässig, zusätzliche Alternativen nicht aufnehmen \}
a) Für Patienten, die in Ihrer Mobilität eingeschränkt sind
b) Für Patienten mit chronischen Krankheiten
c) Als Service für Kunden, z. B. Berufstätige
d) Für Patienten in Gebieten mit einer Unterversorgung mit Apotheken


11) Bitte bewerten Sie folgende Aussagen auf einer Skala von 1 bis 5 , wobei 1 „volle Zustimmung“ und 5 „totale Ablehnung“ ist. (Geschlossene Frage mit Skalierung) \{Int.: Antwortalternativen vorlesen, Mehrfachnennungen sind zulässig, zusätzliche Alternativen nicht aufnehmen $\}$
a) Versandapotheken werden zu einer qualitativ besseren Arzneimittelversorgung führen
b) Versandapotheken werden zu Kosteneinsparungen im Arzneimittelbereich führen.
c) Versandapotheken stellen generell ein Verdrängungspotential für Präsenzapotheken dar
d) Die Einführung dieses Modells der Versandapotheke stellt eine zukunftsträchtige Reformoption dar.

12) Wie, glauben Sie, würde die Einführung der Arzneimitteldistribution über Versandapotheken den Umsatz Ihrer eigene Apotheke beeinflussen? (Alternativfrage)
a) $\quad \square \quad$ positiv (Umsatzsteigerung)
b) $\quad$ gar nicht
c) $\quad \square \quad$ negativ (Umsatzeinbußen)
d) $\square \quad$ Weiß nicht

$\rightarrow$ weiter mit $\rightarrow$ 13)

$\rightarrow$ weiter mit $\rightarrow 13$ )

$\rightarrow$ weiter mit $\rightarrow$ 12.1)

$\rightarrow$ weiter mit $\rightarrow 13$ )

12.1) Sie sagen, die Einführung der Arzneimitteldistribution über Versandapotheken würde den Umsatz ihrer eigene Apotheke negativ beeinflussen. Was, glauben Sie, wäre für Ihre eigene Apotheke die Konsequenz? (Alternativfrage)
a) $\quad \square \quad$ Die Apotheke müßte schließen
b) $\quad$ Umsatzeinbußen - Personal müßte entlassen werden
c) $\quad$ Umsatzeinbußen - Personal müßte aber nicht entlassen werden
d) $\square \quad$ Weiß nicht 
13) Welche der folgenden Reformoptionen - gesetzliche Änderungen vorausgesetzt können Sie sich vorstellen? (Geschlossene Frage und einer Antwortalternative als Offene Frage)

\{lnt: Antwortalternativen vorlesen, Mehrfachnennungen sind zulässig, zusätzliche Alternativen nicht aufnehmen \}

a) Der verordnende Arzt schickt das Rezept nach Einwilligung des Patienten per Datenfernübertragung an die Versandapotheke. Der Patient erscheint nicht mehr persönlich in der Apotheke.
口 Ja
Nein

b) Der Patient erhält das Rezept vom verordnenden Arzt und schickt dies per Brief an die Versandapotheke.
口 Ja
Nein

c) andere Reformoptionen:

\{Int.: bitte Antwortalternative aufnehmen\}

Int.: Ich bedanke mich für Ihre Teilnahme an der Befragung und wünsche Ihnen noch einen angenehmen Tag. 


\section{Fragebogen der Telefonbefragung: Bevölkerung}

Int.: Guten Tag, mein Name ist vom EMNID-Institut für Markt- und Meinungsforschung. Wir führen heute eine repräsentative Umfrage zu verschiedenen Themen durch. Dazu haben wir auch Ihren Haushalt zufällig ausgewählt und möchten Sie bitten, uns einige Fragen zu beantworten, sofern Sie mindestens 14 Jahre alt sind.

1) Sind Sie gesetzlich krankenversichert bzw. mitversichert? \{Int.: Bei Unsicherheit des Befragten bitte folgende Beispiele vorlesen: AOK, BKK, IKK, Krankenversicherung der Rentner, Ersatzkrankenkasse: z. B. Barmer, DAK, TKK\}? (Alternativfrage)
a) $\quad \mathrm{Ja}$
b) $\quad$ Nein
c) $\quad$ Weiß nicht

2) Leiden Sie unter dauerhaften Gesundheitsstörungen, die vom Arzt behandelt werden? (Alternativfrage)
a) $\quad \square \quad \mathrm{Ja}$
weiter mit $\rightarrow$ 2.1)
b) $\quad$ Nein
weiter mit $\rightarrow 3$ )
c) $\square \quad$ Weiß nicht
weiter mit $\rightarrow$ 3) 
2.1) In welche der folgenden Kategorien lässt sich Ihre dauerhafte gesundheitliche Beeinträchtigung einordnen? (Geschlossene Frage)

\{Int.: Antwortalternativen vorlesen, Mehrfachnennungen sind zulässig, andere Altemativen als die angegebenen nicht aufnehmen\}

In die Bereiche
a) $\quad$ Schilddrüsen-Erkrankungen
b) Asthma, Bronchialerkrankungen
c) Allergien
d) $\square \quad$ Krebs
e) Herz-/Kreislauferkrankungen
f) $\square$ Diabetes
g) $\quad \square \quad$ Augendruck, grüner oder grauer Star
h) $\quad$ Neurologische Erkrankungen
i) $\quad$ Sonstige

2.2) Wie viele verschiedene vom Arzt verordnete Arzneimittel - nicht die Anzahl der Tablettenl - nehmen Sie aufgrund der genannten gesundheitlichen Beeinträchtigungen regelmäßig ein? (Geschlossene Frage)
a) $\quad 1$ pro Tag
b) $\quad 2-4$ pro Tag
c) $\quad 5 \quad 5$ oder mehr pro Tag
d) Ich benötige nicht regelmäßig Medikamente
e) Weiß nicht

3) Wie lösen Sie in der Regel Rezepte in der Apotheke ein? (Geschlossene Frage) \{lint.: Antwortaltemativen vorlesen, Mehrfachnennungen sind zulässig, andere Alternativen als die angegebenen nicht aufnehmen\}
a) $\quad$ Ich löse das Rezept selber ein
weiter mit $\rightarrow$ 3.1)
b) $\quad$ Ich bekomme Medikamente häufiger von der Apotheke nach Hause geliefert
weiter mit $\rightarrow$ 3.1)
c) $\quad$ Angehörige, Vertrauenspersonen oder der Arzt lösen für mich die Rezepte ein
weiter mit $\rightarrow$ 4) 
3.1) Wie oft gehen Sie durchschnittlich selbst in eine Apotheke, um ein Rezept einzulösen? (Geschlossene Frage)

\{Int.: Antwortalternativen vorlesen, Mehrfachnennungen sind zulässig, andere Alternativen als die angegebenen nicht aufnehmen\}
a) $\quad$ Mindestens einmal pro Woche
b) $\square \quad$ Ein bis zweimal im Monat
c) Alle zwei bis drei Monate
d) Nicht mehr als zwei- bis dreimal pro Jahr
e) $\square \quad$ Weiß nicht

3.2) Welche Kriterien legen Sie bei der Auswahl der Apotheke zugrunde? Wie wichtig sind Ihnen folgende Aspekte? Bitte bewerten Sie jedes einzelne Kriterium anhand einer Skala von 1 bis 5, wobei 1 „sehr wichtig“ und 5 „völlig unwichtig“ ist. (Geschlossene Frage mit Skalierungen)

\{lnt: Antwortalternativen vorlesen, Mehrfachnennungen sind zulässig, andere

Alternativen als die angegebenen nicht aufnehmen\}
a) Schnelle und gute Erreichbarkeit
b) Umfassende Beratung
c) Kostenloser Heimlieferservice von Medikamenten
d) Bin Stammkunde einer bestimmten Apotheke

3.3) Wie oft berät man Sie in der Apotheke bei der Aushändigung von Medikamenten? (Geschlossene Frage)

\{Int: Antwortkategorien vorlesen und darauf hinweisen, dass dies nur Tendenzen sind.\}
a) $\quad \square \quad$ Bei ungefähr jedem Apothekenbesuch
b) $\quad$ Bei circa jedem zweiten Apothekenbesuch
c) Bei circa jedem dritten Apothekenbesuch
d) Bei fast keinem Apothekenbesuch
e) Kann ich nicht sagen

Int.: Die nun folgenden Fragen befassen sich mit einem neuen, derzeit noch nicht existenten, Apothekenmodell. Dazu möchten wir gerne Ihre Meinung erfragen. 
4) Können Sle sich vorstellen, in einigen Fällen die vom Arzt verordneten Arzneimittel von der Apotheke auch per Post zugeschickt zu bekommen? (Alternativfrage)
a) $\square \mathrm{Ja}$
b) Nein
c) Weiß nicht

$$
\begin{aligned}
& \rightarrow \text { weiter mit } \rightarrow 5 \\
& \rightarrow \text { weiter mit } \rightarrow 8 \\
& \rightarrow \text { weiter mit } \rightarrow 8
\end{aligned}
$$

Int: In einigen europäischen Ländern gibt es bereits parallel zu den herkömmlichen Apotheken solche, die Medikamente auf Rezept landesweit nach Hause versenden. Der Ablauf ist folgender: Das Rezept wird vom Arzt oder dem Patienten an die Versandapotheke geschickt. Diese stellt die Medikamente zusammen und liefert sie - meist per Post und binnen 48 Stunden - nach Hause. Die Apotheke bietet eine telefonische Beratung rund um die Uhr an.

Int: Nur auf Nachfrage zu antworten - Diese Apotheken sind in ihrer Arbeitsweise von den sogenannten Internetapotheken konsequent zu unterscheiden. Im Gegensatz zu den Intemetapotheken benötigen Sie von Ihrem Arzt ein Rezept, um ein Medikament bestellen zu können. 
5) Stellen Sie sich nun vor, es würde eine solche Versandapotheke auch in Deutschland existieren. Stimmen Sie folgenden Aussagen zu? (Geschlossene Frage)

\{Int.: Antwortalternativen vorlesen, Mehrfachnennungen sind zulässig, andere Alternativen als die angegebenen nicht aufnehmen\}

a) „Sofern kein Risiko besteht, wäre eine Versandapotheke eine sinnvolle Alternative für mich".
․ Stimme zu
․ Stimme nicht zu

b) „Wegen der schlechten Erreichbarkeit der nächsten Apotheke wäre eine Versandapotheke eine sinnvolle Alternative für mich“.

$\checkmark$ Stimme zu $\quad \square \quad$ Stimme nicht zu

c) „Bei eingeschränkter Mobilität, wäre eine Versandapotheke eine sinnvolle Alternative für mich“.

S Stimme zu $\quad \square \quad$ Stimme nicht zu

d) „Bei dauerhaften gesundheitlichen Störungen und planbarem Medikamentenkonsum, wäre eine Versandapotheke eine sinnvolle Alternative für mich“.
$\checkmark$ Stimme zu
Stimme nicht zu

e) „Bei akuten Erkrankungen wäre eine Versandapotheke eine sinnvolle Alternative für mich".
( Stimme zu
口 Stimme nicht zu

f) „Da mir die Beratung und der soziale Kontakt in der Apotheke sehr wichtig sind, wäre eine Versandapotheke keine sinnvolle Alternative für mich“.
- Stimme zu
Stimme nicht zu 
6) Welche der folgenden Anforderungen müsste eine solche Versandapotheke Ihrer Meinung nach mindestens erfüllen. Bitte bewerten Sie anhand der Skala von 1 „sehr wichtig“ bis 5 „völlig unwichtig“. (Geschlossene Frage mit Skalierungen) \{Int: Antwortalternativen vorlesen, Mehrfachnennungen sind zulässig, andere Alternativen als die angegebenen nicht aufnehmen\}
a) Kostenlose, sichere Zustellung der Medikamente
b) Medikamentenlieferung binnen 48 Stunden
c) 24 Stunden Telefon-Hotline als Beratungsservice
d) Rezepterinnerung

7) Stimmen Sie der folgenden Aussage zu? „Eine Versandapotheke würde für mich persönlich bei der Versorgung mit Arzneimitteln auf Rezept eine Verbesserung darstellen." (Alternativfrage)
a) $\quad \mathrm{Ja}$
b) $\quad$ Nein
c) $\quad$ Weiß nicht

8) Nutzen Sie privat das Internet? (Alternativfrage)
a) $\square \quad \mathrm{Ja}$
b) Nein

9) Könnten Sie sich vorstellen, Medikamente über eine Versandapotheke zu beziehen? (Alternativfrage)
a) $\quad \mathrm{Ja}$
b) $\quad$ Nein
c) Weiß nicht

Int: Ich bedanke mich für Ihre Teilnahme an der Befragung und wünsche Ihnen noch einen angenehmen Tag. 


\section{Leitfaden \\ zu Expertengesprächen zum Thema "Versandapotheken"}

\begin{tabular}{|l|l|}
\hline Institution: & Ort: \\
\hline \multirow{3}{*}{ Vertreter: } & Datum: \\
\cline { 2 - 2 } & Zeit: \\
\cline { 2 - 2 } & Interviewer: \\
\hline
\end{tabular}

\section{Anrede}

Vielen Dank, dass sie uns für ein Expertengespräch zur Verfügung stehen.

Das Internationale Institut für Empirische Sozioökonomie (INIFES) untersucht derzeit die potentiellen Auswirkungen einer Ausweitung des Pharmaversandes in Deutschland. Die INIFES-Studie hinterfragt dabei die in der Öffentlichkeit diskutierten Argumente pro und contra Pharmaversand unvoreingenommen und überprüft diese empirisch. Sowohl qualitative, finanzielle als auch qualitative und strukturelle Aspekte werden berücksichtigt. Im Mittelpunkt der Studie stehen nationale Modelle des Arzneimittelversandes, die u.a. explizit eine Rezeptübermittlung durch den Arzt beinhalten. Die bereits bestehenden Internet-Apotheken sind somit nicht zentraler Gegenstand des Projektes.

Zur Evaluation der Akzeptanz und der generellen Einstellung niedergelassener Apotheker und Ärzte sowie der Bevölkerung gegenüber einer Versandapotheke haben wir im November und Dezember 2000 eine repräsentative CATI-Befragung durchgeführt, deren Ergebnisse Ihnen in einer ersten vorläufigen Zusammenfassung bereits vorliegen.

Zu diesen Ergebnissen und einer Ausweitung des Pharmaversandes in Deutschland bitte ich (wir) Sie, mir (uns) einige Fragen zu beantworten.

Das Expertengespräch wird leitfadengestützt geführt und zur späteren Auswertung aufgezeichnet. Im Leitfaden haben wir sechs allgemeine Themenkomplexe aufgeführt. Die Diskussion dieser Themen sollte nicht mehr als eine Stunde Ihrer Zeit beanspruchen.

Ihre Antworten bleiben, sofern gewünscht, anonym. 


\section{Teil I - allgemeine Fragen}

1. Ich möchte Sie zunächst nach Ihrer grundsätzlichen Einstellung zu Versandapotheken befragen. Ich lese Ihnen deshalb einige Aussagen bzgl. einer Versandapotheke vor. Bitte nehmen Sie zu diesen Aussagen jeweils kurz Stellung.

a. Versandapotheken werden zu einer qualitativ besseren Arzneimittelversorgung führen.

b. Versandapotheken werden zu Kosteneinsparungen im Arzneimittelbereich führen.

c. Versandapotheken stellen generell ein Verdrängungspotential für Präsenzapotheken dar.

d. Die Einführung des Modells einer Versandapotheke stellt eine zukunftsträchtige Reformoption dar.

2. Die INIFES-Befragung hat u.a. ergeben, dass $42 \%$ der Apotheken bereits jetzt regelmäßig Arzneimittel an Patienten nach Hause liefern. Die Ausnahmeregelung des $\S 17$, II Apothekenbetriebsordnung wird also bereits intensiv genutzt. Sollte vor diesem Hintergrund § 17, II Apothekenbetriebsordnung gelockert werden?

3. Die von INIFES durchgeführte Befragung zeigt, dass innerhalb der Bevölkerung durchaus Interesse darin existiert, verordnete Arzneimittel über eine Versandapotheke zu beziehen ( 39,0 Prozent der Befragten könnten sich vorstellen, Medikamente über eine Versandapotheke zu beziehen). Ist es vor diesem Hintergrund sinnvoll, eine Versandapotheke unter bestimmten Umständen zu erlauben?

4. Welche Anforderungen müßte eine Versandapotheke ggf. erfüllen, um in der Bundesrepublik Deutschland zugelassen zu werden?

5. Für welche der folgenden Zielgruppe sehen Sie ggf. besondere Vorteile oder Nachteile durch die Zulassung von Versandapotheken? Geben Sie bitte jeweils nur eine kurze Begründung.

a. Für Patienten, die in Ihrer Mobilität eingeschränkt sind.

b. Für Patienten mit chronischen Krankheiten.

c. Als Service für Kunden, z.B. Berufstätige

d. Für Patienten in Gebieten mit einer Unterversorgung mit Apotheken.

6 Welche Arbeitsmarkteffekte wären Ihrer Meinung nach durch eine Legalisierung des Arzneimittel-Versandhandels zu erwarten? Können Sie diese Arbeitsmarkteffekte quantifizieren? 


\section{Literaturverzeichnis}

ABDA - Bundesvereinigung Deutscher Apothekerverbände (2001), E-Commerce und Versandhandel mit Arzneimitteln aus Sicht der Apotheker, siehe unter www.abda.de/ABDAartikel, Zugriff am 27.04.2001.

ABDA - Bundesvereinigung Deutscher Apothekerverbände (1999), Die Apotheke: Zahlen, Daten, Fakten, Bonn.

ABDA - Bundesvereinigung Deutscher Apothekerverbände (2000), Die Apotheke: Zahlen, Daten, Fakten, Bonn.

Ahrens, H.J. (2001), Interview des Vorstandsvorsitzenden der AOK mit der Zeitschrift impulse, Rubrik Drei Fragen an ..., siehe: impulse / März 2001.

American Medical Association (1998), Report of the AMA Young Physicians section governing council, siehe unter www.ama.assn.org. Zugriff am 28.07.1998.

AGV - Arbeitsgemeinschaft der Verbraucherverbände e.V. (2000), Medikamentenkauf im Internet, Pressemitteilung (65/00).

AGV - Arbeitsgemeinschaft der Verbraucherverbände e.V. (2000), Internethandel mit Medikamenten: In Europa Qualität sichern \& Chancen für Verbraucher nutzen, S. 5, siehe unter www.agv.de, Zugriff am 24.01.2001.

ATG - Aktionsforum "Telematik im Gesundheitswesen" (2001), Management-Papier zur Sicherheitsinfrastruktur, Köln.

ATG - Aktionsforum Telematik im Gesundheitswesen (2000), Managementpapier "Elektronisches Rezept", Köln.

Atteslander, P. (1995), Methoden der empirischen Sozialforschung, Gruyter-Verlag, Berlin.

Blankenberg, K., (2000), www.zukunft.de? - Einblicke in Amerikas legale OnlineApotheken, in: Pharmazeutische Zeitung Nr. 49, Jg. 145 vom 07.12.2000, S. $25-26$.

BMfG - Bundesministerium für Gesundheit (1998), Warum ist in Deutschland der Versandhandel mit Arzneimitteln verboten - könnte man dadurch nicht viel Geld sparen?, siehe unter www.bmgesundheit.de/aktuell/frage von 31/98.

BMfG- Bundesministerium für Gesundheit (2000), Knapp eine Milliarde Überschuß und stabiles Beitragsniveau: Finanzentwicklung der gesetzlichen Krankenversicherung in 1999 überaus positiv!, siehe unter www.bmgesundheit.de, Pressemitteilung Nr. 20, Zugriff am 15.03.2000.

Boroch, W. (1994), Internationale Wettbewerbsfähigkeit der EU-Arzneimittelindustrie, Duisburger Volkswirtschaftliche Schriften Band 20, S+W Steuer- und Wirtschaftsverlag, Hamburg.

Bortz, J., Döring, N. (1995), Forschungsmethoden und Evaluation, Springer-Verlag, Berlin. 
Boström, H.C., Eichholz-Klein, S. (1998), Apotheke 2010, BBE Handelsbericht, Köln.

BPI - Bundesverband der Pharmazeutischen Industrie e.V. (1999), Broschüre Pharmadaten 1999, Frankfurt / Main.

BPI - Bundesverband der Pharmazeutischen Industrie e.V., (1999), Arzneimittel im Internet, siehe unter www.bpi.de, Zugriff im Dezember 1999.

BPI - Bundesverband der Pharmazeutischen Industrie e.V. (2000), Broschüre Pharmadaten 2000, Frankfurt / Main.

Büchel, D. (1998), Der neue IfH-Monatsbetriebsvergleich für Apotheken, in: Management in Apotheken, Sonderheft 43 des IFH Köln, Köln, S. 87- 105.

Buhle, E. L. (1999), Online Pharmacies. Now, online outlets are alternate retail sites. But if they can help physicians and plans solve medication compliance problems, they will be formidable. Potential for Profit, Potential for Real Change, in: Managed Care July 1999, aus: www.managedcaremag.com, Zugriff am: 01.12.1999.

Bundesgesetzblatt, Vierte Verordnung zur Änderung der Verordnung über die Zuzahlung bei der Abgabe von Arznei- und Verbandsmitteln in der vertragsärztlichen Versorgung, Bundesgesetzblatt 1997, Teil I, S. 2328.

Bundesverband der Betriebskrankenkassen (2000), Papier zur Arzneimittelvertragspolitik, Essen.

Bundesverband der Betriebskrankenkassen, (2001) Pressemitteilung vom 27.06.2001 GKV: Krankenkassen gegen die Abschaffung von Arznei- und Heilmittelbudgets - Ausgaben explodieren - wachsende Beitragssätze, siehe unter www.bkk.de, Zugriff am 27.06.01.

Cassel, D. (2000), Effiziente Distributionswege, in: Wille, E., Albring, M. (Hrsg.), (2000), Rationalisierungsreserven im Gesundheitswesen, Peter Lang Verlag, Frankfurt / Main.

Eckey, H.-F. (1992), Statistik: Grundlagen, Methoden, Beispiele, Gabler Verlag, Wiesbaden.

Eisinger, H. (1998), So funktioniert der Versandhandel, Ueberreuter, Wien

Fenske, D., Krämer, I. (2001), Optimierung der pharmazeutischen Logistik, in: Krankenhauspharmazie, Jg. 22, Nr. 2, S. 62 - 67.

FIND/SVP (1996), The International Market for Mail Service Pharmaceuticals. A Market Intelligence Report, New York.

Finke-Anthe, C. (1997), Die Kirschen aus Nachbars Garten, in: Pharmazeutische Industrie, Jg.59, Heft 3, S. III/68 - III/69.

Franzen, K., u.a. (1995), Apothekenbetriebslehre, 3. neu bearbeitete Auflage, Deutscher Apothekerverlag Stuttgart. 
Fuchs, M. (1994), Umfrageforschung mit Telefon und Computer - Einführung in die computergestützte telefonische Befragung, Weinheim, S. 35.

GEHE AG (2000), Geschäftsbericht GEHE AG 1999, Stuttgart.

Gerste, B., Niemeyer, M., Lauterberg, J. (2000), Wie viel chronisch Kranke gibt es?, in: Arnold, M., Litsch, M., Schwartz, F. W. (Hrsg.) (2000), Krankenhaus-Report 1999, Stuttgart, S. 67 - 92.

Glaeske, G. (2001), Gefahr für die Verbraucher oder für die Apotheken?, in: die Ersatzkasse 2/2001, S. 45.

Hackenbroch, V. (2000), Schwindel auf Rezept, S. 164, in: DER SPIEGEL 33/2000, S. 164 - 168.

Hasan-Boehme, U., u.a., Tätigkeits- und Kostenprofile der Apothekenmitarbeiter, siehe unter: www.pharmazeutische-zeitung.de/pza/1999-39, Zugriff am 21.11.00.

Hasan-Boehme, U., u.a., Tätigkeits- und Kostenprofile der Apothekenmitarbeiter, siehe unter: www.pharmazeutische-zeitung.de/pza/1999-35, Zugriff am 21.11.00.

HBV - Gewerkschaft Handel, Banken und Versicherungen, Landesbezirksleitung Bayern, Lohntarifvertrag Bayern, §1, Löhne ab 01.07.2001.

Hennersdorf, A., Kiani-Kress, R. (2001), Frust am Draht, in: Wirtschaftswoche Nr. 21, vom 17.5.2001, S. $105-115$.

Heyde, I. (2001), Auch öffentliche Apotheken leiden unter Mangel an Arbeitskräften, in: apv-news 02/01, S. 7 - 8.

Hovermann, E. (2001), Versandhandel versus Einzelapotheke, in: Die BKK. Zeitschrift der Betrieblichen Krankenversicherung, Jg. 89, Heft 4, S.174 - 181.

Hovermann, E., zitiert in: Kieselbach, K. (2001), Internet-Apotheken erhalten jetzt Unterstützung, in: DIE WELT vom 12.06.2001, S.16.

Hudetz, K. (1999), Electronic Commerce - Chancen und Risiken für den Großhandel, in: Mitteilungen des IFH, Köln, Jg. 51, Nr. 12, Dezember 1999, S. 251.

Hüsgen, U. (1998) Die öffentliche Apotheke im Gesundheitsmarkt. Darstellung der volkswirtschaftlichen Situation und der gesundheitsökonomischen Bedeutung, in: Management in Apotheken, Sonderheft 43 des IFH, Köln, S. 9 - 31.

IFW - Institut der deutschen Wirtschaft Köln (2000), Zahlen zur wirtschaftlichen Entwicklung der Bundesrepublik Deutschland, Köln.

IGES - Institut für Gesundheits- und Sozialforschung, (2000), Qualitätstest medizinischer Informationsdienste - Abschlußbericht, siehe unter www.almeda.de, Zugriff am 20.03.2001. 
Institut für Sozial- und Präventivmedizin der Universität Zürich; Schweizerische Patienten-Organisation (SPO); Schweizerische Patientendienststelle (DVSP) (1999), Zufriedenheit der Kunden der MediService Apotheke - Schlussbericht, Zürich.

Kaapke, A. (1998), Controlling in Apotheken mit Hilfe des Jahresbetriebsvergleiches des IFH, Köln, in: Management in Apotheken, Sonderheft 43 des IFH, Köln, S. $57-85$.

Kaapke, A. (1999), Der Jahresbetriebsvergleich des Großhandels im Jahr 1998, in: Mitteilungen des IFH, Köln, Jg. 51, Nr. 12,, S. 258 - 268.

Kirschner, K. (2001), Versandhandel für apothekenpflichtige Arzneimittel freigeben, in: Die Ersatzkasse 06/2001, S. 217

Korzilius, H. (1999), "Surfen" in gefährlichen Gewässern - Medikamente im Internet, in: Deutsches Ärzteblatt, Nr. 6, S. A-314.

Krähenbühl, J.-P. (2000), Medikamente direkt nach Hause liefern - eine Innovation im Gesundheitswesen, in: Die BKK. Zeitschrift der Betrieblichen Krankenversicherung, Jg. 88, Heft 5, S. 200.

Lamnek, S. (1995), Qualitative Sozialforschung. Bd.1 Methodologie, PsychologieVerl.-Union, Weinheim.

Laschet, H. (2001), Kasse machen trotz knapper Kassen - die heimlichen Apothekergewinne, S. 6, in: ÄRZTE ZEITUNG, Nr. 163, Donnerstag, 13.09.2001, S. 6 - 7.

May, O. (1994), Parallelimporte - Eine Möglichkeit zur Sicherstellung der Arzneimittelversorgung? - Eine Möglichkeit zur Wettbewerbsbelebung auf dem Arzneimittelmarkt? in: Medizin Mensch Gesellschaft, Jg. 9, S. 82 - 91.

MediaTransfer AG (2001), Fragen und Ergebnisse zum Medikamentenhandel im Netz, siehe unter www.b2b.mediatransfer.de, Zugriff am 16.05.2001.

Mehnert, A. (1998), Regulierung auf europäischen Arzneimittelmärkten. Reihe: Gesundheit und Ökonomie, Bd.7, Peter Lang AG, Bern.

Meyer, C (2000), Viagra aus Kerkrade, in: Der Spiegel, Heft 39, S. 88.

Meyer, H.J. (1995), Pharmazeutischer Großhandel in Europa im Wandel, in: Pharmazeutische Industrie, Jg. 57, Heft 6, S. 431 - 433.

Müller-Hagedorn, L. (1998), Strategische Optionen für die Apotheke, in: Management in Apotheken, Sonderheft 43 des IFH Köln, S. 38 - 47.

o. V. (1993), Mail-Order Pharmacy in Europe, in: Marketletter, July 26.

o. V. (2001), 55 Prozent der Rezepte ohne Zuzahlung, in: Apotheker-Zeitung, Jg. 17, Nr. 16/17 vom 23.04.01, S. 1.

o. V. (2001), Treiben Kassen Missbrauch mit der Rezeptgebühr?, siehe unter www.medical-tribune.de, Zugriff am 09.07.01. 
o.V. (1999), Lagerorientierte Kennziffern, in: Wirtschaftshandbuch für die Apotheke, siehe unter Leseprobe

www.govi.de/rubriken/buchart7buecher/covers/0003.htm, Zugriff am 05.12.00.

o.V. (1999), Beratung in Apotheken - Risiken und Nebenwirkungen, Apothekentest bei Präparaten der Selbstmedikation, in: Stiftung Warentest 7/99, S. 94 - 99.

o.V. (2000), Apotheker verdienen wieder besser, in: Stuttgarter Zeitung vom 11.05.2000.

o.V. (2000), Online Gesetze, Richtlinien und Rechtsgrundlagen der Internetnutzung, siehe unter www.deutsche-bank.de, Rubrik eCommerce, Zugriff am 17.03.00.

o.V. (2001), Phoenix-Chef sieht Marge durch Pharma-Industrie unter Druck, in: Financial Times Deutschland vom 29.01.2001, siehe unter www.ftd.de, Zugriff am 29.01.01.

o.V. (1998), Apotheker warnen neue Regierung: kein Versandhandel und Ketten, in: dpa vom 30.09.98.

o.V. (2001), Arzneimittelversand via Internet - Legal, illegal, völlig egal?, in: Deutsche Apotheker Zeitung vom 22.03.2001, S. 50 - 55.

o.V. (2001), Gehe plant Wachstum in Europa, in: Apothekerzeitung vom 30.04.2001, 17. Jg., Nr. 18, S. 2.

ÖBIG - Österreichisches Bundesinstitut für Gesundheitswesen (1998), Arzneimittelvertrieb in Europa, Wien.

ÖBIG - Österreichisches Bundesinstitut für Gesundheitswesen (1998), Arzneimittel, Steuerung der Märkte in neun europäischen Ländern, Wien.

Pfaff, M., Wassener, D., Sterzel, A., Zweiter Zwischenbericht zum Forschungsvorhaben "Analyse potentieller Auswirkungen einer Ausweitung des Pharmaversandes in Deutschland", Stadtbergen 2001.

PHAGRO (2000), Kennzahlen deutscher Pharmagroßhandel, siehe unter www.phagro.de/Kennzahlen, Zugriff am 05.12.00.

PHAGRO (2001), Arbeitsgruppe der SPD-Fraktion fordert Änderungen in der Arzneimitteldistribution, siehe unter www.phagro.de, Zugriff am 02.05.01.

Rahner, E. (1989), Qualität und Sicherheit von Arzneimittelimporten. Beanstandungen der Apotheker, in: Pharmazeutische Industrie, Jg. 51, Heft 12, S.1332 1333.

Reiblich, M. (2000), Die Schlacht der Statistiker im Gesundheitswesen, in: Medikament \& Meinung, Jg. 24, Nr. 8, S. 3.

Riegl \& Partner GmbH, (1995), Institut für Management im Gesundheitsdienst, Das Image der deutschen Apotheke, Augsburg. 
Rothe, T. (1980), Möglichkeiten und Grenzen einer marktorientierten Führung öffentlicher Apotheken in der Bundesrepublik Deutschland, Berlin.

Rücker, D. (1999), Politik Versandhandel Einsparungen im Promillebereich, siehe unter www.pharmazeutische-zeitung.de, Zugriff am 20.09.99.

Salz, J. (2000), Bedeutung verloren, in: Wirtschaftswoche Heft 4 / 20.01.2000, S. $58-60$.

Schäfers, C., Kaesbach, W. (2000), Internethandel mit Arzneimitteln, in: Die BKK Zeitschrift der Betrieblichen Krankenversicherung, Jg.88, Heft 5, S. 203 - 209.

Schefold, C.P. (1997), Der "graue" Gemeinsame Markt pharmazeutischer Markenartikel, Shaker-Verlag, Aachen.

Schleert, D. (1998), Strukturwandel der pharmazeutischen Industrie - Auswirkungen auf den GKV - Arzneimittelmarkt, in: Die BKK. Zeitschrift der Betrieblichen Krankenversicherung, Jg. 86, Heft 11, S. 566 - 573.

Schleert, D.(2000), zitiert in: Zander, B., Invasion der @-Pillen, in: STERN 24/2000.

Schleert, D., Kaesbach, W. (1997), Arzneimitteldistribution - value for money? Konsequenz aus der Reformdiskussion um die Arzneimittelpreisverordnung, in: Forum für Gesellschaftspolitik, Februar 1997, S.51 - 55.

Schneider, M., Hofmann, U., Biene-Dietrich, P., Späth, B., Mill, D. (1999), Die deutschen Arzneimittelpreise im europäischen Vergleich. Gutachten für den Verband Forschender Arzneimittelhersteller (VFA) und die Bundesregierung Deutscher Apothekenverbände (ABDA), BASYS, Augsburg.

Schöffski, O. (1995), Die Regulierung des deutschen Apothekenwesens. Eine ökonomische Analyse, Nomos Verlag, Baden-Baden.

Schröder, H., Selke, G. W. (2000), Arzneiverordnungen nach Arztgruppen, in: Schwabe, U., Paffrath, D. (Hrsg.), Arzneiverordnungs-Report 1999. Schwerpunkt Naturheilmittel, Berlin, Heidelberg, New York, S.784 - 795.

Schröder, H., Selke, G.W. (2000), Ergänzende statistische Übersicht, in: Schwabe, U., Paffrath, D. (2000), Arzneiverordnungs-Report 1999, Schwerpunkt Naturheilmittel, Berlin, Heidelberg, New York, S. 796 - 881.

Schubert, F.(1999) Das Elektronische Rezept: Chancen, Risiken und Gestaltungsmöglichkeiten. Diplomarbeit der Fachhochschule Heilbronn Heidelberg.

Schwabe, U., Pfaffrath, D. (1993), Arzneiverordnungs-Report 1993. Schwerpunkt: Erste Auswirkungen des Gesundheitsstrukturgesetzes, Gustav Fischer Verlag, Stuttgart, Jena.

Sendatzki, V. (2001), Elektronisches Rezept gewinnt an Kontur, in: Arbeit und Sozialpolitik 1-2/2001 S. 21 - 26. 
Sendatzki, V. (2001), Elektronisches Rezept. Startrampe für die Telematikplattform in der GKV, in: Die BKK. Zeitschrift der Betrieblichen Krankenversicherung, Jg. 89, Heft 3, S. 133.

Sterzel, A., Wassener, D. (2001), Arzneimitteldistribution in Deutschland - Versandapotheken als Reformoption?, in :Arbeit und Sozialpolitik Heft 1-2, Jg. 55,394 S. 14 - 20.

VFA - Verband forschender Arzneimittelhersteller (2000), Broschüre Statistics 2000 Die Arzneimittelindustrie in Deutschland, Frankfurt / Main.

VFA - Verband forschender Arzneimittelhersteller (1997), Einblicke 1997, Jahresbericht, Frankfurt / Main.

Voss, V. (1998), Arzneimittel / Der Versandhandel ist ein Geschäft voller Risiken und Nebenwirkungen. Mit der Gesundheit zahlen, in: Rheinischer Merkur vom 16.10.1998, S. 13.

Wähling, S., Schulenburg, J.-M. Graf v.d. (1996), Regulierung und Eingriffshäufigkeit im Arzneimittelmarkt. Analyse anhand eines Simulationsmodells, in: ifo Studien. Zeitschrift für empirische Wirtschaftsforschung, Jg. 42, S. 449 - 482.

Weitkunat, R., Crispin, A. (2000), Computergestützte Telefoninterviews, in: Zeitschrift für Gesundheitswissenschaften, Jg. 8, Heft 2, S. 106 - 113.

WIdO - Wissenschaftliches Institut der AOK (2000), An der vertragsärztlichen Versorgung teilnehmende Ärzte nach ihrem Teilnehmestatus, siehe unter www.wido.de/ambulanteVersorgung/Aerzte/Arztzahlen/Dlgesamt.html, Zugriff am 12.07.2000.

WidO - Wissenschaftliches Institut der AOK (2001), Versandapotheke - eine preiswerte Alternative, Aktuelle Modellrechnung des WidO berechnet Einsparpotential, siehe unter www.wido.de, Zugriff am 25.06.2001.

Wirtz, K. (2000), Bericht über die Ergebnisse des Betriebsvergleiches der Apotheken im Jahre 1998, in: Beiträge zur betriebswirtschaftlichen Planung und Kontrolle in Apotheken. IFH Köln, Jg. 52, Heft 52, Köln, S. 23 - 25.

Wolters, J., Simon, S.T., Sochert, R. (1998), Arzneimitteldaten - Vorteile ihrer Einbeziehung in die betriebliche Gesundheitsberichterstattung, in: Die BKK. Zeitschrift der Betrieblichen Krankenversicherung, Jg.86, Heft 11, S. 558 - 565.

Ziegler, B. (1980), Arzneimittelversorgung und Wettbewerb: eine Analyse von Marktstruktur, Marktverhalten und Marktergebnis, Vandenhoeck und Ruprecht, Göttingen.

Zuzahlungsverordnung (1997), Verordnung über die Zuzahlung bei der Abgabe von Arznei- und Verbandmitteln in der vertragsärztlichen Versorgung, Vierte Verordnung zur Änderung, Bundesgesetzblatt 1997, Teil I, S. 2328. 


\section{STAATLICHE ALLOKATIONSPOLITIK IM MARKTWIRTSCHAFTLICHEN SYSTEM}

Band 1 Horst Siebert (Hrsg.): Umweltallokation im Raum. 1982.

Band 2 Horst Siebert (Hrsg.): Global Environmental Resources. The Ozone Problem. 1982.

Band 3 Hans-Joachim Schulz: Steuerwirkungen in einem dynamischen Unternehmensmodell. Ein Beitrag zur Dynamisierung der Steuerüberwälzungsanalyse. 1981.

Band 4 Eberhard Wille (Hrsg.): Beiträge zur gesamtwirtschaftlichen Allokation. Allokationsprobleme im intermediären Bereich zwischen öffentlichem und privatem Wirtschaftssektor. 1983.

Band 5 Heinz König (Hrsg.): Ausbildung und Arbeitsmarkt. 1983.

Band 6 Horst Siebert (Hrsg.): Reaktionen auf Energiepreissteigerungen. 1982.

Band 7 Eberhard Wille (Hrsg.): Konzeptionelle Probleme öffentlicher Planung. 1983.

Band 8 Ingeborg Kiesewetter-Wrana: Exporterlösinstabilität. Kritische Analyse eines entwicklungspolitischen Problems. 1982.

Band 9 Ferdinand Dudenhöfer: Mehrheitswahl-Entscheidungen über Umweltnutzungen. Eine Untersuchung von Gleichgewichtszuständen in einem mikroökonomischen Markt- und Abstimmungsmodell. 1983.

Band 10 Horst Siebert (Hrsg.): Intertemporale Allokation. 1984.

Band 11 Helmut Meder: Die intertemporale Allokation erschöpfbarer Naturressourcen bei fehlenden Zukunftsmärkten und institutionalisierten Marktsubstituten. 1984.

Band 12 Ulrich Ring: Öffentliche Planungsziele und staatliche Budgets. Zur Erfüllung öffentlicher Aufgaben durch nicht-staatliche Entscheidungseinheiten. 1985.

Band 13 Ehrentraud Graw: Informationseffizienz von Terminkontraktmärkten für Währungen. Eine empirische Untersuchung. 1984.

Band 14 Rüdiger Pethig (Ed.): Public Goods and Public Allocation Policy. 1985.

Band 15 Eberhard Wille (Hrsg.): Öffentliche Planung auf Landesebene. Eine Analyse von Planungskonzepten in Deutschland, Österreich und der Schweiz. 1986.

Band 16 Helga Gebauer: Regionale Umweltnutzungen in der Zeit. Eine intertemporale Zwei-Regionen-Analyse. 1985.

Band 17 Christine Pfitzer: Integrierte Entwicklungsplanung als Allokationsinstrument auf Landesebene. Eine Analyse der öffentlichen Planung der Länder Hessen, Bayern und Niedersachsen. 1985.

Band 18 Heinz König (Hrsg.): Kontrolltheoretische Ansätze in makroökonometrischen Modellen. 1985.

Band 19 Theo Kempf: Theorie und Empirie betrieblicher Ausbildungsplatzangebote. 1985.

Band 20 Eberhard Wille (Hrsg.): Konkrete Probleme öffentlicher Planung. Grundlegende Aspekte der Zielbildung, Effizienz und Kontrolle. 1986.

Band 21 Eberhard Wille (Hrsg.): Informations- und Planungsprobleme in öffentlichen Aufgabenbereichen. Aspekte der Zielbildung und Outputmessung unter besonderer Berücksichtigung des Gesundheitswesens. 1986.

Band 22 Bernd Gutting: Der Einfluß der Besteuerung auf die Entwicklung der Wohnungs- und Baulandmärkte. Eine intertemporale Analyse der bundesdeutschen Steuergesetze. 1986.

Band 23 Heiner Kuhl: Umweltressourcen als Gegenstand internationaler Verhandlungen. Eine theoretische Transaktionskostenanalyse. 1987. 
Band 24 Hubert Hornbach: Besteuerung, Inflation und Kapitalallokation. Intersektorale und internationale Aspekte. 1987.

Band 25 Peter Müller: Intertemporale Wirkungen der Staatsverschuldung. 1987.

Band 26 Stefan Kronenberger: Die Investitionen im Rahmen der Staatsausgaben. 1988.

Band 27 Armin-Detlef Rieß: Optimale Auslandsverschuldung bei potentiellen Schuldendienstproblemen. 1988.

Band 28 Volker Ulrich: Preis- und Mengeneffekte im Gesundheitswesen. Eine Ausgabenanalyse von GKV-Behandlungsarten. 1988.

Band 29 Hans-Michael Geiger: Informational Efficiency in Speculative Markets. A Theoretical Investigation. Edited by Ehrentraud Graw. 1989.

Band 30 Karl Sputek: Zielgerichtete Ressourcenallokation. Ein Modellentwurf zur Effektivitätsanalyse praktischer Budgetplanung am Beispiel von Berlin (West). 1989.

\section{ALLOKATION IM MARKTWIRTSCHAFTLICHEN SYSTEM}

Band 31 Wolfgang Krader: Neuere Entwicklungen linearer latenter Kovarianzstrukturmodelle mit quantitativen und qualitativen Indikatorvariablen. Theorie und Anwendung auf ein mikroempirisches Modell des Preis-, Produktions- und Lageranpassungsverhaltens von deutschen und französischen Unternehmen des verarbeitenden Gewerbes. 1991.

Band 32 Manfred Erbsland: Die öffentlichen Personalausgaben. Eine empirische Analyse für die Bundesrepublik Deutschland. 1991.

Band 33 Walter Ried: Information und Nutzen der medizinischen Diagnostik. 1992.

Band 34 Anselm U. Römer: Was ist den Bürgern die Verminderung eines Risikos wert? Eine Anwendung des kontingenten Bewertungsansatzes auf das Giftmüllrisiko. 1993.

Band 35 Eberhard Wille, Angelika Mehnert, Jan Philipp Rohweder: Zum gesellschaftlichen Nutzen pharmazeutischer Innovationen. 1994.

Band 36 Peter Schmidt: Die Wahl des Rentenalters. Theoretische und empirische Analyse des Rentenzugangsverhaltens in West- und Ostdeutschland. 1995.

Band 37 Michael Ohmer: Die Grundlagen der Einkommensteuer. Gerechtigkeit und Effizienz. 1997.

Band 38 Evamaria Wagner: Risikomanagement rohstoffexportierender Entwicklungsländer. 1997.

Band 39 Matthias Meier: Das Sparverhalten der privaten Haushalte und der demographische Wandel: Makroökonomische Auswirkungen. Eine Simulation verschiedener Reformen der Rentenversicherung. 1997.

Band 40 Manfred Albring / Eberhard Wille (Hrsg.): Innovationen in der Arzneimitteltherapie. Definition, medizinische Umsetzung und Finanzierung. Bad Orber Gespräche über kontroverse Themen im Gesundheitswesen 25.-27.10.1996. 1997.

Band 41 Eberhard Wille / Manfred Albring (Hrsg.): Reformoptionen im Gesundheitswesen. Bad Orber Gespräche über kontroverse Themen im Gesundheitswesen 7.-8.11.1997. 1998.

Band 42 Manfred Albring / Eberhard Wille (Hrsg.): Szenarien im Gesundheitswesen. Bad Orber Gespräche über kontroverse Themen im Gesundheitswesen 5.-7.11.1998. 1999.

Band 43 Eberhard Wille / Manfred Albring (Hrsg.): Rationalisierungsreserven im deutschen Gesundheitswesen. 2000.

Band 44 Manfred Albring / Eberhard Wille (Hrsg.): Qualitätsorientierte Vergütungssysteme in der ambulanten und stationären Behandlung. 2001. 
Band 45 Martin Pfaff / Dietmar Wassener / Astrid Sterzel / Thomas Neldner: Analyse potentieller Auswirkungen einer Ausweitung des Pharmaversandes in Deutschland. 2002. 


\section{Eberhard Wille / Manfred Albring (Hrsg.) \\ Rationalisierungsreserven im deutschen Gesundheitswesen}

Frankfurt/M., Berlin, Bern, Bruxelles, New York, Oxford, Wien, 2000. 392 S., zahlr. Abb. u. Tab.

Allokation im Marktwirtschaftlichen System. Herausgegeben von Heinz König, Hans-Heinrich Nachtkamp, Ulrich Schlieper und Eberhard Wille. Bd. 43 ISBN 3-631-36757-0 · br. € 37.80*

Der Sammelband enthält die erweiterten Referate eines interdisziplinären Workshops über Rationalisierungsreserven im deutschen Gesundheitswesen, insbesondere in dem Bereich der gesetzlichen Krankenversicherung (GKV). Das Themenspektrum umfasst Effizienzpotentiale im stationären Bereich, Praxisnetze und integrierte Versorgungsformen als innovative Suchprozesse, hausärztliche Versorgung als Ansatz zur Kostensenkung im Gesundheitswesen und die zukünftige Arznei- und Hilfsmittelversorgung. Der Teilnehmerkreis setzte sich aus Vertretern der Ärzteschaft, Krankenkassen und versicherungen, der pharmazeutischen Industrie, der Wissenschaft, der ministerialen Bürokratie und der Politik zusammen.

Frankfurt/M - Berlin · Bern - Bruxelles · New York · Oxford · Wien

Auslieferung: Verlag Peter Lang AG

Jupiterstr. 15, CH-3000 Bern 15

Telefax (004131) 9402131

*inklusive der in Deutschland gültigen Mehrwertsteuer

Preisänderungen vorbehalten

Homepage http://www.peterlang.de 